\title{
Estatística gradiente e refinamento de métodos assintóticos no modelo de regressão Birnbaum-Saunders
}

\author{
Artur José Lemonte
}

TESE APRESENTADA

AO

Instituto De Matemática e Estatística

DA

Universidade de SÃo Paulo

PARA

OBTENÇÃO DO TÍTULO

$\mathrm{DE}$

DOUTOR EM CIÊNCIAS

\author{
Área de Concentração: Estatística \\ Orientador: Profa. Dra. Silvia Lopes de Paula Ferrari
}

Durante o desenvolvimento deste trabalho o autor recebeu auxílio da FAPESP

São Paulo, fevereiro de 2010 


\section{Estatística gradiente \\ e refinamento de métodos \\ assintóticos no modelo de \\ regressão Birnbaum-Saunders}

Este exemplar corresponde à redação

final da tese devidamente corrigida

e defendida por Artur José Lemonte

e aprovada pela Comissão Julgadora.

Banca Examinadora:

- Profa. Dra. Silvia Lopes de Paula Ferrari (orientadora) - IME-USP.

- Prof. Dr. Gilberto Alvarenga Paula - IME-USP.

- Prof. Dr. Francisco Cribari Neto - UFPE.

- Prof. Dr. Gauss Moutinho Cordeiro - UFRPE.

- Prof. Dr. Klaus Leite Pinto Vasconcellos - UFPE. 


\section{Agradecimentos}

Agradeço a minha família: Janaina e Graziela, minhas irmãs queridas, a meu pai, Jayme Lemonte, meus sinceros agradecimentos e, aos meus cunhados, Pedro e Jobson, pela amizade de cunho familiar. Finalmente, a minha mãe, Maria Burini Lemonte (in memoriam), que mesmo não estando presente desde os meus 7 anos, nunca esqueci dos poucos momentos que passamos juntos.

À Professora Silvia L.P. Ferrari, pela orientação.

A Themis Abensur, pelo amor que me tem dedicado.

Ao meu amigo Prof. Dr. Valdério A. Reisen, pela confiança e total apoio.

Aos amigos: Alexandre Patriota, Germán Moreno, Rafael Farias e Michel Helcias, pela convivência e pelos muitos momentos de descontração durante o doutorado.

Ao casal Alessandro e Tatiane, por terem me recebido no meu primeiro ano aqui em SP.

À trindade: $\mathrm{LT}_{\mathrm{E} X} \mathrm{X}, \mathrm{Ox}$ e R.

À FAPESP, pelo apoio financeiro. 


\section{Resumo}

Rieck \& Nedelman (1991) propuseram um modelo de regressão log-linear tendo como base a distribuição Birnbaum-Saunders (Birnbaum \& Saunders, 1969a). O modelo proposto pelos autores vem sendo bastante explorado e tem se mostrado uma ótima alternativa a outros modelos propostos na literatura, como por exemplo, os modelos de regressão Weibull, gama e lognormal. No entanto, até o presente momento, não existe nenhum estudo tratando de refinamentos para as estatísticas da razão de verossimilhanças e escore nesta classe de modelos de regressão. Assim, um dos objetivos desta tese é obter um fator de correção de Bartlett para a estatística da razão de verossimilhanças e um fator de correção tipo-Bartlett para a estatística escore nesse modelo. Estes ajustes melhoram a aproximação da distribuição nula destas estatísticas pela distribuição qui-quadrado de referência. Adicionalmente, objetiva-se obter ajustes para a estatística da razão de verossimilhanças sinalizada. Tais ajustes melhoram a aproximação desta estatística pela distribuição normal padrão.

Recentemente, uma nova estatística de teste foi proposta por Terrell (2002), a qual o autor denomina estatística gradiente. Esta estatística foi derivada a partir da estatística escore e da estatística de Wald modificada (Hayakawa \& Puri, 1985). A combinação daquelas duas estatísticas resulta em uma estatística muito simples de ser calculada, não envolvendo, por exemplo, nenhum cálculo matricial como produto e inversa de matrizes. Esta estatística foi recentemente citada por Rao (2005): "The suggestion by Terrell is attractive as it is simple to compute. It would be of interest to investigate the performance of the [gradient] statistic." Caminhando na direção da sugestão de Rao, outro objetivo da tese é obter uma expansão assintótica para a distribuição da estatística gradiente sob uma sequência de alternativas de Pitman convergindo para a hipótese nula a uma taxa de convergência de $n^{-1 / 2}$ utilizando a metodologia desenvolvida por Peers (1971) e Hayakawa (1975). Em particular, mostramos que, até ordem $n^{-1 / 2}$, a estatística gradiente segue distribuição qui-quadrado central sob a hipótese nula e distribuição qui-quadrado não central sob a hipótese alternativa. Também temos como objetivo comparar o poder local deste teste com o poder local dos testes da razão de verossimilhanças, de Wald e escore. Finalmente, aplicaremos a expansão assintótica derivada na tese em algumas classes particulares de modelos.

Palavras-chave: Distribuição Birnbaum-Saunders, Correção de Bartlett, Correção tipo-Bartlett, Estatística gradiente, Expansões assintóticas, Modelos de regressão, Poder local, Teste da razão de verossimilhanças. 


\section{Abstract}

The Birnbaum-Saunders regression model is commonly used in reliability studies. We address the issue of performing inference in this class of models when the number of observations is small. Our simulation results suggest that the likelihood ratio and score tests tend to be liberal when the sample size is small. We derive Bartlett and Bartlett-type correction factors which reduce the size distortion of the tests. Additionally, we also consider modified signed log-likelihood ratio statistics in this class of models. Finally, the asymptotic expansion of the distribution of the gradient test statistic is derived for a composite hypothesis under a sequence of Pitman alternative hypotheses converging to the null hypothesis at rate $n^{-1 / 2}, n$ being the sample size. Comparisons of the local powers of the gradient, likelihood ratio, Wald and score tests reveal no uniform superiority property.

Keywords: Asymptotic expansions, Bartlett correction, Bartlett-type correction, Birnbaum-Saunders distribution, chi-square distribution, fatigue life distribution, lifetime data, likelihood ratio test, Pitman alternative, reliability data, score test, Wald test. 


\section{Sumário}

Lista de Figuras $\quad$ xi

Lista de Tabelas $\quad$ xiii

1 Introdução 1

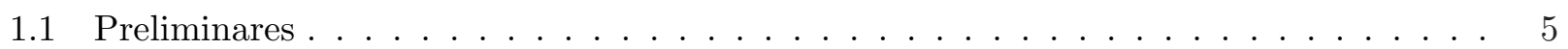

1.1.1 Estimadores de Máxima Verossimilhança . . . . . . . . . . . . . . . . . 7

1.1.2 Identidades de Bartlett . . . . . . . . . . . . . . . . . . 8

1.2 Densidades Birnbaum-Saunders e senh-normal . . . . . . . . . . . . . . . 9

1.3 Modelo de Regressão Birnbaum-Saunders . . . . . . . . . . . . . . . . . . . . 13

1.3.1 Estimação dos Parâmetros . . . . . . . . . . . . . . . . . . . . . . . . 14

1.3.2 Distribuição Assintótica . . . . . . . . . . . . . . . . . . . . . . 15

2 Correção de Bartlett $\quad 17$

2.1 Fator de Correção de Bartlett . . . . . . . . . . . . . . . . . . . . . . . . . . . . 19

2.2 Correção de Bartlett no Modelo Birnbaum-Saunders . . . . . . . . . . . . . . . . . 21

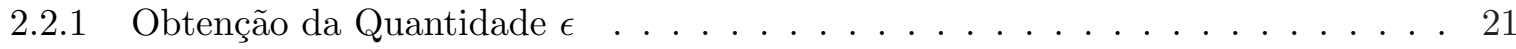

2.2 .2 Alguns Casos Especiais . . . . . . . . . . . . . . . . . . . . 22

2.3 Resultados Numéricos . . . . . . . . . . . . . . . . . . . . . . . . 24

2.4 Uma Aplicação . . . . . . . . . . . . . . . . . . . . . . . . . . . . . . . . . 29

3 Correção tipo-Bartlett $\quad 33$

3.1 Estatística Escore Aperfeiçoada . . . . . . . . . . . . . . . . . 35

3.1 .1 Expansão Assintótica de Harris . . . . . . . . . . . . . . . . . . . . 35 
3.1.2 Melhoramento de Cordeiro-Ferrari . . . . . . . . . . . . . . . . . 37

3.2 Correção Tipo-Bartlett no Modelo Birnbaum-Saunders ～. . . . . . . . . . . . . 38

3.2 .1 Hipótese Sobre um Subconjunto de $\boldsymbol{\beta} \ldots \ldots$. . . . . . . . . . . 39

3.2 .2 Hipótese Sobre $\alpha \ldots \ldots \ldots \ldots$. . . . . . . . . . . . . . . . 42

$3.2 .3 \quad$ Hipótese Sobre $\boldsymbol{\beta} \ldots \ldots \ldots \ldots \ldots$

3.2 .4 Aproximação para $a_{0}(\alpha) \ldots \ldots \ldots \ldots \ldots \ldots \ldots \ldots$

3.3 Resultados Numéricos . . . . . . . . . . . . . . . . . . . . . . . . 44

4 Razão de Verossimilhanças Sinalizada

4.1 Notação e Derivadas com Relação ao Espaço Amostral . . . . . . . . . . . . . . . . . 54

4.2 Fórmula $p^{*}$ de Barndorff-Nielsen $\ldots \ldots \ldots \ldots \ldots$

4.3 Razão de Verossimilhanças Sinalizada $-R \ldots \ldots \ldots \ldots$. . . . . . . 57

4.4 Transformação Normalizadora . . . . . . . . . . . . . . . . . . . . 58

4.5 Razão de Verossimilhanças Sinalizada Modificada $-R^{*} \ldots \ldots$. . . . . . . . . . 59

4.5 .1 Caso Uniparamétrico . . . . . . . . . . . . . . . . . . . . 59

4.5 .2 Caso Multiparamétrico . . . . . . . . . . . . . . . . . . . 60

4.5.3 Distribuição Assintótica de $R^{*} \ldots \ldots \ldots \ldots \ldots$. . . . . . . 63

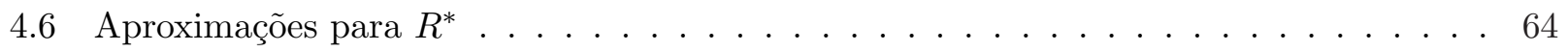

4.6.1 Aproximação de DiCiccio-Martin . . . . . . . . . . . . . . . . . . . . 65

4.6 .2 Aproximações de Skovgaard e Severini . . . . . . . . . . . . . . . . 68

4.6.3 Aproximação de Fraser-Reid-Wu . . . . . . . . . . . . . . . . . . . . . . 74

4.7 Ajustes no Modelo de Regressão Birnbaum-Saunders . . . . . . . . . . . . . . 78

4.7 .1 Inferência sobre $\alpha \ldots \ldots \ldots \ldots \ldots \ldots \ldots \ldots$

$4.7 .2 \quad$ Hipótese sobre $\beta_{r}, r=1,2, \ldots, p \ldots \ldots \ldots \ldots \ldots$

4.8 Resultados Numéricos . . . . . . . . . . . . . . . . . . . . . . . . . 88

5 Estatística Gradiente $\quad 97$

5.1 Estatística Gradiente $-S_{T} \ldots \ldots \ldots \ldots \ldots \ldots \ldots$

5.2 Expansão Assintótica de $S_{T}$ sob uma Hipótese Alternativa Local . . . . . . . . . . 101 


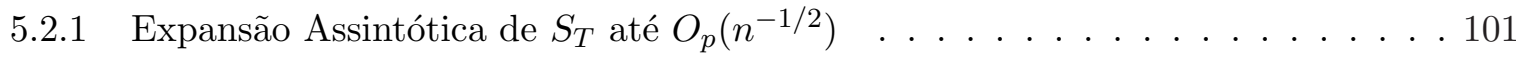

5.2 .2 Função Geradora de Momentos de $S_{T} \ldots \ldots \ldots$. . . . . . . 106

5.2.3 Características de $S_{T}$ em uma Vizinhança de $\mathcal{H}_{0} \ldots \ldots \ldots \ldots$

5.2.4 Comparação de Poder entre os Testes Rivais . . . . . . . . . . . . . . . . . 111

5.3 Expansões Assintóticas na Família Exponencial Uniparamétrica . . . . . . . . . . . . 112

5.4 Expansões Assintóticas no Modelo Birnbaum-Saunders ～. . . . . . . . . . . . . 117

5.5 Expansões Assintóticas em Modelos Lineares Generalizados . . . . . . . . . . . . . . 120

$5.5 .1 \phi$ Conhecido . . . . . . . . . . . . . . . . . . . . 121

$5.5 .2 \phi$ Desconhecido . . . . . . . . . . . . . . . . . . . . . 124

5.5.3 Testes de Hipóteses sobre o Parâmetro de Dispersão . . . . . . . . . . . . . . 125

6 Conclusões e Pesquisas Futuras 129

$\begin{array}{ll}\text { A Derivadas e Cumulantes } & 131\end{array}$

A.1 Função de Log-verossimilhança . . . . . . . . . . . . . . . . . . . . . . . . 131

A.2 Cálculo dos Cumulantes . . . . . . . . . . . . . . . . . . . . . . 132

A.3 Derivadas dos Cumulantes . . . . . . . . . . . . . . . . . . . 133

A.4 Cumulantes Adicionais . . . . . . . . . . . . . . . . . . . . 134

B Fator de Correção de Bartlett 137

C Fator de Correção tipo-Bartlett 143

C.1 Obtenção de $A_{1}, A_{2}$ e $A_{3}$ para testar $\mathcal{H}_{0}: \boldsymbol{\beta}_{1}=\boldsymbol{\beta}_{1}^{(0)} \ldots \ldots \ldots \ldots$

C.2 Obtenção de $A_{1}, A_{2}$ e $A_{3}$ para testar $\mathcal{H}_{0}: \alpha=\alpha^{(0)} \ldots \ldots \ldots \ldots$

C.3 Obtenção de $A_{1}, A_{2}$ e $A_{3}$ para testar $\mathcal{H}_{0}: \boldsymbol{\beta}=\boldsymbol{\beta}^{(0)} \ldots \ldots \ldots \ldots$

D Modelo Birnbaum-Saunders Reparametrizado 151

E Estatística Gradiente $-S_{T} \quad 155$

$\begin{array}{ll}\text { Referências Bibliográficas } & 163\end{array}$ 


\section{Lista de Figuras}

1.1 Densidade Birnbaum-Saunders para diferentes valores de $\alpha(\eta=1.0) \ldots \ldots \ldots$

1.2 Funções de risco da distribuição Birnbaum-Saunders considerando diferentes valores para $\alpha$ fixando $\eta=1.0 . \ldots \ldots \ldots \ldots \ldots 11$

1.3 Densidade senh-normal para diferentes valores de $\alpha(\mu=0$ e $\sigma=2) \ldots \ldots$

1.4 Gráfico de $1 / h(\alpha)$ para diferentes valores de $\alpha \ldots \ldots \ldots \ldots \ldots$

2.1 Quantis assintóticos versus quantis exatos das estatísticas $L R, L R_{b}$ e $L R_{b}^{1} \ldots \ldots$. . . 27

2.2 Discrepância relativa: $n=30, p=6$ e $\alpha=0.5 \ldots \ldots \ldots \ldots$

3.1 Quantis assintóticos versus quantis exatos das estatísticas $S R, S R_{c}, S R_{c}^{1}, S R_{c}^{2}$. . . 50 


\section{Lista de Tabelas}

2.1 Taxas de rejeição nulas dos testes $L R, L R_{b}$ e $L R_{b}^{1} \operatorname{com} \alpha=0.5$ e $n=30 \ldots \ldots$. . . . 25

2.2 Taxas de rejeição nulas dos testes $L R, L R_{b}$ e $L R_{b}^{1} \operatorname{com} \alpha=0.5, p=6$ e diferentes tamanhos amostrais. . . . . . . . . . . . . . . . . 25

2.3 Médias e variâncias (Monte Carlo) das estatísticas $L R, L R_{b}$ e $L R_{b}^{1}$, considerando $p=6$, $\alpha=0.5$ e diferentes tamanhos amostrais $\left(\mathcal{H}_{0}: \beta_{5}=\beta_{6}=0\right) \ldots \ldots \ldots \ldots$

2.4 Quantis $\chi_{2}^{2}$ e das distribuições nulas de $L R, L R_{b}$ e $L R_{b}^{1}$, considerando $p=6, \alpha=0.5$

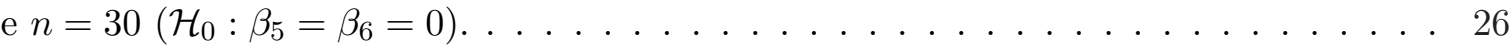

2.5 Taxas de rejeição não-nulas (poder) dos testes $L R_{b}$ e $L R_{b}^{1} \operatorname{com} \alpha=0.5, p=4$ e diferentes tamanhos amostrais. . . . . . . . . . . . . . . . . . 28

2.6 Taxas de rejeição nulas dos testes $L R, L R_{b}$ e $L R_{b}^{1}$; inferência sobre $\alpha \operatorname{com} n=30$ e $p$

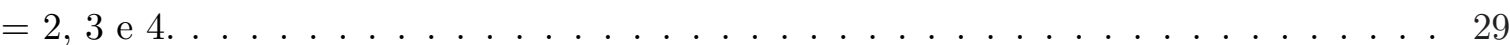

2.7 Rejeição nula de $\mathcal{H}_{0}: \beta_{3}=\beta_{4}=0 ; p=4, n=25$ e diferentes valores para $\alpha . \ldots 30$

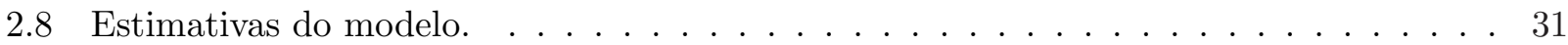

3.1 Taxas de rejeição nulas dos testes $S R, S R_{c}, S R_{c}^{1}, S R_{c}^{2}$ e $S R^{*} \operatorname{com} \alpha=0.5$ e $n=30 . \quad$. 47

3.2 Taxas de rejeição nulas dos testes $S R, S R_{c}, S R_{c}^{1}, S R_{c}^{2}$ e $S R^{*} \operatorname{com} \alpha=1.0$ e $n=30 . \quad$. 48

3.3 Taxas de rejeição nulas dos testes $S R, S R_{c}, S R_{c}^{1}, S R_{c}^{2}$ e $S R^{*} \operatorname{com} \alpha=0.5$ e diferentes tamanhos amostrais. . . . . . . . . . . . . . . . . . 49

3.4 Médias e variâncias (Monte Carlo) das estatísticas $S R, S R_{c}, S R_{c}^{1}$ e $S R_{c}^{2}$ considerando $p=7, \alpha=0.5$ e diferentes tamanhos amostrais $\left(\mathcal{H}_{0}: \beta_{6}=\beta_{7}=0\right) \ldots \ldots \ldots$

3.5 Quantis $\chi_{2}^{2}, S R, S R_{c}, S R_{c}^{1}$ e $S R_{c}^{2}$, considerando $p=6, \alpha=0.5$ e $n=30\left(\mathcal{H}_{0}: \beta_{5}=\right.$

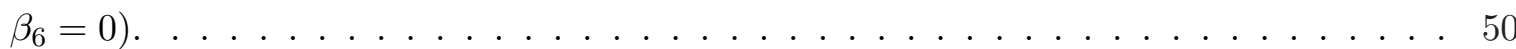

3.6 Taxas de rejeição não-nulas (poder) dos testes $S R_{c}, S R_{c}^{1}, S R_{c}^{2}$ e $S R^{*} \operatorname{com} \alpha=0.5$, $p=6$ e diferentes tamanhos amostrais. . . . . . . . . . . . . . . . 51 
3.7 Taxas de rejeição nulas dos testes $S R, S R_{c}, S R_{c}^{1}, S R_{c}^{2}$ e $S R^{*}$, considerando $p=5$ e diferentes tamanhos amostrais. . . . . . . . . . . . . . . . . 52

4.1 Tamanhos empíricos dos testes $R, R_{o}^{*}, \widehat{R}^{*}$ e $\widetilde{R}^{*} \operatorname{com} \alpha=0.5$ e $n=20 . \quad \ldots \ldots . . .92$

4.2 Tamanhos empíricos dos testes $R, R_{o}^{*}, \widehat{R}^{*}$ e $\widetilde{R}^{*} \operatorname{com} \alpha=1.5$ e $n=20$. . . . . . . 93

4.3 Tamanhos empíricos dos testes $R, R_{o}^{*}, \widehat{R}^{*}$ e $\widetilde{R}^{*}$ com $\alpha=0.5, p=5$ e diferentes tamanhos amostrais. . . . . . . . . . . . . . . . . . . 94

4.4 Poderes dos testes $R_{o}^{*}, \widehat{R}^{*}$ e $\widetilde{R}^{*} \operatorname{com} \alpha=0.5, p=2, \gamma=10 \%$ e diferentes tamanhos

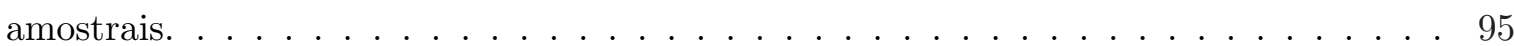

4.5 Tamanhos empíricos dos testes $R, R_{o}^{*}, \widehat{R}^{*}$ e $\widetilde{R}^{*} \operatorname{com} \alpha=0.5, p=6, n=25$ e diferentes

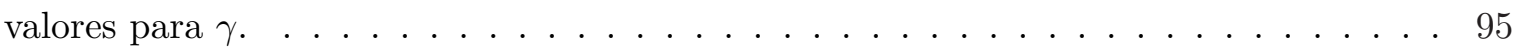

4.6 Tamanhos empíricos dos testes $R, R_{o}^{*}, \widehat{R}^{*}$ e $\widetilde{R}^{*} \operatorname{com} \alpha^{(0)}=0.5$ e $n=20 . \ldots$. . . . 96

4.7 Poderes dos testes $R, R_{o}^{*}, \widehat{R}^{*}$ e $\widetilde{R}^{*} \operatorname{com} \alpha^{(0)}=0.5, p=7$ e $n=20$ considerando a hipótese $\mathcal{H}_{0}: \alpha \leq \alpha^{(0)}$ versus $\mathcal{H}_{1}: \alpha>\delta \ldots \ldots \ldots \ldots$ 


\section{Capítulo 1}

\section{Introdução}

A fadiga é um dano estrutural que ocorre quando um material é exposto a flutuações de estresse e tensão. Modelos estatísticos para processos de fadiga nos possibilitam descrever a variação aleatória dos tempos de falha associados aos materiais expostos à fadiga como um resultado de diferentes padrões e forças cíclicas. Esses materias podem ser caracterizados por parâmetros de distribuições de vida. Dentre os modelos probabilísticos que têm sido popularmente propostos para descrever a vida por fadiga encontram-se as distribuições gama, normal inversa, lognormal e Weibull, sendo que estas distribuições se ajustam com grande precisão na região central da distribuição de vida. Entretanto, o interesse, em geral, está nos percentis mais baixos ou mais altos da distribuição, região em que costuma haver poucos dados, o que conduz a um pobre ajuste dos modelos acima mencionados. Por exemplo, problemas de gerenciamento ou de garantia, doses letais, etc.

Em 1969, motivados por problemas de vibração encontrados nos novos aviões comerciais e por problemas de falhas de materiais, Birnbaum \& Saunders (1969a) propuseram uma nova família de distribuições para modelar o tempo de vida de materiais e equipamentos sujeitos a cargas dinâmicas. Esta distribuição, denominada distribuição Birnbaum-Saunders, foi derivada a partir de um modelo que mostra que as falhas acontecem devido ao desenvolvimento e ao crescimento de uma rachadura dominante. A seguir descreveremos brevemente como esta nova família foi derivada. Birnbaum \& Saunders (1969a) fizeram as seguintes suposições sobre o processo de fadiga:

1. Considere um material que é sujeito a um padrão cíclico de tensão e força. Aqui, está implícito que, em qualquer momento no tempo, 'carga' é uma função que representa o resultado da tensão lançada no material neste tempo;

2. A falha do material ocorre devido ao desenvolvimento e ao crescimento de uma rachadura dominante dentro do material, ou seja, quando o tamanho da rachadura excede certo nível de resistência;

3. A sequência de tensão imposta ao material é a mesma de ciclo para ciclo; (Um ciclo é definido como $m$ oscilações e cada aplicação da $i$-ésima oscilação em um ciclo resulta em uma extensão aleatória da rachadura $X_{i}$.)

4. A extensão incremental da rachadura $X_{i}$ resultante da aplicação da $i$-ésima oscilação de carga é uma variável aleatória com uma distribuição que só depende da rachadura atual causada pela tensão neste ciclo; 
5. A extensão da rachadura devido ao $(j+1)$-ésimo ciclo é dada por

$$
Y_{j+1}=X_{j m+1}+\cdots+X_{j m+m}, \quad j=0,1,2,3, \ldots,
$$

em que $X_{j m+i}, i=1,2, \ldots, m$, é a extensão da rachadura (possivelmente microscópica) após a $i$-ésima oscilação de carga do $(j+1)$-ésimo ciclo;

6. As extensões das rachaduras em diferentes ciclos são independentes;

7. A extensão total da rachadura, $Y_{j}$, devido ao $j$-ésimo ciclo é uma variável aleatória com média $\mu$ e variância $\sigma^{2}$, para todo $j=1,2, \ldots$

8. Os ciclos demoram aproximadamente o mesmo intervalo de tempo.

A extensão total da rachadura após $z$ ciclos é dada pela variável aleatória

$$
W_{z}=\sum_{j=1}^{z} Y_{j}
$$

com função de distribuição $H_{z}(w)=\operatorname{Pr}\left(W_{z} \leq w\right)$, para $z=1,2,3, \ldots$

Seja $N$ o número de ciclos até a falha, onde tal falha ocorre quando o comprimento da rachadura dominante excede um dado comprimento crítico $\omega$, isto é, um certo nível de resistência $\omega$. A função de distribuição da variável aleatória $N$ é

$$
\operatorname{Pr}(N \leq z)=\operatorname{Pr}\left(W_{z}>\omega\right)=\operatorname{Pr}\left(\sum_{j=1}^{z} Y_{j}>\omega\right)=1-H_{z}(\omega)
$$

Assumindo que os $Y_{j}$ 's são variáveis aleatórias independentes e identicamente distribuídas, a função de distribuição de $N$ pode ser aproximada usando o Teorema Central do Limite, isto é,

$$
\operatorname{Pr}(N \leq z)=1-\operatorname{Pr}\left(\sum_{j=1}^{z} \frac{Y_{j}-\mu}{\sigma \sqrt{z}} \leq \frac{\omega-z \mu}{\sigma \sqrt{z}}\right) \doteq 1-\Phi\left(\frac{\omega}{\sigma \sqrt{z}}-\frac{\mu \sqrt{z}}{\sigma}\right)=\Phi\left(\frac{\mu \sqrt{z}}{\sigma}-\frac{\omega}{\sigma \sqrt{z}}\right),
$$

em que ".̈" significa aproximadamente igual e $\Phi(\cdot)$ representa a função distribuição acumulada normal padrão.

Birnbaum \& Saunders (1969a) usaram a equação (1.1) para definir uma distribuição contínua de tempo de vida. Segundo os autores, se $z$ é substituído por uma variável real não-negativa $t$, então a variável aleatória $T$ é a extensão contínua da variável aleatória discreta $N$. Dessa forma, $T$ pode ser considerada como o tempo até que ocorra a falha. Portanto, a função de distribuição pode agora ser escrita como

$$
F_{T}(t)=\Phi\left[\frac{1}{\alpha}\left(\sqrt{\frac{t}{\eta}}-\sqrt{\frac{\eta}{t}}\right)\right], \quad t>0,
$$

em que

$$
\alpha=\frac{\sigma}{\sqrt{\omega \mu}}>0 \quad \text { e } \quad \eta=\frac{\omega}{\mu}>0 .
$$

Dizemos que $T$ segue distribuição Birnbaum-Saunders bi-paramétrica, isto é, $T \sim \mathcal{B}-\mathcal{S}(\alpha, \eta)$. Uma descrição completa e mais detalhada de como este modelo foi derivado pode ser encontrada em 
Birnbaum \& Saunders (1969a).

A distribuição (1.2) possui algumas propriedades interessantes dadas em Birnbaum \& Saunders (1969a). O parâmetro $\alpha$ é um parâmetro de forma. O parâmetro $\eta$ é um parâmetro de escala, isto é, $T / \eta \sim \mathcal{B}-\mathcal{S}(\alpha, 1)$. Adicionalmente, $\eta$ é a mediana da distribuição, pois $F_{T}(\eta)=\Phi(0)=1 / 2$. Para qualquer constante real $k>0$, tem-se que $k T \sim \mathcal{B}-\mathcal{S}(\alpha, k \eta)$. A distribuição $\mathcal{B}-\mathcal{S}(\alpha, \eta)$ também possui a propriedade recíproca, isto é, $T^{-1} \sim \mathcal{B}-\mathcal{S}\left(\alpha, \eta^{-1}\right)$, que pertence à mesma família de distribuições (Saunders, 1974).

A escolha de uma distribuição de tempo de vida para a análise dos dados é feita com base em argumentos teóricos. Um argumento de 'morte/falha por fadiga' justifica o uso da distribuição Birnbaum-Saunders. Adicionalmente, outros argumentos são usados para escolher uma distribuição de vida. Por exemplo, Leiva, Riquelme, Balakrishnan \& Sanhueza (2008) fazem o seguinte comentário: "Typically, lifetime probability models are unimodal and positively skewed. The choice of life distribution is often made on the basis of a theoretical argument that describes the mechanism of failure. For example, an argument of "fatigue or cumulative damage" justifies the use of the Birnbaum-Saunders (BS) distribution. Also, it is known that an argument of "wear-out of type phase", with exponential lifetimes for each phase, justifies a gamma or Erlang distribution. An argument of "first passage time" justifies the inverse Gaussian (IG) distribution, while an argument of "multiplicative degradation" justifies the use of the lognormal (LN) distribution. In addition, an argument of the "extreme values" type justifies the use of the Weibull distribution. However, whatever the justification to choose the life distribution, the model must be logical and possess visual and statistical tests for fitting the data and/or criteria for model selection."

Rieck \& Nedelman (1991) propuseram um modelo de regressão log-linear com base na distribuição Birnbaum-Saunders. Tal modelo pode ser motivado por aplicações em caracterizações de materiais. Além disso, engenheiros têm considerado leis empíricas que descrevem o tempo até a falha de materiais expostos a fadiga sujeitos a diferentes padrões cíclicos de tensão e força. O modelo proposto pelos autores vem sendo bastante explorado e tem se mostrado uma ótima alternativa a outros modelos propostos na literatura, como por exemplo, os modelos de regressão Weibull, gama e lognormal; veja Rieck \& Nedelman $(1991, \S 7)$. Alguns trabalhos referentes a este modelo merecem destaque. Tisionas (2001) faz uma análise bayesiana do modelo de regressão Birnbaum-Saunders, mostrando que a distribuição posteriori marginal dos parâmetros não pode ser analisada usando procedimentos analíticos e utiliza o método MCMC (veja, por exemplo, Gamerman \& Lopes, 2006) para obter tal distribuição. Galea, Leiva \& Paula (2004) apresentam vários métodos de diagnóstico para o modelo de regressão Birnbaum-Saunders. Alguns métodos de influência, tais como influência local, influência local total e alavancagem generalizada são derivados, analisados e discutidos. Adicionalmente, os autores apresentam uma conexão entre os métodos de influência local e a alavancagem generalizada. Leiva, Barros, Paula \& Galea (2007) estenderam os resultados de Galea, Leiva \& Paula (2004) ao considerar métodos de diagnóstico para o modelo de regressão Birnbaum-Saunders com observações censuradas. Os autores apresentam uma interessante aplicação deste modelo com dados da área médica, mostrando que o modelo de regressão Birnbaum-Saunders não se restringe apenas a dados da área de engenharia e confiabilidade. Xie \& Wei (2007) propuseram algumas medidas de diagnóstico para o modelo de regressão Birnbaum-Saunders que podem ser vistas como um suplemento ao trabalho de Galea, Leiva \& Paula (2004). Adicionalmente, apresentam um teste para outliers neste modelo. Recentemente, Lemonte \& Cordeiro (2009) propuseram um modelo de regressão não-linear com base na distribuição Birnbaum-Saunders. A classe de modelos inclui o modelo proposto em Rieck \& Nedelman (1991) como um caso particular. Adicionalmente, os autores encontram o viés de 
segunda ordem dos estimadores de máxima verossimilhança dos parâmetros do modelo.

É interessante ressaltar que a maioria dos trabalhos citados acima sobre o modelo de regressão Birnbaum-Saunders se restringem, basicamente, a técnicas de diagnóstico. Porém, como mencionado, este modelo vem sendo amplamente usado em aplicações práticas, por exemplo, para comparar os valores do parâmetro de locação de algumas populações ou para controlar o efeito de variáveis em situações de testes de vida (veja, por exemplo, Rieck \& Nedelman, 1991). Em tais aplicações, tipicamente testes de hipóteses clássicos baseados nas estatísticas da razão de verossimilhanças e escore são utilizados, isto é, a inferência estatística a respeito da significância de parâmetros no modelo é conduzida sob testes assintóticos.

Sabemos que as estatísticas da razão de verossimilhanças $(L R)$ e escore $\left(S_{R}\right)$ têm distribuição, assintoticamente e sob a hipótese nula, qui-quadrado com número de graus de liberdade igual ao número de restrições impostas pela hipótese nula, com erro de ordem $O\left(n^{-1}\right)$. Porém, esta aproximação é de primeira ordem e pode conduzir a graves distorções de tamanho em amostras de tamanho pequeno e moderado. Isso foi constatado por Rieck \& Nedelman $(1991, \S 5)$ em um breve estudo de simulação Monte Carlo para o teste da razão de verossimilhanças. Os autores observaram uma considerável distorção de tamanho deste teste em pequenas amostras no modelo de regressão Birnbaum-Saunders.

Vale ressaltar que não existe nenhum estudo tratando de refinamentos para as estatísticas da razão de verossimilhanças e escore no modelo de regressão Birnbaum-Saunders. Assim, alguns dos objetivos desta tese são descritos a seguir. Primeiramente, obteremos um fator de correção de Bartlett para a estatística da razão de verossimilhanças e um fator de correção tipo-Bartlett para a estatística escore. Estes ajustes melhoram a aproximação das distribuições destas estatísticas pela distribuição qui-quadrado. Adicionalmente, iremos obter ajustes para a estatística da razão de verossimilhanças sinalizada. Tais ajustes melhoram a aproximação da distributição nula desta estatística pela distribuição normal padrão.

Em seguida, iremos considerar uma estatística recentemente proposta por Terrell (2002), que o autor denomina de estatística gradiente. Obteremos uma expansão assintótica para a distribuição desta estatística sob uma sequência de alternativas de Pitman convergindo para a hipótese nula a uma taxa de convergência de $n^{-1 / 2}$ utilizando a metodologia desenvolvida por Peers (1971) e Hayakawa (1975). Adicionalmente, iremos comparar as funções de poder das estatísticas $L R, W$ e $S_{R}$ com a função de poder da estatística gradiente $\left(S_{T}\right)$. Mostraremos que não é possível, em geral, afirmar que um dos testes $\left(L R, W\right.$ ou $\left.S_{R}\right)$ é mais poderoso que o teste com base na estatística gradiente, ou seja, somente em situações particulares podem ser feitas comparações.

Posteriormente, iremos particularizar a expressão geral da expansão assintótica da distribuição da estatística gradiente até ordem $n^{-1 / 2}$, bem como as expansões assintóticas das funções de poder das estatísticas $L R, W$ e $S_{R}$, em distribuições pertencentes à família exponencial uniparamétrica. Adicionalmente, desenvolveremos expansões assintóticas na classe de modelos de regressão BirnbaumSaunders, assumindo $\alpha$ conhecido e, posteriormente, considerando este parâmetro desconhecido. Mostraremos que as funções de poder das estatísticas $L R, W, S_{R}$ e $S_{T}$, até ordem $n^{-1 / 2}$, são iguais, considerando $\alpha$ conhecido ou desconhecido. Finalmente, iremos obter a expansão assintótica, até ordem $n^{-1 / 2}$, para a função de poder do teste gradiente em modelos lineares generalizados (McCullagh \& Nelder, 1989). As expansões assintóticas, até ordem $n^{-1 / 2}$, das funções de poder das estatísticas $L R, W$ e $S_{R}$ nesta classe de modelos foram derivadas em Cordeiro, Botter \& Ferrari (1994) e Ferrari, Botter \& Cribari-Neto (1997). 


\section{$1.1 \quad$ Preliminares}

O objetivo desta seção é introduzir alguns conceitos básicos para o desenvolvimento do nosso trabalho, assim como definir a notação e terminologia pertinentes. Suponha que $\mathbb{E}\left\{\exp \left(t_{0}|Y|\right)\right\}<\infty$ para algum $t_{0}>0$. Neste caso, $M_{Y}$, definida por

$$
M_{Y}(t)=\mathbb{E}\{\exp (t Y)\}, \quad|t|<t_{0},
$$

é chamada função geradora de momentos de $Y$. Se $M_{Y}$ está definida em uma vizinhança $\left\{t:|t|<t_{0}\right\}$ de zero, então todos os momentos de $Y$ são finitos e

$$
M_{Y}(t)=\sum_{j=0}^{\infty} \frac{\mathbb{E}\left(Y^{j}\right)}{j !} t^{j}=\sum_{j=0}^{\infty} \frac{m_{j}}{j !} t^{j}, \quad|t|<t_{0},
$$

em que $m_{j}=\mathbb{E}\left(Y^{j}\right)$. Além disso, $M_{Y}$ tem derivadas de toda ordem em $t=0 \mathrm{e}$

$$
\left.\frac{\mathrm{d}^{j}}{\mathrm{~d} t^{j}} M_{Y}(t)\right|_{t=0}=\mathbb{E}\left(Y^{j}\right)=m_{j}
$$

A função geradora de momentos caracteriza a distribuição de $Y$, no sentido de que se $M_{Y}(t)$ é finita para $|t|<t_{0}, t_{0}>0$, então $M_{Y}(t)$ e a sequência de momentos $m_{j}(j=1,2, \ldots)$, identificam, unicamente, a distribuição.

Definimos a função geradora de cumulantes de $Y$ como $K_{Y}(t)=\log \left\{M_{Y}(t)\right\}$, sendo possível representá-la por uma expansão de Taylor da forma

$$
K_{Y}(t)=\sum_{j=0}^{\infty} \frac{\kappa_{j}}{j !} t^{j}
$$

em que as constantes $\kappa_{1}, \kappa_{2}, \ldots$ são chamadas de cumulantes de $Y$ e são obtidas pela diferenciação de $K_{Y}(t)$ da forma

$$
\left.\frac{\mathrm{d}^{j}}{\mathrm{~d} t^{j}} K_{Y}(t)\right|_{t=0}=\kappa_{j}
$$

com $\kappa_{0}=K_{Y}(0)=0$. Os dois primeiros cumulantes são a média e a variância de $Y$, isto é, $\kappa_{1}=\mathbb{E}(Y)$ e $\kappa_{2}=\operatorname{Var}(Y)$. O terceiro cumulante de $Y$ é uma medida de assimetria, no sentido de que $\kappa_{3}=\mathbb{E}\left(Y-m_{1}\right)^{3}$ é nulo se $Y$ é uma variável aleatória simétrica. Entretanto, $\kappa_{3}=0$ não implica simetria. Um exemplo de uma distribuição assimétrica em que os cumulantes de ordem ímpar são zero é apresentado em Kendall \& Stuart (1977, Exercício 3.26). Os coeficientes de assimetria e de excesso de curtose são dados, respectivamente, por

$$
\gamma_{1}=\frac{\kappa_{3}}{\kappa_{2}^{3 / 2}} \quad \text { e } \quad \gamma_{2}=\frac{\kappa_{4}}{\kappa_{2}^{2}}
$$

A análise dos cumulantes é importante no estudo assintótico de estatísticas, já que para $j \geq 3$ estes se anulam para a distribuição normal.

Segundo McCullagh (1987), os cumulantes oferencem mais vantagens em termos estatísticos do que os momentos. Entre estas vantagens, tem-se: (i) muitos cálculos estatísticos usando cumulantes são mais fáceis do que os cálculos correspondentes através de momentos; (ii) para variáveis aleatórias 
independentes, os cumulantes de uma soma são, simplesmente, somas dos cumulantes das variáveis individuais; (iii) séries do tipo Edgeworth para aproximar densidades, distribuições e logaritmos de densidades são expressas de forma mais conveniente via cumulantes em vez de momentos; (iv) os cumulantes de inúmeras distribuições podem ter ordens pré-estabelecidas, o que não ocorre com os momentos. Uma discussão detalhada sobre a teoria de cumulantes pode ser encontrada em McCullagh (1987, Capítulos 2, 3 e 4).

Pode-se mostrar que existe uma relação biunívoca entre momentos e cumulantes, já que, em geral, o $j$-ésimo cumulante de $Y$ é uma função dos primeiros $j$-ésimos momentos de $Y$, e vice-versa. Isto pode ser verificado expandindo $M_{Y}(t)$ e $K_{Y}(t)$ nas formas

$$
M_{Y}(t)=1+\sum_{j=1}^{\infty} \frac{m_{j}}{j !} t^{j} \quad \text { e } \quad K_{Y}(t)=\log \left\{M_{Y}(t)\right\}=\log \left(1+\sum_{j=1}^{\infty} \frac{m_{j}}{j !} t^{j}\right) .
$$

Assim, usando uma expansão em série de Taylor de $\log (1+x)$ ao redor do ponto $x=0$ e ignorando potências de $t$ maiores que quatro, temos

$$
\begin{aligned}
\log \left(1+\sum_{j=1}^{\infty} \frac{m_{j}}{j !} t^{j}\right)= & m_{1} t+m_{2} \frac{t^{2}}{2 !}+m_{3} \frac{t^{3}}{3 !}+m_{4} \frac{t^{4}}{4 !}-\frac{1}{2}\left(m_{1} t+m_{2} \frac{t^{2}}{2 !}+m_{3} \frac{t^{3}}{3 !}\right)^{2} \\
& +\frac{1}{3}\left(m_{1} t+m_{2} \frac{t^{2}}{2 !}\right)^{3}-\frac{1}{4} m_{1}^{4} t^{4}+\cdots \\
= & m_{1} t+m_{2} \frac{t^{2}}{2 !}+m_{3} \frac{t^{3}}{3 !}+m_{4} \frac{t^{4}}{4 !}-\frac{1}{2}\left(m_{1}^{2} t^{2}+\frac{1}{4} m_{2}^{2} t^{4}+m_{1} m_{2} t^{3}+\frac{1}{3} m_{1} m_{3} t^{4}\right) \\
& +\frac{1}{3}\left(m_{1}^{3} t^{3}+\frac{3}{2} m_{1}^{2} m_{2} t^{4}\right)-\frac{1}{4} m_{1}^{4} t^{4}+\cdots \\
= & m_{1} t+\left(m_{2}-m_{1}^{2}\right) \frac{t^{2}}{2 !}+\left(m_{3}-3 m_{1} m_{2}+2 m_{1}^{3}\right) \frac{t^{3}}{3 !} \\
& +\left(m_{4}-3 m_{2}^{2}-4 m_{1} m_{3}+12 m_{1}^{2} m_{2}-6 m_{1}^{4}\right) \frac{t^{4}}{4 !}+\cdots
\end{aligned}
$$

de onde se obtém que $\kappa_{1}=m_{1}, \kappa_{2}=m_{2}-m_{1}^{2}, \kappa_{3}=m_{3}-3 m_{1} m_{2}+2 m_{1}^{3}, \kappa_{4}=m_{4}-3 m_{2}^{2}-$ $4 m_{1} m_{3}+12 m_{1}^{2} m_{2}-6 m_{1}^{4}$, e assim por diante. Adicionalmante, os cumulantes são relacionados com os momentos através da equação (McCullagh, 1987, pág. 46)

$$
\kappa_{j}=m_{j}-\sum_{i=1}^{j-1}\left(\begin{array}{c}
j-1 \\
i-1
\end{array}\right) \kappa_{i} m_{j-i}, \quad j=1,2,3, \ldots
$$

Considere um vetor de variáveis aleatórias $\boldsymbol{Y}=\left(Y_{1}, Y_{2}, \ldots, Y_{n}\right)^{\top}$ de dimensão $n$. A função geradora de momentos de $\boldsymbol{Y}$ é dada por

$$
M_{\boldsymbol{Y}}(\boldsymbol{t})=\mathbb{E}\left\{\exp \left(\boldsymbol{t}^{\top} \boldsymbol{Y}\right)\right\}
$$

e a função geradora de cumulantes de $\boldsymbol{Y}$ é dada por $K(\boldsymbol{t})=\log \left\{M_{\boldsymbol{Y}}(\boldsymbol{t})\right\}$, sendo $\boldsymbol{t}=\left(t_{1}, t_{2}, \ldots, t_{n}\right)^{\top} \in$ $\mathbb{R}^{n} ;\|\boldsymbol{t}\|<t_{0}$, sendo $\|\cdot\|$ a norma euclidiana. Os cumulantes conjuntos de $\boldsymbol{Y}$ podem ser obtidos 
diferenciando $K(\boldsymbol{t})$. Especificamente, o cumulante de ordem $\left(j_{1}, \ldots, j_{n}\right)$ é dado por

$$
\kappa_{j_{1} \cdots j_{n}}=\left.\frac{\partial^{j_{1}+\cdots+j_{n}}}{\partial t_{1}^{j_{1}} \cdots \partial t_{n}^{j_{n}}} K(\boldsymbol{t})\right|_{\boldsymbol{t}=(0, \ldots, 0)}
$$

Note que o cumulante de ordem $(j, 0, \ldots, 0)$ é simplesmente o $j$-ésimo cumulante de $Y_{1}$. Cumulantes conjuntos são escritos usando a notação $\operatorname{cum}(\cdot, \ldots, \cdot)$, isto é,

$$
\operatorname{cum}\left(Y_{1}\right)=\kappa_{10 \cdots 0}, \quad \operatorname{cum}\left(Y_{1}, Y_{2}\right)=\kappa_{110 \cdots 0}, \quad \operatorname{cum}\left(Y_{1}, Y_{1}, Y_{2}, Y_{3}\right)=\kappa_{2110 \cdots 0}
$$

e assim por diante. Por exemplo, suponha que $n=2$, ou seja, $\boldsymbol{Y}=\left(Y_{1}, Y_{2}\right)^{\top}$. Então, é fácil mostrar que

$$
\kappa_{10}=\mathbb{E}\left(Y_{1}\right), \quad \kappa_{01}=\mathbb{E}\left(Y_{2}\right), \quad \kappa_{20}=\operatorname{Var}\left(Y_{1}\right), \quad \kappa_{02}=\operatorname{Var}\left(Y_{2}\right) \quad \text { e } \quad \kappa_{11}=\operatorname{Cov}\left(Y_{1}, Y_{2}\right) .
$$

Uma boa discussão sobre funções geradoras de momentos e cumulantes pode ser encontrada em Severini (2000).

\subsubsection{Estimadores de Máxima Verossimilhança}

Seja $\boldsymbol{y}=\left(y_{1}, \ldots, y_{n}\right)^{\top}$ o valor observado de uma variável aleatória $\boldsymbol{Y}=\left(Y_{1}, \ldots, Y_{n}\right)^{\top}$ associada a um experimento aleatório, o qual é representado por um espaço de probabilidade $(\Omega, \mathcal{A}, P)$, sendo $\Omega$ o conjunto de todos os possíveis resultados do experimento, $\mathcal{A}$ uma $\sigma$-álgebra de subconjuntos de $\Omega$ e $P$ uma medida de probabilidade definida nos elementos de $\mathcal{A}$. Suponha que $\boldsymbol{Y}$ é caracterizada por uma função de probabilidade ou densidade com forma analítica $f(\boldsymbol{y} ; \boldsymbol{\theta})$ conhecida mas envolvendo um vetor $\boldsymbol{\theta}=\left(\theta_{1}, \ldots, \theta_{p}\right)^{\top}$ de parâmetros desconhecidos. Seja $\boldsymbol{\Theta} \subseteq \mathbb{R}^{p}$ o espaço paramétrico representando o conjunto dos valores possíveis do vetor $\boldsymbol{\theta}$.

A função de verossimilhança, $L(\boldsymbol{\theta})$, é definida como sendo igual a $f(\boldsymbol{y} ; \boldsymbol{\theta})$, embora seja interpretada diferentemente, como função de $\boldsymbol{\theta}$ para $\boldsymbol{y}$ fixado. Ou seja, a função de verossimilhança para $\boldsymbol{\theta}$ baseada na observação $\boldsymbol{Y}=\boldsymbol{y}$ é dada por

$$
L(\boldsymbol{\theta})=L(\boldsymbol{\theta} ; \boldsymbol{y})=f(\boldsymbol{y} ; \boldsymbol{\theta}), \quad \boldsymbol{\theta} \in \boldsymbol{\Theta}, \quad \boldsymbol{\Theta} \subseteq \mathbb{R}^{p} .
$$

Muito frequentemente, os componentes de $\boldsymbol{Y}$ são mutuamente independentes e a verossimilhança de $\boldsymbol{\theta}$ pode ser escrita como

$$
L(\boldsymbol{\theta})=\prod_{i=1}^{n} f_{i}\left(y_{i} ; \boldsymbol{\theta}\right)
$$

em que $f_{i}$ corresponde à densidade individual da $i$-ésima observação. Dada a função $L(\boldsymbol{\theta})$, o estimador de máxima verossimilhança $\widehat{\boldsymbol{\theta}}$ de $\boldsymbol{\theta}$ é o valor que maximiza $L(\boldsymbol{\theta})$ em $\boldsymbol{\Theta}$. Formalmente, o estimador $\widehat{\boldsymbol{\theta}}$ é definido como a variável aleatória

$$
\widehat{\boldsymbol{\theta}}=\underset{\boldsymbol{\theta} \in \boldsymbol{\Theta}}{\arg \max } L(\boldsymbol{\theta}) \text {. }
$$

É conveniente trabalhar com o logaritmo da função de verossimilhança, também chamada de função suporte, isto é,

$$
\ell(\boldsymbol{\theta})=\ell(\boldsymbol{\theta})=\log \{L(\boldsymbol{\theta})\}=\sum_{i=1}^{n} \log \left\{f_{i}\left(y_{i} ; \boldsymbol{\theta}\right)\right\} .
$$

Como a função logaritmo é monótona crescente, maximizar $L(\boldsymbol{\theta})$ e $\ell(\boldsymbol{\theta})$ em $\boldsymbol{\Theta}$ são processos equiva- 
lentes.

A função escore $\boldsymbol{U}(\boldsymbol{\theta})=\left(U_{1}(\boldsymbol{\theta}), \ldots, U_{p}(\boldsymbol{\theta})\right)^{\top}$ é definida como

$$
U_{r}(\boldsymbol{\theta})=\frac{\partial \ell(\boldsymbol{\theta})}{\partial \theta_{r}}, \quad r=1, \ldots, p,
$$

ou seja,

$$
\boldsymbol{U}(\boldsymbol{\theta})=\nabla_{\boldsymbol{\theta}} \ell(\boldsymbol{\theta}),
$$

em que $\nabla_{\boldsymbol{\theta}}=\left(\partial / \partial \theta_{1}, \ldots, \partial / \partial \theta_{p}\right)^{\top}$ é o operador gradiente. A função escore descreve como a função de log-verossimilhança varia em $\Theta$.

Seja $\boldsymbol{J}(\boldsymbol{\theta})=-\partial^{2} \ell(\boldsymbol{\theta}) / \partial \boldsymbol{\theta} \partial \boldsymbol{\theta}^{\top}$ o negativo da matriz hessiana da função de log-verossimilhança; $\boldsymbol{J}(\boldsymbol{\theta})$ é chamada matriz de informação observada (Fisher, 1925). Sob condições gerais de regularidade (ver, por exemplo, Cox \& Hinkley, 1974, cap. 9; Lehmann, 1999, cap. 7), temos que

$$
\boldsymbol{K}(\boldsymbol{\theta})=\mathbb{E}_{\boldsymbol{\theta}}\left\{\boldsymbol{U}(\boldsymbol{\theta}) \boldsymbol{U}(\boldsymbol{\theta})^{\top}\right\}=\mathbb{E}_{\boldsymbol{\theta}}\{\boldsymbol{J}(\boldsymbol{\theta})\},
$$

em que o subescrito $\boldsymbol{\theta}$ indica que a esperança matemática é calculada supondo que $\boldsymbol{\theta}$ é o verdadeiro valor do parâmetro. A matriz $\boldsymbol{K}(\boldsymbol{\theta})$ é chamada de matriz de informação (esperada) de Fisher de $\boldsymbol{\theta}$. Uma justificativa qualitativa para a palavra "informação" é que quando a matriz $\boldsymbol{J}(\boldsymbol{\theta})$ "cresce", isto é, quando é maior a informação sobre $\boldsymbol{\theta}$, a dispersão de $\widehat{\boldsymbol{\theta}}$ ao redor de $\boldsymbol{\theta}$ decresce (Barndorff-Nielsen \& Cox, 1994, pág. 25).

Sob condições gerais de regularidade, $\ell(\boldsymbol{\theta})$ é diferenciável em $\boldsymbol{\theta}, \widehat{\boldsymbol{\theta}}$ existe e satisfaz a equação de verossimilhança

$$
\boldsymbol{U}(\widehat{\boldsymbol{\theta}})=\nabla_{\boldsymbol{\theta}} \ell(\widehat{\boldsymbol{\theta}})=\mathbf{0}
$$

Adicionalmente,

$$
\ell(\widehat{\boldsymbol{\theta}}) \geq \ell(\boldsymbol{\theta}), \quad \forall \boldsymbol{\theta} \in \boldsymbol{\Theta} .
$$

A equação de verossimilhança (1.5) simplifica-se na forma

$$
\sum_{i=1}^{n} \boldsymbol{\nabla}_{\boldsymbol{\theta}} \log \left\{f_{i}\left(y_{i} ; \widehat{\boldsymbol{\theta}}\right)\right\}=\mathbf{0} .
$$

Lehmann \& Casela (1998, cap. 6) mostram que, sob condições de regularidade, o estimador de máxima verossimilhança $\widehat{\boldsymbol{\theta}}$, obtido como raiz única da equação (1.6), apresenta as seguintes propriedades: (i) é consistente; (ii) é assintoticamente eficiente, isto é, dentre todos os estimadores consistentes de $\boldsymbol{\theta}$, o estimador de máxima verossimilhança possui variância assintótica mínima; (iii) é invariante sob transformações biunívocas, isto é, se $\widehat{\boldsymbol{\theta}}$ é o estimador de máxima verossimilhança de $\boldsymbol{\theta}$ e $g$ é uma função bijetora de $\boldsymbol{\theta}$, então $g(\widehat{\boldsymbol{\theta}})$ é o estimador de máxima verossimilhança de $g(\boldsymbol{\theta})$; (iv) é distribuído assintoticamente como uma variável aleatória normal $p$-variada da forma: $\widehat{\boldsymbol{\theta}} \stackrel{A}{\sim} \mathcal{N}_{p}\left(\boldsymbol{\theta}, \boldsymbol{K}(\boldsymbol{\theta})^{-1}\right) ;(\mathrm{v})$ e, em geral, viesado, embora seja assintoticamente não-viesado.

\subsubsection{Identidades de Bartlett}

Utilizaremos a seguinte notação, dada por Lawley (1956), para as derivadas da log-verossimilhança (1.4), na qual todos os índices variam de 1 a $p: U_{r}=\partial \ell(\boldsymbol{\theta}) / \partial \theta_{r}, U_{r s}=\partial^{2} \ell(\boldsymbol{\theta}) / \partial \theta_{r} \partial \theta_{s}, U_{r s t}=$ $\partial^{3} \ell(\boldsymbol{\theta}) / \partial \theta_{r} \partial \theta_{s} \partial \theta_{t}, U_{r, s}=\left(\partial \ell(\boldsymbol{\theta}) / \partial \theta_{r}\right)\left(\partial \ell(\boldsymbol{\theta}) / \partial \theta_{s}\right)$, etc. Os momentos conjuntos de derivadas de 
$\ell(\boldsymbol{\theta})$ são $\mu_{r}=\mathbb{E}\left(U_{r}\right), \mu_{r s}=\mathbb{E}\left(U_{r s}\right), \mu_{r s t}=\mathbb{E}\left(U_{r s t}\right), \mu_{r, s}=\mathbb{E}\left(U_{r, s}\right), \mu_{r, s t}=\mathbb{E}\left(U_{r} U_{s t}\right)$, e assim por diante. Como $\mu_{r}=0$, os cumulantes conjuntos expressos em termos de momentos são $\kappa_{r s}=\mu_{r s}$, $\kappa_{r, s}=\mu_{r, s}, \kappa_{r s, t}=\mu_{r s, t}, \kappa_{r s, t u}=\mu_{r s, t u}-\mu_{r s} \mu_{t u}, \kappa_{r, s, t}=\mu_{r, s, t}$ e $\kappa_{r, s, t, u}=\mu_{r, s, t, u}-\sum_{(3)} \mu_{r, s} \mu_{t, u}$, onde $\sum_{(j)}$ representa o somatório sobre todas as $j$-ésimas combinações de índices, etc.; por exemplo, $\sum_{(3)} \kappa_{r s, t u}=\kappa_{r s, t u}+\kappa_{r t, s u}+\kappa_{r u, s t}$. Ainda, $-\kappa^{r s}=\kappa^{r, s}$ representa o elemento $(r, s)$ da inversa da matriz de informação $\boldsymbol{K}(\boldsymbol{\theta})^{-1}$. As derivadas dos cumulantes são representadas com sobrescritos: $\kappa_{r s}^{(t)}=\partial \kappa_{r s} / \partial \theta_{t}, \kappa_{r s}^{(t u)}=\partial^{2} \kappa_{r s} / \partial \theta_{t} \partial \theta_{u}, \kappa_{r s t}^{(u)}=\partial \kappa_{r s t} / \partial \theta_{u}$, etc. Os momentos e cumulantes são intra-relacionados, satisfazendo algumas restrições que facilitam seus cálculos. Estas equações, que decorrem de condições de regularidade, são denominadas identidades de Bartlett, as mais conhecidas sendo $\kappa_{r}=0$ e $\kappa_{r s}+\kappa_{r, s}=0$. Adicionalmente, pode-se mostrar que, sobre a amostra total, os cumulantes são de ordem $O(n) .{ }^{1}$ A idéia central na dedução das identidades de Bartlett é a validade em problemas regulares da fórmula

$$
\frac{\partial}{\partial \boldsymbol{\theta}} \mathbb{E}\{T(\boldsymbol{Y})\}=\int T(\boldsymbol{y}) \frac{\partial}{\partial \boldsymbol{\theta}} f(\boldsymbol{y} ; \boldsymbol{\theta}) \mathrm{d} \boldsymbol{y}
$$

para qualquer estatística $T(\boldsymbol{Y})$, isto é, pode-se inverter a ordem das operações de diferenciação em relação a $\boldsymbol{\theta}$ e integração com respeito a $\boldsymbol{y}$. Expressando a identidade (1.7) em termos de momentos e diferenciando sucessivamente com relação aos componentes de $\boldsymbol{\theta}$ encontram-se as ditas identidades. Assim, por exemplo, da definição da função escore temos que $U_{r}=L_{r} / L \operatorname{com} L_{r}=\partial L / \partial \theta_{r}$. Diferenciando $\int L \mathrm{~d} \boldsymbol{y}=1$ em relação a $\theta_{r}$ obtemos $\int L_{r} \mathrm{~d} \boldsymbol{y}=\int U_{r} L \mathrm{~d} \boldsymbol{y}=0$, isto é, $\kappa_{r}=\mathbb{E}\left(U_{r}\right)=$ 0 . Diferenciando esta última expressão em relação a $\theta_{s}$, encontramos $\int\left(U_{r s} L+U_{r} U_{s} L\right) \mathrm{d} \boldsymbol{y}=0$ e, assim, obtemos $\kappa_{r s}+\kappa_{r, s}=0$. Diferenciando novamente a integral em relação a $\theta_{t}$, obtémse $\kappa_{r s t}+\kappa_{r s, t}+\kappa_{r t, s}+\kappa_{r, s t}+\kappa_{r, s, t}=0$ e, de forma análoga, outras identidades são deduzidas: $\kappa_{r, s t}+\kappa_{r s t}-\kappa_{s t}^{(r)}=0, \kappa_{r, s, t}-2 \kappa_{r s t}+\sum_{(3)} \kappa_{r s}^{(t)}=0, \kappa_{r, s, t}^{(u)}=\kappa_{r s t, u}, \kappa_{r, s t u}+\kappa_{r s t u}-\kappa_{s t u}^{(r)}=0$, $\kappa_{r s t u}+\sum_{(4)} \kappa_{r, s t u}+\sum_{(3)} \kappa_{r s, t u}+\sum_{(6)} \kappa_{r, s, t u}+\kappa_{r, s, t, u}=0, \kappa_{r, s, t, u}=-3 \kappa_{r s t u}+2 \sum_{(4)} \kappa_{r s t}^{(u)}+\sum_{(6)} \kappa_{r s}^{(t u)}+$ $\sum_{(3)} \kappa_{r s, t u}, \kappa_{r, s, t u}=\kappa_{r s t u}-\kappa_{r t u}^{(s)}+\kappa_{t u}^{(r s)}-\kappa_{r s, t u}$, etc.

A grande vantagem das identidades de Bartlett é facilitar a obtenção dos cumulantes $\kappa$ 's, já que determinada parametrização pode conduzir a um cálculo simples de alguns cumulantes, sendo os demais obtidos indiretamente através destas identidades. Esses cumulantes têm grande aplicabilidade no cálculo das correções de Bartlett e tipo-Bartlett para as estatísticas da razão de verossimilhanças e escore, respectivamente, como será visto adiante. Uma boa discussão sobre as identidades de Bartlett e suas aplicações pode ser encontrada em Barndorff--Nielsen \& Cox $(1989, \S 5.5)$.

\subsection{Densidades Birnbaum-Saunders e senh-normal}

A função densidade de probabilidade de uma variável aleatória $T$ que segue distribuição BirnbaumSaunders é da forma

$$
f_{T}(t ; \alpha, \eta)=\frac{1}{2 \alpha \eta \sqrt{2 \pi}}\left[\left(\frac{\eta}{t}\right)^{1 / 2}+\left(\frac{\eta}{t}\right)^{3 / 2}\right] \exp \left\{-\frac{1}{2 \alpha^{2}}\left(\frac{t}{\eta}+\frac{\eta}{t}-2\right)\right\}, \quad t, \alpha, \eta>0 .
$$

\footnotetext{
${ }^{1}$ Se $\left\{a_{n}\right\}_{n \geq 1}$ e $\left\{b_{n}\right\}_{n \geq 1}$ são duas sequências de números reais, dizemos que $a_{n}$ é de ordem menor que $b_{n}$ e escrevemos $a_{n}=o\left(b_{n}\right)$ se $\lim _{n \rightarrow \infty} a_{n} / b_{n}=0$. Dizemos que $a_{n}$ é de ordem no máximo igual a $b_{n}$, denotado por $a_{n}=O\left(b_{n}\right)$, se existe um número real $M>0$ tal que $\left|a_{n} / b_{n}\right| \leq M$, isto é, a razão $\left|a_{n} / b_{n}\right|$ é limitada. Observamos que se $a_{n}=o\left(b_{n}\right)$, então $a_{n}=O\left(b_{n}\right)$ e que quando $b_{n} \longrightarrow 0$ a ordem fornece noção da taxa de convergência de $a_{n}$ para zero.
} 


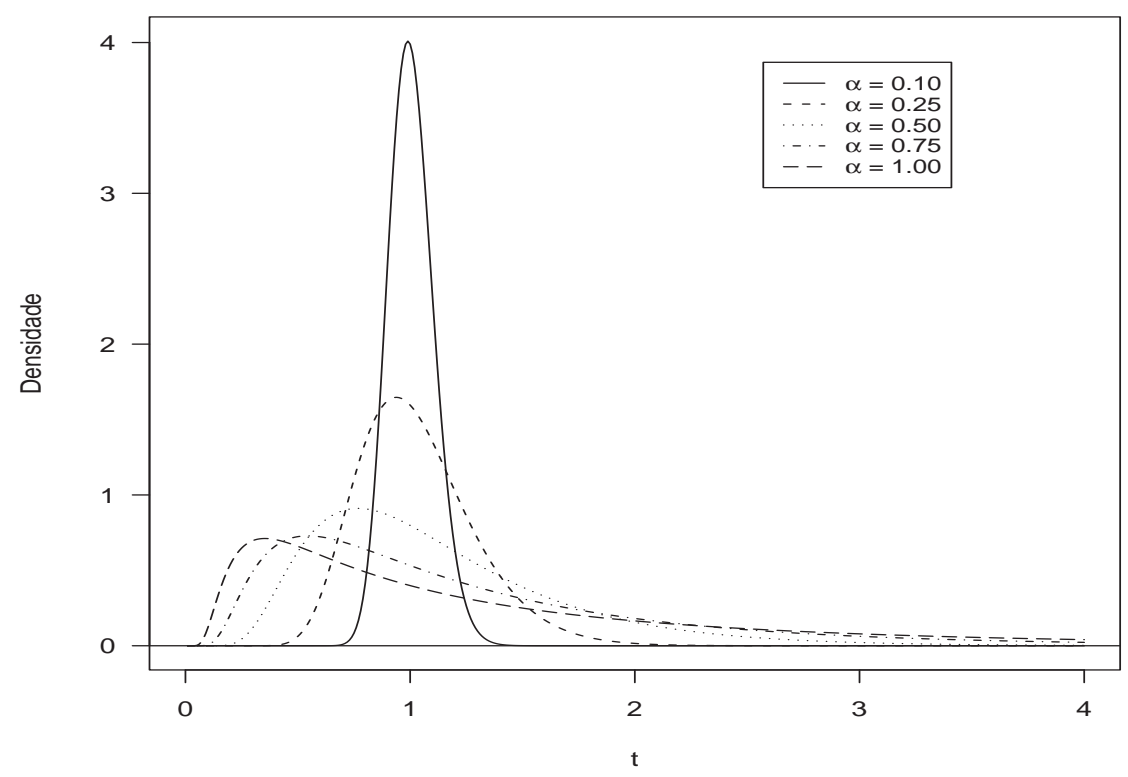

Figura 1.1: Densidade Birnbaum-Saunders para diferentes valores de $\alpha(\eta=1.0)$.

O valor esperado, a variância, a assimetria e a curtose da distribuição Birnbaum-Saunders são dados, respectivamente, por

$$
\begin{gathered}
\mathbb{E}(T)=\eta\left(1+\frac{1}{2} \alpha^{2}\right), \quad \operatorname{Var}(T)=(\alpha \eta)^{2}\left(1+\frac{5}{4} \alpha^{2}\right), \\
\gamma_{1}=\frac{4 \alpha\left(11 \alpha^{2}+6\right)}{\left(5 \alpha^{2}+4\right)^{3 / 2}} \quad \text { e } \quad \gamma_{2}=3+\frac{6 \alpha^{2}\left(93 \alpha^{2}+40\right)}{\left(5 \alpha^{2}+4\right)^{2}} .
\end{gathered}
$$

As quantidades $\gamma_{1}$ e $\gamma_{2}$ medem a assimetria e a largura das caudas da distribuição, respectivamente. Esta densidade é caracteristicamente assimétrica à direita. Entretanto, à medida que o valor de $\alpha$ decresce, particularmente para valores menores que um, a densidade se torna mais simétrica. Na Figura 1.1, apresentamos o gráfico desta densidade para alguns valores de $\alpha$ considerando $\eta=1$. Observe que à medida que o valor de $\alpha$ decresce, a curva tende a ficar simétrica em torno de $\eta$, que é a mediana da distribuição. Da mesma forma, note que a variância também decresce com $\alpha$.

A função de sobrevivência e a função de risco da distribuição $\mathcal{B}-\mathcal{S}(\alpha, \eta)$ são dadas, respectivamente, por

$$
S_{T}(t)=1-F_{T}(t)=1-\Phi\left[\frac{1}{\alpha}\left(\sqrt{\frac{t}{\eta}}-\sqrt{\frac{\eta}{t}}\right)\right] \quad \text { e } \quad \nu(t)=\frac{f_{T}(t)}{S_{T}(t)},
$$

em que $f_{T}(t)$ é a função densidade de probabilidades da distribuição Birnbaum-Saunders. A função de risco $\nu(t)$ assume valor zero em $t=0$, cresce até um valor máximo e depois decresce até uma constante positiva, como pode ser observado na Figura 1.2. Uma comparação entre as funções de risco das distribuições Birnbaum-Saunders e lognormal foi apresentada em Nelson (1990). Mais detalhes sobre a função de sobrevivência da distribuição Birnbaum-Saunders podem ser encontrado em Kundu, Kannan \& Balakrishnan (2008). 


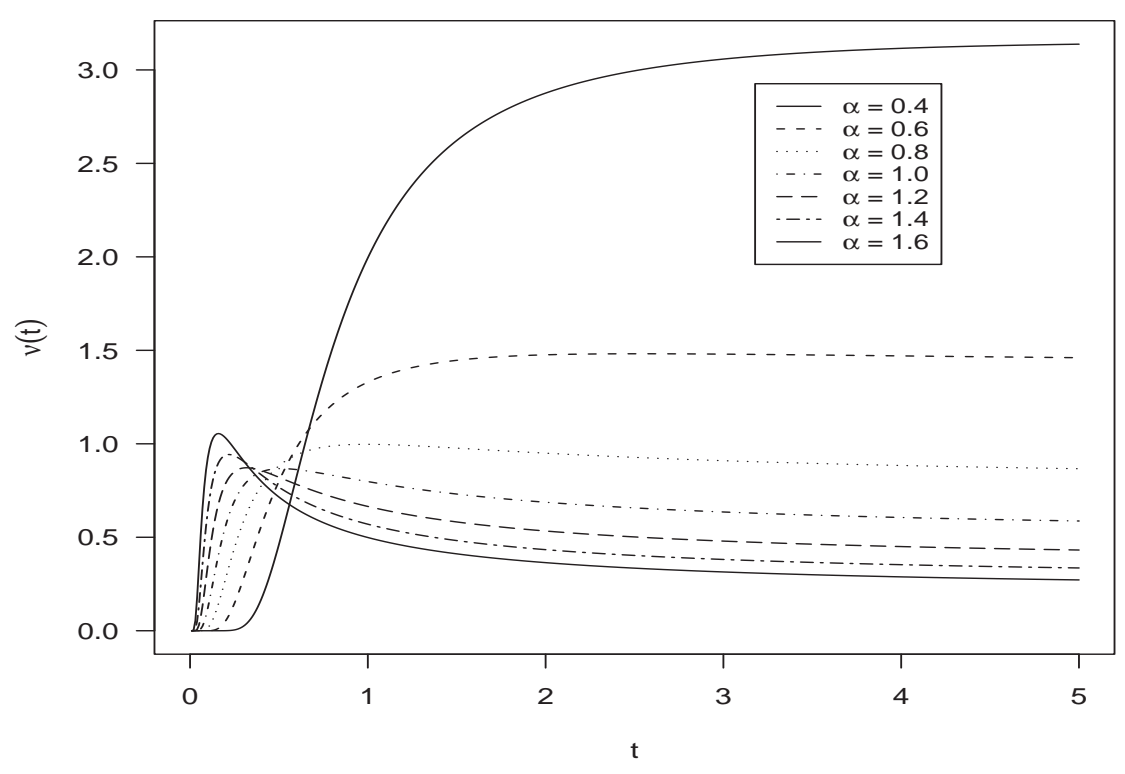

Figura 1.2: Funções de risco da distribuição Birnbaum-Saunders considerando diferentes valores para $\alpha$ fixando $\eta=1.0$.

A distribuição Birnbaum-Saunders bi paramétrica vem sendo amplamente usada em ciências da engenharia. Consequentemente, há vários estudos referentes a esta distribuição. A seguir, listamos alguns trabalhos relevantes. Engelhardt, Bain \& Wright (1981) propuseram intervalos de confiança para $\alpha$ considerando $\eta$ como um parâmetro de perturbação desconhecido e, da mesma forma, para $\eta$ considerando $\alpha$ como um parâmetro de perturbação desconhecido. Eles também apresentaram testes de hipóteses para estes parâmetros. Desmond (1985) derivou a distribuição $\mathcal{B}-\mathcal{S}(\alpha, \eta)$ tendo por base um modelo biológico. O autor também estendeu a justificativa física para o uso desta distribuição, relaxando algumas suposições feitas em Birnbaum \& Saunders (1969a). Desmond (1986) investigou a relação entre a distribuição $\mathcal{B}-\mathcal{S}(\alpha, \eta)$ e a distribuição gaussiana inversa. Achcar (1993) desenvolveu procedimentos de estimação bayesiana para os parâmetros desta distribuição usando aproximações para as distribuições posterioris marginais de $\alpha$ e $\eta$; veja também Xu \& Tang (2010). Rieck (1999) derivou uma função geratriz de momentos para a distribuição senh-normal (seno hiperbólico normal) que pode ser usada para obter momentos de ordem inteira ou fracionária da distribuição $\mathcal{B}-\mathcal{S}(\alpha, \eta)$. McCarter (1999) discutiu a estimação dos parâmetros da distribuição $\mathcal{B}-\mathcal{S}(\alpha, \eta)$ para o caso de amostras censuradas do tipo II. Lemonte, Cribari-Neto \& Vasconcellos (2007) derivaram os vieses de segunda ordem dos estimadores de máxima verossimilhança de $\alpha$ e $\eta$. Os autores também obtiveram um fator de correção de Bartlett que melhora o desempenho do teste da razão de verossimilhanças em amostras finitas para o parâmetro $\alpha$. Lemonte, Simas \& Cribari-Neto (2007) propuseram estimadores modificados para $\alpha$ e $\eta$ baseados em diferentes esquemas de reamostragem bootstrap. Eles verificaram, através de simulações Monte Carlo, que os estimadores baseados em bootstrap apresentam desempenho superior, em termos de viés, do que os estimadores de máxima verossimilhança de $\alpha$ e $\eta$ em amostras de tamanho pequeno e moderado. Outros trabalhos referentes à distribuição BirnbaumSaunders são Chang \& Tang (1993, 1994), Jin \& Kawczak (2003), Ng, Kundu \& Balakrishnan (2003), Owen \& Padgett (1999, 2000), Rieck (1995), Wang, Desmond \& Lu (2006) e Wu \& Wong (2004). 
Uma boa discussão sobre a distribuição Birnbaum-Saunders pode ser encontrada em Johnson, Kotz \& Balakrishnan (1995).

Algumas generalizações e extensões da distribuição $\mathcal{B}-\mathcal{S}(\alpha, \eta)$ tem sido propostas. Owen \& Padgett (1999) desenvolveram a distribuição Birnbaum-Saunders tri paramétrica. Recentemente, DíazGarcía \& Leiva (2005) propuseram uma nova classe de distribuições de vida generalizando a distribuição Birnbaum-Saunders a partir de distribuições de contornos elípticos. Segundo os autores, esta generalização baseia-se, além do aspecto elíptico, na busca de distribuições de vida que possuam caudas com menor ou maior curtose do que a distribuição Birnbaum-Saunders, entre outras propiedades tais como: distribuições de vida que não possuem momentos e que sejam bimodais. É interessante ressaltar que, neste caso, o uso de distribuições elípticas para gerar a distribuição Birnbaum-Saunders generalizada (BSG) não segue uma justificacão física, como no caso clássico (distribuição $\mathcal{B}-\mathcal{S}(\alpha, \eta)$ ), mas razões puramente estatísticas e/ou matemáticas. Além disso, a distribuição $\mathcal{B}-\mathcal{S}(\alpha, \eta)$ é um caso particular da distribuição BSG e muitas propriedades da versão clássica são transferidas para a versão generalizada. Vilca-Labra \& Leiva (2006), baseados em argumentos semelhantes aos empregados por Díaz-Garcia \& Leiva (2005), obtiveram uma maior generalização ao desenvolver a distribuição Birnbaum-Saunders mediante distribuições elípticas assimétricas. Dessa forma, a distribuição Birnbaum-Saunders duplamente generalizada (BSG' ${ }^{\prime \prime}$, como eles denominaram, é mais flexível em relação à curtose e assimetria do que todas as versões anteriores da distribuição Birnbaum-Saunders. Até o presente momento, modelagem estatística sob distribuição BSG não tem recebido muita atenção. Mais recentemente, Guiraud, Leiva \& Fierro (2009) introduziram uma versão não central para a distribuição Birnbaum-Saunders.

Rieck \& Nedelman (1991) mostraram que se $T \sim \mathcal{B}-\mathcal{S}(\alpha, \eta)$, então $Y=\log (T)$ segue distribuição senh-normal (seno hiperbólico normal) com parâmetros de forma, locação e escala, dados por $\alpha$, $\mu=\log (\eta)$ e $\sigma=2$, respectivamente $[Y \sim \mathcal{S N}(\alpha, \mu, \sigma)]$. A função densidade de $Y$ é da forma

$$
f(y)=\frac{2}{\alpha \sigma \sqrt{2 \pi}} \cosh \left(\frac{y-\mu}{\sigma}\right) \exp \left\{-\frac{2}{\alpha^{2}} \operatorname{senh}^{2}\left(\frac{y-\mu}{\sigma}\right)\right\}, \quad y \in \mathbb{R} .
$$

Esta distribuição tem algumas propriedades interessantes (Rieck, 1989): (i) é simétrica em torno do parâmetro de locação $\mu$; (ii) é unimodal para $\alpha \leq 2$ e bimodal para $\alpha>2$; (iii) A média e variância são $\mathrm{E}(Y)=\mu$ e $\operatorname{Var}(Y)=\sigma^{2} w(\alpha)$, respectivamente, em que $w(\alpha)$ é a variância quando $\sigma=1$. Não existe uma expressão em forma "fechada" para $w(\alpha)$, mas Rieck (1989) obteve aproximações assintóticas para $w(\alpha)$ para pequenos e grandes valores de $\alpha$; (iv) se $Y_{\alpha} \sim \mathcal{S} \mathcal{N}(\alpha, \mu, \sigma)$, então $S_{\alpha}=2\left(Y_{\alpha}-\mu\right) /(\alpha \sigma)$ converge em distribuição para a distribuição normal padrão quando $\alpha \rightarrow 0$. Rieck (1989) mostrou que a média e variância da distribuição senh-normal podem ser obtidas usando a função geradora de momentos dada por

$$
M(s)=\left[\frac{B_{a}\left(\alpha^{-2}\right)+B_{b}\left(\alpha^{-2}\right)}{2 B_{1 / 2}\left(\alpha^{-2}\right)}\right] \exp (\mu s)
$$

em que $a=(\sigma s+1) / 2, b=(\sigma s-1) / 2$ e $B_{\lambda}(\cdot)$ é a função de Bessel modificada de terceiro tipo, dada por

$$
B_{\lambda}(x)=\frac{x^{\lambda}}{2^{\lambda+1}} \int_{0}^{\infty} z^{-(\lambda+1)} \exp \left\{-z-\left(x^{2} / 4 z\right)\right\} \mathrm{d} z
$$

veja Gradshteyn \& Ryzhik (2007).

Na Figura 1.3 apresentamos gráficos da densidade de $Y$ para diferentes valores de $\alpha$ considerando $\mu=0$ e $\sigma=2$. Note que a bimodalidade desta densidade fica clara à medida que o valor do parâmetro 


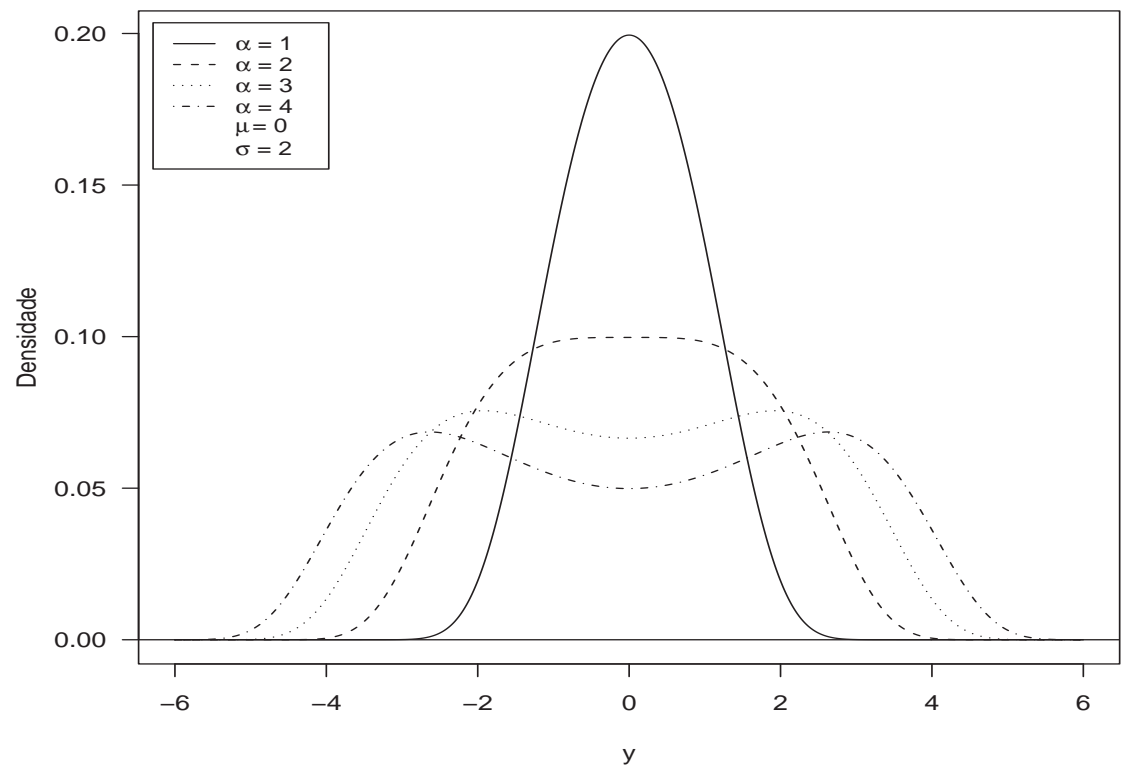

Figura 1.3: Densidade senh-normal para diferentes valores de $\alpha(\mu=0$ e $\sigma=2)$.

$\alpha$ ultrapassa o valor 2. Adicionalmente, note que a distribuição é simétrica em torno do parâmetro $\mu$.

\subsection{Modelo de Regressão Birnbaum-Saunders}

Rieck \& Nedelman (1991) formularam e desenvolveram um modelo log-linear a partir da distribuição Birnbaum-Saunders fazendo algumas suposições sobre os parâmetros $\alpha$ e $\eta$ desta distribuição. Considere uma amostra aleatória $\boldsymbol{T}=\left(T_{1}, T_{2}, \ldots, T_{n}\right)^{\top}$ de tamanho $n$ da distribuição Birnbaum-Saunders, tal que $T_{i} \sim \mathcal{B}-\mathcal{S}\left(\alpha_{i}, \eta_{i}\right)$. Assume-se que a distribuição de $T_{i}$ depende de um conjunto de $p$ variáveis explicativas $(p<n)$, denotadas por $\boldsymbol{x}_{i}^{\top}=\left(x_{i 1}, x_{i 2}, \ldots, x_{i p}\right)$. Assume-se que: (1) $\eta_{i}=\exp \left(\boldsymbol{x}_{i}^{\top} \boldsymbol{\beta}\right)$ para $i=1,2, \ldots, n$, em que $\boldsymbol{\beta}=\left(\beta_{1}, \beta_{2}, \ldots, \beta_{p}\right)^{\top}$ é um vetor de parâmetros desconhecidos; (2) o parâmetro de forma é independente das variáveis explicativas, isto é, $\alpha_{i}=\alpha$ para $i=1,2, \ldots, n$.

Birnbaum \& Saunders (1969b) mostraram que se $k>0$, então $k T_{i}$ segue distribuição BirnbaumSaunders com parâmetro de forma $\alpha$ e parâmetro de escala $k \eta_{i}$. Usando este fato e as suposições mencionadas anteriormente, $T_{i}$ pode ser expressado como $T_{i}=\exp \left(\boldsymbol{x}_{i}^{\top} \boldsymbol{\beta}\right) \delta_{i}$, em que $\delta_{i} \sim \mathcal{B}-\mathcal{S}(\alpha, 1)$. Se $y_{i}=\log \left(T_{i}\right)$, tem-se que $y_{i}=\boldsymbol{x}_{i}^{\top} \boldsymbol{\beta}+\log \left(\delta_{i}\right)$. Se denotarmos $\log \left(\delta_{i}\right)$ por $\varepsilon_{i}$, temos

$$
y_{i}=\boldsymbol{x}_{i}^{\top} \boldsymbol{\beta}+\varepsilon_{i}, \quad i=1,2, \ldots, n,
$$

em que $\varepsilon_{i}$ são variáveis aleatórias independentes e identicamente distribuídas tendo distribuição senh-normal, isto é, $\varepsilon_{i} \sim \mathcal{S N}(\alpha, 0,2)$. 


\subsubsection{Estimação dos Parâmetros}

A função de log-verossimilhança para uma amostra aleatória $\boldsymbol{y}=\left(y_{1}, y_{2}, \ldots, y_{n}\right)^{\top}$ do modelo $(1.8)$ pode ser expressa na forma

$$
\ell(\boldsymbol{\theta})=-\frac{n}{2} \log (8 \pi)+\sum_{i=1}^{n} \log \left(\xi_{i 1}\right)-\frac{1}{2} \sum_{i=1}^{n} \xi_{i 2}^{2},
$$

em que $\boldsymbol{\theta}=\left(\boldsymbol{\beta}^{\top}, \alpha\right)^{\top}$

$$
\xi_{i 1}=\xi_{i 1}(\boldsymbol{\theta})=\frac{2}{\alpha} \cosh \left(\frac{y_{i}-\mu_{i}}{2}\right), \quad \xi_{i 2}=\xi_{i 2}(\boldsymbol{\theta})=\frac{2}{\alpha} \operatorname{senh}\left(\frac{y_{i}-\mu_{i}}{2}\right)
$$

e $\mu_{i}=\boldsymbol{x}_{i}^{\top} \boldsymbol{\beta}, \operatorname{com} i=1,2, \ldots, n$. Diferenciando (1.9) em relação a $\beta_{r}$ e $\alpha$, obtêm-se

$$
\frac{\partial \ell(\boldsymbol{\theta})}{\partial \beta_{r}}=\frac{1}{2} \sum_{i=1}^{n} x_{i r}\left\{\xi_{i 1} \xi_{i 2}-\frac{\xi_{i 2}}{\xi_{i 1}}\right\}, \quad r=1,2, \ldots, p,
$$

e

$$
\frac{\partial \ell(\boldsymbol{\theta})}{\partial \alpha}=-\frac{n}{\alpha}+\frac{1}{\alpha} \sum_{i=1}^{n} \xi_{i 2}^{2} .
$$

Em notação matricial, tem-se que a função escore de $\boldsymbol{\beta}$ pode ser escrita na forma

$$
\boldsymbol{U}_{\boldsymbol{\beta}}(\boldsymbol{\theta})=\boldsymbol{U}_{\boldsymbol{\beta}}=\frac{\partial \ell(\boldsymbol{\theta})}{\partial \boldsymbol{\beta}}=\frac{1}{2} \boldsymbol{X}^{\top} \boldsymbol{s}
$$

em que $\boldsymbol{X}=\left(\boldsymbol{x}_{1}, \boldsymbol{x}_{2}, \ldots, \boldsymbol{x}_{n}\right)^{\top}$ é a matriz modelo de dimensão $n \times p$ e de posto completo e $\boldsymbol{s}=\boldsymbol{s}(\boldsymbol{\theta})$ é um vetor coluna de dimensão $n$ com $i$-ésimo elemento dado por $\xi_{i 1} \xi_{i 2}-\xi_{i 2} / \xi_{i 1}$.

Rieck \& Nedelman (1991) obtiveram uma expressão para o estimador de máxima verossimilhança (EMV) de $\alpha^{2}$ em termos do EMV do vetor $\boldsymbol{\beta}$, solucionando a equação de verossimilhança de $\alpha$ para $\alpha^{2}$, que é dado por

$$
\widehat{\alpha}^{2}=\frac{4}{n} \sum_{i=1}^{n} \operatorname{senh}^{2}\left(\frac{y_{i}-\boldsymbol{x}_{i}^{\top} \widehat{\boldsymbol{\beta}}}{2}\right),
$$

em que $\widehat{\boldsymbol{\beta}}$ é o EMV do vetor $\boldsymbol{\beta}$. Não há uma expressão em forma "fechada" para o EMV de $\boldsymbol{\beta}$. Dessa forma, algum procedimento numérico, tal como Newton-Raphson ou escore de Fisher, deve ser usado para se obter $\widehat{\boldsymbol{\beta}}$.

Rieck (1989) aplicando o método escore de Fisher, obteve o seguinte sistema de equações:

$$
\left(\boldsymbol{X}^{\top} \boldsymbol{X}\right) \boldsymbol{\beta}^{(m+1)}=\boldsymbol{X}^{\top} \boldsymbol{y}^{*}
$$

$\mathrm{e}$

$$
\alpha^{(m+1)}=\frac{\alpha^{(m)}}{2}\left(1+\frac{1}{n} \sum_{i=1}^{n} \xi_{i 2}^{2}\right)
$$

em que $\boldsymbol{y}^{*}=\boldsymbol{X} \boldsymbol{\beta}^{(m)}+\left[2 / h\left(\alpha^{(m)}\right)\right] \boldsymbol{s}$ e $m=0,1,2, \ldots$ As quantidades $\xi_{i 1}$ e $\xi_{i 2}$, definidas anteriormente, devem ser avaliadas em $\boldsymbol{\theta}^{(m)}=\left(\boldsymbol{\beta}^{(m) \top}, \alpha^{(m)}\right)^{\top}$. O sistema de equações para $\boldsymbol{\beta}$ tem a mesma forma das 
equações normais de mínimos quadrados ordinários, exceto que estas equações devem ser solucionadas iterativamente, pois $\boldsymbol{y}^{*}$ depende dos parâmetros. Ainda, Rieck (1989) também compara os métodos Newton-Rapshon e escore de Fisher. Para Newton-Raphson, o número de cálculos por iteração cresce com o tamanho da amostra, que não foi o caso do método escore de Fisher. Ele ainda verificou que, em geral, o método Newton-Rapshon requer menos iterações. Além disso, através de um breve estudo de simulação Monte Carlo (considerando modelos com um ou dois parâmetros e tamanhos de amostra 10 e 20), o autor verificou que o método Newton-Rapshon teve desempenho superior ao método escore de Fisher.

O estimador de mínimos quadrados (EMQ) de $\boldsymbol{\beta}$ é dado pela equação clássica $\widetilde{\boldsymbol{\beta}}=\left(\boldsymbol{X}^{\top} \boldsymbol{X}\right)^{-1} \boldsymbol{X}^{\top} \boldsymbol{y}$. Rieck \& Nedelman (1991) verificam que o EMQ de $\boldsymbol{\beta}$ não é tão eficiente quanto o EMV de $\boldsymbol{\beta}$, com base em simulações que comparam a eficiência relativa em pequenas amostras do EMV e EMQ. Porém, quando o valor de $\alpha$ é pequeno $(\alpha<0.5)$, o EMV e o EMQ de $\boldsymbol{\beta}$ apresentaram a mesma eficiência.

Neste trabalho, para encontrar as estimativas de máxima verossimilhança de $\boldsymbol{\beta}$ e $\alpha$, a função de log-verossimilhança dada em (1.9) foi maximizada utilizando o algoritmo de otimização nãolinear BFGS com derivadas analíticas desenvolvido por Broyden, Fletcher, Goldfarf e Shanno (veja, por exemplo, Press, Teulosky, Vetterling \& Flannery, 1992). Este método está implementado na linguagem de programação Ox através da função MaxBFGS. Como valores iniciais foi considerado $\widetilde{\boldsymbol{\beta}}$ para $\boldsymbol{\beta}$ e $\sqrt{\widetilde{\alpha}^{2}}$ para $\alpha$, em que $\widetilde{\alpha}^{2}$ é obtido a partir de $\widehat{\alpha}^{2} \operatorname{com} \widehat{\boldsymbol{\beta}}$ substituido por $\widetilde{\boldsymbol{\beta}}$.

\subsubsection{Distribuição Assintótica}

Seja $\widehat{\boldsymbol{\theta}}=\left(\widehat{\boldsymbol{\beta}}^{\top}, \widehat{\alpha}\right)^{\top}$ o estimador de máxima verossimilhança do vetor $\boldsymbol{\theta}=\left(\boldsymbol{\beta}^{\top}, \alpha\right)^{\top}$. Rieck \& Nedelman (1991) encontraram a distribuição assintótica de $\widehat{\boldsymbol{\theta}}$ adaptando métodos descritos em Chanda (1954) e Bradley \& Gart (1962). Suponha que à medida que o tamanho da amostra $n$ aumenta: (a) para algum $H$ finito, $\left|x_{i j}\right| \leq H$ para todo $i=1,2, \ldots, n$ e $j=1,2, \ldots, p$; (b) para alguma matriz positiva definida $\boldsymbol{M}$,

$$
\lim _{n \rightarrow \infty} \frac{1}{n} \sum_{i=1}^{n} \boldsymbol{x}_{i} \boldsymbol{x}_{i}^{\top}=\boldsymbol{M}
$$

Sob as condições anteriores, Rieck \& Nedelman (1991) mostraram que

$$
\widehat{\boldsymbol{\theta}} \stackrel{A}{\sim} \mathcal{N}_{p+1}\left(\boldsymbol{\theta}, \boldsymbol{K}(\boldsymbol{\theta})^{-1}\right)
$$

em que $\boldsymbol{K}(\boldsymbol{\theta})$ é a matriz de informação de Fisher e $\boldsymbol{K}(\boldsymbol{\theta})^{-1}$ representa sua inversa. Ainda,

$$
\boldsymbol{K}(\boldsymbol{\theta})=\left[\begin{array}{cc}
h(\alpha)\left(\boldsymbol{X}^{\top} \boldsymbol{X}\right) / 4 & \mathbf{0} \\
\mathbf{0} & 2 n / \alpha^{2}
\end{array}\right] \quad \text { e } \quad \boldsymbol{K}(\boldsymbol{\theta})^{-1}=\left[\begin{array}{cc}
4\left(\boldsymbol{X}^{\top} \boldsymbol{X}\right)^{-1} / h(\alpha) & \mathbf{0} \\
\mathbf{0} & \alpha^{2} / 2 n
\end{array}\right],
$$

em que

$$
h(\alpha)=2+\frac{4}{\alpha^{2}}-\frac{\sqrt{2 \pi}}{\alpha}\left\{1-\operatorname{erf}\left(\frac{\sqrt{2}}{\alpha}\right)\right\} \exp \left(\frac{2}{\alpha^{2}}\right) .
$$

Aqui, erf $(\cdot)$ representa a error function, que é da forma

$$
\operatorname{erf}(x)=\frac{2}{\sqrt{\pi}} \int_{0}^{x} \mathrm{e}^{-t^{2}} \mathrm{~d} t
$$




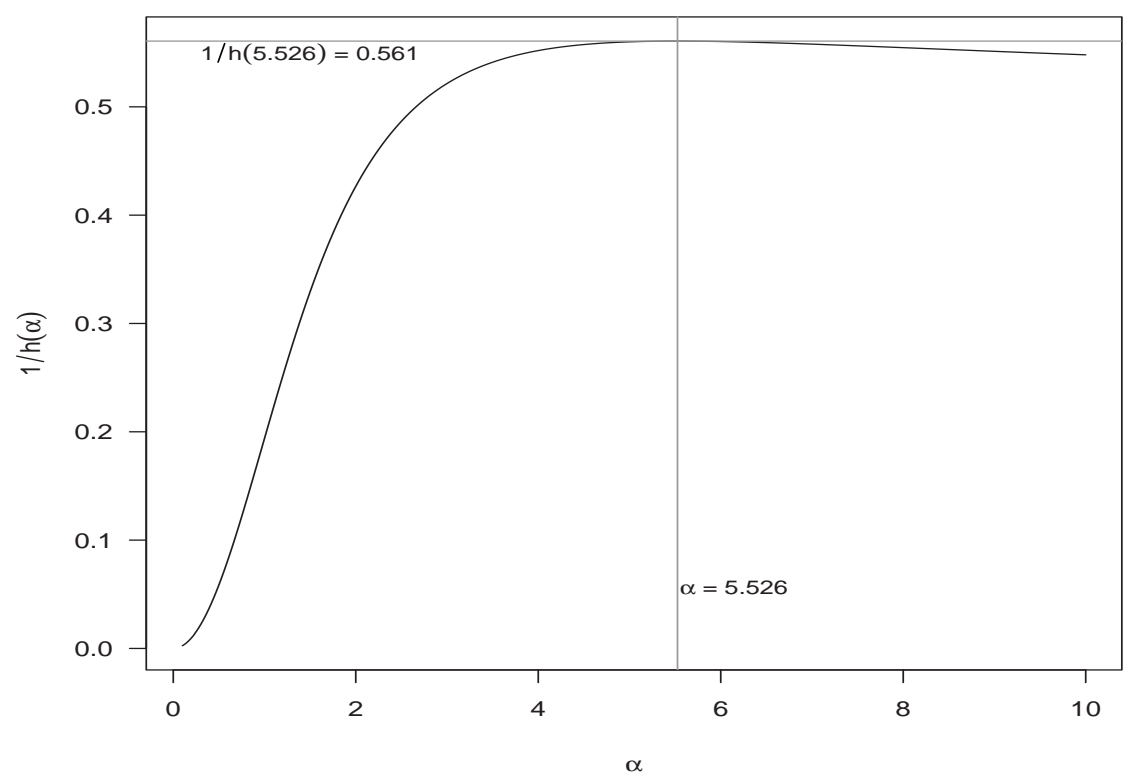

Figura 1.4: Gráfico de $1 / h(\alpha)$ para diferentes valores de $\alpha$.

Detalhes sobre a função erf $(\cdot)$ podem ser encontrados em Gradstein \& Ryshik (2007). Para pequenos valores de $\alpha$, tem-se que $h(\alpha) \approx 1+4 / \alpha^{2}$, e para valores grandes de $\alpha, h(\alpha) \approx 2$ (Rieck, 1989). Na Figura 1.4 apresentamos um gráfico de $1 / h(\alpha)$ considerando diferentes valores para $\alpha$, pois em muitos casos utilizamos $h(\alpha)$ como um divisor. Note que $1 / h(\alpha)$ é uma função crescente até, aproximadamente, $\alpha=5.526$, e uma função decrescente para valores de $\alpha$ maiores que 5.526. Observe ainda que o valor máximo de $1 / h(\alpha)$ é $1 / h(5.526)=0.561$.

Note que a matriz $\boldsymbol{K}(\boldsymbol{\theta})$ é bloco diagonal, ou seja, $\boldsymbol{\beta}$ e $\alpha$ são globalmente ortogonais (Cox \& Reid, 1987), isto é, $\widehat{\boldsymbol{\beta}}$ e $\widehat{\alpha}$ são assintoticamente independentes. Portanto, podemos escrever $\boldsymbol{K}(\boldsymbol{\theta})=$ $\operatorname{diag}\left\{\boldsymbol{K}(\boldsymbol{\beta}), \kappa_{\alpha, \alpha}\right\}$, sendo $\boldsymbol{K}(\boldsymbol{\beta})=h(\alpha)\left(\boldsymbol{X}^{\top} \boldsymbol{X}\right) / 4$ a matriz de informação para $\boldsymbol{\beta}$ e $\kappa_{\alpha, \alpha}=2 n / \alpha^{2}$ a informação para $\alpha$.

Seja $\widetilde{\boldsymbol{X}}$ uma matriz $(n+1) \times(p+1)$ da forma

$$
\widetilde{\boldsymbol{X}}=\left[\begin{array}{cc}
\boldsymbol{X} & \mathbf{0} \\
\mathbf{0} & 1
\end{array}\right]
$$

e seja $\widetilde{\boldsymbol{W}}=\operatorname{diag}\left\{h(\alpha) \boldsymbol{I}_{n} / 4,2 n / \alpha^{2}\right\}$, sendo $\boldsymbol{I}_{n}$ a matriz identidade de ordem $n$. Então, pode-se escrever a matriz de informação total de Fisher para $\boldsymbol{\theta}$ como

$$
\boldsymbol{K}(\boldsymbol{\theta})=\widetilde{\boldsymbol{X}}^{\top} \widetilde{\boldsymbol{W}} \widetilde{\boldsymbol{X}}
$$




\section{Capítulo 2}

\section{Correção de Bartlett}

Testes baseados em aproximações para grandes amostras são frequentementes usados em Estatística, pois testes exatos nem sempre estão disponíveis. Esses testes são denominados "assintóticos de primeira ordem", isto é, são baseados em valores críticos obtidos de uma distribuição nula limite conhecida. Um problema natural que surge é verificar se a aproximação de primeira ordem é adequada para a distribuição nula da estatística de teste em consideração. Os testes assintóticos cujas distribuições de referência são qui-quadrado mais conhecidos são: razão de verossimilhanças, escore e Wald. As estatísticas destes três testes são equivalentes em grandes amostras e, em problemas regulares, convergem, sob a hipótese nula $\mathcal{H}_{0}$, para a distribuição $\chi_{q}^{2}$, onde $q$ é o número de restrições impostas por $\mathcal{H}_{0}$. Em pequenas amostras, a aproximação de primeira ordem pode não ser satisfatória, podendo conduzir a taxas de rejeição sob a hipótese nula bastante distorcidas.

A primeira proposta de melhoria de testes estatísticos foi feita por Bartlett (1937). O autor considerou apenas a estatística da razão de verossimilhanças $(L R)$, calculando seu valor esperado segundo $\mathcal{H}_{0}$ até ordem $O\left(n^{-1}\right)$. Bartlett $(1937,1947,1954)$ obteve fatores de correção na área de Análise Multivariada e estes fatores são usados para melhorar a aproximação qui-quadrado em grandes amostras da distribuição nula da estatística $L R$. Box (1949) usou os resultados de Bartlett (1937) para investigar em detalhes a expressão geral para os momentos da estatística $L R$ em alguns casos.

Em problemas regulares, Lawley (1956) obteve expressões para os momentos de certas derivadas da função de log-verossimilhança e, através de uma demonstração extremamente complicada, apresentou uma fórmula geral para o valor esperado de $L R$ sob a hipótese nula. Além disso, ele mostrou que todos os cumulantes da estatística corrigida pelo fator de correção de Bartlett, para testar uma hipótese nula composta, concordam com os cumulantes da distribuição nula qui-quadrado de referência até ordem $O\left(n^{-1}\right)$.

Uma grande contribuição no melhoramento da estatística $L R$ foi feita por Hayakawa (1977), que obteve uma expansão assintótica da distribuição nula ${ }^{1}$ de $L R$ para testar uma hipótese nula $\mathcal{H}_{0}$ composta contra uma hipótese alternativa $\mathcal{H}_{1}$. Ele mostrou que

$$
\begin{aligned}
\operatorname{Pr}(L R \leq z)= & F_{q}(z)+\frac{1}{24 n}\left[A_{2} F_{q+4}(z)-\left(2 A_{2}-A_{1}\right) F_{q+2}(z)\right. \\
& \left.+\left(A_{2}-A_{1}\right) F_{q}(z)\right]+O\left(n^{-2}\right),
\end{aligned}
$$

em que $F_{s}(\cdot)$ é a função distribuição acumulada de uma variável aleatória qui-quadrado com $s$ graus de

\footnotetext{
${ }^{1}$ Aqui, 'distribuição nula' significa que a distribuição da estatística de teste é calculada sob a hipótese nula.
} 
liberdade. As quantidades $A_{1}$ e $A_{2}$ são funções de alguns cumulantes de certas derivadas da função de log-verossimilhança. O erro em (2.1) é, de fato, $O\left(n^{-2}\right)$ e não $O\left(n^{-3 / 2}\right)$ como é usualmente descrito; veja Barndorff-Nielsen \& Hall (1988) para uma prova deste resultado. Adicionalmente, como o fator de correção de Bartlett é dado por $c=1+A_{1} /(12 n q)$, os resultados de Lawley (1956) e Hayakawa (1977) estão em conflito, a menos que $A_{2}=0 \mathrm{em}$ (2.1). A resposta para este conflito acontece dez anos depois com os artigos de Harris (1986) e Cordeiro (1987); veja também Hayakawa (1987). Harris (1986) mostrou que $A_{2}$ não deve estar presente em (2.1), enquanto Cordeiro (1987) mostrou que $A_{2}$ é igual a zero. Esta discordância foi revisada por Chesher \& Smith (1994). A principal contribuição da equação (2.1) com $A_{2}=0$ é que dela provém uma demonstração relativamente simples de que a estatística corrigida $L R_{b}=L R / c$ tem distribuição $\chi_{q}^{2}$ com erro de ordem $O\left(n^{-2}\right)$ sob a hipótese nula.

Barndorff-Nielsen \& Cox (1984) apresentam um método indireto para calcular correções de Bartlett em modelos paramétricos estabelecendo uma simples conexão entre o fator de correção de Bartlett e a constante normalizadora da expressão geral para a distribuição condicional do estimador de máxima verossimilhança (Barndorff-Nielsen, 1983). Em geral, é mais fácil obter correções de Bartlett usando a fórmula de Lawley (1956) do que a expressão de Barndorff-Nielsen \& Cox (1984), pois a primeira envolve somente momentos de derivadas da função de log-verossimilhança, enquanto a última requer um cálculo exato ou aproximado da distribuição condicional do estimador de máxima verossimilhança.

McCullagh \& Cox (1986), usando o fato de que a estatística da razão de verossimilhanças é invariante sob reparametrização, expressaram o fator de correção de Bartlett em termos de combinações invariantes de cumulantes das primeiras derivadas da função de log-verossimilhança. Adicionalmente, os autores apresentam uma interpretação geométrica para alguns modelos específicos. Além disso, a fórmula geral de McCullagh \& Cox (1986) coincide com a expressão de Lawley (1956). A vantagem da abordagem desses autores é sua interpretação geométrica, enquanto que a principal vantagem da abordagem de Lawley (1956) é que ela pode ser facilmente implementada para obter a correção de Bartlett em casos especiais.

Outro método para obter fatores de correção de Bartlett foi descrito por Barndorff-Nielsen \& Blaesild (1986), que simplifica os cálculos numéricos em situações em que se consideram hipóteses lineares. Entretanto, dentre os métodos descritos até aqui para se obter fatores de correção de Bartlett, o método de Lawley (1956) parece ser o mais atrativo, pois como ressaltam Cribari-Neto \& Cordeiro (1996): "Our experience is that it is usually more convenient to work with Lawley's expression." Dessa forma, utilizaremos o método de Lawley (1956) para obter um fator de correção de Bartlett no modelo de regressão Birnbaum-Saunders.

Vale ressaltar que não existe nenhuma garantia de que a estatística $L R$ corrigida pelo fator de correção de Bartlett para variáveis aleatórias discretas vai produzir um melhoramento na aproximação assintótica pela distribuição qui-quadrado. No entanto, Cordeiro (1982) faz vários estudos de simulação envolvendo as distribuições multinomial e Poisson e verifica que a estatística da razão de verossimilhanças corrigida pelo fator de correção de Bartlett apresentou taxas de rejeição sob a hipótese nula mais próximas dos respectivos níveis nominais do que a estatística da razão de verossimilhanças usual. Por outro lado, Frydenberg \& Jensen (1989) mostraram, através de extensivos cálculos numéricos que, em situações particulares, a correção de Bartlett nem sempre melhora a aproximação assintótica pela distribuição qui-quadrado.

Também é possível usar correções de Bartlett em alguns casos não-paramétricos. Por exemplo, DiCiccio, Hall \& Romano (1991) mostraram que a verossimilhança empírica (Owen, 1988, 1990) é corrigível por Bartlett. Chen \& Hall (1993) estenderam estes resultados considerando verossimi- 
lhança empírica suavizada. Ainda, Bickel \& Ghosh (1990) mostraram que é possível aplicar correções de Bartlett para melhorar inferência bayesiana. Eles mostram que a distribuição a posteriori da estatística da razão de verossimilhanças coincide com a distribuição qui-quadrado de referência com erro de ordem $O\left(n^{-1}\right)$ e que a distribuição posteriori de sua versão corrigida pelo fator de Bartlett é qui-quadrado quando termos de ordem $O\left(n^{-2}\right)$ e menores são negligenciados.

Nos últimos trinta anos houve um grande interesse por parte dos pesquisadores em correções de Bartlett, obtendo expressões em forma fechada para problemas específicos. A seguir, citamos alguns trabalhos nesta área. Cordeiro (1983) derivou uma expressão geral em forma matricial para o fator de correção de Bartlett nos modelos lineares generalizados quando o parâmetro de dispersão é conhecido. Cordeiro (1987) generaliza este resultado considerando o parâmetro de dispersão desconhecido. Cordeiro \& Paula (1989) obtiveram uma expressão matricial geral para o fator de correção de Bartlett nos modelos não-lineares da família exponencial. Cordeiro (1993a) apresenta uma fórmula matricial geral para calcular correções de Bartlett. Attfield (1991) e Cordeiro (1993b) mostram como corrigir a estatística $L R$ em modelos de regressão heteroscedásticos. Wong (1991) obteve um fator de correção de Bartlett para testar algumas inclinações em modelos de regressão cujas variáveis independentes são sujeitas a erros. Um algoritimo para calcular correções de Bartlett foi proposto por Jensen (1993); veja também Andrews \& Stafford (1993) e Stafford \& Andrews (1993).

Cordeiro, Paula \& Botter (1994) derivaram correções de Bartlett na classe de modelos de dispersão propostos por Jørgensen (1987, 1997). Cribari-Neto \& Ferrari (1995a) e Cribari-Neto \& Zarkos (1995) derivaram correções de Bartlett em modelos lineares de regressão heteroscedáticos e regressão multivariada, respectivamente. Uma correção em modelos de regressão com erros $t$-Student foi obtida por Ferrari \& Arellano-Valle (1996). Cordeiro, Cribari-Neto, Aubin \& Ferrari (1995) derivaram correções de Bartlett em modelos da família exponencial uniparamétrica. Eles aplicaram seus resultados em 24 distribuições nesta família, algumas das quais são amplamente usadas em aplicações empíricas em uma variedade de campos do conhecimento.

Cordeiro (2004) obteve um fator de correção de Bartlett em modelos de regressão não-lineares simétricos. Este artigo generaliza os resultados apresentados em Ferrari \& Arellano-Valle (1996) e Ferrari \& Uribe-Opazo (2001). Lagos \& Morettin (2004) obtiveram um fator de correção de Bartlett para a estatística da razão de verossimilhanças em modelos ARMA. Barroso \& Cordeiro (2005) derivaram uma fórmula geral para o fator de correção de Bartlett em modelos de regressão heteroscedásticos com erros $t$-Student quando ambos os parâmetros de locação e dispersão variam com as observações. Este artigo pode ser visto como uma extensão dos resultados em Cordeiro (1993b) e Botter \& Cordeiro (1997). Cordeiro, Cysneiros \& Cysneiros (2006) obtiveram fatores de correção de Bartlett em modelos lineares generalizados com superdispersão, generalizando os resultados de Botter \& Cordeiro (1998) e Cordeiro (1983). Finalmente, uma excelente referência para uma revisão sobre correções de Bartlett é Cribari-Neto \& Cordeiro (1996). Além disso, existe uma home-page na internet, mantida pelo Prof. Dr. Francisco Cribari-Neto da Universidade Federal de Pernambuco (UFPE), destinada a correções de Bartlett e extensões: http://www.de.ufpe.br/ cribari/bartlett.html.

\subsection{Fator de Correção de Bartlett}

Considere um modelo paramétrico $f(\boldsymbol{y} ; \boldsymbol{\theta})$ e seja $\ell(\boldsymbol{\theta})$ a respectiva função de log-verossimilhança, em que $\boldsymbol{\theta}=\left(\boldsymbol{\theta}_{1}^{\top}, \boldsymbol{\theta}_{2}^{\top}\right)^{\top}$ é um vetor de parâmetros desconhecido de dimensão $p$. As dimensões de $\boldsymbol{\theta}_{1}$ e $\boldsymbol{\theta}_{2}$ são $q$ e $p-q$, respectivamente. Suponha que se deseja testar a hipótese nula composta $\mathcal{H}_{0}: \boldsymbol{\theta}_{1}=\boldsymbol{\theta}_{1}^{(0)}$ versus $\mathcal{H}_{1}: \boldsymbol{\theta}_{1} \neq \boldsymbol{\theta}_{1}^{(0)}$, sendo $\boldsymbol{\theta}_{2}$ um vetor de parâmetros de perturbação e $\boldsymbol{\theta}_{1}^{(0)}$ um 
vetor de constantes conhecidas. Seja $L R$ a estatística da razão de verossimilhanças definida por

$$
L R=2\{\ell(\widehat{\boldsymbol{\theta}})-\ell(\widetilde{\boldsymbol{\theta}})\},
$$

em que $\widehat{\boldsymbol{\theta}}=\left(\widehat{\boldsymbol{\theta}}_{1}^{\top}, \widehat{\boldsymbol{\theta}}_{2}^{\top}\right)^{\top}$ e $\widetilde{\boldsymbol{\theta}}=\left(\boldsymbol{\theta}_{1}^{(0) \top}, \widetilde{\boldsymbol{\theta}}_{2}^{\top}\right)^{\top}$ são os estimadores de máxima verossimilhança de $\boldsymbol{\theta}=\left(\boldsymbol{\theta}_{1}^{\top}, \boldsymbol{\theta}_{2}^{\top}\right)^{\top}$ correspondentes às maximizações de $\ell(\boldsymbol{\theta})$ segundo $\mathcal{H}_{1}$ e $\mathcal{H}_{0}$, respectivamente.

Bartlett (1937) calculou o valor esperado de $L R$ segundo $\mathcal{H}_{0}$ até ordem $n^{-1}$ como

$$
\mathbb{E}(L R)=q+B(\boldsymbol{\theta})+O\left(n^{-2}\right),
$$

em que $B(\boldsymbol{\theta})$ é uma constante de ordem $O\left(n^{-1}\right)$ que pode ser estimada segundo a hipótese nula $\mathcal{H}_{0}$. É possível mostrar que, sob a hipótese nula, a estatística da razão de verossimilhanças modificada

$$
L R_{b}=\frac{L R}{1+B(\boldsymbol{\theta}) / q}
$$

tem valor esperado $q$, exceto por termos de ordem $O\left(n^{-2}\right)$. Adicionalmente, enquanto $\operatorname{Pr}(L R \leq z)=$ $\operatorname{Pr}\left(\chi_{q}^{2} \leq z\right)+O\left(n^{-1}\right)$ tem-se o melhoramento $\operatorname{Pr}\left(L R_{b} \leq z\right)=\operatorname{Pr}\left(\chi_{q}^{2} \leq z\right)+O\left(n^{-2}\right)$. O fator de correção

$$
c=1+\frac{B(\boldsymbol{\theta})}{q}
$$

tornou-se conhecido como fator de correção de Bartlett, sendo usado para definir uma estatística da razão de verossimilhanças aperfeiçoada que tem distribuição, segundo a hipótese nula, mais próxima da distribuição $\chi_{q}^{2}$ de referência do que a distribuição da estatística da razão de verossimilhanças $L R$ usual.

Em problemas regulares, Lawley (1956) deduziu uma fórmula geral para $B(\boldsymbol{\theta})$ em termos de cumulantes da função de log-verossimilhança, que são valores esperados de produtos de derivadas da função de log-verossimilhança. Adicionalmente, através de uma demonstração extremamente complicada, Lawley concluiu que os momentos de $L R_{b}$ concordam com os correspondentes momentos da distribuição $\chi_{q}^{2}$ exceto por termos de ordem $O\left(n^{-2}\right)$. Devido a esta complexidade nos cálculos, Lawley faz o seguinte comentário: "We have not, however, been able to discover a better method." Este resultado é muito importante, pois mostra que a simples correção do primeiro momento de $L R$ possibilita obter uma estatística de teste aperfeiçoada, cujos momentos (segundo $\mathcal{H}_{0}$ ) concordam, até termos de ordem $O\left(n^{-1}\right)$, com os correspondentes momentos da distribuição qui-quadrado de referência.

Pode-se escrever $L R$ na equação $(2.2)$ do teste de $\mathcal{H}_{0}: \boldsymbol{\theta}_{1}=\boldsymbol{\theta}_{1}^{(0)}$ versus $\mathcal{H}_{1}: \boldsymbol{\theta}_{1} \neq \boldsymbol{\theta}_{1}^{(0)}$ como

$$
L R=2\{\ell(\widehat{\boldsymbol{\theta}})-\ell(\boldsymbol{\theta})\}-2\{\ell(\widetilde{\boldsymbol{\theta}})-\ell(\boldsymbol{\theta})\},
$$

em que $\ell(\boldsymbol{\theta})$ é a função de log-verossimilhança avaliada no verdadeiro vetor de parâmetros. Lawley (1956) demonstrou que

$$
2 \mathbb{E}[\ell(\widehat{\boldsymbol{\theta}})-\ell(\boldsymbol{\theta})]=p+\epsilon_{p}+O\left(n^{-2}\right),
$$

em que $\epsilon_{p}$ é um termo de ordem $n^{-1}$ dado por

$$
\epsilon_{p}=\sum^{\prime}\left(\lambda_{r s t u}-\lambda_{\text {rstuvw }}\right)
$$


sendo $\sum^{\prime}$ o somatório sobre todos os componentes do vetor $\boldsymbol{\theta}$, isto é, os índices $r, s, t, u, v$ e $w$ variam sobre todos os $p$ parâmetros, e os $\lambda$ 's são dados por

$$
\begin{aligned}
\lambda_{r s t u} & =\kappa^{r s} \kappa^{t u}\left\{\frac{\kappa_{r s t u}}{4}-\kappa_{r s t}^{(u)}+\kappa_{r t}^{(s u)}\right\}, \\
\lambda_{r s t u v w} & =\kappa^{r s} \kappa^{t u} \kappa^{v w}\left\{\kappa_{r t v}\left(\frac{\kappa_{s u w}}{6}-\kappa_{s w}^{(u)}\right)+\kappa_{r t u}\left(\frac{\kappa_{s v w}}{4}-\kappa_{s w}^{(v)}\right)+\kappa_{r t}^{(v)} \kappa_{s w}^{(u)}+\kappa_{r t}^{(u)} \kappa_{s w}^{(v)}\right\},
\end{aligned}
$$

a partir dos cumulantes $\kappa$ 's definidos na Seção 1.1.2. Lawley (1956) demonstrou também que

$$
2 \mathbb{E}[\ell(\widetilde{\boldsymbol{\theta}})-\ell(\boldsymbol{\theta})]=p-q+\epsilon_{p-q}+O\left(n^{-2}\right),
$$

em que $\epsilon_{p-q}$ é um termo de ordem $O\left(n^{-1}\right)$ sendo deduzido da equação (2.3) com o somatório $\sum^{\prime}$ estendendo-se apenas sobre as componentes de $\boldsymbol{\theta}_{2}$, isto é, sobre os $p-q$ parâmetros de perturbação, uma vez que $\boldsymbol{\theta}_{1}$ está fixado em $\mathcal{H}_{0}$.

Dessa forma, segundo a hipótese nula $\mathcal{H}_{0}$, o valor esperado da estatística da razão de verossimilhanças é dado por

$$
\mathbb{E}(L R)=q+\epsilon_{p}-\epsilon_{p-q}+O\left(n^{-2}\right) .
$$

Assim, pode-se melhorar a aproximação da estatística de teste pela distribuição $\chi_{q}^{2}$ utilizando $L R_{b}=$ $L R / c$ em vez de $L R$, sendo o fator de correção de Bartlett dado por

$$
c=1+\frac{\epsilon_{p}-\epsilon_{p-q}}{q}
$$

isto é, $c=1+B(\boldsymbol{\theta}) / q$, em que $B(\boldsymbol{\theta})=\epsilon_{p}-\epsilon_{p-q}$. A estatística corrigida $L R_{b}$ tem distribuição $\chi_{q}^{2}$ até ordem $O\left(n^{-1}\right)$ sob $\mathcal{H}_{0}$. O teste aperfeiçoado compara $L R_{b}$ com o quantil apropriado da distribuição $\chi_{q}^{2}$ de referência. A dificuldade do aperfeiçoamento reside no cálculo de $\epsilon_{p}$ e $\epsilon_{p-q}$ a partir das equações (2.3) e (2.4). Quando o fator de correção de Bartlett depender de parâmetros desconhecidos, estes devem ser substituídos por suas respectivas estimativas de máxima verossimilhança segundo $\mathcal{H}_{0}$, o que não afeta a ordem da aproximação resultante. O inconveniente no uso da fórmula de Lawley (2.5), na prática, é o cálculo do grande número de produtos de cumulantes em testes envolvendo três ou mais parâmetros.

A estatística corrigida pelo fator de correção de Bartlett é usualmente definida como $L R_{b}=$ $L R /\{1+B(\boldsymbol{\theta}) / q\}$. Entretanto, existem algumas definições alternativas à correção de Bartlett que são equivalentes até ordem $O\left(n^{-1}\right)$. Por exemplo, $L R_{b}^{1}=L R \exp \{-B(\boldsymbol{\theta}) / q\}$ e $L R_{b}^{2}=L R\{1-B(\boldsymbol{\theta}) / q\}$ são equivalentes a $L R_{b}$ se termos de ordens menores que $O\left(n^{-1}\right)$ são ignorados. Note que $L R_{b}^{1}$ tem a vantagem de ser uma estatística corrigida que sempre assume valores positivos.

Vale ressaltar que o primeiro pesquisador a utilizar a metodologia de Lawley (1956) foi o brasileiro Gauss M. Cordeiro durante o desenvolvimento de sua tese de doutorado na Inglaterra. Um artigo pioneiro que utiliza esta metodologia é Cordeiro (1983), que obteve uma fórmula matricial geral para o fator de correção de Bartlett em modelos lineares generalizados.

\subsection{Correção de Bartlett no Modelo de Regressão Birnbaum-Saunders}

\subsubsection{Obtenção da Quantidade $\epsilon$}

Nós derivamos a seguir uma expressão geral para o fator de correção de Bartlett no modelo de regressão Birnbaum-Saunders. O vetor de parâmetros $\boldsymbol{\theta}=\left(\boldsymbol{\beta}^{\top}, \alpha\right)^{\top}$ é $(p+1)$-dimensional, dessa 
forma, devemos encontrar $\epsilon_{p+1}$ a partir da expressão (2.3), com os índices daquela expressão variando de 1 até $p+1$. Devido à ortogonalidade entre $\boldsymbol{\beta}$ e $\alpha$ (veja Seção 1.3), muitos cálculos são simplificados.

Sejam as matrizes $\boldsymbol{Z}=\boldsymbol{X}\left(\boldsymbol{X}^{\top} \boldsymbol{X}\right)^{-1} \boldsymbol{X}^{\top}=\left\{z_{i j}\right\}, \boldsymbol{Z}_{d}=\operatorname{diag}\left\{z_{11}, z_{22}, \ldots, z_{n n}\right\}$ e considere $\boldsymbol{Z}^{(2)}=$

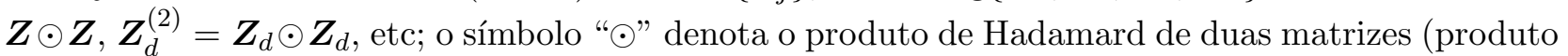
direto). Após um longo trabalho algébrico, veja Apêndice B, obtemos

$$
\epsilon_{p+1}=\epsilon(\alpha, p, \boldsymbol{X})=\epsilon_{\alpha}(\alpha, p)+\epsilon_{\boldsymbol{\beta}}(\alpha, \boldsymbol{X}),
$$

com

$$
\epsilon_{\alpha}(\alpha, p)=\frac{1}{n}\left\{\frac{1}{3}+\delta_{1}(\alpha) p+\delta_{2}(\alpha) p^{2}\right\} \quad \text { e } \quad \epsilon_{\boldsymbol{\beta}}(\alpha, \boldsymbol{X})=\delta_{3}(\alpha) \operatorname{tr}\left(\boldsymbol{Z}_{d}^{(2)}\right),
$$

em que $\operatorname{tr}(\cdot)$ representa o operador traço de uma matriz e

$$
\begin{gathered}
\delta_{0}(\alpha)=\frac{2+\alpha^{2}}{\psi_{1}(\alpha) \alpha^{2}}, \quad \delta_{1}(\alpha)=4 \delta_{0}(\alpha)\left\{\frac{2}{2+\alpha^{2}}+\delta_{0}(\alpha)-\frac{2 \alpha \psi_{3}(\alpha)}{\psi_{1}(\alpha)}\right\}, \\
\delta_{2}(\alpha)=2 \delta_{0}(\alpha)^{2}, \quad \delta_{3}(\alpha)=\frac{4 \psi_{2}(\alpha)}{\psi_{1}(\alpha)^{2}}, \quad \psi_{0}(\alpha)=\left\{1-\operatorname{erf}\left(\frac{\sqrt{2}}{\alpha}\right)\right\} \exp \left(\frac{2}{\alpha^{2}}\right), \\
\psi_{1}(\alpha)=2+\frac{4}{\alpha^{2}}-\frac{\sqrt{2 \pi}}{\alpha} \psi_{0}(\alpha), \quad \psi_{2}(\alpha)=-\frac{1}{4}\left\{2+\frac{7}{\alpha^{2}}-\sqrt{\frac{\pi}{2}}\left(\frac{1}{2 \alpha}+\frac{6}{\alpha^{3}}\right) \psi_{0}(\alpha)\right\}
\end{gathered}
$$

$\mathrm{e}$

$$
\psi_{3}(\alpha)=\frac{3}{\alpha^{3}}-\frac{\sqrt{2 \pi}}{4 \alpha^{2}}\left(1+\frac{4}{\alpha^{2}}\right) \psi_{0}(\alpha)
$$

Nós escrevemos $\epsilon_{p+1}$ como a soma de dois termos, $\epsilon_{\alpha}(\alpha, p)$ e $\epsilon_{\boldsymbol{\beta}}(\alpha, \boldsymbol{X})$. A quantidade $\epsilon_{\boldsymbol{\beta}}(\alpha, \boldsymbol{X})$ refere-se à contribuição do parâmetro $\boldsymbol{\beta}$, isto é, como se $\alpha$ fosse conhecido. A quantidade $\epsilon_{\alpha}(\alpha, p)$ é a contribuição adicional produzida pelo fato do parâmetro $\alpha$ ser desconhecido. Note que $\epsilon_{\alpha}(\alpha, p)$ depende da matriz modelo somente através do seu posto. Mais especificamente, ela é um polinômio de segundo grau em $p$ dividido por $n$. Assim, $\epsilon_{\alpha}(\alpha, p)$ pode ser não negligível se a dimensão do vetor de parâmetros $\boldsymbol{\beta}$ não é consideravelmente menor que o tamanho da amostra. Observe também que $\epsilon(\alpha, p, \boldsymbol{X})$ depende de $\alpha$ mas não de $\boldsymbol{\beta}$. A dependência de $\epsilon(\alpha, p, \boldsymbol{X})$ sobre $\alpha$ ocorre através de $\delta_{1}(\alpha)$, $\delta_{2}(\alpha)$ e $\delta_{3}(\alpha)$. Adicionalmente, $\operatorname{tr}\left(\boldsymbol{Z}_{d}^{(2)}\right)$ estabelece a dependência de $\epsilon(\alpha, p, \boldsymbol{X})$ sobre $\boldsymbol{X}$. Em outras palavras, $\epsilon(\alpha, p, \boldsymbol{X})$ depende da soma de quadrados dos elementos da diagonal da matriz "chapéu" $\boldsymbol{Z}$. Em particular, se $p=1$, ou seja, $\boldsymbol{X}$ tem uma simples coluna, $\boldsymbol{x}=\left(x_{1}, \ldots, x_{n}\right)^{\top}$ digamos, $\operatorname{tr}\left(\boldsymbol{Z}_{d}^{(2)}\right)=\sum_{i=1}^{n} x_{i}^{4} /\left(\sum_{i=1}^{n} x_{i}^{2}\right)^{2}$, a curtose amostral de $\boldsymbol{x}$.

Observe que a expressão (2.6) é muito simples e pode ser facilmente implementada em qualquer linguagem de programação que execute operações matriciais, tais como $\mathrm{R}$ ( $\mathrm{R}$ Development Core Team, 2008), Ox (Cribari-Neto \& Zarkos, 2003; Doornik, 2006), MAPLE (Abell \& Braselton, 1994), entre outras.

\subsubsection{Alguns Casos Especiais}

Nesta seção apresentamos fatores de correções de Bartlett em testes de hipóteses sobre o vetor de parâmetros $\boldsymbol{\theta}=\left(\boldsymbol{\beta}^{\top}, \alpha\right)^{\top}$ que são de interesse prático. A partir da expressão geral dada em (2.6), torna-se muito simples obter estes fatores de correções para o teste de algumas hipóteses particulares. 


\section{Hipótese Sobre $\alpha$}

Estamos interessados em testar a hipótese

$$
\mathcal{H}_{0}: \alpha=\alpha^{(0)} \text { contra } \mathcal{H}_{1}: \alpha \neq \alpha^{(0)},
$$

em que $\alpha^{(0)}$ é uma constante positiva conhecida e $\boldsymbol{\beta}$ um vetor de parâmetros de perturbação. Neste caso, o fator de correção de Bartlett é dado por $c=1+B(\boldsymbol{\theta})$, em que $B(\boldsymbol{\theta})=\epsilon(\alpha, p, \boldsymbol{X})-\epsilon_{\boldsymbol{\beta}}(\alpha, \boldsymbol{X})$, e assim, $B(\boldsymbol{\theta})=\epsilon_{\alpha}(\alpha, p)$. Observe que o fator de correção só depende da matriz modelo $\boldsymbol{X}$ através de seu posto. Em particular, quando $p=1$ (caso i.i.d.), temos

$$
B(\boldsymbol{\theta})=\frac{1}{n}\left\{\frac{1}{3}+\delta_{1}(\alpha)+\delta_{2}(\alpha)\right\}
$$

Essa fórmula corrige a equação (14) em Lemonte, Cribari-Neto \& Vasconcellos (2007), que contém um erro.

\section{Hipótese Sobre $\beta$}

Estamos interessados em testar a hipótese

$$
\mathcal{H}_{0}: \boldsymbol{\beta}=\boldsymbol{\beta}^{(0)} \text { contra } \mathcal{H}_{1}: \boldsymbol{\beta} \neq \boldsymbol{\beta}^{(0)},
$$

em que $\boldsymbol{\beta}^{(0)}$ é um vetor $p$-dimensional de constantes conhecidas e $\alpha$ um parâmetro de perturbação. Neste caso, o fator de correção de Bartlett é dado por $c=1+B(\boldsymbol{\theta}) / p$, em que $B(\boldsymbol{\theta})=\epsilon(\alpha, p, \boldsymbol{X})-$ $\epsilon_{\alpha}(\alpha, 0)$, que produz

$$
B(\boldsymbol{\theta})=\frac{1}{n}\left\{\delta_{1}(\alpha) p+\delta_{2}(\alpha) p^{2}\right\}+\delta_{3}(\alpha) \operatorname{tr}\left(\boldsymbol{Z}_{d}^{(2)}\right) .
$$

\section{Hipótese Sobre um Subconjunto de $\beta$}

Na maioria dos problemas práticos, as restrições em um teste envolvem um subconjunto do vetor de parâmetros $\boldsymbol{\beta}$; por exemplo, pode-se estar interessado em saber se um subconjunto de variáveis explicativas (independentes) são significativas no modelo. Particionando $\boldsymbol{\beta}$ da forma $\boldsymbol{\beta}=\left(\boldsymbol{\beta}_{1}^{\top}, \boldsymbol{\beta}_{2}^{\top}\right)^{\top}$, em que $\boldsymbol{\beta}_{1}=\left(\beta_{1}, \beta_{2}, \ldots, \beta_{p-q}\right)^{\top}$ e $\boldsymbol{\beta}_{2}=\left(\beta_{p-q+1}, \beta_{p-q+2}, \ldots, \beta_{p}\right)^{\top}$ são vetores de dimensão $(p-q) \times 1$ e $q \times 1$, respectivamente, o interesse é testar a hipótese

$$
\mathcal{H}_{0}: \boldsymbol{\beta}_{2}=\boldsymbol{\beta}_{2}^{(0)} \quad \text { contra } \quad \mathcal{H}_{1}: \boldsymbol{\beta}_{2} \neq \boldsymbol{\beta}_{2}^{(0)},
$$

em que $\boldsymbol{\beta}_{2}^{(0)}$ é um vetor $q$-dimensional de constantes conhecidas. Em geral, em aplicações práticas, considera-se $\boldsymbol{\beta}_{2}^{(0)}=\mathbf{0}$. Neste caso, $\boldsymbol{\beta}_{1}$ e $\alpha$ são parâmetros de perturbação. A hipótese anterior induz a uma partição da matriz modelo da forma $\boldsymbol{X}=\left[\begin{array}{ll}\boldsymbol{X}_{1} & \boldsymbol{X}_{2}\end{array}\right]$, em que as dimensões de $\boldsymbol{X}_{1}$ e $\boldsymbol{X}_{2}$ são $n \times(p-q)$ e $n \times q$, respectivamente, ambas as matrizes sendo de posto completo.

Sejam as matrizes $\boldsymbol{Z}_{1}=\boldsymbol{X}_{1}\left(\boldsymbol{X}_{1}^{\top} \boldsymbol{X}_{1}\right)^{-1} \boldsymbol{X}_{1}^{\top}=\left\{z_{1 i j}\right\}$ e $\boldsymbol{Z}_{1 d}=\operatorname{diag}\left\{z_{111}, z_{122}, \ldots, z_{1 n n}\right\}$. O fator de correção de Bartlett é dado por $c=1+B(\boldsymbol{\theta}) / q$, em que $B(\boldsymbol{\theta})=\epsilon(\alpha, p, \boldsymbol{X})-\epsilon\left(\alpha, p-q, \boldsymbol{X}_{1}\right)$. É fácil ver que

$$
B(\boldsymbol{\theta})=\frac{1}{n}\left\{\delta_{1}(\alpha) q+\delta_{2}(\alpha) q(2 p-q)\right\}+\delta_{3}(\alpha) \operatorname{tr}\left(\boldsymbol{Z}_{d}^{(2)}-\boldsymbol{Z}_{1 d}^{(2)}\right) .
$$




\section{$2.3 \quad$ Resultados Numéricos}

Apresentamos a seguir alguns resultados de simulações Monte Carlo para avaliar a eficácia da correção de Bartlett para o teste da razão de verossimilhanças no modelo de regressão BirnbaumSaunders. Comparamos os desempenhos de três testes, o teste da razão de verossimilhanças usual, $L R$, o teste da razão de verossimilhanças corrigido pelo fator de correção de Bartlett, $L R_{b}$, e uma das suas versões assintoticamente equivalentes, $L R_{b}^{1}$, uma vez que esta versão corrigida sempre assume valores positivos. Não apresentamos os resultados referentes ao teste $L R_{b}^{2}$, pois suas taxas de rejeição empíricas foram muito similares às do teste $L R_{b}^{1}$. Vale recordar que $L R_{b}=L R /\{1+B(\boldsymbol{\theta}) / q\}$ e $L R_{b}^{1}=L R \exp \{-B(\boldsymbol{\theta}) / q\}$. O desempenho de cada teste é avaliado em função da proximidade das probabilidades de rejeição da hipótese nula, sendo esta verdadeira (probabilidade do erro tipo I), aos respectivos níveis nominais dos testes. As simulações realizadas foram baseadas no modelo de regressão

$$
y_{i}=\beta_{1} x_{i 1}+\beta_{2} x_{i 2}+\cdots+\beta_{p} x_{i p}+\varepsilon_{i}
$$

em que $x_{i 1}=1$ e $\varepsilon_{i} \sim \mathcal{S N}(\alpha, 0,2), i=1,2, \ldots, n$. As covariáveis foram geradas como amostras aleatórias da distribuição $\mathcal{U}(0,1)$. Estas covariáveis foram mantidas fixas no esquema Monte Carlo.

O número de réplicas Monte Carlo foi fixado em 10000 e foram considerados os seguintes níveis nominais: $\gamma=10 \%, 5 \%$ e $1 \%$. Todas as simulações foram realizadas usando a linguagem de programação matricial 0x (Doornik, 2006). ${ }^{2}$ Para cada tamanho amostral e cada nível considerado, calculamos as taxas de rejeição de cada teste, isto é, estimamos, via simulação, $\operatorname{Pr}\left(L R>z_{\gamma}\right)$, $\operatorname{Pr}\left(L R_{b}>z_{\gamma}\right)$ e $\operatorname{Pr}\left(L R_{b}^{1}>z_{\gamma}\right)$, em que $z_{\gamma}$ é o quantil $1-\gamma$ apropriado da distribuição qui-quadrado de referência. Todas as entradas das tabelas apresentadas são porcentagens.

$\mathrm{Na}$ Tabela 2.1 apresentamos as taxas de rejeição empíricas considerando a hipótese nula $\mathcal{H}_{0}$ : $\beta_{p-1}=\beta_{p}=0$, tamanho de amostra $n=30$ e $\alpha=0.5$. Adicionalmente, diferentes valores para $p$ foram considerados. Variamos $p$ para analisar o efeito do número de covariáveis presentes no modelo sobre os diferentes testes. Ainda, sem perda de generalidade, a variável resposta foi gerada assumindo que $\beta_{1}=\beta_{2}=\cdots=\beta_{p-2}=1$. Observe que o teste da razão de verossimilhanças usual, $L R$, é extremamente liberal, isto é, suas taxas de rejeição estão muito acima dos níveis de significância considerados, principalmente quando o número de regressores presentes no modelo aumenta. Por exemplo, quando $p=8$ e $\gamma=10 \%$, a taxa de rejeição do teste foi $18.78 \%$, quase o dobro do nível de significância considerado, ou seja, uma distorção de tamanho considerável. A tendência do teste de rejeitar com frequência demasiada a hipótese nula é atenuada pela correção de Bartlett de forma que o teste da razão de verossimilhanças corrigido, $L R_{b}$, e sua versão assintoticamente equivalente, $L R_{b}^{1}$, apresentam apenas uma leve distorção de tamanho. Por exemplo, as taxas de rejeição foram $11.82 \%$ e $11.13 \%$, para os testes $L R_{b}$ e $L R_{b}^{1}$, respectivamente, considerando a mesma situação anterior.

Na Tabela 2.2, fixamos o valor de $p$ em 6 e variamos o tamanho de amostra em $n=20,30,40$, 50, 100 e 200, considerando $\alpha=0.5$. Neste caso, a hipótese nula é dada por $\mathcal{H}_{0}: \beta_{5}=\beta_{6}=0$. Com o aumento do tamanho da amostra, as taxas de rejeição empíricas dos três testes se aproximam do nível nominal, como esperado. Observe também que, até mesmo para $n=100$, o teste da razão de verossimilhanças usual apresenta taxas de rejeição acima dos níveis considerados. Quando $n=50$ e $\gamma=5 \%$, as taxas de rejeição empíricas dos testes $L R, L R_{b}$ e $L R_{b}^{1}$ foram $7.49 \%, 5.32 \%$ e $5.17 \%$, respectivamente. É claro que $n=30$ equivale ao caso da Tabela 2.1 quando $p=6$. Observe ainda que com o aumento do tamanho da amostra, as taxas de rejeição empíricas dos testes $L R_{b}$ e $L R_{b}^{1}$ ficam muito próximas, evidenciando a equivalência assintótica destes dois testes. É claro que, na realidade,

\footnotetext{
${ }^{2}$ Veja http://www.doornik. com para mais detalhes sobre esta linguagem de programação.
} 
Tabela 2.1: Taxas de rejeição nulas dos testes $L R, L R_{b}$ e $L R_{b}^{1} \operatorname{com} \alpha=0.5$ e $n=30$.

\begin{tabular}{c|ccc|ccc|ccc}
\hline \multirow{3}{*}{$p$} & \multicolumn{3}{|c|}{$\gamma=10 \%$} & \multicolumn{3}{c|}{$\gamma=5 \%$} & \multicolumn{3}{c}{$\gamma=1 \%$} \\
\cline { 2 - 10 } & $L R$ & $L R_{b}$ & $L R_{b}^{1}$ & $L R$ & $L R_{b}$ & $L R_{b}^{1}$ & $L R$ & $L R_{b}$ & $L R_{b}^{1}$ \\
\hline 3 & 12.69 & 10.36 & 10.22 & 6.51 & 4.98 & 4.90 & 1.75 & 1.25 & 1.23 \\
4 & 13.44 & 10.27 & 10.07 & 7.46 & 5.41 & 5.32 & 1.90 & 1.10 & 1.04 \\
5 & 14.77 & 10.74 & 10.45 & 8.25 & 5.53 & 5.31 & 2.21 & 1.18 & 1.14 \\
6 & 15.94 & 11.07 & 10.53 & 9.14 & 5.55 & 5.23 & 2.54 & 1.24 & 1.17 \\
7 & 17.28 & 11.55 & 10.88 & 10.13 & 5.69 & 5.42 & 2.95 & 1.29 & 1.19 \\
8 & 18.78 & 11.82 & 11.13 & 11.15 & 6.44 & 5.83 & 3.38 & 1.48 & 1.31 \\
9 & 19.92 & 12.11 & 11.00 & 12.00 & 6.33 & 5.66 & 3.82 & 1.49 & 1.25 \\
\hline
\end{tabular}

Tabela 2.2: Taxas de rejeição nulas dos testes $L R, L R_{b}$ e $L R_{b}^{1}$ com $\alpha=0.5, p=6$ e diferentes tamanhos amostrais.

\begin{tabular}{c|ccc|ccc|ccc}
\hline \multirow{3}{*}{$n$} & \multicolumn{3}{|c|}{$\gamma=10 \%$} & \multicolumn{3}{c|}{$\gamma=5 \%$} & \multicolumn{3}{c}{$\gamma=1 \%$} \\
\cline { 2 - 10 } & $L R$ & $L R_{b}$ & $L R_{b}^{1}$ & $L R$ & $L R_{b}$ & $L R_{b}^{1}$ & $L R$ & $L R_{b}$ & $L R_{b}^{1}$ \\
\hline 20 & 19.54 & 12.04 & 11.08 & 11.97 & 6.54 & 5.87 & 4.05 & 1.58 & 1.38 \\
30 & 15.94 & 11.07 & 10.53 & 9.14 & 5.55 & 5.23 & 2.54 & 1.24 & 1.17 \\
40 & 13.57 & 10.14 & 9.97 & 7.45 & 4.99 & 4.81 & 1.79 & 1.03 & 1.01 \\
50 & 13.36 & 10.72 & 10.51 & 7.49 & 5.32 & 5.17 & 1.51 & 1.02 & 0.99 \\
100 & 11.86 & 10.46 & 10.44 & 5.90 & 4.92 & 4.88 & 1.25 & 1.04 & 1.03 \\
200 & 10.92 & 10.14 & 10.12 & 5.57 & 5.07 & 5.07 & 1.04 & 0.96 & 0.96 \\
\hline
\end{tabular}

os três testes considerados são equivalentes assintoticamente, ou seja, para $n$ suficientemente grande, os resultados destes testes serão praticamente os mesmos, porém, como mostrado na Tabela 2.2, no caso do modelo de regressão Birnbaum-Saunders, precisamos de um tamanho amostral maior que 200 para que esta equivalência se verifique. Entretanto, na prática, um tamanho amostral desta magnitude raramente acontece, surgindo a necessidade de se corrigir a estatística da razão de verossimilhanças usual para que, em amostras de tamanho pequeno ou moderado, haja uma diminuição nas distorções de tamanho do teste.

Na Tabela 2.3 apresentamos as médias e variâncias estimadas das estatísticas $L R, L R_{b}$ e $L R_{b}^{1}$. Observe que as médias e variâncias de $L R_{b}$ e $L R_{b}^{1}$ estão mais próximas da média e variância da distribuição qui-quadrado de referência do que as médias e variâncias da estatística $L R$. Na Tabela 2.4 comparamos os quantis amostrais das estatísticas $L R, L R_{b}$ e $L R_{b}^{1}$ com os da distribuição $\chi_{2}^{2}$. Consideramos $n=30, p=6$ e $\alpha=0.5$. Observe que os quantis amostrais de $L R$ estão bem acima dos quantis da distribuição $\chi_{2}^{2}$, enquanto os quantis amostrais das estatísticas corrigidas pelo fator de correção de Bartlett estão muito próximos destes quantis $\chi_{2}^{2}$ de referência. Por exemplo, para o quantil $95 \%$, caso em que o quantil da $\chi_{2}^{2}$ vale 6.0 , os quantis amostrais de $L R, L R_{b}$ e $L R_{b}^{1}$ foram 7.43, 6.20 e 6.09 , respectivamente.

Na Figura 2.1 apresentamos gráficos dos quantis das estatísticas $L R$ (linha tracejada), $L R_{b}$ (linha ponto) e $L R_{b}^{1}$ (linha ponto traço) versus os quantis assintóticos. Consideramos $p=5, \alpha=0.5$, diferentes tamanhos amostrais e como hipótese nula $\mathcal{H}_{0}: \beta_{4}=\beta_{5}=0$, ou seja, os quantis assintóticos 
Tabela 2.3: Médias e variâncias (Monte Carlo) das estatísticas $L R, L R_{b}$ e $L R_{b}^{1}$, considerando $p=6, \alpha=0.5$ e diferentes tamanhos amostrais $\left(\mathcal{H}_{0}: \beta_{5}=\beta_{6}=0\right)$.

\begin{tabular}{c|cc|cc|cc|cc}
\hline \multirow{2}{*}{$n$} & \multicolumn{2}{|c|}{$\chi_{2}^{2}$} & \multicolumn{2}{c|}{$L R$} & \multicolumn{2}{c|}{$L R_{b}$} & \multicolumn{2}{c}{$L R_{b}^{1}$} \\
\cline { 2 - 9 } & $\mathrm{E}\left(\chi_{2}^{2}\right)$ & $\operatorname{Var}\left(\chi_{2}^{2}\right)$ & $\mathrm{E}(L R)$ & $\operatorname{Var}(L R)$ & $\mathrm{E}\left(L R_{b}\right)$ & $\operatorname{Var}\left(L R_{b}\right)$ & $\mathrm{E}\left(L R_{b}^{1}\right)$ & $\operatorname{Var}\left(L R_{b}^{1}\right)$ \\
\hline 20 & 2.0 & 4.0 & 2.84 & 8.18 & 2.19 & 4.86 & 2.11 & 4.51 \\
30 & 2.0 & 4.0 & 2.51 & 6.42 & 2.10 & 4.47 & 2.06 & 4.32 \\
40 & 2.0 & 4.0 & 2.33 & 5.37 & 2.03 & 4.07 & 2.01 & 3.99 \\
50 & 2.0 & 4.0 & 2.30 & 5.18 & 2.05 & 4.14 & 2.04 & 4.09 \\
100 & 2.0 & 4.0 & 2.13 & 4.47 & 2.01 & 3.98 & 2.01 & 3.97 \\
\hline
\end{tabular}

Tabela 2.4: Quantis $\chi_{2}^{2}$ e das distribuições nulas de $L R, L R_{b}$ e $L R_{b}^{1}$, considerando $p=6, \alpha=0.5$ e $n=30$ $\left(\mathcal{H}_{0}: \beta_{5}=\beta_{6}=0\right)$.

\begin{tabular}{c|cccc}
\hline quantis (\%) & $\chi_{2}^{2}$ & $L R$ & $L R_{b}$ & $L R_{b}^{1}$ \\
\hline 80.0 & 3.2 & 4.03 & 3.36 & 3.30 \\
85.0 & 3.8 & 4.72 & 3.94 & 3.87 \\
90.0 & 4.6 & 5.73 & 4.78 & 4.70 \\
95.0 & 6.0 & 7.43 & 6.20 & 6.09 \\
97.0 & 7.0 & 8.74 & 7.29 & 7.16 \\
99.0 & 9.2 & 11.48 & 9.59 & 9.43 \\
99.5 & 10.6 & 13.18 & 11.00 & 10.81 \\
\hline
\end{tabular}

referem-se aos quantis da distribuição qui-quadrado com dois graus de liberdade. A linha cheia representa a identidade, ou seja, os quantis assintóticos são iguais aos quantis exatos. Observe que a estatística do teste da razão de verossimilhanças usual $L R$ apresenta quantis bem superiores aos quantis da distribuição qui-quadrado, principalmente quando o tamanho da amostra é pequeno. Porém, à medida que o tamanho da amostra aumenta, os quantis da estatística $L R$ tendem aos quantis da distribuição qui-quadrado, ou seja, a linha tracejada aproxima-se da linha sólida. Note ainda que a estatística corrigida pelo fator de correção de Bartlett $L R_{b}$ e sua versão assintoticamente equivalente $L R_{b}^{1}$ apresentam quantis bem próximos dos quantis da distribuição qui-quadrado em todos os tamanhos de amostra, evidenciando que as distribuições nulas das estatísticas $L R_{b}$ e $L R_{b}^{1}$ são bem aproximadas pela distribuição assintótica utilizada no teste.

Na Figura 2.2 apresentamos um gráfico dos quantis assintóticos versus a discrepância relativa dos quantis. A discrepância relativa é definida como a diferença entre o quantil exato (estimado via simulação) e o quantil assintótico dividido por este último. Consideramos a hipótese nula $\mathcal{H}_{0}: \beta_{5}=$ $\beta_{6}=0$, ou seja, os quantis assintóticos referem-se aos quantis da distribuição qui-quadrado com dois graus de liberade. Quanto mais próxima de zero estiver a discrepância, melhor a aproximação da distribuição exata sob a hipótese nula da estatística de teste pela distribuição limite $\chi_{2}^{2}$. Observe que as discrepâncias relativas com base na estatística $L R$ estão em torno de $25 \%$, isto é, o teste é demasiadamente liberal, evidenciando que a distribuição de $L R$ sob a hipótese nula não está sendo bem aproximada pela distribuição assintótica utilizada no teste. Por outro lado, as discrepâncias com base em $L R_{b}$ e $L R_{b}^{1}$ estão em torno de $5 \%$, evidenciando que as distribuições sob a hipótese nula 

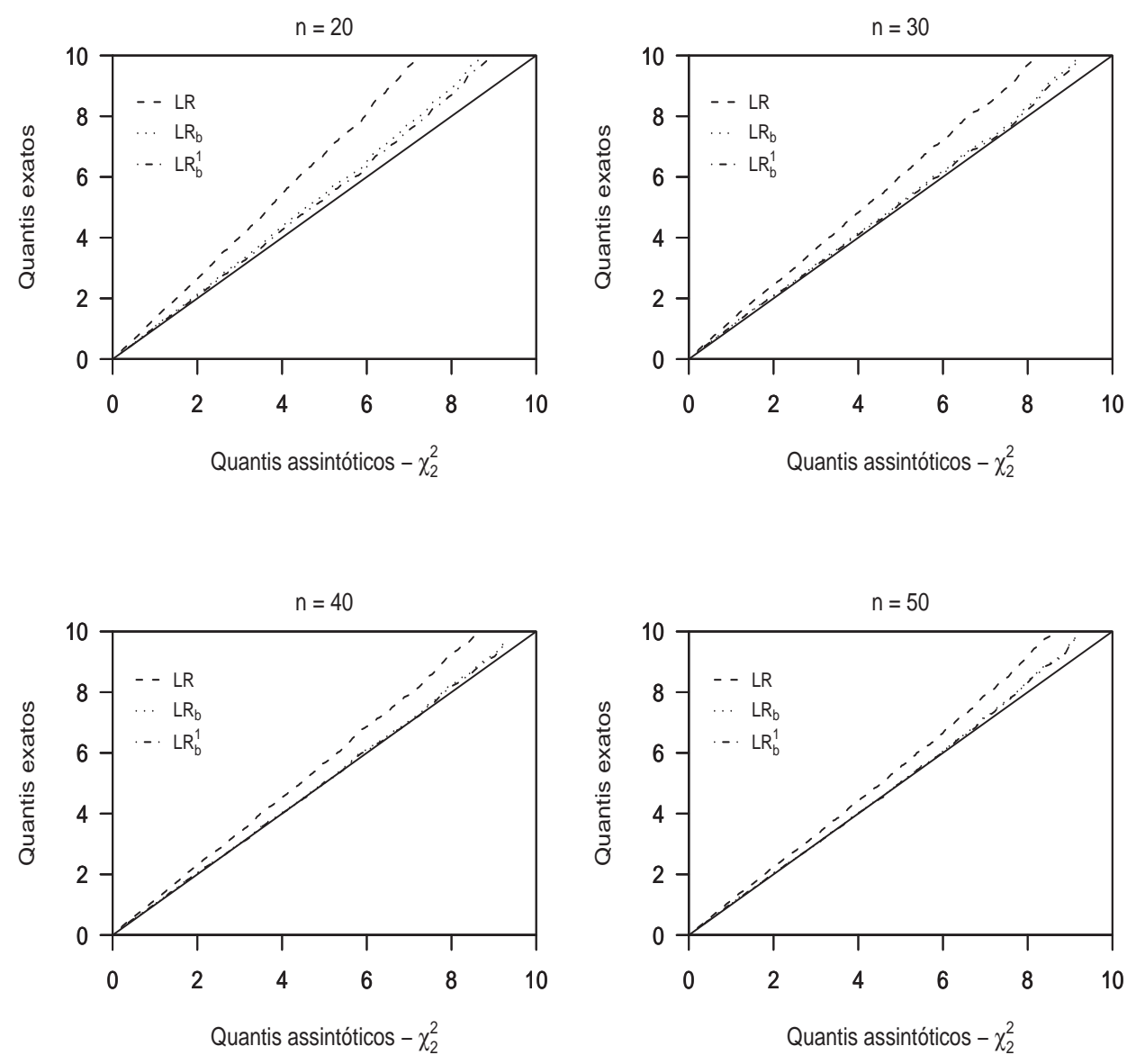

Figura 2.1: Quantis assintóticos versus quantis exatos das estatísticas $L R, L R_{b}$ e $L R_{b}^{1}$.

destas estatísticas estão sendo bem aproximadas pela distribuição $\chi_{2}^{2}$.

Na Tabela 2.5 apresentamos os resultados de simulação para as taxas de rejeição não-nulas (poder). Fixamos $p=4, \alpha=0.5$ e consideramos diferentes tamanhos amostrais: $n=30,50$ e 100 . As taxas de rejeição foram obtidas levando em consideração a hipótese alternativa $\mathcal{H}_{1}: \beta_{3}=\beta_{4}=\delta$, em que diferentes valores para $\delta$ são considerados. Adicionalmente, foi usado como ponto crítico para os testes o percentil da distribuição qui-quadrado com dois graus de liberdade. Somente consideramos os poderes do teste da razão de verossimilhanças corrigido pelo fator de correção de Bartlett, $L R_{b}$, e sua versão assintoticamente equivalente, $L R_{b}^{1}$, uma vez que o teste da razão de verossimilhanças usual, $L R$, é consideravelmente liberal, isto é, apresenta taxas de rejeição empíricas bem acima dos níveis nominais e, portanto, não deve ser recomendado. Os resultados evidenciam que não há perda de poder (em relação ao teste $L R$ usual) pelo fato de usar o fator de correção de Bartlett derivado neste capítulo; por exemplo, os poderes do teste $L R$ considerando a mesma situação da Tabela 2.5 com $n=30$ e $\delta=0.1$ foram $12.62 \%, 6.70 \%$ e $1.32 \%$ para os níveis nominais $\gamma=10 \%, 5 \%$ e $1 \%$, respectivamente. (A distribuição empírica da estatística $L R$ foi obtida com base em 100000 amostras Monte Carlo e os quantis amostrais de ordem 90\%, 95\% e 99\% foram calculados com base nesta amostra.) Os poderes dos dois testes são semelhantes, com leve vantagem para a estatística corrigida 


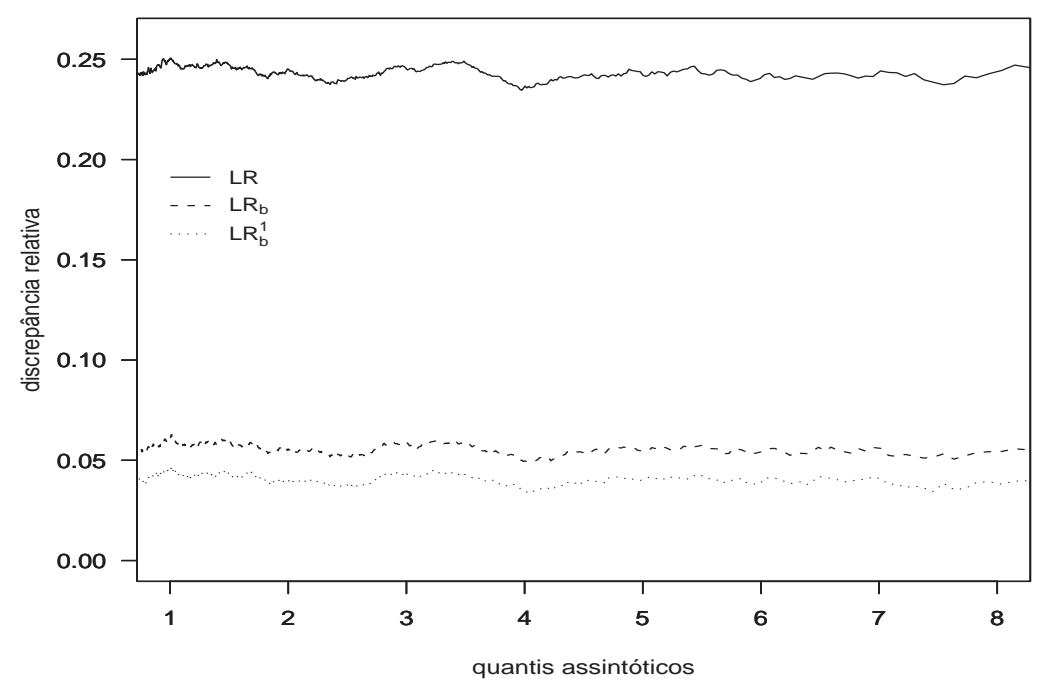

Figura 2.2: Discrepância relativa: $n=30, p=6$ e $\alpha=0.5$.

$L R_{b}$; por exemplo, quando $n=30, \gamma=5 \%$ e $\delta=0.5$, os poderes dos testes $L R_{b}$ e $L R_{b}^{1}$ foram $51.59 \%$ e $51.19 \%$, respectivamente. Observe também que à medida que o tamanho da amostra aumenta, os poderes crescem, como esperado.

Tabela 2.5: Taxas de rejeição não-nulas (poder) dos testes $L R_{b}$ e $L R_{b}^{1} \operatorname{com} \alpha=0.5, p=4$ e diferentes tamanhos amostrais.

\begin{tabular}{cc|ccc|ccc}
\hline \multirow{2}{*}{$n$} & & \multicolumn{3}{|c|}{$L R_{b}$} & \multicolumn{3}{|c}{$L R_{b}^{1}$} \\
\cline { 3 - 8 } & $10 \%$ & $5 \%$ & $1 \%$ & $10 \%$ & $5 \%$ & $1 \%$ \\
\hline 30 & 0.1 & 13.20 & 6.91 & 1.57 & 13.01 & 6.73 & 1.52 \\
& 0.2 & 20.66 & 12.22 & 3.46 & 20.40 & 12.02 & 3.30 \\
& 0.3 & 33.07 & 21.63 & 7.49 & 32.73 & 21.28 & 7.33 \\
& 0.4 & 48.36 & 35.57 & 14.96 & 48.08 & 35.20 & 14.61 \\
& 0.5 & 65.11 & 51.59 & 26.42 & 64.72 & 51.19 & 25.99 \\
\hline 50 & 0.1 & 13.82 & 7.63 & 2.03 & 13.71 & 7.60 & 1.99 \\
& 0.2 & 25.89 & 16.03 & 5.11 & 25.81 & 15.96 & 5.03 \\
& 0.3 & 45.00 & 32.06 & 13.15 & 44.86 & 31.95 & 13.07 \\
& 0.4 & 65.07 & 52.09 & 28.18 & 64.97 & 51.96 & 28.03 \\
& 0.5 & 82.31 & 72.39 & 48.01 & 82.14 & 72.28 & 47.88 \\
\hline 100 & 0.1 & 18.66 & 11.02 & 2.91 & 18.65 & 11.01 & 2.90 \\
& 0.2 & 43.63 & 31.29 & 13.05 & 43.61 & 31.28 & 13.02 \\
& 0.3 & 73.47 & 62.39 & 37.40 & 73.47 & 62.35 & 37.34 \\
& 0.4 & 92.12 & 86.39 & 69.15 & 92.11 & 86.37 & 69.11 \\
& 0.5 & 98.76 & 97.34 & 89.98 & 98.76 & 97.33 & 89.93 \\
\hline
\end{tabular}


Na Tabela 2.6 apresentamos os resultados de simulações para testes de hipóteses sobre o parâmetro $\alpha$. Consideramos $n=30$ e $p=2,3$ e 4 . As hipóteses consideradas foram $\mathcal{H}_{0}: \alpha=0.5$ e $\mathcal{H}_{0}: \alpha=1.0$. Note nesta tabela que o teste da razão de verossimilhanças usual é muito liberal, principalmente quando o valor de $p$ aumenta. Observe que os testes $L R_{b}$ e $L R_{b}^{1}$ apresentam distorções de tamanho bem inferiores. Por exemplo, quando $p=4, \gamma=5 \%$ e o parâmetro testado na hipótese nula é $\alpha=1.0$, as taxas de rejeição empíricas dos testes $L R, L R_{b}$ e $L R_{b}^{1}$ foram $12.03 \%, 5.20 \%$ e $4.02 \%$, respectivamente.

Tabela 2.6: Taxas de rejeição nulas dos testes $L R, L R_{b}$ e $L R_{b}^{1}$; inferência sobre $\alpha$ com $n=30$ e $p=2,3$ e 4 .

\begin{tabular}{cc|ccc|ccc}
\hline \multirow{2}{*}{$p$} & & \multicolumn{3}{|c|}{$\mathcal{H}_{0}: \alpha=0.5$} & \multicolumn{3}{c}{$\mathcal{H}_{0}: \alpha=1.0$} \\
\cline { 3 - 8 }$p$ & & $10 \%$ & $5 \%$ & $1 \%$ & $10 \%$ & $5 \%$ & $1 \%$ \\
\hline 2 & $L R$ & 12.76 & 6.99 & 1.82 & 13.55 & 7.26 & 1.93 \\
& $L R_{b}$ & 10.13 & 5.15 & 1.21 & 10.52 & 5.10 & 1.08 \\
& $L R_{b}^{1}$ & 9.90 & 5.02 & 1.20 & 10.31 & 4.94 & 1.05 \\
\hline 3 & $L R$ & 15.16 & 8.64 & 2.45 & 16.10 & 9.35 & 2.70 \\
& $L R_{b}$ & 10.46 & 5.43 & 1.16 & 10.53 & 5.06 & 0.97 \\
& $L R_{b}^{1}$ & 9.77 & 5.02 & 1.02 & 9.65 & 4.68 & 0.81 \\
\hline 4 & $L R$ & 18.16 & 10.72 & 3.39 & 19.86 & 12.03 & 3.73 \\
& $L R_{b}$ & 10.45 & 5.37 & 0.98 & 10.73 & 5.20 & 0.75 \\
& $L R_{b}^{1}$ & 9.29 & 4.57 & 0.72 & 8.77 & 4.02 & 0.43 \\
\hline
\end{tabular}

Nossos resultados de simulações Monte Carlo em testes sobre os parâmetros de regressão foram obtidos considerando $\alpha=0.5$. Na prática, valores de $\alpha$ entre 0 (zero) e 1 (um) cobrem a maioria das aplicações; veja, por exemplo, Rieck \& Nedelman (1991). É claro que valores de $\alpha$ maiores que 1 (um) podem ocorrer na prática. Na Tabela 2.7, apresentamos resultados de simulações para diferentes valores de $\alpha$, com $\alpha=0.1,0.3,0.5,0.7,0.9,1.2,2,10,50$ e 100. Observe nesta tabela que os desempenhos dos testes corrigidos $L R_{b}$ e $L R_{b}^{1}$ não se alteram, ou seja, suas taxas de rejeição empíricas continuam próximas dos verdadeiros níveis nominais. É interessante notar que, à medida que o valor do parâmetro $\alpha$ aumenta, as taxas de rejeição empíricas do teste $L R$ tendem aos verdadeiros níveis nominais.

Em linhas gerais, o teste da razão de verossimilhanças usual pode ser severamente liberal em amostras de tamanho pequeno e moderado, rejeitando a hipótese nula muito mais frequentemente do que o que seria esperado com base no nível nominal selecionado. O fator de correção de Bartlett para o teste da razão de verossimilhanças derivado neste capítulo faz com que a taxa de rejeição empírica fique mais próxima do nível nominal do teste. Portanto, dentre os testes considerados neste capítulo, recomendamos os testes $L R_{b}$ ou $L R_{b}^{1}$ (já que seus resultados foram bastante similares), pois apresentaram os melhores resultados em todos os casos considerados. Em geral, a estatística corrigida pelo fator de correção de Bartlett $L R_{b}$ é a mais comumente usada na prática, por outro lado, $L R_{b}^{1}$ tem a vantagem de sempre assumir valores positivos. Dessa forma, deixamos sob a responsabilidade do pesquisador a escolha entre as estatísticas de testes $L R_{b}$ ou $L R_{b}^{1}$ em pesquisas aplicadas.

\subsection{Uma Aplicação}

A seguir, iremos aplicar a metodologia desenvolvida nas seções anteriores em um conjunto de dados reais. Vamos considerar um estudo feito em Lepadatu et al. (2005), no qual o objetivo é 
Tabela 2.7: Rejeição nula de $\mathcal{H}_{0}: \beta_{3}=\beta_{4}=0 ; p=4, n=25$ e diferentes valores para $\alpha$.

\begin{tabular}{|c|c|c|c|c|c|c|}
\hline \multirow[b]{2}{*}{$\gamma$} & \multicolumn{3}{|c|}{$\alpha=0.1$} & \multicolumn{3}{|c|}{$\alpha=0.3$} \\
\hline & $L R$ & $L R_{b}$ & $L R_{b}^{1}$ & $L R$ & $L R_{b}$ & $L R_{b}^{1}$ \\
\hline $10 \%$ & 14.33 & 11.42 & 11.32 & 14.45 & 10.70 & 10.44 \\
\hline $5 \%$ & 7.88 & 5.82 & 5.74 & 8.01 & 5.49 & 5.22 \\
\hline \multirow[t]{2}{*}{$1 \%$} & 1.95 & 1.29 & 1.23 & 2.07 & 1.20 & 1.13 \\
\hline & \multicolumn{3}{|c|}{$\alpha=0.5$} & \multicolumn{3}{|c|}{$\alpha=0.7$} \\
\hline$\gamma$ & $L R$ & $L R_{b}$ & $L R_{b}^{1}$ & $L R$ & $L R_{b}$ & $L R_{b}^{1}$ \\
\hline $10 \%$ & 14.25 & 10.23 & 10.01 & 14.17 & 10.61 & 10.36 \\
\hline $5 \%$ & 7.69 & 5.17 & 5.02 & 8.09 & 5.35 & 5.17 \\
\hline \multirow[t]{2}{*}{$1 \%$} & 1.89 & 0.91 & 0.85 & 2.07 & 1.12 & 1.06 \\
\hline & \multicolumn{3}{|c|}{$\alpha=0.9$} & \multicolumn{3}{|c|}{$\alpha=1.2$} \\
\hline$\gamma$ & $L R$ & $L R_{b}$ & $L R_{b}^{1}$ & $L R$ & $L R_{b}$ & $L R_{b}^{1}$ \\
\hline $10 \%$ & 13.96 & 10.80 & 10.60 & 13.49 & 10.45 & 10.29 \\
\hline $5 \%$ & 8.03 & 5.77 & 5.61 & 7.51 & 5.37 & 5.26 \\
\hline \multirow[t]{2}{*}{$1 \%$} & 2.29 & 1.30 & 1.26 & 1.90 & 1.18 & 1.16 \\
\hline & \multicolumn{3}{|c|}{$\alpha=2$} & \multicolumn{3}{|c|}{$\alpha=10$} \\
\hline$\gamma$ & $L R$ & $L R_{b}$ & $L R_{b}^{1}$ & $L R$ & $L R_{b}$ & $L R_{b}^{1}$ \\
\hline $10 \%$ & 13.21 & 10.87 & 10.78 & 12.44 & 11.23 & 11.20 \\
\hline $5 \%$ & 7.29 & 5.61 & 5.53 & 6.59 & 5.81 & 5.79 \\
\hline \multirow[t]{2}{*}{$1 \%$} & 1.63 & 1.08 & 1.06 & 1.42 & 1.17 & 1.17 \\
\hline & \multicolumn{3}{|c|}{$\alpha=50$} & \multicolumn{3}{|c|}{$\alpha=100$} \\
\hline$\gamma$ & $L R$ & $L R_{b}$ & $L R_{b}^{1}$ & $L R$ & $L R_{b}$ & $L R_{b}^{1}$ \\
\hline $10 \%$ & 11.26 & 10.45 & 10.44 & 10.87 & 10.17 & 10.13 \\
\hline $5 \%$ & 5.60 & 5.21 & 5.21 & 5.67 & 5.11 & 5.10 \\
\hline $1 \%$ & 1.19 & 1.06 & 1.06 & 1.23 & 1.07 & 1.07 \\
\hline
\end{tabular}

modelar o tempo de vida $(T)$ de um certo produto no processo de extrusão de metal. Como notado pelos autores (p. 38), "the estimation of tool life (fatigue life) in the extrusion operation is important for scheduling tool changing times, for adaptive process control and for tool cost evaluation." Eles também ressaltam (p. 39) que "die fatigue cracks are caused by the repeat application of loads which individually would be too small to cause failure." Lembre que falhas por fadiga devido a uma propagação de uma quebra inicial foram a principal motivação em considerar a distribuição Birnbaum-Saunders.

Segundo Lepadatu et al. (2005), as covariáveis que afetam o tempo de vida são o coeficiente de fricção $\left(x_{1}\right)$, o ângulo de falha $\left(x_{2}\right)$ e a temperatura $\left(x_{3}\right)$. Considere um modelo de regressão que também inclui efeitos de interação, isto é,

$$
y_{i}=\beta_{0}+\beta_{1} x_{1 i}+\beta_{2} x_{2 i}+\beta_{3} x_{3 i}+\beta_{4} x_{1 i} x_{2 i}+\beta_{5} x_{1 i} x_{3 i}+\beta_{6} x_{2 i} x_{3 i}+\varepsilon_{i},
$$

em que $y_{i}=\log \left(T_{i}\right)$ e $\varepsilon_{i} \sim \mathcal{S N}(\alpha, 0,2), i=1,2, \ldots, n$. As estimativas de máxima verossimilhança, erros-padrão assintóticos, estatísticas $z$ e $p$-valores marginais estão apresentados na Tabela 2.8. 
Tabela 2.8: Estimativas do modelo.

\begin{tabular}{lrrrr}
\hline \multicolumn{5}{c}{ Tabela 2.8: Estimativas do modelo. } \\
\cline { 1 - 4 }$\beta_{0}$ & Estimativa & E.P. & Estat- $z$ & $p$-valor \\
$\beta_{1}$ & 0.3675 & 2.6095 & 0.1408 & 0.8880 \\
$\beta_{2}$ & 38.7766 & 15.9885 & 2.4253 & 0.0153 \\
$\beta_{3}$ & 0.1085 & 0.0854 & 1.2704 & 0.2039 \\
$\beta_{4}$ & 0.0117 & 0.0033 & 3.5287 & 0.0004 \\
$\beta_{5}$ & -0.3539 & 0.4180 & -0.8467 & 0.3972 \\
$\beta_{6}$ & -0.0404 & 0.0157 & -2.5657 & 0.0103 \\
\cline { 1 - 3 }$\alpha$ & -0.0001 & 0.0001 & -0.8601 & 0.3897 \\
\cline { 1 - 3 } & 0.1599 & 0.029198 & & \\
\hline \multicolumn{4}{r}{} \\
\cline { 1 - 2 } & & & & \\
\end{tabular}

Existem apenas 15 observações $(n=15)$ e o objetivo é inferir sobre a significância do efeito das interações, isto é, o interesse é testar a hipótese $\mathcal{H}_{0}: \beta_{4}=\beta_{5}=\beta_{6}=0$. A estatística da razão de verossimilhanças usual $(L R)$ foi igual a 6.387 ( $p$-valor 0.094$), L R_{b}=4.724$ ( $p$-valor 0.193 ) e $L R_{b}^{1}=4.492$ ( $p$-valor 0.213 ). Note que rejeitamos a hipótese nula ao nível de significância $10 \%$ quando a inferência é baseada no teste da razão de verossimilhanças usual, mas uma inferência (conclusão) diferente é encontrada quando os testes modificados (corrigidos por Bartlett) são usados. Lembre das seções prévias que o teste não modificado é muito distorcido (liberal) quando o tamanho da amostra é pequeno (aqui, $n=15$ ), o que nos leva a desconfiar da conclusão imposta pelo teste da razão de verossimilhanças usual.

Nós procedemos removendo o efeito das interações (como sugerido pelos testes modificados) do modelo (2.7). Assim, estimamos

$$
y_{i}=\beta_{0}+\beta_{1} x_{1 i}+\beta_{2} x_{2 i}+\beta_{3} x_{3 i}+\varepsilon_{i},
$$

$i=1,2, \ldots, 15$. As estimativas pontuais são (erros-padrão assintóticos em parênteses): $\widehat{\beta}_{0}=$ 5.9011 (0.488), $\widehat{\beta}_{1}=0.7917(1.777), \widehat{\beta}_{2}=0.0098(0.012), \widehat{\beta}_{3}=0.0052(0.001)$ e $\widehat{\alpha}=0.1982(0.036)$. A hipótese nula $\mathcal{H}_{0}: \beta_{3}=0$ é fortemente rejeitada pelos três testes (não modificado e modificados) aos níveis de significância usuais. Todos os testes também sugerem a exclusão individual e conjunta de $x_{1}$ e $x_{2}$ do modelo de regressão. Assim, finalizamos com o modelo reduzido

$$
y_{i}=\beta_{0}+\beta_{3} x_{3 i}+\varepsilon_{i}
$$

$i=1,2, \ldots, 15$. As estimativas pontuais são (erros-padrão assintóticos em parênteses): $\widehat{\beta}_{0}=$ $6.2453(0.326), \widehat{\beta}_{3}=0.0052(0.001)$ e $\widehat{\alpha}=0.2039$ (0.037). É notório que o modelo final estimado conduz a uma interessante interpretação: o tempo de vida esperado deve crescer $\exp \{0.0052\} \times 100 \%$ (aproximadamente 5\%) quando a temperatura é acrescida de uma unidade.

Finalmente, nós retornamos ao modelo (2.7) e testamos $\mathcal{H}_{0}: \beta_{1}=\beta_{2}=\beta_{4}=\beta_{5}=\beta_{6}=0$ (exclusão de todas as variáveis menos $x_{3}$ ). A hipótese nula não é rejeitada ao nível nominal de $10 \%$ por todos os testes, mas notamos que os testes corrigidos apresentaram $p$-valores consideravelmente maiores. As estatísticas de teste são $L R=7.229, L R_{b}=5.610$ e $L R_{b}^{1}=5.417$, os correspondentes $p$-valores sendo $0.204,0.346$ e 0.367 . 


\section{Capítulo 3}

\section{Correção tipo-Bartlett}

No Capítulo 2 apresentamos uma estatística de teste da razão de verossimilhanças corrigida por um fator de correção denominado fator de correção de Bartlett. Vários métodos foram discutidos para se obter este fator de correção e, em especial, foi dada ênfase à metodologia desenvolvida por Lawley (1956). O problema em se desenvolver uma correção similar à correção de Bartlett para a estatística escore foi primeiramente apresentado por Cox (1988) e solucionado três anos depois em generalidade por Cordeiro \& Ferrari (1991), e por Chandra \& Mukerjee (1991) e Taniguchi (1991) para certos casos especiais; veja também Mukerjee (1992).

O teste escore foi originalmente introduzido por Rao (1948) e posteriormente por Aitchison \& Silvey (1958) e Silvey (1959) sob a denominação de teste de multipicadores de Lagrange. No passado, os testes da razão de verossimilhanças e de Wald foram mais difundidos que o teste escore. Entretanto, mais recentemente, pesquisas envolvendo o teste escore tiveram grande impulso quer em teoria quer em aplicações. Adicionalmente, em muitas situações, o teste escore tem grande vantagem computacional sobre os demais, pois os testes da razão de verossimilhanças e de Wald envolvem estimação dos parâmetros sob a hipótese alternativa, enquanto o teste escore requer estimação somente sob a hipótese nula. Dessa forma, nos casos em que a estimação sob a hipótese alternativa é muito complicada, o teste escore pode apresentar custo computacional bastante inferior aos demais. Além disso, no caso em que a hipótese nula é simples, o teste escore não requer estimação alguma.

Antes dos artigos de Chandra \& Mukerjee (1991), Cordeiro \& Ferrari (1991) e Taniguchi (1991), citava-se frequentemente na literatura que a estatística escore, $S R$, não admitia um fator de correção simples como no caso da estatística da razão de verossimilhanças (Barndorff-Nielsen, 1990a; Cox, 1988; Harris, 1985). Harris (1985), seguindo as idéias de Hayakawa (1977), desenvolveu uma expansão assintótica até ordem $O\left(n^{-1}\right)$ para a distribuição da estatística escore. Dada a impossibilidade de se obter um fator de correção simples para $S R$, Harris propôs um teste escore aperfeiçoado baseado em uma expansão, até ordem $O\left(n^{-1}\right)$, para seus quantis, ou seja, em lugar de modificar a estatística de teste $S R$, ele propôs a modificação do valor crítico obtido da distribuição qui-quadrado de referência para um nível de significância fixado, já que, em problemas regulares, $S R$ converge, sob a hipótese nula, para distribuição qui-quadrado com número de graus de liberdade igual ao número de restrições impostas sob a hipótese nula.

A aproximação da distribuição nula de $S R$ pela qui-quadrado pode não ser satisfatória em pequenas amostras, podendo conduzir a taxas de rejeição sob a hipótese nula bastante distorcidas. Dessa forma, foram obtidos alguns refinamentos com o objetivo de melhorar a aproximação pela distribuição qui-quadrado: Taniguchi (1991) apresentou uma estatística escore modificada tendo distribuição quiquadrado até ordem $O\left(n^{-1}\right)$, porém, esta estatística é válida apenas para o caso uniparamétrico. 
Chandra \& Mukerjee (1991) encontraram uma estatística escore modificada tendo distribuição quiquadrado até ordem $O\left(n^{-1}\right)$ que é válida para os casos uni e multiparamétricos. Entretanto, eles não obtiveram tal estatística para situações mais gerais como, por exemplo, aquela em que a hipótese nula é composta e há mais de um parâmetro de perturbação.

Por outro lado, Cordeiro \& Ferrari (1991) mostraram que é sempre possível encontrar uma estatística escore aperfeiçoada tendo distribuição qui-quadrado até ordem $O\left(n^{-1}\right)$. A estatística modificada que eles obtiveram é dada por $S R$ multiplicada por um fator de correção que envolve um polinômio de segundo grau na própria estatística e cujos coeficientes são, em geral, funções de parâmetros desconhecidos que devem ser substituídos por suas respectivas estimativas de máxima verossimilhança sob a hipótese nula. Os resultados obtidos por Cordeiro \& Ferrari (1991) são bastante abrangentes no sentido de que são válidos para problemas uni e multiparamétricos, admitindo, ainda, a presença de parâmetros de perturbação; isto é, os resultados de Cordeiro \& Ferrari (1991) generalizam os resultados de Chandra \& Mukerjee (1991) e Taniguchi (1991).

As estatísticas escore aperfeiçoadas obtidas por Chandra \& Mukerjee (1991), Cordeiro \& Ferrari (1991) e Taniguchi (1991) não são estatísticas genuinamente corrigidas por um fator de correção de Bartlett, pois os fatores de correção obtidos por estes autores envolvem a própria estatística $S R$. Devido a isso, essas correções foram denominadas de correções tipo-Bartlett. Dada a generalidade dos resultados obtidos por Cordeiro \& Ferrari (1991), vamos nos concentrar em sua metodologia neste capítulo para obtermos um fator de correção tipo-Bartlett para a estatística escore no modelo de regressão Birnbaum-Saunders.

Rao \& Mukerjee (1995) apresentam um estudo comparando, sob alternativas contíguas, ${ }^{1}$ as três estatísticas escore corrigidas propostas por Chandra \& Mukerjee (1991), Cordeiro \& Ferrari (1991) e Taniguchi (1991). Os autores verificaram que em alguns modelos específicos é possível indicar qual das três estatísticas corrigidas apresenta maior poder.

Seguindo a metodologia de Cordeiro \& Ferrari (1991), vários autores obtiveram uma estatística escore aperfeiçoada em modelos específicos, entre eles: Cordeiro, Ferrari \& Paula (1993) e CribariNeto \& Ferrari (1995b) obtiveram correções tipo-Bartlett para o teste escore em modelos lineares generalizados para o caso em que o parâmetro de dispersão é conhecido e desconhecido, respectivamente. Correções similares para o teste escore em modelos de regressão multivariados foram obtidas por Cribari-Neto \& Zarkos (1995). Correções tipo-Bartlett do teste escore para heteroscedasticidade foram obtidas por Cribari-Neto \& Ferrari (1995c). Ferrari \& Arellano-Valle (1993) derivaram uma estatística de teste escore melhorada em modelos de regressão com erros $t$-Student. Uma fórmula matricial geral para encontrar fatores de correções tipo-Bartlett está dada em Ferrari \& Cordeiro (1994). Ferrari \& Cordeiro (1996) derivaram uma estatística escore melhorada em modelos nãolineares da família exponencial. Os resultados obtidos pelos autores são uma extensão dos resultados apresentados em Cordeiro, Ferrari \& Paula (1993).

Correções para o teste escore em modelos próprios de dispersão (Jørgensen, 1997) foram obtidas por Cordeiro \& Ferrari (1996). Entretanto, seus resultados cobrem apenas testes sobre todos os parâmetros da regressão supondo o parâmetro de dispersão desconhecido. Ferrari, Cordeiro \& Cribari-Neto (2001) estenderam estes resultados para o caso em que há interesse em se testar um subconjunto dos parâmetros da regressão. Cordeiro, Botter, Barroso \& Ferrari (2003) obtiveram uma

\footnotetext{
${ }^{1}$ Uma hipótese alternativa contígua à hipótese nula $\mathcal{H}_{0}: \theta=\theta^{(0)}$ tem a forma $\mathcal{H}_{1}: \theta=\theta^{(0)}+\epsilon$, em que $\epsilon$ é de ordem $O\left(n^{-1 / 2}\right)$ sendo $n$ o tamanho da amostra. Aqui $\theta$ é um parâmetro desconhecido e $\theta^{(0)}$ é um número real fixado; veja Cox \& Hinkley (1974) para mais detalhes. Esta definição é estendida de maneira evidente para o caso multidimensional.
} 
estatística escore melhorada em modelos lineares generalizados com o parâmetro de dispersão dependendo de covariáveis. Correções tipo-Bartlett do teste escore em modelos de regressão von Mises foram obtidas por Barroso, Cordeiro \& Vasconcellos (2001). Tu, Chen, Shi \& Wu (2005) obtiveram um teste escore melhorado no modelo de regressão de Cox. Uribe-Opazo, Ferrari \& Cordeiro (2008) obtiveram um teste escore aperfeiçoado em modelos lineares simétricos. Lagos, Morettin \& Barroso (2009) derivaram correções tipo-Bartlett do teste escore em modelos de séries temporais. Uma boa revisão sobre correção tipo-Bartlett está dada em Cribari-Neto \& Cordeiro (1996).

\subsection{Estatística Escore Aperfeiçoada}

\subsubsection{Expansão Assintótica de Harris}

Considere o vetor $\boldsymbol{y}=\left(y_{1}, y_{2}, \ldots, y_{n}\right)^{\top}$ composto de $n$ observações independentes com densidades contínuas e cuja função de log-verossimilhança $\ell(\boldsymbol{\theta})$ depende de um vetor de parâmetros desconhecidos $\boldsymbol{\theta}=\left(\theta_{1}, \theta_{2}, \ldots, \theta_{p}\right)^{\top}$ com $p$ componentes. Assume-se que $\ell(\boldsymbol{\theta})$ seja regular em relação às derivadas com respeito aos componentes de $\boldsymbol{\theta}$ até a quarta ordem. Vamos considerar o problema de testar uma hipótese nula simples ou composta $\mathcal{H}_{0}: \boldsymbol{\theta}_{1}=\boldsymbol{\theta}_{1}^{(0)}$ contra a hipótese alternativa composta $\mathcal{H}_{1}: \boldsymbol{\theta}_{1} \neq \boldsymbol{\theta}_{1}^{(0)}$, em que $\boldsymbol{\theta}=\left(\boldsymbol{\theta}_{1}^{\top}, \boldsymbol{\theta}_{2}^{\top}\right)^{\top}$, sendo $\boldsymbol{\theta}_{1}=\left(\theta_{1}, \theta_{2}, \ldots, \theta_{q}\right)^{\top}$ um vetor $q$ dimensional e $\boldsymbol{\theta}_{2}=\left(\theta_{q+1}, \theta_{q+2}, \ldots, \theta_{p}\right)^{\top}$ um vetor $p-q$ dimensional. Neste caso, $\boldsymbol{\theta}_{2}$ é um vetor de parâmetros de perturbação e $\boldsymbol{\theta}_{1}^{(0)}$ é um vetor de constantes conhecidas de dimensão $q$.

Seja $\boldsymbol{U}(\boldsymbol{\theta})=\partial \ell(\boldsymbol{\theta}) / \partial \boldsymbol{\theta}=\left(\boldsymbol{U}_{1}(\boldsymbol{\theta})^{\top}, \boldsymbol{U}_{2}(\boldsymbol{\theta})^{\top}\right)^{\top}$ a função escore total de $\boldsymbol{\theta}$ particionada da mesma forma que $\boldsymbol{\theta}$. Considere $\widehat{\boldsymbol{\theta}}=\left(\widehat{\boldsymbol{\theta}}_{1}^{\top}, \widehat{\boldsymbol{\theta}}_{2}^{\top}\right)^{\top}$ o estimador de máxima verossimilhança irrestrito de $\boldsymbol{\theta}$ e $\widetilde{\boldsymbol{\theta}}=\left(\boldsymbol{\theta}_{1}^{(0) \top}, \widetilde{\boldsymbol{\theta}}_{2}^{\top}\right)^{\top}$ o estimador de máxima verossimilhança restrito sob a hipótese nula. A partição $\boldsymbol{\theta}=\left(\boldsymbol{\theta}_{1}^{\top}, \boldsymbol{\theta}_{2}^{\top}\right)^{\top}$ conduz às seguintes partições:

$$
\boldsymbol{K}(\boldsymbol{\theta})=\left[\begin{array}{ll}
\boldsymbol{K}_{11} & \boldsymbol{K}_{12} \\
\boldsymbol{K}_{21} & \boldsymbol{K}_{22}
\end{array}\right] \quad \text { e } \quad \boldsymbol{K}(\boldsymbol{\theta})^{-1}=\left[\begin{array}{ll}
\boldsymbol{K}^{11} & \boldsymbol{K}^{12} \\
\boldsymbol{K}^{21} & \boldsymbol{K}^{22}
\end{array}\right]
$$

em que $\boldsymbol{K}(\boldsymbol{\theta})=\mathbb{E}\left\{\boldsymbol{U}(\boldsymbol{\theta}) \boldsymbol{U}(\boldsymbol{\theta})^{\top}\right\}$ é a matriz de informação de Fisher e $\boldsymbol{K}(\boldsymbol{\theta})^{-1}$ representa sua inversa.

A estatística escore $S R\left(\right.$ Rao, 1948) para testar $\mathcal{H}_{0}$ contra $\mathcal{H}_{1}$ é definida como

$$
S R=\boldsymbol{U}(\widetilde{\boldsymbol{\theta}})^{\top} \boldsymbol{K}(\widetilde{\boldsymbol{\theta}})^{-1} \boldsymbol{U}(\widetilde{\boldsymbol{\theta}}) .
$$

Buse (1982) apresenta uma interpretação geométrica, no caso de hipóteses simples, para a estatística $S R$ e compara-a com as estatísticas da razão de verossimilhanças e de Wald. Cox \& Hinkley (1974, $\S 9.3)$ expressam $S R$ como

$$
S R=\boldsymbol{U}_{1}(\widetilde{\boldsymbol{\theta}})^{\top} \boldsymbol{K}^{11}(\widetilde{\boldsymbol{\theta}}) \boldsymbol{U}_{1}(\widetilde{\boldsymbol{\theta}}),
$$

em que $\boldsymbol{K}^{11}(\boldsymbol{\theta})$ é a matriz de covariância assintótica de $\widehat{\boldsymbol{\theta}}_{1}$ obtida de (3.1). A equivalência das duas expressões para $S R$ é observada notando-se que $\boldsymbol{U}_{2}(\widetilde{\boldsymbol{\theta}})$ é igual a um vetor nulo. Em grandes amostras, a distribuição de $S R$, sob a hipótese nula, é aproximadamente $\chi_{q}^{2}$, ou seja, qui-quadrado com $q$ graus de liberdade.

Harris (1985), utilizando um procedimento análogo ao de Hayakawa (1977), obteve uma expansão 
assintótica, sob a hipótese nula, para a estatística $S R$. Ele mostrou que

$$
\begin{aligned}
\operatorname{Pr}(S R \leq z)= & F_{q}(z)+\frac{1}{24}\left[A_{3} F_{q+6}(z)+\left(A_{2}-3 A_{3}\right) F_{q+4}(z)\right. \\
& \left.+\left(3 A_{3}-2 A_{2}+A_{1}\right) F_{q+2}(z)+\left(A_{2}-A_{1}-A_{3}\right) F_{q}(z)\right]+O\left(n^{-2}\right),
\end{aligned}
$$

em que $F_{s}(\cdot)$ é a função distribuição acumulada de uma variável aleatória qui-quadrado com $s$ graus de liberdade. Ainda,

$$
\begin{gathered}
A_{1}=3 \sum^{\prime}\left(\kappa_{i j k}+2 \kappa_{i, j k}\right)\left(\kappa_{r s t}+2 \kappa_{r s, t}\right) a_{i j} a_{s t} m_{k r} \\
-6 \sum^{\prime}\left(\kappa_{i j k}+2 \kappa_{i, j k}\right) \kappa_{r, s, t} a_{i j} a_{k r} m_{s t} \\
+6 \sum^{\prime}\left(\kappa_{i, j k}-\kappa_{i, j, k}\right)\left(\kappa_{r s t}+2 \kappa_{r s, t}\right) a_{j s} a_{k t} m_{i r} \\
-6 \sum^{\prime}\left(\kappa_{i, j, k, r}+\kappa_{i, j, k r}\right) a_{k r} m_{i j}, \\
A_{2}=-3 \sum^{\prime} \kappa_{i, j, k} \kappa_{r, s, t} a_{k r} m_{i j} m_{s t}+6 \sum^{\prime}\left(\kappa_{i j k}+2 \kappa_{i, j k}\right) \kappa_{r, s, t} a_{i j} m_{k r} m_{s t} \\
-6 \sum^{\prime} \kappa_{i, j, k} \kappa_{r, s, t} a_{k t} m_{i r} m_{j s}+3 \sum^{\prime} \kappa_{i, j, k, r} m_{i j} m_{k r}
\end{gathered}
$$

$\mathrm{e}$

$$
A_{3}=3 \sum^{\prime} \kappa_{i, j, k} \kappa_{r, s, t} m_{i j} m_{k r} m_{s t}+2 \sum^{\prime} \kappa_{i, j, k} \kappa_{r, s, t} m_{i r} m_{j s} m_{k t}
$$

sendo $a_{i j}$ e $m_{i j}$ os elementos $(i, j)$ das matrizes

$$
\boldsymbol{A}=\left[\begin{array}{cc}
\mathbf{0} & \mathbf{0} \\
\mathbf{0} & \boldsymbol{K}_{22}^{-1}
\end{array}\right] \quad \text { e } \quad \boldsymbol{M}=\boldsymbol{K}(\boldsymbol{\theta})^{-1}-\boldsymbol{A},
$$

respectivamente. Note que $\boldsymbol{K}_{22}^{-1}$ é a matriz de covariância assintótica de $\widetilde{\boldsymbol{\theta}}_{2}$. A soma $\sum^{\prime}$ indica que o somatório é tomado sobre todos os componentes do vetor $\boldsymbol{\theta}$, e os $\boldsymbol{\kappa}$ 's estão definidos na Seção 1.1.2. Adicionalmente, $A_{1}, A_{2}$ e $A_{3}$ são de ordem $O\left(n^{-1}\right)$, sendo avaliados sob a hipótese nula. Harris observou que, no caso de uma hipótese nula simples, $A_{1}=0$ e $A_{2}$ e $A_{3}$ refletem aspectos de nãonormalidade da distribuição nula da função escore. Já no caso de hipóteses nulas compostas a interpretação de $A_{1}, A_{2}$ e $A_{3}$ é complicada (ver Harris, 1985, p. 665).

Harris (1985) também obteve expansões para os três primeiros momentos de $S R$. Ele mostrou que, até ordem $O\left(n^{-1}\right)$ e sob a hipótese nula, os três primeiros momentos de $S R$ são

$$
\begin{aligned}
\mathbb{E}(S R) & =q+\frac{A_{1}}{12}, \\
\mathbb{E}\left(S R^{2}\right) & =q(q+2)+\frac{A_{1}(q+2)+2 A_{2}}{6}, \\
\mathbb{E}\left(S R^{3}\right) & =q(q+2)(q+4)+\frac{A_{1}(q+2)(q+4)+4 A_{2}(q+4)+8 A_{3}}{6} .
\end{aligned}
$$

Consequentemente, tem-se que, até ordem $O\left(n^{-1}\right)$ e sob a hipótese nula, $\operatorname{Var}(S R)=2 q+\left(A_{1}+A_{2}\right) / 3$.

Harris $(1985, \S 5)$ ainda observou que não é possível obter um fator de correção simples para a estatística $S R$ de tal forma que a estatística modificada, dada por $S R$ multiplicada por este fator, 
tenha distribuição qui-quadrado até ordem $O\left(n^{-1}\right)$. Esta mesma observação foi feita por Cox (1988). Dessa forma, em lugar de modificar a estatística $S R$, Harris obteve uma expansão para os quantis de $S R$. Através da fórmula de inversão de expansões de Hill \& Davis (1968), ele mostrou que, até ordem $O\left(n^{-1}\right)$, pode-se escrever

$$
\begin{aligned}
z_{\gamma}=x_{\gamma}+\frac{1}{12}[ & \frac{A_{3} x_{\gamma}}{q(q+2)(q+4)}\left\{x_{\gamma}^{2}+(q+4) x_{\gamma}+(q+2)(q+4)\right\} \\
& \left.+\frac{x_{\gamma}\left(x_{\gamma}+q+2\right)}{q(q+2)}\left(A_{2}-3 A_{3}\right)+\frac{x_{\gamma}}{q}\left(3 A_{3}-2 A_{2}+A_{1}\right)\right],
\end{aligned}
$$

em que $\operatorname{Pr}\left(S R>z_{\gamma}\right)=\gamma$ e $\operatorname{Pr}\left(\chi_{q}^{2}>x_{\gamma}\right)=\gamma$. Assim, desde que os $A$ 's possam ser calculados a partir das expressões (3.3)-(3.5), um teste escore aperfeiçoado pode ser conduzido encontrando aproximadamente o nível descritivo do teste fixando-se um nível de significância $\gamma$ e comparando o valor observado de $S R$ com o valor crítico modificado $z_{\gamma}$ apresentado em (3.7).

\subsubsection{Melhoramento de Cordeiro-Ferrari}

Cordeiro \& Ferrari (1991) obtiveram uma estatística escore modificada por um fator de correção que envolve constantes de ordem $O\left(n^{-1}\right)$ e um polinômio de segundo grau na própria estatística $S R$, produzindo uma estatística ajustada com distribuição qui-quadrado até ordem $O\left(n^{-1}\right)$ segundo a hipótese nula.

Cordeiro \& Ferrari (1991), escrevendo a expansão assintótica (3.2) em função da densidade de $S R$ e usando a relação de recorrência $f_{q+2}(z)=z q^{-1} f_{q}(z)$, em que $f_{s}(\cdot)$ denota a função densidade de probabilidades de uma variável aleatória qui-quadrado com $s$ graus de liberdade, mostraram que até ordem $O\left(n^{-1}\right)$ a densidade de $S R$ pode ser escrita na forma

$$
g(z)=f_{q}(z)\left(1+B_{0}+B_{1} z+B_{2} z^{2}+B_{3} z^{3}\right),
$$

em que

$$
\begin{aligned}
& B_{0}=\frac{A_{2}-A_{1}-A_{3}}{24}, \quad B_{1}=\frac{3 A_{3}-2 A_{2}+A_{1}}{24 q}, \\
& B_{2}=\frac{A_{2}-3 A_{3}}{24 q(q+2)} \quad \text { e } \quad B_{3}=\frac{A_{3}}{24 q(q+2)(q+4)} .
\end{aligned}
$$

Observe que a função densidade de $S R$ apresentada em (3.8) envolve um polinômio multiplicativo de terceiro grau com coeficientes dependendo de três constantes. Dessa forma, Cordeiro \& Ferrari (1991) definem uma estatística $S R$ modificada da forma

$$
S R_{c}=S R\left\{1-\left(c+b S R+a S R^{2}\right)\right\}
$$

em que o fator de correção entre chaves é uma função da própria estatística $S R$. Eles obtiveram as constantes $a, b$ e $c$ de tal forma que a função densidade da estatística $S R_{c}$ apresentada em (3.9) coincida com $f_{q}(z)$ até ordem $O\left(n^{-1}\right)$. Os autores mostraram que

$$
a=\frac{A_{3}}{12 q(q+2)(q+4)}, \quad b=\frac{A_{2}-2 A_{3}}{12 q(q+2)} \quad \text { e } \quad c=\frac{A_{1}-A_{2}+A_{3}}{12 q} .
$$

Portanto, de (3.9) e (3.10), conclui-se que uma estatística escore modificada tendo distribuição qui- 
quadrado até ordem $O\left(n^{-1}\right)$ é da forma

$$
S R_{c}=S R\left\{1-B\left(S R, A_{1}, A_{2}, A_{3}, q\right)\right\},
$$

em que

$$
B\left(t, A_{1}, A_{2}, A_{3}, q\right)=\frac{A_{1}-A_{2}+A_{3}}{12 q}+\frac{A_{2}-2 A_{3}}{12 q(q+2)} t+\frac{A_{3}}{12 q(q+2)(q+4)} t^{2} .
$$

O fator $1-B\left(S R, A_{1}, A_{2}, A_{3}, q\right)$ é um tipo de correção de Bartlett envolvendo cumulantes de derivadas da $\log$-verossimilhança e um polinômio de segundo grau na própria estatística $S R$. Devido ao fato deste fator depender de $S R$, esta correção é denominada de correção tipo-Bartlett. Uma vez que os $A$ 's possam ser calculados através das expressões (3.3)-(3.5), um teste escore aperfeiçoado pode ser conduzido utilizando-se a estatística modificada $S R_{c}$ e a distribuição qui-quadrado com $q$ graus de liberdade como referência.

Em geral, $S R_{c}$ vai depender de parâmetros desconhecidos. Neste caso, Cordeiro \& Ferrari (1991) mostram que se substituirmos estes parâmetros por suas respectivas estimativas de máxima verossimilhança sob a hipótese nula, a distribuição da estatística modificada ainda será qui-quadrado com $q$ graus de liberdade até ordem $O\left(n^{-1}\right)$.

Vale ressaltar que os resultados obtidos por Cordeiro \& Ferrari (1991) são bastante abrangentes no sentido de que são válidos para hipóteses simples e compostas, para parâmetros uni e multidimensionais, ortogonais ou não-ortogonais, admitindo ainda a presença de parâmetros de perturbação. Os resultados obtidos pelos autores generalizam os resultados apresentados em Chandra \& Mukerjee (1991), que encontraram uma estatística escore aperfeiçoada tendo distribuição qui-quadrado até ordem $O\left(n^{-1}\right)$ para testar hipóteses nulas simples e compostas. Eles não trataram, no entanto, de situações mais gerais como, por exemplo, aquela em que a hipótese nula é composta e há mais do que um parâmetro de perturbação. Chandra \& Mukerjee (1991, p. 110) fazem a seguinte observação: "In this paper, we have considered simple null hypotheses. In the presence of nuisance parameter(s) the position is as follows: Let $\theta$ be the parameter of interest and $m$ be the nuisance parameter. If $\theta$ is one-dimensional then combining the methods in this paper with those in Mukerjee [23] it should be possible to derive appropriate modifications for Rao's statistic. The problem, however, becomes much more complex for multidimensional $\theta$ - in particular, if $\theta$ and $m$ are both multidimensional then in general one cannot employ parametric orthogonality (Cox and Reid [17]) and tensor methods (McCullagh [19, 20]) should be useful. These aspects deserve further attention."

A estatística corrigida pelo fator de correção tipo-Bartlett é usualmente definida como $S R_{c}=$ $S R\left\{1-B\left(S R, A_{1}, A_{2}, A_{3}, q\right)\right\}$. Entretanto, existem algumas definições alternativas à correção tipoBartlett que são equivalentes até ordem $O\left(n^{-1}\right)$. Por exemplo, $S R_{c}^{1}=S R \exp \left\{-B\left(S R, A_{1}, A_{2}, A_{3}, q\right)\right\}$ e $S R_{c}^{2}=S R /\left\{1+B\left(S R, A_{1}, A_{2}, A_{3}, q\right)\right\}$ são equivalentes a $S R_{c}$ se termos de ordens menores que $O\left(n^{-1}\right)$ são ignorados. Note que $S R_{c}^{1}$ tem a vantagem de ser uma estatística escore corrigida que sempre apresenta valores positivos.

\subsection{Correção tipo-Bartlett no Modelo de Regressão Birnbaum-Saunders}

Nesta seção apresentamos fatores de correções tipo-Bartlett em testes de hipóteses sobre o vetor de parâmetros $\boldsymbol{\theta}=\left(\boldsymbol{\beta}^{\top}, \alpha\right)^{\top}$ que são de grande interesse na prática. Primeiramente, derivamos um fator de correção tipo-Bartlett para testes de hipóteses sobre um subconjunto do vetor de parâmetros $\boldsymbol{\beta}$. Em seguida, derivamos um fator de correção tipo-Bartlett para testar o parâmetro $\alpha$. Finalizamos esta seção apresentando um fator de correção para o teste sobre o vetor de parâmetros $\boldsymbol{\beta}$. 


\subsubsection{Hipótese Sobre um Subconjunto de $\beta$}

Na maioria dos problemas práticos, as restrições sobre um teste envolvem um subconjunto do vetor de parâmetros $\boldsymbol{\beta}$. Particionando $\boldsymbol{\beta}$ da forma $\boldsymbol{\beta}=\left(\boldsymbol{\beta}_{1}^{\top}, \boldsymbol{\beta}_{2}^{\top}\right)^{\top}$, em que $\boldsymbol{\beta}_{1}=\left(\beta_{1}, \beta_{2}, \ldots, \beta_{q}\right)^{\top}$ e $\boldsymbol{\beta}_{2}=\left(\beta_{q+1}, \beta_{q+2}, \ldots, \beta_{p}\right)^{\top}$ são vetores $q$ e $p-q$ dimensionais, respectivamente, o interesse é testar a hipótese

$$
\mathcal{H}_{0}: \boldsymbol{\beta}_{1}=\boldsymbol{\beta}_{1}^{(0)} \quad \text { contra } \quad \mathcal{H}_{1}: \boldsymbol{\beta}_{1} \neq \boldsymbol{\beta}_{1}^{(0)}
$$

em que $\boldsymbol{\beta}_{1}^{(0)}$ é um vetor $q$-dimensional de constantes conhecidas. Neste caso, $\boldsymbol{\beta}_{2}$ e $\alpha$ são parâmetros de perturbação. Em geral, em aplicações práticas, considera-se $\boldsymbol{\beta}_{1}^{(0)}=\mathbf{0}$. A hipótese anterior induz a uma partição da matriz modelo da forma $\boldsymbol{X}=\left[\begin{array}{ll}\boldsymbol{X}_{1} & \boldsymbol{X}_{2}\end{array}\right]$, cujas dimensões de $\boldsymbol{X}_{1}$ e $\boldsymbol{X}_{2}$ são $n \times q$ e $n \times(p-q)$, respectivamente, sendo essas matrizes de posto completo. Adicionalmente, considere $\boldsymbol{U}(\boldsymbol{\theta})=\left(\boldsymbol{U}_{\boldsymbol{\beta}}(\boldsymbol{\theta})^{\top}, U_{\alpha}(\boldsymbol{\theta})\right)^{\top}$ a função escore total de $\boldsymbol{\theta}$, em que $\boldsymbol{U}_{\boldsymbol{\beta}}(\boldsymbol{\theta})$ e $U_{\alpha}(\boldsymbol{\theta})$ são as funções escore de $\boldsymbol{\beta}$ e $\alpha$, respectivamente. A função $\boldsymbol{U}_{\boldsymbol{\beta}}(\boldsymbol{\theta})$ pode ser particionada da mesma forma que $\boldsymbol{\beta}$ como $\boldsymbol{U}_{\boldsymbol{\beta}}(\boldsymbol{\theta})=\left(\boldsymbol{U}_{1}(\boldsymbol{\theta})^{\top}, \boldsymbol{U}_{2}(\boldsymbol{\theta})^{\top}\right)^{\top}$, em que

$$
\boldsymbol{U}_{1}(\boldsymbol{\theta})=\frac{1}{2} \boldsymbol{X}_{1}^{\top} \boldsymbol{s} \quad \text { e } \quad \boldsymbol{U}_{2}(\boldsymbol{\theta})=\frac{1}{2} \boldsymbol{X}_{2}^{\top} \boldsymbol{s}
$$

o vetor $s=\boldsymbol{s}(\boldsymbol{\theta})$ foi definido na Seção 1.3 .

Devido à ortogonalidade global entre $\boldsymbol{\beta}$ e $\alpha$ (veja a Seção 1.3), a matriz de informação total de Fisher $\boldsymbol{K}(\boldsymbol{\theta})$ para $\boldsymbol{\theta}$ e sua inversa $\boldsymbol{K}(\boldsymbol{\theta})^{-1}$ são bloco-diagonais e podem ser escritas, como consequência da partição da matriz $\boldsymbol{X}$ que a hipótese anterior induz, na forma

$$
\boldsymbol{K}(\boldsymbol{\theta})=\left[\begin{array}{ccc}
\boldsymbol{K}_{11} & \boldsymbol{K}_{12} & \mathbf{0} \\
\boldsymbol{K}_{21} & \boldsymbol{K}_{22} & \mathbf{0} \\
\mathbf{0} & \mathbf{0} & \kappa_{\alpha, \alpha}
\end{array}\right] \quad \text { e } \boldsymbol{K}(\boldsymbol{\theta})^{-1}=\left[\begin{array}{ccc}
\boldsymbol{K}^{11} & \boldsymbol{K}^{12} & \mathbf{0} \\
\boldsymbol{K}^{21} & \boldsymbol{K}^{22} & \mathbf{0} \\
\mathbf{0} & \mathbf{0} & \kappa_{\alpha, \alpha}^{-1}
\end{array}\right]
$$

em que

$$
\boldsymbol{K}_{11}=h(\alpha)\left(\boldsymbol{X}_{1}^{\top} \boldsymbol{X}_{1}\right) / 4, \quad \boldsymbol{K}_{12}=\boldsymbol{K}_{21}^{\top}=h(\alpha)\left(\boldsymbol{X}_{1}^{\top} \boldsymbol{X}_{2}\right) / 4 \quad \text { e } \quad \boldsymbol{K}_{22}=h(\alpha)\left(\boldsymbol{X}_{2}^{\top} \boldsymbol{X}_{2}\right) / 4 ;
$$

veja Seção 1.3 para uma descrição da função $h(\alpha)$. Ainda, $\kappa_{\alpha, \alpha}=-\mathbb{E}\left\{\partial^{2} \ell(\boldsymbol{\theta}) / \partial \alpha^{2}\right\}=2 n / \alpha^{2}$. As matrizes $\boldsymbol{K}^{11}, \boldsymbol{K}^{12}, \boldsymbol{K}^{21}$ e $\boldsymbol{K}^{22}$ podem ser escritas como

$$
\begin{aligned}
& \boldsymbol{K}^{11}=\left(\boldsymbol{R}^{\top} \boldsymbol{R}\right)^{-1}, \\
& \boldsymbol{K}^{12}=\boldsymbol{K}^{21 \top}=-\left(\boldsymbol{R}^{\top} \boldsymbol{R}\right)^{-1} \boldsymbol{C}^{\top}, \\
& \boldsymbol{K}^{22}=\left(\boldsymbol{X}_{2}^{\top} \boldsymbol{X}_{2}\right)^{-1}+\boldsymbol{C}\left(\boldsymbol{R}^{\top} \boldsymbol{R}\right)^{-1} \boldsymbol{C}^{\top},
\end{aligned}
$$

em que $\boldsymbol{R}=\boldsymbol{X}_{1}-\boldsymbol{X}_{2} \boldsymbol{C}$ com $\boldsymbol{C}=\left(\boldsymbol{X}_{2}^{\top} \boldsymbol{X}_{2}\right)^{-1} \boldsymbol{X}_{2}^{\top} \boldsymbol{X}_{1}$ representando uma matriz $(p-q) \times q$ cujas colunas são vetores dos coeficientes de uma regressão linear das colunas de $\boldsymbol{X}_{1}$ sobre a matriz modelo $\boldsymbol{X}_{2}$; veja Rao (1973, p. 29) para mais detalhes sobre inversas de matrizes particionadas. É claro que as matrizes $\boldsymbol{K}^{11}, \boldsymbol{K}^{12}, \boldsymbol{K}^{21}$ e $\boldsymbol{K}^{22}$ devem ser multiplicadas por $4 / h(\alpha)$. Dessa forma, a estatística escore $S R$ para testar $\mathcal{H}_{0}$ pode ser escrita na forma

$$
S R=h(\widetilde{\alpha})^{-1} \widetilde{\boldsymbol{s}}^{\top} \boldsymbol{X}_{1}\left(\boldsymbol{R}^{\top} \boldsymbol{R}\right)^{-1} \boldsymbol{X}_{1}^{\top} \widetilde{\boldsymbol{s}}
$$

em que $\widetilde{\boldsymbol{s}}=\boldsymbol{s}(\widetilde{\boldsymbol{\theta}})$ e $\widetilde{\boldsymbol{\theta}}=\left(\boldsymbol{\beta}_{1}^{(0) \top}, \widetilde{\boldsymbol{\beta}}_{2}^{\top}, \widetilde{\alpha}\right)^{\top}$ é a estimativa de $\boldsymbol{\theta}$ sob a hipótese nula. Assintoticamente e 
sob $\mathcal{H}_{0}, S R$ converge em distribuição para $\chi_{q}^{2}$. Rejeitamos a hipótese nula $\mathcal{H}_{0}$ se $S R>q_{1-\gamma}$, em que $q_{1-\gamma}$ representa o quantil $1-\gamma$ da distribuição $\chi_{q}^{2}$ e $\gamma$ o nível de significância adotado, $0<\gamma<1$.

A seguir, iremos definir algumas matrizes que são usadas para o cálculo do fator de correção tipo-Bartlett:

$$
\boldsymbol{A}_{\boldsymbol{\beta}}=\left[\begin{array}{cc}
\mathbf{0} & \mathbf{0} \\
\mathbf{0} & \boldsymbol{K}_{22}^{-1}
\end{array}\right], \boldsymbol{K}_{\boldsymbol{\beta}}^{-1}=\left[\begin{array}{ll}
\boldsymbol{K}^{11} & \boldsymbol{K}^{12} \\
\boldsymbol{K}^{21} & \boldsymbol{K}^{22}
\end{array}\right], \boldsymbol{A}=\left[\begin{array}{cc}
\boldsymbol{A}_{\boldsymbol{\beta}} & \mathbf{0} \\
\mathbf{0} & \kappa_{\alpha, \alpha}^{-1}
\end{array}\right] \quad \text { e } \quad \boldsymbol{M}=\left[\begin{array}{cc}
\boldsymbol{M}_{\boldsymbol{\beta}} & \mathbf{0} \\
\mathbf{0} & 0
\end{array}\right]
$$

em que $\boldsymbol{M}_{\boldsymbol{\beta}}=\boldsymbol{K}_{\boldsymbol{\beta}}^{-1}-\boldsymbol{A}_{\boldsymbol{\beta}}$. Sejam $m_{r \alpha}$ e $a_{r \alpha}$ os $(r, p+1)$-ésimos elementos das matrizes $\boldsymbol{M}$ e $\boldsymbol{A}$, respectivamente, e sejam $m_{\alpha \alpha}$ e $a_{\alpha \alpha}$ os $(p+1, p+1)$-ésimos elementos das matrizes $\boldsymbol{M}$ e $\boldsymbol{A}$, respectivamente. Dessa forma, temos que $m_{r \alpha}=m_{\alpha r}=m_{\alpha \alpha}=0, a_{r \alpha}=a_{\alpha r}=0(r=1,2, \ldots, p) \mathrm{e}$ $a_{\alpha \alpha}=\kappa_{\alpha, \alpha}^{-1}=\alpha^{2} / 2 n$.

Similarmente ao que Cribari-Neto \& Ferrari (1995a) desenvolveram para a classe de modelos lineares generalizados (McCullagh \& Nelder, 1989), é possível mostrar que, admitindo $\alpha$ desconhecido e pelo fato deste parâmetro ser globalmente ortogonal a $\boldsymbol{\beta}$,

$$
A_{1}=A_{1, \boldsymbol{\beta}}+A_{1, \boldsymbol{\beta} \alpha}, \quad A_{2}=A_{2, \boldsymbol{\beta}}+A_{2, \boldsymbol{\beta} \alpha} \quad \text { e } \quad A_{3}=A_{3, \boldsymbol{\beta}}+A_{3, \boldsymbol{\beta} \alpha},
$$

em que $A_{1, \boldsymbol{\beta}}, A_{2, \boldsymbol{\beta}}$ e $A_{3, \boldsymbol{\beta}}$ são como nas equações (3.3), (3.4) e (3.5), respectivamente. Essas quantidades correspondem às contribuições quando o parâmetro $\alpha$ é conhecido. As quantidades $A_{1, \boldsymbol{\beta} \alpha}$, $A_{2, \boldsymbol{\beta} \alpha}$ e $A_{3, \boldsymbol{\beta} \alpha}$ representam contribuições adicionais pelo fato do parâmetro $\alpha$ ser desconhecido. As quantidades $A_{1, \boldsymbol{\beta} \alpha}, A_{2, \boldsymbol{\beta} \alpha}$ e $A_{3, \boldsymbol{\beta} \alpha}$ são obtidas das expressões (3.3), (3.4) e (3.5), respectivamente, variando convenientemente os índices dessas equações, isto é,

$$
\begin{aligned}
& A_{1, \boldsymbol{\beta} \alpha}=3 \sum^{\prime}\left(\kappa_{\alpha \alpha k}+2 \kappa_{\alpha, \alpha k}\right)\left(\kappa_{r s t}+2 \kappa_{r s, t}\right) a_{\alpha \alpha} a_{s t} m_{k r} \\
&+3 \sum^{\prime}\left(\kappa_{i j k}+2 \kappa_{i, j k}\right)\left(\kappa_{r \alpha \alpha}+2 \kappa_{r \alpha, \alpha}\right) a_{i j} a_{\alpha \alpha} m_{k r} \\
&+3 \sum^{\prime}\left(\kappa_{\alpha \alpha k}+2 \kappa_{\alpha, \alpha k}\right)\left(\kappa_{r \alpha \alpha}+2 \kappa_{r \alpha, \alpha}\right) a_{\alpha \alpha}^{2} m_{k r} \\
&-6 \sum^{\prime}\left(\kappa_{\alpha \alpha k}+2 \kappa_{\alpha, \alpha k}\right) \kappa_{r, s, t} a_{\alpha \alpha} a_{k r} m_{s t} \\
&-6 \sum^{\prime}\left(\kappa_{i j \alpha}+2 \kappa_{i, j \alpha}\right) \kappa_{\alpha, s, t} a_{i j} a_{\alpha \alpha} m_{s t} \\
&-6 \sum^{\prime}\left(\kappa_{\alpha \alpha \alpha}+2 \kappa_{\alpha, \alpha \alpha}\right) \kappa_{\alpha, s, t} a_{\alpha \alpha}^{2} m_{s t} \\
&+6 \sum^{\prime}\left(\kappa_{i, \alpha k}-\kappa_{i, \alpha, k}\right)\left(\kappa_{r \alpha t}+2 \kappa_{r \alpha, t}\right) a_{\alpha \alpha} a_{k t} m_{i r} \\
&+6 \sum^{\prime}\left(\kappa_{i, j \alpha}-\kappa_{i, j, \alpha}\right)\left(\kappa_{r s \alpha}+2 \kappa_{r s, \alpha}\right) a_{j s} a_{\alpha \alpha} m_{i r} \\
&+6 \sum^{\prime}\left(\kappa_{i, \alpha \alpha}-\kappa_{i, \alpha, \alpha}\right)\left(\kappa_{r \alpha \alpha}+2 \kappa_{r \alpha, \alpha}\right) a_{\alpha \alpha}^{2} m_{i r} \\
&-6 \sum^{\prime}\left(\kappa_{i, j, \alpha, \alpha}+\kappa_{i, j, \alpha \alpha}\right) a_{\alpha \alpha} m_{i j}, \\
& A_{2, \boldsymbol{\beta} \alpha}=-3 \sum^{\prime} \kappa_{i, j, \alpha} \kappa_{\alpha, s, t} a_{\alpha \alpha} m_{i j} m_{s t}+6 \sum^{\prime}\left(\kappa_{\alpha \alpha k}+2 \kappa_{\alpha, \alpha k}\right) \kappa_{r, s, t} a_{\alpha \alpha} m_{k r} m_{s t} \\
&-6 \sum_{i, j, \alpha}^{\prime} \kappa_{r, s, \alpha} a_{\alpha \alpha} m_{i r} m_{j s}
\end{aligned}
$$


$\mathrm{e}$

$$
A_{3, \boldsymbol{\beta} \alpha}=0,
$$

já que $m_{r \alpha}=m_{\alpha r}=m_{\alpha \alpha}=0(r=1,2, \ldots, p)$. Ainda, $a_{i j}$ e $m_{i j}(i, j=1,2, \ldots, p)$ são os $(i, j)$-ésimos elementos das matrizes $\boldsymbol{A}$ e $\boldsymbol{M}$, respectivamente. A notação introduzida acima referese aos cumulantes envolvendo o parâmetro $\alpha$, dada como segue: $\kappa_{\alpha \alpha}=\mathbb{E}\left\{\partial^{2} \ell(\boldsymbol{\theta}) / \partial \alpha^{2}\right\}, \kappa_{r s \alpha}=$ $\mathbb{E}\left\{\partial^{3} \ell(\boldsymbol{\theta}) / \partial \beta_{r} \partial \beta_{s} \partial \alpha\right\}, \kappa_{r s, \alpha}=\mathbb{E}\left\{\left(\partial^{2} \ell(\boldsymbol{\theta}) / \partial \beta_{r} \partial \beta_{s}\right)(\partial \ell(\boldsymbol{\theta}) / \partial \alpha)\right\}$, etc.

Sejam as matrizes

$$
\begin{aligned}
\boldsymbol{Z} & =\boldsymbol{X}\left(\boldsymbol{X}^{\top} \boldsymbol{X}\right)^{-1} \boldsymbol{X}^{\top}=\left\{z_{i j}\right\}, \quad \boldsymbol{Z}_{d}=\operatorname{diag}\left\{z_{11}, z_{22}, \ldots, z_{n n}\right\} \\
\boldsymbol{Z}_{2} & =\boldsymbol{X}_{2}\left(\boldsymbol{X}_{2}^{\top} \boldsymbol{X}_{2}\right)^{-1} \boldsymbol{X}_{2}^{\top}=\left\{z_{2 i j}\right\}, \quad \boldsymbol{Z}_{2 d}=\operatorname{diag}\left\{z_{211}, z_{222}, \ldots, z_{2 n n}\right\}
\end{aligned}
$$

e considere $\boldsymbol{Z}^{(2)}=\boldsymbol{Z} \odot \boldsymbol{Z}, \boldsymbol{Z}_{d}^{(2)}=\boldsymbol{Z}_{d} \odot \boldsymbol{Z}_{d}$, etc; o símbolo $\odot$ denota o produto de Hadamard de duas matrizes. Após longo e tedioso trabalho algébrico, veja Apêndice $\mathrm{C}$, obtemos como expressões para $A_{1, \boldsymbol{\beta}}, A_{2, \boldsymbol{\beta}}$ e $A_{3, \boldsymbol{\beta}}$ as quantidades

$$
\begin{aligned}
& A_{1, \boldsymbol{\beta}}=g_{1}(\alpha) \operatorname{tr}\left\{\left(\boldsymbol{Z}-\boldsymbol{Z}_{2}\right)_{d} \boldsymbol{Z}_{2 d}\right\} \\
& A_{2, \boldsymbol{\beta}}=g_{2}(\alpha) \operatorname{tr}\left\{\left(\boldsymbol{Z}-\boldsymbol{Z}_{2}\right)_{d}^{(2)}\right\} \\
& A_{3, \boldsymbol{\beta}}=0
\end{aligned}
$$

e para $A_{1, \boldsymbol{\beta} \alpha}, A_{2, \boldsymbol{\beta} \alpha}$ e $A_{3, \boldsymbol{\beta} \alpha}$ as expressões

$$
\begin{aligned}
& A_{1, \boldsymbol{\beta} \alpha}=\frac{12 q}{n a_{1}(\alpha)}\left\{\frac{4\left(2+\alpha^{2}\right) s_{3}(\alpha)}{a_{1}(\alpha) \alpha}(p-q)+s_{3}(\alpha) \alpha-s_{4}(\alpha) \alpha^{2}\right\} \\
& A_{2, \boldsymbol{\beta} \alpha}=-\frac{24 s_{3}(\alpha)^{2} \alpha^{2}}{n a_{1}(\alpha)^{2}} q(q+2), \\
& A_{3, \boldsymbol{\beta} \alpha}=0
\end{aligned}
$$

em que $\operatorname{tr}(\cdot)$ representa o operador traço de uma matriz e

$$
\begin{gathered}
g_{1}(\alpha)=-\frac{96 s_{1}(\alpha)}{a_{1}(\alpha)^{2}}, \quad g_{2}(\alpha)=\frac{48 s_{2}(\alpha)}{a_{1}(\alpha)^{2}}, \\
s_{1}(\alpha)=-2 a_{4}(\alpha)+\frac{1}{8}\left\{s_{0}(\alpha)-a_{1}(\alpha)^{2}\right\}, \quad s_{2}(\alpha)=-3 a_{4}(\alpha)+\frac{3}{16}\left\{s_{0}(\alpha)-a_{1}(\alpha)^{2}\right\}, \\
s_{3}(\alpha)=\frac{2\left(2+\alpha^{2}\right)}{\alpha^{3}}-a_{2}(\alpha) \quad \text { e } \quad s_{4}(\alpha)=-\left\{\frac{4\left(1-2 \alpha^{2}\right)}{\alpha^{4}}+a_{3}(\alpha)\right\} .
\end{gathered}
$$

Adicionalmente, considere $a_{0}(\alpha)=\{1-\operatorname{erf}(\sqrt{2} / \alpha)\} \exp \left(2 / \alpha^{2}\right)$, assim, $s_{0}(\alpha), a_{1}(\alpha), a_{2}(\alpha), a_{3}(\alpha)$ e $a_{4}(\alpha)$ são expressos nas formas

$$
\begin{gathered}
s_{0}(\alpha)=12+\frac{2}{\alpha^{2}}+\frac{16}{\alpha^{4}}+\sqrt{\frac{\pi}{2}}\left(\frac{1}{\alpha}+\frac{12}{\alpha^{3}}\right) a_{0}(\alpha), \quad a_{1}(\alpha)=2+\frac{4}{\alpha^{2}}-\frac{\sqrt{2 \pi}}{\alpha} a_{0}(\alpha), \\
a_{2}(\alpha)=\frac{3}{\alpha^{3}}-\frac{\sqrt{2 \pi}}{4 \alpha^{2}}\left(1+\frac{4}{\alpha^{2}}\right) a_{0}(\alpha), \quad a_{3}(\alpha)=-\frac{10}{\alpha^{4}}-\frac{4}{\alpha^{6}}+\frac{1}{\alpha^{7}} \sqrt{\frac{\pi}{2}}\left(\alpha^{4}+10 \alpha^{2}+8\right) a_{0}(\alpha) \quad \text { е }
\end{gathered}
$$




$$
a_{4}(\alpha)=-\frac{1}{4}\left\{2+\frac{7}{\alpha^{2}}-\sqrt{\frac{\pi}{2}}\left(\frac{1}{2 \alpha}+\frac{6}{\alpha^{3}}\right) a_{0}(\alpha)\right\} .
$$

Observe que as expressões para $A_{1, \boldsymbol{\beta}}$ e $A_{2, \boldsymbol{\beta}}$ apresentadas em (3.15) são funções das matrizes $\left(\boldsymbol{Z}-\boldsymbol{Z}_{2}\right)_{d}, \boldsymbol{Z}_{2 d}$ e do parâmetro $\alpha$, não depende do vetor de parâmetros deconhecido $\boldsymbol{\beta}$. Além disso, $A_{3, \boldsymbol{\beta}}=0$. Note que estas quantidades envolvem operações matriciais bem simples e podem ser facilmente implementadas em qualquer linguagem de programação que execute operações matriciais, tais como R (R Development Core Team, 2008), Ox (Cribari-Neto \& Zarkos, 2003; Doornik, 2006), MAPLE (Abell \& Braselton, 1994), entre outras. É interessante notar que as expressões para $A_{1, \boldsymbol{\beta} \alpha} \mathrm{e}$ $A_{2, \boldsymbol{\beta} \alpha}$ apresentadas em (3.16) são funções apenas do número total de parâmetros $p$, do número de restrições sob a hipótese nula $q$ e do parâmetro $\alpha$. Em particular, estas quantidades não dependem da matriz modelo $\boldsymbol{X}$, exceto através do seu posto. Adicionalmente, $A_{3, \boldsymbol{\beta} \alpha}=0$. As quantidades apresentadas em (3.15) e (3.16) não são fáceis de serem interpretadas.

Portanto, uma estatística escore aperfeiçoada é obtida como em (3.11) através dos $A$ 's obtidos acima, isto é, $A_{1}=A_{1, \boldsymbol{\beta}}+A_{1, \boldsymbol{\beta} \alpha}, A_{2}=A_{2, \boldsymbol{\beta}}+A_{2, \boldsymbol{\beta} \alpha}$ e $A_{3}=0$. É importante ressaltar que essas expressões devem ser calculadas sob a hipótese nula. Note que o fator de correção tipo-Bartlett é um polinônio de primeiro grau na estatística escore $S R$, uma vez que $A_{3}=0$.

\subsubsection{Hipótese Sobre $\alpha$}

Estamos interessados em testar a hipótese

$$
\mathcal{H}_{0}: \alpha=\alpha^{(0)} \quad \text { contra } \quad \mathcal{H}_{1}: \alpha \neq \alpha^{(0)},
$$

em que $\alpha^{(0)}$ é uma constante positiva conhecida e $\boldsymbol{\beta}$ um vetor $p$ dimensional de parâmetros de perturbação. A função escore de $\alpha$ tem a forma

$$
U_{\alpha}(\boldsymbol{\theta})=-\frac{n}{\alpha}+\frac{1}{\alpha} \sum_{i=1}^{n} \xi_{i 2}^{2},
$$

em que $\xi_{i 2}=\xi_{i 2}(\boldsymbol{\theta})$ foi definido na Seção 1.3. A estatística escore para testar $\mathcal{H}_{0}$ pode ser escrita na forma

$$
S R=n\left\{\bar{\xi}_{2}-1\right\}^{2} / 2,
$$

em que $\bar{\xi}_{2}=\bar{\xi}_{2}(\widetilde{\boldsymbol{\theta}})=\sum_{i=1}^{n} \xi_{i 2}^{2} / n$ e $\widetilde{\boldsymbol{\theta}}=\left(\widetilde{\boldsymbol{\beta}}^{\top}, \alpha^{(0)}\right)^{\top}$ é a estimativa de máxima verossimilhança de $\boldsymbol{\theta}$ sob a hipótese nula. Assintoticamente e sob a hipótese nula, $S R$ converge para a distribuição $\chi_{1}^{2}$.

Devido à ortogonalidade entre $\boldsymbol{\beta}$ e $\alpha$, a matriz particionada $\boldsymbol{M}$ para $\boldsymbol{\beta}$ e $\alpha$ apresentada em (3.12) considerando a hipótese nula $\mathcal{H}_{0}: \alpha=\alpha^{(0)}$ tem apenas o $(p+1, p+1)$-ésimo elemento diferente de zero, sendo este $\alpha^{2} / 2 n$. Isto implica considerável redução para várias somas em (3.3)-(3.5) envolvendo permutações dos parâmetros $\boldsymbol{\beta}$ e $\alpha$. Notando que $\operatorname{tr}(\boldsymbol{Z})=\operatorname{tr}(\boldsymbol{Z} \boldsymbol{Z})=p$ (a matriz $\boldsymbol{Z}$ está definida em (3.14)), o posto da matriz modelo $\boldsymbol{X}$, segue após algumas manipulações algébricas, veja Apêndice 
C, que

$$
\begin{aligned}
& A_{1}=\frac{24 p}{n a_{1}(\alpha)^{2} \alpha^{4}}\left\{\left(2+\alpha^{2}\right)^{2}(p+6)-\alpha^{2}\left[4\left(2+\alpha^{2}\right) a_{2}(\alpha) \alpha+\left(4+5 \alpha^{2}\right) a_{1}(\alpha)\right]\right\}, \\
& A_{2}=\frac{12}{n}\left\{3-\frac{4\left(2+\alpha^{2}\right)}{a_{1}(\alpha) \alpha^{2}} p\right\} \\
& A_{3}=\frac{40}{n}
\end{aligned}
$$

em que $a_{1}(\alpha)$ e $a_{2}(\alpha)$ foram definidos anteriormente. Note que as expressões apresentadas para os $A$ 's em (3.17) são muito simples, de fácil implementação computacional e não envolvem a matriz modelo $\boldsymbol{X}$, exceto através de seu posto. Além disso, essas expressões dependem apenas do parâmetro $\alpha$, não envolvendo o vetor de parâmetros desconhecido $\boldsymbol{\beta}$, ou seja, só envolvem quantidades conhecidas. É claro que os $A$ 's devem ser avaliados em $\alpha^{(0)}$ especificado na hipótese nula.

\subsubsection{Hipótese Sobre $\beta$}

Estamos interessados em testar a hipótese

$$
\mathcal{H}_{0}: \boldsymbol{\beta}=\boldsymbol{\beta}^{(0)} \quad \text { contra } \quad \mathcal{H}_{1}: \boldsymbol{\beta} \neq \boldsymbol{\beta}^{(0)},
$$

em que $\boldsymbol{\beta}^{(0)}$ é um vetor $p$ dimensional de constantes conhecidas e $\alpha$ é um parâmetro de perturbação. A função escore de $\boldsymbol{\beta}$ tem a forma

$$
\boldsymbol{U}_{\boldsymbol{\beta}}(\boldsymbol{\theta})=\frac{1}{2} \boldsymbol{X}^{\top} \boldsymbol{s}
$$

em que $\boldsymbol{s}=\boldsymbol{s}(\boldsymbol{\theta})$ está definido na Seção 1.3. A estatística escore para testar $\mathcal{H}_{0}$ pode ser escrita na forma

$$
S R=h(\widetilde{\alpha})^{-1} \widetilde{\boldsymbol{s}}^{\top} \boldsymbol{X}\left(\boldsymbol{X}^{\top} \boldsymbol{X}\right)^{-1} \boldsymbol{X}^{\top} \widetilde{\boldsymbol{s}}
$$

em que $\widetilde{\boldsymbol{s}}=\boldsymbol{s}(\widetilde{\boldsymbol{\theta}})$ e $\widetilde{\boldsymbol{\theta}}=\left(\boldsymbol{\beta}^{(0)^{\top}}, \widetilde{\alpha}\right)^{\top}$ é a estimativa de $\boldsymbol{\theta}$ sob a hipótese nula; veja Seção 1.3 para uma descrição da função $h(\alpha)$. Assintoticamente e sob a hipótese nula, $S R$ convege para uma distribuição $\chi_{p}^{2}$.

É possível obter expressões simples para os $A$ 's devido à ortogonalidade entre $\boldsymbol{\beta}$ e $\alpha$. De (3.3)-(3.5) segue após alguma álgebra, veja o Apêndice $\mathrm{C}$, que

$$
\begin{aligned}
& A_{1}=\frac{12 p}{n a_{1}(\alpha)}\left\{s_{3}(\alpha) \alpha-s_{4}(\alpha) \alpha^{2}\right\}, \\
& A_{2}=-\frac{24 s_{3}(\alpha)^{2} \alpha^{2}}{n a_{1}(\alpha)^{2}} p(p+2)+\frac{48 s_{2}(\alpha)}{a_{1}(\alpha)^{2}} \operatorname{tr}\left(\boldsymbol{Z}_{d}^{(2)}\right), \\
& A_{3}=0
\end{aligned}
$$

em que $a_{1}(\alpha), s_{2}(\alpha), s_{3}(\alpha)$ e $s_{4}(\alpha)$ foram definidos anteriormente, $\boldsymbol{Z}_{d}$ está dado em (3.14) e $\operatorname{tr}(\cdot)$ representa o operador traço de uma matriz. Observe que as expressões para os $A$ 's apresentadas em (3.18) são bem simples e podem ser implementadas em qualquer linguagem de programação que execute operações matriciais. Além disso, estas quantidades devem ser calculadas sob a hipótese nula. Note que essas expressões não dependem do vetor de parâmetros desconhecido $\boldsymbol{\beta}$, dependendo apenas de $\alpha$ e da matriz modelo $\boldsymbol{X}$, além, evidentemente, do seu posto. É claro que, como esperado, as expressões apresentadas em (3.18) coincidem com $A_{1}=A_{1, \boldsymbol{\beta}}+A_{1, \boldsymbol{\beta} \alpha}, A_{2}=A_{2, \boldsymbol{\beta}}+A_{2, \boldsymbol{\beta} \alpha}$ e 
$A_{3}=A_{3, \boldsymbol{\beta}}+A_{3, \boldsymbol{\beta} \alpha}$, apresentadas em (3.15)-(3.16), quando $q=p$.

\subsubsection{Aproximação para $a_{0}(\alpha)$}

Note que muitas quantidades que são funções do parâmetro $\alpha$ apresentadas anteriormente dependem de

$$
a_{0}(\alpha)=\left\{1-\operatorname{erf}\left(\frac{\sqrt{2}}{\alpha}\right)\right\} \exp \left(\frac{2}{\alpha^{2}}\right)
$$

Para valores pequenos de $\alpha$, é possível aproximar $a_{0}(\alpha)$ como (Abramowitz \& Stegun, 1970, p. 298)

$$
a_{0}(\alpha)=\frac{\alpha}{\sqrt{2 \pi}}\left\{1-\frac{\alpha^{2}}{4}+\frac{3 \alpha^{4}}{16}\right\}+O\left(\alpha^{7}\right)
$$

Em avaliações numéricas, recomendamos utilizar a aproximação dada em (3.19) para $a_{0}(\alpha)$ quando $\alpha$ é pequeno, $\alpha<0.5$ digamos.

\subsection{Resultados Numéricos}

Apresentamos alguns resultados de simulações Monte Carlo para avaliar a eficácia da correção tipo-Bartlett para o teste escore no modelo de regressão Birnbaum-Saunders. Comparamos os desempenhos de cinco testes: o teste escore usual, $S R$, o teste escore corrigido pelo fator de correção tipo-Bartlett, $S R_{c}$, duas de suas versões assintoticamente equivalentes, $S R_{c}^{1}$ e $S R_{c}^{2}$, e o teste escore usual $S R$ utilizando como ponto crítico o quantil modificado apresentado em (3.7), isto é, calculamos $\operatorname{Pr}\left(S R>z_{\gamma}\right)$. Este teste está denotado nas tabelas como $S R^{*}$. Vale recordar que $S R_{c}=S R\left\{1-B\left(S R, A_{1}, A_{2}, A_{3}, q\right)\right\}, S R_{c}^{1}=S R \exp \left\{-B\left(S R, A_{1}, A_{2}, A_{3}, q\right)\right\}$ e $S R_{c}^{2}=S R /\{1+$ $\left.B\left(S R, A_{1}, A_{2}, A_{3}, q\right)\right\}$. O desempenho de cada teste é avaliado em função da proximidade das probabilidades de rejeição da hipótese nula, sendo esta verdadeira (probabilidade do erro tipo I), aos respectivos níveis nominais. As simulações realizadas foram baseadas no modelo de regressão

$$
y_{i}=\beta_{1} x_{i 1}+\beta_{2} x_{i 2}+\cdots+\beta_{p} x_{i p}+\varepsilon_{i}
$$

em que $x_{i 1}=1$ e $\varepsilon_{i} \sim \mathcal{S N}(\alpha, 0,2), i=1,2, \ldots, n$. As covariáveis foram geradas como amostras aleatórias da distribuição $\mathcal{U}(0,1)$. Estas covariáveis foram mantidas fixas no laço de Monte Carlo.

O número de réplicas Monte Carlo foi fixado em 10000 e foram considerados os seguintes níveis nominais: $\gamma=10 \%, 5 \%, 1 \%$ e $0.5 \%$. Todas as simulações foram realizadas usando a linguagem de programação matricial 0x(Doornik, 2006). ${ }^{2}$ Para cada tamanho amostral e cada nível considerado, calculamos as taxas de rejeição de cada teste, isto é, estimamos, via simulação, $\operatorname{Pr}\left(S R>x_{\gamma}\right)$, $\operatorname{Pr}\left(S R_{c}>x_{\gamma}\right), \operatorname{Pr}\left(S R_{c}^{1}>x_{\gamma}\right), \operatorname{Pr}\left(S R_{c}^{2}>x_{\gamma}\right)$ e $\operatorname{Pr}\left(S R>z_{\gamma}\right)$ em que $x_{\gamma}$ é o quantil $1-\gamma$ apropriado da distribuição qui-quadrado de referência e $z_{\gamma}$ é o quantil modificado proposto em Harris (1985). Todas as entradas das tabelas apresentadas são porcentagens.

Na Tabela 3.1, apresentamos as taxas de rejeição empíricas considerando a hipótese nula $\mathcal{H}_{0}$ : $\beta_{p-1}=\beta_{p}=0$, tamanho de amostra $n=30$ e $\alpha=0.5$. Além disso, diferentes valores para $p$ foram considerados. Variamos $p$ para analisar o efeito do número de covariáveis presentes no modelo nos diferentes testes. Ainda, sem perda de generalidade, a variável resposta foi gerada assumindo que $\beta_{1}=\beta_{2}=\cdots=\beta_{p-2}=1$. Observe que o teste escore usual, $S R$, apresenta alta distorção de tamanho, isto é, suas taxas de rejeição estão muito afastadas dos níveis de significância considerados,

\footnotetext{
${ }^{2}$ Veja http://www.doornik. com para mais detalhes sobre esta linguagem de programação.
} 
principalmente quando o número de regressores presentes no modelo aumenta. Por exemplo, quando $p=10$ e $\gamma=5 \%$, a taxa de rejeição do teste foi $10.30 \%$, mais que o dobro do nível de significância considerado, ou seja, uma distorção de tamanho considerável. A tendência do teste em rejeitar com frequência demasiada a hipótese nula é atenuada pela correção tipo-Bartlett de forma que os testes escore corrigidos, $S R_{c}, S R_{c}^{1}, S R_{c}^{2}$ e $S R^{*}$, apresentam leve distorção de tamanho. Por exemplo, as taxas de rejeição foram $5.50 \%, 5.94 \%, 6.23 \%$ e $5.93 \%$, para os testes $S R_{c}, S R_{c}^{1}, S R_{c}^{2}$ e $S R^{*}$, respectivamente, considerando a mesma situação anterior.

Ao compararmos as Tabelas 3.1 e 3.2, ou seja, quando passamos de $\alpha=0.5$ para $\alpha=1.0$, notamos que os comportamentos dos testes não se altera. Observe que as taxas de rejeição empíricas do teste $S R$ estão acima dos níveis nominais considerados e, novamente, os testes $S R_{c}, S R_{c}^{1}, S R_{c}^{2}$ e $S R^{*}$ apresentam taxas de rejeição muito próximas dos níveis nominais. Por exemplo, quando $p=9$ e $\gamma=5 \%$, as taxas de rejeição foram $7.62 \%, 5.66 \%, 5.73 \%, 5.78 \%$ e $5.64 \%$ para os testes $S R, S R_{c}$, $S R_{c}^{1}, S R_{c}^{2}$ e $S R^{*}$, respectivamente (veja a Tabela 3.2 ).

Na Tabela 3.3, fixamos o valor de $p$ em 7 e variamos o tamanho de amostra em $n=20,30,40,50$, 100, 200 e 500, considerando $\alpha=0.5$. Neste caso, a hipótese nula considerada é $\mathcal{H}_{0}: \beta_{6}=\beta_{7}=0$. Com o aumento do tamanho da amostra, as taxas de rejeição empíricas dos testes ficam mais próximas do nível nominal, como esperado. Observe também que há necessidade de um tamanho amostral relativamente grande $(n>50)$ para que o teste escore usual $S R$ apresente uma pequena distorção de tamanho; por exemplo, quando $n=50$ e $\gamma=10 \%$, as taxas de rejeição empíricas dos testes $S R$, $S R_{c}, S R_{c}^{1}, S R_{c}^{2}$ e $S R^{*}$ foram, respectivamente, $12.74 \%, 10.75 \%, 10.80 \%, 10.84 \%$ e $10.80 \%$. Observe que quando $n=20$ o teste escore usual apresentou taxas de rejeição muito acima dos níveis nominais considerados, enquanto os testes corrigidos, mesmo para um tamanho amostral dessa maginitude, apresentaram taxas de rejeição empíricas muito próximas dos níveis nominais. Observe também que para um tamanho amostral relativamente grande $(n \geq 200)$, os testes corrigidos apresentaram os mesmos valores para as taxas de rejeição empíricas em todos os níveis nominais considerados, evidenciando, assim, a equivalência assintótica destes testes. É claro que $n=30$ equivale ao caso da Tabela 3.1 quando $p=7$.

Na Tabela 3.4 apresentamos as médias e variâncias de Monte Carlo das estatísticas $S R, S R_{c}$, $S R_{c}^{1}$ e $S R_{c}^{2}$. Observe que as médias e variâncias de $S R_{c}, S R_{c}^{1}$ e $S R_{c}^{2}$ estão mais próximas da média e variância da distribuição qui-quadrado de referência do que as médias e variâncias da estatística $S R$; por exemplo, quando $n=30$, as médias e variâncias Monte Carlo das estatísticas $S R, S R_{c}$, $S R_{c}^{1}$ e $S R_{c}^{2}$ foram $2.3,2.0,2.1$ e $2.1 ; 4.8,4.1,4.1$ e 4.2 , respectivamente. Adicionalmente, à medida que o tamanho da amostra aumenta, as médias e variâncias de todas as estatísticas se aproximam, respectivamente, da média e variância da distribuição $\chi_{2}^{2}$. Na Tabela 3.5 comparamos os quantis amostrais das estatísticas $S R, S R_{c}, S R_{c}^{1}$ e $S R_{c}^{2}$ com os da distribuição $\chi_{2}^{2}$. Consideramos $n=30$, $p=6$ e $\alpha=0.5$. Observe que os quantis amostrais de $S R$ estão acima dos quantis da $\chi_{2}^{2}$, enquanto os quantis amostrais das estatísticas corrigidas pelo fator de correção tipo-Bartlett estão muito próximos dos quantis da distribuição $\chi_{2}^{2}$.

Na Figura 3.1 apresentamos gráficos dos quantis das estatísticas $S R, S R_{c}, S R_{c}^{1}$ e $S R_{c}^{2}$ versus os quantis assintóticos. Consideramos $p=6, \alpha=0.5$, diferentes tamanhos amostrais e como hipótese nula $\mathcal{H}_{0}: \beta_{5}=\beta_{6}=0$, ou seja, os quantis assintóticos referem-se aos quantis da distribuição quiquadrado com dois graus de liberdade. A linha cheia representa a identidade, ou seja, os quantis assintóticos são iguais aos quantis exatos. Observe que a estatística do teste escore usual $S R$ apresenta quantis superiores aos quantis da distribuição qui-quadrado, principalmente quando o tamanho da amostra é pequeno. Porém, à medida que o tamanho da amostra aumenta, os quantis da estatística 
$S R$ tendem aos quantis da distribuição qui-quadrado. Note ainda que as estatísticas corrigidas pelo fator de correção tipo-Bartlett apresentam quantis bem próximos dos quantis da distribuição quiquadrado em todos os tamanhos de amostra, evidenciando que as distribuições nulas das estatísticas $S R_{c}, S R_{c}^{1}$ e $S R_{c}^{2}$ são bem aproximadas pela distribuição assintótica utilizada no teste.

$\mathrm{Na}$ Tabela 3.6 apresentamos os resultados de simulação para as taxas de rejeição não-nulas (poder). Fixamos $p=6, \alpha=0.5, \gamma=10 \%, 5 \%$ e 1\%, e consideramos diferentes tamanhos amostrais: $n=30,50$ e 100 . As taxas de rejeição foram obtidas levando em consideração a hipótese alternativa $\mathcal{H}_{1}: \beta_{5}=\beta_{6}=\delta$, em que diferentes valores para $\delta$ são considerados. Adicionalmente, foi usado como ponto crítico para os testes $S R_{c}, S R_{c}^{1}$ e $S R_{c}^{2}$ o percentil da distribuição qui-quadrado com dois graus de liberdade. Somente consideramos os poderes dos testes escore corrigidos pelo fator de correção tipo-Bartlett, uma vez que o teste escore usual, $S R$, apresentou taxas de rejeição empíricas sob a hipótese nula bastantes distorcidas e, portanto, não pode ser recomendado. Os resultados evidenciam que não há perda de poder pelo fato de se usar o fator de correção tipo-Bartlett derivado neste capítulo. Os poderes dos quatro testes são bastante semelhentes; por exemplo, quando $n=30$, $\gamma=10 \%$ e $\delta=0.4$, os poderes dos testes $S R_{c}, S R_{c}^{1}, S R_{c}^{2}$ e $S R^{*}$ foram $38.83 \%, 39.04 \%, 39.29 \%$ e $38.89 \%$, respectivamente. Observe também que à medida que o tamanho da amostra aumenta, o poder cresce, como era esperado.

Na Tabela 3.7 apresentamos os resultados de simulações para testes de hipóteses sobre o parâmetro $\alpha$. Consideramos $n=20,30,40,50$ e 100, sendo que o número de covariáveis no modelo foi fixado em $p=5$. As hipóteses consideradas foram $\mathcal{H}_{0}: \alpha=0.5$ e $\mathcal{H}_{0}: \alpha=1.0$. Note nesta tabela que o teste escore usual apresentou taxas de rejeição empíricas bastantes distorcidas quando o tamanho da amostra é pequeno $(n=20,30,40$ e 50$)$. Observe que os testes $S R_{c}, S R_{c}^{1}, S R_{c}^{2}$ e $S R^{*}$ apresentam distorções de tamanho bem inferiores ao teste escore usual em todos os casos considerados. Por exemplo, quando $n=30, \gamma=10 \%$ e o parâmetro testado na hipótese nula é $\alpha=0.5$, as taxas de rejeição empíricas dos testes $S R, S R_{c}, S R_{c}^{1}, S R_{c}^{2}$ e $S R^{*}$ foram $15.13 \%, 9.77 \%, 10.02 \%, 10.40 \%$ e $9.21 \%$, respectivamente. Observe que ao passar de $\alpha=0.5$ para $\alpha=1.0$, as taxas de rejeição empíricas do teste $S R$ ficaram mais distorcidas. Da mesma forma, os testes corrigidos $S R_{c}, S R_{c}^{1}$, $S R_{c}^{2}$ e $S R^{*}$ também foram afetados com o aumento do parâmetro $\alpha$. Por exemplo, quando $n=20$ e $\gamma=5 \%$, as taxas de rejeição dos testes $S R, S R_{c}, S R_{c}^{1}, S R_{c}^{2}$ e $S R^{*}$ foram, respectivamente, $7.33 \%, 4.25 \%, 4.31 \%, 4.38 \%$ e $3.29 \%$ ( $\alpha=0.5$ ), e $8.26 \%, 3.34 \%, 3.61 \%, 3.81 \%$ e $2.29 \%(\alpha=1.0)$. Adicionalmente, note que à medida que o tamanho da amostra aumenta, a distorção de tamanho diminui, como era esperado.

Concluímos destes resultados que o teste escore usual pode apresentar taxas de rejeição empíricas sob a hipótese nula bastante distorcidas em amostras de tamanho pequeno e moderado. Dessa forma, este teste não deve ser recomendado em aplicações práticas se o tamanho da amostra não for relativamente grande. Por outro lado, o fator de correção tipo-Bartlett, derivado neste capítulo, faz com que esta taxa de rejeição fique mais próxima do nível nominal do teste. É difícil escolher um dentre os testes escore corrigidos apresentados neste capítulo, isto é, escolher entre $S R_{c}, S R_{c}^{1}, S R_{c}^{2}$ e $S R^{*}$, uma vez que todos foram eficazes em diminuir a distorção de tamanho do teste escore usual. Além disso, em termos de poder, estes testes apresentaram resultados equivalentes. Entretanto, o teste escore baseado na estatística de teste $S R_{c}$ foi levemente superior aos demais testes levandose em conta as taxas de rejeição empíricas sob a hipótese nula (compare as Tabelas 3.1 até 3.4). Portanto, dentre os testes corrigidos pelo fator de correção tipo-Bartlett, recomendamos o teste $S R_{c}$ em aplicações práticas. 
Tabela 3.1: Taxas de rejeição nulas dos testes $S R, S R_{c}, S R_{c}^{1}, S R_{c}^{2}$ e $S R^{*} \operatorname{com} \alpha=0.5$ e $n=30$.

\begin{tabular}{|c|c|c|c|c|c|c|c|c|c|c|}
\hline \multirow[b]{2}{*}{$p$} & \multicolumn{5}{|c|}{$\gamma=10 \%$} & \multicolumn{5}{|c|}{$\gamma=5 \%$} \\
\hline & $S R$ & $S R_{c}$ & $S R_{c}^{1}$ & $S R_{c}^{2}$ & $S R^{*}$ & $S R$ & $S R_{c}$ & $S R_{c}^{1}$ & $S R_{c}^{2}$ & $S R^{*}$ \\
\hline 3 & 10.30 & 10.00 & 10.00 & 10.00 & 9.97 & 4.56 & 4.72 & 4.72 & 4.72 & 4.72 \\
\hline 4 & 11.12 & 10.09 & 10.10 & 10.12 & 10.07 & 5.37 & 5.17 & 5.18 & 5.18 & 5.16 \\
\hline 5 & 12.34 & 10.13 & 10.24 & 10.28 & 10.12 & 5.97 & 5.18 & 5.21 & 5.22 & 5.13 \\
\hline 6 & 13.15 & 10.35 & 10.50 & 10.58 & 10.40 & 6.73 & 5.01 & 5.05 & 5.08 & 5.00 \\
\hline 7 & 14.53 & 10.56 & 10.72 & 10.87 & 10.71 & 7.51 & 5.16 & 5.25 & 5.31 & 5.18 \\
\hline 8 & 15.86 & 10.49 & 10.84 & 11.19 & 10.83 & 8.57 & 5.38 & 5.55 & 5.74 & 5.46 \\
\hline 9 & 16.98 & 10.51 & 10.94 & 11.29 & 10.98 & 9.03 & 5.25 & 5.50 & 5.67 & 5.44 \\
\hline \multirow[t]{2}{*}{10} & 18.11 & 10.64 & 11.32 & 11.84 & 11.51 & 10.30 & 5.50 & 5.94 & 6.23 & 5.93 \\
\hline & \multicolumn{5}{|c|}{$\gamma=1 \%$} & \multicolumn{5}{|c|}{$\gamma=0.5 \%$} \\
\hline$p$ & $S R$ & $S R_{c}$ & $S R_{c}^{1}$ & $S R_{c}^{2}$ & $S R^{*}$ & $S R$ & $S R_{c}$ & $S R_{c}^{1}$ & $S R_{c}^{2}$ & $S R^{*}$ \\
\hline 3 & 0.73 & 1.12 & 1.14 & 1.14 & 1.21 & 0.29 & 0.44 & 0.45 & 0.45 & 0.52 \\
\hline 4 & 0.77 & 0.94 & 0.94 & 0.95 & 1.01 & 0.27 & 0.40 & 0.40 & 0.42 & 0.44 \\
\hline 5 & 0.95 & 0.98 & 0.98 & 0.98 & 0.99 & 0.51 & 0.57 & 0.57 & 0.57 & 0.58 \\
\hline 6 & 1.11 & 1.01 & 1.01 & 1.01 & 0.96 & 0.55 & 0.53 & 0.53 & 0.53 & 0.52 \\
\hline 7 & 1.36 & 1.04 & 1.05 & 1.06 & 1.04 & 0.67 & 0.54 & 0.55 & 0.57 & 0.53 \\
\hline 8 & 1.65 & 1.16 & 1.16 & 1.17 & 1.12 & 0.83 & 0.54 & 0.55 & 0.56 & 0.54 \\
\hline 9 & 1.93 & 1.00 & 1.07 & 1.12 & 1.01 & 0.84 & 0.54 & 0.55 & 0.56 & 0.52 \\
\hline 10 & 2.43 & 1.15 & 1.25 & 1.32 & 1.14 & 1.25 & 0.63 & 0.65 & 0.68 & 0.60 \\
\hline
\end{tabular}


Tabela 3.2: Taxas de rejeição nulas dos testes $S R, S R_{c}, S R_{c}^{1}, S R_{c}^{2}$ e $S R^{*} \operatorname{com} \alpha=1.0$ e $n=30$.

\begin{tabular}{|c|c|c|c|c|c|c|c|c|c|c|}
\hline \multirow[b]{2}{*}{$P$} & \multicolumn{5}{|c|}{$\gamma=10 \%$} & \multicolumn{5}{|c|}{$\gamma=5 \%$} \\
\hline & $S R$ & $S R_{c}$ & $S R_{c}^{1}$ & $S R_{c}^{2}$ & $B^{*}$ & $S R$ & $N 10_{c}$ & $S R_{c}^{1}$ & $S R_{c}^{2}$ & $S R$ \\
\hline 3 & 9.23 & 9.96 & 9.99 & 10.00 & 10.06 & 4.20 & 4.77 & 4.79 & 4.80 & 4.86 \\
\hline 4 & 0.11 & .24 & 10.24 & 10.24 & 10.27 & 69 & 5.00 & 5.01 & 5.01 & 5.03 \\
\hline 5 & 10.70 & 10.33 & 10.36 & 10.38 & 10.31 & 5.14 & 5.32 & 5.33 & 5.33 & 5.32 \\
\hline 6 & .71 & .47 & 10.52 & 10. & 10 & 82 & 5.29 & 5.29 & 5.31 & 5.27 \\
\hline 7 & 12.85 & 10.88 & 10.93 & 11.04 & 10.90 & 6.35 & 5.51 & 5.54 & 5.56 & 5.47 \\
\hline 8 & 13.96 & 11.05 & 11.22 & 11.37 & 11.13 & 7.16 & 5.48 & 5.52 & 5.57 & 5.44 \\
\hline 9 & .79 & .25 & 1. & 11 & & .62 & 5.66 & 5.73 & 5. & 5.64 \\
\hline \multirow[t]{2}{*}{10} & 17 & 3 & 11.51 & 11 & 3 & 13 & 5.83 & 6.13 & 6. & 6.07 \\
\hline & \multicolumn{5}{|c|}{$\gamma=1 \%$} & \multicolumn{5}{|c|}{$\gamma=0.5 \%$} \\
\hline$p$ & $S R$ & $S R_{c}$ & $S R_{c}^{1}$ & $S R$ & $S R^{*}$ & $S R$ & $S R_{c}$ & $S R_{c}^{1}$ & $S R_{c}^{2}$ & $S R^{*}$ \\
\hline 3 & 0.54 & 1.04 & 1.05 & 1.09 & 1.18 & 0.25 & 0.42 & 0.42 & 0.44 & 0.51 \\
\hline 4 & 0.68 & 0.96 & 0.97 & 0.98 & 1.04 & 0.34 & 0.50 & 0.50 & 0.51 & 0.53 \\
\hline 5 & 0.78 & 0.97 & 0.98 & 0.98 & 1.02 & 0.42 & 0.59 & 0.60 & 0.60 & 0.65 \\
\hline 6 & 0.94 & 1.09 & 1.09 & 1.09 & 1.10 & 0.35 & 0.52 & 0.52 & 0.52 & 0.55 \\
\hline 7 & 1.08 & 1.04 & 1.05 & 1.05 & 1.04 & 0.52 & 0.58 & 0.58 & 0.58 & 0.59 \\
\hline 8 & 1.27 & 1.29 & 1.29 & 1.2 & 1.28 & 0.49 & 0.55 & 0.55 & 0.55 & 0.56 \\
\hline 9 & 1.38 & 1.08 & 1 & 1.0 & 1.0 & 9 & 0.53 & 0.53 & 0.54 & 0.53 \\
\hline 10 & 1.80 & 1.10 & 1.15 & 1.17 & 1.08 & 0.90 & 0.58 & 0.58 & 0.60 & 0.56 \\
\hline
\end{tabular}


Tabela 3.3: Taxas de rejeição nulas dos testes $S R, S R_{c}, S R_{c}^{1}, S R_{c}^{2}$ e $S R^{*}$ com $\alpha=0.5$ e diferentes tamanhos amostrais.

\begin{tabular}{|c|c|c|c|c|c|c|c|c|c|c|}
\hline \multirow[b]{2}{*}{$n$} & \multicolumn{5}{|c|}{$\gamma=10 \%$} & \multicolumn{5}{|c|}{$\gamma=5 \%$} \\
\hline & $S R$ & $S R_{c}$ & $S R_{c}^{1}$ & $S R_{c}^{2}$ & $S R^{*}$ & $S R$ & $S R_{c}$ & $S R_{c}^{1}$ & $S R_{c}^{2}$ & $S R^{*}$ \\
\hline 20 & 17.29 & 11.10 & 11.63 & 11.94 & 11.62 & 9.15 & 5.62 & 5.84 & 6.04 & 5.82 \\
\hline 30 & 14.53 & 10.56 & 10.72 & 10.87 & 10.71 & 7.51 & 5.16 & 5.25 & 5.31 & 5.18 \\
\hline 40 & 13.19 & 10.45 & 10.57 & 10.66 & 10.57 & 6.78 & 5.18 & 5.25 & 5.28 & 5.18 \\
\hline 50 & 12.74 & 10.75 & 10.80 & 10.84 & 10.80 & 6.64 & 5.34 & 5.39 & 5.42 & 5.34 \\
\hline 100 & 10.81 & 9.87 & 9.89 & 9.92 & 9.89 & 5.48 & 5.00 & 5.00 & 5.01 & 5.00 \\
\hline 200 & 10.42 & 10.00 & 10.01 & 10.01 & 10.00 & 5.09 & 4.82 & 4.82 & 4.82 & 4.82 \\
\hline \multirow[t]{2}{*}{500} & 9.78 & 9.61 & 9.61 & 9.61 & 9.61 & 4.95 & 4.85 & 4.85 & 4.85 & 4.85 \\
\hline & \multicolumn{5}{|c|}{$\gamma=1 \%$} & \multicolumn{5}{|c|}{$\gamma=0.5 \%$} \\
\hline$n$ & $S R$ & $S R_{c}$ & $S R_{c}^{1}$ & $S R_{c}^{2}$ & $S R^{*}$ & $S R$ & $S R_{c}$ & $S R_{c}^{1}$ & $S R_{c}^{2}$ & $S R^{*}$ \\
\hline 20 & 1.54 & 1.28 & 1.36 & 1.38 & 1.57 & 0.72 & 0.69 & 0.77 & 0.82 & 1.02 \\
\hline 30 & 1.36 & 1.04 & 1.05 & 1.06 & 1.04 & 0.67 & 0.54 & 0.55 & 0.57 & 0.53 \\
\hline 40 & 1.26 & 1.08 & 1.10 & 1.10 & 1.05 & 0.60 & 0.54 & 0.54 & 0.54 & 0.51 \\
\hline 50 & 1.21 & 1.07 & 1.07 & 1.07 & 1.07 & 0.58 & 0.50 & 0.50 & 0.50 & 0.49 \\
\hline 100 & 1.17 & 1.09 & 1.09 & 1.09 & 1.09 & 0.63 & 0.62 & 0.62 & 0.62 & 0.62 \\
\hline 200 & 0.97 & 0.94 & 0.94 & 0.94 & 0.94 & 0.46 & 0.44 & 0.44 & 0.44 & 0.44 \\
\hline 500 & 0.97 & 0.96 & 0.96 & 0.96 & 0.96 & 0.47 & 0.47 & 0.47 & 0.47 & 0.47 \\
\hline
\end{tabular}

Tabela 3.4: Médias e variâncias (Monte Carlo) das estatísticas $S R, S R_{c}, S R_{c}^{1}$ e $S R_{c}^{2}$ considerando $p=7$, $\alpha=0.5$ e diferentes tamanhos amostrais $\left(\mathcal{H}_{0}: \beta_{6}=\beta_{7}=0\right)$.

\begin{tabular}{cccccc|ccccc}
\hline & \multicolumn{5}{c|}{ Média } & \multicolumn{5}{c}{ Variância } \\
\cline { 2 - 11 }$n$ & $\chi^{2}$ & $S R$ & $S R_{c}$ & $S R_{c}^{1}$ & $S R_{c}^{2}$ & $\chi^{2}$ & $S R$ & $S R_{c}$ & $S R_{c}^{1}$ & $S R_{c}^{2}$ \\
\hline 20 & 2.0 & 2.6 & 2.1 & 2.2 & 1.6 & 4.0 & 5.2 & 4.4 & 4.8 & 4.2 \\
30 & 2.0 & 2.3 & 2.0 & 2.1 & 2.1 & 4.0 & 4.8 & 4.1 & 4.1 & 4.2 \\
40 & 2.0 & 2.3 & 2.0 & 2.0 & 2.0 & 4.0 & 4.6 & 4.1 & 4.1 & 4.1 \\
50 & 2.0 & 2.2 & 2.0 & 2.0 & 2.1 & 4.0 & 4.5 & 4.1 & 4.1 & 4.1 \\
100 & 2.0 & 2.1 & 2.0 & 2.0 & 2.0 & 4.0 & 4.2 & 4.1 & 4.1 & 4.1 \\
200 & 2.0 & 2.0 & 2.0 & 2.0 & 2.0 & 4.0 & 4.0 & 3.9 & 3.9 & 3.9 \\
\hline
\end{tabular}


Tabela 3.5: Quantis $\chi_{2}^{2}, S R, S R_{c}, S R_{c}^{1}$ e $S R_{c}^{2}$, considerando $p=6, \alpha=0.5$ e $n=30\left(\mathcal{H}_{0}: \beta_{5}=\beta_{6}=0\right)$.

\begin{tabular}{c|ccccc}
\hline quantis (\%) & $\chi_{2}^{2}$ & $S R$ & $S R_{c}$ & $S R_{c}^{1}$ & $S R_{c}^{2}$ \\
\hline 80.0 & 3.2 & 3.7 & 3.2 & 3.3 & 3.3 \\
85.0 & 3.8 & 4.3 & 3.8 & 3.8 & 3.8 \\
90.0 & 4.6 & 5.2 & 4.6 & 4.7 & 4.7 \\
95.0 & 6.0 & 6.5 & 6.0 & 6.0 & 6.0 \\
97.0 & 7.0 & 7.4 & 7.0 & 7.0 & 7.0 \\
99.0 & 9.2 & 9.2 & 9.0 & 9.0 & 9.0 \\
99.5 & 10.6 & 10.4 & 10.3 & 10.3 & 10.3 \\
\hline
\end{tabular}
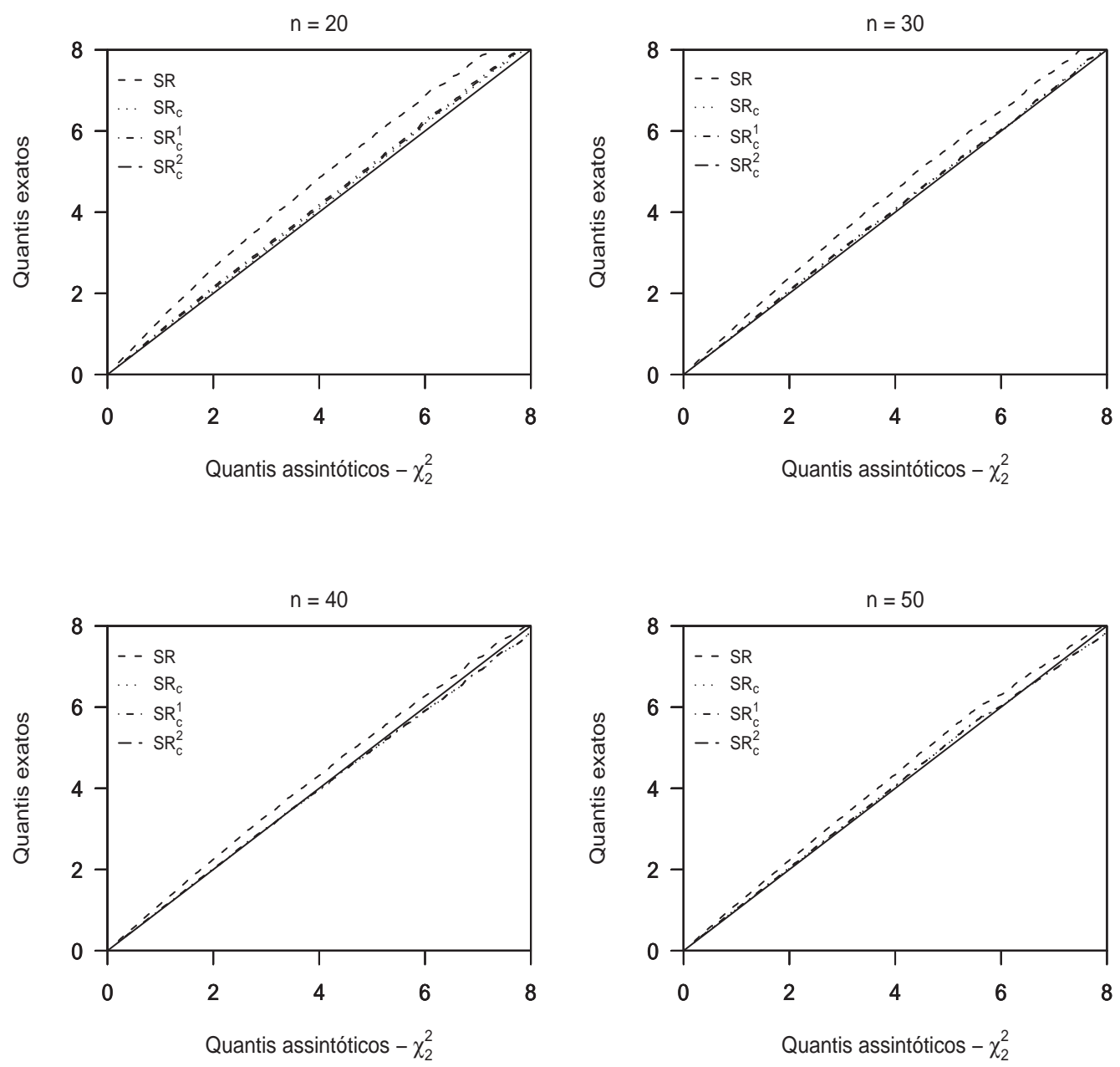

Figura 3.1: Quantis assintóticos versus quantis exatos das estatísticas $S R, S R_{c}, S R_{c}^{1}, S R_{c}^{2}$. 


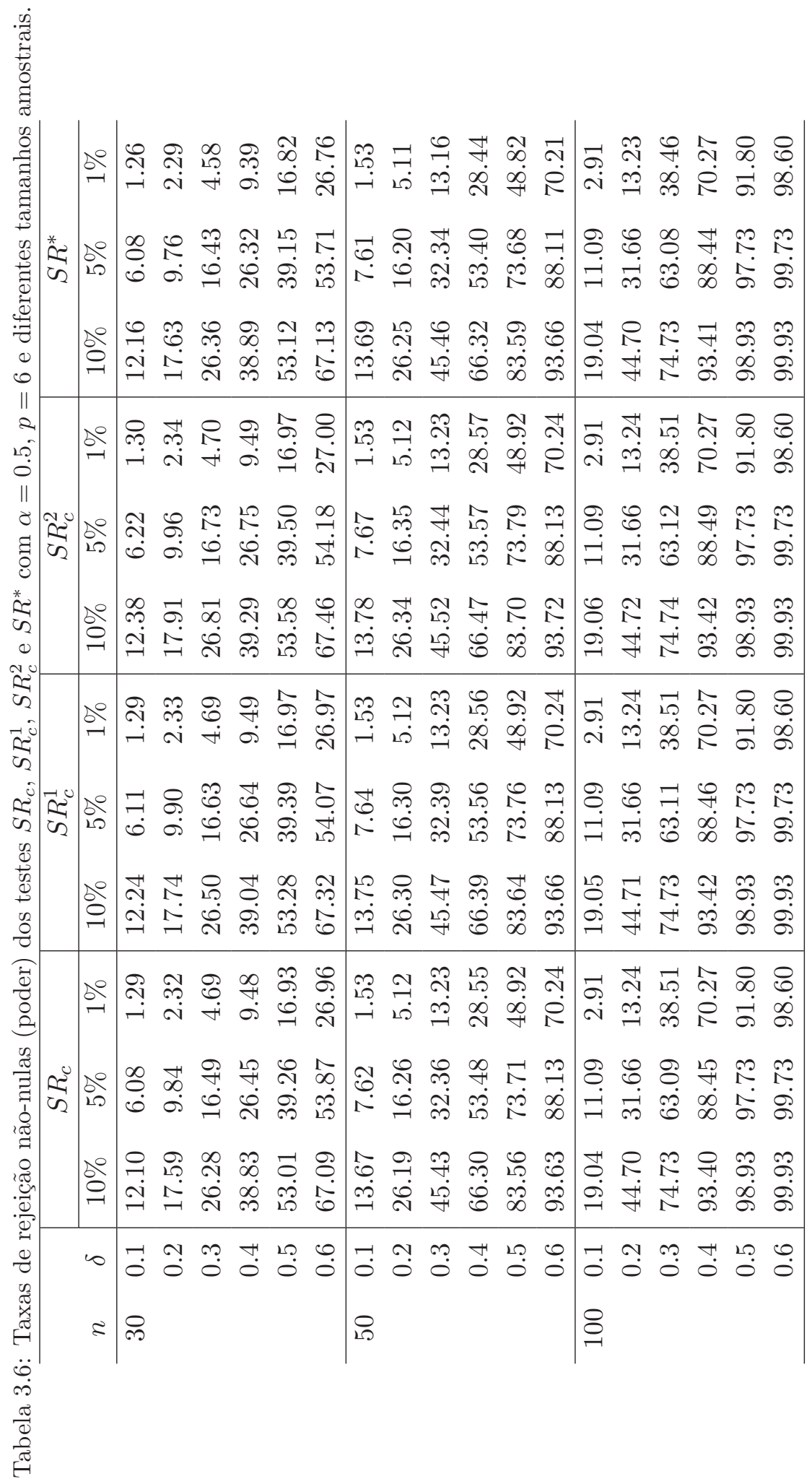


Tabela 3.7: Taxas de rejeição nulas dos testes $S R, S R_{c}, S R_{c}^{1}, S R_{c}^{2}$ e $S R^{*}$, considerando $p=5$ e diferentes tamanhos amostrais.

\begin{tabular}{cc|cccc|cccc}
\hline \multirow{2}{*}{$n$} & & \multicolumn{4}{|c|}{$\mathcal{H}_{0}: \alpha=0.5$} & \multicolumn{4}{c}{$\mathcal{H}_{0}: \alpha=1.0$} \\
\cline { 3 - 10 } 20 & Estatística & $10 \%$ & $5 \%$ & $1 \%$ & $0.5 \%$ & $10 \%$ & $5 \%$ & $1 \%$ & $0.5 \%$ \\
\hline & $S R$ & 17.81 & 7.33 & 0.42 & 0.14 & 19.65 & 8.26 & 0.40 & 0.15 \\
& $S R_{c}$ & 8.81 & 4.25 & 0.73 & 0.33 & 7.50 & 3.34 & 0.55 & 0.27 \\
& $S R_{c}^{1}$ & 9.52 & 4.31 & 0.75 & 0.35 & 8.57 & 3.61 & 0.55 & 0.27 \\
& $S R_{c}^{2}$ & 10.13 & 4.38 & 0.75 & 0.35 & 9.33 & 3.81 & 0.55 & 0.28 \\
& $S R^{*}$ & 7.50 & 3.29 & 1.06 & 0.70 & 6.18 & 2.29 & 0.64 & 0.46 \\
\hline 30 & $S R$ & 15.13 & 6.84 & 0.65 & 0.19 & 16.24 & 7.65 & 0.57 & 0.19 \\
& $S R_{c}$ & 9.77 & 4.99 & 0.91 & 0.36 & 8.77 & 4.61 & 0.69 & 0.27 \\
& $S R_{c}^{1}$ & 10.02 & 5.05 & 0.91 & 0.37 & 9.35 & 4.88 & 0.69 & 0.27 \\
& $S R_{c}^{2}$ & 10.40 & 5.12 & 0.91 & 0.37 & 9.78 & 5.04 & 0.69 & 0.27 \\
& $S R^{*}$ & 9.21 & 4.52 & 1.03 & 0.54 & 8.40 & 3.95 & 0.72 & 0.37 \\
\hline 40 & $S R$ & 13.41 & 6.19 & 0.78 & 0.25 & 14.70 & 6.71 & 0.67 & 0.31 \\
& $S R_{c}$ & 9.75 & 4.88 & 1.01 & 0.46 & 9.21 & 4.43 & 0.82 & 0.43 \\
& $S R_{c}^{1}$ & 9.99 & 4.97 & 1.02 & 0.47 & 9.60 & 4.51 & 0.82 & 0.43 \\
& $S R_{c}^{2}$ & 10.18 & 5.01 & 1.03 & 0.47 & 10.06 & 4.56 & 0.82 & 0.43 \\
& $S R^{*}$ & 9.52 & 4.65 & 1.12 & 0.54 & 8.98 & 4.15 & 0.83 & 0.47 \\
\hline 50 & $S R$ & 12.79 & 6.09 & 0.79 & 0.30 & 13.34 & 6.39 & 0.83 & 0.41 \\
& $S R_{c}$ & 9.68 & 5.07 & 0.99 & 0.44 & 9.14 & 4.78 & 0.92 & 0.49 \\
& $S R_{c}^{1}$ & 9.84 & 5.13 & 0.99 & 0.44 & 9.47 & 4.82 & 0.92 & 0.52 \\
& $S R_{c}^{2}$ & 9.98 & 5.14 & 0.99 & 0.44 & 9.72 & 4.87 & 0.92 & 0.52 \\
& $S R^{*}$ & 9.54 & 4.82 & 1.03 & 0.54 & 9.04 & 4.63 & 0.97 & 0.56 \\
\hline 100 & $S R$ & 11.03 & 5.16 & 0.79 & 0.46 & 11.44 & 5.54 & 0.92 & 0.43 \\
& $S R_{c}$ & 9.49 & 4.66 & 0.86 & 0.54 & 9.31 & 4.79 & 0.98 & 0.47 \\
& $S R_{c}^{1}$ & 9.55 & 4.67 & 0.86 & 0.54 & 9.40 & 4.80 & 0.98 & 0.47 \\
& $S R_{c}^{2}$ & 9.61 & 4.67 & 0.86 & 0.54 & 9.49 & 4.82 & 0.98 & 0.47 \\
& $S R^{*}$ & 9.47 & 4.64 & 0.86 & 0.54 & 9.31 & 4.74 & 0.98 & 0.48 \\
\hline & & & & & & & & &
\end{tabular}




\section{Capítulo 4}

\section{Razão de Verossimilhanças Sinalizada}

Nos Capítulos 2 e 3, respectivamente, derivamos uma estatística de teste da razão de verossimilhanças corrigida por um fator de correção de Bartlett e uma estatística de teste escore corrigida por um fator de correção tipo-Bartlett. Tais ajustes melhoram a aproximação da distribuição da estatística de teste, sob a hipótese nula, pela distribuição qui-quadrado de referência. Estas estatísticas são utilizadas, em geral, para testar hipóteses do tipo $\mathcal{H}_{0}: \boldsymbol{\theta}=\boldsymbol{\theta}^{(0)}$ contra $\mathcal{H}_{1}: \boldsymbol{\theta} \neq \boldsymbol{\theta}^{(0)}$, sendo $\boldsymbol{\theta}$ um vetor $k$-dimensional e $\boldsymbol{\theta}^{(0)}$ um vetor de constantes conhecidas.

Quando o interesse reside em considerar hipóteses apenas sobre um parâmetro (possivelmente na presença de parâmetros de perturbação), uma alternativa aos testes da razão de verissimilhanças e escore é o teste da razão de verossimilhanças sinalizada, em que a estatística de teste, $R$, tem, assintoticamente e sob a hipótese nula, distribuição normal padrão com erro de ordem $O\left(n^{-1 / 2}\right)$. Esse teste é muito utilizado, por exemplo, quando estamos interessados em testar uma hipótese unilateral, isto é, $\mathcal{H}_{0}: \theta_{j} \leq \theta_{j}^{(0)}$ contra $\mathcal{H}_{1}: \theta_{j}>\theta_{j}^{(0)}$ ou $\mathcal{H}_{0}: \theta_{j} \geq \theta_{j}^{(0)}$ contra $\mathcal{H}_{1}: \theta_{j}<\theta_{j}^{(0)}$ $(j=1,2, \ldots, k)$. Além disso, claro, podemos testar $\mathcal{H}_{0}: \theta_{j}=\theta_{j}^{(0)}$ contra $\mathcal{H}_{1}: \theta_{j} \neq \theta_{j}^{(0)}$.

Como $R$ tem, sob a hipótese nula, distribuição (assintótica) normal padrão com erro de ordem $O\left(n^{-1 / 2}\right)$, pode acontecer que em amostras de tamanho pequeno e moderado esta aproximação seja ruim, podendo conduzir a taxas de rejeição bastante distorcidas sob a hipótese nula. Com o intuito de melhorar a qualidade da aproximação da estatística $R$ pela normal padrão, vários ajustes foram propostos. Tais ajustes têm longa data e iniciaram-se com Lawley (1956) que considera um ajuste para $R$ a fim de melhorar a acurácia da aproximação normal; nessa mesma linha, tem-se ainda os trabalhos de DiCiccio (1984), McCullagh (1984) e Jensen (1986, 1987). Entretanto, a estatística da razão de verossimilhanças sinalizada modificada, $R^{*}$, considerada neste capítulo, foi primeiramente proposta por Barndorff-Nielsen (1986). O autor mostra que $R^{*}$ segue (assintoticamente) distribuição normal padrão com erro de ordem $O\left(n^{-3 / 2}\right)$. Posteriormente, $R^{*}$ foi tratada por Fraser (1990), Barndorff-Nielsen $(1991,1995)$ e Jensen $(1992,1993,1997)$. Discussões gerais sobre $R^{*}$ e suas propriedades são dadas em Barndorff-Nielsen \& Cox (1994), Jensen (1995), Reid (1996) e Skovgaard $(1996,2001)$.

Aplicações de $R^{*}$ em alguns modelos particulares podem ser encontradas em Barndorff-Nielsen (1986, § 3.6), Barndorff-Nielsen (1990a, b), Barndorff-Nielsen (1991, § 6), Bebu \& Mathew (2008), Chen \& Zhou (2006), Fraser, Wong \& Wu (1999), Larsen, Blaesild \& Sørensen (2002), Wu, Jiang, Wong \& Sun (2002), Wong \& Wu (2002) e Wu, Wong \& Jiang (2003). Em todos estes artigos foi possível obter $R^{*}$ explicitamente, uma vez que os modelos considerados nos artigos mencionados pertencem, em sua grande maioria, à família exponencial de distribuições. No entanto, isso nem sempre é possível. No modelo de regressão Birnbaum-Saunders, por exemplo, não é possível obter 
$R^{*}$ explicitamente, sendo necessário considerar aproximações para $R^{*}$.

Devido a algumas dificuldades em se obter $R^{*}$ explicitamente, algumas aproximações têm sido propostas. Tais dificuldades consistem em especificar uma estatística ancilar $\boldsymbol{a}$ tal que uma estatística suficiente possa ser escrita na forma $(\widehat{\boldsymbol{\theta}}, \boldsymbol{a})$, em que $\widehat{\boldsymbol{\theta}}$ é a estimativa de máxima verossimilhança de $\boldsymbol{\theta}$. Esta condição é muito difícil ou até impossível de ser verificada em muitos modelos, em particular, no modelo de regressão Birnbaum-Saunders. As aproximações de $R^{*}$ caracterizam-se por não depender de uma especificação para $\boldsymbol{a}$. Neste capítulo, apresentaremos quatro destas aproximações, a saber: aproximação $R_{o}^{*}$ proposta por DiCiccio \& Martin (1993) no caso em que o parâmetro de interesse e os parâmetros de perturbação são globalmente ortogonais; aproximação $\bar{R}^{*}$, derivada em Skovgaard (1996), que é baseada em covariâncias entre a função de log-verossimilhança e a função escore; aproximação $\widehat{R}^{*}$, devida a Severini (1999), baseada em covariâncias empíricas; aproximação $\widetilde{R}^{*}$, derivada em Fraser, Reid \& Wu (1999), tendo por base uma estatística aproximadamente ancilar. Uma descrição completa e detalhada sobre as aproximações $\bar{R}^{*}$ e $\widehat{R}^{*}$ está dada em He \& Severini (2007).

Algumas das aproximações mencionadas anteriormente vêm sendo aplicadas em muitos modelos particulares nos quais não é possível obter $R^{*}$ explicitamente; por exemplo, Wu \& Wong (2004), Wu, Jiang \& Wei (2006), Wu, Wong \& Wei (2006) e Wu \& Jiang (2007). Neste capítulo, obteremos as aproximações de $R^{*}$ no modelo de regressão Birnbaum-Saunders, com o objetivo de melhorar a inferência sobre os parâmetros do modelo.

\subsection{Notação e Derivadas com Relação ao Espaço Amostral}

O objetivo desta seção é introduzir alguns conceitos básicos para o desenvolvimento de alguns resultados apresentados ao longo do capítulo, assim como definir algumas notações que serão utilizadas ao longo do texto. Algumas notações que serão apresentadas aqui foram introduzidas em BarndorffNielsen (1986) e são utilizadas muito frequentemente no contexto de ajustes para a estatística da razão de verossimilhanças sinalizada.

Inicialmente, considere uma amostra aleatória $\boldsymbol{y}=\left(y_{1}, \ldots, y_{n}\right)$ de dimensão $n$ de um modelo estatístico hipotético $f(\boldsymbol{y} ; \boldsymbol{\theta})$ com vetor paramétrico $\boldsymbol{\theta}=\left(\theta_{1}, \ldots, \theta_{k}\right)$ de dimensão $k$. Seja $l(\boldsymbol{\theta} ; \boldsymbol{y})=$ $\log \{f(\boldsymbol{y} ; \boldsymbol{\theta})\}$ a função de $\log$-verossimilhança vista como uma função de $\boldsymbol{\theta}$ para $\boldsymbol{y}$ fixado. Assuma que existe uma transformação um-a-um de $\boldsymbol{y}$ a $(\boldsymbol{s}, \boldsymbol{a})$ tal que

$$
f(\boldsymbol{y} ; \boldsymbol{\theta})=f(\boldsymbol{s}, \boldsymbol{a} ; \boldsymbol{\theta})\left|\partial(\boldsymbol{s}, \boldsymbol{a}) / \partial \boldsymbol{y}^{\top}\right| \propto f(\boldsymbol{s}, \boldsymbol{a} ; \boldsymbol{\theta})=f(\boldsymbol{s} ; \boldsymbol{\theta}) f(\boldsymbol{a} \mid \boldsymbol{s}) \propto f(\boldsymbol{s} ; \boldsymbol{\theta}) .
$$

Assim, $\boldsymbol{s}=\boldsymbol{s}(\boldsymbol{y})$ é suficiente para $\boldsymbol{\theta}$ e a inferência sobre $\boldsymbol{\theta}$ é baseada na densidade marginal de $\boldsymbol{s}$. Na expressão acima, o determinante do jacobiano da transformação de $\boldsymbol{y}$ a $(\boldsymbol{s}, \boldsymbol{a}),\left|\partial(\boldsymbol{s}, \boldsymbol{a}) / \partial \boldsymbol{y}^{\top}\right|$, é absorvido pela constante de proporcionalidade. Além disso, a densidade condicional $f(\boldsymbol{a} \mid \boldsymbol{s})$ não depende de parâmetros, pois $s$ é suficiente. Com isso, podemos escrever a função de log-verossimilhança como uma função da estatística $\boldsymbol{s}$ e do parâmetro $\boldsymbol{\theta}$, isto é, $l(\boldsymbol{\theta} ; \boldsymbol{s})$. Adicionalmente, se as dimensões de $\boldsymbol{s}$ e $\boldsymbol{\theta}$ são iguais, a estimativa de máxima verossimilhança de $\boldsymbol{\theta}, \widehat{\boldsymbol{\theta}}$, é usualmente uma função um-a-um de $\boldsymbol{s}$ e, então, $\widehat{\boldsymbol{\theta}}$ é suficiente minimal se, e somente se, $\boldsymbol{s}$ for suficiente minimal, consequentemente, a função de log-verossimilhança pode ser escrita na forma $l(\boldsymbol{\theta} ; \widehat{\boldsymbol{\theta}})$. Por outro lado, seja $\boldsymbol{s}=(\boldsymbol{t}, \boldsymbol{a})$ uma estatística suficiente em que $\boldsymbol{t}$ tem a mesma dimensão de $\boldsymbol{\theta}$ e $\boldsymbol{a}$ é uma estatística ancilar (veja próximo parágrafo) de dimensão $n-k$ de forma que a transformação de $\boldsymbol{y}$ a $\boldsymbol{s}=(\boldsymbol{t}, \boldsymbol{a})$ seja um-a-um. Então, a função de log-verossimilhança pode ser escrita na forma $l(\boldsymbol{\theta} ; \boldsymbol{t}, \boldsymbol{a})$.

Estatística ancilar, uma das contribuições mais fundamentais de Sir Ronald Fisher para a in- 
ferência estatística, é uma estatística cuja distribuição não depende dos parâmetros do modelo. Entretanto, como mencionado por Fisher (1925, 1934, 1935), embora uma estatística ancilar por si só não forneça qualquer informação sobre os parâmetros, ela, em conjunção com alguma outra estatística, tipicamente a estimativa de máxima verossimilhança, pode fornecer uma informação valiosa sobre os parâmetros do modelo. Em outras palavras, quando $\widehat{\boldsymbol{\theta}}$ por si só não é suficiente, há perda de informação no sentido fisheriano. Assim, como recuperar esta aparente perda de informação? Se $\boldsymbol{a}$ é uma estatística ancilar e $(\widehat{\boldsymbol{\theta}}, \boldsymbol{a})$ é suficiente, então $\boldsymbol{a}$ é referida como um complemento ancilar de $\widehat{\boldsymbol{\theta}}$, ou seja, recuperamos a informação contida em $\widehat{\boldsymbol{\theta}}$, através de uma estatística ancilar. Tomando $\boldsymbol{s}=(\widehat{\boldsymbol{\theta}}, \boldsymbol{a})$, a função de log-verossimilhança pode ser escrita na forma $l(\boldsymbol{\theta} ; \widehat{\boldsymbol{\theta}}, \boldsymbol{a})$; veja Barndorff-Nielsen \& Cox $(1994, \S 2.5,5.2,5.4)$ para mais detalhes.

É claro que muitas vezes não é possível recuperar toda a informação contida em $\widehat{\boldsymbol{\theta}}$. Por exemplo, existem situações em que há mais de uma estatística ancilar, assim, surge o problema de qual estatística ancilar tomar; veja Basu (1964) e Cox (1971). Em outros casos, tal estatística pode não existir; veja Peña, Rohatgi \& Székely (1992). Além disso, em alguns casos não é fácil mostrar que o par $(\widehat{\boldsymbol{\theta}}, \boldsymbol{a})$ é suficiente. Estes problemas não serão discutidos no presente capítulo. Uma excelente revisão sobre estatística ancilar está dada em Ghosh, Reid \& Fraser (2009).

De agora em diante vamos assumir que a função de log-verossimilhança pode ser escrita na forma $l(\boldsymbol{\theta} ; \widehat{\boldsymbol{\theta}}, \boldsymbol{a})$. A informação de Fisher observada pode ser escrita como

$$
\boldsymbol{j}(\boldsymbol{\theta} ; \widehat{\boldsymbol{\theta}}, \boldsymbol{a})=-\frac{\partial^{2} l(\boldsymbol{\theta} ; \widehat{\boldsymbol{\theta}}, \boldsymbol{a})}{\partial \boldsymbol{\theta} \partial \boldsymbol{\theta}^{\top}} .
$$

Usualmente esta quantidade é avaliada em $\boldsymbol{\theta}=\widehat{\boldsymbol{\theta}}$, isto é, $\boldsymbol{j}(\widehat{\boldsymbol{\theta}})=\boldsymbol{j}(\widehat{\boldsymbol{\theta}} ; \widehat{\boldsymbol{\theta}}, \boldsymbol{a})$ (informação observada estimada). Em muitos casos, vamos nos referir a $\boldsymbol{j}(\widehat{\boldsymbol{\theta}})$ como $\widehat{\boldsymbol{j}}$. Adicionalmente, a informação de Fisher esperada é calculada da forma

$$
\boldsymbol{i}(\boldsymbol{\theta})=\mathbb{E}_{\boldsymbol{\theta}}\{\boldsymbol{j}(\boldsymbol{\theta} ; \widehat{\boldsymbol{\theta}}, \boldsymbol{a})\} .
$$

O subescrito $\boldsymbol{\theta}$ indica que a esperança matemática é calculada considerando $\boldsymbol{\theta}$ como o verdadeiro valor do parâmetro. A notação $\widehat{\boldsymbol{i}}$ ou $\boldsymbol{i}(\widehat{\boldsymbol{\theta}})$ indica que $\boldsymbol{i}(\boldsymbol{\theta})$ é avaliado em $\boldsymbol{\theta}=\widehat{\boldsymbol{\theta}}$ (informação esperada estimada).

Derivadas com relação aos parâmetros do modelo serão denotadas da forma

$$
\boldsymbol{l}_{\boldsymbol{\theta}}(\boldsymbol{\theta})=\boldsymbol{l}_{\boldsymbol{\theta}}(\boldsymbol{\theta} ; \widehat{\boldsymbol{\theta}}, \boldsymbol{a})=\frac{\partial l(\boldsymbol{\theta} ; \widehat{\boldsymbol{\theta}}, \boldsymbol{a})}{\partial \boldsymbol{\theta}^{\top}}, \quad \boldsymbol{l}_{\boldsymbol{\theta} \boldsymbol{\theta}}(\boldsymbol{\theta})=\boldsymbol{l}_{\boldsymbol{\theta} \boldsymbol{\theta}}(\boldsymbol{\theta} ; \widehat{\boldsymbol{\theta}}, \boldsymbol{a})=\frac{\partial^{2} l(\boldsymbol{\theta} ; \widehat{\boldsymbol{\theta}}, \boldsymbol{a})}{\partial \boldsymbol{\theta} \partial \boldsymbol{\theta}^{\top}}, \quad \text { etc }
$$

em particular, $\boldsymbol{j}(\boldsymbol{\theta} ; \widehat{\boldsymbol{\theta}}, \boldsymbol{a})=-\boldsymbol{l}_{\boldsymbol{\theta} \boldsymbol{\theta}}(\boldsymbol{\theta})$. Uma definição importante que será muito utilizada neste capítulo é a das derivadas com respeito a $\widehat{\boldsymbol{\theta}}$, considerando $\boldsymbol{a}$ fixado. Estas derivadas são denominadas de derivadas do espaço amostral, pois a diferenciação é com respeito a certas funções da amostra, mantendo outras funções da amostra fixadas. Derivadas com relação a funções dos dados, tais como $\widehat{\boldsymbol{\theta}}$, vão ser denotadas da forma

$$
\boldsymbol{l}_{; \widehat{\boldsymbol{\theta}}}(\boldsymbol{\theta})=\boldsymbol{l}_{; \widehat{\boldsymbol{\theta}}}(\boldsymbol{\theta} ; \widehat{\boldsymbol{\theta}}, \boldsymbol{a})=\frac{\partial l(\boldsymbol{\theta} ; \widehat{\boldsymbol{\theta}}, \boldsymbol{a})}{\partial \widehat{\boldsymbol{\theta}}}, \quad \boldsymbol{l}_{\boldsymbol{\theta} ; \widehat{\boldsymbol{\theta}}}(\boldsymbol{\theta})=\boldsymbol{l}_{\boldsymbol{\theta} ; \widehat{\boldsymbol{\theta}}}(\boldsymbol{\theta} ; \widehat{\boldsymbol{\theta}}, \boldsymbol{a})=\frac{\partial^{2} l(\boldsymbol{\theta} ; \widehat{\boldsymbol{\theta}}, \boldsymbol{a})}{\partial \boldsymbol{\theta}^{\top} \partial \widehat{\boldsymbol{\theta}}}=\frac{\partial \boldsymbol{l}_{\boldsymbol{\theta}}(\boldsymbol{\theta} ; \widehat{\boldsymbol{\theta}}, \boldsymbol{a})}{\partial \widehat{\boldsymbol{\theta}}}, \quad \text { etc. }
$$

Em particular, note que $\boldsymbol{l}_{\boldsymbol{\theta}}(\boldsymbol{\theta})$ é um vetor coluna $k$-dimensional, enquanto que $\boldsymbol{l}_{; \widehat{\boldsymbol{\theta}}}(\boldsymbol{\theta})$ é um vetor linha $k$-dimensional. Adicionalmente, $\boldsymbol{l}_{\boldsymbol{\theta} \boldsymbol{\theta}}(\boldsymbol{\theta})$ e $\boldsymbol{l}_{\boldsymbol{\theta} ; \hat{\boldsymbol{\theta}}}(\boldsymbol{\theta})$ são matrizes $k \times k$. Todas estas derivadas podem 
ser avaliadas em $\widehat{\boldsymbol{\theta}}=\boldsymbol{\theta}_{0}$, em que $\boldsymbol{\theta}_{0}$ é um elemento arbitrário do espaço paramétrico. Por exemplo,

$$
\boldsymbol{l}_{\boldsymbol{\theta} ; \hat{\boldsymbol{\theta}}}\left(\boldsymbol{\theta}, \boldsymbol{\theta}_{0}, \boldsymbol{a}\right)=\left.\frac{\partial^{2} l(\boldsymbol{\theta} ; \widehat{\boldsymbol{\theta}}, \boldsymbol{a})}{\partial \boldsymbol{\theta}^{\top} \partial \widehat{\boldsymbol{\theta}}}\right|_{\widehat{\boldsymbol{\theta}}=\boldsymbol{\theta}_{0}} .
$$

Note que, como $\boldsymbol{a}$ está fixado, $\boldsymbol{l}_{\boldsymbol{\theta} ; \hat{\boldsymbol{\theta}}}\left(\boldsymbol{\theta}, \boldsymbol{\theta}_{0}, \boldsymbol{a}\right)$ é uma constante. Uma relação interessante entre as derivadas de $l(\boldsymbol{\theta} ; \widehat{\boldsymbol{\theta}}, \boldsymbol{a})$ com relação a $\boldsymbol{\theta}$ e $\widehat{\boldsymbol{\theta}}$, avaliadas em $\boldsymbol{\theta}=\widehat{\boldsymbol{\theta}}$, é

$$
\boldsymbol{l}_{\boldsymbol{\theta} \boldsymbol{\theta}}(\widehat{\boldsymbol{\theta}})+\boldsymbol{l}_{\boldsymbol{\theta} ; \widehat{\boldsymbol{\theta}}}(\widehat{\boldsymbol{\theta}})=\mathbf{0}
$$

isto é, $\boldsymbol{l}_{\boldsymbol{\theta} ; \widehat{\boldsymbol{\theta}}}(\widehat{\boldsymbol{\theta}})=-\boldsymbol{l}_{\boldsymbol{\theta} \boldsymbol{\theta}}(\widehat{\boldsymbol{\theta}})=\boldsymbol{j}(\widehat{\boldsymbol{\theta}})$; veja Barndorff-Nielsen \& Cox (1994, cap. 5) para mais detalhes.

\subsection{Fórmula $p^{*}$ de Barndorff-Nielsen}

A fórmula $p^{*}$ derivada em Barndorff-Nielsen $(1980,1983)$ consiste de uma aproximação para a densidade do estimador de máxima verossimilhança condicionalmente a uma estatística ancilar $\boldsymbol{a}$. A fórmula $p^{*}$ é definida por

$$
p^{*}(\widehat{\boldsymbol{\theta}} \mid \boldsymbol{a} ; \boldsymbol{\theta})=c|\widehat{\boldsymbol{j}}|^{1 / 2} \exp \{l(\boldsymbol{\theta} ; \widehat{\boldsymbol{\theta}}, \boldsymbol{a})-l(\widehat{\boldsymbol{\theta}} ; \widehat{\boldsymbol{\theta}}, \boldsymbol{a})\},
$$

em que $|\widehat{\boldsymbol{j}}|$ é o determinante da matriz de informação observada estimada e $c$ é uma constante normalizadora, isto é, $c=c(\boldsymbol{\theta}, \boldsymbol{a})$ é escolhida de tal forma que a integral de (4.2) com respeito a $\widehat{\boldsymbol{\theta}}$, mantendo $\boldsymbol{a}$ fixado, é igual a um. Em geral, $c$ é determinado numericamente. De (4.1), fazendo $\boldsymbol{s}=(\widehat{\boldsymbol{\theta}}, \boldsymbol{a})$, note que

$$
f(\boldsymbol{y} ; \boldsymbol{\theta}) \propto f(\widehat{\boldsymbol{\theta}} \mid \boldsymbol{a} ; \boldsymbol{\theta}) f(\boldsymbol{a}) .
$$

Assim, o fato de $\boldsymbol{a}$ ser uma estatística ancilar assegura que toda informação sobre $\boldsymbol{\theta}$ está contida na densidade condicional $f(\widehat{\boldsymbol{\theta}} \mid \boldsymbol{a} ; \boldsymbol{\theta})$. (Note que há uma redução de dimensionalidade de $n$ para $k$.) Adicionalmente, se $\boldsymbol{a}$ é uma estatística aproximadamente ancilar, a equação (4.2) ainda é válida, isto é, acredita-se que exista pouca informação sobre $\boldsymbol{\theta}$ em $f(\boldsymbol{a})$, assim, espera-se que a distribuição condicional de $\widehat{\boldsymbol{\theta}}$ dado $\boldsymbol{a}$ forneça "boa" inferência sobre $\boldsymbol{\theta}$; veja Reid $(1995,2003)$ para mais detalhes. McCullagh (1987, pág. 241) faz o seguinte comentário em relação à expressão (4.2): "One peculiar aspect of the formula is that the ancillary is not specified and the formula appears to be correct for a wide range of ancillary statistics. For this reason the description 'magical mystery formula' is sometimes used."

Antes de discutir a exatidão de $p^{*}(\widehat{\boldsymbol{\theta}} \mid \boldsymbol{a} ; \boldsymbol{\theta})$ como uma aproximação para a verdadeira densidade condicional $p(\widehat{\boldsymbol{\theta}} \mid \boldsymbol{a} ; \boldsymbol{\theta})$, apresentamos a seguir três propriedades interessantes da fórmula $p^{*}$ : (i) é invariante sob transformações um-a-um dos dados, isto é, pode-se extrair qualquer fator da verossimilhança original que dependa apenas de elementos da amostra; (ii) é invariante sob reparametrizações; (iii) a constante $c$ é invariante sob reparametrizações. A propriedade (ii) pode ser reformulada como: (ii') se $\boldsymbol{\theta}$ e $\boldsymbol{\omega}$ são parametrizações alternativas, então, em uma notação natural,

$$
p^{*}(\widehat{\boldsymbol{\omega}} \mid \boldsymbol{a} ; \boldsymbol{\omega})=p^{*}(\widehat{\boldsymbol{\theta}} \mid \boldsymbol{a} ; \boldsymbol{\theta})\left|\partial \widehat{\boldsymbol{\theta}} / \partial \widehat{\boldsymbol{\omega}}^{\top}\right| .
$$

A validade da expressão anterior e de que a constante $c$ não depende da parametrização utilizada [propriedade (iii)], seguem do fato de que

$$
\left|\widehat{\boldsymbol{j}}_{\boldsymbol{\omega}}(\widehat{\boldsymbol{\omega}})\right|=\left|\partial \widehat{\boldsymbol{\theta}} / \partial \widehat{\boldsymbol{\omega}}^{\top}\right|^{2}\left|\widehat{\boldsymbol{j}}_{\boldsymbol{\theta}}(\widehat{\boldsymbol{\theta}})\right|
$$


ou seja, reparametrizar o modelo e calcular $p^{*}$ a partir da nova função de log-verossimilhança, equivale a calcular $p^{*}$ inicialmente e transformá-la segundo regras de mudança de variáveis (jacobiano).

Como uma aproximação para $p(\widehat{\boldsymbol{\theta}} \mid \boldsymbol{a} ; \boldsymbol{\theta})$, a fórmula $p^{*}(\widehat{\boldsymbol{\theta}} \mid \boldsymbol{a} ; \boldsymbol{\theta})$ dada em (4.2) é, em geral, precisa até ordem $O\left(n^{-3 / 2}\right)$; ver Skovgaard (1990). Mais precisamente,

$$
p(\widehat{\boldsymbol{\theta}} \mid \boldsymbol{a} ; \boldsymbol{\theta})=p^{*}(\widehat{\boldsymbol{\theta}} \mid \boldsymbol{a} ; \boldsymbol{\theta})\left\{1+O\left(n^{-3 / 2}\right)\right\} .
$$

Pode-se mostrar que $c(\boldsymbol{\theta}, \boldsymbol{a})=(2 \pi)^{-k / 2}\left\{1+O\left(n^{-1}\right)\right\}$, sendo $k$ a dimensão do vetor $\boldsymbol{\theta}$; Pace \& Salvan (1997, cap. 11). Assim, tal constante é muitas vezes tomada como sendo $(2 \pi)^{-k / 2}$. Neste caso, obtém-se uma aproximação para $p(\widehat{\boldsymbol{\theta}} \mid \boldsymbol{a} ; \boldsymbol{\theta})$ com erro relativo de ordem $O\left(n^{-1}\right)$, ou seja,

$$
p(\widehat{\boldsymbol{\theta}} \mid \boldsymbol{a} ; \boldsymbol{\theta})=p^{\dagger}(\widehat{\boldsymbol{\theta}} \mid \boldsymbol{a} ; \boldsymbol{\theta})\left\{1+O\left(n^{-1}\right)\right\},
$$

com

$$
p^{\dagger}(\widehat{\boldsymbol{\theta}} \mid \boldsymbol{a} ; \boldsymbol{\theta})=(2 \pi)^{-k / 2}|\widehat{\boldsymbol{j}}|^{1 / 2} \exp \{l(\boldsymbol{\theta} ; \widehat{\boldsymbol{\theta}}, \boldsymbol{a})-l(\widehat{\boldsymbol{\theta}} ; \widehat{\boldsymbol{\theta}}, \boldsymbol{a})\}
$$

Vale ressaltar que os resultados apresentados continuam inalterados ao substituir a informação observada, $\boldsymbol{j}$, pela informação esperada, $\boldsymbol{i}$, na expressão (4.2). Vale salientar também que a fórmula $p^{*}$ é exata em alguns casos, por exemplo, em modelos da família de locação e escala. Uma discussão detalhada sobre esta aproximação está dada em Barndorff-Nielsen \& Cox (1994, cap. 6).

\subsection{Razão de Verossimilhanças Sinalizada $-R$}

Seja o parâmetro $\boldsymbol{\theta}=(\psi, \boldsymbol{\lambda})$, em que $\psi$, o parâmetro de interesse, é unidimensional e o parâmetro de perturbação $\boldsymbol{\lambda}$ é de dimensão $1 \times(k-1)$. A estatística da razão de verossimilhanças sinalizada para fazer inferência sobre o parâmetro $\psi$ é definida da forma

$$
R=R(\psi)=\operatorname{sgn}(\widehat{\psi}-\psi)[2\{l(\widehat{\boldsymbol{\theta}})-l(\widetilde{\boldsymbol{\theta}})\}]^{1 / 2},
$$

em que $\operatorname{sgn}(\widehat{\psi}-\psi)$ representa o sinal de $\widehat{\psi}-\psi, \widehat{\boldsymbol{\theta}}=(\widehat{\psi}, \widehat{\boldsymbol{\lambda}})$ e $\widetilde{\boldsymbol{\theta}}=(\psi, \widetilde{\boldsymbol{\lambda}})$ são os estimadores de máxima verossimilhança irrestrito e restrito de $\boldsymbol{\theta}$, respectivamente. O estimador $\tilde{\boldsymbol{\lambda}}$ é obtido maximizando a função de $\log$-verossimilhança $l(\boldsymbol{\theta})$ com relação a $\boldsymbol{\lambda}$ supondo $\psi$ constante (fixado). A estatística (4.3) pode ser usada para testar hipóteses do tipo: (i) $\mathcal{H}_{0}: \psi \leq \psi_{0}$ versus $\mathcal{H}_{1}: \psi>\psi_{0}$; (ii) $\mathcal{H}_{0}: \psi \geq \psi_{0}$ versus $\mathcal{H}_{1}: \psi<\psi_{0}$; (iii) $\mathcal{H}_{0}: \psi=\psi_{0}$ versus $\mathcal{H}_{1}: \psi \neq \psi_{0}$. Aqui, $\psi_{0}$ é uma constante conhecida.

A fim de utilizar a estatística da razão de verossimilhanças sinalizada $R$ para fazer inferência sobre o parâmetro $\psi$, devemos obter, exatamente ou aproximadamente, sua distribuição. Pode ser mostrado que, sob a distribuição com parâmetro $\psi$, a estatística $R$ segue, assintoticamente, distribuição normal padrão com erro de ordem $O\left(n^{-1 / 2}\right)$; veja Severini (2000, $\S 4.3$ e 4.4) para uma demonstração deste resultado. Em muitos casos, entretanto, a precisão da aproximação normal é questionável e, assim, inferência sobre $\psi$ utilizando $R$ pode não ser confiável. Devido a isso, algumas versões modificadas de $R$ têm sido propostas a fim de melhorar a aproximação pela distribuição normal padrão; veja a Seção 4.5.

A abordagem básica para construir uma estatística, digamos $R^{*}$, que seja melhor aproximada pela distribuição normal padrão, é aproximar a distribuição condicional de $R$ dada uma estatística ancilar $\boldsymbol{a}$; veja a Seção 4.5 . Se $F(\cdot \mid \boldsymbol{a})$ denota a função distribuição condicional aproximada de $R$, então, $F(R \mid \boldsymbol{a})$ é distribuída, aproximadamente, como uma variável aleatória uniforme no intervalo $(0,1)$, isto é, $F(R \mid \boldsymbol{a}) \dot{\sim} \mathcal{U}(0,1)$, em que o símbolo ' $\dot{\sim}$ ' denota aproximadamente distribuído. Assim, $\Phi^{-1}(F(R \mid \boldsymbol{a}))$ 
é aproximadamente uma variável aleatória normal padrão, ou seja, $\Phi^{-1}(F(R \mid \boldsymbol{a})) \dot{\sim} \mathcal{N}(0,1)$, em que $\Phi(\cdot)$ representa a função de distribuição da normal padrão. A precisão da aproximação normal padrão de $\Phi^{-1}(F(R \mid \boldsymbol{a}))$ depende da precisão de $F$ como uma aproximação da função distribuição condicional de $R$. Segue que, como $R$ é aproximadamente normal padrão, $\Phi^{-1}(F(R \mid \boldsymbol{a}))=R+O_{p}\left(n^{-1 / 2}\right)$.

\subsection{Transformação Normalizadora}

A seguir apresentamos uma transformação normalizadora para uma variável aleatória que tem distribuição aproximadamente normal. Esta transformação tem como objetivo melhorar a ordem de aproximação pela distribuição normal. Seja $X$ uma variável aleatória contínua $O_{p}(1)$ com densidade da forma

$$
\bar{c} h(x / \sqrt{n}) \phi(x)
$$

em que $h$ é $O(1), h(0)=1, \phi(\cdot)$ denota a densidade da normal padrão e $\bar{c}$ é uma constante satisfazendo $\bar{c}=1+O\left(n^{-1}\right)$. [Note que $X$ tem distribuição normal com erro de ordem $O\left(n^{-1 / 2}\right)$. Verifica-se este fato notando que $h(x / \sqrt{n})=1+O\left(n^{-1 / 2}\right)$.] Uma aproximação para a função distribuição de $X$ é dada por

$$
\bar{c} \int_{-\infty}^{t} h(x / \sqrt{n}) \phi(x) \mathrm{d} x=1-\bar{c} \int_{t / \sqrt{n}}^{\infty} h(s) \sqrt{n} \phi(s \sqrt{n}) \mathrm{d} s .
$$

Esta integral pode ser aproximada por (Severini, 2000, § 2.5)

$$
F_{n}(t)=\Phi(t)-\phi(t) \frac{h(t / \sqrt{n})-1}{t},
$$

com erro de ordem $O\left(n^{-3 / 2}\right)$. Assim, a variável aleatória tansformada

$$
F_{n}(X)=\Phi(X)-\phi(X) \frac{h(X / \sqrt{n})-1}{X}
$$

é uniformemente distribuída com erro de ordem $O\left(n^{-3 / 2}\right)$.

A fim de obter uma variável aleatória que é aproximadamente distribuída de acordo com uma normal padrão, é necessário considerar a transformação $\Phi^{-1}\left(F_{n}(X)\right)$. Usando uma expansão em série de Taylor, a função $\Phi^{-1}$ avaliada em $t+c / \sqrt{n}$ pode ser expandida como

$$
\begin{aligned}
\Phi^{-1}(t+c / \sqrt{n}) & =\Phi^{-1}(t)+\frac{1}{\phi\left(\Phi^{-1}(t)\right)} \frac{c}{\sqrt{n}}+\frac{1}{2} \frac{\Phi^{-1}(t)}{\phi\left(\Phi^{-1}(t)\right)^{2}} \frac{c^{2}}{n}+O\left(n^{-3 / 2}\right) \\
& =\Phi^{-1}(t)-\frac{1}{\Phi^{-1}(t)} \log \left[1-\frac{\Phi^{-1}(t)}{\phi\left(\Phi^{-1}(t)\right)} \frac{c}{\sqrt{n}}\right]+O\left(n^{-3 / 2}\right) .
\end{aligned}
$$

A equivalência das duas igualdades é obtida ao se expandir $\log (1+w)$ em série de Taylor em torno do ponto $w=0$, $\operatorname{com} w=-c \Phi^{-1}(t) /\left\{\phi\left(\Phi^{-1}(t)\right) \sqrt{n}\right\}$. Como $\log (1+w)=w-w^{2} / 2+\cdots$, temos que $\log \left(1-c \Phi^{-1}(t) / \phi\left(\Phi^{-1}(t)\right) \sqrt{n}\right)=-c \Phi^{-1}(t) /\left\{\phi\left(\Phi^{-1}(t)\right) \sqrt{n}\right\}-\left[c \Phi^{-1}(t) / \phi\left(\Phi^{-1}(t)\right) \sqrt{n}\right]^{2}+O\left(n^{-3 / 2}\right)$. Assim, a igualdade é verificada.

Fazendo $t=\Phi(X)$ e

$$
c=-\sqrt{n} \phi(X) \frac{h(X / \sqrt{n})-1}{X}
$$


segue que uma transformação normalizadora de $X$ é dada por

$$
X^{*}=X-\frac{\log \{h(X / \sqrt{n})\}}{X} .
$$

A variável aleatória $X^{*}$, em geral, tem distribuição normal padrão com erro de ordem $O\left(n^{-3 / 2}\right)$.

\subsection{Razão de Verossimilhanças Sinalizada Modificada $-R^{*}$}

Nesta seção, apresentaremos uma estatística da razão de verossimilhanças sinalizada modificada, $R^{*}$, proposta por Barndorff-Nielsen $(1986,1991)$, que tem erro de aproximação pela normal padrão menor que o erro de aproximação pela normal padrão da estatística $R$, que é de ordem $O\left(n^{-1 / 2}\right)$. Espera-se, portanto, que em amostras de tamanho pequeno ou moderado, a inferência estatística com base em $R^{*}$ seja mais confiável. Consideraremos inicialmente o caso uniparamétrico.

\subsubsection{Caso Uniparamétrico}

No caso uniparamétrico, a estatística $R$ apresentada em (4.3) tem a forma

$$
R=\operatorname{sgn}(\widehat{\theta}-\theta)[2\{l(\widehat{\theta})-l(\theta)\}]^{1 / 2}
$$

Primeiramente, vamos derivar uma aproximação para a densidade de $R$ com base na fórmula $p^{*}$ apresentada na Seção 4.2. A fórmula $p^{*}$ no caso uniparamétrico pode ser escrita como

$$
p^{*}(\widehat{\theta} \mid \boldsymbol{a} ; \theta)=c \widehat{j}^{1 / 2} \exp \{l(\theta)-l(\widehat{\theta})\},
$$

em que $c=(2 \pi)^{-1 / 2} \bar{c}$ e $\bar{c}=1+O\left(n^{-1}\right)$. A estatística da razão de verossimilhanças sinalizada é uma função de $\widehat{\theta}$. Vamos assumir que esta relação seja um-a-um. Note que

$$
\frac{1}{2} R^{2}=l(\widehat{\theta})-l(\theta)
$$

assim, a densidade condicional de $R$ pode ser aproximada por

$$
p^{*}(r \mid \boldsymbol{a} ; \theta)=c \widehat{j}^{1 / 2} \exp \left\{-\frac{1}{2} r^{2}\right\}\left|\frac{\partial \widehat{\theta}}{\partial r}\right|,
$$

em que $\widehat{j}$ é visto como uma função de $r$ e $\partial \widehat{\theta} / \partial r$ é o jacobiano da transformação de $\widehat{\theta}$ a $r$. Usando (4.5), lembrando que $l(\theta)=l(\theta ; \widehat{\theta}, \boldsymbol{a})$, sendo $\boldsymbol{a}$ uma estatística ancilar que está fixada, segue que

$$
r \frac{\partial r}{\partial \widehat{\theta}}=l_{; \widehat{\theta}}(\widehat{\theta})-l_{; \widehat{\theta}}(\theta)
$$

Assim,

$$
\left|\frac{\partial \widehat{\theta}}{\partial r}\right|=\frac{|r|}{\left|l_{; \widehat{\theta}}(\widehat{\theta})-l_{; \widehat{\theta}}(\theta)\right|}
$$


Portanto, uma aproximação para a densidade condicional de $R$, notando que $\sqrt{2 \pi} \phi(r)=\exp \left\{-r^{2} / 2\right\}$, é dada por

$$
p^{*}(r \mid \boldsymbol{a} ; \theta)=\bar{c} \frac{\widehat{j}^{1 / 2}|r|}{\left|l_{\widehat{\theta} \hat{\theta}}(\widehat{\theta})-l_{; \widehat{\theta}}(\theta)\right|} \phi(r) .
$$

Nesta expressão, $\widehat{\theta}$ é interpretado como uma função de $r$. Observe que esta expressão é da forma (4.4) com

$$
h(r / \sqrt{n})=\frac{\widehat{j}^{1 / 2}|r|}{\left|l_{; \hat{\theta}}(\widehat{\theta})-l_{; \widehat{\theta}}(\theta)\right|} .
$$

Assim, a versão normalizada de $R$ é dada por

$$
R^{*}=R-\frac{1}{R} \log \{h(R / \sqrt{n})\}=R+\frac{1}{R} \log (U / R),
$$

em que

$$
U=\widehat{j}^{-1 / 2}\left\{l_{; \widehat{\theta}}(\widehat{\theta})-l_{; \widehat{\theta}}(\theta)\right\}
$$

O sinal de $U$ é determinado de forma a ser o mesmo que o sinal de $R$ fazendo com que $U / R$ nunca seja negativo; veja Barndorff-Nielsen (1991, § 3).

\subsubsection{Caso Multiparamétrico}

Seja $\boldsymbol{\theta}=(\psi, \boldsymbol{\lambda})$, em que $\psi$, o parâmetro de interesse, é unidimensional e o parâmetro de perturbação $\boldsymbol{\lambda}$ é de dimensão $1 \times(k-1)$. A estatística da razão de verossimilhanças sinalizada para fazer inferência sobre o parâmetro $\psi$ é da forma

$$
R=R(\psi)=\operatorname{sgn}(\widehat{\psi}-\psi)[2\{l(\widehat{\boldsymbol{\theta}})-l(\widetilde{\boldsymbol{\theta}})\}]^{1 / 2},
$$

em que, como antes, $\widetilde{\boldsymbol{\theta}}=(\psi, \widetilde{\boldsymbol{\lambda}})$ denota o estimador de máxima verossimilhança de $\boldsymbol{\theta}$ com $\psi$ fixado. Para obter a estatística da razão de verossimilhanças sinalizada modificada, $R^{*}$, procedemos como no caso uniparamétrico.

A densidade condicional de $R$ dado $\boldsymbol{a}$ pode ser escrita na forma

$$
p(r \mid \boldsymbol{a} ; \boldsymbol{\theta})=\frac{p(r, \widetilde{\boldsymbol{\lambda}} \mid \boldsymbol{a} ; \boldsymbol{\theta})}{p(\widetilde{\boldsymbol{\lambda}} \mid r, \boldsymbol{a} ; \boldsymbol{\theta})}
$$

Assim, precisamos aproximar as densidades do lado direito desta expressão. Para aproximar a densidade $p(r, \widetilde{\boldsymbol{\lambda}} \mid \boldsymbol{a} ; \boldsymbol{\theta})$, utilizamos a distribuição condicional aproximada de $\widehat{\boldsymbol{\theta}}$ dado $\boldsymbol{a}, p^{*}(\widehat{\boldsymbol{\theta}} \mid \boldsymbol{a} ; \boldsymbol{\theta})$. Esta aproximação é então transformada para obter uma aproximação $p^{*}$ para a densidade condicional de $(R, \widetilde{\boldsymbol{\lambda}})$ dado $\boldsymbol{a}$. Adicionalmente, no modelo em que $\psi$ está fixo, $(R, \widetilde{\boldsymbol{\lambda}}, \boldsymbol{a})$ é suficiente e $R$ é aproximadamente ancilar; Severini $(2000, \S 6.6 .5)$. Assim, $p^{*}(\widetilde{\boldsymbol{\lambda}} \mid r, \boldsymbol{a} ; \boldsymbol{\theta})$ pode ser usado para aproximar a distribuição condicional de $\widetilde{\boldsymbol{\lambda}}$ dado $r$ e $\boldsymbol{a}$. Estas aproximações podem ser usadas para construir a estatística ajustada $R^{*}$.

A fórmula $p^{*}$ da distribuição condicional de $\widehat{\boldsymbol{\theta}}$ dada uma estatística ancilar $\boldsymbol{a}$ é dada por (veja a Seção 4.2)

$$
p^{*}(\widehat{\boldsymbol{\theta}} \mid \boldsymbol{a} ; \boldsymbol{\theta})=\frac{\bar{c}}{(2 \pi)^{k / 2}}|\widehat{\boldsymbol{j}}|^{1 / 2} \exp \{l(\boldsymbol{\theta})-l(\widehat{\boldsymbol{\theta}})\} .
$$


Esta densidade pode ser transformada para aproximar a densidade de $(R, \widetilde{\boldsymbol{\lambda}})$ :

$$
\begin{aligned}
p^{*}(r, \widetilde{\boldsymbol{\lambda}} \mid \boldsymbol{a} ; \boldsymbol{\theta}) & =\frac{\bar{c}}{(2 \pi)^{k / 2}}|\widehat{\boldsymbol{j}}|^{1 / 2} \exp \{l(\boldsymbol{\theta})-l(\widehat{\boldsymbol{\theta}})\}|\boldsymbol{J}| \\
& =\frac{\bar{c}}{(2 \pi)^{k / 2}}|\widehat{\boldsymbol{j}}|^{1 / 2} \exp \{l(\boldsymbol{\theta})-l(\widetilde{\boldsymbol{\theta}})-[l(\widehat{\boldsymbol{\theta}})-l(\widetilde{\boldsymbol{\theta}})]\}|\boldsymbol{J}| \\
& =\frac{\bar{c}}{(2 \pi)^{k / 2}}|\widehat{\boldsymbol{j}}|^{1 / 2} \exp \{l(\boldsymbol{\theta})-l(\widetilde{\boldsymbol{\theta}})\} \exp \{-[l(\widehat{\boldsymbol{\theta}})-l(\widetilde{\boldsymbol{\theta}})]\}|\boldsymbol{J}| \\
& =\frac{\bar{c}}{(2 \pi)^{k / 2}}|\widehat{\boldsymbol{j}}|^{1 / 2} \exp \{l(\boldsymbol{\theta})-l(\widetilde{\boldsymbol{\theta}})\} \exp \left\{-\frac{1}{2} r^{2}\right\}|\boldsymbol{J}|,
\end{aligned}
$$

em que $|\boldsymbol{J}|$ denota o determinante do jacobiano da transformação. Note que usamos o fato de que $r^{2} / 2=l(\widehat{\boldsymbol{\theta}})-l(\widetilde{\boldsymbol{\theta}})$. Em vez de calcular $\boldsymbol{J}=\partial \widehat{\boldsymbol{\theta}} / \partial(r, \widetilde{\boldsymbol{\lambda}})^{\top}$, vamos calcular $\boldsymbol{J}^{-1}=\partial(r, \widetilde{\boldsymbol{\lambda}})^{\top} / \partial \widehat{\boldsymbol{\theta}}$ e, então, usar o fato de que $\left|\boldsymbol{J}^{-1}\right|=|\boldsymbol{J}|^{-1}$, sendo $\boldsymbol{J}^{-1}$ da forma

$$
\boldsymbol{J}^{-1}=\left[\begin{array}{cc}
\partial r / \partial \widehat{\psi} & \partial r / \partial \widehat{\boldsymbol{\lambda}} \\
\partial \widetilde{\boldsymbol{\lambda}}^{\top} / \partial \widehat{\psi} & \partial \widetilde{\boldsymbol{\lambda}}^{\top} / \partial \widehat{\boldsymbol{\lambda}}
\end{array}\right]
$$

Como $r^{2} / 2=l(\widehat{\boldsymbol{\theta}})-l(\widetilde{\boldsymbol{\theta}})$, temos que

$$
r \frac{\partial r}{\partial \widehat{\psi}}=l_{; \widehat{\psi}}(\widehat{\boldsymbol{\theta}})-l_{; \widehat{\psi}}(\widetilde{\boldsymbol{\theta}}) \quad \text { e } \quad r \frac{\partial r}{\partial \widehat{\boldsymbol{\lambda}}}=\boldsymbol{l}_{; \widehat{\boldsymbol{\lambda}}}(\widehat{\boldsymbol{\theta}})-\boldsymbol{l}_{; \widehat{\boldsymbol{\lambda}}}(\widetilde{\boldsymbol{\theta}})
$$

Para completar a especificacão de $\boldsymbol{J}^{-1}$, precisamos obter $\partial \tilde{\boldsymbol{\lambda}}^{\top} / \partial \widehat{\psi}$ e $\partial \tilde{\boldsymbol{\lambda}}^{\top} / \partial \widehat{\boldsymbol{\lambda}}$. Estas quantidades podem ser obtidas através da equação de verossimilhança usada para determinar $\widetilde{\lambda}$ :

$$
\boldsymbol{l}_{\boldsymbol{\lambda}}(\widetilde{\boldsymbol{\theta}} ; \widehat{\psi}, \widehat{\boldsymbol{\lambda}})=\{\boldsymbol{0}\}_{(k-1) \times 1},
$$

em que a notação $\{\mathbf{0}\}_{l \times m}$ denota uma matriz de zeros com $l$ linhas e $m$ colunas. Diferenciando esta expressão em relação a $\widehat{\psi}$ e $\widehat{\boldsymbol{\lambda}}$ é possível mostrar que (Barndorff-Nielsen, 1991; Barndorff-Nielsen \& Cox, 1994, § 6.6.1)

$$
\boldsymbol{l}_{\boldsymbol{\lambda} \boldsymbol{\lambda}}(\widetilde{\boldsymbol{\theta}}) \frac{\partial \widetilde{\boldsymbol{\lambda}}^{\top}}{\partial \widehat{\psi}}+\boldsymbol{l}_{\boldsymbol{\lambda} ; \widehat{\psi}}(\widetilde{\boldsymbol{\theta}})=\{\mathbf{0}\}_{(k-1) \times 1} \quad \text { e } \quad \boldsymbol{l}_{\boldsymbol{\lambda} \boldsymbol{\lambda}}(\widetilde{\boldsymbol{\theta}}) \frac{\partial \widetilde{\boldsymbol{\lambda}}^{\top}}{\partial \widehat{\boldsymbol{\lambda}}}+\boldsymbol{l}_{\boldsymbol{\lambda} ; \widehat{\boldsymbol{\lambda}}}(\widetilde{\boldsymbol{\theta}})=\{\mathbf{0}\}_{(k-1) \times(k-1)} .
$$

Assim,

$$
\frac{\partial \widetilde{\boldsymbol{\lambda}}^{\top}}{\partial \widehat{\psi}}=\boldsymbol{j}_{\boldsymbol{\lambda} \boldsymbol{\lambda}}(\widetilde{\boldsymbol{\theta}})^{-1} \boldsymbol{l}_{\boldsymbol{\lambda} ; \tilde{\psi}}(\widetilde{\boldsymbol{\theta}}) \quad \text { e } \frac{\partial \widetilde{\boldsymbol{\lambda}}^{\top}}{\partial \widehat{\boldsymbol{\lambda}}}=\boldsymbol{j}_{\boldsymbol{\lambda} \boldsymbol{\lambda}}(\widetilde{\boldsymbol{\theta}})^{-1} \boldsymbol{l}_{\boldsymbol{\lambda} ; \widehat{\boldsymbol{\lambda}}}(\widetilde{\boldsymbol{\theta}})
$$

Usamos o fato de que $\boldsymbol{j}_{\boldsymbol{\lambda} \boldsymbol{\lambda}}=-\boldsymbol{l}_{\boldsymbol{\lambda} \boldsymbol{\lambda}}$, a matriz de informação observada do vetor $\boldsymbol{\lambda}$.

Dessa forma, temos que

$$
\boldsymbol{J}^{-1}=\left[\begin{array}{cc}
\partial r / \partial \widehat{\psi} & \partial r / \partial \widehat{\boldsymbol{\lambda}} \\
\partial \widetilde{\boldsymbol{\lambda}}^{\top} / \partial \widehat{\psi} & \partial \widetilde{\boldsymbol{\lambda}}^{\top} / \partial \widehat{\boldsymbol{\lambda}}
\end{array}\right]=\left[\begin{array}{cc}
r^{-1}\left\{l_{; \widehat{\psi}}(\widehat{\boldsymbol{\theta}})-l_{; \widehat{\psi}}(\widetilde{\boldsymbol{\theta}})\right\} & r^{-1}\left\{\boldsymbol{l}_{; \widehat{\boldsymbol{\lambda}}}(\widehat{\boldsymbol{\theta}})-\boldsymbol{l}_{; \widehat{\boldsymbol{\lambda}}}(\widetilde{\boldsymbol{\theta}})\right\} \\
\boldsymbol{j}_{\boldsymbol{\lambda} \boldsymbol{\lambda}}(\widetilde{\boldsymbol{\theta}})^{-1} \boldsymbol{l}_{\boldsymbol{\lambda} ; \hat{\psi}}(\widetilde{\boldsymbol{\theta}}) & \boldsymbol{j}_{\boldsymbol{\lambda} \boldsymbol{\lambda}}(\widetilde{\boldsymbol{\theta}})^{-1} \boldsymbol{l}_{\boldsymbol{\lambda} ; \widehat{\boldsymbol{\lambda}}}(\widetilde{\boldsymbol{\theta}})
\end{array}\right]
$$


que pode ser escrito na forma

$$
\left[\begin{array}{cc}
r & \{\mathbf{0}\}_{1 \times(k-1)} \\
\{\mathbf{0}\}_{(k-1) \times 1} & \boldsymbol{j}_{\boldsymbol{\lambda \lambda}}(\widetilde{\boldsymbol{\theta}})
\end{array}\right]^{-1}\left[\begin{array}{cc}
l_{; \widehat{\psi}}(\widehat{\boldsymbol{\theta}})-l_{i \widehat{\psi}}(\widetilde{\boldsymbol{\theta}}) & \boldsymbol{l}_{; \widehat{\boldsymbol{\lambda}}}(\widehat{\boldsymbol{\theta}})-\boldsymbol{l}_{; \widehat{\boldsymbol{\lambda}}}(\widetilde{\boldsymbol{\theta}}) \\
\boldsymbol{l}_{\boldsymbol{\lambda} ; \widehat{\psi}} & \boldsymbol{l}_{\boldsymbol{\lambda} ; \widehat{\boldsymbol{\boldsymbol { \lambda }}}}(\tilde{\boldsymbol{\theta}})
\end{array}\right]
$$

Assim,

$$
|\boldsymbol{J}|=r\left|\boldsymbol{j}_{\boldsymbol{\lambda} \boldsymbol{\lambda}}(\widetilde{\boldsymbol{\theta}})\right| /\left|\begin{array}{cc}
l_{; \widehat{\psi}}(\widehat{\boldsymbol{\theta}})-l_{; \widehat{\psi}}(\widetilde{\boldsymbol{\theta}}) & \boldsymbol{l}_{; \widehat{\boldsymbol{\lambda}}}(\widehat{\boldsymbol{\theta}})-\boldsymbol{l}_{; \widehat{\boldsymbol{\lambda}}}(\widetilde{\boldsymbol{\theta}}) \\
\boldsymbol{l}_{\boldsymbol{\lambda} ; \widehat{\psi}}(\tilde{\boldsymbol{\theta}}) & \boldsymbol{l}_{\boldsymbol{\lambda} ; \widehat{\boldsymbol{\lambda}}}(\tilde{\boldsymbol{\theta}})
\end{array}\right|=r\left|\boldsymbol{j}_{\boldsymbol{\lambda} \boldsymbol{\lambda}}(\widetilde{\boldsymbol{\theta}})\right| /\left|\begin{array}{c}
\boldsymbol{l}_{; \widehat{\boldsymbol{\theta}}}(\widehat{\boldsymbol{\theta}})-\boldsymbol{l}_{; \widehat{\boldsymbol{\theta}}}(\widetilde{\boldsymbol{\theta}}) \\
\boldsymbol{l}_{\boldsymbol{\lambda} ; \widehat{\boldsymbol{\theta}}}(\widetilde{\tilde{\boldsymbol{\theta}}})
\end{array}\right|
$$

Seja

$$
u=\left|\begin{array}{c}
\boldsymbol{l}_{; \widehat{\boldsymbol{\theta}}}(\widehat{\boldsymbol{\theta}})-\boldsymbol{l}_{; \widehat{\boldsymbol{\theta}}}(\widetilde{\boldsymbol{\theta}}) \\
\boldsymbol{l}_{\boldsymbol{\lambda} ; \hat{\boldsymbol{\theta}}}(\tilde{\boldsymbol{\theta}})
\end{array}\right| /\left|\boldsymbol{j}_{\lambda \boldsymbol{\lambda}}(\widetilde{\boldsymbol{\theta}})\right|^{1 / 2}|\widehat{\boldsymbol{j}}|^{1 / 2}
$$

então,

$$
p^{*}(r, \widetilde{\boldsymbol{\lambda}} \mid \boldsymbol{a} ; \boldsymbol{\theta})=\frac{\bar{c}}{(2 \pi)^{k / 2}} \frac{r\left|\boldsymbol{j}_{\boldsymbol{\lambda} \boldsymbol{\lambda}}(\widetilde{\boldsymbol{\theta}})\right|^{1 / 2}}{u} \exp \{l(\boldsymbol{\theta})-l(\widetilde{\boldsymbol{\theta}})\} \exp \left\{-\frac{1}{2} r^{2}\right\}
$$

Adicionalmente, no modelo em que $\psi$ está fixado, podemos usar $p^{*}(\widetilde{\boldsymbol{\lambda}} \mid r, \boldsymbol{a} ; \boldsymbol{\theta})$ para aproximar a densidade condicional de $\widetilde{\boldsymbol{\lambda}}$ dado $r$ e $\boldsymbol{a}$. É possível mostrar que (Barndorff-Nielsen \& Cox, 1994, $\S 6.6 .1)$

$$
p^{*}(\widetilde{\boldsymbol{\lambda}} \mid r, \boldsymbol{a} ; \boldsymbol{\theta})=\frac{\bar{c}_{\psi}}{(2 \pi)^{(k-1) / 2}}\left|\boldsymbol{j}_{\boldsymbol{\lambda} \boldsymbol{\lambda}}(\widetilde{\boldsymbol{\theta}})\right|^{1 / 2} \exp \{l(\boldsymbol{\theta})-l(\widetilde{\boldsymbol{\theta}})\},
$$

em que $\bar{c}_{\psi}=1+O\left(n^{-1}\right)$. Portanto, as aproximações $p^{*}(r, \widetilde{\boldsymbol{\lambda}} \mid \boldsymbol{a} ; \boldsymbol{\theta})$ e $p^{*}(\widetilde{\boldsymbol{\lambda}} \mid r, \boldsymbol{a} ; \boldsymbol{\theta})$ levam à seguinte aproximação para a densidade condicional de $R$ dado $\boldsymbol{a}$ :

$$
p^{*}(r \mid \boldsymbol{a} ; \boldsymbol{\theta})=\frac{p(r, \widetilde{\boldsymbol{\lambda}} \mid \boldsymbol{a} ; \boldsymbol{\theta})}{p(\widetilde{\boldsymbol{\lambda}} \mid r, \boldsymbol{a} ; \boldsymbol{\theta})}=\frac{\bar{c}}{\bar{c}_{\psi}} \frac{1}{(2 \pi)^{1 / 2}} \frac{r}{u} \exp \left\{-\frac{1}{2} r^{2}\right\}=\frac{\bar{c}}{\bar{c}_{\psi}} \frac{r}{u} \phi(r)
$$

Observe que (4.6) é da forma (4.4) com a função $h$ da forma $h(r / \sqrt{n})=r / u$, em que $u$ é visto como uma função de $r$. Assim, a estatística da razão de verossimilhanças sinalizada ajustada é dada por

$$
R^{*}=R+\frac{1}{R} \log (U / R)
$$

em que $U$ é da forma

$$
U=\left|\begin{array}{c}
\boldsymbol{l}_{; \widehat{\boldsymbol{\theta}}}(\widehat{\boldsymbol{\theta}})-\boldsymbol{l}_{; \widehat{\boldsymbol{\theta}}}(\widetilde{\boldsymbol{\theta}}) \\
\boldsymbol{l}_{\boldsymbol{\lambda} ; \widehat{\boldsymbol{\theta}}}(\tilde{\boldsymbol{\theta}})
\end{array}\right| /\left|\boldsymbol{j}_{\boldsymbol{\lambda} \boldsymbol{\lambda}}(\widetilde{\boldsymbol{\theta}})\right|^{1 / 2}|\widehat{\boldsymbol{j}}|^{1 / 2}
$$

Usando a fórmula do determinante de uma matriz particionada (Rao, 1973, p. 32), uma forma alternativa para a expressão de $U$ dada em (4.7) é

$$
U=\frac{\left|\boldsymbol{l}_{\boldsymbol{\lambda} ; \widehat{\boldsymbol{\lambda}}}(\widetilde{\boldsymbol{\theta}})\right|\left|l_{; \widehat{\psi}}(\widehat{\boldsymbol{\theta}})-l_{; \widehat{\psi}}(\widetilde{\boldsymbol{\theta}})-\left[\boldsymbol{l}_{; \widehat{\boldsymbol{\lambda}}}(\widehat{\boldsymbol{\theta}})-\boldsymbol{l}_{; \widehat{\boldsymbol{\lambda}}}(\widetilde{\boldsymbol{\theta}})\right] \boldsymbol{l}_{\boldsymbol{\lambda} ; \widehat{\boldsymbol{\lambda}}}(\widetilde{\boldsymbol{\theta}})^{-1} \boldsymbol{l}_{\boldsymbol{\lambda} ; \widehat{\psi}}(\widetilde{\boldsymbol{\theta}})\right|}{\left|\boldsymbol{j}_{\boldsymbol{\lambda} \boldsymbol{\lambda}}(\widetilde{\boldsymbol{\theta}})\right|^{1 / 2}|\widehat{\boldsymbol{j}}|^{1 / 2}}
$$


A expressão (4.8) ainda pode ser esrcita na forma

$$
U=\frac{\left|\boldsymbol{l}_{\boldsymbol{\lambda} ; \widehat{\boldsymbol{\lambda}}}(\widetilde{\boldsymbol{\theta}})\right|}{\left|\boldsymbol{j}_{\boldsymbol{\lambda} \boldsymbol{\lambda}}(\widetilde{\boldsymbol{\theta}})\right|^{1 / 2}\left|\widehat{\boldsymbol{j}}_{\boldsymbol{\lambda} \boldsymbol{\lambda}}\right|^{1 / 2}} \frac{\left|l_{; \widehat{\psi}}(\widehat{\boldsymbol{\theta}})-l_{; \widehat{\psi}}(\widetilde{\boldsymbol{\theta}})-\left[\boldsymbol{l}_{; \widehat{\boldsymbol{\lambda}}}(\widehat{\boldsymbol{\theta}})-\boldsymbol{l}_{; \widehat{\boldsymbol{\lambda}}}(\widetilde{\boldsymbol{\theta}})\right]_{\boldsymbol{\lambda} ; \hat{\boldsymbol{\lambda}}}(\widetilde{\boldsymbol{\theta}})^{-1} \boldsymbol{l}_{\boldsymbol{\lambda} ; \widehat{\psi}}(\widetilde{\boldsymbol{\theta}})\right|}{\left|\hat{j}_{\psi \psi}-\widehat{\boldsymbol{j}}_{\psi \boldsymbol{\lambda}} \hat{\boldsymbol{j}}_{\boldsymbol{\lambda} \boldsymbol{\lambda}}^{-1} \widehat{\boldsymbol{j}}_{\boldsymbol{\lambda} \psi}\right|^{1 / 2}}
$$

Como no caso uniparamétrico, o sinal de $U$ é determinado de forma a ser o mesmo que o sinal de $R$.

\subsubsection{Distribuição Assintótica de $R^{*}$}

As propriedades assintóticas de $R^{*}$ tanto no caso uniparamétrico quanto no caso multiparamétrico são as mesmas, isto é, em ambos os casos, temos a convergência (assintótica) de $R^{*}$ para a distribuição normal padrão sob a hipótese nula. Assim, em geral,

$$
\operatorname{Pr}\left(R^{*} \leq t\right)=\Phi(t)\left\{1+O\left(n^{-3 / 2}\right)\right\}
$$

para valores fixos de $t$. Note que a distribuição assintótica de $R^{*}$ é melhor aproximada pela normal padrão do que a distribuição assintótica de $R$, em que o erro é de ordem $O\left(n^{-1 / 2}\right)$. De acordo com Barndorff-Nielsen \& Cox (1994, § 6.6.2), a aproximação (4.9) acontece se $r$ é $O(1)$, sendo $r$ o valor observado da estatística da razão de verossimilhanças sinalizada, $R$. Neste caso, $r$ é $O(1)$ se $\widehat{\theta}=\theta+O\left(n^{-1 / 2}\right),{ }^{1}$ isto é, $|\widehat{\theta}-\theta| \leq \delta / \sqrt{n}$ para alguma constante fixa $\delta$.

Suponha, por exemplo, o caso unidimensional. Não é difícil verificar que $r$ é $O(1)$ se $\widehat{\theta}=\theta+$ $O\left(n^{-1 / 2}\right)$. (No caso multidimensional o procedimento é o mesmo.) Temos que

$$
r= \pm[2\{l(\widehat{\theta})-l(\theta)\}]^{1 / 2}
$$

em que, neste caso, $\widehat{\theta}$ é a estimativa de máxima verossimilhança de $\theta$. Se expandirmos $l(\theta)$ em torno de $\theta=\widehat{\theta}$, temos que

$$
l(\theta)=l(\widehat{\theta})+(\theta-\widehat{\theta}) l_{\theta}(\widehat{\theta})+\frac{1}{2}(\theta-\widehat{\theta})^{2} l_{\theta \theta}(\widehat{\theta})+\cdots .
$$

Como $l_{\theta}(\widehat{\theta})=0$ e $\widehat{j}=-l_{\theta \theta}(\widehat{\theta})$ (informação de Fisher observada estimada), temos

$$
l(\widehat{\theta})-l(\theta)=\frac{1}{2}(\widehat{\theta}-\theta)^{2} \widehat{j}+\cdots .
$$

Como $\widehat{\theta}-\theta=O\left(n^{-1 / 2}\right)$, temos que $(\widehat{\theta}-\theta)^{2}=O\left(n^{-1}\right)$. Como $\widehat{j}$ é de ordem $O(n)$, a quantidade $(\widehat{\theta}-\theta)^{2} \widehat{j}$ é de ordem $O(1)$, implicando que $l(\widehat{\theta})-l(\theta)=O(1)$ e, consequentemente, $r$ é $O(1)$.

Em geral, estamos interessados em calcular $p$-valores a partir da probabilidade $\operatorname{Pr}\left(R^{*} \leq r^{*}\right)$ para verificar a significância de um parâmetro de interesse no modelo em estudo. A probabilidade $\operatorname{Pr}\left(R^{*} \leq\right.$ $\left.r^{*}\right)$ pode ser aproximada por $\operatorname{Pr}\left(R^{*} \leq r^{*}\right) \doteq \Phi\left(r^{*}\right)$, em que o símbolo ' $\doteq$ ' indica aproximadamente igual. Entretanto, pode ser mostrado que

$$
\Phi\left(r^{*}\right) \doteq \Phi(r)+\phi(r)\left[\frac{1}{r}-\frac{1}{u}\right]
$$

em que $r$ e $u$ são os valores observados de $R$ e $U$, respectivamente; veja Barndorff-Nielsen (1990a,

\footnotetext{
${ }^{1}$ Note que, neste caso, estamos considerando $\widehat{\theta}$ como a estimativa de $\theta$ e não um estimador. Neste capítulo esta distinção será indicada por $O(\cdot)$ e $O_{p}(\cdot)$, respectivamente.
} 
b, c), Barndorff-Nielsen (1991) e Fraser (1990). A expressão $\Phi(r)+\phi(r)\left[r^{-1}-u^{-1}\right]$ é equivalente à aproximação de Lugannani \& Rice (1980) no caso de modelos uniparamétricos da família exponencial e, neste caso, $\Phi\left(r^{*}\right)=\Phi(r)+\phi(r)\left[r^{-1}-u^{-1}\right]$; veja Barndorff-Nielsen (1991). Portanto, podemos aproximar $\operatorname{Pr}\left(R^{*} \leq r^{*}\right)$ de duas formas, considerando $\Phi\left(r^{*}\right)$ ou $\Phi(r)+\phi(r)\left[r^{-1}-u^{-1}\right]$. Adicionalmente, Barndorff-Nielsen (1991) mostra que se $\widehat{\psi} \rightarrow \pm \infty,|r| \rightarrow \infty$,

$$
\frac{\log (u / r)}{r} \rightarrow 0 \quad \text { e } \quad \frac{u}{r^{3}} \rightarrow 0,
$$

então, em ambas as extremidades, a aproximação de $\operatorname{Pr}\left(R^{*} \leq r^{*}\right)$ por $\Phi\left(r^{*}\right)$ ou $\Phi(r)+\phi(r)\left[r^{-1}-u^{-1}\right]$ tende a um, isto é, as duas aproximações dão o mesmo resultado. Note que esta afirmação se refere ao caso em que $\widehat{\psi} \rightarrow \pm \infty$, não o tamanho amostral indo para o infinito. Segundo o autor, quando a condição (4.10) não é satisfeita, $\Phi\left(r^{*}\right)$ forneça uma melhor aproximação para $\operatorname{Pr}\left(R^{*} \leq r^{*}\right)$, caso contrário, $\Phi(r)+\phi(r)[1 / r-1 / u]$ será melhor. Uma boa discussão sobre este fato está dada em Barndorff-Nielsen \& Cox $(1994, \S 6.7)$.

\subsection{Aproximações para $R^{*}$}

Embora $R^{*}$ seja, em geral, um melhoramento sobre a estatística da razão de verossimilhanças sinalizada usual, $R$, existe o inconveniente de que a estatística $U$ requerida para o seu cálculo é difícil de se obter. A dificuldade consiste, principalmente, na determinação de derivadas com respeito ao espaço amostral; veja as Seções 4.5.1 e 4.5.2. Cálculos de derivadas em relação ao espaço amostral, tais como $\boldsymbol{l}_{: \hat{\boldsymbol{\theta}}}(\boldsymbol{\theta})$ ou $\boldsymbol{l}_{\boldsymbol{\theta} ; \hat{\boldsymbol{\theta}}}(\boldsymbol{\theta})$, requerem a especificação de uma estatística ancilar $\boldsymbol{a}$ e que, além disso, a estatística suficiente possa ser escrita na forma $(\widehat{\boldsymbol{\theta}}, \boldsymbol{a})$. Entretanto, isso só é possível em certas classes especiais de modelos, tais como os modelos da família exponencial e modelos de transformação (locação e escala), por exemplo. Em modelos gerais, os cálculos destas derivadas podem ser extremamente difíceis ou até impossíveis. A fim de contornar esta dificuldade, algumas aproximações para as derivadas com relação ao espaço amostral têm sido propostas. Tais aproximações caracterizam-se por não dependerem de uma especificação para $\boldsymbol{a}$.

As quantidades $\boldsymbol{l}_{; \widehat{\boldsymbol{\theta}}}(\boldsymbol{\theta})$ e $\boldsymbol{l}_{\boldsymbol{\theta} ; \hat{\boldsymbol{\theta}}}(\boldsymbol{\theta})$ podem ser expandidas em série de Taylor em torno do ponto $\boldsymbol{\theta}=\widehat{\boldsymbol{\theta}}$, ou seja,

$$
\left[\boldsymbol{l}_{; \widehat{\boldsymbol{\theta}}}(\boldsymbol{\theta})-\boldsymbol{l}_{; \widehat{\boldsymbol{\theta}}}(\widehat{\boldsymbol{\theta}})\right]^{\top}=\widehat{\boldsymbol{j}}(\boldsymbol{\theta}-\widehat{\boldsymbol{\theta}})^{\top}+\frac{1}{2}(\boldsymbol{\theta}-\widehat{\boldsymbol{\theta}})^{\top} \boldsymbol{l}_{\boldsymbol{\theta} \boldsymbol{\theta} ; \widehat{\boldsymbol{\theta}}}(\widehat{\boldsymbol{\theta}})(\boldsymbol{\theta}-\widehat{\boldsymbol{\theta}})+\cdots
$$

e

$$
\begin{aligned}
\boldsymbol{l}_{\boldsymbol{\theta} ; \widehat{\boldsymbol{\theta}}}(\boldsymbol{\theta}) & =\boldsymbol{l}_{\boldsymbol{\theta} ; \hat{\boldsymbol{\theta}}}(\widehat{\boldsymbol{\theta}})+(\boldsymbol{\theta}-\widehat{\boldsymbol{\theta}})^{\top} \boldsymbol{l}_{\boldsymbol{\theta \theta} ; \widehat{\boldsymbol{\theta}}}(\widehat{\boldsymbol{\theta}})+\cdots \\
& =\widehat{\boldsymbol{j}}+(\boldsymbol{\theta}-\widehat{\boldsymbol{\theta}})^{\top} \boldsymbol{l}_{\boldsymbol{\theta} \boldsymbol{\theta} ; \widehat{\boldsymbol{\theta}}}(\widehat{\boldsymbol{\theta}})+\cdots
\end{aligned}
$$

Estas expressões serão usadas nas seções seguintes para avaliar as propriedades das aproximações de $\boldsymbol{l}_{; \widehat{\boldsymbol{\theta}}}(\boldsymbol{\theta})$ e $\boldsymbol{l}_{\boldsymbol{\theta} ; \widehat{\boldsymbol{\theta}}}(\boldsymbol{\theta})$, respectivamente. Note que $\boldsymbol{l}_{\boldsymbol{\theta} ; \widehat{\boldsymbol{\theta}}}$ é uma matriz $k \times k$ e que $\boldsymbol{l}_{\boldsymbol{\theta} ; \hat{\boldsymbol{\theta}}}$ é uma array tridimensional, sendo $(\boldsymbol{\theta}-\widehat{\boldsymbol{\theta}})^{\top} \boldsymbol{l}_{\boldsymbol{\theta \theta} ; \widehat{\boldsymbol{\theta}}}(\widehat{\boldsymbol{\theta}})(\boldsymbol{\theta}-\widehat{\boldsymbol{\theta}})$ um vetor e $(\boldsymbol{\theta}-\widehat{\boldsymbol{\theta}})^{\top} \boldsymbol{l}_{\boldsymbol{\theta} \boldsymbol{\theta} ; \widehat{\boldsymbol{\theta}}}(\widehat{\boldsymbol{\theta}})$ uma matriz. Vale ressaltar que não estaremos interessados diretamente nestas multiplicações de arrays nos desenvolvimentos apresentados nas seções a seguir. Detalhes sobre multiplicação de arrays podem ser encontrados em Wei (1998).

A seguir, diferentes métodos de aproximação são apresentados. Na Seção 4.6.1, a aproximação 
é baseada em parâmetros ortogonais (DiCiccio \& Martin, 1993). Na Seção 4.6.2, a aproximação é baseada em covariâncias e covariâncias empíricas (Skovgaard, 1996; Severini, 1999). Finalmente, na Seção 4.6.3, a aproximação é baseado em uma estatística aproximadamente ancilar (Fraser \& Reid, 1995, 1999; Fraser, Reid \& Wu, 1999).

\subsubsection{Aproximação de DiCiccio-Martin}

Seja $\boldsymbol{\theta}=(\psi, \boldsymbol{\lambda})$, sendo $\psi$ o parâmetro de interesse (unidimensional) e $\boldsymbol{\lambda}$ de dimensão $k-1$ o vetor de parâmetros de perturbação. Suponha que $\psi$ e $\boldsymbol{\lambda}$ sejam ortogonais, isto é, $\boldsymbol{i}_{\boldsymbol{\lambda} \psi}(\boldsymbol{\theta})=$ $\mathbb{E}\left(\partial^{2} l(\boldsymbol{\theta}) / \partial \boldsymbol{\lambda} \partial \psi\right)=\{\boldsymbol{0}\}_{1 \times(k-1)}$, o que significa que a matriz de informação de Fisher é bloco diagonal. Seja $\widehat{\psi}=\psi+O_{p}\left(n^{-1 / 2}\right)$. Da relação entre $\widetilde{\boldsymbol{\lambda}}$ e $\widehat{\boldsymbol{\lambda}}$, isto é, $\widetilde{\boldsymbol{\lambda}}^{\top}=\widehat{\boldsymbol{\lambda}}^{\top}+\boldsymbol{i}_{\boldsymbol{\lambda} \boldsymbol{\lambda}}^{-1} \boldsymbol{i}_{\boldsymbol{\lambda} \psi}^{\top}(\widehat{\psi}-\psi)+O_{p}\left(n^{-1}\right)$, e usando o fato de que $\boldsymbol{i}_{\boldsymbol{\lambda} \psi}=\{\mathbf{0}\}_{1 \times(k-1)}$, temos que (Cox \& Reid, 1987)

$$
\widetilde{\boldsymbol{\lambda}}^{\top}=\widehat{\boldsymbol{\lambda}}^{\top}+O_{p}\left(n^{-1}\right)
$$

Dai, tem-se que

$$
\frac{\partial \widetilde{\boldsymbol{\lambda}}^{\top}}{\partial \widehat{\psi}}=O_{p}\left(n^{-1}\right) \quad \text { e } \quad \frac{\partial \widetilde{\boldsymbol{\lambda}}^{\top}}{\partial \widehat{\boldsymbol{\lambda}}}=\boldsymbol{D}+O_{p}\left(n^{-1}\right),
$$

em que $\boldsymbol{D}$ denota uma matriz identidade de dimensão $(k-1) \times(k-1)$. Como

$$
\frac{\partial \widetilde{\boldsymbol{\lambda}}^{\top}}{\partial \widehat{\psi}}=\boldsymbol{j}_{\boldsymbol{\lambda} \boldsymbol{\lambda}}(\widetilde{\boldsymbol{\theta}})^{-1} \boldsymbol{l}_{\boldsymbol{\lambda} ; \widehat{\psi}}(\widetilde{\boldsymbol{\theta}}) \quad \text { e } \frac{\partial \widetilde{\boldsymbol{\lambda}}^{\top}}{\partial \widehat{\boldsymbol{\lambda}}}=\boldsymbol{j}_{\boldsymbol{\lambda} \boldsymbol{\lambda}}(\widetilde{\boldsymbol{\theta}})^{-1} \boldsymbol{l}_{\boldsymbol{\lambda} ; \widehat{\boldsymbol{\lambda}}}(\widetilde{\boldsymbol{\theta}})
$$

(veja a Seção 4.5.2), segue que

$$
\boldsymbol{l}_{\boldsymbol{\lambda} ; \widehat{\psi}}(\widetilde{\boldsymbol{\theta}})=O_{p}(1) \quad \text { e } \quad \boldsymbol{l}_{\boldsymbol{\lambda} ; \hat{\boldsymbol{\lambda}}}(\widetilde{\boldsymbol{\theta}})=\boldsymbol{j}_{\boldsymbol{\lambda} \boldsymbol{\lambda}}(\widetilde{\boldsymbol{\theta}})+O_{p}(1) .
$$

Usando estes resultados e a equação (4.8), $U$ pode ser escrito na forma (Barndorff-Nielsen, 1991, § 4)

$$
U=\frac{\left|\boldsymbol{j}_{\boldsymbol{\lambda} \boldsymbol{\lambda}}(\widetilde{\boldsymbol{\theta}})\right|^{1 / 2}}{|\widehat{\boldsymbol{j}}|^{1 / 2}}\left\{l_{; \widehat{\psi}}(\widehat{\boldsymbol{\theta}})-l_{; \widehat{\psi}}(\widetilde{\boldsymbol{\theta}})\right\}+O_{p}\left(n^{-1}\right) .
$$

Assim, para aproximar $U$ (ou seja, $R^{*}$ ), é necessário aproximar

$$
l_{; \widehat{\psi}}(\widehat{\boldsymbol{\theta}})-l_{; \widehat{\psi}}(\widetilde{\boldsymbol{\theta}})=l_{; \widehat{\psi}}(\widehat{\psi}, \widehat{\boldsymbol{\lambda}})-l_{; \widehat{\psi}}(\psi, \widetilde{\boldsymbol{\lambda}}) .
$$

Fazendo uma expansão em série de Taylor de $l_{; \widehat{\psi}}(\psi, \widetilde{\boldsymbol{\lambda}})$ e usando o fato que $\widetilde{\boldsymbol{\lambda}}=\widehat{\boldsymbol{\lambda}}+O_{p}\left(n^{-1}\right)$, temos que

$$
\begin{aligned}
l_{; \widehat{\psi}}(\psi, \widetilde{\boldsymbol{\lambda}})-l_{; \widehat{\psi}}(\widehat{\psi}, \widehat{\boldsymbol{\lambda}}) & =l_{\psi ; \widehat{\psi}}(\widehat{\boldsymbol{\theta}})(\psi-\widehat{\psi})+\frac{1}{2} l_{\psi \psi ; \widehat{\psi}}(\widehat{\boldsymbol{\theta}})(\psi-\widehat{\psi})^{2}+\frac{1}{6} l_{\psi \psi \psi ; \widehat{\psi}}(\widehat{\boldsymbol{\theta}})(\psi-\widehat{\psi})^{3}+\cdots \\
& =l_{\psi ; \widehat{\psi}}(\widehat{\boldsymbol{\theta}})(\psi-\widehat{\psi})+\frac{1}{2} l_{\psi \psi ; \widehat{\psi}}(\widehat{\boldsymbol{\theta}})(\psi-\widehat{\psi})^{2}+O_{p}\left(n^{-1 / 2}\right) .
\end{aligned}
$$

Se denotarmos por $\kappa_{i j k}(\boldsymbol{\theta})$ o $(i, j, k)$-ésimo cumulante de $\left(l_{\psi}(\boldsymbol{\theta}), l_{\psi \psi}(\boldsymbol{\theta}), l_{\psi \psi \psi}(\boldsymbol{\theta})\right)$, isto é, $\kappa_{100}(\boldsymbol{\theta})=$ $\mathbb{E}\left\{l_{\psi}(\boldsymbol{\theta})\right\}, \kappa_{101}(\boldsymbol{\theta})=\mathbb{E}\left\{l_{\psi}(\boldsymbol{\theta}) l_{\psi \psi \psi}(\boldsymbol{\theta})\right\}$, etc., e usando resultados de Severini (2000, $\S$ 6.4.2), que 
relacionam derivadas do espaço amostral a quantidades da forma $\kappa_{i j k}$, pode ser mostrado que

$$
l_{\psi \psi ; \widehat{\psi}}(\boldsymbol{\theta})=\kappa_{110}(\boldsymbol{\theta})+O_{p}\left(n^{1 / 2}\right)
$$

e, consequentemente,

$$
l_{\psi \psi ; \widehat{\psi}}(\widehat{\boldsymbol{\theta}})=\kappa_{110}(\widehat{\boldsymbol{\theta}})+O_{p}\left(n^{1 / 2}\right) .
$$

Este resultado, juntamente com o fato de que $l_{\psi ; \widehat{\psi}}(\widehat{\boldsymbol{\theta}})=\widehat{j}_{\psi \psi}$, implica que

$$
l_{; \widehat{\psi}}(\psi, \widetilde{\boldsymbol{\lambda}})-l_{; \widehat{\psi}}(\widehat{\psi}, \widehat{\boldsymbol{\lambda}})=\widehat{j}_{\psi \psi}(\psi-\widehat{\psi})+\frac{1}{2} \kappa_{110}(\widehat{\boldsymbol{\theta}})(\psi-\widehat{\psi})^{2}+O_{p}\left(n^{-1 / 2}\right)
$$

Fazendo uma expansão similar para a função escore de $\psi, l_{\psi}(\psi, \widetilde{\boldsymbol{\lambda}})$, temos que

$$
\begin{aligned}
l_{\psi}(\psi, \widetilde{\boldsymbol{\lambda}})-l_{\psi}(\widehat{\psi}, \widehat{\boldsymbol{\lambda}}) & =l_{\psi \psi}(\widehat{\boldsymbol{\theta}})(\psi-\widehat{\psi})+\frac{1}{2} l_{\psi \psi \psi}(\widehat{\boldsymbol{\theta}})(\psi-\widehat{\psi})^{2}+O_{p}\left(n^{-1 / 2}\right) \\
& =-\widehat{j}_{\psi \psi}(\psi-\widehat{\psi})+\frac{1}{2} \kappa_{001}(\widehat{\boldsymbol{\theta}})(\psi-\widehat{\psi})^{2}+O_{p}\left(n^{-1 / 2}\right) .
\end{aligned}
$$

Assim, notando que o primeiro termo da expansão $l_{; \widehat{\psi}}(\psi, \widetilde{\boldsymbol{\lambda}})-l_{; \widehat{\psi}}(\widehat{\psi}, \widehat{\boldsymbol{\lambda}})$ é igual ao primeiro termo da expansão $l_{\psi}(\psi, \tilde{\boldsymbol{\lambda}})-l_{\psi}(\widehat{\psi}, \widehat{\boldsymbol{\lambda}})$, a menos do sinal negativo, e que $l_{\psi}(\widehat{\psi}, \widehat{\boldsymbol{\lambda}})=0$, temos que

$$
l_{; \widehat{\psi}}(\psi, \widetilde{\boldsymbol{\lambda}})-l_{; \widehat{\psi}}(\widehat{\psi}, \widehat{\boldsymbol{\lambda}})=-l_{\psi}(\psi, \widetilde{\boldsymbol{\lambda}})+\frac{1}{2}\left[\kappa_{110}(\widehat{\boldsymbol{\theta}})+\kappa_{001}(\widehat{\boldsymbol{\theta}})\right](\psi-\widehat{\psi})^{2}+O_{p}\left(n^{-1 / 2}\right)
$$

Note que

$$
\begin{aligned}
\frac{\partial}{\partial \psi} i_{\psi \psi}(\boldsymbol{\theta}) & =-\frac{\partial}{\partial \psi} \int l_{\psi \psi}(\boldsymbol{\theta}) f(\boldsymbol{y} ; \boldsymbol{\theta}) \mathrm{d} \boldsymbol{y}=-\int \frac{\partial}{\partial \psi} l_{\psi \psi}(\boldsymbol{\theta}) f(\boldsymbol{y} ; \boldsymbol{\theta}) \mathrm{d} \boldsymbol{y} \\
& =-\int\left[l_{\psi \psi \psi}(\boldsymbol{\theta}) f(\boldsymbol{y} ; \boldsymbol{\theta})+l_{\psi \psi}(\boldsymbol{\theta}) f^{\prime}(\boldsymbol{y} ; \boldsymbol{\theta})\right] \mathrm{d} \boldsymbol{y} \\
& =-\int\left[l_{\psi \psi \psi}(\boldsymbol{\theta}) f(\boldsymbol{y} ; \boldsymbol{\theta})+l_{\psi \psi}(\boldsymbol{\theta}) \frac{f^{\prime}(\boldsymbol{y} ; \boldsymbol{\theta})}{f(\boldsymbol{y} ; \boldsymbol{\theta})} f(\boldsymbol{y} ; \boldsymbol{\theta})\right] \mathrm{d} \boldsymbol{y} \\
& =-\int\left[l_{\psi \psi \psi}(\boldsymbol{\theta}) f(\boldsymbol{y} ; \boldsymbol{\theta})+l_{\psi \psi}(\boldsymbol{\theta}) l_{\psi}(\boldsymbol{\theta}) f(\boldsymbol{y} ; \boldsymbol{\theta})\right] \mathrm{d} \boldsymbol{y} \\
& =-\left[\mathbb{E}\left\{l_{\psi \psi \psi}(\boldsymbol{\theta})\right\}+\mathbb{E}\left\{l_{\psi}(\boldsymbol{\theta}) l_{\psi \psi}(\boldsymbol{\theta})\right\}\right]=-\left[\kappa_{001}(\boldsymbol{\theta})+\kappa_{110}(\boldsymbol{\theta})\right]
\end{aligned}
$$

Consequentemente, temos que

$$
-\left.\frac{\partial}{\partial \psi} i_{\psi \psi}(\boldsymbol{\theta})\right|_{\boldsymbol{\theta}=\widehat{\boldsymbol{\theta}}}=\kappa_{001}(\widehat{\boldsymbol{\theta}})+\kappa_{110}(\widehat{\boldsymbol{\theta}}) .
$$

Expandindo $i_{\psi \psi}(\psi, \widetilde{\boldsymbol{\lambda}})$ em série de Taylor e usando o fato de que $\widetilde{\boldsymbol{\lambda}}=\widehat{\boldsymbol{\lambda}}+O_{p}\left(n^{-1}\right)$, temos que

$$
\begin{aligned}
i_{\psi \psi}(\psi, \widetilde{\boldsymbol{\lambda}})-i_{\psi \psi}(\widehat{\psi}, \widehat{\boldsymbol{\lambda}}) & =(\psi-\widehat{\psi}) \frac{\partial}{\partial \psi} i_{\psi \psi}(\widehat{\psi}, \widehat{\boldsymbol{\lambda}})+\frac{1}{2}(\psi-\widehat{\psi})^{2} \frac{\partial^{2}}{\partial \psi^{2}} i_{\psi \psi}(\widehat{\psi}, \widehat{\boldsymbol{\lambda}})+\cdots \\
& =(\psi-\widehat{\psi}) \frac{\partial}{\partial \psi} i_{\psi \psi}(\widehat{\psi}, \widehat{\boldsymbol{\lambda}})+O_{p}(1) \\
& =-(\psi-\widehat{\psi})\left[\kappa_{001}(\widehat{\boldsymbol{\theta}})+\kappa_{110}(\widehat{\boldsymbol{\theta}})\right]+O_{p}(1),
\end{aligned}
$$


isto é,

$$
-(\psi-\widehat{\psi})\left[\kappa_{001}(\widehat{\boldsymbol{\theta}})+\kappa_{110}(\widehat{\boldsymbol{\theta}})\right]=i_{\psi \psi}(\psi, \tilde{\boldsymbol{\lambda}})-i_{\psi \psi}(\widehat{\psi}, \widehat{\boldsymbol{\lambda}})+O_{p}(1)
$$

Usando este fato, a equação (4.14) pode ser escrita na forma

$$
l_{; \widehat{\psi}}(\psi, \widetilde{\boldsymbol{\lambda}})-l_{; \widehat{\psi}}(\widehat{\psi}, \widehat{\boldsymbol{\lambda}})=-l_{\psi}(\psi, \tilde{\boldsymbol{\lambda}})-\frac{1}{2}\left[i_{\psi \psi}(\psi, \widetilde{\boldsymbol{\lambda}})-i_{\psi \psi}(\widehat{\psi}, \widehat{\boldsymbol{\lambda}})\right](\psi-\widehat{\psi})+O_{p}\left(n^{-1 / 2}\right) .
$$

Agora, usando a expansão (4.13), temos que

$$
l_{\psi}(\psi, \tilde{\boldsymbol{\lambda}})=-\widehat{j}_{\psi \psi}(\psi-\widehat{\psi})+O_{p}(1)=-\widehat{i}_{\psi \psi}(\psi-\widehat{\psi})+O_{p}(1) .
$$

Note que na segunda igualdade usamos o fato de que, em geral, derivadas de alta ordem são de ordem $O_{p}(n)$ e, além disso, são iguais a uma constante de ordem $O(n)$ mais um termo de ordem $O_{p}\left(n^{1 / 2}\right)$; veja Severini (2000, cap. 3) para uma discussão de alguns resultados deste tipo. Em particular,

$$
\boldsymbol{j}(\boldsymbol{\theta})=\boldsymbol{i}(\boldsymbol{\theta})+O_{p}\left(n^{1 / 2}\right)
$$

e, consequentemente,

$$
\boldsymbol{j}(\widehat{\boldsymbol{\theta}})=\boldsymbol{i}(\widehat{\boldsymbol{\theta}})+O_{p}\left(n^{1 / 2}\right) .
$$

Dai, segue que $\widehat{j}_{\psi \psi}=\widehat{i}_{\psi \psi}+O_{p}\left(n^{1 / 2}\right)$. Então, temos que

$$
-(\psi-\widehat{\psi})=\frac{l_{\psi}(\psi, \tilde{\boldsymbol{\lambda}})}{\widehat{j}_{\psi \psi}}+O_{p}\left(n^{-1}\right)=\frac{l_{\psi}(\psi, \tilde{\boldsymbol{\lambda}})}{i_{\psi \psi}(\widehat{\psi}, \widehat{\boldsymbol{\lambda}})}+O_{p}\left(n^{-1}\right) .
$$

Assim,

$$
\begin{aligned}
l_{; \widehat{\psi}}(\psi, \widetilde{\boldsymbol{\lambda}})-l_{; \widehat{\psi}}(\widehat{\psi}, \widehat{\boldsymbol{\lambda}}) & =-l_{\psi}(\psi, \widetilde{\boldsymbol{\lambda}})+\frac{1}{2}\left[i_{\psi \psi}(\psi, \widetilde{\boldsymbol{\lambda}})-i_{\psi \psi}(\widehat{\psi}, \widehat{\boldsymbol{\lambda}})\right] \frac{l_{\psi}(\psi, \widetilde{\boldsymbol{\lambda}})}{i_{\psi \psi}(\widehat{\psi}, \widehat{\boldsymbol{\lambda}})}+O_{p}\left(n^{-1 / 2}\right) \\
& =-l_{\psi}(\psi, \widetilde{\boldsymbol{\lambda}})\left\{1-\frac{1}{2}\left[\frac{i_{\psi \psi}(\psi, \widetilde{\boldsymbol{\lambda}})}{i_{\psi \psi}(\widehat{\psi}, \widehat{\boldsymbol{\lambda}})}-1\right]\right\}+O_{p}\left(n^{-1 / 2}\right)
\end{aligned}
$$

Expandindo $g(x)=1 / \sqrt{x}$ em torno de $x=1$, isto é, $1 / \sqrt{x}=1-(x-1) / 2+\cdots$, a expressão anterior pode ser escrita na forma (DiCiccio \& Martin, 1993)

$$
l_{; \widehat{\psi}}(\psi, \widetilde{\boldsymbol{\lambda}})-l_{; \widehat{\psi}}(\widehat{\psi}, \widehat{\boldsymbol{\lambda}})=-l_{\psi}(\psi, \widetilde{\boldsymbol{\lambda}}) \frac{i_{\psi \psi}(\widehat{\psi}, \widehat{\boldsymbol{\lambda}})^{1 / 2}}{i_{\psi \psi}(\psi, \widetilde{\boldsymbol{\lambda}})^{1 / 2}}+O_{p}\left(n^{-1 / 2}\right)
$$

Portanto, temos a aproximação

$$
l_{; \widehat{\psi}}(\widehat{\boldsymbol{\theta}})-l_{; \widehat{\psi}}(\widetilde{\boldsymbol{\theta}})=l_{\psi}(\psi, \widetilde{\boldsymbol{\lambda}}) \frac{i_{\psi \psi}(\widehat{\psi}, \widehat{\boldsymbol{\lambda}})^{1 / 2}}{i_{\psi \psi}(\psi, \widetilde{\boldsymbol{\lambda}})^{1 / 2}}+O_{p}\left(n^{-1 / 2}\right) .
$$

Assim, $U$ pode ser aproximado por

$$
U_{o}=l_{\psi}(\widetilde{\boldsymbol{\theta}}) \frac{\left|\boldsymbol{j}_{\boldsymbol{\lambda} \boldsymbol{\lambda}}(\widetilde{\boldsymbol{\theta}})\right|^{1 / 2} i_{\psi \psi}(\widehat{\boldsymbol{\theta}})^{1 / 2}}{|\widehat{\boldsymbol{j}}|^{1 / 2} i_{\psi \psi}(\widetilde{\boldsymbol{\theta}})^{1 / 2}}
$$


e $R^{*}$ é aproximado por

$$
R_{o}^{*}=R+\frac{1}{R} \log \left(U_{o} / R\right)
$$

De acordo com DiCiccio \& Martin (1993), em geral, $U_{o}=U+O_{p}\left(n^{-1}\right)$, assim, $R_{o}^{*}=R^{*}+O_{p}\left(n^{-1}\right)$.

Vale ressaltar que esta aproximação para $R^{*}$ apresenta uma restrição no cálculo de $U_{o}$ e, consequentemente, na obtenção de $R_{o}^{*}$. Para o seu cálculo, é necessário que o parâmetro de interesse seja ortogonal aos parâmetros de perturbação. Embora uma parametrização ortogonal sempre exista quando $\psi$ é escalar, muitas vezes resolver as equações diferenciais necessárias pode não ser trivial; veja Cox \& Reid $(1987,1993)$.

\subsubsection{Aproximações de Skovgaard e Severini}

Inicialmente, iremos apresentar a derivação de uma aproximação para

$$
\boldsymbol{l}_{; \widehat{\theta}}\left(\boldsymbol{\theta} ; \boldsymbol{\theta}_{0}, \boldsymbol{a}\right)-\boldsymbol{l}_{; \widehat{\theta}}\left(\boldsymbol{\theta}_{0} ; \boldsymbol{\theta}_{0}, \boldsymbol{a}\right)
$$

em que $\boldsymbol{\theta}_{0}$ denota um ponto específico do espaço paramétrico e $\boldsymbol{\theta}=\boldsymbol{\theta}_{0}+O\left(n^{-1 / 2}\right)$, isto é, $\boldsymbol{\theta}=$ $\boldsymbol{\theta}_{0}+n^{-1 / 2} \boldsymbol{\delta}$. Assim, fazendo $\boldsymbol{\theta}_{0}=\widehat{\boldsymbol{\theta}}$, obtemos uma aproximação para $\boldsymbol{l}_{\hat{;}}(\boldsymbol{\theta} ; \widehat{\boldsymbol{\theta}}, \boldsymbol{a})-\boldsymbol{l}_{; \widehat{\boldsymbol{\theta}}}(\widehat{\boldsymbol{\theta}} ; \widehat{\boldsymbol{\theta}}, \boldsymbol{a})=$ $\boldsymbol{l}_{; \widehat{\boldsymbol{\theta}}}(\boldsymbol{\theta})-\boldsymbol{l}_{; \widehat{\boldsymbol{\theta}}}(\widehat{\boldsymbol{\theta}})$.

Expandindo $l(\boldsymbol{\theta} ; \widehat{\boldsymbol{\theta}}, \boldsymbol{a})$ em série de Taylor em torno do ponto $\widehat{\boldsymbol{\theta}}=\boldsymbol{\theta}_{0}$, temos que

$$
l(\boldsymbol{\theta} ; \widehat{\boldsymbol{\theta}}, \boldsymbol{a})=l\left(\boldsymbol{\theta} ; \boldsymbol{\theta}_{0}, \boldsymbol{a}\right)+\left(\widehat{\boldsymbol{\theta}}-\boldsymbol{\theta}_{0}\right) \boldsymbol{l}_{\widehat{\widehat{\boldsymbol{\theta}}}}\left(\boldsymbol{\theta} ; \boldsymbol{\theta}_{0}, \boldsymbol{a}\right)^{\top}+\cdots .
$$

Da mesma forma, temos que

$$
l\left(\boldsymbol{\theta}_{0} ; \widehat{\boldsymbol{\theta}}, \boldsymbol{a}\right)=l\left(\boldsymbol{\theta}_{0} ; \boldsymbol{\theta}_{0}, \boldsymbol{a}\right)+\left(\widehat{\boldsymbol{\theta}}-\boldsymbol{\theta}_{0}\right) \boldsymbol{l}_{\widehat{\widehat{\boldsymbol{\theta}}}}\left(\boldsymbol{\theta}_{0} ; \boldsymbol{\theta}_{0}, \boldsymbol{a}\right)^{\top}+\cdots .
$$

Assim,

$$
l(\boldsymbol{\theta} ; \widehat{\boldsymbol{\theta}}, \boldsymbol{a})-l\left(\boldsymbol{\theta}_{0} ; \widehat{\boldsymbol{\theta}}, \boldsymbol{a}\right)=l\left(\boldsymbol{\theta} ; \boldsymbol{\theta}_{0}, \boldsymbol{a}\right)-l\left(\boldsymbol{\theta}_{0} ; \boldsymbol{\theta}_{0}, \boldsymbol{a}\right)+\left(\widehat{\boldsymbol{\theta}}-\boldsymbol{\theta}_{0}\right)\left[\boldsymbol{l}_{\hat{⿰} \widehat{\boldsymbol{\theta}}}\left(\boldsymbol{\theta} ; \boldsymbol{\theta}_{0}, \boldsymbol{a}\right)-\boldsymbol{l}_{; \widehat{\boldsymbol{\theta}}}\left(\boldsymbol{\theta}_{0} ; \boldsymbol{\theta}_{0}, \boldsymbol{a}\right)\right]^{\top}+\cdots
$$

Segue que,

$$
\begin{aligned}
\mathbb{E}_{\boldsymbol{\theta}_{0}}\left[l(\boldsymbol{\theta} ; \widehat{\boldsymbol{\theta}}, \boldsymbol{a})-l\left(\boldsymbol{\theta}_{0} ; \widehat{\boldsymbol{\theta}}, \boldsymbol{a}\right) \mid \boldsymbol{a}\right] & =l\left(\boldsymbol{\theta} ; \boldsymbol{\theta}_{0}, \boldsymbol{a}\right)-l\left(\boldsymbol{\theta}_{0} ; \boldsymbol{\theta}_{0}, \boldsymbol{a}\right) \\
& +\mathbb{E}_{\boldsymbol{\theta}_{0}}\left\{\left(\widehat{\boldsymbol{\theta}}-\boldsymbol{\theta}_{0}\right)\left[\boldsymbol{l}_{\hat{\boldsymbol{\theta}}}\left(\boldsymbol{\theta} ; \boldsymbol{\theta}_{0}, \boldsymbol{a}\right)-\boldsymbol{l}_{; \widehat{\boldsymbol{\theta}}}\left(\boldsymbol{\theta}_{0} ; \boldsymbol{\theta}_{0}, \boldsymbol{a}\right)\right]^{\top} \mid \boldsymbol{a}\right\}+\cdots
\end{aligned}
$$

sendo que

$$
\mathbb{E}_{\boldsymbol{\theta}_{0}}\left\{\left(\widehat{\boldsymbol{\theta}}-\boldsymbol{\theta}_{0}\right)\left[\boldsymbol{l}_{; \widehat{\boldsymbol{\theta}}}\left(\boldsymbol{\theta} ; \boldsymbol{\theta}_{0}, \boldsymbol{a}\right)-\boldsymbol{l}_{; \widehat{\boldsymbol{\theta}}}\left(\boldsymbol{\theta}_{0} ; \boldsymbol{\theta}_{0}, \boldsymbol{a}\right)\right]^{\top} \mid \boldsymbol{a}\right\}=\mathbb{E}_{\boldsymbol{\theta}_{0}}\left(\widehat{\boldsymbol{\theta}}-\boldsymbol{\theta}_{0}\right)\left[\boldsymbol{l}_{\widehat{\boldsymbol{\theta}}}\left(\boldsymbol{\theta} ; \boldsymbol{\theta}_{0}, \boldsymbol{a}\right)-\boldsymbol{l}_{; \widehat{\boldsymbol{\theta}}}\left(\boldsymbol{\theta}_{0} ; \boldsymbol{\theta}_{0}, \boldsymbol{a}\right)\right]^{\top}
$$

pois, condicionalmente em $\boldsymbol{a}$, ou seja, mantendo $\boldsymbol{a}$ fixado, $l\left(\boldsymbol{\theta} ; \boldsymbol{\theta}_{0}, \boldsymbol{a}\right)-l\left(\boldsymbol{\theta}_{0} ; \boldsymbol{\theta}_{0}, \boldsymbol{a}\right)$ e $\boldsymbol{l}_{; \widehat{\boldsymbol{\theta}}}\left(\boldsymbol{\theta} ; \boldsymbol{\theta}_{0}, \boldsymbol{a}\right)-$ $\boldsymbol{l}_{; \widehat{\boldsymbol{\theta}}}\left(\boldsymbol{\theta}_{0} ; \boldsymbol{\theta}_{0}, \boldsymbol{a}\right)$ são constantes. Adicionalmente, $\mathbb{E}_{\boldsymbol{\theta}_{0}}\left(\widehat{\boldsymbol{\theta}}-\boldsymbol{\theta}_{0}\right)$ é de ordem $O\left(n^{-1}\right)$ e $\boldsymbol{l}_{; \widehat{\boldsymbol{\theta}}}\left(\boldsymbol{\theta} ; \boldsymbol{\theta}_{0}, \boldsymbol{a}\right)-$ $\boldsymbol{l}_{; \widehat{\boldsymbol{\theta}}}\left(\boldsymbol{\theta}_{0} ; \boldsymbol{\theta}_{0}, \boldsymbol{a}\right)$ é de ordem $O\left(n^{1 / 2}\right)$, já que esta diferença pode ser expandida da forma

$$
\boldsymbol{l}_{; \widehat{\boldsymbol{\theta}}}\left(\boldsymbol{\theta} ; \boldsymbol{\theta}_{0}, \boldsymbol{a}\right)-\boldsymbol{l}_{\hat{;} \boldsymbol{\theta}}\left(\boldsymbol{\theta}_{0} ; \boldsymbol{\theta}_{0}, \boldsymbol{a}\right)=\left(\boldsymbol{\theta}-\boldsymbol{\theta}_{0}\right) \boldsymbol{l}_{\boldsymbol{\theta} ; \widehat{\boldsymbol{\theta}}}\left(\boldsymbol{\theta}_{0} ; \widehat{\boldsymbol{\theta}}, \boldsymbol{a}\right)+\cdots=\left(\boldsymbol{\theta}-\boldsymbol{\theta}_{0}\right) \boldsymbol{j}\left(\boldsymbol{\theta}_{0}\right)+\cdots
$$

e como, em geral, $\boldsymbol{j}\left(\boldsymbol{\theta}_{0}\right)$ (matriz de informação observada) é de ordem $O(n)$, segue que $\boldsymbol{l}_{; \widehat{\boldsymbol{\theta}}}\left(\boldsymbol{\theta} ; \boldsymbol{\theta}_{0}, \boldsymbol{a}\right)-$ 
$\boldsymbol{l}_{; \widehat{\boldsymbol{\theta}}}\left(\boldsymbol{\theta}_{0} ; \boldsymbol{\theta}_{0}, \boldsymbol{a}\right)$ é de ordem $O\left(n^{1 / 2}\right)$. Estamos assumindo que $\boldsymbol{\theta}=\boldsymbol{\theta}_{0}+O\left(n^{-1 / 2}\right)$. Assim,

$$
\mathbb{E}_{\boldsymbol{\theta}_{0}}\left(\widehat{\boldsymbol{\theta}}-\boldsymbol{\theta}_{0}\right)\left[\boldsymbol{l}_{\widehat{;}}\left(\boldsymbol{\theta} ; \boldsymbol{\theta}_{0}, \boldsymbol{a}\right)-\boldsymbol{l}_{; \widehat{\boldsymbol{\theta}}}\left(\boldsymbol{\theta}_{0} ; \boldsymbol{\theta}_{0}, \boldsymbol{a}\right)\right]^{\top}=O\left(n^{-1 / 2}\right)
$$

Deste resultado, segue que

$$
\mathbb{E}_{\boldsymbol{\theta}_{0}}\left[l(\boldsymbol{\theta} ; \widehat{\boldsymbol{\theta}}, \boldsymbol{a})-l\left(\boldsymbol{\theta}_{0} ; \widehat{\boldsymbol{\theta}}, \boldsymbol{a}\right) \mid \boldsymbol{a}\right]=l\left(\boldsymbol{\theta} ; \boldsymbol{\theta}_{0}, \boldsymbol{a}\right)-l\left(\boldsymbol{\theta}_{0} ; \boldsymbol{\theta}_{0}, \boldsymbol{a}\right)+O\left(n^{-1 / 2}\right) .
$$

Severini (1999) mostra que, dado $\boldsymbol{a}$,

$$
\begin{aligned}
{\left[\boldsymbol{l}_{; \widehat{\boldsymbol{\theta}}}\left(\boldsymbol{\theta} ; \boldsymbol{\theta}_{0}, \boldsymbol{a}\right)-\boldsymbol{l}_{; \widehat{\boldsymbol{\theta}}}\left(\boldsymbol{\theta}_{0} ; \boldsymbol{\theta}_{0}, \boldsymbol{a}\right)\right]^{\top}=} & \mathbb{E}_{\boldsymbol{\theta}_{0}}\left[\boldsymbol{l}_{\boldsymbol{\theta}}\left(\boldsymbol{\theta}_{0} ; \widehat{\boldsymbol{\theta}}, \boldsymbol{a}\right) l(\boldsymbol{\theta} ; \widehat{\boldsymbol{\theta}}, \boldsymbol{a}) \mid \boldsymbol{a}\right] \\
& -\mathbb{E}_{\boldsymbol{\theta}_{0}}\left[\boldsymbol{l}_{\boldsymbol{\theta}}\left(\boldsymbol{\theta}_{0} ; \widehat{\boldsymbol{\theta}}, \boldsymbol{a}\right) l\left(\boldsymbol{\theta}_{0} ; \widehat{\boldsymbol{\theta}}, \boldsymbol{a}\right) \mid \boldsymbol{a}\right]+O\left(n^{-1 / 2}\right) \\
= & \mathbb{E}_{\boldsymbol{\theta}_{0}}\left[\boldsymbol{l}_{\boldsymbol{\theta}}\left(\boldsymbol{\theta}_{0} ; \widehat{\boldsymbol{\theta}}, \boldsymbol{a}\right)\left\{l(\boldsymbol{\theta} ; \widehat{\boldsymbol{\theta}}, \boldsymbol{a})-l\left(\boldsymbol{\theta}_{0} ; \widehat{\boldsymbol{\theta}}, \boldsymbol{a}\right)\right\} \mid \boldsymbol{a}\right]+O\left(n^{-1 / 2}\right) \\
= & \mathbb{E}_{\boldsymbol{\theta}_{0}}\left[\boldsymbol{l}_{\boldsymbol{\theta}}\left(\boldsymbol{\theta}_{0}\right)\left\{l(\boldsymbol{\theta})-l\left(\boldsymbol{\theta}_{0}\right)\right\} \mid \boldsymbol{a}\right]+O\left(n^{-1 / 2}\right) .
\end{aligned}
$$

Expandindo $l(\boldsymbol{\theta})$ em série de Taylor em torno do ponto $\boldsymbol{\theta}=\boldsymbol{\theta}_{0}$, temos que

$$
l(\boldsymbol{\theta})-l\left(\boldsymbol{\theta}_{0}\right)=\left(\boldsymbol{\theta}-\boldsymbol{\theta}_{0}\right) \boldsymbol{l}_{\boldsymbol{\theta}}\left(\boldsymbol{\theta}_{0}\right)+\cdots=\left(\frac{\boldsymbol{\delta}}{\sqrt{n}} \boldsymbol{l}_{\boldsymbol{\theta}}\left(\boldsymbol{\theta}_{0}\right)\right)^{\top}+\cdots
$$

Assim,

$$
\mathbb{E}_{\boldsymbol{\theta}_{0}}\left[\boldsymbol{l}_{\boldsymbol{\theta}}\left(\boldsymbol{\theta}_{0}\right)\left\{l(\boldsymbol{\theta})-l\left(\boldsymbol{\theta}_{0}\right)\right\} \mid \boldsymbol{a}\right]=\mathbb{E}_{\boldsymbol{\theta}_{0}}\left[\boldsymbol{l}_{\boldsymbol{\theta}}\left(\boldsymbol{\theta}_{0}\right) \boldsymbol{l}_{\boldsymbol{\theta}}\left(\boldsymbol{\theta}_{0}\right)^{\top} \mid \boldsymbol{a}\right] \frac{\boldsymbol{\delta}^{\top}}{\sqrt{n}}+\cdots
$$

Adicionalmente,

$$
\mathbb{E}_{\boldsymbol{\theta}_{0}}\left[\boldsymbol{l}_{\boldsymbol{\theta}}\left(\boldsymbol{\theta}_{0}\right)\left\{l(\boldsymbol{\theta})-l\left(\boldsymbol{\theta}_{0}\right)\right\}\right]=\mathbb{E}_{\boldsymbol{\theta}_{0}}\left[\boldsymbol{l}_{\boldsymbol{\theta}}\left(\boldsymbol{\theta}_{0}\right) \boldsymbol{l}_{\boldsymbol{\theta}}\left(\boldsymbol{\theta}_{0}\right)^{\top}\right] \frac{\boldsymbol{\delta}^{\top}}{\sqrt{n}}+\cdots
$$

Assim, temos que

$$
\begin{aligned}
\mathbb{E}_{\boldsymbol{\theta}_{0}}\left[\boldsymbol{l}_{\boldsymbol{\theta}}\left(\boldsymbol{\theta}_{0}\right)\left\{l(\boldsymbol{\theta})-l\left(\boldsymbol{\theta}_{0}\right)\right\} \mid \boldsymbol{a}\right]-\mathbb{E}_{\boldsymbol{\theta}_{0}}\left[\boldsymbol{l}_{\boldsymbol{\theta}}\left(\boldsymbol{\theta}_{0}\right)\left\{l(\boldsymbol{\theta})-l\left(\boldsymbol{\theta}_{0}\right)\right\}\right] \\
=\mathbb{E}_{\boldsymbol{\theta}_{0}}\left[\boldsymbol{l}_{\boldsymbol{\theta}}\left(\boldsymbol{\theta}_{0}\right) \boldsymbol{l}_{\boldsymbol{\theta}}\left(\boldsymbol{\theta}_{0}\right)^{\top} \mid \boldsymbol{a}\right] \frac{\boldsymbol{\delta}^{\top}}{\sqrt{n}}-\mathbb{E}_{\boldsymbol{\theta}_{0}}\left[\boldsymbol{l}_{\boldsymbol{\theta}}\left(\boldsymbol{\theta}_{0}\right) \boldsymbol{l}_{\boldsymbol{\theta}}\left(\boldsymbol{\theta}_{0}\right)^{\top}\right] \frac{\boldsymbol{\delta}^{\top}}{\sqrt{n}}+O\left(n^{-1 / 2}\right) \\
=\left\{\mathbb{E}_{\boldsymbol{\theta}_{0}}\left[\boldsymbol{l}_{\boldsymbol{\theta}}\left(\boldsymbol{\theta}_{0}\right) \boldsymbol{l}_{\boldsymbol{\theta}}\left(\boldsymbol{\theta}_{0}\right)^{\top} \mid \boldsymbol{a}\right]-\mathbb{E}_{\boldsymbol{\theta}_{0}}\left[\boldsymbol{l}_{\boldsymbol{\theta}}\left(\boldsymbol{\theta}_{0}\right) \boldsymbol{l}_{\boldsymbol{\theta}}\left(\boldsymbol{\theta}_{0}\right)^{\top}\right]\right\} \frac{\boldsymbol{\delta}^{\top}}{\sqrt{n}}+O\left(n^{-1 / 2}\right) .
\end{aligned}
$$

Barndorff-Nielsen \& Cox $(1994, \S 6.3)$ mostram que

$$
\mathbb{E}_{\boldsymbol{\theta}_{0}}\left[\boldsymbol{l}_{\boldsymbol{\theta}}\left(\boldsymbol{\theta}_{0}\right) \boldsymbol{l}_{\boldsymbol{\theta}}\left(\boldsymbol{\theta}_{0}\right)^{\top} \mid \boldsymbol{a}\right]=-\boldsymbol{l}_{\boldsymbol{\theta} \boldsymbol{\theta}}\left(\boldsymbol{\theta}_{0} ; \boldsymbol{\theta}_{0}, \boldsymbol{a}\right)+O(1) .
$$

Adicionalmente, note que

$$
\mathbb{E}_{\boldsymbol{\theta}_{0}}\left[\boldsymbol{l}_{\boldsymbol{\theta}}\left(\boldsymbol{\theta}_{0}\right) \boldsymbol{l}_{\boldsymbol{\theta}}\left(\boldsymbol{\theta}_{0}\right)^{\top}\right]=\boldsymbol{i}\left(\boldsymbol{\theta}_{0}\right),
$$

em que $\boldsymbol{i}\left(\boldsymbol{\theta}_{0}\right)$ denota a matriz de informação de Fisher avaliada em $\boldsymbol{\theta}=\boldsymbol{\theta}_{0}$. Dessa forma, temos que

$$
\begin{aligned}
\mathbb{E}_{\boldsymbol{\theta}_{0}}\left[\boldsymbol{l}_{\boldsymbol{\theta}}\left(\boldsymbol{\theta}_{0}\right)\left\{l(\boldsymbol{\theta})-l\left(\boldsymbol{\theta}_{0}\right)\right\} \mid \boldsymbol{a}\right]= & \mathbb{E}_{\boldsymbol{\theta}_{0}}\left[\boldsymbol{l}_{\boldsymbol{\theta}}\left(\boldsymbol{\theta}_{0}\right)\left\{l(\boldsymbol{\theta})-l\left(\boldsymbol{\theta}_{0}\right)\right\}\right] \\
& -\left\{\boldsymbol{l}_{\boldsymbol{\theta} \boldsymbol{\theta}}\left(\boldsymbol{\theta}_{0} ; \boldsymbol{\theta}_{0}, \boldsymbol{a}\right)+\boldsymbol{i}\left(\boldsymbol{\theta}_{0}\right)\right\} \frac{\boldsymbol{\delta}^{\top}}{\sqrt{n}}+O\left(n^{-1 / 2}\right) .
\end{aligned}
$$


Seja o vetor linha $k$-dimensional

$$
\boldsymbol{Q}\left(\boldsymbol{\theta} ; \boldsymbol{\theta}_{0}\right)=\mathbb{E}_{\boldsymbol{\theta}_{0}}\left[l(\boldsymbol{\theta}) \boldsymbol{l}_{\boldsymbol{\theta}}\left(\boldsymbol{\theta}_{0}\right)^{\top}\right] .
$$

Então, (4.15) pode ser escrito na forma

$$
\begin{aligned}
\mathbb{E}_{\boldsymbol{\theta}_{0}}\left[\boldsymbol{l}_{\boldsymbol{\theta}}\left(\boldsymbol{\theta}_{0}\right)\left\{l(\boldsymbol{\theta})-l\left(\boldsymbol{\theta}_{0}\right)\right\} \mid \boldsymbol{a}\right]= & {\left[\boldsymbol{Q}\left(\boldsymbol{\theta} ; \boldsymbol{\theta}_{0}\right)-\boldsymbol{Q}\left(\boldsymbol{\theta}_{0} ; \boldsymbol{\theta}_{0}\right)\right]^{\top} } \\
& -\left\{\boldsymbol{l}_{\boldsymbol{\theta} \boldsymbol{\theta}}\left(\boldsymbol{\theta}_{0} ; \boldsymbol{\theta}_{0}, \boldsymbol{a}\right)+\boldsymbol{i}\left(\boldsymbol{\theta}_{0}\right)\right\} \frac{\boldsymbol{\delta}^{\top}}{\sqrt{n}}+O\left(n^{-1 / 2}\right) .
\end{aligned}
$$

Note que, como $l(\boldsymbol{\theta})-l\left(\boldsymbol{\theta}_{0}\right)=\left(\boldsymbol{\theta}-\boldsymbol{\theta}_{0}\right) \boldsymbol{l}_{\boldsymbol{\theta}}\left(\boldsymbol{\theta}_{0}\right)+O_{p}(1)$, temos que

$$
\begin{aligned}
{\left[\boldsymbol{Q}(\boldsymbol{\theta} ; \boldsymbol{\theta})-\boldsymbol{Q}\left(\boldsymbol{\theta} ; \boldsymbol{\theta}_{0}\right)\right]^{\top} } & =\mathbb{E}_{\boldsymbol{\theta}_{0}}\left[\left\{l(\boldsymbol{\theta})-l\left(\boldsymbol{\theta}_{0}\right)\right\} \boldsymbol{l}_{\boldsymbol{\theta}}\left(\boldsymbol{\theta}_{0}\right)^{\top}\right]^{\top}=\mathbb{E}_{\boldsymbol{\theta}_{0}}\left[\left(\boldsymbol{\theta}-\boldsymbol{\theta}_{0}\right) \boldsymbol{l}_{\boldsymbol{\theta}}\left(\boldsymbol{\theta}_{0}\right) \boldsymbol{l}_{\boldsymbol{\theta}}\left(\boldsymbol{\theta}_{0}\right)^{\top}\right]^{\top}+O(1) \\
& =\mathbb{E}_{\boldsymbol{\theta}_{0}}\left[\boldsymbol{l}_{\boldsymbol{\theta}}\left(\boldsymbol{\theta}_{0}\right) \boldsymbol{l}_{\boldsymbol{\theta}}\left(\boldsymbol{\theta}_{0}\right)^{\top}\right]^{\top}\left(\boldsymbol{\theta}-\boldsymbol{\theta}_{0}\right)^{\top}+O(1)=\boldsymbol{i}\left(\boldsymbol{\theta}_{0}\right)\left(\boldsymbol{\theta}-\boldsymbol{\theta}_{0}\right)^{\top}+O(1) .
\end{aligned}
$$

Daí, segue que

$$
\left(\boldsymbol{\theta}-\boldsymbol{\theta}_{0}\right)^{\top}=\boldsymbol{i}\left(\boldsymbol{\theta}_{0}\right)^{-1}\left[\boldsymbol{Q}\left(\boldsymbol{\theta} ; \boldsymbol{\theta}_{0}\right)-\boldsymbol{Q}\left(\boldsymbol{\theta}_{0} ; \boldsymbol{\theta}_{0}\right)\right]^{\top}+O\left(n^{-1}\right) .
$$

Assim, notando que $\boldsymbol{\delta}^{\top} / \sqrt{n}=\left(\boldsymbol{\theta}-\boldsymbol{\theta}_{0}\right)^{\top}$, a equação (4.16) pode ser escrita na forma

$$
\mathbb{E}_{\boldsymbol{\theta}_{0}}\left[\boldsymbol{l}_{\boldsymbol{\theta}}\left(\boldsymbol{\theta}_{0}\right)\left\{l(\boldsymbol{\theta})-l\left(\boldsymbol{\theta}_{0}\right)\right\} \mid \boldsymbol{a}\right]=-\boldsymbol{l}_{\boldsymbol{\theta} \boldsymbol{\theta}}\left(\boldsymbol{\theta}_{0} ; \boldsymbol{\theta}_{0}, \boldsymbol{a}\right) \boldsymbol{i}\left(\boldsymbol{\theta}_{0}\right)^{-1}\left[\boldsymbol{Q}\left(\boldsymbol{\theta} ; \boldsymbol{\theta}_{0}\right)-\boldsymbol{Q}\left(\boldsymbol{\theta}_{0} ; \boldsymbol{\theta}_{0}\right)\right]^{\top}+O\left(n^{-1 / 2}\right) .
$$

Portanto,

$$
\begin{aligned}
\boldsymbol{l}_{; \widehat{\boldsymbol{\theta}}}\left(\boldsymbol{\theta} ; \boldsymbol{\theta}_{0}, \boldsymbol{a}\right)-\boldsymbol{l}_{; \widehat{\boldsymbol{\theta}}}\left(\boldsymbol{\theta}_{0} ; \boldsymbol{\theta}_{0}, \boldsymbol{a}\right) & =\left[\boldsymbol{Q}\left(\boldsymbol{\theta} ; \boldsymbol{\theta}_{0}\right)-\boldsymbol{Q}\left(\boldsymbol{\theta}_{0} ; \boldsymbol{\theta}_{0}\right)\right] \boldsymbol{i}\left(\boldsymbol{\theta}_{0}\right)^{-1}\left[-\boldsymbol{l}_{\boldsymbol{\theta} \boldsymbol{\theta}}\left(\boldsymbol{\theta}_{0} ; \boldsymbol{\theta}_{0}, \boldsymbol{a}\right)\right]+O\left(n^{-1 / 2}\right) \\
& =\left[\boldsymbol{Q}\left(\boldsymbol{\theta} ; \boldsymbol{\theta}_{0}\right)-\boldsymbol{Q}\left(\boldsymbol{\theta}_{0} ; \boldsymbol{\theta}_{0}\right)\right] \boldsymbol{i}\left(\boldsymbol{\theta}_{0}\right)^{-1} \boldsymbol{j}_{\boldsymbol{\theta} \boldsymbol{\theta}}\left(\boldsymbol{\theta}_{0}\right)+O\left(n^{-1 / 2}\right) .
\end{aligned}
$$

Fazendo $\boldsymbol{\theta}_{0}=\widehat{\boldsymbol{\theta}}$, segue que $\boldsymbol{l}_{; \widehat{\boldsymbol{\theta}}}(\widehat{\boldsymbol{\theta}})-\boldsymbol{l}_{; \widehat{\boldsymbol{\theta}}}(\boldsymbol{\theta})$ pode ser aproximado por

$$
\overline{\boldsymbol{l}}_{; \widehat{\boldsymbol{\theta}}}(\widehat{\boldsymbol{\theta}} ; \widehat{\boldsymbol{\theta}}, \boldsymbol{a})-\overline{\boldsymbol{l}}_{; \widehat{\boldsymbol{\theta}}}(\boldsymbol{\theta} ; \widehat{\boldsymbol{\theta}}, \boldsymbol{a})=\overline{\boldsymbol{l}}_{; \widehat{\boldsymbol{\theta}}}(\widehat{\boldsymbol{\theta}})-\overline{\boldsymbol{l}}_{; \widehat{\boldsymbol{\theta}}}(\boldsymbol{\theta})=[\boldsymbol{Q}(\widehat{\boldsymbol{\theta}} ; \widehat{\boldsymbol{\theta}})-\boldsymbol{Q}(\boldsymbol{\theta} ; \widehat{\boldsymbol{\theta}})] \boldsymbol{i}(\widehat{\boldsymbol{\theta}})^{-1} \boldsymbol{j}_{\boldsymbol{\theta} \boldsymbol{\theta}}(\widehat{\boldsymbol{\theta}}) \mathrm{.}
$$

Note que podemos escrever $\overline{\boldsymbol{l}}_{; \widehat{\boldsymbol{\theta}}}(\widehat{\boldsymbol{\theta}})-\overline{\boldsymbol{l}}_{; \widehat{\boldsymbol{\theta}}}(\boldsymbol{\theta})$ em $(4.17)$ como

$$
\overline{\boldsymbol{l}}_{; \widehat{\boldsymbol{\theta}}}(\widehat{\boldsymbol{\theta}})-\overline{\boldsymbol{l}}_{; \widehat{\boldsymbol{\theta}}}(\boldsymbol{\theta})=(\widehat{\boldsymbol{\theta}}-\boldsymbol{\theta}) \boldsymbol{j}_{\boldsymbol{\theta} \boldsymbol{\theta}}(\widehat{\boldsymbol{\theta}})+O_{p}\left(n^{-1 / 2}\right),
$$

uma vez que $\boldsymbol{Q}(\boldsymbol{\theta} ; \widehat{\boldsymbol{\theta}})=\boldsymbol{Q}(\widehat{\boldsymbol{\theta}} ; \widehat{\boldsymbol{\theta}})+(\boldsymbol{\theta}-\widehat{\boldsymbol{\theta}}) \boldsymbol{Q}^{\prime}(\widehat{\boldsymbol{\theta}} ; \widehat{\boldsymbol{\theta}})+\cdots=\boldsymbol{Q}(\widehat{\boldsymbol{\theta}} ; \widehat{\boldsymbol{\theta}})+(\boldsymbol{\theta}-\widehat{\boldsymbol{\theta}}) \boldsymbol{i}(\widehat{\boldsymbol{\theta}})+\cdots$, pois $\boldsymbol{Q}^{\prime}(\widehat{\boldsymbol{\theta}} ; \widehat{\boldsymbol{\theta}})=$ $\boldsymbol{i}(\widehat{\boldsymbol{\theta}})$, sendo $\boldsymbol{Q}^{\prime}\left(\boldsymbol{\theta} ; \boldsymbol{\theta}_{0}\right)=\partial \boldsymbol{Q}\left(\boldsymbol{\theta} ; \boldsymbol{\theta}_{0}\right) / \partial \boldsymbol{\theta}^{\top}$, isto é, $\boldsymbol{Q}(\boldsymbol{\theta} ; \widehat{\boldsymbol{\theta}})-\boldsymbol{Q}(\widehat{\boldsymbol{\theta}} ; \widehat{\boldsymbol{\theta}})=(\boldsymbol{\theta}-\widehat{\boldsymbol{\theta}}) \boldsymbol{i}(\widehat{\boldsymbol{\theta}})+O_{p}(1)$. Assim, comparando com a equação (4.11), temos que

$$
\overline{\boldsymbol{l}}_{; \widehat{\boldsymbol{\theta}}}(\widehat{\boldsymbol{\theta}})-\overline{\boldsymbol{l}}_{; \widehat{\boldsymbol{\theta}}}(\boldsymbol{\theta})=\boldsymbol{l}_{; \widehat{\boldsymbol{\theta}}}(\widehat{\boldsymbol{\theta}})-\boldsymbol{l}_{; \widehat{\boldsymbol{\theta}}}(\boldsymbol{\theta})+O_{p}(1)
$$

Adicionalmente, como estamos supondo que $\widehat{\boldsymbol{\theta}}-\boldsymbol{\theta}=O_{p}\left(n^{-1 / 2}\right)$, temos que $\boldsymbol{l}_{; \widehat{\boldsymbol{\theta}}}(\widehat{\boldsymbol{\theta}})-\boldsymbol{l}_{; \widehat{\boldsymbol{\theta}}}(\boldsymbol{\theta})$ é de ordem $O_{p}\left(n^{1 / 2}\right)$ [veja equação (4.11)]. Assim, podemos escrever

$$
\overline{\boldsymbol{l}}_{; \widehat{\boldsymbol{\theta}}}(\widehat{\boldsymbol{\theta}})-\overline{\boldsymbol{l}}_{; \widehat{\boldsymbol{\theta}}}(\boldsymbol{\theta})=\left[\boldsymbol{l}_{; \widehat{\boldsymbol{\theta}}}(\widehat{\boldsymbol{\theta}})-\boldsymbol{l}_{; \widehat{\boldsymbol{\theta}}}(\boldsymbol{\theta})\right]\left\{1+O_{p}\left(n^{-1 / 2}\right)\right\} .
$$


Uma aproximação similar pode ser desenvolvida para $\boldsymbol{l}_{\boldsymbol{\theta} ; \hat{\boldsymbol{\theta}}}\left(\boldsymbol{\theta} ; \boldsymbol{\theta}_{0}, \boldsymbol{a}\right)$. Seja

$$
\boldsymbol{I}\left(\boldsymbol{\theta} ; \boldsymbol{\theta}_{0}\right)=\mathbb{E}_{\boldsymbol{\theta}_{0}}\left[\boldsymbol{l}_{\boldsymbol{\theta}}(\boldsymbol{\theta}) \boldsymbol{l}_{\boldsymbol{\theta}}\left(\boldsymbol{\theta}_{0}\right)^{\top}\right] .
$$

Expandindo $\boldsymbol{l}_{\boldsymbol{\theta} ; \widehat{\boldsymbol{\theta}}}(\boldsymbol{\theta} ; \widehat{\boldsymbol{\theta}}, \boldsymbol{a})$ e $\boldsymbol{l}_{\boldsymbol{\theta} ; \widehat{\boldsymbol{\theta}}}\left(\boldsymbol{\theta}_{0} ; \widehat{\boldsymbol{\theta}}, \boldsymbol{a}\right)$ em série de Taylor em torno do ponto $\widehat{\boldsymbol{\theta}}=\boldsymbol{\theta}_{0}$, e procedendo da mesma forma que antes, pode ser mostrado que (Severini, 1999)

$$
\boldsymbol{l}_{\boldsymbol{\theta} ; \hat{\boldsymbol{\theta}}}\left(\boldsymbol{\theta} ; \boldsymbol{\theta}_{0}, \boldsymbol{a}\right)=\boldsymbol{l}_{\boldsymbol{\theta} ; \hat{\boldsymbol{\theta}}}\left(\boldsymbol{\theta}_{0} ; \boldsymbol{\theta}_{0}, \boldsymbol{a}\right)+\boldsymbol{I}\left(\boldsymbol{\theta} ; \boldsymbol{\theta}_{0}\right)-\boldsymbol{I}\left(\boldsymbol{\theta}_{0} ; \boldsymbol{\theta}_{0}\right)+O(1)
$$

Note que $\boldsymbol{l}_{\boldsymbol{\theta} ; \hat{\boldsymbol{\theta}}}\left(\boldsymbol{\theta}_{0} ; \boldsymbol{\theta}_{0}, \boldsymbol{a}\right)=\boldsymbol{j}\left(\boldsymbol{\theta}_{0} ; \boldsymbol{\theta}_{0}, \boldsymbol{a}\right)=\boldsymbol{j}\left(\boldsymbol{\theta}_{0}\right)$ e $\boldsymbol{I}\left(\boldsymbol{\theta}_{0} ; \boldsymbol{\theta}_{0}\right)=\boldsymbol{i}\left(\boldsymbol{\theta}_{0} ; \boldsymbol{\theta}_{0}, \boldsymbol{a}\right)=\boldsymbol{i}\left(\boldsymbol{\theta}_{0}\right)$, assim,

$$
\boldsymbol{I}\left(\boldsymbol{\theta} ; \boldsymbol{\theta}_{0}\right)+\boldsymbol{l}_{\boldsymbol{\theta} ; \hat{\boldsymbol{\theta}}}\left(\boldsymbol{\theta}_{0} ; \boldsymbol{\theta}_{0}, \boldsymbol{a}\right)-\boldsymbol{I}\left(\boldsymbol{\theta}_{0} ; \boldsymbol{\theta}_{0}\right)=\boldsymbol{I}\left(\boldsymbol{\theta} ; \boldsymbol{\theta}_{0}\right)+\boldsymbol{j}\left(\boldsymbol{\theta}_{0}\right)-\boldsymbol{i}\left(\boldsymbol{\theta}_{0}\right) .
$$

Seja $\boldsymbol{D}$ a matriz identidade de ordem $k$, então

$$
\boldsymbol{I}\left(\boldsymbol{\theta} ; \boldsymbol{\theta}_{0}\right)+\boldsymbol{j}\left(\boldsymbol{\theta}_{0}\right)-\boldsymbol{i}\left(\boldsymbol{\theta}_{0}\right)=\boldsymbol{I}\left(\boldsymbol{\theta} ; \boldsymbol{\theta}_{0}\right)+\boldsymbol{i}\left(\boldsymbol{\theta}_{0}\right)\left\{\boldsymbol{i}\left(\boldsymbol{\theta}_{0}\right)^{-1} \boldsymbol{j}\left(\boldsymbol{\theta}_{0}\right)-\boldsymbol{D}\right\} .
$$

Como $\boldsymbol{I}\left(\boldsymbol{\theta} ; \boldsymbol{\theta}_{0}\right)=\boldsymbol{I}\left(\boldsymbol{\theta}_{0} ; \boldsymbol{\theta}_{0}\right)+\left(\boldsymbol{\theta}-\boldsymbol{\theta}_{0}\right)^{\top} \boldsymbol{I}^{\prime}\left(\boldsymbol{\theta}_{0} ; \boldsymbol{\theta}_{0}\right)+\cdots=\boldsymbol{i}\left(\boldsymbol{\theta}_{0}\right)+O\left(n^{1 / 2}\right)$, segue que

$$
\begin{aligned}
\boldsymbol{I}\left(\boldsymbol{\theta} ; \boldsymbol{\theta}_{0}\right)+\boldsymbol{j}\left(\boldsymbol{\theta}_{0}\right)-\boldsymbol{i}\left(\boldsymbol{\theta}_{0}\right) & =\boldsymbol{I}\left(\boldsymbol{\theta} ; \boldsymbol{\theta}_{0}\right)+\left[\boldsymbol{I}\left(\boldsymbol{\theta} ; \boldsymbol{\theta}_{0}\right)+O\left(n^{1 / 2}\right)\right]\left\{\boldsymbol{i}\left(\boldsymbol{\theta}_{0}\right)^{-1} \boldsymbol{j}\left(\boldsymbol{\theta}_{0}\right)-\boldsymbol{D}\right\} \\
& =\boldsymbol{I}\left(\boldsymbol{\theta} ; \boldsymbol{\theta}_{0}\right)+\boldsymbol{I}\left(\boldsymbol{\theta} ; \boldsymbol{\theta}_{0}\right)\left\{\boldsymbol{i}\left(\boldsymbol{\theta}_{0}\right)^{-1} \boldsymbol{j}\left(\boldsymbol{\theta}_{0}\right)-\boldsymbol{D}\right\}+O\left(n^{1 / 2}\right)\left\{\boldsymbol{i}\left(\boldsymbol{\theta}_{0}\right)^{-1} \boldsymbol{j}\left(\boldsymbol{\theta}_{0}\right)-\boldsymbol{D}\right\} \\
& =\boldsymbol{I}\left(\boldsymbol{\theta} ; \boldsymbol{\theta}_{0}\right) \boldsymbol{i}\left(\boldsymbol{\theta}_{0}\right)^{-1} \boldsymbol{j}\left(\boldsymbol{\theta}_{0}\right)+O(1) .
\end{aligned}
$$

Usamos o resultado: $\boldsymbol{j}\left(\boldsymbol{\theta}_{0}\right)=\boldsymbol{i}\left(\boldsymbol{\theta}_{0}\right)+O\left(n^{1 / 2}\right)$, isto implica que $\boldsymbol{i}\left(\boldsymbol{\theta}_{0}\right)^{-1} \boldsymbol{j}\left(\boldsymbol{\theta}_{0}\right)=\boldsymbol{D}+O\left(n^{-1 / 2}\right)$; veja Severini (2000, cap. 3).

Fazendo $\boldsymbol{\theta}_{0}=\widehat{\boldsymbol{\theta}}, \boldsymbol{l}_{\boldsymbol{\theta} ; \widehat{\boldsymbol{\theta}}}\left(\boldsymbol{\theta} ; \boldsymbol{\theta}_{0}, \boldsymbol{a}\right)$ pode ser aproximado por

$$
\overline{\boldsymbol{l}}_{\boldsymbol{\theta} ; \widehat{\boldsymbol{\theta}}}(\boldsymbol{\theta} ; \widehat{\boldsymbol{\theta}}, \boldsymbol{a})=\overline{\boldsymbol{l}}_{\boldsymbol{\theta} ; \widehat{\boldsymbol{\theta}}}(\boldsymbol{\theta})=\boldsymbol{I}(\boldsymbol{\theta} ; \widehat{\boldsymbol{\theta}}) \boldsymbol{i}(\widehat{\boldsymbol{\theta}})^{-1} \boldsymbol{j}(\widehat{\boldsymbol{\theta}})
$$

Note que, expandindo $\boldsymbol{I}(\boldsymbol{\theta} ; \widehat{\boldsymbol{\theta}})$, temos

$$
\boldsymbol{I}(\boldsymbol{\theta} ; \widehat{\boldsymbol{\theta}})=\boldsymbol{I}(\widehat{\boldsymbol{\theta}} ; \widehat{\boldsymbol{\theta}})+(\boldsymbol{\theta}-\widehat{\boldsymbol{\theta}})^{\top} \boldsymbol{I}^{\prime}(\widehat{\boldsymbol{\theta}} ; \widehat{\boldsymbol{\theta}})+O_{p}(1),
$$

isto é,

$$
\boldsymbol{I}(\boldsymbol{\theta} ; \widehat{\boldsymbol{\theta}}) \boldsymbol{i}(\widehat{\boldsymbol{\theta}})^{-1} \boldsymbol{j}(\widehat{\boldsymbol{\theta}})=\boldsymbol{I}(\widehat{\boldsymbol{\theta}} ; \widehat{\boldsymbol{\theta}}) \boldsymbol{i}(\widehat{\boldsymbol{\theta}})^{-1} \boldsymbol{j}(\widehat{\boldsymbol{\theta}})+(\boldsymbol{\theta}-\widehat{\boldsymbol{\theta}})^{\top} \boldsymbol{I}^{\prime}(\widehat{\boldsymbol{\theta}} ; \widehat{\boldsymbol{\theta}}) \boldsymbol{i}(\widehat{\boldsymbol{\theta}})^{-1} \boldsymbol{j}(\widehat{\boldsymbol{\theta}})+O_{p}(1) .
$$

Como $\boldsymbol{I}(\widehat{\boldsymbol{\theta}} ; \widehat{\boldsymbol{\theta}})=\boldsymbol{i}(\widehat{\boldsymbol{\theta}})$ e, de acordo com Severini $\left(2000\right.$, cap. 3), $\boldsymbol{I}^{\prime}(\widehat{\boldsymbol{\theta}} ; \widehat{\boldsymbol{\theta}})=\boldsymbol{l}_{\boldsymbol{\theta \boldsymbol { \theta }} ; \widehat{\boldsymbol{\theta}}}(\widehat{\boldsymbol{\theta}})+O_{p}\left(n^{1 / 2}\right)$ e $\boldsymbol{j}(\widehat{\boldsymbol{\theta}})=\boldsymbol{i}(\widehat{\boldsymbol{\theta}})+O_{p}\left(n^{1 / 2}\right)$, segue que

$$
\boldsymbol{I}(\boldsymbol{\theta} ; \widehat{\boldsymbol{\theta}}) \boldsymbol{i}(\widehat{\boldsymbol{\theta}})^{-1} \boldsymbol{j}(\widehat{\boldsymbol{\theta}})=\boldsymbol{j}(\widehat{\boldsymbol{\theta}})+(\boldsymbol{\theta}-\widehat{\boldsymbol{\theta}})^{\top} \boldsymbol{l}_{\boldsymbol{\theta} \boldsymbol{\theta} ; \widehat{\boldsymbol{\theta}}}(\widehat{\boldsymbol{\theta}})+O_{p}(1) .
$$

Comparando esta expressão com a equação (4.12), temos que

$$
\overline{\boldsymbol{l}}_{\boldsymbol{\theta} ; \widehat{\boldsymbol{\theta}}}(\boldsymbol{\theta})=\boldsymbol{l}_{\boldsymbol{\theta} ; \widehat{\boldsymbol{\theta}}}(\boldsymbol{\theta})+O_{p}(1) .
$$


Adicionalmente, como $\boldsymbol{l}_{\boldsymbol{\theta} ; \widehat{\boldsymbol{\theta}}}(\boldsymbol{\theta})$ é de ordem $O_{p}(n)$, podemos escrever a expressão anterior da forma

$$
\overline{\boldsymbol{l}}_{\boldsymbol{\theta} ; \widehat{\boldsymbol{\theta}}}(\boldsymbol{\theta})=\boldsymbol{l}_{\boldsymbol{\theta} ; \widehat{\boldsymbol{\theta}}}(\boldsymbol{\theta})\left\{1+O_{p}\left(n^{-1}\right)\right\}
$$

Se $\bar{U}$ denota a estatística $U$ [equação (4.7)] baseada nas aproximações (4.17) e (4.18), a estatística $R^{*}$ pode ser aproximada por

$$
\bar{R}^{*}=R+\frac{1}{R} \log (\bar{U} / R)
$$

De acordo com Skovgaard (1996), $\bar{R}^{*}=R^{*}+O_{p}\left(n^{-1}\right)$; veja também Severini (2000, cap. 7).

As aproximações (4.17) e (4.18) foram derivadas primeiramente em Skovgaard (1996) usando um argumento geométrico com base em um modelo da família exponencial que pode ser usado para aproximar um modelo mais geral. Uma derivação alternativa deste resultado, que foi apresentada aqui, é devida a Severini $(1998,1999)$. Vale ressaltar que estas aproximações são, basicamente, baseadas em covariâncias, por exemplo: $\boldsymbol{I}\left(\boldsymbol{\theta} ; \boldsymbol{\theta}_{0}\right)=\mathbb{E}_{\boldsymbol{\theta}_{0}}\left[\boldsymbol{l}_{\boldsymbol{\theta}}(\boldsymbol{\theta}) \boldsymbol{l}_{\boldsymbol{\theta}}\left(\boldsymbol{\theta}_{0}\right)^{\top}\right]$ é a covariância entre $\boldsymbol{l}_{\boldsymbol{\theta}}(\boldsymbol{\theta})$ e $\boldsymbol{l}_{\boldsymbol{\theta}}\left(\boldsymbol{\theta}_{0}\right)$, já que $\mathbb{E}_{\boldsymbol{\theta}_{0}}\left[\boldsymbol{l}_{\boldsymbol{\theta}}\left(\boldsymbol{\theta}_{0}\right)\right]=\mathbf{0}$. Deste fato, a aproximação denomina-se, algumas vezes, aproximação baseada em covariâncias.

Severini (1999) apresenta uma aproximação alternativa para $R^{*}, \widehat{R}^{*}$, em que as esperanças matemáticas com relação à distribuição com parâmetro $\boldsymbol{\theta}_{0}$, usadas para obter (4.17) e (4.18), são substituídas pelas esperanças com relação à distribuição empírica dos dados. Esta aproximação é motivada pelo fato de que muitas vezes não é possível encontrar fórmulas explícitas para as esperanças $\boldsymbol{Q}\left(\boldsymbol{\theta} ; \boldsymbol{\theta}_{0}\right)=\mathbb{E}_{\boldsymbol{\theta}_{0}}\left[l(\boldsymbol{\theta}) \boldsymbol{l}_{\boldsymbol{\theta}}\left(\boldsymbol{\theta}_{0}\right)^{\top}\right]$ e $\boldsymbol{I}\left(\boldsymbol{\theta} ; \boldsymbol{\theta}_{0}\right)=\mathbb{E}_{\boldsymbol{\theta}_{0}}\left[\boldsymbol{l}_{\boldsymbol{\theta}}(\boldsymbol{\theta}) \boldsymbol{l}_{\boldsymbol{\theta}}\left(\boldsymbol{\theta}_{0}\right)^{\top}\right]$.

Suponha $n$ observações independentes. Se $l^{(j)}(\boldsymbol{\theta})$ denota a função de log-verossimilhança com base apenas na $j$-ésima observação, podemos escrever a função de log-verossimilhança total na forma $l(\boldsymbol{\theta})=\sum_{j=1}^{n} l^{(j)}(\boldsymbol{\theta})$. Note que $\boldsymbol{Q}\left(\boldsymbol{\theta} ; \boldsymbol{\theta}_{0}\right)$ pode ser escrito como

$$
\boldsymbol{Q}\left(\boldsymbol{\theta} ; \boldsymbol{\theta}_{0}\right)=\mathbb{E}_{\boldsymbol{\theta}_{0}}\left\{\sum l^{(j)}(\boldsymbol{\theta}) \boldsymbol{l}_{\boldsymbol{\theta}}^{(j)}\left(\boldsymbol{\theta}_{0}\right)^{\top}\right\}
$$

Quando $\boldsymbol{\theta}_{0}$ é o verdadeiro valor do parâmetro, temos que

$$
\frac{1}{n} \sum l^{(j)}(\boldsymbol{\theta}) \boldsymbol{l}_{\boldsymbol{\theta}}^{(j)}\left(\boldsymbol{\theta}_{0}\right)^{\top}=\frac{1}{n} \mathbb{E}_{\boldsymbol{\theta}_{0}}\left\{\sum l^{(j)}(\boldsymbol{\theta}) \boldsymbol{l}_{\boldsymbol{\theta}}^{(j)}\left(\boldsymbol{\theta}_{0}\right)^{\top}\right\}+O_{p}\left(n^{-1 / 2}\right),
$$

ou seja, a média empírica converge para o seu valor esperado. Assim, $\boldsymbol{Q}\left(\boldsymbol{\theta} ; \boldsymbol{\theta}_{0}\right)$ pode ser aproximado por

$$
\widehat{\boldsymbol{Q}}\left(\boldsymbol{\theta} ; \boldsymbol{\theta}_{0}\right)=\sum l^{(j)}(\boldsymbol{\theta}) \boldsymbol{l}_{\boldsymbol{\theta}}^{(j)}\left(\boldsymbol{\theta}_{0}\right)^{\top} .
$$

De forma similar, $\boldsymbol{I}\left(\boldsymbol{\theta} ; \boldsymbol{\theta}_{0}\right)$ pode ser aproximado por

$$
\widehat{\boldsymbol{I}}\left(\boldsymbol{\theta} ; \boldsymbol{\theta}_{0}\right)=\sum \boldsymbol{l}_{\boldsymbol{\theta}}^{(j)}(\boldsymbol{\theta}) \boldsymbol{l}_{\boldsymbol{\theta}}^{(j)}\left(\boldsymbol{\theta}_{0}\right)^{\top} .
$$

$\operatorname{Sejam} \widehat{\boldsymbol{i}}(\widehat{\boldsymbol{\theta}})=\widehat{\boldsymbol{I}}(\widehat{\boldsymbol{\theta}} ; \widehat{\boldsymbol{\theta}})$,

$$
\widehat{\boldsymbol{l}}_{; \widehat{\boldsymbol{\theta}}}(\boldsymbol{\theta})=\widehat{\boldsymbol{Q}}(\boldsymbol{\theta} ; \widehat{\boldsymbol{\theta}}) \widehat{\boldsymbol{i}}(\widehat{\boldsymbol{\theta}})^{-1} \widehat{\boldsymbol{j}} \quad \mathrm{e} \quad \widehat{\boldsymbol{l}}_{; \widehat{\boldsymbol{\theta}}}(\widehat{\boldsymbol{\theta}})=\widehat{\boldsymbol{Q}}(\widehat{\boldsymbol{\theta}} ; \widehat{\boldsymbol{\theta}}) \widehat{\boldsymbol{i}}(\widehat{\boldsymbol{\theta}})^{-1} \widehat{\boldsymbol{j}}
$$


Note que

$$
\left[\widehat{\boldsymbol{l}}_{; \widehat{\boldsymbol{\theta}}}(\boldsymbol{\theta})-\widehat{\boldsymbol{l}}_{; \widehat{\boldsymbol{\theta}}}(\widehat{\boldsymbol{\theta}})\right]^{\top}=\widehat{\boldsymbol{j} \boldsymbol{i}}(\widehat{\boldsymbol{\theta}})^{-1}[\widehat{\boldsymbol{Q}}(\boldsymbol{\theta} ; \widehat{\boldsymbol{\theta}})-\widehat{\boldsymbol{Q}}(\widehat{\boldsymbol{\theta}} ; \widehat{\boldsymbol{\theta}})]^{\top}
$$

Expandindo $\widehat{\boldsymbol{Q}}(\boldsymbol{\theta} ; \widehat{\boldsymbol{\theta}})$ em série de Taylor em torno do ponto $\boldsymbol{\theta}=\widehat{\boldsymbol{\theta}}$, temos que

$$
\widehat{\boldsymbol{Q}}(\boldsymbol{\theta} ; \widehat{\boldsymbol{\theta}})=\widehat{\boldsymbol{Q}}(\widehat{\boldsymbol{\theta}} ; \widehat{\boldsymbol{\theta}})+(\boldsymbol{\theta}-\widehat{\boldsymbol{\theta}}) \widehat{\boldsymbol{Q}}^{\prime}(\widehat{\boldsymbol{\theta}} ; \widehat{\boldsymbol{\theta}})+\frac{1}{2}(\boldsymbol{\theta}-\widehat{\boldsymbol{\theta}})^{\top} \widehat{\boldsymbol{Q}}^{\prime \prime}(\widehat{\boldsymbol{\theta}} ; \widehat{\boldsymbol{\theta}})(\boldsymbol{\theta}-\widehat{\boldsymbol{\theta}})+O_{p}\left(n^{-1 / 2}\right),
$$

em que

$$
\widehat{\boldsymbol{Q}}^{\prime}\left(\boldsymbol{\theta} ; \boldsymbol{\theta}_{0}\right)=\frac{\partial \widehat{\boldsymbol{Q}}\left(\boldsymbol{\theta} ; \boldsymbol{\theta}_{0}\right)}{\partial \boldsymbol{\theta}^{\top}}=\widehat{\boldsymbol{I}}\left(\boldsymbol{\theta} ; \boldsymbol{\theta}_{0}\right) \quad \text { e } \quad \widehat{\boldsymbol{Q}}^{\prime \prime}\left(\boldsymbol{\theta} ; \boldsymbol{\theta}_{0}\right)=\frac{\partial \widehat{\boldsymbol{Q}}^{\prime}\left(\boldsymbol{\theta} ; \boldsymbol{\theta}_{0}\right)}{\partial \boldsymbol{\theta}}
$$

Dessa forma,

$$
\begin{aligned}
{\left[\widehat{\boldsymbol{l}}_{; \widehat{\boldsymbol{\theta}}}(\boldsymbol{\theta})-\widehat{\boldsymbol{l}}_{\hat{\widehat{\boldsymbol{\theta}}}}(\widehat{\boldsymbol{\theta}})\right]^{\top} } & =\widehat{\boldsymbol{j}} \widehat{\boldsymbol{i}}(\widehat{\boldsymbol{\theta}})^{-1}\left[(\boldsymbol{\theta}-\widehat{\boldsymbol{\theta}}) \widehat{\boldsymbol{Q}}^{\prime}(\widehat{\boldsymbol{\theta}} ; \widehat{\boldsymbol{\theta}})+\frac{1}{2}(\boldsymbol{\theta}-\widehat{\boldsymbol{\theta}})^{\top} \widehat{\boldsymbol{Q}}^{\prime \prime}(\widehat{\boldsymbol{\theta}} ; \widehat{\boldsymbol{\theta}})(\boldsymbol{\theta}-\widehat{\boldsymbol{\theta}})+O_{p}\left(n^{-1 / 2}\right)\right]^{\top} \\
& =\widehat{\boldsymbol{j}} \widehat{\boldsymbol{i}}(\widehat{\boldsymbol{\theta}})^{-1}\left[\widehat{\boldsymbol{Q}}^{\prime}(\widehat{\boldsymbol{\theta}} ; \widehat{\boldsymbol{\theta}})(\boldsymbol{\theta}-\widehat{\boldsymbol{\theta}})^{\top}+\frac{1}{2}(\boldsymbol{\theta}-\widehat{\boldsymbol{\theta}})^{\top} \widehat{\boldsymbol{Q}}^{\prime \prime}(\widehat{\boldsymbol{\theta}} ; \widehat{\boldsymbol{\theta}})(\boldsymbol{\theta}-\widehat{\boldsymbol{\theta}})+O_{p}\left(n^{-1 / 2}\right)\right]
\end{aligned}
$$

Observe que $\widehat{\boldsymbol{Q}}^{\prime}(\widehat{\boldsymbol{\theta}} ; \widehat{\boldsymbol{\theta}})=\widehat{\boldsymbol{I}}(\widehat{\boldsymbol{\theta}} ; \widehat{\boldsymbol{\theta}})=\widehat{\boldsymbol{i}}(\widehat{\boldsymbol{\theta}})$. Assim,

$$
\widehat{\boldsymbol{j}} \widehat{\boldsymbol{i}}(\widehat{\boldsymbol{\theta}})^{-1} \widehat{\boldsymbol{Q}}^{\prime}(\widehat{\boldsymbol{\theta}} ; \widehat{\boldsymbol{\theta}})=\widehat{\boldsymbol{j} \boldsymbol{i}}(\widehat{\boldsymbol{\theta}})^{-1} \widehat{\boldsymbol{i}}(\widehat{\boldsymbol{\theta}})=\widehat{\boldsymbol{j}}=\boldsymbol{l}_{\boldsymbol{\theta} ; \widehat{\boldsymbol{\theta}}}(\widehat{\boldsymbol{\theta}})
$$

Por outro lado, temos que

$$
\widehat{\boldsymbol{j i}}(\widehat{\boldsymbol{\theta}})^{-1} \boldsymbol{Q}^{\prime}(\widehat{\boldsymbol{\theta}} ; \widehat{\boldsymbol{\theta}})=\widehat{\boldsymbol{j} \boldsymbol{i}}(\widehat{\boldsymbol{\theta}})^{-1} \boldsymbol{i}(\widehat{\boldsymbol{\theta}})=\widehat{\boldsymbol{j}}=\boldsymbol{l}_{\boldsymbol{\theta} ; \widehat{\boldsymbol{\theta}}}(\widehat{\boldsymbol{\theta}}),
$$

ou seja, $\widehat{\boldsymbol{j} \boldsymbol{i}}(\widehat{\boldsymbol{\theta}})^{-1} \widehat{\boldsymbol{Q}}^{\prime}(\widehat{\boldsymbol{\theta}} ; \widehat{\boldsymbol{\theta}})=\widehat{\boldsymbol{j} \boldsymbol{i}}(\widehat{\boldsymbol{\theta}})^{-1} \boldsymbol{Q}^{\prime}(\widehat{\boldsymbol{\theta}} ; \widehat{\boldsymbol{\theta}})=\boldsymbol{l}_{\boldsymbol{\theta} ; \widehat{\boldsymbol{\theta}}}(\widehat{\boldsymbol{\theta}})$ exatamente. Adicionalmente, de forma análoga à expressão obtida para $\left[\widehat{\boldsymbol{l}}_{; \widehat{\boldsymbol{\theta}}}(\boldsymbol{\theta})-\widehat{\boldsymbol{l}}_{; \widehat{\boldsymbol{\theta}}}(\widehat{\boldsymbol{\theta}})\right]^{\top}$, temos que

$$
\left[\overline{\boldsymbol{l}}_{; \widehat{\boldsymbol{\theta}}}(\boldsymbol{\theta})-\overline{\boldsymbol{l}}_{; \widehat{\boldsymbol{\theta}}}(\widehat{\boldsymbol{\theta}})\right]^{\top}=\widehat{\boldsymbol{j} \boldsymbol{i}}(\widehat{\boldsymbol{\theta}})^{-1}\left[\boldsymbol{Q}^{\prime}(\widehat{\boldsymbol{\theta}} ; \widehat{\boldsymbol{\theta}})(\boldsymbol{\theta}-\widehat{\boldsymbol{\theta}})^{\top}+\frac{1}{2}(\boldsymbol{\theta}-\widehat{\boldsymbol{\theta}})^{\top} \boldsymbol{Q}^{\prime \prime}(\widehat{\boldsymbol{\theta}} ; \widehat{\boldsymbol{\theta}})(\boldsymbol{\theta}-\widehat{\boldsymbol{\theta}})+O_{p}\left(n^{-1 / 2}\right)\right]
$$

Como $\widehat{\boldsymbol{j}} \widehat{\boldsymbol{i}}(\widehat{\boldsymbol{\theta}})^{-1} \widehat{\boldsymbol{Q}}^{\prime}(\widehat{\boldsymbol{\theta}} ; \widehat{\boldsymbol{\theta}})=\widehat{\boldsymbol{j} \boldsymbol{i}}(\widehat{\boldsymbol{\theta}})^{-1} \boldsymbol{Q}^{\prime}(\widehat{\boldsymbol{\theta}} ; \widehat{\boldsymbol{\theta}})$, temos que

$$
\left[\widehat{\boldsymbol{l}}_{; \widehat{\boldsymbol{\theta}}}(\boldsymbol{\theta})-\widehat{\boldsymbol{l}}_{; \widehat{\boldsymbol{\theta}}}(\widehat{\boldsymbol{\theta}})\right]^{\top}=\left[\overline{\boldsymbol{l}}_{; \widehat{\boldsymbol{\theta}}}(\boldsymbol{\theta})-\overline{\boldsymbol{l}}_{; \widehat{\boldsymbol{\theta}}}(\widehat{\boldsymbol{\theta}})\right]^{\top}+\frac{1}{2}(\boldsymbol{\theta}-\widehat{\boldsymbol{\theta}})^{\top}\left[\widehat{\boldsymbol{Q}}^{\prime \prime}(\widehat{\boldsymbol{\theta}} ; \widehat{\boldsymbol{\theta}})-\boldsymbol{Q}^{\prime \prime}(\widehat{\boldsymbol{\theta}} ; \widehat{\boldsymbol{\theta}})\right](\boldsymbol{\theta}-\widehat{\boldsymbol{\theta}})+O_{p}\left(n^{-1 / 2}\right) .
$$

Usando o fato de que $\widehat{\boldsymbol{Q}}^{\prime \prime}(\widehat{\boldsymbol{\theta}} ; \widehat{\boldsymbol{\theta}})=\boldsymbol{Q}^{\prime \prime}(\widehat{\boldsymbol{\theta}} ; \widehat{\boldsymbol{\theta}})+O_{p}\left(n^{1 / 2}\right)$ (veja Severini, 2000, cap. 3), segue que

$$
\widehat{\boldsymbol{l}}_{; \widehat{\boldsymbol{\theta}}}(\boldsymbol{\theta})-\widehat{\boldsymbol{l}}_{; \widehat{\boldsymbol{\theta}}}(\widehat{\boldsymbol{\theta}})=\overline{\boldsymbol{l}}_{; \widehat{\boldsymbol{\theta}}}(\boldsymbol{\theta})-\overline{\boldsymbol{l}}_{; \widehat{\boldsymbol{\theta}}}(\widehat{\boldsymbol{\theta}})+O_{p}\left(n^{-1 / 2}\right) .
$$

Porém, como foi mostrado anteriormente que $\overline{\boldsymbol{l}}_{; \widehat{\boldsymbol{\theta}}}(\boldsymbol{\theta})-\overline{\boldsymbol{l}}_{; \widehat{\boldsymbol{\theta}}}(\widehat{\boldsymbol{\theta}})=\boldsymbol{l}_{; \widehat{\boldsymbol{\theta}}}(\boldsymbol{\theta})-\boldsymbol{l}_{; \widehat{\boldsymbol{\theta}}}(\widehat{\boldsymbol{\theta}})+O_{p}(1)$, segue que

$$
\widehat{\boldsymbol{l}}_{; \widehat{\boldsymbol{\theta}}}(\boldsymbol{\theta})-\widehat{\boldsymbol{l}}_{; \widehat{\boldsymbol{\theta}}}(\widehat{\boldsymbol{\theta}})=\boldsymbol{l}_{; \widehat{\boldsymbol{\theta}}}(\boldsymbol{\theta})-\boldsymbol{l}_{; \widehat{\boldsymbol{\theta}}}(\widehat{\boldsymbol{\theta}})+O_{p}(1),
$$

ou seja,

$$
\widehat{\boldsymbol{l}}_{; \widehat{\boldsymbol{\theta}}}(\boldsymbol{\theta})-\widehat{\boldsymbol{l}}_{; \widehat{\boldsymbol{\theta}}}(\widehat{\boldsymbol{\theta}})=\left[\boldsymbol{l}_{; \widehat{\boldsymbol{\theta}}}(\boldsymbol{\theta})-\boldsymbol{l}_{; \widehat{\boldsymbol{\theta}}}(\widehat{\boldsymbol{\theta}})\right]\left\{1+O_{p}\left(n^{-1 / 2}\right)\right\}
$$


Portanto, $\boldsymbol{l}_{; \widehat{\boldsymbol{\theta}}}(\widehat{\boldsymbol{\theta}})-\boldsymbol{l}_{; \widehat{\boldsymbol{\theta}}}(\boldsymbol{\theta})$ pode ser aproximado por

$$
\widehat{\boldsymbol{l}}_{; \widehat{\boldsymbol{\theta}}}(\widehat{\boldsymbol{\theta}})-\widehat{\boldsymbol{l}}_{; \widehat{\boldsymbol{\theta}}}(\boldsymbol{\theta})=[\widehat{\boldsymbol{Q}}(\widehat{\boldsymbol{\theta}} ; \widehat{\boldsymbol{\theta}})-\widehat{\boldsymbol{Q}}(\boldsymbol{\theta} ; \widehat{\boldsymbol{\theta}})] \widehat{\boldsymbol{i}}(\widehat{\boldsymbol{\theta}})^{-1} \widehat{\boldsymbol{j}}
$$

Similarmente, uma aproximação para $\boldsymbol{l}_{\boldsymbol{\theta} ; \widehat{\boldsymbol{\theta}}}(\boldsymbol{\theta})$ é dada por

$$
\widehat{\boldsymbol{l}}_{\boldsymbol{\theta} ; \widehat{\boldsymbol{\theta}}}(\boldsymbol{\theta})=\widehat{\boldsymbol{I}}(\boldsymbol{\theta} ; \widehat{\boldsymbol{\theta}}) \widehat{\boldsymbol{i}}(\widehat{\boldsymbol{\theta}})^{-1} \widehat{\boldsymbol{j}}
$$

De (4.12), temos que

$$
\boldsymbol{l}_{\boldsymbol{\theta} ; \widehat{\boldsymbol{\theta}}}(\boldsymbol{\theta})=\widehat{\boldsymbol{j}}+(\boldsymbol{\theta}-\widehat{\boldsymbol{\theta}})^{\top} \boldsymbol{l}_{\boldsymbol{\theta} \boldsymbol{\theta} ; \widehat{\boldsymbol{\theta}}}(\widehat{\boldsymbol{\theta}})+O_{p}(1) .
$$

Expandindo $\widehat{\boldsymbol{I}}(\boldsymbol{\theta} ; \widehat{\boldsymbol{\theta}})$ em série de Taylor em torno do ponto $\boldsymbol{\theta}=\widehat{\boldsymbol{\theta}}$, temos que

$$
\widehat{\boldsymbol{I}}(\boldsymbol{\theta} ; \widehat{\boldsymbol{\theta}})=\widehat{\boldsymbol{I}}(\widehat{\boldsymbol{\theta}} ; \widehat{\boldsymbol{\theta}})+(\boldsymbol{\theta}-\widehat{\boldsymbol{\theta}})^{\top} \widehat{\boldsymbol{I}}^{\prime}(\widehat{\boldsymbol{\theta}} ; \widehat{\boldsymbol{\theta}})+O_{p}(1),
$$

em que $\widehat{\boldsymbol{I}}^{\prime}\left(\boldsymbol{\theta} ; \boldsymbol{\theta}_{0}\right)=\partial \widehat{\boldsymbol{I}}\left(\boldsymbol{\theta} ; \boldsymbol{\theta}_{0}\right) / \partial \boldsymbol{\theta}$. Como $\widehat{\boldsymbol{I}}(\widehat{\boldsymbol{\theta}} ; \widehat{\boldsymbol{\theta}})=\widehat{\boldsymbol{i}}(\widehat{\boldsymbol{\theta}})$, temos

$$
\begin{aligned}
\widehat{\boldsymbol{l}}_{\boldsymbol{\theta} ; \widehat{\boldsymbol{\theta}}}(\boldsymbol{\theta}) & =\left\{\widehat{\boldsymbol{i}}(\widehat{\boldsymbol{\theta}})+(\boldsymbol{\theta}-\widehat{\boldsymbol{\theta}})^{\top} \widehat{\boldsymbol{I}}^{\prime}(\widehat{\boldsymbol{\theta}} ; \widehat{\boldsymbol{\theta}})+O_{p}(1)\right\} \widehat{\boldsymbol{i}}(\widehat{\boldsymbol{\theta}})^{-1} \widehat{\boldsymbol{j}} \\
& =\widehat{\boldsymbol{j}}+(\boldsymbol{\theta}-\widehat{\boldsymbol{\theta}})^{\top} \widehat{\boldsymbol{I}}^{\prime}(\widehat{\boldsymbol{\theta}} ; \widehat{\boldsymbol{\theta}})+O_{p}(1) .
\end{aligned}
$$

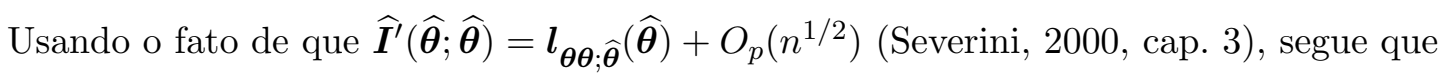

$$
\widehat{\boldsymbol{l}}_{\boldsymbol{\theta} ; \widehat{\boldsymbol{\theta}}}(\boldsymbol{\theta})=\widehat{\boldsymbol{j}}+(\boldsymbol{\theta}-\widehat{\boldsymbol{\theta}})^{\top} \boldsymbol{l}_{\boldsymbol{\theta} \boldsymbol{\theta} ; \widehat{\boldsymbol{\theta}}}(\widehat{\boldsymbol{\theta}})+O_{p}(1)
$$

ou seja, comparando com a equação (4.12), temos que $\widehat{\boldsymbol{l}}_{\boldsymbol{\theta} ; \widehat{\boldsymbol{\theta}}}(\boldsymbol{\theta})=\boldsymbol{l}_{\boldsymbol{\theta} ; \widehat{\boldsymbol{\theta}}}(\boldsymbol{\theta})+O_{p}(1)$, isto é,

$$
\widehat{\boldsymbol{l}}_{\boldsymbol{\theta} ; \widehat{\boldsymbol{\theta}}}(\boldsymbol{\theta})=\boldsymbol{l}_{\boldsymbol{\theta} ; \widehat{\boldsymbol{\theta}}}(\boldsymbol{\theta})\left\{1+O_{p}\left(n^{-1}\right)\right\}
$$

Se $\widehat{U}$ denota a estatística $U$ [equação (4.7)] baseada nas aproximações (4.19) e (4.20), a estatística $R^{*}$ pode ser aproximada por

$$
\widehat{R}^{*}=R+\frac{1}{R} \log (\widehat{U} / R)
$$

De acordo com Severini (1999), $\widehat{R}^{*}=R^{*}+O_{p}\left(n^{-1}\right)$.

\subsubsection{Aproximação de Fraser-Reid-Wu}

Assuma que a função de log-verossimilhança $l(\boldsymbol{\theta})$ depende dos dados $\boldsymbol{y}=\left(y_{1}, y_{2}, \ldots, y_{n}\right)$ somente através da estatística suficiente $(\widehat{\boldsymbol{\theta}}, \boldsymbol{a})$, em que, como antes, $\widehat{\boldsymbol{\theta}}$ é a estimativa de máxima verossimilhança de $\boldsymbol{\theta}=(\psi, \boldsymbol{\lambda})$ e $\boldsymbol{a}$ é uma estatística ancilar. Seja $\boldsymbol{\theta}=\left(\theta_{1}, \ldots, \theta_{k}\right)$ um vetor linha $k$-dimensional e assuma que $\boldsymbol{a}$ seja de dimensão $m \leq n-k$, sendo $n$ o tamanho da amostra. A função de logverossimilhança $l(\boldsymbol{\theta})$ pode ser escrita como $l(\boldsymbol{\theta} ; \boldsymbol{y})$ ou $l(\boldsymbol{\theta} ; \widehat{\boldsymbol{\theta}}, \boldsymbol{a})$. Inicialmente, vamos considerar o cálculo exato de $\boldsymbol{l}_{; \widehat{\boldsymbol{\theta}}}(\boldsymbol{\theta})$.

Usando a regra da cadeia, a derivada

$$
\boldsymbol{l}_{; \boldsymbol{y}}(\boldsymbol{\theta})=\frac{\partial l(\boldsymbol{\theta} ; \boldsymbol{y})}{\partial \boldsymbol{y}}=\frac{\partial l(\boldsymbol{\theta} ; \widehat{\boldsymbol{\theta}}, \boldsymbol{a})}{\partial \boldsymbol{y}}
$$


pode ser escrita como

$$
\boldsymbol{l}_{; \boldsymbol{y}}(\boldsymbol{\theta})=\boldsymbol{l}_{; \widehat{\boldsymbol{\theta}}}(\boldsymbol{\theta}) \frac{\partial \widehat{\boldsymbol{\theta}}^{\top}}{\partial \boldsymbol{y}}+\boldsymbol{l}_{; \boldsymbol{a}}(\boldsymbol{\theta}) \frac{\partial \boldsymbol{a}^{\top}}{\partial \boldsymbol{y}}=\left[\boldsymbol{l}_{; \widehat{\boldsymbol{\theta}}}(\boldsymbol{\theta}) \boldsymbol{l}_{; \boldsymbol{a}}(\boldsymbol{\theta})\right]\left(\begin{array}{c}
\frac{\partial \widehat{\boldsymbol{\theta}}^{\top}}{\partial \boldsymbol{y}} \\
\frac{\partial \boldsymbol{a}^{\top}}{\partial \boldsymbol{y}}
\end{array}\right)
$$

Logo,

$\boldsymbol{l}_{; \boldsymbol{y}}(\boldsymbol{\theta})\left(\begin{array}{c}\frac{\partial \widehat{\boldsymbol{\theta}}^{\top}}{\partial \boldsymbol{y}} \\ \frac{\partial \boldsymbol{a}^{\top}}{\partial \boldsymbol{y}}\end{array}\right)^{-}\left(\begin{array}{c}\frac{\partial \widehat{\boldsymbol{\theta}}^{\top}}{\partial \boldsymbol{y}} \\ \frac{\partial \boldsymbol{a}^{\top}}{\partial \boldsymbol{y}}\end{array}\right)=\left[\boldsymbol{l}_{; \hat{\boldsymbol{\theta}}}(\boldsymbol{\theta}) \boldsymbol{l}_{; \boldsymbol{a}}(\boldsymbol{\theta})\right]\left(\begin{array}{c}\frac{\partial \widehat{\boldsymbol{\theta}}^{\top}}{\partial \boldsymbol{y}} \\ \frac{\partial \boldsymbol{a}^{\top}}{\partial \boldsymbol{y}}\end{array}\right)\left(\begin{array}{c}\frac{\partial \widehat{\boldsymbol{\theta}}^{\top}}{\partial \boldsymbol{y}} \\ \frac{\partial \boldsymbol{a}^{\top}}{\partial \boldsymbol{y}}\end{array}\right)^{-}\left(\begin{array}{c}\frac{\partial \widehat{\boldsymbol{\theta}}^{\top}}{\partial \boldsymbol{y}} \\ \frac{\partial \boldsymbol{a}^{\top}}{\partial \boldsymbol{y}}\end{array}\right)=\left[\boldsymbol{l}_{; \widehat{\boldsymbol{\theta}}}(\boldsymbol{\theta}) \boldsymbol{l}_{; \boldsymbol{a}}(\boldsymbol{\theta})\right]\left(\begin{array}{c}\frac{\partial \widehat{\boldsymbol{\theta}}^{\top}}{\partial \boldsymbol{y}} \\ \frac{\partial \boldsymbol{a}^{\top}}{\partial \boldsymbol{y}}\end{array}\right)$

em que $\boldsymbol{M}^{-}$denota qualquer inversa generalizada de $\boldsymbol{M}$. [Se $\boldsymbol{M}^{-}$é inversa generalizada de $\boldsymbol{M}$, então, $\boldsymbol{M} \boldsymbol{M}^{-} \boldsymbol{M}=\boldsymbol{M}$. Além disso, se $\boldsymbol{M}$ é de dimensão $r \times s$, então $\boldsymbol{M}^{-}$tem dimensão $s \times r$; veja Rao \& Mitra (1971) para mais detalhes sobre inversas generalizadas.] Desta igualdade, temos

$$
\left[\boldsymbol{l}_{; \widehat{\boldsymbol{\theta}}}(\boldsymbol{\theta}) \boldsymbol{l}_{; \boldsymbol{a}}(\boldsymbol{\theta})\right]=\boldsymbol{l}_{; \boldsymbol{y}}(\boldsymbol{\theta})\left(\begin{array}{c}
\frac{\partial \widehat{\boldsymbol{\theta}}^{\top}}{\partial \boldsymbol{y}} \\
\frac{\partial \boldsymbol{a}^{\top}}{\partial \boldsymbol{y}}
\end{array}\right)^{-}
$$

Seja

$$
\left(\begin{array}{ll}
\boldsymbol{V}_{1} & \boldsymbol{V}_{2}
\end{array}\right)=\left(\begin{array}{c}
\frac{\partial \widehat{\boldsymbol{\theta}}^{\top}}{\partial \boldsymbol{y}} \\
\frac{\partial \boldsymbol{a}^{\top}}{\partial \boldsymbol{y}}
\end{array}\right)^{-}
$$

em que $\boldsymbol{V}_{1}$ e $\boldsymbol{V}_{2}$ são matrizes de dimensões $n \times k$ e $n \times m$, respectivamente. Então,

$$
\boldsymbol{l}_{; \widehat{\theta}}(\boldsymbol{\theta})=\boldsymbol{l}_{; \boldsymbol{y}}(\boldsymbol{\theta}) \boldsymbol{V}_{1}
$$

Note que se $\boldsymbol{V}_{1}$ for conhecido, obtém-se a derivada $\boldsymbol{l}_{\boldsymbol{\theta} ; \widehat{\boldsymbol{\theta}}}(\boldsymbol{\theta})=\partial \boldsymbol{l}_{; \widehat{\boldsymbol{\theta}}}(\boldsymbol{\theta}) / \partial \boldsymbol{\theta}^{\top}=\boldsymbol{l}_{\boldsymbol{\theta} ; \boldsymbol{y}}(\boldsymbol{\theta}) \boldsymbol{V}_{1}$. Adicionalmente, $\boldsymbol{V}_{1}$ e $\boldsymbol{V}_{2}$ satisfazem

$$
\left(\begin{array}{c}
\frac{\partial \widehat{\boldsymbol{\theta}}^{\top}}{\partial \boldsymbol{y}} \\
\frac{\partial \boldsymbol{a}^{\top}}{\partial \boldsymbol{y}}
\end{array}\right)\left(\begin{array}{ll}
\boldsymbol{V}_{1} & \boldsymbol{V}_{2}
\end{array}\right)\left(\begin{array}{c}
\frac{\partial \widehat{\boldsymbol{\theta}}^{\top}}{\partial \boldsymbol{y}} \\
\frac{\partial \boldsymbol{a}^{\top}}{\partial \boldsymbol{y}}
\end{array}\right)=\left(\begin{array}{c}
\frac{\partial \widehat{\boldsymbol{\theta}}^{\top}}{\partial \boldsymbol{y}} \\
\frac{\partial \boldsymbol{a}^{\top}}{\partial \boldsymbol{y}}
\end{array}\right),
$$

isto é,

$$
\frac{\partial \widehat{\boldsymbol{\theta}}^{\top}}{\partial \boldsymbol{y}} \boldsymbol{V}_{1} \frac{\partial \widehat{\boldsymbol{\theta}}^{\top}}{\partial \boldsymbol{y}}+\frac{\partial \widehat{\boldsymbol{\theta}}^{\top}}{\partial \boldsymbol{y}} \boldsymbol{V}_{2} \frac{\partial \boldsymbol{a}^{\top}}{\partial \boldsymbol{y}}=\frac{\partial \widehat{\boldsymbol{\theta}}^{\top}}{\partial \boldsymbol{y}} \quad \mathrm{e} \quad \frac{\partial \boldsymbol{a}^{\top}}{\partial \boldsymbol{y}} \boldsymbol{V}_{1} \frac{\partial \widehat{\boldsymbol{\theta}}^{\top}}{\partial \boldsymbol{y}}+\frac{\partial \boldsymbol{a}^{\top}}{\partial \boldsymbol{y}} \boldsymbol{V}_{2} \frac{\partial \boldsymbol{a}^{\top}}{\partial \boldsymbol{y}}=\frac{\partial \boldsymbol{a}^{\top}}{\partial \boldsymbol{y}}
$$


Suponha que $\boldsymbol{V}$ seja uma matriz de dimensão $n \times k$ que satisfaça

$$
\frac{\partial \boldsymbol{a}^{\top}}{\partial \boldsymbol{y}} \boldsymbol{V}=\{\mathbf{0}\}_{m \times k} \quad \text { e }\left|\frac{\partial \widehat{\boldsymbol{\theta}}^{\top}}{\partial \boldsymbol{y}} \boldsymbol{V}\right| \neq 0
$$

A segunda condição é equivalente a $\left(\partial \widehat{\boldsymbol{\theta}}^{\top} / \partial \boldsymbol{y}\right) \boldsymbol{V}$ ser invertível. Multiplicando ambos os lados da primeira condição de (4.21) à direita pela matriz $\boldsymbol{V}$, obtém-se

$$
\frac{\partial \widehat{\boldsymbol{\theta}}^{\top}}{\partial \boldsymbol{y}} \boldsymbol{V}_{1} \frac{\partial \widehat{\boldsymbol{\theta}}^{\top}}{\partial \boldsymbol{y}} \boldsymbol{V}=\frac{\partial \widehat{\boldsymbol{\theta}}^{\top}}{\partial \boldsymbol{y}} \boldsymbol{V}
$$

Assim, uma proposta viável para $\boldsymbol{V}_{1}$ é

$$
\boldsymbol{V}_{1}=\boldsymbol{V}\left(\frac{\partial \widehat{\boldsymbol{\theta}}^{\top}}{\partial \boldsymbol{y}} \boldsymbol{V}\right)^{-1}
$$

Substituindo esta proposta para $\boldsymbol{V}_{1}$ em (4.21), vem

$$
\frac{\partial \widehat{\boldsymbol{\theta}}^{\top}}{\partial \boldsymbol{y}} \boldsymbol{V}_{2} \frac{\partial \boldsymbol{a}^{\top}}{\partial \boldsymbol{y}}=\{\mathbf{0}\}_{k \times n} \quad \text { e } \quad \frac{\partial \boldsymbol{a}^{\top}}{\partial \boldsymbol{y}} \boldsymbol{V}_{2} \frac{\partial \boldsymbol{a}^{\top}}{\partial \boldsymbol{y}}=\frac{\partial \boldsymbol{a}^{\top}}{\partial \boldsymbol{y}} .
$$

Note que a matriz $\boldsymbol{V}_{2}$ pode ser uma inversa generalizada da matriz $\partial \boldsymbol{a}^{\top} / \partial \boldsymbol{y}(m \times n)$ satisfazendo

$$
\frac{\partial \widehat{\boldsymbol{\theta}}^{\top}}{\partial \boldsymbol{y}} \boldsymbol{V}_{2}=\{\mathbf{0}\}_{k \times m} .
$$

Tomando $\boldsymbol{V}_{1}$ e $\boldsymbol{V}_{2}$ como acima, (4.21) é satisfeito. Então,

$$
\boldsymbol{l}_{; \widehat{\boldsymbol{\theta}}}(\boldsymbol{\theta})=\boldsymbol{l}_{; \boldsymbol{y}}(\boldsymbol{\theta}) \boldsymbol{V}\left(\frac{\partial \widehat{\boldsymbol{\theta}}^{\top}}{\partial \boldsymbol{y}} \boldsymbol{V}\right)^{-1}
$$

Se é possível expressar explicitamente o vetor dos dados $\boldsymbol{y}$ em termos de $(\widehat{\boldsymbol{\theta}}, \boldsymbol{a})$, podemos tomar $\boldsymbol{V}$ da forma

$$
\boldsymbol{V}=\frac{\partial \boldsymbol{y}^{\top}}{\partial \widehat{\boldsymbol{\theta}}}
$$

Como $\widehat{\boldsymbol{\theta}}$ satisfaz $\boldsymbol{l}_{\boldsymbol{\theta}}(\widehat{\boldsymbol{\theta}} ; \boldsymbol{y})=\mathbf{0}$, isto é,

$$
\left[\frac{\partial l(\boldsymbol{\theta} ; \boldsymbol{y})}{\partial \boldsymbol{\theta}^{\top}}\right]_{\boldsymbol{\theta}=\widehat{\boldsymbol{\theta}}}=\{\boldsymbol{0}\}_{k \times 1},
$$

podemos diferenciar ambos os lados desta expressão em relação a $\boldsymbol{y}$ e avaliá-la em $\boldsymbol{\theta}=\widehat{\boldsymbol{\theta}}$. Assim, usando a regra da cadeia e pelo fato de $\widehat{\boldsymbol{\theta}}$ depender de $\boldsymbol{y}$, temos que (Fraser, Reid \& Wu, 1999)

$$
\boldsymbol{l}_{\boldsymbol{\theta} \boldsymbol{\theta}}(\widehat{\boldsymbol{\theta}}) \frac{\partial \widehat{\boldsymbol{\theta}}^{\top}}{\partial \boldsymbol{y}}+\boldsymbol{l}_{\boldsymbol{\theta} ; \boldsymbol{y}}(\widehat{\boldsymbol{\theta}})=\{\boldsymbol{0}\}_{k \times n}
$$

ou seja,

$$
\frac{\partial \widehat{\boldsymbol{\theta}}^{\top}}{\partial \boldsymbol{y}}=\left\{-\boldsymbol{l}_{\boldsymbol{\theta} \boldsymbol{\theta}}(\widehat{\boldsymbol{\theta}})\right\}^{-1} \boldsymbol{l}_{\boldsymbol{\theta} ; \boldsymbol{y}}(\widehat{\boldsymbol{\theta}})=\widehat{\boldsymbol{j}}^{-1} \boldsymbol{l}_{\boldsymbol{\theta} ; \boldsymbol{y}}(\widehat{\boldsymbol{\theta}})
$$


Assim, de (4.23) temos a expressão

$$
\boldsymbol{l}_{; \widehat{\boldsymbol{\theta}}}(\boldsymbol{\theta})=\boldsymbol{l}_{; \boldsymbol{y}}(\boldsymbol{\theta}) \boldsymbol{V}\left(\boldsymbol{l}_{\boldsymbol{\theta} ; \boldsymbol{y}}(\widehat{\boldsymbol{\theta}}) \boldsymbol{V}\right)^{-1} \widehat{\boldsymbol{j}}
$$

Diferenciando (4.24) em relação a $\boldsymbol{\theta}$, obtemos

$$
\boldsymbol{l}_{\boldsymbol{\theta} ; \widehat{\boldsymbol{\theta}}}(\boldsymbol{\theta})=\boldsymbol{l}_{\boldsymbol{\theta} ; \boldsymbol{y}}(\boldsymbol{\theta}) \boldsymbol{V}\left(\boldsymbol{l}_{\boldsymbol{\theta} ; \boldsymbol{y}}(\widehat{\boldsymbol{\theta}}) \boldsymbol{V}\right)^{-1} \widehat{\boldsymbol{j}}
$$

Note que (4.24) e (4.25) são expressões exatas, e como $\boldsymbol{l}_{\boldsymbol{y}}(\boldsymbol{\theta})$ e $\boldsymbol{l}_{\boldsymbol{\theta} ; \boldsymbol{y}}(\boldsymbol{\theta})$ são relativamente simples de serem obtidas, a determinação das derivadas com relação ao espaço amostral requer apenas a determinação da matriz $\boldsymbol{V}$.

Vale ressaltar que assumimos que $\boldsymbol{a}$ é uma estatística ancilar $m$ dimensional tal que $(\widehat{\boldsymbol{\theta}}, \boldsymbol{a})$ seja suficiente e que $m \leq n-k$. Entretanto, suponha que existe uma estatística ancilar $\boldsymbol{b}$, de dimensão arbitrária $d$, tal que $\boldsymbol{a}=g(\boldsymbol{b})$ para alguma função $g(\cdot)$. Note que, se $\boldsymbol{V}$ satisfaz

$$
\frac{\partial \boldsymbol{b}^{\top}}{\partial \boldsymbol{y}} \boldsymbol{V}=\{\boldsymbol{0}\}_{d \times k}
$$

então, pela regra da cadeia, a primeira condição de (4.22) é satisfeita. [Usando a regra da cadeia, $\partial \boldsymbol{a}^{\top} / \partial \boldsymbol{y}=\left(\partial g(\boldsymbol{b})^{\top} / \partial \boldsymbol{b}\right) \partial \boldsymbol{b}^{\top} / \partial \boldsymbol{y}$.] Assim, em (4.24) e (4.25), podemos assumir que $\boldsymbol{a}$ é de dimensão arbitrária.

Como, em geral, na maioria dos modelos estatísticos não é possível determinar uma estatística ancilar exata, a idéia é construir a matriz $\boldsymbol{V}$ com base em uma estatística aproximadamente ancilar. Seja $\widehat{\boldsymbol{a}}$ uma estatística ancilar aproximada. Suponha que $\widehat{\boldsymbol{a}}$ seja aproximadamante ancilar até ordem $n^{-1 / 2}$. Assuma ainda que, neste caso, a estatística ancilar exata $\boldsymbol{a}$ satisfaz

$$
\widehat{\boldsymbol{a}}=\boldsymbol{a}+O_{p}\left(n^{-1 / 2}\right) .
$$

Então, podemos usar a matriz

$$
\widehat{\boldsymbol{V}}=\frac{\partial \boldsymbol{y}^{\top}}{\partial \widehat{\boldsymbol{\theta}}}
$$

em que $\boldsymbol{y}$ é visto como uma função de $(\widehat{\boldsymbol{\theta}}, \widehat{\boldsymbol{a}})$ no cálculo das derivadas do espaço amostral. As expressões resultantes,

$$
\widetilde{\boldsymbol{l}}_{; \widehat{\boldsymbol{\theta}}}(\boldsymbol{\theta})=\boldsymbol{l}_{; \boldsymbol{y}}(\boldsymbol{\theta}) \widehat{\boldsymbol{V}}\left(\boldsymbol{l}_{\boldsymbol{\theta} ; \boldsymbol{y}}(\widehat{\boldsymbol{\theta}}) \widehat{\boldsymbol{V}}\right)^{-1} \widehat{\boldsymbol{j}}
$$

e

$$
\widetilde{\boldsymbol{l}}_{\boldsymbol{\theta} ; \widehat{\boldsymbol{\theta}}}(\boldsymbol{\theta})=\boldsymbol{l}_{\boldsymbol{\theta} ; \boldsymbol{y}}(\boldsymbol{\theta}) \widehat{\boldsymbol{V}}\left(\boldsymbol{l}_{\boldsymbol{\theta} ; \boldsymbol{y}}(\widehat{\boldsymbol{\theta}}) \widehat{\boldsymbol{V}}\right)^{-1} \widehat{\boldsymbol{j}},
$$

são aproximações para as derivadas exatas em relação ao espaço amostral. Adicionalmente, Fraser, Reid \& Wu (1999) mostram que

$$
\tilde{\boldsymbol{l}}_{; \widehat{\boldsymbol{\theta}}}(\widehat{\boldsymbol{\theta}})-\widetilde{\boldsymbol{l}}_{; \widehat{\boldsymbol{\theta}}}(\boldsymbol{\theta})=\left[\boldsymbol{l}_{\widehat{\boldsymbol{\theta}}}(\widehat{\boldsymbol{\theta}})-\boldsymbol{l}_{; \widehat{\boldsymbol{\theta}}}(\boldsymbol{\theta})\right]\left\{1+O_{p}\left(n^{-3 / 2}\right)\right\}
$$

e

$$
\widetilde{\boldsymbol{l}}_{\boldsymbol{\theta} ; \widehat{\boldsymbol{\theta}}}(\boldsymbol{\theta})=\boldsymbol{l}_{\boldsymbol{\theta} ; \widehat{\boldsymbol{\theta}}}(\boldsymbol{\theta})\left\{1+O_{p}\left(n^{-3 / 2}\right)\right\} .
$$

A seguir, apresentaremos uma estatística aproximadamente ancilar sugerida por Fraser, Reid \& $\mathrm{Wu}$ (1999). Adicionalmente, ao construir a matriz $\widehat{\boldsymbol{V}}$, assuma que podemos usar uma estatística 
aproximadamente ancilar $n$-dimensional, $\boldsymbol{b}$, e que existe uma função $\widehat{\boldsymbol{a}}$ de $\boldsymbol{b}$ tal que $\widehat{\boldsymbol{a}}$ seja aproximadamente ancilar e que $(\widehat{\boldsymbol{\theta}}, \widehat{\boldsymbol{a}})$ seja suficiente.

Embora muitas estatísticas aproximadamente ancilares possam, em princípio, ser usadas neste contexto, apresentaremos uma escolha simples sugerida por Fraser, Reid \& Wu (1999). Suponha que $Y_{1}, Y_{2}, \ldots, Y_{n}$ sejam variáveis aleatórias independentes com função de distribuiçao $F_{j}\left(y_{j} ; \boldsymbol{\theta}\right)$. A quantidade pivotal $F_{j}\left(Y_{j} ; \boldsymbol{\theta}\right)$ tem distribuição uniforme. Pode ser mostrado que se tomarmos $\boldsymbol{b}=\left(b_{1}, \ldots, b_{n}\right)^{\top}$, sendo $b_{j}=F_{j}\left(y_{j} ; \widehat{\boldsymbol{\theta}}\right) \operatorname{com} j=1,2, \ldots, n, b_{j}$ é aproximadamente ancilar; veja, por exemplo, Severini $(2000, \S 6)$. Então, como mostrado por Fraser, Reid \& Wu (1999),

$$
\frac{\partial b_{j}}{\partial \widehat{\boldsymbol{\theta}}}=\frac{\partial F_{j}\left(y_{j} ; \widehat{\boldsymbol{\theta}}\right)}{\partial y_{j}} \frac{\partial y_{j}}{\partial \widehat{\boldsymbol{\theta}}}+\frac{\partial F_{j}\left(y_{j} ; \widehat{\boldsymbol{\theta}}\right)}{\partial \widehat{\boldsymbol{\theta}}}=\{\mathbf{0}\}_{1 \times k}, \quad j=1,2, \ldots, n .
$$

Segue que

$$
\frac{\partial y_{j}}{\partial \widehat{\boldsymbol{\theta}}}=-\frac{\partial F_{j}\left(y_{j} ; \widehat{\boldsymbol{\theta}}\right) / \partial \widehat{\boldsymbol{\theta}}}{f_{j}\left(y_{j} ; \widehat{\boldsymbol{\theta}}\right)}, \quad j=1,2, \ldots, n,
$$

em que $f_{j}\left(y_{j} ; \boldsymbol{\theta}\right)$ é a densidade de $Y_{j}$. Assim, a matriz $\widehat{\boldsymbol{V}}=\partial \boldsymbol{y}^{\top} / \partial \widehat{\boldsymbol{\theta}}$ pode ser tomada da forma

$$
\widehat{\boldsymbol{V}}=\left[\begin{array}{c}
-\frac{\partial F_{1}\left(y_{1} ; \widehat{\boldsymbol{\theta}}\right) / \partial \widehat{\boldsymbol{\theta}}}{f_{1}\left(y_{1} ; \widehat{\boldsymbol{\theta}}\right)} \\
\vdots \\
-\frac{\partial F_{n}\left(y_{n} ; \widehat{\boldsymbol{\theta}}\right) / \partial \widehat{\boldsymbol{\theta}}}{f_{n}\left(y_{n} ; \widehat{\boldsymbol{\theta}}\right)}
\end{array}\right] .
$$

Portanto, como sugerido por Fraser, Reid \& Wu (1999), $\widehat{\boldsymbol{V}}$ tomado como acima é usado no cálculo das aproximações (4.26) e (4.27). Adicionalmente, segundo Fraser, Reid \& Wu (1999), $\widehat{\boldsymbol{V}}$ é obtido da seguinte forma: primeiramente calcula-se

$$
\frac{\partial F_{j}\left(y_{j} ; \boldsymbol{\theta}\right) / \partial \boldsymbol{\theta}}{f_{j}\left(y_{j} ; \boldsymbol{\theta}\right)}, \quad j=1,2, \ldots, n,
$$

e, posteriormente, substituem-se as quantidades desconhecidas pelas suas respectivas estimativas de máxima verossimilhança. Em outras palavras, $\widehat{\boldsymbol{V}}=\left.\boldsymbol{V}\right|_{\boldsymbol{\theta}=\widehat{\boldsymbol{\theta}}}$.

Se $\widetilde{U}$ denota a estatística $U$ [equação (4.7)] baseada nas aproximações (4.26) e (4.27), a estatística $R^{*}$ pode ser aproximada por

$$
\widetilde{R}^{*}=R+\frac{1}{R} \log (\widetilde{U} / R)
$$

De acordo com Fraser, Reid \& Wu (1999), $\widetilde{R}^{*}=R^{*}+O_{p}\left(n^{-3 / 2}\right)$. Note que $R^{*}$ é melhor aproximada por $\widetilde{R}^{*}$ do que por $R_{o}^{*}, \bar{R}^{*}$ e $\widehat{R}^{*}$ [veja Seções 4.6.1 e 4.6.2], uma vez que o erro em se aproximar $R^{*}$ por $R_{o}^{*}, \bar{R}^{*}$ e $\widehat{R}^{*}$ é de ordem $O_{p}\left(n^{-1}\right)$, o erro de aproximação no caso de $\widetilde{R}^{*}$ é de ordem $O_{p}\left(n^{-3 / 2}\right)$.

\subsection{Ajustes no Modelo de Regressão Birnbaum-Saunders}

Nesta seção, derivaremos as aproximações para a estatística da razão de verossimlhanças sinalizada modificada, $R^{*}$, apresentadas nas seções anteriores, no modelo de regressão Birnbaum-Saunders, 
uma vez que não é possível obter $R^{*}$ exatamente nesta classe de modelos. Vale ressaltar que ao obter as aproximações necessárias para o cálculo de $\bar{R}^{*}$ (aproximação de Skovgaard; veja Seção 4.6.2), não foi possível encontrar expressões explícitas para as quantidades $\boldsymbol{Q}\left(\boldsymbol{\theta} ; \boldsymbol{\theta}_{0}\right)$ e $\boldsymbol{I}\left(\boldsymbol{\theta} ; \boldsymbol{\theta}_{0}\right)$, ficando estas em função de cálculos envolvendo muitas integrais numéricas. Por esse motivo, não apresentaremos os cálculos referentes à aproximação $\bar{R}^{*}$ e não a consideraremos nos estudos de simulações Monte Carlo apresentados na Seção 4.8.

Seguindo a mesma notação da Seção 1.3 , seja $\boldsymbol{\theta}=(\alpha, \boldsymbol{\beta})$ o vetor de parâmetros do modelo de regressão Birnbaum-Saunders de dimensão $p+1$, em que $\alpha$ é unidimensional e estritamente positivo e $\boldsymbol{\beta}=\left(\beta_{1}, \beta_{2}, \ldots, \beta_{p}\right)$ é de dimensão $1 \times p$, isto é, o espaço paramétrico é da forma $\boldsymbol{\Omega}=\mathbb{R}^{+} \times \mathbb{R}^{p}$. A matriz de informação de Fisher observada é dada por

$$
\boldsymbol{j}(\boldsymbol{\theta})=\left[\begin{array}{cc}
j_{\alpha \alpha} & \boldsymbol{j}_{\alpha \boldsymbol{\beta}} \\
\boldsymbol{j}_{\boldsymbol{\beta} \alpha} & \boldsymbol{j}_{\boldsymbol{\beta} \boldsymbol{\beta}}
\end{array}\right]=\left[\begin{array}{cc}
\nu & \boldsymbol{k}^{\top} \boldsymbol{X} \\
\boldsymbol{X}^{\top} \boldsymbol{k} & \boldsymbol{X}^{\top} \boldsymbol{W} \boldsymbol{X}
\end{array}\right]
$$

em que $\boldsymbol{X}=\left(\begin{array}{llll}\boldsymbol{x}_{1} & \boldsymbol{x}_{2} & \cdots & \boldsymbol{x}_{n}\end{array}\right)^{\top}$ é a matriz modelo de posto completo, sendo $\boldsymbol{x}_{i}^{\top}=\left(x_{i 1}, x_{i 2}, \ldots, x_{i p}\right)$, $\boldsymbol{W}=\boldsymbol{W}(\boldsymbol{\theta})=\operatorname{diag}\left\{w_{1}, w_{2}, \ldots, w_{n}\right\} \operatorname{com} w_{i}=w_{i}(\boldsymbol{\theta})=-0.25 \operatorname{sech}^{2}\left[\left(y_{i}-\mu_{i}\right) / 2\right]+\alpha^{-2} \cosh \left(y_{i}-\mu_{i}\right)$, $\boldsymbol{k}=\boldsymbol{k}(\boldsymbol{\theta})=\left(k_{1}, k_{2}, \ldots, k_{n}\right)^{\top} \operatorname{com} k_{i}=k_{i}(\boldsymbol{\theta})=2 \alpha^{-3} \operatorname{senh}\left(y_{i}-\mu_{i}\right)$ e $\nu=\nu(\boldsymbol{\theta})=n \alpha^{-2}\left(3 \bar{\xi}_{2}-1\right)$ com $\bar{\xi}_{2}=\bar{\xi}_{2}(\boldsymbol{\theta})=\sum_{i=1}^{n} \xi_{i 2}^{2} / n$ e $\mu_{i}=\boldsymbol{\beta} \boldsymbol{x}_{i}$. Adicionalmente, a matriz de informação de Fisher esperada é da forma

$$
\boldsymbol{i}(\boldsymbol{\theta})=\left[\begin{array}{cc}
i_{\alpha \alpha} & \boldsymbol{i}_{\alpha \boldsymbol{\beta}} \\
\boldsymbol{i}_{\boldsymbol{\beta} \alpha} & \boldsymbol{i}_{\boldsymbol{\beta} \boldsymbol{\beta}}
\end{array}\right]=\left[\begin{array}{cc}
2 n / \alpha^{2} & \{\mathbf{0}\}_{1 \times p} \\
\{\mathbf{0}\}_{p \times 1} & h(\alpha)\left(\boldsymbol{X}^{\top} \boldsymbol{X}\right) / 4
\end{array}\right],
$$

em que

$$
h(\alpha)=2+\frac{4}{\alpha^{2}}-\frac{\sqrt{2 \pi}}{\alpha}\{1-\operatorname{erf}(\sqrt{2} / \alpha)\} \exp \left(2 / \alpha^{2}\right) ;
$$

veja a Seção 1.3. De (4.29), note que $\alpha$ e $\boldsymbol{\beta}$ são globalmente ortogonais. O determinante da matriz $\boldsymbol{j}(\boldsymbol{\theta})$ dada em (4.28) pode ser calculado, por exemplo, usando a fórmula do determinante de uma matriz particionada, da forma

$$
|\boldsymbol{j}(\boldsymbol{\theta})|=\nu\left|\boldsymbol{X}^{\top} \boldsymbol{W} \boldsymbol{X}-\nu^{-1} \boldsymbol{X}^{\top} \boldsymbol{k} \boldsymbol{k}^{\top} \boldsymbol{X}\right|=\nu\left|\boldsymbol{X}^{\top}\left(\boldsymbol{W}-\nu^{-1} \boldsymbol{k} \boldsymbol{k}^{\top}\right) \boldsymbol{X}\right|=\nu\left|\boldsymbol{X}^{\top} \boldsymbol{H} \boldsymbol{X}\right|,
$$

em que $\boldsymbol{H}=\boldsymbol{H}(\boldsymbol{\theta})=\boldsymbol{W}-\nu^{-1} \boldsymbol{k} \boldsymbol{k}^{\top}$. Assim, por exemplo, $|\boldsymbol{j}(\boldsymbol{\theta})|^{1 / 2}=\nu^{1 / 2}\left|\boldsymbol{X}^{\top} \boldsymbol{H} \boldsymbol{X}\right|^{1 / 2}$.

\subsubsection{Inferência sobre $\alpha$}

Como $\alpha$ é globalmente ortogonal a $\boldsymbol{\beta}$, podemos usar a aproximação proposta em DiCiccio \& Martin (1993) descrita na Seção 4.6.1, além, claro, das outras aproximações apresentadas nas Seções 4.6.2 e 4.6.3 que não requerem restrições sobre os parâmetros do modelo. Como foi mencionado anteriormente [veja a Seção 4.3], podemos testar hipóteses do tipo:

$$
\begin{array}{lll}
\mathcal{H}_{0}: \alpha \leq \alpha^{(0)} & \text { versus } & \mathcal{H}_{1}: \alpha>\alpha^{(0)} \\
\mathcal{H}_{0}: \alpha \geq \alpha^{(0)} & \text { versus } & \mathcal{H}_{1}: \alpha<\alpha^{(0)} \\
\mathcal{H}_{0}: \alpha=\alpha^{(0)} & \text { versus } & \mathcal{H}_{1}: \alpha \neq \alpha^{(0)}
\end{array}
$$

Aqui, $\alpha^{(0)}$ é uma constante conhecida estritamente positiva. Neste caso, $\boldsymbol{\beta}$ é um vetor de parâmetros de perturbação. Adicionalmente, sejam $\widehat{\boldsymbol{\theta}}=(\widehat{\alpha}, \widehat{\boldsymbol{\beta}})$ o estimador de máxima verossimilhança irrestrito de $\boldsymbol{\theta}$ e $\widetilde{\boldsymbol{\theta}}=\left(\alpha^{(0)}, \widetilde{\boldsymbol{\beta}}\right)$ o estimador de máxima verossimilhança de $\boldsymbol{\theta}$ restrito sob a hipótese nula. Vale 
lembrar que neste caso a estatística da razão de verossimilhanças sinalizada é dada por

$$
R=\operatorname{sgn}\left(\widehat{\alpha}-\alpha^{(0)}\right)[2\{l(\widehat{\boldsymbol{\theta}})-l(\widetilde{\boldsymbol{\theta}})\}]^{1 / 2}
$$

veja a Seção 4.3 .

\section{Aproximação $R_{o}^{*}$}

A estatística $R_{o}^{*}$ é da forma

$$
R_{o}^{*}=R+\frac{1}{R} \log \left(U_{o} / R\right)
$$

em que

$$
U_{o}=l_{\alpha}(\widetilde{\boldsymbol{\theta}}) \frac{\left|\boldsymbol{j}_{\boldsymbol{\beta} \boldsymbol{\beta}}(\widetilde{\boldsymbol{\theta}})\right|^{1 / 2} i_{\alpha \alpha}(\widehat{\boldsymbol{\theta}})^{1 / 2}}{|\widehat{\boldsymbol{j}}|^{1 / 2} i_{\alpha \alpha}(\widetilde{\boldsymbol{\theta}})^{1 / 2}} .
$$

As quantidades necessárias para obter $U_{o}$ são dadas a seguir. Inicialmente, note que a função escore de $\alpha$ pode ser escrita na forma [veja Apêndice A]

$$
l_{\alpha}(\boldsymbol{\theta})=\frac{n}{\alpha}\left(\bar{\xi}_{2}-1\right) .
$$

As outras quantidades são $|\boldsymbol{j}(\boldsymbol{\theta})|^{1 / 2}=\nu^{1 / 2}\left|\boldsymbol{X}^{\top} \boldsymbol{H} \boldsymbol{X}\right|^{1 / 2},\left|\boldsymbol{j}_{\boldsymbol{\beta} \boldsymbol{\beta}}(\boldsymbol{\theta})\right|^{1 / 2}=\left|\boldsymbol{X}^{\top} \boldsymbol{W} \boldsymbol{X}\right|^{1 / 2}$ e $i_{\alpha \alpha}(\boldsymbol{\theta})=$ $2 n / \alpha^{2}$. Assim, após uma simples álgebra, $U_{o}$ pode ser escrito na forma

$$
U_{o}=\sqrt{n} \frac{\left\{\bar{\xi}_{2}(\widetilde{\boldsymbol{\theta}})-1\right\}}{\left\{3 \bar{\xi}_{2}(\widehat{\boldsymbol{\theta}})-1\right\}^{1 / 2}} \frac{\left|\boldsymbol{X}^{\top} \widetilde{\boldsymbol{W}} \boldsymbol{X}\right|^{1 / 2}}{\left|\boldsymbol{X}^{\top} \widehat{\boldsymbol{H}} \boldsymbol{X}\right|^{1 / 2}},
$$

em que $\widehat{\boldsymbol{H}}=\boldsymbol{H}(\widehat{\boldsymbol{\theta}})$ e $\widetilde{\boldsymbol{W}}=\boldsymbol{W}(\widetilde{\boldsymbol{\theta}})$. Portanto, podemos calcular $R_{o}^{*}$ dado em (4.30) com $U_{o}$ dado em (4.31).

\section{Aproximação $\widehat{R}^{*}$}

A estatística $\widehat{R}^{*}$ é da forma

$$
\widehat{R}^{*}=R+\frac{1}{R} \log (\widehat{U} / R)
$$

em que

$$
\widehat{U}=\left|\begin{array}{c}
\widehat{\boldsymbol{l}}_{; \widehat{\boldsymbol{\theta}}}(\widehat{\boldsymbol{\theta}})-\widehat{\boldsymbol{l}}_{: \widehat{\boldsymbol{\theta}}}(\widetilde{\boldsymbol{\theta}}) \\
\widehat{\boldsymbol{l}}_{\boldsymbol{\beta} ; \widehat{\boldsymbol{\theta}}}(\tilde{\boldsymbol{\theta}})
\end{array}\right| /\left|\boldsymbol{j}_{\boldsymbol{\beta} \boldsymbol{\beta}}(\widetilde{\boldsymbol{\theta}})\right|^{1 / 2}|\widehat{\boldsymbol{j}}|^{1 / 2},
$$

com

$$
\widehat{\boldsymbol{l}}_{; \widehat{\boldsymbol{\theta}}}(\widehat{\boldsymbol{\theta}})-\widehat{\boldsymbol{l}}_{; \widehat{\boldsymbol{\theta}}}(\widetilde{\boldsymbol{\theta}})=[\widehat{\boldsymbol{Q}}(\widehat{\boldsymbol{\theta}} ; \widehat{\boldsymbol{\theta}})-\widehat{\boldsymbol{Q}}(\widetilde{\boldsymbol{\theta}} ; \widehat{\boldsymbol{\theta}})] \widehat{\boldsymbol{i}}(\widehat{\boldsymbol{\theta}})^{-1} \widehat{\boldsymbol{j}} \quad \text { e } \quad \widehat{\boldsymbol{l}}_{\boldsymbol{\theta} ; \widehat{\boldsymbol{\theta}}}(\widetilde{\boldsymbol{\theta}})=\widehat{\boldsymbol{I}}(\widetilde{\boldsymbol{\theta}} ; \widehat{\boldsymbol{\theta}}) \widehat{\boldsymbol{i}}(\widehat{\boldsymbol{\theta}})^{-1} \widehat{\boldsymbol{j}}
$$

Adicionalmente,

$$
\widehat{\boldsymbol{Q}}\left(\boldsymbol{\theta} ; \boldsymbol{\theta}_{0}\right)=\sum l^{(i)}(\boldsymbol{\theta}) \boldsymbol{l}_{\boldsymbol{\theta}}^{(i)}\left(\boldsymbol{\theta}_{0}\right)^{\top}, \quad \widehat{\boldsymbol{I}}\left(\boldsymbol{\theta} ; \boldsymbol{\theta}_{0}\right)=\sum \boldsymbol{l}_{\boldsymbol{\theta}}^{(i)}(\boldsymbol{\theta}) \boldsymbol{l}_{\boldsymbol{\theta}}^{(i)}\left(\boldsymbol{\theta}_{0}\right)^{\top} \text { e } \widehat{\boldsymbol{i}}(\widehat{\boldsymbol{\theta}})=\widehat{\boldsymbol{I}}(\widehat{\boldsymbol{\theta}} ; \widehat{\boldsymbol{\theta}}),
$$

em que $\boldsymbol{\theta}_{0}=\left(\alpha_{0}, \boldsymbol{\beta}_{0}\right)$.

A função de log-verossimilhança do modelo de regressão Birnbaum-Saunders com base apenas 
na $i$-ésima observação é da forma

$$
l^{(i)}(\boldsymbol{\theta})=-\frac{1}{2} \log (8 \pi)+\log \left(\xi_{i 1}\right)-\frac{1}{2} \xi_{i 2}^{2},
$$

em que $\xi_{i 1}=\xi_{i 1}(\boldsymbol{\theta})=2 \alpha^{-1} \cosh \left[\left(y_{i}-\mu_{i}\right) / 2\right], \xi_{i 2}=\xi_{i 2}(\boldsymbol{\theta})=2 \alpha^{-1} \operatorname{senh}\left[\left(y_{i}-\mu_{i}\right) / 2\right]$ e $\mu_{i}=\boldsymbol{\beta} \boldsymbol{x}_{i}$, sendo $\boldsymbol{x}_{i}^{\top}=\left(x_{i 1}, x_{i 2}, \ldots, x_{i p}\right)$ a $i$-ésima linha da matriz $\boldsymbol{X}$. Assim,

$$
l_{\alpha}^{(i)}(\boldsymbol{\theta})=\frac{\partial l^{(i)}(\boldsymbol{\theta})}{\partial \alpha}=-\frac{1}{\alpha}\left(1-\xi_{i 2}^{2}\right) \quad \text { e } \quad \boldsymbol{l}_{\boldsymbol{\beta}}^{(i)}(\boldsymbol{\theta})=\frac{\partial l^{(i)}(\boldsymbol{\theta})}{\partial \boldsymbol{\beta}^{\top}}=\frac{1}{2}\left\{\xi_{i 1} \xi_{i 2}-\xi_{i 2} / \xi_{i 1}\right\} \boldsymbol{x}_{i} .
$$

Dai, temos que

$$
\boldsymbol{l}_{\boldsymbol{\theta}}^{(i)}(\boldsymbol{\theta})=\frac{\partial l^{(i)}(\boldsymbol{\theta})}{\partial \boldsymbol{\theta}^{\top}}=\left(\begin{array}{c}
l_{\alpha}^{(i)}(\boldsymbol{\theta}) \\
\boldsymbol{l}_{\boldsymbol{\beta}}^{(i)}(\boldsymbol{\theta})
\end{array}\right)=\left(\begin{array}{c}
-\frac{\left(1-\xi_{i 2}^{2}\right)}{\alpha} \\
\frac{1}{2}\left\{\xi_{i 1} \xi_{i 2}-\xi_{i 2} / \xi_{i 1}\right\} \boldsymbol{x}_{i}
\end{array}\right)
$$

O vetor $\widehat{\boldsymbol{Q}}\left(\boldsymbol{\theta} ; \boldsymbol{\theta}_{0}\right)$ de dimensão $1 \times(p+1)$ é da forma

$$
\begin{aligned}
& \widehat{\boldsymbol{Q}}\left(\boldsymbol{\theta} ; \boldsymbol{\theta}_{0}\right)=\sum l^{(i)}(\boldsymbol{\theta}) \boldsymbol{l}_{\boldsymbol{\theta}}^{(i)}\left(\boldsymbol{\theta}_{0}\right)^{\top}=\sum l^{(i)}(\boldsymbol{\theta})\left[l_{\alpha}^{(i)}\left(\boldsymbol{\theta}_{0}\right) \quad \boldsymbol{l}_{\boldsymbol{\beta}}^{(i)}\left(\boldsymbol{\theta}_{0}\right)^{\top}\right] \\
& =\left[\sum l^{(i)}(\boldsymbol{\theta}) l_{\alpha}^{(i)}\left(\boldsymbol{\theta}_{0}\right) \quad \sum l^{(i)}(\boldsymbol{\theta}) \boldsymbol{l}_{\boldsymbol{\beta}}^{(i)}\left(\boldsymbol{\theta}_{0}\right)^{\top}\right] .
\end{aligned}
$$

Note que

$$
\sum l^{(i)}(\boldsymbol{\theta}) l_{\alpha}^{(i)}\left(\boldsymbol{\theta}_{0}\right)=\sum l^{(i)}(\boldsymbol{\theta})\left\{-\frac{\left(1-\xi_{i 20}^{2}\right)}{\alpha_{0}}\right\}=\sum z_{1 i}=\boldsymbol{z}_{1}^{\top} \mathbf{1}
$$

em que $\boldsymbol{z}_{1}=\boldsymbol{z}_{1}\left(\boldsymbol{\theta} ; \boldsymbol{\theta}_{0}\right)=\left(z_{11}, z_{12}, \ldots, z_{1 n}\right)^{\top} \operatorname{com} z_{1 i}=z_{1 i}\left(\boldsymbol{\theta} ; \boldsymbol{\theta}_{0}\right)=-l^{(i)}(\boldsymbol{\theta})\left\{1-\xi_{i 20}^{2}\right\} / \alpha_{0}, \xi_{i 20}^{2}=$ $\xi_{i 2}^{2}\left(\boldsymbol{\theta}_{0}\right)$ e $\mathbf{1}=(1,1, \ldots, 1)^{\top}$ é um vetor $n$-dimensional de 1's. Da mesma forma,

$$
\sum l^{(i)}(\boldsymbol{\theta}) \boldsymbol{l}_{\boldsymbol{\beta}}^{(i)}\left(\boldsymbol{\theta}_{0}\right)^{\top}=\sum l^{(i)}(\boldsymbol{\theta})\left[\frac{1}{2}\left\{\xi_{i 10} \xi_{i 20}-\xi_{i 20} / \xi_{i 10}\right\} \boldsymbol{x}_{i}^{\top}\right]=\sum z_{2 i} \boldsymbol{x}_{i}^{\top}=\boldsymbol{z}_{2}^{\top} \boldsymbol{X},
$$

em que $\boldsymbol{z}_{2}=\boldsymbol{z}_{2}\left(\boldsymbol{\theta} ; \boldsymbol{\theta}_{0}\right)=\left(z_{21}, z_{22}, \ldots, z_{2 n}\right)^{\top} \operatorname{com} z_{2 i}=z_{2 i}\left(\boldsymbol{\theta} ; \boldsymbol{\theta}_{0}\right)=l^{(i)}(\boldsymbol{\theta})\left\{\xi_{i 10} \xi_{i 20}-\xi_{i 20} / \xi_{i 10}\right\} / 2 \mathrm{e}$ $\xi_{i 10}=\xi_{i 1}\left(\boldsymbol{\theta}_{0}\right)$. Assim,

$$
\widehat{\boldsymbol{Q}}\left(\boldsymbol{\theta} ; \boldsymbol{\theta}_{0}\right)=\left[\begin{array}{ll}
\boldsymbol{z}_{1}^{\top} \mathbf{1} & \boldsymbol{z}_{2}^{\top} \boldsymbol{X}
\end{array}\right] .
$$

A matriz $\widehat{\boldsymbol{I}}\left(\boldsymbol{\theta} ; \boldsymbol{\theta}_{0}\right)$ de dimensão $(p+1) \times(p+1)$ é da forma

$$
\widehat{\boldsymbol{I}}\left(\boldsymbol{\theta} ; \boldsymbol{\theta}_{0}\right)=\left[\begin{array}{cc}
\sum l_{\alpha}^{(i)}(\boldsymbol{\theta}) l_{\alpha}^{(i)}\left(\boldsymbol{\theta}_{0}\right) & \sum l_{\alpha}^{(i)}(\boldsymbol{\theta}) \boldsymbol{l}_{\boldsymbol{\beta}}^{(i)}\left(\boldsymbol{\theta}_{0}\right)^{\top} \\
\sum \boldsymbol{l}_{\boldsymbol{\beta}}^{(i)}(\boldsymbol{\theta}) \sum l_{\alpha}^{(i)}\left(\boldsymbol{\theta}_{0}\right) & \sum \boldsymbol{l}_{\boldsymbol{\beta}}^{(i)}(\boldsymbol{\theta}) \boldsymbol{l}_{\boldsymbol{\beta}}^{(i)}\left(\boldsymbol{\theta}_{0}\right)^{\top}
\end{array}\right] .
$$

Vamos obter cada parcela da matriz $\widehat{\boldsymbol{I}}\left(\boldsymbol{\theta} ; \boldsymbol{\theta}_{0}\right)$ separadamente. Observe que

$$
\sum l_{\alpha}^{(i)}(\boldsymbol{\theta}) l_{\alpha}^{(i)}\left(\boldsymbol{\theta}_{0}\right)=\sum\left\{-\frac{1-\xi_{i 2}^{2}}{\alpha}\right\}\left\{-\frac{1-\xi_{i 20}^{2}}{\alpha_{0}}\right\}=\sum \frac{\left(1-\xi_{i 2}^{2}\right)}{\alpha} \frac{\left(1-\xi_{i 20}^{2}\right)}{\alpha_{0}}=\sum z_{3 i}=\boldsymbol{z}_{3}^{\top} \mathbf{1},
$$

em que $\boldsymbol{z}_{3}=\boldsymbol{z}_{3}\left(\boldsymbol{\theta} ; \boldsymbol{\theta}_{0}\right)=\left(z_{31}, z_{32}, \ldots, z_{3 n}\right)^{\top} \operatorname{com} z_{3 i}=z_{3 i}\left(\boldsymbol{\theta} ; \boldsymbol{\theta}_{0}\right)=\left(1-\xi_{i 2}^{2}\right)\left(1-\xi_{i 20}^{2}\right) / \alpha \alpha_{0}$. Adicional- 
mente,

$$
\begin{aligned}
\sum l_{\alpha}^{(i)}(\boldsymbol{\theta}) \boldsymbol{l}_{\boldsymbol{\beta}}^{(i)}\left(\boldsymbol{\theta}_{0}\right)^{\top} & =\sum\left\{-\frac{1-\xi_{i 2}^{2}}{\alpha}\right\}\left\{\frac{1}{2}\left\{\xi_{i 10} \xi_{i 20}-\xi_{i 20} / \xi_{i 10}\right\} \boldsymbol{x}_{i}^{\top}\right\} \\
& =-\sum \frac{1}{2} \frac{\left\{1-\xi_{i 2}^{2}\right\}}{\alpha}\left\{\xi_{i 10} \xi_{i 20}-\xi_{i 20} / \xi_{i 10}\right\} \boldsymbol{x}_{i}^{\top} \\
& =\sum z_{4 i} \boldsymbol{x}_{i}^{\top}=\boldsymbol{z}_{4}^{\top} \boldsymbol{X}
\end{aligned}
$$

em que $\boldsymbol{z}_{4}=\boldsymbol{z}_{4}\left(\boldsymbol{\theta} ; \boldsymbol{\theta}_{0}\right)=\left(z_{41}, z_{42}, \ldots, z_{4 n}\right)^{\top} \operatorname{com} z_{4 i}=z_{4 i}\left(\boldsymbol{\theta} ; \boldsymbol{\theta}_{0}\right)=-\left\{1-\xi_{i 2}^{2}\right\}\left\{\xi_{i 10} \xi_{i 20}-\xi_{i 20} / \xi_{i 10}\right\} / 2 \alpha$. Da mesma forma,

$$
\begin{aligned}
\sum \boldsymbol{l}_{\boldsymbol{\beta}}^{(i)}(\boldsymbol{\theta}) l_{\alpha}^{(i)}\left(\boldsymbol{\theta}_{0}\right) & =\sum\left\{\frac{1}{2}\left\{\xi_{i 1} \xi_{i 2}-\xi_{i 2} / \xi_{i 1}\right\} \boldsymbol{x}_{i}\right\}\left\{-\frac{1-\xi_{i 20}^{2}}{\alpha_{0}}\right\} \\
& =-\sum \frac{1}{2} \frac{\left\{1-\xi_{i 20}^{2}\right\}}{\alpha_{0}}\left\{\xi_{i 1} \xi_{i 2}-\xi_{i 2} / \xi_{i 1}\right\} \boldsymbol{x}_{i} \\
& =\sum z_{5 i} \boldsymbol{x}_{i}^{\top}=\boldsymbol{X}^{\top} \boldsymbol{z}_{5},
\end{aligned}
$$

em que $\boldsymbol{z}_{5}=\boldsymbol{z}_{5}\left(\boldsymbol{\theta} ; \boldsymbol{\theta}_{0}\right)=\left(z_{51}, z_{52}, \ldots, z_{5 n}\right)^{\top} \operatorname{com} z_{5 i}=z_{5 i}\left(\boldsymbol{\theta} ; \boldsymbol{\theta}_{0}\right)=-\left\{1-\xi_{i 20}^{2}\right\}\left\{\xi_{i 1} \xi_{i 2}-\xi_{i 2} / \xi_{i 1}\right\} / 2 \alpha_{0}$. Finalmente,

$$
\begin{aligned}
\sum \boldsymbol{l}_{\boldsymbol{\beta}}^{(i)}(\boldsymbol{\theta}) \boldsymbol{l}_{\boldsymbol{\beta}}^{(i)}\left(\boldsymbol{\theta}_{0}\right)^{\top} & =\sum \frac{1}{2}\left\{\xi_{i 1} \xi_{i 2}-\xi_{i 2} / \xi_{i 1}\right\} \boldsymbol{x}_{i} \frac{1}{2}\left\{\xi_{i 10} \xi_{i 20}-\xi_{i 20} / \xi_{i 10}\right\} \boldsymbol{x}_{i}^{\top} \\
& =\sum \boldsymbol{x}_{i} \frac{1}{4}\left\{\xi_{i 1} \xi_{i 2}-\xi_{i 2} / \xi_{i 1}\right\}\left\{\xi_{i 10} \xi_{i 20}-\xi_{i 20} / \xi_{i 10}\right\} \boldsymbol{x}_{i}^{\top} \\
& =\sum \boldsymbol{x}_{i} z_{6 i} \boldsymbol{x}_{i}^{\top}=\boldsymbol{X}^{\top} \boldsymbol{Z} \boldsymbol{X}
\end{aligned}
$$

em que $\boldsymbol{Z}=\boldsymbol{Z}\left(\boldsymbol{\theta} ; \boldsymbol{\theta}_{0}\right)=\operatorname{diag}\left\{z_{61}, z_{62}, \ldots, z_{6 n}\right\}$ com $z_{6 i}=z_{6 i}\left(\boldsymbol{\theta} ; \boldsymbol{\theta}_{0}\right)=\left\{\xi_{i 1} \xi_{i 2}-\xi_{i 2} / \xi_{i 1}\right\}\left\{\xi_{i 10} \xi_{i 20}-\right.$ $\left.\xi_{i 20} / \xi_{i 10}\right\} / 4$. Portanto, a matriz $\widehat{\boldsymbol{I}}\left(\boldsymbol{\theta} ; \boldsymbol{\theta}_{0}\right)$ é da forma

$$
\widehat{\boldsymbol{I}}\left(\boldsymbol{\theta} ; \boldsymbol{\theta}_{0}\right)=\left[\begin{array}{cc}
\boldsymbol{z}_{3}^{\top} \mathbf{1} & \boldsymbol{z}_{4}^{\top} \boldsymbol{X} \\
\boldsymbol{X}^{\top} \boldsymbol{z}_{5} & \boldsymbol{X}^{\top} \boldsymbol{Z} \boldsymbol{X}
\end{array}\right]
$$

Assim, tendo sido as quantidades $\widehat{\boldsymbol{Q}}\left(\boldsymbol{\theta} ; \boldsymbol{\theta}_{0}\right)$ e $\widehat{\boldsymbol{I}}\left(\boldsymbol{\theta} ; \boldsymbol{\theta}_{0}\right)$, que são usadas para obter $\widehat{U}$, calculadas, notando que $\widehat{\boldsymbol{l}}_{\boldsymbol{\beta} ; \widehat{\boldsymbol{\theta}}}(\boldsymbol{\theta})$ é obtido de $\widehat{\boldsymbol{l}}_{\boldsymbol{\theta} ; \widehat{\boldsymbol{\theta}}}(\boldsymbol{\theta})$ excluindo-se a primeira linha dessa matriz, calculamos $\widehat{R}^{*}$ como em (4.32) considerando $\widehat{U}$ como em (4.33). Note que $\widehat{\boldsymbol{l}}_{\boldsymbol{\beta} ; \widehat{\boldsymbol{\theta}}}(\boldsymbol{\theta})$ é de dimensão $p \times(p+1)$.

\section{Aproximação $\widetilde{R}^{*}$}

A estatística $\widetilde{R}^{*}$ é da forma

$$
\widetilde{R}^{*}=R+\frac{1}{R} \log (\widetilde{U} / R)
$$

em que

$$
\widetilde{U}=\left|\begin{array}{c}
\widetilde{\boldsymbol{l}}_{; \widehat{\boldsymbol{\theta}}}(\widehat{\widehat{\boldsymbol{\theta}}})-\widetilde{\boldsymbol{l}}_{; \widehat{\boldsymbol{\theta}}}(\widetilde{\boldsymbol{\theta}}) \\
\widetilde{\boldsymbol{l}}_{\boldsymbol{\beta} ; \hat{\boldsymbol{\theta}}}(\tilde{\boldsymbol{\theta}})
\end{array}\right| /\left|\boldsymbol{j}_{\boldsymbol{\beta} \boldsymbol{\beta}}(\widetilde{\boldsymbol{\theta}})\right|^{1 / 2}|\widehat{\boldsymbol{j}}|^{1 / 2},
$$

com

$$
\widetilde{\boldsymbol{l}}_{; \widehat{\boldsymbol{\theta}}}(\boldsymbol{\theta})=\boldsymbol{l}_{; \boldsymbol{y}}(\boldsymbol{\theta}) \widehat{\boldsymbol{V}}\left(\boldsymbol{l}_{\boldsymbol{\theta} ; \boldsymbol{y}}(\widehat{\boldsymbol{\theta}}) \widehat{\boldsymbol{V}}\right)^{-1} \widehat{\boldsymbol{j}} \quad \text { e } \quad \widetilde{\boldsymbol{l}}_{\boldsymbol{\theta} ; \hat{\boldsymbol{\theta}}}(\boldsymbol{\theta})=\boldsymbol{l}_{\boldsymbol{\theta} ; \boldsymbol{y}}(\boldsymbol{\theta}) \widehat{\boldsymbol{V}}\left(\boldsymbol{l}_{\boldsymbol{\theta} ; \boldsymbol{y}}(\widehat{\boldsymbol{\theta}}) \widehat{\boldsymbol{V}}\right)^{-1} \widehat{\boldsymbol{j}}
$$


Adicionalmente,

$\mathrm{e}$

$$
\boldsymbol{l}_{; \boldsymbol{y}}(\boldsymbol{\theta})=\frac{\partial l(\boldsymbol{\theta})}{\partial \boldsymbol{y}}, \quad \boldsymbol{l}_{\boldsymbol{\theta} ; \boldsymbol{y}}(\boldsymbol{\theta})=\frac{\partial \boldsymbol{l}_{\boldsymbol{y}}(\boldsymbol{\theta})}{\partial \boldsymbol{\theta}^{\top}}
$$

$$
\boldsymbol{V}=\left[\begin{array}{c}
-\frac{\partial F_{1}\left(y_{1} ; \boldsymbol{\theta}\right) / \partial \boldsymbol{\theta}}{f_{1}\left(y_{1} ; \boldsymbol{\theta}\right)} \\
\vdots \\
-\frac{\partial F_{n}\left(y_{n} ; \boldsymbol{\theta}\right) / \partial \boldsymbol{\theta}}{f_{n}\left(y_{n} ; \boldsymbol{\theta}\right)}
\end{array}\right]
$$

sendo $\widehat{\boldsymbol{V}}=\left.\boldsymbol{V}\right|_{\boldsymbol{\theta}=\widehat{\boldsymbol{\theta}}}$.

Vamos obter primeiramente $\boldsymbol{l}_{; \boldsymbol{y}}(\boldsymbol{\theta})$ e $\boldsymbol{l}_{\boldsymbol{\theta} ; \boldsymbol{y}}(\boldsymbol{\theta})$. Da Seção 1.3, temos que a função de log-verossimilhança do modelo de regressão Birnbaum-Saunders é da forma

$$
l(\boldsymbol{\theta})=-\frac{n}{2} \log (8 \pi)+\sum_{i=1}^{n} \log \left(\xi_{i 1}\right)-\frac{1}{2} \sum_{i=1}^{n} \xi_{i 2}^{2} .
$$

Observe que

$$
\boldsymbol{l}_{; \boldsymbol{y}}(\boldsymbol{\theta})=\left(\begin{array}{llll}
\frac{\partial l(\boldsymbol{\theta})}{\partial y_{1}} & \frac{\partial l(\boldsymbol{\theta})}{\partial y_{2}} & \ldots & \frac{\partial l(\boldsymbol{\theta})}{\partial y_{n}}
\end{array}\right)
$$

em que

$$
\frac{\partial l(\boldsymbol{\theta})}{\partial y_{i}}=\frac{\partial}{\partial y_{i}} \log \left(\xi_{i 1}\right)-\frac{1}{2} \frac{\partial}{\partial y_{i}} \xi_{i 2}^{2}=\frac{1}{\xi_{i 1}} \frac{\partial \xi_{i 1}}{\partial y_{i}}-\xi_{i 2} \frac{\partial \xi_{i 2}}{\partial y_{i}}, \quad i=1,2, \ldots, n .
$$

Agora,

$$
\frac{\partial \xi_{i 1}}{\partial y_{i}}=\frac{\partial}{\partial y_{i}}\left\{\frac{2}{\alpha} \cosh \left(\frac{y_{i}-\mu_{i}}{2}\right)\right\}=\frac{1}{\alpha} \operatorname{senh}\left(\frac{y_{i}-\mu_{i}}{2}\right)=\frac{\xi_{i 2}}{2} .
$$

Da mesma forma,

$$
\frac{\partial \xi_{i 2}}{\partial y_{i}}=\frac{\partial}{\partial y_{i}}\left\{\frac{2}{\alpha} \operatorname{senh}\left(\frac{y_{i}-\mu_{i}}{2}\right)\right\}=\frac{1}{\alpha} \cosh \left(\frac{y_{i}-\mu_{i}}{2}\right)=\frac{\xi_{i 1}}{2} .
$$

Assim,

$$
\frac{\partial l(\boldsymbol{\theta})}{\partial y_{i}}=\frac{1}{\xi_{i 1}} \frac{\xi_{i 2}}{2}-\xi_{i 2} \frac{\xi_{i 1}}{2}=-\frac{1}{2}\left\{\xi_{i 1} \xi_{i 2}-\xi_{i 2} / \xi_{i 1}\right\}, \quad i=1,2, \ldots, n
$$

Portanto,

$$
\boldsymbol{l}_{; \boldsymbol{y}}(\boldsymbol{\theta})=\boldsymbol{z}_{7}(\boldsymbol{\theta})
$$

em que $\boldsymbol{z}_{7}(\boldsymbol{\theta})=\left(z_{71}, \ldots, z_{7 n}\right) \operatorname{com} z_{7 i}=-\left\{\xi_{i 1} \xi_{i 2}-\xi_{i 2} / \xi_{i 1}\right\} / 2$.

Note que

em que

$$
\boldsymbol{l}_{\boldsymbol{\theta} ; \boldsymbol{y}}(\boldsymbol{\theta})=\frac{\partial \boldsymbol{l}_{\boldsymbol{y}}(\boldsymbol{\theta})}{\partial \boldsymbol{\theta}^{\top}}=\frac{\partial \boldsymbol{l}_{\boldsymbol{\theta}}(\boldsymbol{\theta})}{\partial \boldsymbol{y}},
$$

$$
\boldsymbol{l}_{\boldsymbol{\theta}}(\boldsymbol{\theta})=\left(\begin{array}{c}
-\frac{1}{\alpha} \sum_{i=1}^{n}\left(1-\xi_{i 2}^{2}\right) \\
\frac{1}{2} \boldsymbol{X}^{\top} \boldsymbol{s}
\end{array}\right)
$$


sendo $\boldsymbol{s}=\boldsymbol{s}(\boldsymbol{\theta})=\left(s_{1}, \ldots, s_{n}\right)^{\top} \operatorname{com} s_{i}=\xi_{i 1} \xi_{i 2}-\xi_{i 2} / \xi_{i 1}$; veja a Seção 1.3. Então, $\boldsymbol{l}_{\boldsymbol{\theta} ; \boldsymbol{y}}(\boldsymbol{\theta})$ é uma matriz $(p+1) \times n$ da forma

$$
\boldsymbol{l}_{\boldsymbol{\theta} ; \boldsymbol{y}}(\boldsymbol{\theta})=\frac{\partial \boldsymbol{l}_{\boldsymbol{\theta}}(\boldsymbol{\theta})}{\partial \boldsymbol{y}}=\left[\begin{array}{ccc}
\frac{1}{\alpha} \frac{\partial \xi_{12}^{2}}{\partial y_{1}} & \ldots & \frac{1}{\alpha} \frac{\partial \xi_{n 2}^{2}}{\partial y_{n}} \\
\frac{x_{11}}{2} \frac{\partial\left\{\xi_{11} \xi_{12}-\xi_{12} / \xi_{11}\right\}}{\partial y_{1}} & \ldots & \frac{x_{n 1}}{2} \frac{\partial\left\{\xi_{n 1} \xi_{n 2}-\xi_{n 2} / \xi_{n 1}\right\}}{\partial y_{n}} \\
\vdots & & \vdots \\
\frac{x_{1 p}}{2} \frac{\partial\left\{\xi_{11} \xi_{12}-\xi_{12} / \xi_{11}\right\}}{\partial y_{1}} & \ldots & \frac{x_{n p}}{2} \frac{\partial\left\{\xi_{n 1} \xi_{n 2}-\xi_{n 2} / \xi_{n 1}\right\}}{\partial y_{n}}
\end{array}\right]
$$

Após álgebra simples, é possível mostrar que

$$
\frac{\partial \xi_{i 2}^{2}}{\partial y_{i}}=\frac{\partial}{\partial y_{i}}\left[\frac{4}{\alpha^{2}} \operatorname{senh}^{2}\left(\frac{y_{i}-\mu_{i}}{2}\right)\right]=\xi_{i 1} \xi_{i 2}, \quad i=1,2, \ldots, n .
$$

Da mesma forma, notando que

$$
\frac{\partial \xi_{i 1} \xi_{i 2}}{\partial y_{i}}=\frac{\xi_{i 1}^{2}+\xi_{i 2}^{2}}{2} \quad \text { e } \frac{\partial}{\partial y_{i}}\left(\frac{\xi_{i 2}}{\xi_{i 1}}\right)=\frac{1}{2} \frac{\xi_{i 1}^{2}-\xi_{i 2}^{2}}{\xi_{i 1}^{2}}
$$

temos que

$$
\frac{\partial\left\{\xi_{i 1} \xi_{i 2}-\xi_{i 2} / \xi_{i 1}\right\}}{\partial y_{i}}=\frac{1}{2}\left\{\frac{4}{\alpha^{2}}+2 \xi_{i 2}^{2}-\frac{4}{\alpha^{2} \xi_{i 1}^{2}}\right\}, \quad i=1,2, \ldots, n .
$$

Usamos o fato de que $\cosh ^{2}(x)-\operatorname{senh}^{2}(x)=1$. Seja o vetor linha $n$-dimensional $\boldsymbol{\zeta}=\boldsymbol{\zeta}(\boldsymbol{\theta})$ da forma

$$
\boldsymbol{\zeta}=\boldsymbol{\zeta}(\boldsymbol{\theta})=\frac{1}{\alpha}\left(\begin{array}{llll}
\xi_{11} \xi_{12} & \xi_{21} \xi_{22} & \ldots & \xi_{n 1} \xi_{n 2}
\end{array}\right) .
$$

Adicionalmente, seja a matriz $\boldsymbol{\Delta}=\boldsymbol{\Delta}(\boldsymbol{\theta})=\operatorname{diag}\left\{\delta_{1}, \delta_{2}, \ldots, \delta_{n}\right\}$ de dimensão $n \times n$, em que $\delta_{i}=$ $\delta_{i}(\boldsymbol{\theta})=\left\{4 / \alpha^{2}+2 \xi_{i 2}^{2}-4 /\left(\alpha^{2} \xi_{i 1}^{2}\right)\right\} / 4$. Assim, temos que

$$
l_{\theta ; \boldsymbol{y}}(\boldsymbol{\theta})=\left[\begin{array}{c}
\boldsymbol{\zeta} \\
\boldsymbol{X}^{\top} \boldsymbol{\Delta}
\end{array}\right]
$$

Vamos obter a quantidade $\widehat{\boldsymbol{V}}$. A função de distribuição acumulada de uma variável aleatória $Y_{i}$ que tem distribuição seno-hiperbólico normal é

$$
F_{i}\left(y_{i} ; \boldsymbol{\theta}\right)=\Phi\left[\frac{2}{\alpha} \operatorname{senh}\left(\frac{y_{i}-\mu_{i}}{2}\right)\right]=\Phi\left(\xi_{i 2}\right), \quad i=1,2, \ldots, n,
$$

em que $\Phi(\cdot)$ representa a função de distribuição acumulada da normal padrão; veja Rieck \& Nedelman (1991). Consequentemente, a densidade é da forma

$$
f_{i}\left(y_{i} ; \boldsymbol{\theta}\right)=\frac{1}{\alpha \sqrt{2 \pi}} \cosh \left(\frac{y_{i}-\mu_{i}}{2}\right) \exp \left\{-\frac{2}{\alpha^{2}} \operatorname{senh}^{2}\left(\frac{y_{i}-\mu_{i}}{2}\right)\right\}=\frac{\xi_{i 1}}{2 \sqrt{2 \pi}} \exp \left\{-\frac{1}{2} \xi_{i 2}^{2}\right\}
$$


veja a Seção 1.2. Note que a matriz $\boldsymbol{V}$, de dimensão $n \times(p+1)$, é da forma

$$
\boldsymbol{V}=\left[\begin{array}{cc}
-\frac{\partial F_{1}\left(y_{1} ; \boldsymbol{\theta}\right) / \partial \alpha}{f_{1}\left(y_{1} ; \boldsymbol{\theta}\right)} & -\frac{\partial F_{1}\left(y_{1} ; \boldsymbol{\theta}\right) / \partial \boldsymbol{\beta}}{f_{1}\left(y_{1} ; \boldsymbol{\theta}\right)} \\
\vdots & \vdots \\
-\frac{\partial F_{n}\left(y_{n} ; \boldsymbol{\theta}\right) / \partial \alpha}{f_{n}\left(y_{n} ; \boldsymbol{\theta}\right)} & -\frac{\partial F_{n}\left(y_{n} ; \boldsymbol{\theta}\right) / \partial \boldsymbol{\beta}}{f_{n}\left(y_{n} ; \boldsymbol{\theta}\right)}
\end{array}\right]
$$

Vamos obter primeiramente

$$
\frac{\partial F_{i}\left(y_{i} ; \boldsymbol{\theta}\right) / \partial \boldsymbol{\beta}}{f_{i}\left(y_{i} ; \boldsymbol{\theta}\right)}, \quad i=1,2, \ldots, n
$$

Note que

$$
\frac{\partial F_{i}\left(y_{i} ; \boldsymbol{\theta}\right)}{\partial \boldsymbol{\beta}}=\frac{\partial \Phi\left(\xi_{i 2}\right)}{\partial \boldsymbol{\beta}}=-\frac{\xi_{i 1}}{2 \sqrt{2 \pi}} \exp \left\{-\frac{1}{2} \xi_{i 2}^{2}\right\} \boldsymbol{x}_{i}^{\top}=-f_{i}\left(y_{i} ; \boldsymbol{\theta}\right) \boldsymbol{x}_{i}^{\top}
$$

Daí, temos

$$
\frac{\partial F_{i}\left(y_{i} ; \boldsymbol{\theta}\right) / \partial \boldsymbol{\beta}}{f_{i}\left(y_{i} ; \boldsymbol{\theta}\right)}=\frac{-f_{i}\left(y_{i} ; \boldsymbol{\theta}\right) \boldsymbol{x}_{i}^{\top}}{f_{i}\left(y_{i} ; \boldsymbol{\theta}\right)}=-\boldsymbol{x}_{i}^{\top}, \quad i=1,2, \ldots, n
$$

Da mesma forma,

$$
\frac{\partial F_{i}\left(y_{i} ; \boldsymbol{\theta}\right)}{\partial \alpha}=-\frac{\xi_{i 2}}{\alpha \sqrt{2 \pi}} \exp \left\{-\frac{1}{2} \xi_{i 2}^{2}\right\}
$$

Dai, temos que

$$
\frac{\partial F_{i}\left(y_{i} ; \boldsymbol{\theta}\right) / \partial \alpha}{f_{i}\left(y_{i} ; \boldsymbol{\theta}\right)}=-\frac{\xi_{i 2}}{\alpha \sqrt{2 \pi}} \exp \left\{-\frac{1}{2} \xi_{i 2}^{2}\right\} / f_{i}\left(y_{i} ; \boldsymbol{\theta}\right)=-\frac{2}{\alpha} \frac{\xi_{i 2}}{\xi_{i 1}}, \quad i=1,2, \ldots, n .
$$

Seja o vetor $n$-dimensional $\varphi=\varphi(\theta)$ definido como

$$
\boldsymbol{\varphi}=\boldsymbol{\varphi}(\boldsymbol{\theta})=\frac{2}{\alpha}\left(\begin{array}{llll}
\frac{\xi_{12}}{\xi_{11}} & \frac{\xi_{22}}{\xi_{21}} & \cdots & \frac{\xi_{n 2}}{\xi_{n 1}}
\end{array}\right)^{\top} .
$$

Então, a matriz $\boldsymbol{V}=\boldsymbol{V}(\boldsymbol{\theta})$ pode ser escrita na forma

$$
\boldsymbol{V}=\boldsymbol{V}(\boldsymbol{\theta})=\left[\begin{array}{ll}
\varphi & \boldsymbol{X}
\end{array}\right]
$$

A matriz $\widehat{\boldsymbol{V}}$ é obtida avaliando-se $\boldsymbol{V}$ em $\boldsymbol{\theta}=\widehat{\boldsymbol{\theta}}$, isto é, $\widehat{\boldsymbol{V}}=\left.\boldsymbol{V}\right|_{\boldsymbol{\theta}=\widehat{\boldsymbol{\theta}}}$.

Assim, como as quantidades $\boldsymbol{l}_{;}(\boldsymbol{\theta}), \boldsymbol{l}_{\boldsymbol{\theta} ; \boldsymbol{y}}(\boldsymbol{\theta})$ e $\boldsymbol{V}(\boldsymbol{\theta})$, que são usadas para obter $\widetilde{U}$, foram calculadas, notando que $\widetilde{\boldsymbol{l}}_{\boldsymbol{\beta} ; \widehat{\boldsymbol{\theta}}}(\boldsymbol{\theta})$ é obtido de $\widetilde{\boldsymbol{l}}_{\boldsymbol{\theta} ; \widehat{\boldsymbol{\theta}}}(\boldsymbol{\theta})$ excluindo-se a primeira linha dessa matriz, podemos obter $\widetilde{R}^{*}$ como em (4.36) considerando $\widetilde{U}$ como em (4.37). Note que $\widetilde{\boldsymbol{l}}_{\boldsymbol{\beta} ; \widehat{\boldsymbol{\theta}}}(\boldsymbol{\theta})$ é de dimensão $p \times(p+1)$.

\subsubsection{Hipótese sobre $\beta_{r}, r=1,2, \ldots, p$}

Vimos na Seção 1.3 que $\beta_{r}(r=1,2, \ldots, p)$ é ortogonal a $\alpha$. Porém, $\beta_{r}$ não é ortogonal a $\beta_{s}$ com $r \neq s(r, s=1,2, \ldots, p)$. Entretanto, usando resultados de Cox \& Reid $(1987,1993)$, mostraremos que é possível encontrar uma parametrização ortogonal de forma que $\beta_{r}$ ( $r$-ésimo parâmetro de interesse) seja globalmente ortogonal aos parâmetros de perturbação do modelo. Assim, a aproximação proposta por DiCiccio \& Martin (1993) pode ser aplicada para fazer inferência sobre $\beta_{r}$. Além, claro, das outras 
aproximações.

Como foi mencionado anteriormente [veja a Seção 4.3], podemos testar hipóteses do tipo:

$$
\begin{array}{lll}
\mathcal{H}_{0}: \beta_{r} \leq \beta_{r}^{(0)} & \text { versus } & \mathcal{H}_{1}: \beta_{r}>\beta_{r}^{(0)} \\
\mathcal{H}_{0}: \beta_{r} \geq \beta_{r}^{(0)} & \text { versus } & \mathcal{H}_{1}: \beta_{r}<\beta_{r}^{(0)} \\
\mathcal{H}_{0}: \beta_{r}=\beta_{r}^{(0)} & \text { versus } & \mathcal{H}_{1}: \beta_{r} \neq \beta_{r}^{(0)}
\end{array}
$$

Aqui, $\beta_{r}^{(0)}$ é uma constante conhecida. Neste caso, $\boldsymbol{\theta}^{*}=\left(\alpha, \boldsymbol{\beta}^{*}\right)$ é um vetor linha $p$-dimensional de parâmetros de perturbação, sendo $\boldsymbol{\beta}^{*}=\left(\beta_{1}, \ldots, \beta_{r-1}, \beta_{r+1}, \ldots, \beta_{p}\right)$. Adicionalmente, seja $\widehat{\boldsymbol{\theta}}=$ $(\widehat{\alpha}, \widehat{\boldsymbol{\beta}})$ o estimador de máxima verossimihança irrestrito de $\boldsymbol{\theta}$ e $\widetilde{\boldsymbol{\theta}}=(\widetilde{\alpha}, \widetilde{\boldsymbol{\beta}})$ o estimador de máxima verossimilhança de $\boldsymbol{\theta}$ restrito sob a hipótese nula, sendo $\widetilde{\boldsymbol{\beta}}=\left(\widetilde{\beta}_{1}, \ldots, \widetilde{\beta}_{r-1}, \beta_{r}^{(0)}, \widetilde{\beta}_{r+1}, \ldots, \widetilde{\beta}_{p}\right)$. Vale lembrar que neste caso a estatística da razão de verossimilhanças sinalizada é dada por

$$
R=\operatorname{sgn}\left(\widehat{\beta}_{r}-\beta_{r}^{(0)}\right)[2\{l(\widehat{\boldsymbol{\theta}})-l(\widetilde{\boldsymbol{\theta}})\}]^{1 / 2} ;
$$

veja a Seção 4.3.

Seja $\boldsymbol{X}^{*}=\left(\boldsymbol{x}_{1}^{*} \boldsymbol{x}_{2}^{*} \cdots \boldsymbol{x}_{n}^{*}\right)^{\top}$ uma matriz $n \times(p-1)$, em que $\boldsymbol{x}_{i}^{* \top}=\left(x_{i 1}, \ldots, x_{i(r-1)}, x_{i(r+1)}, \ldots, x_{i p}\right)$ representa sua $i$-ésima linha, ou seja, $\boldsymbol{X}^{*}$ é a matriz $\boldsymbol{X}$ sem a $r$-ésima coluna. De (4.28), temos que a matriz de informação observada para o vetor de parâmetros $\boldsymbol{\theta}^{*}=\left(\alpha, \boldsymbol{\beta}^{*}\right)$ pode ser escrita na forma

$$
\boldsymbol{j}_{\boldsymbol{\theta}^{*} \boldsymbol{\theta}^{*}}(\boldsymbol{\theta})=\left[\begin{array}{cc}
j_{\alpha \alpha} & \boldsymbol{j}_{\alpha \boldsymbol{\beta}^{*}} \\
\boldsymbol{j}_{\boldsymbol{\beta}^{* \alpha}} & \boldsymbol{j}_{\boldsymbol{\beta}^{*} \boldsymbol{\beta}^{*}}
\end{array}\right]=\left[\begin{array}{cc}
\nu & \boldsymbol{k}^{\top} \boldsymbol{X}^{*} \\
\boldsymbol{X}^{* \top} \boldsymbol{k} & \boldsymbol{X}^{* \top} \boldsymbol{W} \boldsymbol{X}^{*}
\end{array}\right],
$$

em que $\nu, \boldsymbol{k}$ e $\boldsymbol{W}$ foram definidos anteriormente.

\section{Aproximação $\widehat{R}^{*}$}

A estatística $\widehat{R}^{*}$ é da forma

$$
\widehat{R}^{*}=R+\frac{1}{R} \log (\widehat{U} / R)
$$

em que

$$
\widehat{U}=\left|\begin{array}{c}
\widehat{\boldsymbol{l}}_{; \widehat{\boldsymbol{\theta}}}(\widehat{\boldsymbol{\theta}})-\widehat{\boldsymbol{l}}_{: \widehat{\boldsymbol{\theta}}}(\widetilde{\boldsymbol{\theta}}) \\
\widehat{\boldsymbol{l}}_{\boldsymbol{\theta}^{*} ; \widehat{\boldsymbol{\theta}}}(\tilde{\tilde{\boldsymbol{\theta}}})
\end{array}\right| /\left|\boldsymbol{j}_{\boldsymbol{\theta}^{*} \boldsymbol{\theta}^{*}}(\widetilde{\boldsymbol{\theta}})\right|^{1 / 2}|\widehat{\boldsymbol{j}}|^{1 / 2}
$$

com

$$
\widehat{\boldsymbol{l}}_{; \widehat{\boldsymbol{\theta}}}(\widehat{\boldsymbol{\theta}})-\widehat{\boldsymbol{l}}_{; \widehat{\boldsymbol{\theta}}}(\widetilde{\boldsymbol{\theta}})=[\widehat{\boldsymbol{Q}}(\widehat{\boldsymbol{\theta}} ; \widehat{\boldsymbol{\theta}})-\widehat{\boldsymbol{Q}}(\widetilde{\boldsymbol{\theta}} ; \widehat{\boldsymbol{\theta}})] \widehat{\boldsymbol{i}}(\widehat{\boldsymbol{\theta}})^{-1} \widehat{\boldsymbol{j}} \quad \text { e } \quad \widehat{\boldsymbol{l}}_{\boldsymbol{\theta} ; \widehat{\boldsymbol{\theta}}}(\widetilde{\boldsymbol{\theta}})=\widehat{\boldsymbol{I}}(\widetilde{\boldsymbol{\theta}} ; \widehat{\boldsymbol{\theta}}) \widehat{\boldsymbol{i}}(\widehat{\boldsymbol{\theta}})^{-1} \widehat{\boldsymbol{j}}
$$

As quantidades $\widehat{\boldsymbol{Q}}\left(\boldsymbol{\theta} ; \boldsymbol{\theta}_{0}\right)$ e $\widehat{\boldsymbol{I}}\left(\boldsymbol{\theta} ; \boldsymbol{\theta}_{0}\right)$, que são usadas no cálculo de $\widehat{U}$, já foram calculadas anteriormente, expressões (4.34) e (4.35), respectivamente. A quantidade $\widehat{\boldsymbol{l}}_{\boldsymbol{\theta}^{*} ; \widehat{\boldsymbol{\theta}}}(\boldsymbol{\theta})$ é obtida de $\widehat{\boldsymbol{l}}_{\boldsymbol{\theta} ; \widehat{\boldsymbol{\theta}}}(\boldsymbol{\theta})$ excluindo-se a $(r+1)$-ésima linha dessa matriz, com $r=1,2, \ldots, p$. Portanto, calculamos $\widehat{R}^{*}$ como em (4.41) considerando $\widehat{U}$ como em (4.42). Note que $\widehat{\boldsymbol{l}}_{\boldsymbol{\theta}^{*} ; \widehat{\boldsymbol{\theta}}}(\boldsymbol{\theta})$ é de dimensão $p \times(p+1)$.

\section{Aproximação $\widetilde{R}^{*}$}

A estatística $\widetilde{R}^{*}$ é da forma

$$
\widetilde{R}^{*}=R+\frac{1}{R} \log (\widetilde{U} / R),
$$


em que

$$
\widetilde{U}=\left|\begin{array}{c}
\widetilde{\boldsymbol{l}}_{; \widehat{\boldsymbol{\theta}}}(\widehat{\boldsymbol{\theta}})-\widetilde{\boldsymbol{l}}_{; \widehat{\boldsymbol{\theta}}}(\widetilde{\boldsymbol{\theta}}) \\
\widetilde{\boldsymbol{l}}_{\boldsymbol{\theta}^{*} ; \widehat{\boldsymbol{\theta}}}(\tilde{\boldsymbol{\theta}})
\end{array}\right| /\left|\boldsymbol{j}_{\boldsymbol{\theta}^{*} \boldsymbol{\theta}^{*}}(\widetilde{\boldsymbol{\theta}})\right|^{1 / 2}|\widehat{\boldsymbol{j}}|^{1 / 2}
$$

com

$$
\widetilde{\boldsymbol{l}}_{; \widehat{\boldsymbol{\theta}}}(\boldsymbol{\theta})=\boldsymbol{l}_{\boldsymbol{y} \boldsymbol{y}}(\boldsymbol{\theta}) \widehat{\boldsymbol{V}}\left(\boldsymbol{l}_{\boldsymbol{\theta} ; \boldsymbol{y}}(\widehat{\boldsymbol{\theta}}) \widehat{\boldsymbol{V}}\right)^{-1} \widehat{\boldsymbol{j}} \quad \text { e } \quad \widetilde{\boldsymbol{l}}_{\boldsymbol{\theta} ; \widehat{\boldsymbol{\theta}}}(\boldsymbol{\theta})=\boldsymbol{l}_{\boldsymbol{\theta} ; \boldsymbol{y}}(\boldsymbol{\theta}) \widehat{\boldsymbol{V}}\left(\boldsymbol{l}_{\boldsymbol{\theta} ; \boldsymbol{y}}(\widehat{\boldsymbol{\theta}}) \widehat{\boldsymbol{V}}\right)^{-1} \widehat{\boldsymbol{j}}
$$

Adicionalmente,

$$
\boldsymbol{l}_{; \boldsymbol{y}}(\boldsymbol{\theta})=\frac{\partial l(\boldsymbol{\theta})}{\partial \boldsymbol{y}}, \quad \boldsymbol{l}_{\boldsymbol{\theta} ; \boldsymbol{y}}(\boldsymbol{\theta})=\frac{\partial \boldsymbol{l}_{; \boldsymbol{y}}(\boldsymbol{\theta})}{\partial \boldsymbol{\theta}^{\top}}
$$

$\mathrm{e}$

$$
\boldsymbol{V}=\left[\begin{array}{c}
-\frac{\partial F_{1}\left(y_{1} ; \boldsymbol{\theta}\right) / \partial \boldsymbol{\theta}}{f_{1}\left(y_{1} ; \boldsymbol{\theta}\right)} \\
\vdots \\
-\frac{\partial F_{n}\left(y_{n} ; \boldsymbol{\theta}\right) / \partial \boldsymbol{\theta}}{f_{n}\left(y_{n} ; \boldsymbol{\theta}\right)}
\end{array}\right]
$$

sendo $\widehat{\boldsymbol{V}}=\left.\boldsymbol{V}\right|_{\boldsymbol{\theta}=\widehat{\boldsymbol{\theta}}}$.

As quantidades $\boldsymbol{l}_{; \boldsymbol{y}}(\boldsymbol{\theta}), \boldsymbol{l}_{\boldsymbol{\theta} ; \boldsymbol{y}}(\boldsymbol{\theta})$ e $\boldsymbol{V}(\boldsymbol{\theta})$, que são usadas no cálculo de $\widetilde{U}$, já foram calculadas anteriormente, expressões (4.38), (4.39) e (4.40), respectivamente. A quantidade $\widetilde{\boldsymbol{l}}_{\boldsymbol{\theta}^{*} ; \hat{\boldsymbol{\theta}}}(\boldsymbol{\theta})$ é obtida de $\widetilde{\boldsymbol{l}}_{\boldsymbol{\theta} ; \widehat{\boldsymbol{\theta}}}(\boldsymbol{\theta})$ excluindo-se a $(r+1)$-ésima linha dessa matriz, com $r=1,2, \ldots, p$. Portanto, calculamos $\widetilde{R}^{*}$ como em (4.43) considerando $\widetilde{U}$ como em (4.44). Note que $\widetilde{\boldsymbol{l}}_{\boldsymbol{\theta}^{*} ; \widehat{\boldsymbol{\theta}}}(\boldsymbol{\theta})$ é de dimensão $p \times(p+1)$.

\section{Aproximação $R_{o}^{*}$}

Inicialmente, apresentaremos uma parametrização ortogonal de forma a tornar $\beta_{r}$ ( $r$-ésimo parâmetro de interesse) ortogonal aos parâmetros de perturbação. É necessário obter uma transformação $\boldsymbol{\theta}=\left(\alpha, \boldsymbol{\beta}^{*}, \beta_{r}\right) \rightarrow \boldsymbol{\psi}=\left(\alpha, \boldsymbol{\gamma}, \beta_{r}\right)$ de modo que $\mathbb{E}\left(-\partial l^{*} / \partial \beta_{r} \partial \boldsymbol{\gamma}\right)=\{\mathbf{0}\}_{1 \times p}$. Se denotarmos a $r$-ésima coluna $(r=1,2, \ldots, p)$ da matriz $\boldsymbol{X}$ por $\widetilde{\boldsymbol{x}}_{r}=\left(x_{1 r}, x_{2 r}, \ldots, x_{n r}\right)^{\top}$, a matriz de informação de Fisher $\boldsymbol{i}(\boldsymbol{\theta})$ dada em (4.29) pode ser reescrita na forma

$$
\boldsymbol{i}\left(\alpha, \boldsymbol{\beta}^{*}, \beta_{r}\right)=\left[\begin{array}{ccc}
i_{\alpha \alpha} & \boldsymbol{i}_{\alpha \beta^{*}} & i_{\alpha \beta_{r}} \\
\boldsymbol{i}_{\boldsymbol{\beta}^{*} \alpha} & \boldsymbol{i}_{\boldsymbol{\beta}^{*} \boldsymbol{\beta}^{*}} & \boldsymbol{i}_{\boldsymbol{\beta}^{*} \beta_{r}} \\
i_{\beta_{r} \alpha} & \boldsymbol{i}_{\beta_{r} \boldsymbol{\beta}^{*}} & i_{\beta_{r} \beta_{r}}
\end{array}\right]=\left[\begin{array}{ccc}
2 n / \alpha^{2} & \{\mathbf{0}\}_{1 \times(p-1)} & 0 \\
\{\boldsymbol{0}\}_{(p-1) \times 1} & h(\alpha) \boldsymbol{X}^{* \top} \boldsymbol{X}^{*} / 4 & h(\alpha) \boldsymbol{X}^{* \top} \widetilde{\boldsymbol{x}}_{r} / 4 \\
0 & h(\alpha) \widetilde{\boldsymbol{x}}_{r}^{\top} \boldsymbol{X}^{*} / 4 & h(\alpha) \widetilde{\boldsymbol{x}}_{r}^{\top} \widetilde{\boldsymbol{x}}_{r} / 4
\end{array}\right] .
$$

Seguindo Cox \& Reid $(1987,1993)$, chega-se à transformação desejada através de uma solução do sistema de equações diferenciais

$$
\frac{\partial \boldsymbol{\beta}^{*}}{\partial \beta_{r}}=-\boldsymbol{i}_{\beta_{r} \boldsymbol{\beta}^{*}} \boldsymbol{i}_{\boldsymbol{\beta}^{*} \boldsymbol{\beta}^{*}}^{-1}
$$

ou seja,

$$
\frac{\partial \boldsymbol{\beta}^{*}}{\partial \beta_{r}}=-\widetilde{\boldsymbol{x}}_{r}^{\top} \boldsymbol{X}^{*}\left(\boldsymbol{X}^{* \top} \boldsymbol{X}^{*}\right)^{-1}
$$

Uma possível solução para a equação diferencial acima é

$$
\boldsymbol{\gamma}=\boldsymbol{\beta}^{*}+\beta_{r} \widetilde{\boldsymbol{x}}_{r}^{\top} \boldsymbol{X}^{*}\left(\boldsymbol{X}^{* \top} \boldsymbol{X}^{*}\right)^{-1}
$$


Note que $\gamma$ é um vetor linha de dimensão $p-1$. A função de log-verossimilhança para o modelo reparametrizado é da forma

$$
l^{*}(\boldsymbol{\psi})=-\frac{n}{2} \log (8 \pi)+\sum_{i=1}^{n} \log \left(\xi_{i 1}^{*}\right)-\frac{1}{2} \sum_{i=1}^{n} \xi_{i 2}^{* 2},
$$

em que $\boldsymbol{\psi}=\left(\alpha, \gamma, \beta_{r}\right)$,

$$
\xi_{i 1}^{*}=\xi_{i 1}^{*}(\boldsymbol{\psi})=\frac{2}{\alpha} \cosh \left(\frac{y_{i}-\mu_{i}^{*}}{2}\right), \quad \xi_{i 2}^{*}=\xi_{i 2}^{*}(\boldsymbol{\psi})=\frac{2}{\alpha} \operatorname{senh}\left(\frac{y_{i}-\mu_{i}^{*}}{2}\right)
$$

e $\mu_{i}^{*}=\boldsymbol{\gamma} \boldsymbol{x}_{i}^{*}+\beta_{r}\left(x_{i r}-\widetilde{\boldsymbol{x}}_{r}^{\top} \boldsymbol{X}^{*}\left(\boldsymbol{X}^{* \top} \boldsymbol{X}^{*}\right)^{-1} \boldsymbol{x}_{i}^{*}\right)$, com $i=1,2, \ldots, n$. As derivadas de primeira e segunda ordens e as matrizes de informação observada e esperada do modelo reparametrizado estão dadas no Apêndice D.

Como foi mostrado no Apêndice $\mathrm{D}, \beta_{r}$ é globalmente ortogonal ao vetor de parâmetros de perturbação $\boldsymbol{\lambda}=(\alpha, \gamma)$. Deste modo, a estatística $R_{o}^{*}$ é da forma

$$
R_{o}^{*}=R+\frac{1}{R} \log \left(U_{o} / R\right)
$$

em que

$$
U_{o}=l_{\beta_{r}}^{*}(\widetilde{\boldsymbol{\psi}}) \frac{\left|\boldsymbol{j}_{\lambda \boldsymbol{\lambda}}^{*}(\widetilde{\boldsymbol{\psi}})\right|^{1 / 2} i_{\beta_{r} \beta_{r}}^{*}(\widehat{\boldsymbol{\psi}})^{1 / 2}}{\left|\boldsymbol{j}^{*}(\widehat{\boldsymbol{\psi}})\right|^{1 / 2} i_{\beta_{r} \beta_{r}}^{*}(\widetilde{\boldsymbol{\psi}})^{1 / 2}},
$$

em que $\widehat{\boldsymbol{\psi}}=\left(\widehat{\alpha}, \widehat{\boldsymbol{\gamma}}, \widehat{\beta}_{r}\right)$ e $\widetilde{\boldsymbol{\psi}}=\left(\widetilde{\alpha}, \widetilde{\boldsymbol{\gamma}}, \beta_{r}^{(0)}\right)$ são, respectivamente, os estimadores de máxima verossimilhança irrestrito e restrito de $\boldsymbol{\psi}$.

As quantidades necessárias para se obter $U_{o}$ são dadas a seguir. Inicialmente, note que a função escore de $\beta_{r}$ pode ser escrita na forma [veja Apêndice D]

$$
l_{\beta_{r}}^{*}(\psi)=\frac{1}{2} \overline{\boldsymbol{x}}_{r}^{\top} \boldsymbol{s}^{*}
$$

em que $\boldsymbol{s}^{*}=\boldsymbol{s}^{*}(\boldsymbol{\psi})$ é um vetor $n$-dimensional com $i$-ésimo elemento dado por $\xi_{i 1}^{*} \xi_{i 2}^{*}-\xi_{i 2}^{*} / \xi_{i 1}^{*}$. As outras quantidades são $\left|\boldsymbol{j}^{*}(\boldsymbol{\psi})\right|^{1 / 2}=\nu^{* 1 / 2}\left|\boldsymbol{Z}^{* \top} \boldsymbol{H}^{*} \boldsymbol{Z}^{*}\right|^{1 / 2},\left|\boldsymbol{j}_{\boldsymbol{\lambda} \boldsymbol{\lambda}}^{*}(\boldsymbol{\psi})\right|^{1 / 2}=\nu^{* 1 / 2}\left|\boldsymbol{X}^{* \top} \boldsymbol{H}^{*} \boldsymbol{X}^{*}\right|^{1 / 2}$ e $i_{\beta_{r} \beta_{r}}^{*}(\boldsymbol{\psi})=$ $h(\alpha) \overline{\boldsymbol{x}}_{r}^{\top} \overline{\boldsymbol{x}}_{r} / 4$; veja Apêndice D para a definição das quantidades com "*". Assim, após álgebra simples, $U_{o}$ pode ser escrito na forma

$$
U_{o}=\frac{\overline{\boldsymbol{x}}_{r}^{\top} \widetilde{\boldsymbol{s}}^{*}}{2}\left(\frac{\widetilde{\nu}^{*} \widehat{h}}{\widehat{\nu}^{*} \widetilde{h}}\right)^{1 / 2} \frac{\left|\boldsymbol{X}^{* \top} \widetilde{\boldsymbol{H}}^{*} \boldsymbol{X}^{*}\right|^{1 / 2}}{\left|\boldsymbol{Z}^{* \top} \widehat{\boldsymbol{H}}^{*} \boldsymbol{Z}^{*}\right|^{1 / 2}},
$$

em que $\widetilde{\boldsymbol{s}}^{*}=\boldsymbol{s}^{*}(\widetilde{\boldsymbol{\psi}}), \widetilde{\nu}^{*}=\nu^{*}(\widetilde{\boldsymbol{\psi}}), \widehat{\nu}^{*}=\nu^{*}(\widehat{\boldsymbol{\psi}}), \widehat{h}=h(\widehat{\alpha}), \widetilde{h}=h(\widetilde{\alpha}), \widehat{\boldsymbol{H}}^{*}=\boldsymbol{H}^{*}(\widehat{\boldsymbol{\psi}})$ e $\widetilde{\boldsymbol{H}}^{*}=\boldsymbol{H}^{*}(\widetilde{\boldsymbol{\psi}})$. Portanto, podemos calcular $R_{o}^{*}$ dado em (4.46) com $U_{o}$ dado em (4.47).

\subsection{Resultados Numéricos}

Apresentamos a seguir alguns resultados de simulações Monte Carlo para avaliar o desempenho da estatística da razão de verossimilhanças sinalizada, $R$, e das versões aproximadas de $R^{*}$, ou seja, $R_{o}^{*}, \widehat{R}^{*}$ e $\widetilde{R}^{*}$, no modelo de regressão Birnbaum-Saunders em amostras finitas. O desempenho de 
cada teste é avaliado em função da proximidade das probabilidades de rejeição da hipótese nula, sendo esta verdadeira (probabilidade do erro tipo I), aos respectivos níveis nominais dos testes e dos poderes dos testes.

As simulações realizadas foram baseadas no modelo de regressão

$$
y_{i}=\beta_{1} x_{i 1}+\beta_{2} x_{i 2}+\cdots+\beta_{p} x_{i p}+\varepsilon_{i}
$$

em que $x_{i 1}=1$ e $\varepsilon_{i} \sim \mathcal{S N}(\alpha, 0,2), i=1,2, \ldots, n$. As covariáveis foram geradas como amostras aleatórias da distribuição $\mathcal{U}(0,1)$. Estas covariáveis foram mantidas fixas no esquema Monte Carlo. O número de réplicas Monte Carlo foi fixado em 10000 e foram considerados os seguintes níveis nominais: $\gamma=10 \%, 5 \%$ e $1 \%$. Todas as simulações foram realizadas utilizando a linguagem de programação matricial 0x (Doornik, 2006). Todas as entradas das tabelas apresentadas são porcentagens.

Em particular, consideramos hipóteses do tipo: (i) $\mathcal{H}_{0}: \beta_{p} \leq 0$ versus $\mathcal{H}_{1}: \beta_{p}>0 ; \mathcal{H}_{0}: \beta_{p} \geq 0$ versus $\mathcal{H}_{1}: \beta_{p}<0$; e $\mathcal{H}_{0}: \beta_{p}=0$ versus $\mathcal{H}_{1}: \beta_{p} \neq 0$. Neste caso, sem perda de generalidade, a variável resposta foi gerada assumindo que $\beta_{1}=\beta_{2}=\cdots=\beta_{p-1}=1$ considerando $\alpha=0.5$ e 1.5 . (ii) $\mathcal{H}_{0}: \alpha \leq \alpha^{(0)}$ versus $\mathcal{H}_{1}: \alpha>\alpha^{(0)} ; \mathcal{H}_{0}: \alpha \geq \alpha^{(0)}$ versus $\mathcal{H}_{1}: \alpha<\alpha^{(0)} ;$ e $\mathcal{H}_{0}: \alpha=\alpha^{(0)}$ versus $\mathcal{H}_{1}: \alpha \neq \alpha^{(0)}$. Neste caso, a variável resposta foi gerada assumindo que $\beta_{1}=\beta_{2}=\cdots=\beta_{p}=1 \mathrm{e}$ $\alpha=\alpha^{(0)}, \operatorname{com} \alpha^{(0)}=0.5$.

Nas Tabelas 4.1 e 4.2, apresentamos as taxas de rejeição empíricas dos testes com base na estatística da razão de verossimilhanças sinalizada, $R$, e três de suas versões modificadas, $R_{o}^{*}, \widehat{R}^{*}$ e $\widetilde{R}^{*}$, considerando hipóteses sobre o parâmetro $\beta_{p}$ para um tamanho amostral $n=20$ com $\alpha=0.5$ e 1.5. Adicionalmente, diferentes valores para $p$ (dimensão do vetor $\boldsymbol{\beta}$ ) foram considerados. Variamos $p$ para analisar o efeito do número de covariáveis presentes no modelo sobre os diferentes testes. Na Tabela 4.1, caso em que $\alpha=0.5$, observe que o teste da razão de verossimilhanças sinalizada usual, $R$, é extremamente liberal, isto é, suas taxas de rejeição estão muito acima dos níveis de significância considerados, principalmente quando o número de regressores presentes no modelo aumenta. Por exemplo, quando $p=8, \gamma=5 \%$ e $\mathcal{H}_{0}: \beta_{p} \leq 0$, a taxa de rejeição do teste foi $10.43 \%$, mais que o dobro do nível nominal considerado, ou seja, uma distorção de tamanho considerável. A tendência do teste de rejeitar com frequência demasiada a hipótese nula é atenuada pelo ajuste aplicado à estatística $R$ de forma que os testes da razão de verossimilhanças sinalizadas corrigidos, $R_{o}^{*}, \widehat{R}^{*}$ e $\widetilde{R}^{*}$, apresentam taxas de rejeição mais próximas dos níveis de significância considerados. Entretanto, assim como $R$, note que o teste com base em $\widehat{R}^{*}$ também é afetado pelo número de regressores presentes no modelo, porém, em direção contrária, isto é, à medida que o valor de $p$ aumenta, suas taxas de rejeição empíricas diminuem. Por outro lado, $R_{o}^{*}$ e $\widetilde{R}^{*}$ não sofrem tal influência e apresentam taxas de rejeição muito próximas dos respectivos níveis nominais em todos os casos. Por exemplo, as taxas de rejeição foram $5.51 \%, 2.78 \%$ e $5.59 \%$ para os testes $R_{o}^{*}, \widehat{R}^{*}$ e $\widetilde{R}^{*}$, respectivamente, considerando a mesma situação anterior. Na Tabela 4.2, caso em que $\alpha=1.5$, note que os resultados são similares aos da Tabela 4.1; compare as duas tabelas.

Na Tabela 4.3, fixamos o valor de $p$ em 5 e variamos o tamanho da amostra em $n=10,20,30$, 40, 50, 100, 200 e 500, considerando $\alpha=0.5$. Note que, com o aumento do tamanho da amostra, as taxas de rejeição empíricas do teste com base em $R$ se aproximam do nível nominal, como esperado. Adicionalmente, em todos os tamanhos de amostra, os testes $R_{o}^{*}, \widehat{R}^{*}$ e $\widetilde{R}^{*}$ apresentam taxas de rejeição muito próximas do níveis nominais considerados. Por exemplo, quando $n=30, \gamma=10 \%$ e $\mathcal{H}_{0}: \beta_{5} \geq 0$, as taxas de rejeição empíricas dos testes $R, R_{o}^{*}, \widehat{R}^{*}$ e $\widetilde{R}^{*}$ foram $12.34 \%, 10.19 \%, 9.95 \%$ e $10.27 \%$, respectivamente. É claro que $n=20$ equivale ao caso da Tabela 4.1 quando $p=5$. Observe 
ainda que até mesmo para uma tamanho amostral pequeno, $n=10$, as taxas de rejeição empíricas dos testes $R_{o}^{*}, \widehat{R}^{*}$ e $\widetilde{R}^{*}$ estão próximas dos níveis nominais, enquanto que o teste $R$ é extremamente liberal. Por exemplo, quando $n=10, \gamma=5 \%$ e $\mathcal{H}_{0}: \beta_{5} \leq 0$, as taxas de rejeição empíricas dos testes $R, R_{o}^{*}, \widehat{R}^{*}$ e $\widetilde{R}^{*}$ foram $12.88 \%, 6.06 \%, 5.26 \%$ e $6.17 \%$, respectivamente.

$\mathrm{Na}$ Tabela 4.4, apresentamos os resultados de simulação para as taxas de rejeição empíricas não nulas (poder). Fixamos $p=2, \alpha=0.5$ e consideramos diferentes tamanhos amostrais, isto é, $n=10,20$ e 30. As taxas de rejeição foram obtidas levando-se em consideração as hipóteses alternativas $\mathcal{H}_{1}: \beta_{2}>\delta_{1}, \mathcal{H}_{1}: \beta_{2}<\delta_{2}$ e $\mathcal{H}_{1}: \beta_{2} \neq \delta_{3}$, em que diferentes valores para $\delta_{1}, \delta_{2}$ e $\delta_{3}$ são considerados. Adicionalmente, foi usado como ponto crítico para os testes o percentil da distribuição normal padrão. Somente consideramos os poderes dos testes $R_{o}^{*}, \widehat{R}^{*}$ e $\widetilde{R}^{*}$, uma vez que o teste da razão de verossimilhanças sinalizada usual, $R$, é consideravelmente distorcido, isto é, apresenta erros do tipo I empíricos distantes dos níveis nominais; veja Tabelas 4.1, 4.2 e 4.3. Observe que os poderes dos três testes são similares, com leve vantagem para o teste com base na estatística $\widetilde{R}^{*}$. Por exemplo, quando $n=30, \mathcal{H}_{1}: \beta_{2}<\delta_{2}$, com $\delta_{2}=-0.5$, os poderes dos testes $R_{o}^{*}$, $\widehat{R}^{*}$ e $\widetilde{R}^{*}$ foram $83.77 \%, 83.78 \%$ e $83.81 \%$, respectivamente. Observe também que à medida que o tamanho da amostra aumenta, os poderes crescem, como esperado.

Na Tabela 4.5, fixamos $p$ em 6 e variamos o valor de $\gamma$ (nível de significância) para um tamanho amostral $n=25$. Variamos $\gamma$ para observar como se comportam os testes com base nas estatísticas $R, R_{o}^{*}, \widehat{R}^{*}$ e $\widetilde{R}^{*}$ nas extremidades da distribuição. A variável resposta foi gerada assumindo que $\beta_{1}=\beta_{2}=\cdots=\beta_{5}=1 \mathrm{com} \alpha=0.5$. O número de réplicas Monte Carlo foi 100000. As hipóteses consideradas foram $\mathcal{H}_{0}: \beta_{6} \leq 0$ versus $\mathcal{H}_{1}: \beta_{6}>0$ e $\mathcal{H}_{0}: \beta_{6} \geq 0$ versus $\mathcal{H}_{1}: \beta_{6}<0$. Observe na Tabela 4.5 que, em todos os casos, o teste com base em $R$ é demasiadamente liberal. Por outro lado, os testes com base em $R_{o}^{*}, \widehat{R}^{*}$ e $\widetilde{R}^{*}$ apresentam taxas de rejeição empíricas muito próximas dos níveis nominais considerados, mesmo quando o valor de $\gamma$ é muito baixo. Por exemplo, para $\mathcal{H}_{0}: \beta_{6} \leq 0$ e $\gamma=0.05 \%$, as taxas de rejeição empíricas dos testes $R, R_{o}^{*}, \widehat{R}^{*}$ e $\widetilde{R}^{*}$ foram $0.23 \%, 0.05 \%, 0.03 \%$ e $0.05 \%$, respectivamente. Estes resultados evidenciam que, mesmo nas extremidades da distribuição, os testes com base nas estatísticas $R_{o}^{*}, \widehat{R}^{*}$ e $\widetilde{R}^{*}$ apresentam comportamento satisfatório.

$\mathrm{Na}$ Tabela 4.6, apresentamos as taxas de rejeição empíricas dos testes com base na estatística da razão de verossimilhanças sinalizada, $R$, e três de suas versões modificadas, $R_{o}^{*}, \widehat{R}^{*}$ e $\widetilde{R}^{*}$, considerando hipóteses sobre o parâmetro $\alpha$ para tamanho amostral $n=20$. Adicionalmente, consideramos diferentes valores para $p$. Observe que para a hipótese $\mathcal{H}_{0}: \alpha \leq \alpha^{(0)}$, o teste $R$ é bastante conservador, apresentando taxas de rejeição empíricas bem abaixo dos níveis nominais considerados e, à medida que o valor de $p$ aumenta, suas taxas de rejeição deterioram-se consideravelmente. Entretanto, os testes $R_{o}^{*}, \widehat{R}^{*}$ e $\widetilde{R}^{*}$ apresentam taxas de rejeição empíricas mais próximas dos níveis nominais, deteriorando-se lentamente à medida que $p$ cresce. Por exemplo, quando $p=3$ e $\gamma=5 \%$, as taxas de rejeição dos testes $R, R_{o}^{*}, \widehat{R}^{*}$ e $\widetilde{R}^{*}$ foram $1.56 \%, 7.81 \%, 7.87 \%$ e $7.79 \%$, respectivamente. Por outro lado, note que para a hipótese $\mathcal{H}_{0}: \alpha \geq \alpha^{(0)}$, o teste $R$ é extremamente liberal, apresentando taxas de rejeição empíricas muito acima dos níveis nominais considerados e, à medida que o valor de $p$ aumenta, suas taxas de rejeição deterioram-se demasiadamente. No entanto, os testes $R_{o}^{*}, \widehat{R}^{*}$ e $\widetilde{R}^{*}$ apresentam taxas de rejeição bem próximas dos níveis nominais, deteriorando-se muito lentamente à medida que $p$ cresce. Por exemplo, para $p=5$ e $\gamma=10 \%$, as taxas de rejeição dos testes $R, R_{o}^{*}, \widehat{R}^{*} \mathrm{e}$ $\widetilde{R}^{*}$ foram $41.04 \%, 12.91 \%, 12.90 \%$ e $12.93 \%$, respectivamente. Note ainda que em ambos as hipóteses, $\mathcal{H}_{0}: \alpha \leq \alpha^{(0)}$ e $\mathcal{H}_{0}: \alpha \geq \alpha^{(0)}$, os testes $R_{o}^{*}, \widehat{R}^{*}$ e $\widetilde{R}^{*}$ apresentam desempenhos muito similares, isto é, não foi possível destacar (selecionar) uma dentre estas três estatísticas de teste para inferência sobre $\alpha$, ao contrário dos testes sobre parâmetros de regressão, nos quais foi possível destacar (selecionar) 
$R_{o}$ e $\widetilde{R}^{*}$.

Uma justificativa ao fato de $R$ ser conservador para $\mathcal{H}_{0}: \alpha \leq \alpha^{(0)}$ e liberal para $\mathcal{H}_{0}: \alpha \geq \alpha^{(0)}$ é dada a seguir. Rieck \& Nedelman (1991) mostraram que o viés de segunda ordem de $\widehat{\alpha}$ é negativo ( $\alpha$ é subestimado por $\widehat{\alpha}$ ) e proporcional a $p$, isto é, se o valor de $p$ cresce, o viés de $\widehat{\alpha}$ aumenta (em valor absoluto). Como $R$ é da forma

$$
R=\operatorname{sgn}\left(\widehat{\alpha}-\alpha^{(0)}\right)[2\{l(\widehat{\boldsymbol{\theta}})-l(\widetilde{\boldsymbol{\theta}})\}]^{1 / 2},
$$

temos que, na maioria dos casos, $r$, o valor observado de $R$, será negativo, uma vez que $\operatorname{sgn}\left(\widehat{\alpha}-\alpha^{(0)}\right)$ será negativo na maioria das vezes. Como $\mathcal{H}_{0}: \alpha \leq \alpha^{(0)}$ é rejeitada se $r>z_{\gamma}$ e $\mathcal{H}_{0}: \alpha \geq \alpha^{(0)}$ é rejeitada se $r<-z_{\gamma}$, sendo $z_{\gamma}$ o quantil de ordem $100 \times(1-\gamma) \%$ da distribuição normal padrão, segue que, consequentemente, no primeiro caso, $R$ será conservador, enquanto que no segundo caso $R$ será liberal. Isto está de acordo com os resultados apresentados na Tabela 4.6.

Na Tabela 4.7, apresentamos os resultados de simulação para as taxas de rejeição empíricas não nulas (poder) considerando inferência sobre $\alpha$. Fixamos $p=7, n=20$ e $\alpha^{(0)}=0.5$. As taxas de rejeição foram obtidas levando-se em consideração a hipótese alternativa $\mathcal{H}_{1}: \alpha>\delta$, em que diferentes valores para $\delta$ são considerados. Consideramos tal hipótese pelo fato do teste com base em $R$ ter sido extremamente conservador nesta situação (veja Tabela 4.6). (À primeira vista, pode parecer uma característica desejável o fato de um teste ser conservativo, uma vez que nessa situação o erro tipo I ocorre com baixa probabilidade. No entanto, testes conservativos apresentam baixo poder.) Deste modo, busca-se identificar testes que tenham tamanhos empíricos razoavelmente próximos (ou menores) ao nível de significância nominal e, ao mesmo tempo, tenham um alto poder. Observe na Tabela 4.7 que o teste com base em $R$ foi o menos poderoso entre os quatro, como era de se esperar. Note ainda que os poderes dos testes com base em $R_{o}^{*}, \widehat{R}^{*}$ e $\widetilde{R}^{*}$ são similares. Por exemplo, para $\gamma=5 \%$ e $\delta=0.9$, os poderes dos testes $R, R_{o}^{*}, \widehat{R}^{*}$ e $\widetilde{R}^{*}$ foram $64.83 \%, 88.81 \%, 88.69 \%$ e $88.78 \%$, respectivamente. É claro que $\delta=0.5$ equivale ao caso da Tabela 4.6 quando $p=7$ e $\mathcal{H}_{0}: \alpha \leq \alpha^{(0)}$.

Em linhas gerais, o teste da razão de verossimilhanças sinalizada usual, $R$, apresentou taxas de rejeição empíricas bastantes distorcidas em amostras de tamanho pequeno e moderado; veja as Tabelas 4.1, 4.2, 4.3, 4.5 e 4.6. As aproximações para $R^{*}$, derivadas neste capítulo, fazem com que a taxa de rejeição empírica fique mais próxima do nível nominal do teste. Em relação a testes de hipóteses sobre um componente do vetor $\boldsymbol{\beta}$, observamos que os testes com base em $R_{o}^{*}$ e $\widetilde{R}^{*}$ apresentaram os melhores resultados, tanto em tamanho quanto em poder, do que teste com base em $\widehat{R}^{*}$; veja as Tabelas 4.1 a 4.4. Dessa forma, em aplicações práticas, recomendamos os testes com base em $R_{o}^{*}$ e $\widetilde{R}^{*}$ para fazer inferência sobre um componente de $\boldsymbol{\beta}$. Entretanto, deixamos sob a responsabilidade do pesquisador a escolha entre a utilização das estatísticas de testes $R_{o}^{*}$ ou $\widetilde{R}^{*}$, em pesquisas aplicadas. Em relação a testes de hipóteses sobre $\alpha$, observamos que os testes $R_{o}^{*}$, $\widehat{R}^{*}$ e $\widetilde{R}^{*}$ apresentaram desempenhos similares (veja Tabela 4.6). Assim, novamente, deixamos sob a responsabilidade do pesquisador a escolha dentre as estatísticas de testes $R_{o}^{*}, \widehat{R}^{*}$ ou $\widetilde{R}^{*}$, em pesquisas aplicadas. 
Tabela 4.1: Tamanhos empíricos dos testes $R, R_{o}^{*}, \widehat{R}^{*}$ e $\widetilde{R}^{*} \operatorname{com} \alpha=0.5$ e $n=20$.

\begin{tabular}{|c|c|c|c|c|c|c|c|c|c|c|c|c|}
\hline \multirow[b]{3}{*}{$p$} & \multicolumn{12}{|c|}{$\mathcal{H}_{0}: \beta_{p} \leq 0$ versus $\mathcal{H}_{1}: \beta_{p}>0$} \\
\hline & \multicolumn{4}{|c|}{$\gamma=10 \%$} & \multicolumn{4}{|c|}{$\gamma=5 \%$} & \multicolumn{4}{|c|}{$\gamma=1 \%$} \\
\hline & $R$ & $R_{o}^{*}$ & $\widehat{R}^{*}$ & $\widetilde{R}^{*}$ & $R$ & $R_{o}^{*}$ & $\widehat{R}^{*}$ & $\widetilde{R}^{*}$ & $R$ & $R_{o}^{*}$ & $\widehat{R}^{*}$ & $\widetilde{R}^{*}$ \\
\hline 2 & 11.63 & 10.20 & 10.12 & 10.29 & 6.53 & 5.16 & 5.22 & 5.20 & 1.57 & 1.12 & 1.13 & 1.13 \\
\hline 3 & 11.97 & 10.02 & 9.92 & 10.10 & 6.71 & 5.16 & 4.92 & 5.21 & 1.97 & 1.26 & 1.03 & 1.29 \\
\hline 4 & 12.95 & 10.00 & 8.95 & 10.13 & 7.06 & 4.96 & 4.07 & 4.98 & 1.80 & 0.90 & 0.62 & 0.92 \\
\hline 5 & 13.64 & 10.02 & 8.44 & 10.11 & 7.63 & 5.04 & 3.85 & 5.06 & 2.44 & 1.11 & 0.82 & 1.11 \\
\hline 6 & 14.80 & 10.33 & 9.22 & 10.43 & 8.82 & 5.12 & 4.24 & 5.19 & 2.85 & 1.10 & 0.72 & 1.12 \\
\hline 7 & 14.90 & 10.09 & 6.67 & 10.16 & 9.03 & 5.08 & 2.65 & 5.16 & 3.05 & 1.04 & 0.43 & 1.08 \\
\hline 8 & 16.41 & 10.64 & 6.91 & 10.71 & 10.43 & 5.51 & 2.78 & 5.59 & 3.89 & 1.28 & 0.47 & 1.29 \\
\hline \multicolumn{13}{|c|}{$\mathcal{H}_{0}: \beta_{p} \geq 0$ versus $\mathcal{H}_{1}: \beta_{p}<0$} \\
\hline & \multicolumn{4}{|c|}{$\gamma=10 \%$} & \multicolumn{4}{|c|}{$\gamma=5 \%$} & \multicolumn{4}{|c|}{$\gamma=1 \%$} \\
\hline$p$ & $R$ & $R_{o}^{*}$ & $\widehat{R}^{*}$ & $\widetilde{R}^{*}$ & $R$ & $R_{o}^{*}$ & $\widehat{R}^{*}$ & $\widetilde{R}^{*}$ & $R$ & $R_{o}^{*}$ & $\widehat{R}^{*}$ & $\widetilde{R}^{*}$ \\
\hline 2 & 11.81 & 10.14 & 10.24 & 10.25 & 6.39 & 5.20 & 5.16 & 5.26 & 1.50 & 0.99 & 1.01 & 1.02 \\
\hline 3 & 12.04 & 9.98 & 9.82 & 10.08 & 6.56 & 4.90 & 4.83 & 4.91 & 1.77 & 0.94 & 0.89 & 0.96 \\
\hline 4 & 12.97 & 10.07 & 9.28 & 10.19 & 7.12 & 4.88 & 4.28 & 4.95 & 1.89 & 1.04 & 0.83 & 1.11 \\
\hline 5 & 13.56 & 10.28 & 9.32 & 10.42 & 8.16 & 5.12 & 4.36 & 5.28 & 2.41 & 1.35 & 1.10 & 1.39 \\
\hline 6 & 14.59 & 10.57 & 9.00 & 10.66 & 8.83 & 5.29 & 4.41 & 5.37 & 2.81 & 1.21 & 0.86 & 1.25 \\
\hline 7 & 15.21 & 10.44 & 6.74 & 10.58 & 9.42 & 5.44 & 3.11 & 5.52 & 3.27 & 1.19 & 0.63 & 1.20 \\
\hline 8 & 16.31 & 10.51 & 6.64 & 10.67 & 10.29 & 5.49 & 2.72 & 5.51 & 3.80 & 1.10 & 0.40 & 1.10 \\
\hline \multicolumn{13}{|c|}{$\mathcal{H}_{0}: \beta_{p}=0$ versus $\mathcal{H}_{1}: \beta_{p} \neq 0$} \\
\hline & \multicolumn{4}{|c|}{$\gamma=10 \%$} & \multicolumn{4}{|c|}{$\gamma=5 \%$} & \multicolumn{4}{|c|}{$\gamma=1 \%$} \\
\hline$p$ & $R$ & $R_{o}^{*}$ & $\widehat{R}^{*}$ & $\widetilde{R}^{*}$ & $R$ & $R_{o}^{*}$ & $\widehat{R}^{*}$ & $\widetilde{R}^{*}$ & $R$ & $R_{o}^{*}$ & $\widehat{R}^{*}$ & $\widetilde{R}^{*}$ \\
\hline 2 & 12.92 & 10.36 & 10.38 & 10.46 & 7.00 & 5.33 & 5.39 & 5.41 & 1.62 & 1.08 & 1.10 & 1.11 \\
\hline 3 & 13.27 & 10.06 & 9.75 & 10.12 & 7.35 & 4.96 & 4.99 & 5.11 & 2.07 & 0.99 & 0.96 & 1.05 \\
\hline 4 & 14.18 & 9.84 & 8.35 & 9.93 & 8.20 & 4.89 & 4.17 & 5.02 & 2.20 & 0.91 & 0.60 & 0.94 \\
\hline 5 & 15.79 & 10.16 & 8.21 & 10.34 & 9.18 & 5.34 & 4.14 & 5.46 & 3.02 & 1.32 & 1.01 & 1.39 \\
\hline 6 & 17.65 & 10.41 & 8.65 & 10.56 & 10.59 & 5.46 & 3.90 & 5.58 & 3.54 & 1.27 & 0.77 & 1.34 \\
\hline 7 & 18.45 & 10.52 & 5.76 & 10.68 & 11.58 & 5.43 & 2.63 & 5.49 & 3.97 & 1.11 & 0.55 & 1.13 \\
\hline 8 & 20.72 & 11.00 & 5.50 & 11.10 & 13.63 & 5.79 & 2.54 & 5.86 & 4.93 & 1.27 & 0.33 & 1.28 \\
\hline
\end{tabular}


Tabela 4.2: Tamanhos empíricos dos testes $R, R_{o}^{*}, \widehat{R}^{*}$ e $\widetilde{R}^{*} \operatorname{com} \alpha=1.5$ e $n=20$.

\begin{tabular}{|c|c|c|c|c|c|c|c|c|c|c|c|c|}
\hline \multirow[b]{3}{*}{$p$} & \multicolumn{12}{|c|}{$\mathcal{H}_{0}: \beta_{p} \leq 0$ versus $\mathcal{H}_{1}: \beta_{p}>0$} \\
\hline & \multicolumn{4}{|c|}{$\gamma=10 \%$} & \multicolumn{4}{|c|}{$\gamma=5 \%$} & \multicolumn{4}{|c|}{$\gamma=1 \%$} \\
\hline & $R$ & $R_{o}^{*}$ & $\widehat{R}^{*}$ & $\widetilde{R}^{*}$ & $R$ & $R_{o}^{*}$ & $\widehat{R}^{*}$ & $\widetilde{R}^{*}$ & $R$ & $R_{o}^{*}$ & $\widehat{R}^{*}$ & $\widetilde{R}^{*}$ \\
\hline 2 & 11.50 & 9.35 & 10.05 & 10.20 & 6.35 & 4.59 & 5.16 & 5.42 & 1.45 & 0.77 & 1.03 & 1.12 \\
\hline 3 & 11.86 & 9.70 & 9.29 & 9.94 & 6.53 & 4.87 & 4.55 & 5.07 & 1.84 & 0.91 & 1.00 & 1.15 \\
\hline 4 & 12.57 & 9.33 & 7.92 & 10.17 & 6.87 & 4.46 & 3.40 & 4.87 & 1.63 & 0.97 & 0.38 & 0.96 \\
\hline 5 & 13.03 & 9.80 & 7.02 & 10.17 & 7.55 & 4.90 & 3.11 & 4.99 & 2.20 & 1.01 & 0.64 & 1.17 \\
\hline 6 & 14.37 & 9.45 & 7.51 & 10.39 & 8.47 & 4.77 & 3.18 & 5.20 & 2.75 & 0.90 & 0.53 & 1.18 \\
\hline 7 & 14.01 & 9.75 & 4.07 & 9.98 & 8.40 & 5.04 & 1.33 & 5.05 & 2.74 & 1.19 & 0.15 & 1.03 \\
\hline \multirow[t]{3}{*}{8} & 16.02 & 10.13 & 4.44 & 10.55 & 9.93 & 4.95 & 1.70 & 5.48 & 3.55 & 1.11 & 0.21 & 1.23 \\
\hline & \multicolumn{12}{|c|}{$\mathcal{H}_{0}: \beta_{p} \geq 0$ versus $\mathcal{H}_{1}: \beta_{p}<0$} \\
\hline & \multicolumn{4}{|c|}{$\gamma=10 \%$} & \multicolumn{4}{|c|}{$\gamma=5 \%$} & \multicolumn{4}{|c|}{$\gamma=1 \%$} \\
\hline$p$ & $R$ & $R_{o}^{*}$ & $\widehat{R}^{*}$ & $\widetilde{R}^{*}$ & $R$ & $R_{o}^{*}$ & $\widehat{R}^{*}$ & $\widetilde{R}^{*}$ & $R$ & $R_{o}^{*}$ & $\widehat{R}^{*}$ & $\widetilde{R}^{*}$ \\
\hline 2 & 11.56 & 9.78 & 9.95 & 10.35 & 6.12 & 4.80 & 4.91 & 5.16 & 1.37 & 0.97 & 1.01 & 1.01 \\
\hline 3 & 11.84 & 9.30 & 9.39 & 10.11 & 6.42 & 4.39 & 4.46 & 5.00 & 1.51 & 0.75 & 0.80 & 0.99 \\
\hline 4 & 12.46 & 9.47 & 8.13 & 10.03 & 6.85 & 4.68 & 3.78 & 4.96 & 1.73 & 1.04 & 0.52 & 1.04 \\
\hline 5 & 13.40 & 10.01 & 7.41 & 10.38 & 7.66 & 4.95 & 3.50 & 5.25 & 2.24 & 0.92 & 0.64 & 1.36 \\
\hline 6 & 14.39 & 9.76 & 7.42 & 10.49 & 8.56 & 4.77 & 3.32 & 5.19 & 2.56 & 0.98 & 0.51 & 1.30 \\
\hline 7 & 14.47 & 10.00 & 4.35 & 10.39 & 9.00 & 5.18 & 1.78 & 5.41 & 2.92 & 1.08 & 0.27 & 1.15 \\
\hline 8 & 16.05 & 9.96 & 4.52 & 10.74 & 10.07 & 4.93 & 1.67 & 5.32 & 3.43 & 1.04 & 0.19 & 1.07 \\
\hline \multicolumn{13}{|c|}{$\mathcal{H}_{0}: \beta_{p}=0$ versus $\mathcal{H}_{1}: \beta_{p} \neq 0$} \\
\hline & \multicolumn{4}{|c|}{$\gamma=10 \%$} & \multicolumn{4}{|c|}{$\gamma=5 \%$} & \multicolumn{4}{|c|}{$\gamma=1 \%$} \\
\hline$p$ & $R$ & $R_{o}^{*}$ & $\widehat{R}^{*}$ & $\widetilde{R}^{*}$ & $R$ & $R_{o}^{*}$ & $\widehat{R}^{*}$ & $\widetilde{R}^{*}$ & $R$ & $R_{o}^{*}$ & $\widehat{R}^{*}$ & $\widetilde{R}^{*}$ \\
\hline 2 & 12.47 & 9.39 & 10.07 & 10.58 & 6.67 & 4.55 & 4.76 & 5.21 & 1.60 & 0.80 & 1.11 & 1.17 \\
\hline 3 & 12.95 & 9.26 & 9.01 & 10.07 & 7.19 & 4.50 & 4.46 & 5.00 & 1.90 & 0.84 & 0.86 & 1.08 \\
\hline 4 & 13.72 & 9.14 & 7.18 & 9.83 & 7.55 & 4.70 & 3.03 & 4.89 & 1.96 & 1.17 & 0.42 & 0.99 \\
\hline 5 & 15.21 & 9.85 & 6.61 & 10.24 & 8.66 & 4.82 & 2.90 & 5.46 & 2.80 & 1.01 & 0.63 & 1.27 \\
\hline 6 & 17.03 & 9.54 & 6.50 & 10.39 & 10.06 & 4.63 & 3.04 & 5.55 & 3.26 & 1.01 & 0.47 & 1.26 \\
\hline 7 & 17.40 & 10.22 & 3.11 & 10.46 & 10.61 & 5.25 & 1.15 & 5.52 & 3.50 & 1.12 & 0.22 & 1.21 \\
\hline 8 & 20.00 & 9.88 & 3.37 & 10.80 & 12.87 & 4.96 & 1.33 & 5.50 & 4.46 & 0.99 & 0.24 & 1.26 \\
\hline
\end{tabular}


Tabela 4.3: Tamanhos empíricos dos testes $R, R_{o}^{*}, \widehat{R}^{*}$ e $\widetilde{R}^{*} \operatorname{com} \alpha=0.5, p=5$ e diferentes tamanhos amostrais.

\begin{tabular}{|c|c|c|c|c|c|c|c|c|c|c|c|c|}
\hline \multirow[b]{3}{*}{$n$} & \multicolumn{12}{|c|}{$\mathcal{t}_{0}: \beta_{5} \leq 0$ versus $\mathcal{H}_{1}: \beta_{5}>0$} \\
\hline & \multicolumn{4}{|c|}{$\gamma=10 \%$} & \multicolumn{4}{|c|}{$\gamma=5 \%$} & \multicolumn{4}{|c|}{$\gamma=1 \%$} \\
\hline & $R$ & $R_{o}^{*}$ & $\widehat{R}^{*}$ & $\widetilde{R}^{*}$ & $R$ & $R_{o}^{*}$ & $\widehat{R}^{*}$ & $\widetilde{R}^{*}$ & $R$ & $R_{o}^{*}$ & $\widehat{R}^{*}$ & $\widetilde{R}^{*}$ \\
\hline 10 & 18.82 & 11.39 & 9.00 & .56 & 12.88 & 6.06 & 5.26 & 6.17 & 51 & 1.36 & 1.65 & 1.41 \\
\hline 20 & 13.64 & 10.02 & 8.44 & .11 & 63 & 5.04 & 3.85 & 5.06 & 44 & .11 & 0.82 & .11 \\
\hline 30 & 2.71 & 10.52 & 10.08 & 9 & 7.01 & 5.06 & 4.75 & 5.10 & 62 & 0.96 & 0.87 & 0.97 \\
\hline 40 & 1.62 & 10.14 & 10.12 & 5 & 6.37 & 5.03 & 4.88 & 5.06 & 48 & 1.02 & 0.94 & 1.03 \\
\hline 50 & 1 & 10.11 & 10.14 & 10 & 6.42 & 5.46 & 5.51 & 5.52 & 62 & 1.05 & 0.99 & 1.09 \\
\hline 100 & 1 & 1 & 11.11 & 1 & 44 & & 5.84 & 5.89 & 33 & 1.19 & 1.20 & 1.19 \\
\hline 200 & 10.66 & 1 & 10.34 & 1 & 32 & & 5.51 & 5.50 & 11 & 1.30 & 1.30 & .30 \\
\hline \multirow[t]{3}{*}{500} & & 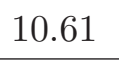 & 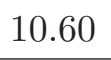 & 0.61 & 5.51 & & 5.43 & 5.41 & 0 & 5 & 5 & .95 \\
\hline & \multicolumn{12}{|c|}{$\mathcal{H}_{0}: \beta_{5} \geq 0$ versus $\mathcal{H}_{1}: \beta_{5}<0$} \\
\hline & \multicolumn{4}{|c|}{$\gamma=10 \%$} & \multicolumn{4}{|c|}{$\gamma=5 \%$} & \multicolumn{4}{|c|}{$\gamma=1 \%$} \\
\hline$n$ & $R$ & $R_{o}^{*}$ & $\widehat{R}^{*}$ & $\widetilde{R}^{*}$ & $R$ & & $\widehat{R}^{*}$ & $\widetilde{R}^{*}$ & $R$ & $R_{o}^{*}$ & $\widehat{R}^{*}$ & $\widetilde{R}^{*}$ \\
\hline 10 & 19.27 & 11.65 & 9.00 & 11.78 & 10.41 & & 5.12 & 6.53 & 0.84 & 1.35 & 1.62 & 1.38 \\
\hline 20 & $1:$ & 10 & 9.3 & 2 & 16 & & 4.50 & 5.28 & 1 & 1.35 & 1.10 & 1.39 \\
\hline 30 & 12 & 1 & 9.9 & 7 & 6.87 & 5. & 4.91 & 5.18 & .99 & 1.09 & 1.07 & 1.13 \\
\hline 40 & 1 & 10 & 10.09 & 1 & 56 & & 5.18 & 5.20 & 57 & 0.99 & 0.93 & 1.04 \\
\hline 50 & 0 & 10.08 & 10.02 & 10.12 & 97 & 4. & 4.97 & 4.91 & 59 & 1.14 & 1.09 & 1.16 \\
\hline 100 & 1 & & 9 & & 19 & & 4.71 & 4.77 & .97 & 0.86 & 0.83 & 0.87 \\
\hline 200 & 10.29 & 10.00 & 10.00 & 10.00 & 07 & 4. & 4.91 & 4.92 & .96 & 0.92 & 0.92 & 0.93 \\
\hline \multirow[t]{3}{*}{500} & & & 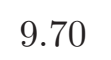 & 9.73 & 4.89 & 4.76 & 4.78 & 4.76 & 0.92 & 0.89 & 0.90 & 0.89 \\
\hline & \multicolumn{12}{|c|}{$\mathcal{H}_{0}: \beta_{5}=0$ versus $\mathcal{H}_{1}: \beta_{5} \neq 0$} \\
\hline & \multicolumn{4}{|c|}{$\gamma=10 \%$} & \multicolumn{4}{|c|}{$\gamma=5 \%$} & \multicolumn{4}{|c|}{$\gamma=1 \%$} \\
\hline$n$ & $R$ & $R_{o}^{*}$ & $\widehat{R}^{*}$ & $R^{*}$ & $R$ & $R_{o}^{*}$ & $\widehat{R}^{*}$ & $\widetilde{R}^{*}$ & $R$ & $R_{o}^{*}$ & $\widehat{R}^{*}$ & $\widetilde{R}^{*}$ \\
\hline 10 & 26.29 & 12.48 & 10.38 & 12.70 & 18.45 & 6.40 & 6.09 & 6.62 & 8.03 & 1.51 & 2.22 & 1.56 \\
\hline 20 & 15.79 & 10.16 & 8.21 & 10.34 & 9.18 & 5.34 & 4.14 & 5.46 & 3.02 & 1.32 & 1.01 & 1.39 \\
\hline 30 & 13.88 & 10.16 & 9.66 & 10.28 & 7.59 & 5.01 & 4.77 & 5.11 & 1.98 & 1.08 & 1.05 & 1.14 \\
\hline 40 & 12.93 & 10.20 & 10.06 & 10.26 & 7.01 & 5.19 & 5.03 & 5.21 & 1.61 & 1.05 & 0.98 & 1.09 \\
\hline 50 & 12.39 & 10.36 & 10.48 & 10.43 & 6.89 & 5.37 & 5.38 & 5.42 & 1.57 & 0.97 & 0.94 & 0.99 \\
\hline 100 & 11.63 & 10.61 & 10.55 & 10.66 & 6.10 & 5.31 & 5.28 & 5.33 & 1.27 & 1.04 & 1.03 & 1.05 \\
\hline 200 & 10.89 & 10.41 & 10.42 & 10.42 & 5.58 & 5.35 & 5.36 & 5.36 & 1.13 & 1.05 & 1.05 & 1.06 \\
\hline 500 & 10.40 & 10.17 & 10.21 & 10.17 & 5.14 & 4.99 & 4.98 & 4.99 & 0.94 & 0.91 & 0.91 & 0.91 \\
\hline
\end{tabular}


Tabela 4.4: Poderes dos testes $R_{o}^{*}, \widehat{R}^{*}$ e $\widetilde{R}^{*} \operatorname{com} \alpha=0.5, p=2, \gamma=10 \%$ e diferentes tamanhos amostrais.

\begin{tabular}{ccccc|rccc|rccc}
\hline & \multicolumn{4}{c|}{$\mathcal{H}_{1}: \beta_{2}>\delta_{1}$} & \multicolumn{4}{c|}{$\mathcal{H}_{1}: \beta_{2}<\delta_{2}$} & \multicolumn{4}{c}{$\mathcal{H}_{1}: \beta_{2} \neq \delta_{3}$} \\
\cline { 2 - 12 }$n$ & $\delta_{1}$ & $R_{o}^{*}$ & $\widehat{R}^{*}$ & $\widetilde{R}^{*}$ & \multicolumn{1}{c}{$\delta_{2}$} & $R_{o}^{*}$ & $\widehat{R}^{*}$ & $\widetilde{R}^{*}$ & \multicolumn{1}{c}{$\delta_{3}$} & $R_{o}^{*}$ & $\widehat{R}^{*}$ & $\widetilde{R}^{*}$ \\
\hline 10 & 0.1 & 23.17 & 22.85 & 23.29 & -0.1 & 22.81 & 22.47 & 22.97 & -0.7 & 86.47 & 85.80 & 86.49 \\
& 0.3 & 62.87 & 62.59 & 62.90 & -0.3 & 62.97 & 62.72 & 62.99 & -0.3 & 77.60 & 77.54 & 77.78 \\
& 0.5 & 73.11 & 72.96 & 73.15 & -0.5 & 72.88 & 72.59 & 72.93 & 0.0 & 9.85 & 9.74 & 10.15 \\
& 0.7 & 80.94 & 80.44 & 80.98 & -0.7 & 80.45 & 79.91 & 80.51 & 0.3 & 76.76 & 76.60 & 76.89 \\
& 0.9 & 86.07 & 85.40 & 86.14 & -0.9 & 86.23 & 85.35 & 86.32 & 0.7 & 87.17 & 86.63 & 87.20 \\
\hline 20 & 0.1 & 27.64 & 27.74 & 27.71 & -0.1 & 27.80 & 27.83 & 27.91 & -0.7 & 87.86 & 87.95 & 87.91 \\
& 0.3 & 68.39 & 68.44 & 68.39 & -0.3 & 68.20 & 68.22 & 68.20 & -0.3 & 84.24 & 84.26 & 84.28 \\
& 0.5 & 78.73 & 78.76 & 78.73 & -0.5 & 78.64 & 78.67 & 78.66 & 0.0 & 10.36 & 10.38 & 10.46 \\
& 0.7 & 87.00 & 87.00 & 87.06 & -0.7 & 87.29 & 87.27 & 87.36 & 0.3 & 84.57 & 84.50 & 84.61 \\
& 0.9 & 92.84 & 92.81 & 92.87 & -0.9 & 92.62 & 92.69 & 92.70 & 0.7 & 88.09 & 88.01 & 88.11 \\
\hline 30 & 0.1 & 33.92 & 34.01 & 33.98 & -0.1 & 33.59 & 33.61 & 33.67 & -0.7 & 90.30 & 90.30 & 90.31 \\
& 0.3 & 72.46 & 72.49 & 72.47 & -0.3 & 72.32 & 72.35 & 72.32 & -0.3 & 85.44 & 85.38 & 85.47 \\
& 0.5 & 84.05 & 84.12 & 84.07 & -0.5 & 83.77 & 83.78 & 83.81 & 0.0 & 10.36 & 10.38 & 10.46 \\
& 0.7 & 92.17 & 92.21 & 92.24 & -0.7 & 92.50 & 92.52 & 92.52 & 0.3 & 86.04 & 86.07 & 86.07 \\
& 0.9 & 96.83 & 96.85 & 96.91 & -0.9 & 96.67 & 96.81 & 96.78 & 0.7 & 89.83 & 89.80 & 89.85 \\
\hline
\end{tabular}

Tabela 4.5: Tamanhos empíricos dos testes $R, R_{o}^{*}, \widehat{R}^{*}$ e $\widetilde{R}^{*} \operatorname{com} \alpha=0.5, p=6, n=25$ e diferentes valores para $\gamma$.

\begin{tabular}{ccccc|cccc}
\hline & \multicolumn{4}{c|}{$\mathcal{H}_{0}: \beta_{6} \leq 0$} & \multicolumn{4}{c}{$\mathcal{H}_{0}: \beta_{6} \geq 0$} \\
\cline { 2 - 8 }$\gamma$ & $R$ & $R_{o}^{*}$ & $\widehat{R}^{*}$ & $\widetilde{R}^{*}$ & $R$ & $R_{o}^{*}$ & $\widehat{R}^{*}$ & $\widetilde{R}^{*}$ \\
\hline $15 \%$ & 18.62 & 15.19 & 14.29 & 15.26 & 18.44 & 15.13 & 14.26 & 15.22 \\
$10 \%$ & 13.39 & 10.15 & 9.39 & 10.21 & 13.40 & 10.08 & 9.24 & 10.17 \\
$5 \%$ & 7.86 & 5.18 & 4.52 & 5.23 & 7.70 & 4.99 & 4.35 & 5.05 \\
$3 \%$ & 5.30 & 3.15 & 2.68 & 3.19 & 5.10 & 2.97 & 2.49 & 3.02 \\
$1 \%$ & 2.34 & 1.11 & 0.84 & 1.13 & 2.16 & 0.98 & 0.76 & 1.01 \\
$0.5 \%$ & 1.37 & 0.54 & 0.40 & 0.56 & 1.24 & 0.51 & 0.36 & 0.52 \\
$0.1 \%$ & 0.40 & 0.10 & 0.07 & 0.11 & 0.37 & 0.12 & 0.08 & 0.13 \\
$0.05 \%$ & 0.23 & 0.05 & 0.03 & 0.05 & 0.22 & 0.07 & 0.04 & 0.07 \\
\hline
\end{tabular}


Tabela 4.6: Tamanhos empíricos dos testes $R, R_{o}^{*}, \widehat{R}^{*}$ e $\widetilde{R}^{*} \operatorname{com} \alpha^{(0)}=0.5$ e $n=20$.

\begin{tabular}{|c|c|c|c|c|c|c|c|c|c|c|c|c|}
\hline \multirow[b]{3}{*}{$p$} & \multicolumn{12}{|c|}{$\mathcal{H}_{0}: \alpha \leq \alpha^{(0)}$ versus $\mathcal{H}_{1}: \alpha>\alpha^{(0)}$} \\
\hline & \multicolumn{4}{|c|}{$\gamma=10 \%$} & \multicolumn{4}{|c|}{$\gamma=5 \%$} & \multicolumn{4}{|c|}{$\gamma=1 \%$} \\
\hline & $R$ & $R_{o}^{*}$ & $\widehat{R}^{*}$ & $\widetilde{R}^{*}$ & $R$ & $R_{o}^{*}$ & $\widehat{R}^{*}$ & $\widetilde{R}^{*}$ & $R$ & $R_{o}^{*}$ & $\widehat{R}^{*}$ & $\widetilde{R}^{*}$ \\
\hline 2 & 4.65 & & & 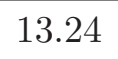 & 2.20 & 8.18 & 8.16 & 8.15 & 0.39 & .81 & 3.83 & 3.76 \\
\hline 3 & & & 10.10 & 1 & 1.56 & 7.81 & 7.87 & 7.79 & 0.29 & 53 & 3.56 & 3.55 \\
\hline 4 & 2.31 & 1 & 12.45 & 12.40 & 1.00 & 7.30 & 7.35 & 7.31 & 0.15 & 3.14 & 3.15 & 3.12 \\
\hline 5 & 1.53 & & 11.66 & 11.71 & 0.65 & 6.95 & 6.91 & 6.89 & 0.11 & 3.07 & 3.12 & 3.04 \\
\hline 6 & 0.96 & 1 & 10.89 & 10.87 & 0.42 & 6.72 & 6.75 & 6.69 & 0.03 & 2.63 & 2.75 & 2.64 \\
\hline 7 & 0 & 8 & 8.93 & 8.74 & 0.27 & 5.68 & 5.73 & 5.64 & 0.05 & 1. & 1.93 & 1.85 \\
\hline 8 & 0 & 7 & 7 & 7.59 & 0.15 & 5.10 & 5.03 & 5.02 & 0.02 & 1 & 1.80 & 1.69 \\
\hline 9 & 0 & & 7.30 & 6.74 & 0.06 & 3 & 3.87 & 3 & 0 & 1.44 & 1 & 1.44 \\
\hline \multirow[t]{3}{*}{10} & & & & 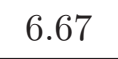 & 0.02 & 3.19 & 3.43 & 3.17 & 0. & 1.24 & 1. & - \\
\hline & \multicolumn{12}{|c|}{$\mathcal{H}_{0}: \alpha \geq \alpha^{(0)}$ versus $\mathcal{H}_{1}: \alpha<\alpha^{(0)}$} \\
\hline & \multicolumn{4}{|c|}{$\gamma=10 \%$} & \multicolumn{4}{|c|}{$\gamma=5 \%$} & \multicolumn{4}{|c|}{$\gamma=1 \%$} \\
\hline$p$ & $R$ & $R_{o}^{*}$ & $\widehat{R}^{*}$ & $\pi$ & $R$ & $R_{o}^{*}$ & $\widehat{R}^{*}$ & $\widetilde{R}^{*}$ & $R$ & $R_{o}^{*}$ & $\widehat{R}^{*}$ & $\widetilde{R}^{*}$ \\
\hline 2 & 21.20 & 12.8 & 12.80 & 12.81 & 12.34 & 7.36 & 7.38 & 7.41 & 3.50 & 3.14 & 3.17 & 3.17 \\
\hline 3 & 27.04 & 12.5 & 12.54 & 12.59 & 17.04 & 7.03 & 7.08 & 7.07 & 4.84 & 2.93 & 2.95 & 2.95 \\
\hline 4 & 33.67 & 12.4 & 12.39 & 12.42 & 21.96 & 7.38 & 7.43 & 7.41 & 7.46 & 2.74 & 2.79 & 2.77 \\
\hline 5 & 41.04 & 12.91 & 12.90 & 12.93 & 28.35 & 7.25 & 7.25 & 7.27 & 11.41 & 2.50 & 2.54 & 2.53 \\
\hline 6 & 48.17 & 12.5 & 12.51 & 12.53 & 34.95 & 7.04 & 7.05 & 7.05 & 15.33 & 2.29 & 2.32 & 2.30 \\
\hline 7 & 56.36 & 12.6 & 12.65 & 12.71 & 43.13 & 6.62 & 6.61 & 6.62 & 21.24 & 2.17 & 2.18 & 2.18 \\
\hline 8 & 64.36 & 13.6 & 13.63 & 13.70 & 52.19 & 7.20 & 7.19 & 7.21 & 27.90 & 2.13 & 2.14 & 2.14 \\
\hline 9 & 72.27 & 14.30 & 14.21 & 14.32 & 60.20 & 7.87 & 7.85 & 7.88 & 36.11 & 2.03 & 2.05 & 2.03 \\
\hline 10 & 79.49 & 15.59 & 15.55 & 15.59 & 69.19 & 8.84 & 8.80 & 8.84 & 46.05 & 2.32 & 2.32 & 2.32 \\
\hline
\end{tabular}

Tabela 4.7: Poderes dos testes $R, R_{o}^{*}, \widehat{R}^{*}$ e $\widetilde{R}^{*} \operatorname{com} \alpha^{(0)}=0.5, p=7$ e $n=20$ considerando a hipótese $\mathcal{H}_{0}: \alpha \leq \alpha^{(0)}$ versus $\mathcal{H}_{1}: \alpha>\delta$.

\begin{tabular}{ccccc|cccc}
\hline & \multicolumn{4}{c|}{$\gamma=10 \%$} & \multicolumn{4}{c}{$\gamma=5 \%$} \\
\cline { 2 - 9 }$\delta$ & $R$ & $R_{o}^{*}$ & $\widehat{R}^{*}$ & $\widetilde{R}^{*}$ & $R$ & $R_{o}^{*}$ & $\widehat{R}^{*}$ & $\widetilde{R}^{*}$ \\
\hline 0.5 & 0.59 & 8.84 & 8.93 & 8.74 & 0.27 & 5.68 & 5.73 & 5.64 \\
0.7 & 28.62 & 63.38 & 63.29 & 63.23 & 19.87 & 55.24 & 54.91 & 55.17 \\
0.9 & 72.86 & 92.04 & 91.86 & 92.00 & 64.83 & 88.81 & 88.69 & 88.78 \\
1.1 & 92.41 & 98.21 & 98.19 & 98.18 & 89.18 & 97.38 & 97.31 & 97.38 \\
1.3 & 97.84 & 99.64 & 99.64 & 99.64 & 96.82 & 99.40 & 99.42 & 99.40 \\
1.5 & 99.34 & 99.89 & 99.89 & 99.89 & 98.96 & 99.84 & 99.83 & 99.84 \\
\hline
\end{tabular}




\section{Capítulo 5}

\section{Estatística Gradiente}

A grande maioria dos testes de hipóteses estatísticos em problemas envolvendo grandes amostras é baseada em um dos seguintes princípios: razão de verossimilhanças (Wilks, 1938), Wald (Wald, 1943) e escore (Rao, 1948). Sabe-se que, em problemas regulares, as estatísticas da razão de verossimilhanças $(L R)$, de Wald $(W)$ e escore de Rao $\left(S_{R}\right)$ têm propriedades assintóticas idênticas até primeira ordem tanto sob a hipótese nula quanto sob alternativas de Pitman (sequência de alternativas convergindo para a hipótese nula a uma taxa de convergência de $n^{-1 / 2}$, em que $n$ é o tamanho da amostra). Uma vez que os testes baseados nas três estatísticas têm o mesmo poder, sob esta classe de alternativas, até primeira ordem e o mesmo tamanho até ordem $n^{-1 / 2}$, um critério de escolha entre eles pode se basear, então, na comparação de seus poderes até ordem $n^{-1 / 2}$. As expansões assintóticas sob esta classe de alternativas de Pitman até esta ordem de aproximação para os poderes dos testes da razão de verossimilhanças e de Wald foram desenvolvidas por Hayakawa (1975), estendendo os resultados previamente obtidos por Peers (1971). Um resultado análogo para o teste escore foi obtido por Harris \& Peers (1980). A validade da expansão assintótica da distribuição de algumas destas estatísticas sob alternativas de Pitman foi mostrada por Chandra \& Ghosh (1980).

Neste capítulo, iremos considerar uma estatística recentemente proposta por Terrell (2002), denominada estatística gradiente. Esta estatística, que será apresentada na próxima seção, foi derivada a partir da estatística escore de Rao e da estatística do teste de Wald modificada (Hayakawa \& Puri, 1985). A combinação destas duas estatísticas resulta em uma estatística muito simples de ser calculada, não envolvendo, por exemplo, nenhum cálculo matricial como produto e inversa de matrizes. Esta estatística foi recentemente citada por Rao (2005) no artigo em que ele diz: "The suggestion by Terrell is attractive as it is simple to compute. It would be of interest to investigate the performance of the [gradient] statistic."

Alguns dos objetivos deste capítulo são descritos a seguir. Primeiramente, vamos obter uma expansão assintótica para a distribuição da estatística gradiente $\left(S_{T}\right)$ sob uma sequência de alternativas de Pitman convergindo para a hipótese nula a uma taxa de convergência de $n^{-1 / 2}$ utilizando a metodologia desenvolvida por Peers (1971) e Hayakawa (1975). Adicionalmente, iremos comparar as funções de poder dos testes que usam as estatísticas $L R, W$ e $S_{R}$ com a função de poder do teste gradiente. Mostraremos que não é possível, em geral, afirmar que um dos testes $(L R, W$ ou $S_{R}$ ) é mais poderoso que o teste com base na estatística gradiente, ou seja, somente em situações particulares podem ser feitas comparações.

Posteriormente, iremos particularizar a expressão geral da expansão assintótica da distribuição da estatística gradiente até ordem $n^{-1 / 2}$, bem como as expansões assintóticas das estatísticas $L R, W$ e $S_{R}$, em distribuições pertencentes à família exponencial uniparamétrica. Adicionalmente, desen- 
volveremos expansões assintóticas na classe de modelos de regressão Birnbaum-Saunders, introduzida no Capítulo 1, assumindo $\alpha$ conhecido e, posteriormente, considerando este parâmetro desconhecido. Mostraremos que as funções de poder dos testes $L R, W, S_{R}$ e $S_{T}$, até ordem $n^{-1 / 2}$, são iguais, considerando $\alpha$ conhecido ou desconhecido. Finalmente, iremos obter a expansão assintótica, até ordem $n^{-1 / 2}$, para a função de poder do teste gradiente em modelos lineares generalizados (McCullagh \& Nelder, 1989). As expansões assintóticas, até ordem $n^{-1 / 2}$, das funções de poder dos testes $L R, W$ e $S_{R}$ nesta classe de modelos foram derivadas em Cordeiro, Botter \& Ferrari (1994) e Ferrari, Botter \& Cribari-Neto (1997).

\subsection{Estatística Gradiente $-S_{T}$}

Considere o vetor $\boldsymbol{x}=\left(x_{1}, x_{2}, \ldots, x_{n}\right)^{\top}$ representando $n$ observações independentes mas não necessariamente identicamente distribuídas. Seja $\ell(\boldsymbol{\theta})$ a função de log-verossimilhança total, dado $\boldsymbol{x}$, que depende de uma parâmetro desconhecido $\boldsymbol{\theta}=\left(\theta_{1}, \theta_{2}, \ldots, \theta_{p}\right)^{\top} \operatorname{com} p$ componentes. Seja $\pi(\cdot ; \boldsymbol{\theta})$ a função densidade da distribuição da qual a amostra foi gerada. A log-verossimilhança é escrita como

$$
\ell(\boldsymbol{\theta})=\sum_{l=1}^{n} \log \left\{\pi_{l}\left(x_{l} ; \boldsymbol{\theta}\right)\right\},
$$

em que $\pi_{l}$ corresponde à densidade individual da l-ésima observação. Sejam $\boldsymbol{U}(\boldsymbol{\theta})=\partial \ell(\boldsymbol{\theta}) / \partial \boldsymbol{\theta}$ e $\boldsymbol{K}(\boldsymbol{\theta})=\mathbb{E}\left\{\boldsymbol{U}(\boldsymbol{\theta}) \boldsymbol{U}(\boldsymbol{\theta})^{\top}\right\}$ a função escore e a matriz de informação de Fisher para $\boldsymbol{\theta}$, respectivamente. O problema considerado é o de testar uma hipótese nula simples ou composta

$$
\mathcal{H}_{0}: \boldsymbol{\theta}_{2}=\boldsymbol{\theta}_{20}
$$

contra a hipótese alternativa bilateral $\mathcal{H}_{1}: \boldsymbol{\theta}_{2} \neq \boldsymbol{\theta}_{20}$, em que $\boldsymbol{\theta}=\left(\boldsymbol{\theta}_{1}^{\top}, \boldsymbol{\theta}_{2}^{\top}\right)^{\top}, \boldsymbol{\theta}_{1}=\left(\theta_{1}, \theta_{2}, \ldots, \theta_{q}\right)^{\top}$, $\boldsymbol{\theta}_{2}=\left(\theta_{q+1}, \theta_{q+2}, \ldots, \theta_{p}\right)^{\top}$, sendo $\boldsymbol{\theta}_{20}$ um vetor $(p-q)$-dimensional especificado. A partição de $\boldsymbol{\theta}$ induz as correspondentes partições $\boldsymbol{U}(\boldsymbol{\theta})=\left(\boldsymbol{U}_{1}(\boldsymbol{\theta})^{\top}, \boldsymbol{U}_{2}(\boldsymbol{\theta})^{\top}\right)^{\top}$,

$$
\boldsymbol{K}=\left[\begin{array}{ll}
\boldsymbol{K}_{11} & \boldsymbol{K}_{12} \\
\boldsymbol{K}_{21} & \boldsymbol{K}_{22}
\end{array}\right] \quad \text { e } \quad \boldsymbol{K}^{-1}=\left[\begin{array}{ll}
\boldsymbol{K}^{11} & \boldsymbol{K}^{12} \\
\boldsymbol{K}^{21} & \boldsymbol{K}^{22}
\end{array}\right]
$$

em que $\boldsymbol{K}^{-1}$ representa a inversa de $\boldsymbol{K}$.

As estatísticas $L R, W$ e $S_{R}$ para testar (5.1) podem ser escritas como

$$
\begin{gathered}
L R=2\{\ell(\widehat{\boldsymbol{\theta}})-\ell(\widetilde{\boldsymbol{\theta}})\}, \\
W=\left(\widehat{\boldsymbol{\theta}}_{2}-\boldsymbol{\theta}_{20}\right)^{\top} \boldsymbol{K}^{22}(\widehat{\boldsymbol{\theta}})^{-1}\left(\widehat{\boldsymbol{\theta}}_{2}-\boldsymbol{\theta}_{20}\right), \\
S_{R}=\boldsymbol{U}_{2}(\widetilde{\boldsymbol{\theta}})^{\top} \boldsymbol{K}^{22}(\widetilde{\boldsymbol{\theta}}) \boldsymbol{U}_{2}(\widetilde{\boldsymbol{\theta}}),
\end{gathered}
$$

em que $\widehat{\boldsymbol{\theta}}=\left(\widehat{\boldsymbol{\theta}}_{1}^{\top}, \widehat{\boldsymbol{\theta}}_{2}^{\top}\right)^{\top}$ e $\widetilde{\boldsymbol{\theta}}=\left(\widetilde{\boldsymbol{\theta}}_{1}^{\top}, \boldsymbol{\theta}_{20}^{\top}\right)^{\top}$ são os estimadores de máxima verossimilhança de $\boldsymbol{\theta}$ sob $\mathcal{H}_{1}$ e $\mathcal{H}_{0}$, respectivamente, e $\boldsymbol{K}^{22}=\left(\boldsymbol{K}_{22}-\boldsymbol{K}_{21} \boldsymbol{K}_{11}^{-1} \boldsymbol{K}_{12}\right)^{-1}$. Note que $\boldsymbol{K}^{22}$ é a matriz de covariância assintótica de $\widehat{\boldsymbol{\theta}}_{2}$ obtida de $\boldsymbol{K}^{-1}$. Para grandes amostras e em problemas regulares, a distribuição das três estatísticas sob a hipótese nula é aproximadamente qui-quadrado central com $p-q$ graus de liberdade, $\chi_{p-q}^{2}(\lambda=0)$, sendo $\lambda$ o parâmetro de não-centralidade. Hayakawa \& Puri (1985) propuseram uma estatística de Wald modificada, denotada aqui por $\tilde{W}$, tendo a forma

$$
\tilde{W}=\left(\widehat{\boldsymbol{\theta}}_{2}-\boldsymbol{\theta}_{20}\right)^{\top} \boldsymbol{K}^{22}(\widetilde{\boldsymbol{\theta}})^{-1}\left(\widehat{\boldsymbol{\theta}}_{2}-\boldsymbol{\theta}_{20}\right) .
$$


Note que $W$ difere de $\tilde{W}$ somente através da matriz $\boldsymbol{K}^{22}(\boldsymbol{\theta})$, isto é, a primeira considera $\boldsymbol{K}^{22}(\widehat{\boldsymbol{\theta}})^{-1}$ enquanto a segunda toma $\boldsymbol{K}^{22}(\widetilde{\boldsymbol{\theta}})^{-1}$. A estatística $\tilde{W}$ tem propriedades assintóticas idênticas até primeira ordem àquelas das estatísticas $L R, W$ e $S_{R}$.

A fim de mostrar a definição da estatística gradiente, proposta por Terrell (2002), e estabelecer sua relação com as estatísticas $S_{R}$ e $\tilde{W}$, considere o caso em que a hipótese nula (5.1) é simples, isto é, $\mathcal{H}_{0}: \boldsymbol{\theta}=\boldsymbol{\theta}_{0}$. Neste caso, podemos escrever $S_{R}$ e $\tilde{W}$ da forma

$$
S_{R}=\boldsymbol{U}\left(\theta_{0}\right)^{\top} \boldsymbol{K}\left(\boldsymbol{\theta}_{0}\right)^{-1} \boldsymbol{U}\left(\boldsymbol{\theta}_{0}\right) \quad \text { e } \quad \tilde{W}=\left(\widehat{\boldsymbol{\theta}}-\boldsymbol{\theta}_{0}\right)^{\top} \boldsymbol{K}\left(\boldsymbol{\theta}_{0}\right)\left(\widehat{\boldsymbol{\theta}}-\boldsymbol{\theta}_{0}\right) .
$$

A proposta de Terrell é como segue: escolha qualquer raiz quadrada $\boldsymbol{L}$ da matriz de informação de Fisher, isto é, encontre uma matriz quadrada $p \times p$ tal que $\boldsymbol{L}^{\top} \boldsymbol{L}=\boldsymbol{K}(\boldsymbol{\theta})$. Note que podemos escrever

$$
S_{R}=\left[\left(\boldsymbol{L}^{-1}\right)^{\top} \boldsymbol{U}\left(\boldsymbol{\theta}_{0}\right)\right]^{\top}\left(\boldsymbol{L}^{-1}\right)^{\top} \boldsymbol{U}\left(\boldsymbol{\theta}_{0}\right) \quad \text { e } \quad \tilde{W}=\left[\boldsymbol{L}\left(\widehat{\boldsymbol{\theta}}-\boldsymbol{\theta}_{0}\right)\right]^{\top} \boldsymbol{L}\left(\widehat{\boldsymbol{\theta}}-\boldsymbol{\theta}_{0}\right) .
$$

Observe que cada um dos vetores padronizados

$$
\left(\boldsymbol{L}^{-1}\right)^{\top} \boldsymbol{U}\left(\boldsymbol{\theta}_{0}\right) \text { e } \boldsymbol{L}\left(\widehat{\boldsymbol{\theta}}-\boldsymbol{\theta}_{0}\right)
$$

possui distribuição $\mathcal{N}_{p}\left(\mathbf{0}, \boldsymbol{I}_{p}\right)$, para $n$ suficientemente grande, em que $\boldsymbol{I}_{p}$ denota uma matriz identidade de ordem $p$. Então, como $\left\{\left(\boldsymbol{L}^{-1}\right)^{\top} \boldsymbol{U}\left(\boldsymbol{\theta}_{0}\right)-\boldsymbol{L}\left(\widehat{\boldsymbol{\theta}}-\boldsymbol{\theta}_{0}\right)\right\} \stackrel{P}{\rightarrow} 0$, o produto interno destes dois vetores padronizados, tem, assintoticamente, distribuição qui-quadrado central com $p$ graus de liberdade:

$$
\left[\left(\boldsymbol{L}^{-1}\right)^{\top} \boldsymbol{U}\left(\boldsymbol{\theta}_{0}\right)\right]^{\top} \boldsymbol{L}\left(\widehat{\boldsymbol{\theta}}-\boldsymbol{\theta}_{0}\right)=\boldsymbol{U}\left(\boldsymbol{\theta}_{0}\right)^{\top} \boldsymbol{L}^{-1} \boldsymbol{L}\left(\widehat{\boldsymbol{\theta}}-\boldsymbol{\theta}_{0}\right)=\boldsymbol{U}\left(\boldsymbol{\theta}_{0}\right)^{\top}\left(\widehat{\boldsymbol{\theta}}-\boldsymbol{\theta}_{0}\right) .
$$

Observe que esta última expressão é consideravelmente mais simples de ser calculada do que as estatísticas do qual ela foi derivada, não envolvendo estimação da matriz de informação e nem operações matricias.

Defrinição 1. (Terrell, 2002) A estatística gradiente, $S_{T}$, para testar a hipótese nula simples $\mathcal{H}_{0}$ : $\boldsymbol{\theta}=\boldsymbol{\theta}_{0}$ contra a alternativa bilateral $\mathcal{H}_{1}: \boldsymbol{\theta} \neq \boldsymbol{\theta}_{0}$, é da forma

$$
S_{T}=\boldsymbol{U}\left(\boldsymbol{\theta}_{0}\right)^{\top}\left(\widehat{\boldsymbol{\theta}}-\boldsymbol{\theta}_{0}\right) .
$$

Exemplo 1. (Terrell, 2002) Seja $n_{i}, i=1,2, \ldots, k$, a contagem observada em um conjunto de $k$ categorias independentes. A hipótese a ser testada é se $n_{i} \sim \operatorname{Poisson}\left(m_{i}\right)$. A função de $\log -$ verossimilhança, a menos de uma constante não importante, é da forma $\sum_{i=1}^{k}\left(n_{i} \log m_{i}-m_{i}\right)$. O estimador de máxima verossimilhança de $m_{i}$ é $\widehat{m}_{i}=n_{i}, i=1,2, \ldots, k$. Assim, a estatística gradiente tem a forma

$$
S_{T}=\sum_{i=1}^{k} \frac{\left(n_{i}-m_{i}\right)^{2}}{m_{i}},
$$

isto é, $S_{T}$ é igual a estatística de aderência de Pearson.

A estatística $S_{T}$ em (5.2) tem uma peculiaridade: não fica evidente que tal estatística seja nãonegativa, mesmo que ela deva ser não-negativa assintoticamente. Como as estatísticas $L R, W, S_{R}$ e $\tilde{W}$ são, obviamente, não-negativas, a questão é natural. Terrell (2002, p. 207) faz o seguinte comentário: "At the seminar at which the statistic was introduced, this was the first question from the floor." 
Teorema 1. (Terrell, 2002) Se $\ell(\boldsymbol{\theta})$ é unimodal e diferenciável em $\boldsymbol{\theta}_{0}$, então

$$
S_{T}=\boldsymbol{U}\left(\boldsymbol{\theta}_{0}\right)^{\top}\left(\widehat{\boldsymbol{\theta}}-\boldsymbol{\theta}_{0}\right) \geq 0 .
$$

Prova: Terrell (2002, p. 208).

Um fato interessante sobre a estatística $S_{T}$ segue da prova padrão da desigualdade da informação (limite inferior de Cramér-Rao). Considere o caso em que $\widehat{\boldsymbol{\theta}}$ é um estimador não-viesado de $\boldsymbol{\theta}$, isto é, $\mathbb{E}_{\boldsymbol{\theta}}(\widehat{\boldsymbol{\theta}})=\boldsymbol{\theta}$, para todo $\boldsymbol{\theta}$. Então,

$$
\operatorname{Cov}(\widehat{\boldsymbol{\theta}}, \boldsymbol{U}(\boldsymbol{\theta}))=\int \widehat{\boldsymbol{\theta}} \frac{\partial \ell(\boldsymbol{\theta})}{\partial \boldsymbol{\theta}^{\top}} L(\boldsymbol{\theta}) d \boldsymbol{x}=\int \widehat{\boldsymbol{\theta}} \frac{\partial L(\boldsymbol{\theta})}{\partial \boldsymbol{\theta}^{\top}} d \boldsymbol{x},
$$

em que $L(\boldsymbol{\theta})$ é a função de verossimilhança, dado $\boldsymbol{x}$. Invertendo a ordem das operações de diferenciação em relação a $\boldsymbol{\theta}$ e integração com respeito a $\boldsymbol{x}$, temos

$$
\int \widehat{\boldsymbol{\theta}} \frac{\partial L(\boldsymbol{\theta})}{\partial \boldsymbol{\theta}^{\top}} d \boldsymbol{x}=\frac{\partial}{\partial \boldsymbol{\theta}^{\top}} \int \widehat{\boldsymbol{\theta}} L(\boldsymbol{\theta}) d \boldsymbol{x}=\frac{\partial \boldsymbol{\theta}}{\partial \boldsymbol{\theta}^{\top}}=\boldsymbol{I}_{p} .
$$

Usamos o fato de que $\widehat{\boldsymbol{\theta}}$ é uma estimador não-viesado para $\boldsymbol{\theta}$. Agora considere a estatística gradiente:

$$
\begin{aligned}
\mathbb{E}_{\boldsymbol{\theta}_{0}}\left(S_{T}\right) & =\mathbb{E}_{\boldsymbol{\theta}_{0}}\left(\boldsymbol{U}\left(\boldsymbol{\theta}_{0}\right)^{\top}\left(\widehat{\boldsymbol{\theta}}-\boldsymbol{\theta}_{0}\right)\right)=\mathbb{E}_{\boldsymbol{\theta}_{0}}\left(\operatorname{tr}\left\{\boldsymbol{U}\left(\boldsymbol{\theta}_{0}\right)^{\top}\left(\widehat{\boldsymbol{\theta}}-\boldsymbol{\theta}_{0}\right)\right\}\right) \\
& =\mathbb{E}_{\boldsymbol{\theta}_{0}}\left(\operatorname{tr}\left\{\left(\widehat{\boldsymbol{\theta}}-\boldsymbol{\theta}_{0}\right) \boldsymbol{U}\left(\boldsymbol{\theta}_{0}\right)^{\top}\right\}\right)=\operatorname{tr}\left\{\mathbb{E}_{\boldsymbol{\theta}_{0}}\left(\left(\widehat{\boldsymbol{\theta}}-\boldsymbol{\theta}_{0}\right) \boldsymbol{U}\left(\boldsymbol{\theta}_{0}\right)^{\top}\right)\right\} \\
& =\operatorname{tr}\{\operatorname{Cov}(\widehat{\boldsymbol{\theta}}, \boldsymbol{U}(\boldsymbol{\theta}))\} \\
& =\operatorname{tr}\left\{\boldsymbol{I}_{p}\right\} \\
& =p .
\end{aligned}
$$

Portanto, para $\widehat{\boldsymbol{\theta}}$ não-viesado, temos o seguinte teorema:

Teorema 2. (Terrell, 2002) Se $\widehat{\boldsymbol{\theta}}$ é não-viesado, então $\mathbb{E}_{\boldsymbol{\theta}_{0}}\left(S_{T}\right)=p$.

Vale ressaltar que $S_{T}$ é bem atrativa pela simplicidade de seu cálculo. Em problemas complexos, não ter que calcular, estimar ou inverter uma matriz de informação é uma grande vantagem. Adicionalmente, o Teorema 2 aponta outra questão importante: ele sugere que podemos, em geral, melhorar a aproximação da estatística gradiente pela distribuição qui-quadrado utilizando uma estimativa menos viesada para $\boldsymbol{\theta}$. Por outro lado, ao contrário das estatísticas $L R$ e $S_{R}$, a estatística gradiente $S_{T}$ não é invariante sob reparametrizações não-lineares de $\boldsymbol{\theta}$ (Terrell, 2002), assim como $W$.

Exemplo 2. (Terrell, 2002) No caso de um modelo de Poisson para contagens, a parametrização mais usual é um modelo log-linear: $\eta_{i}=\log \left(m_{i}\right)$. Neste caso a função de log-verossilhança, a menos de uma constante não importante, é da forma $\sum_{i=1}^{k}\left(n_{i} \eta_{i}-\exp \left(\eta_{i}\right)\right)$. O estimador de máxima verossimilhança de $\eta_{i}$ é $\widehat{\eta}_{i}=\log \left(n_{i}\right), i=1,2, \ldots, k$. Então, a estatística (de aderência) gradiente tem a forma

$$
S_{T}=\sum_{i=1}^{k}\left(n_{i}-m_{i}\right) \log \left(\frac{n_{i}}{m_{i}}\right) .
$$


Note que $S_{T}$ é parecida com a familiar estatística da razão de verossimilhanças:

$$
L R=2 \sum_{i=1}^{k}\left\{n_{i} \log \left(\frac{n_{i}}{m_{i}}\right)-\left(n_{i}-m_{i}\right)\right\},
$$

porém, ligeiramente mais simples.

Exemplo 3. Seja $\boldsymbol{x}=\left(x_{1}, \ldots, x_{n}\right)^{\top}$ uma amostra aleatória de tamanho $n$ de uma distribuição exponencial da forma $f(x ; \mu)=\exp \{-x / \mu\} / \mu$. Considere a hipótese $H_{0}: \mu=\mu_{0}$. Para testar $H_{0}$, as estatísticas $L R, W, S_{R}$ e $S_{T}$ assumem as formas:

$$
\begin{gathered}
L R=2 n\left[\log \left(\frac{\mu_{0}}{\bar{x}}\right)+\frac{\bar{x}}{\mu_{0}}-1\right], \quad W=n\left(\frac{\bar{x}-\mu_{0}}{\bar{x}}\right)^{2}, \\
S_{R}=n\left(\frac{\bar{x}-\mu_{0}}{\mu_{0}}\right)^{2} \text { e } \quad S_{T}=n\left(\frac{\bar{x}-\mu_{0}}{\mu_{0}}\right)^{2},
\end{gathered}
$$

em que $\bar{x}=(1 / n) \sum_{i=1}^{n} x_{i}$. Note, em particular, que $S_{T}$ é igual a $S_{R}$.

DEFINIÇÃo 2. (Terrell, 2002) A estatística gradiente, $S_{T}$, para testar a hipótese nula composta $\mathcal{H}_{0}: \boldsymbol{\theta}_{2}=\boldsymbol{\theta}_{20}$ contra a alternativa bilateral $\mathcal{H}_{0}: \boldsymbol{\theta}_{2} \neq \boldsymbol{\theta}_{20}$ é definida como

$$
S_{T}=\boldsymbol{U}_{2}(\widetilde{\boldsymbol{\theta}})^{\top}\left(\widehat{\boldsymbol{\theta}}_{2}-\boldsymbol{\theta}_{20}\right) .
$$

\subsection{Expansão Assintótica de $S_{T}$ sob uma Hipótese Alternativa Local}

Neste seção, iremos derivar a expansão assintótica da distribuição de $S_{T}$ para uma hipótese composta sob uma sequência de alternativas convergindo para a hipótese nula a uma taxa de convergência de $n^{-1 / 2}$, em que $n$ é o tamanho da amostra. Mostraremos que a distribuição assintótica da estatística $S_{T}$, expandida até ordem $O_{p}\left(n^{-1 / 2}\right),{ }^{1}$ é qui-quadrado central com $p-q$ graus de liberdade sob $\mathcal{H}_{0}$ e qui-quadrado não-central com os mesmos graus de liberdade e parâmetro de não-centralidade $\lambda$ (que será definido posteriormente) sob $\mathcal{H}_{1}$.

Vamos examinar a distribuição limite sob a sequência particular de alternativas

$$
\mathcal{H}_{1 n}: \boldsymbol{\theta}_{2}=\boldsymbol{\theta}_{20}+\frac{\boldsymbol{\epsilon}}{\sqrt{n}}
$$

em que $\boldsymbol{\epsilon}=\left(\epsilon_{q+1}, \epsilon_{q+2}, \ldots, \epsilon_{p}\right)^{\top}$.

\subsubsection{Expansão Assintótica de $S_{T}$ até $O_{p}\left(n^{-1 / 2}\right)$}

Vamos considerar a mesma notação introduzida por Hayakawa (1975):

(a) A menos que seja indicado, os índices $r, s, t$ variam de 1 até $q$ e $r^{\prime}, s^{\prime}, t^{\prime}$ variam de $q+1$ até $p$, respectivamente. Por exemplo:

$$
\sum \theta_{r}=\theta_{1}+\cdots+\theta_{q} \quad \text { e } \sum \theta_{r^{\prime}}=\theta_{q+1}+\cdots+\theta_{p}
$$

\footnotetext{
1 "Até ordem $O_{p}\left(n^{-1 / 2}\right)$ " significa que termos de ordem $n^{-1 / 2}$ são inclusos na expansão.
} 
(b) A função de log-verossimilhanças é regular com respeito a suas derivadas em relação a $\boldsymbol{\theta}$ até e incluindo as de terceira ordem;

(c) Qualquer função avaliada no ponto $\boldsymbol{\theta}=\widehat{\boldsymbol{\theta}}$ vai ser distinguida pela adição de um circunflexo;

(d) Qualquer função avaliada no ponto $\boldsymbol{\theta}_{1}=\widetilde{\boldsymbol{\theta}}_{1}$ e $\boldsymbol{\theta}_{2}=\boldsymbol{\theta}_{20}$ vai ser distinguida pela adição de um til;

(e) Qualquer função avaliada no ponto $\boldsymbol{\theta}_{1}=\boldsymbol{\theta}_{1}$ e $\boldsymbol{\theta}_{2}=\boldsymbol{\theta}_{20}$ vai ser distinguida pela adição de "†";

(f) Sejam

$$
\begin{array}{cl}
v_{r}=\sqrt{n}\left(\widehat{\theta}_{r}-\theta_{r}\right), & r=1,2, \ldots, p, \\
\epsilon_{r^{\prime}}=\sqrt{n}\left(\theta_{r^{\prime}}-\theta_{r^{\prime} 0}\right), & r^{\prime}=q+1, \ldots, p, \\
w_{r}=\sqrt{n}\left(\widetilde{\theta}_{r}-\theta_{r}\right), & r=1,2, \ldots, q ;
\end{array}
$$

(g) Sejam

$$
\begin{gathered}
y_{r}=n^{-1 / 2} \frac{\partial \ell(\boldsymbol{\theta})}{\partial \theta_{r}}, \quad y_{r s}=n^{-1} \frac{\partial^{2} \ell(\boldsymbol{\theta})}{\partial \theta_{r} \partial \theta_{s}}, \quad y_{r s t}=n^{-3 / 2} \frac{\partial^{3} \ell(\boldsymbol{\theta})}{\partial \theta_{r} \partial \theta_{s} \partial \theta_{t}}, \quad \kappa_{r s}=\mathbb{E}\left(y_{r s}\right), \\
\kappa_{r, s}=\mathbb{E}\left(y_{r} y_{s}\right), \quad \kappa_{r s t}=\sqrt{n} \mathbb{E}\left(y_{r s t}\right), \quad \kappa_{r, s t}=\sqrt{n} \mathbb{E}\left(y_{r} y_{s t}\right) \quad \text { e } \quad \kappa_{r, s, t}=\sqrt{n} \mathbb{E}\left(y_{r} y_{s} y_{t}\right),
\end{gathered}
$$

para $r, s, t=1,2, \ldots, p$. Considere também

$$
\begin{gathered}
\boldsymbol{y}=\left(y_{1}, y_{2}, \ldots, y_{p}\right)^{\top}, \quad \boldsymbol{Y}=\left\{y_{r s}\right\}=\left[\begin{array}{ll}
\boldsymbol{Y}_{11} & \boldsymbol{Y}_{12} \\
\boldsymbol{Y}_{21} & \boldsymbol{Y}_{22}
\end{array}\right], \\
\boldsymbol{K}=\left\{\kappa_{r, s}\right\}=\left[\begin{array}{ll}
\boldsymbol{K}_{11} & \boldsymbol{K}_{12} \\
\boldsymbol{K}_{21} & \boldsymbol{K}_{22}
\end{array}\right]=\mathbb{E}\left(\boldsymbol{y} \boldsymbol{y}^{\top}\right)=-\mathbb{E}(\boldsymbol{Y}),
\end{gathered}
$$

em que as dimensões das matrizes $\boldsymbol{Y}_{11}, \boldsymbol{Y}_{12}, \boldsymbol{Y}_{21}$ e $\boldsymbol{Y}_{22}$ são $q \times q, q \times(p-q),(p-q) \times q$ e $(p-q) \times(p-q)$, respectivamente, sendo a matriz $\boldsymbol{K}$ particionada correspondentemente;

(h) Para as quantidades com três índices, a seguinte notação de soma vai ser adotada: seja $\boldsymbol{A}=$ $\left\{a_{r s}\right\}$ uma matriz $p \times p$ e $\boldsymbol{a}=\left(a_{1}, a_{2}, \ldots, a_{p}\right)^{\top}, \boldsymbol{b}=\left(b_{1}, b_{2}, \ldots, b_{p}\right)^{\top}$ e $\boldsymbol{c}=\left(c_{1}, c_{2}, \ldots, c_{p}\right)^{\top}$ vetores colunas $p \times 1$. Então,

$$
\boldsymbol{K}_{\ldots} \circ \boldsymbol{a} \circ \boldsymbol{b} \circ \boldsymbol{c}=\sum_{r, s, t=1}^{p} \kappa_{r s t} a_{r} b_{s} c_{t},
$$

um escalar,

$$
\boldsymbol{K}_{\ldots} \circ \boldsymbol{a} \circ \boldsymbol{b}=\left(\sum_{s, t=1}^{p} \kappa_{r s t} a_{s} b_{t}\right),
$$

um vetor coluna com $p$ componentes designados pelo índice $r$,

$$
\boldsymbol{K}_{\ldots} \circ \boldsymbol{a}=\left(\sum_{t=1}^{p} \kappa_{r s t} a_{t}\right),
$$


uma matriz $p \times p$ com $(r, s)$-ésimo elemento $\sum \kappa_{r s t} a_{t}$,

$$
\boldsymbol{K}_{\ldots} \circ \boldsymbol{A} \circ \boldsymbol{b}=\sum_{r, s, t=1}^{p} \kappa_{r s t} a_{r s} b_{t},
$$

um escalar. Também, $\boldsymbol{A} \boldsymbol{K}_{\ldots} \circ \boldsymbol{a} \circ \boldsymbol{b}=\boldsymbol{A}\left(\boldsymbol{K}_{\ldots} \circ \boldsymbol{a} \circ \boldsymbol{b}\right)$, implicando que o produto usual de matrizes e vetores será feito após a operação $\circ$. Seja o conjunto de índices $\{1,2, \ldots, p\}$ particionado em $\{1,2, \ldots, q\}$ e $\{q+1, q+2, \ldots, p\}$, e sejam os vetores e matrizes particionados correspondentemente, isto é, $\boldsymbol{a}=\left(\boldsymbol{a}_{1}^{\top}, \boldsymbol{a}_{2}^{\top}\right)^{\top}$, etc. As somas com respeito aos índices incluídos no conjunto dividido vão ser denotadas como segue:

$$
\begin{gathered}
\boldsymbol{K}_{111} \circ \boldsymbol{a}_{1} \circ \boldsymbol{b}_{1} \circ \boldsymbol{c}_{1}=\sum_{r, s, t=1}^{q} \kappa_{r s t} a_{r} b_{s} c_{t}, \\
\boldsymbol{K}_{112} \circ \boldsymbol{a}_{1} \circ \boldsymbol{b}_{1} \circ \boldsymbol{c}_{2}=\sum_{r, s=1}^{q} \sum_{r^{\prime}=q+1}^{p} \kappa_{r s r^{\prime}} a_{r} b_{s} c_{r^{\prime}}, \\
\boldsymbol{K}_{212} \circ \boldsymbol{A}_{21} \circ \boldsymbol{a}_{2}=\sum_{r=1}^{q} \sum_{s^{\prime}, t^{\prime}=q+1}^{p} \kappa_{s^{\prime} r t^{\prime}} a_{s^{\prime} r} a_{t^{\prime}} .
\end{gathered}
$$

Utilizaremos as abreviações:

$$
\boldsymbol{K}_{\ldots} \circ \boldsymbol{a} \circ \boldsymbol{a} \circ \boldsymbol{a}=\boldsymbol{K}_{\ldots}(\circ \boldsymbol{a})^{3}, \quad \boldsymbol{K}_{., .} \circ \boldsymbol{a} \circ \boldsymbol{a} \circ \boldsymbol{a}=\boldsymbol{K}_{., . .}(\circ \boldsymbol{a})^{3}, \quad \text { etc. }
$$

Sejam $\boldsymbol{y}=\left(\boldsymbol{y}_{1}^{\top}, \boldsymbol{y}_{2}^{\top}\right)^{\top}, \boldsymbol{v}=\sqrt{n}(\widehat{\boldsymbol{\theta}}-\boldsymbol{\theta})=\left(\boldsymbol{v}_{1}^{\top}, \boldsymbol{v}_{2}^{\top}\right)^{\top}, \boldsymbol{\epsilon}=\sqrt{n}\left(\boldsymbol{\theta}_{2}-\boldsymbol{\theta}_{20}\right)$ e $\boldsymbol{w}=\sqrt{n}\left(\widetilde{\boldsymbol{\theta}}_{1}-\boldsymbol{\theta}_{1}\right)$. Note que podemos escrever a estatística gradiente $S_{T}$ em (5.2) da forma

$$
S_{T}=\widetilde{\boldsymbol{y}}^{\top}(\boldsymbol{v}-\boldsymbol{u}),
$$

em que $\boldsymbol{u}=\left(\boldsymbol{w}^{\top},-\boldsymbol{\epsilon}^{\top}\right)^{\top}$. Notando que $\widetilde{\boldsymbol{y}}_{1}=\boldsymbol{y}_{1}(\widetilde{\boldsymbol{\theta}})=\mathbf{0}$, temos que

$$
S_{T}=\widetilde{\boldsymbol{y}}_{2}^{\top}\left(\boldsymbol{v}_{2}+\boldsymbol{\epsilon}\right)=\widetilde{\boldsymbol{y}}_{2}^{\top} \boldsymbol{v}_{2}+\widetilde{\boldsymbol{y}}_{2}^{\top} \boldsymbol{\epsilon} .
$$

A equação satisfeita para $\boldsymbol{v}$ pode ser escrita na forma

$$
\mathbf{0}=\widehat{\boldsymbol{y}}=\boldsymbol{y}+\boldsymbol{Y} \boldsymbol{v}+\frac{1}{2} \boldsymbol{Y}_{\ldots} \circ \boldsymbol{v} \circ \boldsymbol{v}+O_{p}\left(n^{-1}\right) .
$$

Invertendo esta equação com respeito a $\boldsymbol{v}$, recursivamente, temos que

$$
\boldsymbol{v}=-\boldsymbol{Y}^{-1} \boldsymbol{y}-\frac{1}{2} \boldsymbol{Y}^{-1}\left(\boldsymbol{Y}_{\ldots} \circ \boldsymbol{Y}^{-1} \boldsymbol{y} \circ \boldsymbol{Y}^{-1} \boldsymbol{y}\right)+O_{p}\left(n^{-1}\right) .
$$

Fazendo

$$
\boldsymbol{Y}^{-1}=\left[\begin{array}{ll}
\boldsymbol{Y}^{11} & \boldsymbol{Y}^{12} \\
\boldsymbol{Y}^{21} & \boldsymbol{Y}^{22}
\end{array}\right]=\left[\begin{array}{l}
\boldsymbol{Y}^{(1)} \\
\boldsymbol{Y}^{(2)}
\end{array}\right]
$$


note que

$$
\boldsymbol{v}=\left[\begin{array}{l}
\boldsymbol{v}_{1} \\
\boldsymbol{v}_{2}
\end{array}\right]=\left[\begin{array}{l}
-\boldsymbol{Y}^{(1)} \boldsymbol{y}-\frac{1}{2} \boldsymbol{Y}^{(1)}\left(\boldsymbol{Y}_{\ldots} \circ \boldsymbol{Y}^{-1} \boldsymbol{y} \circ \boldsymbol{Y}^{-1} \boldsymbol{y}\right) \\
-\boldsymbol{Y}^{(2)} \boldsymbol{y}-\frac{1}{2} \boldsymbol{Y}^{(2)}\left(\boldsymbol{Y}_{\ldots} \circ \boldsymbol{Y}^{-1} \boldsymbol{y} \circ \boldsymbol{Y}^{-1} \boldsymbol{y}\right)
\end{array}\right]+O_{p}\left(n^{-1}\right) .
$$

Assim, temos que

$$
\boldsymbol{v}_{2}=-\boldsymbol{Y}^{(2)} \boldsymbol{y}-\frac{1}{2} \boldsymbol{Y}^{(2)}\left(\boldsymbol{Y}_{\ldots} \circ \boldsymbol{Y}^{-1} \boldsymbol{y} \circ \boldsymbol{Y}^{-1} \boldsymbol{y}\right)+O_{p}\left(n^{-1}\right) .
$$

Expandindo $\widetilde{\boldsymbol{y}}$ em série de Taylor em torno do ponto $\widetilde{\boldsymbol{\theta}}=\boldsymbol{\theta}$, temos

$$
\widetilde{\boldsymbol{y}}=\left[\begin{array}{l}
\widetilde{\boldsymbol{y}}_{1} \\
\widetilde{\boldsymbol{y}}_{2}
\end{array}\right]=\left[\begin{array}{c}
\mathbf{0} \\
\widetilde{\boldsymbol{y}}_{2}
\end{array}\right]=\left[\begin{array}{l}
\boldsymbol{y}_{1}+\boldsymbol{Y}_{11} \boldsymbol{w}-\boldsymbol{Y}_{12} \boldsymbol{\epsilon}+\frac{1}{2} \boldsymbol{Y}_{1 . .} \circ \boldsymbol{u} \circ \boldsymbol{u} \\
\boldsymbol{y}_{2}+\boldsymbol{Y}_{21} \boldsymbol{w}-\boldsymbol{Y}_{22} \boldsymbol{\epsilon}+\frac{1}{2} \boldsymbol{Y}_{2 . .} \circ \boldsymbol{u} \circ \boldsymbol{u}
\end{array}\right]+O_{p}\left(n^{-1}\right) .
$$

Considere primeiramente

$$
\mathbf{0}=\boldsymbol{y}_{1}+\boldsymbol{Y}_{11} \boldsymbol{w}-\boldsymbol{Y}_{12} \boldsymbol{\epsilon}+\frac{1}{2} \boldsymbol{Y}_{1 . .} \circ \boldsymbol{u} \circ \boldsymbol{u}+O_{p}\left(n^{-1}\right) .
$$

Da equação anterior,

$$
\boldsymbol{w}=-\boldsymbol{Y}_{11}^{-1} \boldsymbol{y}_{1}+\boldsymbol{Y}_{11}^{-1} \boldsymbol{Y}_{12} \boldsymbol{\epsilon}-\frac{1}{2} \boldsymbol{Y}_{11}^{-1}\left(\boldsymbol{Y}_{1 . .} \circ \boldsymbol{u} \circ \boldsymbol{u}\right)+O_{p}\left(n^{-1}\right)
$$

Notando que

$$
\boldsymbol{Y}_{1 . .} \circ \boldsymbol{u} \circ \boldsymbol{u}=\boldsymbol{Y}_{111} \circ \boldsymbol{w} \circ \boldsymbol{w}-\boldsymbol{Y}_{112} \circ \boldsymbol{w} \circ \boldsymbol{\epsilon}-\boldsymbol{Y}_{121} \circ \boldsymbol{\epsilon} \circ \boldsymbol{w}+\boldsymbol{Y}_{122} \circ \boldsymbol{\epsilon} \circ \boldsymbol{\epsilon},
$$

$\boldsymbol{w}$ pode ser escrito na forma

$$
\begin{aligned}
\boldsymbol{w}= & -\boldsymbol{Y}_{11}^{-1}\left(\boldsymbol{y}_{1}-\boldsymbol{Y}_{12} \boldsymbol{\epsilon}\right)-\frac{1}{2} \boldsymbol{Y}_{11}^{-1}\left\{\boldsymbol{Y}_{111} \circ \boldsymbol{w} \circ \boldsymbol{w}-\boldsymbol{Y}_{112} \circ \boldsymbol{w} \circ \boldsymbol{\epsilon}-\boldsymbol{Y}_{121} \circ \boldsymbol{\epsilon} \circ \boldsymbol{w}\right. \\
& \left.+\boldsymbol{Y}_{122} \circ \boldsymbol{\epsilon} \circ \boldsymbol{\epsilon}\right\}+O_{p}\left(n^{-1}\right) .
\end{aligned}
$$

Invertendo esta equação com respeito a $\boldsymbol{w}$, recursivamente, vem

$$
\begin{aligned}
\boldsymbol{w}= & -\boldsymbol{Y}_{11}^{-1}\left(\boldsymbol{y}_{1}-\boldsymbol{Y}_{12} \boldsymbol{\epsilon}\right)-\frac{1}{2} \boldsymbol{Y}_{11}^{-1}\left\{\boldsymbol{Y}_{111} \circ \boldsymbol{Y}_{11}^{-1}\left(\boldsymbol{y}_{1}-\boldsymbol{Y}_{12} \boldsymbol{\epsilon}\right) \circ \boldsymbol{Y}_{11}^{-1}\left(\boldsymbol{y}_{1}-\boldsymbol{Y}_{12} \boldsymbol{\epsilon}\right)\right. \\
& \left.+\boldsymbol{Y}_{112} \circ \boldsymbol{Y}_{11}^{-1}\left(\boldsymbol{y}_{1}-\boldsymbol{Y}_{12} \boldsymbol{\epsilon}\right) \circ \boldsymbol{\epsilon}+\boldsymbol{Y}_{121} \circ \boldsymbol{\epsilon} \circ \boldsymbol{Y}_{11}^{-1}\left(\boldsymbol{y}_{1}-\boldsymbol{Y}_{12} \boldsymbol{\epsilon}\right)+\boldsymbol{Y}_{122} \circ \boldsymbol{\epsilon} \circ \boldsymbol{\epsilon}\right\}+O_{p}\left(n^{-1}\right) .
\end{aligned}
$$

Observe que se considerarmos

$$
\boldsymbol{Z}_{0}=\left[\begin{array}{cc}
\boldsymbol{Y}_{11}^{-1} & \mathbf{0} \\
\mathbf{0} & \mathbf{0}
\end{array}\right] \quad \text { e } \quad \boldsymbol{\xi}=\left[\begin{array}{c}
\boldsymbol{Y}_{11}^{-1} \boldsymbol{Y}_{12} \\
-\boldsymbol{I}_{p-q}
\end{array}\right] \boldsymbol{\epsilon}
$$

em que $\boldsymbol{I}_{p-q}$ denota uma matriz identidade de ordem $p-q, \boldsymbol{w}$ pode ser escrito na forma resumida

$$
\boldsymbol{w}=-\boldsymbol{Y}_{11}^{-1}\left(\boldsymbol{y}_{1}-\boldsymbol{Y}_{12} \boldsymbol{\epsilon}\right)-\frac{1}{2} \boldsymbol{Y}_{11}^{-1}\left\{\boldsymbol{Y}_{1 . .} \circ\left(\boldsymbol{Z}_{0} \boldsymbol{y}-\boldsymbol{\xi}\right) \circ\left(\boldsymbol{Z}_{0} \boldsymbol{y}-\boldsymbol{\xi}\right)\right\}+O_{p}\left(n^{-1}\right) .
$$

Note que $\boldsymbol{u}=\left(\boldsymbol{w}^{\top},-\boldsymbol{\epsilon}^{\top}\right)^{\top}$ pode ser relacionado com as variáveis aleatórias $\boldsymbol{y}, \boldsymbol{Y}$ e $\boldsymbol{Y} \ldots$ da forma

$$
\boldsymbol{u}=-\boldsymbol{Z}_{0} \boldsymbol{y}+\boldsymbol{\xi}-\frac{1}{2} \boldsymbol{Z}_{0} \boldsymbol{g}+O_{p}\left(n^{-1}\right)
$$


em que

$$
\boldsymbol{g}=\left[\begin{array}{c}
\boldsymbol{Y}_{1 . .} \circ\left(\boldsymbol{Z}_{0} \boldsymbol{y}-\boldsymbol{\xi}\right) \circ\left(\boldsymbol{Z}_{0} \boldsymbol{y}-\boldsymbol{\xi}\right) \\
\mathbf{0}
\end{array}\right]
$$

Inserindo $\boldsymbol{w}$ na equação

$$
\widetilde{\boldsymbol{y}}_{2}=\boldsymbol{y}_{2}+\boldsymbol{Y}_{21} \boldsymbol{w}-\boldsymbol{Y}_{22} \boldsymbol{\epsilon}+\frac{1}{2} \boldsymbol{Y}_{2 . .} \circ \boldsymbol{u} \circ \boldsymbol{u}
$$

e notando que

$$
\boldsymbol{Y}_{2 . .} \circ \boldsymbol{u} \circ \boldsymbol{u}=\boldsymbol{Y}_{211} \circ \boldsymbol{w} \circ \boldsymbol{w}-\boldsymbol{Y}_{212} \circ \boldsymbol{w} \circ \boldsymbol{\epsilon}-\boldsymbol{Y}_{221} \circ \boldsymbol{\epsilon} \circ \boldsymbol{w}+\boldsymbol{Y}_{222} \circ \boldsymbol{\epsilon} \circ \boldsymbol{\epsilon}
$$

temos que

$$
\widetilde{\boldsymbol{y}}_{2}=\boldsymbol{y}_{2}-\boldsymbol{Y}_{21} \boldsymbol{Y}_{11}^{-1} \boldsymbol{y}_{1}-\boldsymbol{Y}_{22.1} \boldsymbol{\epsilon}-\frac{1}{2}\left[\begin{array}{c}
\boldsymbol{Y}_{11}^{-1} \boldsymbol{Y}_{12} \\
-\boldsymbol{I}_{p-q}
\end{array}\right]^{\top} \boldsymbol{Y}_{\ldots} \circ\left(\boldsymbol{Z}_{0} \boldsymbol{y}-\boldsymbol{\xi}\right) \circ\left(\boldsymbol{Z}_{0} \boldsymbol{y}-\boldsymbol{\xi}\right)+O_{p}\left(n^{-1}\right),
$$

em que

$$
\boldsymbol{Y}_{22.1}=\boldsymbol{Y}_{22}-\boldsymbol{Y}_{21} \boldsymbol{Y}_{11}^{-1} \boldsymbol{Y}_{12}
$$

Assim, $\widetilde{\boldsymbol{y}}_{2}$ e $\boldsymbol{v}_{2}$ estão em função das variáveis aleatórias $\boldsymbol{y}, \boldsymbol{Y}$ e $\boldsymbol{Y}_{\ldots}$.

Como a estatística gradiente é da forma

$$
S_{T}=\widetilde{\boldsymbol{y}}_{2}^{\top} \boldsymbol{v}_{2}+\widetilde{\boldsymbol{y}}_{2}^{\top} \boldsymbol{\epsilon}
$$

podemos escrevê-la em função das variáveis aleatórias $\boldsymbol{y}, \boldsymbol{Y}$ e $\boldsymbol{Y} \ldots$. Após alguma álgebra, temos que a expansão assintótica de $S_{T}$, até ordem $O_{p}\left(n^{-1 / 2}\right)$, é da forma

$$
\begin{aligned}
S_{T}= & -\boldsymbol{y}^{\top} \boldsymbol{Z} \boldsymbol{y}-2 \boldsymbol{y}^{\top} \boldsymbol{\xi}-\boldsymbol{\epsilon}^{\top} \boldsymbol{Y}_{22.1} \boldsymbol{\epsilon} \\
& -\frac{1}{2} \boldsymbol{Y}_{\ldots} \circ \boldsymbol{Z} \boldsymbol{y} \circ \boldsymbol{Y}^{-1} \boldsymbol{y} \circ \boldsymbol{Y}^{-1} \boldsymbol{y}-\frac{1}{2} \boldsymbol{Y}_{\ldots} \circ \boldsymbol{\xi} \circ \boldsymbol{Y}^{-1} \boldsymbol{y} \circ \boldsymbol{Y}^{-1} \boldsymbol{y} \\
& -\frac{1}{2} \boldsymbol{Y}_{\ldots} \circ \boldsymbol{Z} \boldsymbol{y} \circ\left(\boldsymbol{Z}_{0} \boldsymbol{y}-\boldsymbol{\xi}\right) \circ\left(\boldsymbol{Z}_{0} \boldsymbol{y}-\boldsymbol{\xi}\right) \\
& -\frac{1}{2} \boldsymbol{Y}_{\ldots} \circ\left(\boldsymbol{Z}_{0} \boldsymbol{y}-\boldsymbol{\xi}\right) \circ\left(\boldsymbol{Z}_{0} \boldsymbol{y}-\boldsymbol{\xi}\right) \circ \boldsymbol{\xi}+O_{p}\left(n^{-1}\right),
\end{aligned}
$$

em que $\boldsymbol{Z}=\boldsymbol{Y}^{-1}-\boldsymbol{Z}_{0}$. Notando que

$$
\boldsymbol{Z}^{\top} \boldsymbol{Y} \boldsymbol{Z}=\boldsymbol{Z}, \quad \boldsymbol{Z} \boldsymbol{Y} \boldsymbol{Z}_{0}=\mathbf{0}, \quad \boldsymbol{Z}_{0} \boldsymbol{Y} \boldsymbol{\xi}=\mathbf{0} \quad \text { e } \quad \boldsymbol{Z} \boldsymbol{Y} \boldsymbol{\xi}=\boldsymbol{\xi},
$$

podemos escrever a expansão assintótica de $S_{T}$ da forma

$$
\begin{aligned}
S_{T}= & -(\boldsymbol{Z} \boldsymbol{y}+\boldsymbol{\xi})^{\top} \boldsymbol{Y}(\boldsymbol{Z} \boldsymbol{y}+\boldsymbol{\xi})-\frac{1}{2} \boldsymbol{Y}_{\ldots} \circ(\boldsymbol{Z} \boldsymbol{y}+\boldsymbol{\xi}) \circ \boldsymbol{Y}^{-1} \boldsymbol{y} \circ \boldsymbol{Y}^{-1} \boldsymbol{y} \\
& -\frac{1}{2} \boldsymbol{Y}_{\ldots} \circ(\boldsymbol{Z} \boldsymbol{y}+\boldsymbol{\xi}) \circ\left(\boldsymbol{Z}_{0} \boldsymbol{y}-\boldsymbol{\xi}\right) \circ\left(\boldsymbol{Z}_{0} \boldsymbol{y}-\boldsymbol{\xi}\right)+O_{p}\left(n^{-1}\right) .
\end{aligned}
$$

Como todos os cumulantes de $y_{r s t}(r, s, t=1,2, \ldots, p)$ são de ordem $O\left(n^{-1}\right)$, exceto o primeiro que é de ordem $O\left(n^{-1 / 2}\right)$, podemos substituir $y_{r s t}$ por $n^{-1 / 2} \kappa_{r s t}$, isto é,

$$
y_{r s t}=\frac{\kappa_{r s t}}{\sqrt{n}}+O_{p}\left(n^{-1}\right),
$$


na expressão (5.4) e ainda teremos a expansão correta até ordem $O_{p}\left(n^{-1 / 2}\right)$. Assim,

$$
\begin{aligned}
S_{T}= & -(\boldsymbol{Z} \boldsymbol{y}+\boldsymbol{\xi})^{\top} \boldsymbol{Y}(\boldsymbol{Z} \boldsymbol{y}+\boldsymbol{\xi})-\frac{1}{2 \sqrt{n}} \boldsymbol{K}_{\ldots} \circ(\boldsymbol{Z} \boldsymbol{y}+\boldsymbol{\xi}) \circ \boldsymbol{Y}^{-1} \boldsymbol{y} \circ \boldsymbol{Y}^{-1} \boldsymbol{y} \\
& -\frac{1}{2 \sqrt{n}} \boldsymbol{K}_{\ldots} \circ(\boldsymbol{Z} \boldsymbol{y}+\boldsymbol{\xi}) \circ\left(\boldsymbol{Z}_{0} \boldsymbol{y}-\boldsymbol{\xi}\right) \circ\left(\boldsymbol{Z}_{0} \boldsymbol{y}-\boldsymbol{\xi}\right)+O_{p}\left(n^{-1}\right) .
\end{aligned}
$$

\subsubsection{Função Geradora de Momentos de $S_{T}$}

Para obter a função geradora de momentos (fgm) de $S_{T}$, vamos utilizar a expansão Edgeworth multivariada da densidade conjunta das variáveis aleatórias $\boldsymbol{y}$ e $\boldsymbol{Y}$. Esta expansão foi derivada por Peers (1971) e é da forma

$$
\begin{aligned}
f_{1}= & f_{0}\left[1+\frac{1}{6 \sqrt{n}}\left(\boldsymbol{K}_{., ., .} \circ \boldsymbol{K}^{-1} \boldsymbol{y} \circ \boldsymbol{K}^{-1} \boldsymbol{y} \circ \boldsymbol{K}^{-1} \boldsymbol{y}-3 \boldsymbol{K}_{., .,} \circ \boldsymbol{K}^{-1} \circ \boldsymbol{K}^{-1} \boldsymbol{y}\right)\right. \\
& \left.-\frac{1}{\sqrt{n}} \boldsymbol{K}_{., . .} \circ \boldsymbol{K}^{-1} \boldsymbol{y} \circ \boldsymbol{D}\right]+O\left(n^{-1}\right)
\end{aligned}
$$

em que

$$
f_{0}=(2 \pi)^{-p / 2}|\boldsymbol{K}|^{-1 / 2} \exp \left\{-\frac{1}{2} \boldsymbol{y}^{\top} \boldsymbol{K}^{-1} \boldsymbol{y}\right\} \prod_{r, s=1}^{p} \delta\left(y_{r s}-\kappa_{r s}\right),
$$

$\boldsymbol{D}=\left\{d_{b c}\right\}, \operatorname{com} d_{b c}=\delta^{\prime}\left(y_{b c}-\kappa_{b c}\right) / \delta\left(y_{b c}-\kappa_{b c}\right)$, sendo $\delta(\cdot)$ a função delta de Dirac (Bracewell, 1999).

A fgm de $S_{T}, M(t)$, é dada por

$$
\begin{aligned}
M(t) & =\mathbb{E}\left(e^{t S_{T}}\right)=\int \cdots \int e^{t S_{T}} f_{1} \mathrm{~d} \boldsymbol{y} \mathrm{d} \boldsymbol{Y}+O\left(n^{-1}\right) \\
& =\int \cdots \int\left[\int \cdots \int e^{t S_{T}} f_{1} \mathrm{~d} \boldsymbol{Y}\right] \mathrm{d} \boldsymbol{y}+O\left(n^{-1}\right) \\
& =\int \cdots \int A(\boldsymbol{y}) \mathrm{d} \boldsymbol{y}+O\left(n^{-1}\right),
\end{aligned}
$$

em que

$$
A(\boldsymbol{y})=\int \cdots \int e^{t S_{T}} f_{1} \mathrm{~d} \boldsymbol{Y}
$$

Após alguma álgebra (veja Apêndice E), temos que

$$
\begin{aligned}
A(\boldsymbol{y})= & (2 \pi)^{-p / 2}|\boldsymbol{K}|^{-1 / 2} \exp \left\{-\frac{1}{2} \boldsymbol{y}^{\top} \boldsymbol{K}^{-1} \boldsymbol{y}\right\} \exp \left\{t\left(\boldsymbol{M} \boldsymbol{y}-\boldsymbol{\epsilon}^{*}\right)^{\top} \boldsymbol{K}\left(\boldsymbol{M} \boldsymbol{y}-\boldsymbol{\epsilon}^{*}\right)\right\} \\
& {\left[1+\frac{1}{6 \sqrt{n}}\left(\boldsymbol{K}_{., ., .} \circ \boldsymbol{K}^{-1} \boldsymbol{y} \circ \boldsymbol{K}^{-1} \boldsymbol{y} \circ \boldsymbol{K}^{-1} \boldsymbol{y}-3 \boldsymbol{K}_{., . .} \circ \boldsymbol{K}^{-1} \circ \boldsymbol{K}^{-1} \boldsymbol{y}\right)\right.} \\
& +\frac{t}{\sqrt{n}} \boldsymbol{K}_{., . .} \circ \boldsymbol{K}^{-1} \boldsymbol{y} \circ\left[\boldsymbol{K}^{-1} \boldsymbol{y} \boldsymbol{y}^{\top} \boldsymbol{K}^{-1}-\left(\boldsymbol{A} \boldsymbol{y}+\boldsymbol{\epsilon}^{*}\right)\left(\boldsymbol{A} \boldsymbol{y}+\boldsymbol{\epsilon}^{*}\right)^{\top}\right] \\
& \left.+\frac{t}{2 \sqrt{n}}\left[\boldsymbol{K}_{\ldots} \circ\left(\boldsymbol{M} \boldsymbol{y}-\boldsymbol{\epsilon}^{*}\right) \circ \boldsymbol{K}^{-1} \boldsymbol{y} \circ \boldsymbol{K}^{-1} \boldsymbol{y}+\boldsymbol{K}_{\ldots} \circ\left(\boldsymbol{M} \boldsymbol{y}-\boldsymbol{\epsilon}^{*}\right) \circ\left(\boldsymbol{A} \boldsymbol{y}+\boldsymbol{\epsilon}^{*}\right) \circ\left(\boldsymbol{A} \boldsymbol{y}+\boldsymbol{\epsilon}^{*}\right)\right]\right] \\
& +O_{p}\left(n^{-1}\right),
\end{aligned}
$$


em que

$$
\boldsymbol{M}=\boldsymbol{K}^{-1}-\boldsymbol{A}, \quad \boldsymbol{A}=\left[\begin{array}{cc}
\boldsymbol{K}_{11}^{-1} & \mathbf{0} \\
\mathbf{0} & \mathbf{0}
\end{array}\right] \quad \text { e } \quad \boldsymbol{\epsilon}^{*}=\left[\begin{array}{c}
\boldsymbol{K}_{11}^{-1} \boldsymbol{K}_{12} \\
-\boldsymbol{I}_{p-q}
\end{array}\right] \boldsymbol{\epsilon}
$$

Observe que se tomarmos

$$
\boldsymbol{\Sigma}=\frac{1}{1-2 t} \boldsymbol{K}-\frac{2 t}{1-2 t} \boldsymbol{K} \boldsymbol{A} \boldsymbol{K}, \quad \boldsymbol{\eta}=2 t \boldsymbol{\Sigma} \boldsymbol{M} \boldsymbol{K} \boldsymbol{\epsilon}^{*}=\frac{2 t}{1-2 t} \boldsymbol{K} \boldsymbol{\epsilon}^{*}, \quad \boldsymbol{K}_{22.1}=\boldsymbol{K}_{22}-\boldsymbol{K}_{21} \boldsymbol{K}_{11}^{-1} \boldsymbol{K}_{12},
$$

e usarmos o fato de que

$$
M K M=M
$$

o termo exponencial de $A(\boldsymbol{y})$ pode ser escrito como

$$
\exp \left\{-\frac{1}{2}(\boldsymbol{y}+\boldsymbol{\eta})^{\top} \boldsymbol{\Sigma}^{-1}(\boldsymbol{y}+\boldsymbol{\eta})\right\} \exp \left\{\frac{t}{1-2 t} \boldsymbol{\epsilon}^{\top} \boldsymbol{K}_{22.1} \boldsymbol{\epsilon}\right\} .
$$

Então,

$$
\begin{aligned}
M(t)= & \int \ldots \int A(\boldsymbol{y}) d \boldsymbol{y}+O\left(n^{-1}\right) \\
= & (2 \pi)^{-p / 2}|\boldsymbol{K}|^{-1 / 2} \exp \left\{\frac{t}{1-2 t} \boldsymbol{\epsilon}^{\top} \boldsymbol{K}_{22.1} \boldsymbol{\epsilon}\right\} \int \ldots \int \exp \left\{-\frac{1}{2}(\boldsymbol{y}+\boldsymbol{\eta})^{\top} \boldsymbol{\Sigma}^{-1}(\boldsymbol{y}+\boldsymbol{\eta})\right\} \\
& {\left[1+\frac{1}{6 \sqrt{n}}\left(\boldsymbol{K}_{., ., .} \circ \boldsymbol{K}^{-1} \boldsymbol{y} \circ \boldsymbol{K}^{-1} \boldsymbol{y} \circ \boldsymbol{K}^{-1} \boldsymbol{y}-3 \boldsymbol{K}_{., .,} \circ \boldsymbol{K}^{-1} \circ \boldsymbol{K}^{-1} \boldsymbol{y}\right)\right.} \\
& +\frac{t}{\sqrt{n}} \boldsymbol{K}_{., . .} \circ \boldsymbol{K}^{-1} \boldsymbol{y} \circ\left[\boldsymbol{K}^{-1} \boldsymbol{y} \boldsymbol{y}^{\top} \boldsymbol{K}^{-1}-\left(\boldsymbol{A} \boldsymbol{y}+\boldsymbol{\epsilon}^{*}\right)\left(\boldsymbol{A} \boldsymbol{y}+\boldsymbol{\epsilon}^{*}\right)^{\top}\right] \\
& \left.+\frac{t}{2 \sqrt{n}}\left[\boldsymbol{K}_{\ldots} \circ\left(\boldsymbol{M} \boldsymbol{y}-\boldsymbol{\epsilon}^{*}\right) \circ \boldsymbol{K}^{-1} \boldsymbol{y} \circ \boldsymbol{K}^{-1} \boldsymbol{y}+\boldsymbol{K}_{\ldots} \circ\left(\boldsymbol{M} \boldsymbol{y}-\boldsymbol{\epsilon}^{*}\right) \circ\left(\boldsymbol{A} \boldsymbol{y}+\boldsymbol{\epsilon}^{*}\right) \circ\left(\boldsymbol{A} \boldsymbol{y}+\boldsymbol{\epsilon}^{*}\right)\right]\right] \mathrm{d} \boldsymbol{y} \\
& +O\left(n^{-1}\right) .
\end{aligned}
$$

Para facilitar a resolução desta integral, faremos uma transformação de $\boldsymbol{y}$ a $\boldsymbol{z}$, em que

$$
\boldsymbol{y}=\boldsymbol{\Sigma}^{1 / 2} \boldsymbol{z}-2 t \boldsymbol{\Sigma} \boldsymbol{M} \boldsymbol{K} \boldsymbol{\epsilon}^{*}, \quad \boldsymbol{\Sigma}=\boldsymbol{\Sigma}^{1 / 2} \boldsymbol{\Sigma}^{1 / 2} .
$$

O jacobiano da transformação é

$$
\frac{\partial \boldsymbol{y}}{\partial \boldsymbol{z}}=\boldsymbol{\Sigma}^{1 / 2} \quad \text { e } \quad|\boldsymbol{\Sigma}|^{1 / 2}=(1-2 t)^{-\frac{1}{2}(p-q)}|\boldsymbol{K}|^{1 / 2}
$$

Após longa e tediosa álgebra (veja o Apêndice E), utilizando a transformação em (5.6), $M(t)$ pode ser escrito na forma

$$
M(t)=(1-2 t)^{-\frac{1}{2}(p-q)} \exp \left(\frac{t}{1-2 t} \boldsymbol{\epsilon}^{\top} \boldsymbol{K}_{22.1} \boldsymbol{\epsilon}\right)\left[1+\frac{1}{\sqrt{n}}\left(A_{1} d+A_{2} d^{2}+A_{3} d^{3}\right)\right]+O\left(n^{-1}\right)
$$

em que $d=2 t /(1-2 t)$ e

$$
A_{1}=-\frac{1}{4}\left(\boldsymbol{K}_{\ldots} \circ \boldsymbol{K}^{-1} \circ \boldsymbol{\epsilon}^{*}+4 \boldsymbol{K}_{., . .} \circ \boldsymbol{A} \circ \boldsymbol{\epsilon}^{*}+\boldsymbol{K}_{\ldots} \circ \boldsymbol{A} \circ \boldsymbol{\epsilon}^{*}+\boldsymbol{K}_{\ldots}\left(\circ \boldsymbol{\epsilon}^{*}\right)^{3}\right),
$$




$$
A_{2}=-\frac{1}{4}\left(\boldsymbol{K}_{\ldots} \circ \boldsymbol{K}^{-1} \circ \boldsymbol{\epsilon}^{*}-\boldsymbol{K}_{\ldots} \circ \boldsymbol{A} \circ \boldsymbol{\epsilon}^{*}-2 \boldsymbol{K}_{., . .}\left(\circ \boldsymbol{\epsilon}^{*}\right)^{3}\right) \quad \text { e } \quad A_{3}=-\frac{1}{12} \boldsymbol{K}_{\ldots}\left(\circ \boldsymbol{\epsilon}^{*}\right)^{3}
$$

Quando $n \rightarrow \infty, M(t) \rightarrow(1-2 t)^{-(p-q) / 2} \exp \{2 t \lambda /(1-2 t)\}$, em que $\lambda=\boldsymbol{\epsilon}^{\top} \boldsymbol{K}_{22.1} \boldsymbol{\epsilon} / 2$, assim a distribuição assintótica de $S_{T}$ é qui-quadrado não central com $p-q$ graus de liberdade e parâmetro de não centralidade $\lambda$. Sob $\mathcal{H}_{0}$, i.e. quando $\boldsymbol{\epsilon}=\mathbf{0}, M(t)=(1-2 t)^{-(p-q) / 2}+O\left(n^{-1}\right)$ e, como esperado, $S_{T}$ tem distribuição qui-quadrado central com $p-q$ graus de liberdade com erro de ordem $n^{-1}$. Também, de $M(t)$ nós podemos obter os três primeiros momentos de $S_{T}$ até ordem $n^{-1 / 2}$ como

$$
\mu_{1}^{\prime}\left(S_{T}\right)=p-q+\lambda+\frac{2 A_{1}}{\sqrt{n}}, \quad \mu_{2}\left(S_{T}\right)=2(p-q+2 \lambda)+\frac{8\left(A_{1}+A_{2}\right)}{\sqrt{n}}
$$

e

$$
\mu_{3}\left(S_{T}\right)=8(p-q+3 \lambda)+\frac{6\left(A_{1}+2 A_{2}+A_{3}\right)}{\sqrt{n}} .
$$

\subsubsection{Características de $S_{T}$ em uma Vizinhança de $\mathcal{H}_{0}$}

Vamos examinar as características da estatística gradiente, $S_{T}$, em uma vizinhança do valor hipotético $\boldsymbol{\theta}_{2}=\boldsymbol{\theta}_{20}$. Para tal propósito, precisamos ter os $\boldsymbol{\kappa}$ 's da expressão $M(t)$ em (5.7) avaliados em $\boldsymbol{\theta}_{2}=\boldsymbol{\theta}_{20}$. As relações necessárias são, com $r, s, t=1,2, \ldots, p$ e $r^{\prime}=q+1, q+2, \ldots, p$,

$$
\kappa_{r, s}=\left(\kappa_{r, s}\right)^{\dagger}+\frac{1}{\sqrt{n}}\left(\frac{\partial \kappa_{r, s}}{\partial \theta_{r^{\prime}}}\right)^{\dagger} \epsilon_{r^{\prime}}+O\left(n^{-1}\right)
$$

mas como

$$
-\frac{\partial \kappa_{r, s}}{\partial \theta_{r^{\prime}}}=\kappa_{r^{\prime}, r s}+\kappa_{r^{\prime} r s}
$$

temos que

$$
\kappa_{r, s}=\left(\kappa_{r, s}\right)^{\dagger}-\frac{1}{\sqrt{n}}\left(\kappa_{r^{\prime}, r s}+\kappa_{r^{\prime} r s}\right)^{\dagger} \epsilon_{r^{\prime}}+O\left(n^{-1}\right) .
$$

Da mesma forma, temos

$$
\kappa_{r s t}=\left(\kappa_{r s t}\right)^{\dagger}+O\left(n^{-1 / 2}\right), \quad \kappa_{r, s t}=\left(\kappa_{r, s t}\right)^{\dagger}+O\left(n^{-1 / 2}\right) \quad \text { e } \quad \kappa_{r, s, t}=\left(\kappa_{r, s, t}\right)^{\dagger}+O\left(n^{-1 / 2}\right) .
$$

Então, reescrevendo estas relações, vem

$$
\boldsymbol{K}=\boldsymbol{K}^{\dagger}-\frac{1}{\sqrt{n}} \boldsymbol{F}^{\dagger}+O\left(n^{-1}\right)
$$

em que

$$
\boldsymbol{F}^{\dagger}=\left(\boldsymbol{K}_{2, . .}+\boldsymbol{K}_{2 . .}\right)^{\dagger} \circ \boldsymbol{\epsilon}
$$

Também,

$$
\boldsymbol{K}_{\ldots}=\boldsymbol{K}_{. .}^{\dagger}+O\left(n^{-1 / 2}\right), \quad \boldsymbol{K}_{., .}=\boldsymbol{K}_{., .}^{\dagger}+O\left(n^{-1 / 2}\right) \quad \text { e } \quad \boldsymbol{K}_{., ., .}=\boldsymbol{K}_{., ., .}^{\dagger}+O\left(n^{-1 / 2}\right) .
$$

Note que

$$
\boldsymbol{K}_{22.1}=\boldsymbol{K}_{22}-\boldsymbol{K}_{21} \boldsymbol{K}_{11}^{-1} \boldsymbol{K}_{12}
$$


pode ser escrito como

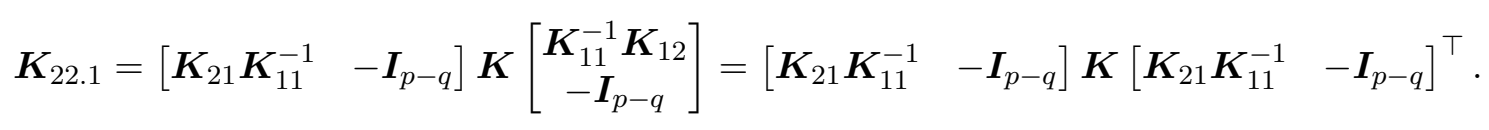

Como

$$
\boldsymbol{K}=\boldsymbol{K}^{\dagger}-\frac{1}{\sqrt{n}} \boldsymbol{F}^{\dagger}+O\left(n^{-1}\right)
$$

temos que

$$
\boldsymbol{K}_{22.1}=\boldsymbol{K}_{22.1}^{\dagger}-\frac{1}{\sqrt{n}}\left[\boldsymbol{K}_{21} \boldsymbol{K}_{11}^{-1} \quad-\boldsymbol{I}_{p-q}\right] \boldsymbol{F}^{\dagger}\left[\boldsymbol{K}_{21} \boldsymbol{K}_{11}^{-1} \quad-\boldsymbol{I}_{p-q}\right]^{\top}+O\left(n^{-1}\right) .
$$

Assim,

$$
\begin{aligned}
\boldsymbol{\epsilon}^{\top} \boldsymbol{K}_{22.1} \boldsymbol{\epsilon} & =\boldsymbol{\epsilon}^{\top} \boldsymbol{K}_{22.1}^{\dagger} \boldsymbol{\epsilon}-\left(\boldsymbol{\epsilon}^{*}\right)^{\dagger \top} \boldsymbol{F}^{\dagger}\left(\boldsymbol{\epsilon}^{*}\right)^{\dagger}+O\left(n^{-1}\right) \\
& =\boldsymbol{\epsilon}^{\top} \boldsymbol{K}_{22.1}^{\dagger} \boldsymbol{\epsilon}-\frac{1}{\sqrt{n}}\left\{\boldsymbol{K}_{2, . .}^{\dagger} \circ \boldsymbol{\epsilon} \circ\left(\boldsymbol{\epsilon}^{*}\right)^{\dagger} \circ\left(\boldsymbol{\epsilon}^{*}\right)^{\dagger}+\boldsymbol{K}_{2 . .}^{\dagger} \circ \boldsymbol{\epsilon} \circ\left(\boldsymbol{\epsilon}^{*}\right)^{\dagger} \circ\left(\boldsymbol{\epsilon}^{*}\right)^{\dagger}\right\}+O\left(n^{-1}\right) \\
& =\boldsymbol{\epsilon}^{\top} \boldsymbol{K}_{22.1}^{\dagger} \boldsymbol{\epsilon}-\frac{1}{\sqrt{n}}\left(\boldsymbol{K}_{2, . .}+\boldsymbol{K}_{2 . .}\right)^{\dagger} \circ \boldsymbol{\epsilon} \circ\left(\boldsymbol{\epsilon}^{*}\right)^{\dagger} \circ\left(\boldsymbol{\epsilon}^{*}\right)^{\dagger}+O\left(n^{-1}\right) .
\end{aligned}
$$

Utilizando a relação anterior, o termo exponencial de (5.7) é da forma

$$
\begin{aligned}
& \exp \left(\frac{t}{1-2 t} \boldsymbol{\epsilon}^{\top} \boldsymbol{K}_{22.1} \boldsymbol{\epsilon}\right)= \\
& \quad=\exp \left(\frac{t}{1-2 t}\left[\boldsymbol{\epsilon}^{\top} \boldsymbol{K}_{22.1}^{\dagger} \boldsymbol{\epsilon}-\frac{1}{\sqrt{n}}\left(\boldsymbol{K}_{2, . .}+\boldsymbol{K}_{2 . .}\right)^{\dagger} \circ \boldsymbol{\epsilon} \circ\left(\boldsymbol{\epsilon}^{*}\right)^{\dagger} \circ\left(\boldsymbol{\epsilon}^{*}\right)^{\dagger}+O\left(n^{-1}\right)\right]\right) .
\end{aligned}
$$

Expandindo em série de Taylor esta última expressão, temos que

$$
\begin{aligned}
& \exp \left(\frac{t}{1-2 t}\left[\boldsymbol{\epsilon}^{\top} \boldsymbol{K}_{22.1}^{\dagger} \boldsymbol{\epsilon}-\frac{1}{\sqrt{n}}\left(\boldsymbol{K}_{2, . .}+\boldsymbol{K}_{2 . .}\right)^{\dagger} \circ \boldsymbol{\epsilon} \circ\left(\boldsymbol{\epsilon}^{*}\right)^{\dagger} \circ\left(\boldsymbol{\epsilon}^{*}\right)^{\dagger}+O\left(n^{-1}\right)\right]\right)= \\
& =\exp \left(\frac{t}{1-2 t} \boldsymbol{\epsilon}^{\top} \boldsymbol{K}_{22.1}^{\dagger} \boldsymbol{\epsilon}\right)\left[1-n^{-1 / 2} \frac{t}{1-2 t}\left(\boldsymbol{K}_{2, . .}+\boldsymbol{K}_{2 . .}\right)^{\dagger} \circ \boldsymbol{\epsilon} \circ\left(\boldsymbol{\epsilon}^{*}\right)^{\dagger} \circ\left(\boldsymbol{\epsilon}^{*}\right)^{\dagger}\right]+O\left(n^{-1}\right) .
\end{aligned}
$$

Denote por $\bar{M}(t)$ a fgm da estatística gradiente $S_{T}$, expandida até ordem $O_{p}\left(n^{-1 / 2}\right)$, em uma vizinhança de $\boldsymbol{\theta}_{2}=\boldsymbol{\theta}_{20}$. Da expressão (5.7), temos que

$$
\begin{aligned}
\bar{M}(t)= & (1-2 t)^{-\frac{1}{2}(p-q)} \exp \left(\frac{t}{1-2 t} \boldsymbol{\epsilon}^{\top} \boldsymbol{K}_{22.1}^{\dagger} \boldsymbol{\epsilon}\right)\left[1-n^{-1 / 2} \frac{t}{1-2 t}\left(\boldsymbol{K}_{2, . .}+\boldsymbol{K}_{2 . .}\right)^{\dagger} \circ \boldsymbol{\epsilon} \circ\left(\boldsymbol{\epsilon}^{*}\right)^{\dagger} \circ\left(\boldsymbol{\epsilon}^{*}\right)^{\dagger}\right] \\
& {\left[1+\frac{1}{\sqrt{n}}\left(A_{1}^{\dagger} d+A_{2}^{\dagger} d^{2}+A_{3}^{\dagger} d^{3}\right)\right]+O\left(n^{-1}\right) } \\
= & (1-2 t)^{-\frac{1}{2}(p-q)} \exp \left(\frac{t}{1-2 t} \boldsymbol{\epsilon}^{\top} \boldsymbol{K}_{22.1}^{\dagger} \boldsymbol{\epsilon}\right) \\
& {\left[1+\frac{1}{\sqrt{n}}\left\{\left[-\frac{1}{2}\left(\boldsymbol{K}_{2, . .}+\boldsymbol{K}_{2 . .}\right)^{\dagger} \circ \boldsymbol{\epsilon} \circ\left(\boldsymbol{\epsilon}^{*}\right)^{\dagger} \circ\left(\boldsymbol{\epsilon}^{*}\right)^{\dagger}+A_{1}^{\dagger}\right] d+A_{2}^{\dagger} d^{2}+A_{3}^{\dagger} d^{3}\right\}\right]+O\left(n^{-1}\right) . }
\end{aligned}
$$


Usando frações parciais, temos que

$$
\begin{gathered}
d=\frac{2 t}{1-2 t}=-1+\frac{1}{1-2 t}, \quad d^{2}=\left(\frac{2 t}{1-2 t}\right)^{2}=1-\frac{2}{1-2 t}+\frac{1}{(1-2 t)^{2}}, \\
d^{3}=\left(\frac{2 t}{1-2 t}\right)^{3}=-1+\frac{3}{1-2 t}-\frac{3}{(1-2 t)^{2}}+\frac{1}{(1-2 t)^{3}} .
\end{gathered}
$$

Após alguma álgebra, $\bar{M}(t)$ pode ser escrita na forma equivalente

$$
\bar{M}(t)=(1-2 t)^{-\frac{1}{2}(p-q)} \exp \left(\frac{t}{1-2 t} \boldsymbol{\epsilon}^{\top} \boldsymbol{K}_{22.1}^{\dagger} \boldsymbol{\epsilon}\right)\left[1+\frac{1}{\sqrt{n}} \sum_{k=0}^{3} a_{k}(1-2 t)^{-k}\right]+O\left(n^{-1}\right),
$$

em que

$$
\begin{aligned}
a_{1}= & \frac{1}{4}\left\{\boldsymbol{K}_{\ldots . .}^{\dagger} \circ\left(\boldsymbol{K}^{-1}\right)^{\dagger} \circ\left(\boldsymbol{\epsilon}^{*}\right)^{\dagger}-2\left(\boldsymbol{K}_{\ldots}+2 \boldsymbol{K}_{., . .}\right)^{\dagger} \circ\left(\boldsymbol{\epsilon}^{*}\right)^{\dagger} \circ\left(\boldsymbol{\epsilon}^{*}\right)^{\dagger} \circ\left(\boldsymbol{\epsilon}^{*}\right)^{\dagger}\right. \\
& \left.-\left(4 \boldsymbol{K}_{. . .}+3 \boldsymbol{K}_{\ldots}\right)^{\dagger} \circ \boldsymbol{A}^{\dagger} \circ\left(\boldsymbol{\epsilon}^{*}\right)^{\dagger}-2\left(\boldsymbol{K}_{2 . .}+\boldsymbol{K}_{2, . .}\right)^{\dagger} \circ \boldsymbol{\epsilon} \circ\left(\boldsymbol{\epsilon}^{*}\right)^{\dagger} \circ\left(\boldsymbol{\epsilon}^{*}\right)^{\dagger}\right\}, \\
a_{2}= & -\frac{1}{4}\left\{\boldsymbol{K}_{\ldots}^{\dagger} \circ\left(\boldsymbol{K}^{-1}-\boldsymbol{A}\right)^{\dagger} \circ\left(\boldsymbol{\epsilon}^{*}\right)^{\dagger}-\left(\boldsymbol{K}_{\ldots}+2 \boldsymbol{K}_{. . . .}\right)^{\dagger} \circ\left(\boldsymbol{\epsilon}^{*}\right)^{\dagger} \circ\left(\boldsymbol{\epsilon}^{*}\right)^{\dagger} \circ\left(\boldsymbol{\epsilon}^{*}\right)^{\dagger}\right\}, \\
a_{3}= & -\frac{1}{12} \boldsymbol{K}_{\ldots}^{\dagger} \circ\left(\boldsymbol{\epsilon}^{*}\right)^{\dagger} \circ\left(\boldsymbol{\epsilon}^{*}\right)^{\dagger} \circ\left(\boldsymbol{\epsilon}^{*}\right)^{\dagger},
\end{aligned}
$$

e o coeficiente $a_{0}$ pode ser obtido da forma $a_{0}=-\left(a_{1}+a_{2}+a_{3}\right)$, como esperado. Invertendo a fgm dada em (5.8), temos o seguinte teorema:

Teorema 3. A expansão assintótica da distribuição da estatística gradiente $\left(S_{T}\right)$ para uma hipótese composta sob uma sequência de alternativas locais convergindo para a hipótese nula a uma taxa de convergência de $n^{-1 / 2}$ é como segue:

$$
\operatorname{Pr}\left(S_{T} \leq x\right)=\operatorname{Pr}\left(\chi_{f, \lambda}^{2} \leq x\right)+\frac{1}{\sqrt{n}} \sum_{k=0}^{3} a_{k} \operatorname{Pr}\left(\chi_{f+2 k, \lambda}^{2} \leq x\right)+O\left(n^{-1}\right),
$$

em que $\chi_{f, \lambda}^{2}$ é uma variável aleatória qui-quadrado não-central com $f$ graus de liberdade e parâmetro de não-centralidade $\lambda$. Aqui, $f=p-q, \lambda=\boldsymbol{\epsilon}^{\top} \boldsymbol{K}_{22.1}^{\dagger} \boldsymbol{\epsilon} / 2$ e os a k $^{\prime}$ s estão dados em (5.9).

Note que se $q=0$, a hipótese nula considerada inicialmente em (5.1) reduz-se ao caso simples, assim $\boldsymbol{\epsilon}^{*}=-\boldsymbol{\epsilon}$ e $\boldsymbol{A}=\mathbf{0}$. Então, uma consequência imediata do teorema anterior é como segue:

Corolário. A expansão assintótica da distribuição da estatística gradiente $\left(S_{T}\right)$ para uma hipótese simples sob uma sequência de alternativas locais convergindo para a hipótese nula a uma taxa de convergência de $n^{-1 / 2}$ é como segue:

$$
\operatorname{Pr}\left(S_{T} \leq x\right)=\operatorname{Pr}\left(\chi_{p, \lambda}^{2} \leq x\right)+\frac{1}{\sqrt{n}} \sum_{k=0}^{3} a_{k} \operatorname{Pr}\left(\chi_{p+2 k, \lambda}^{2} \leq x\right)+O\left(n^{-1}\right),
$$

em que $\lambda=\boldsymbol{\epsilon}^{\top} \boldsymbol{K}^{\dagger} \boldsymbol{\epsilon} / 2$ e

$$
a_{0}=\frac{1}{6} \boldsymbol{K}_{\ldots}^{\dagger} \circ \boldsymbol{\epsilon} \circ \boldsymbol{\epsilon} \circ \boldsymbol{\epsilon}, \quad a_{1}=-\frac{1}{4}\left\{\boldsymbol{K}_{\ldots}^{\dagger} \circ\left(\boldsymbol{K}^{-1}\right)^{\dagger} \circ \boldsymbol{\epsilon}-2 \boldsymbol{K}_{., . .}^{\dagger} \circ \boldsymbol{\epsilon} \circ \boldsymbol{\epsilon} \circ \boldsymbol{\epsilon}\right\},
$$




$$
a_{2}=\frac{1}{4}\left\{\boldsymbol{K}_{\ldots}^{\dagger} \circ\left(\boldsymbol{K}^{-1}\right)^{\dagger} \circ \boldsymbol{\epsilon}-\left(\boldsymbol{K}_{\ldots}+2 \boldsymbol{K}_{., . .}\right)^{\dagger} \circ \boldsymbol{\epsilon} \circ \boldsymbol{\epsilon} \circ \boldsymbol{\epsilon}\right\} \quad e \quad a_{3}=\frac{1}{12} \boldsymbol{K}_{\ldots}^{\dagger} \circ \boldsymbol{\epsilon} \circ \boldsymbol{\epsilon} \circ \boldsymbol{\epsilon} .
$$

\subsubsection{Comparação de Poder entre os Testes Rivais}

Seja $S_{i}(i=1,2,3,4)$ representando, respectivamente, a estatística da razão de verossimilhanças, de Wald, escore de Rao e estatística gradiente. De Hayakawa (1975) e Harris \& Peers (1980), temos

$$
\operatorname{Pr}\left(S_{i} \leq x\right)=\operatorname{Pr}\left(\chi_{f, \lambda}^{2} \leq x\right)+\frac{1}{\sqrt{n}} \sum_{k=0}^{3} a_{i k} \operatorname{Pr}\left(\chi_{f+2 k, \lambda}^{2} \leq x\right)+O\left(n^{-1}\right),
$$

em que

$$
\begin{aligned}
& a_{11}=-\frac{1}{6}\left\{\left(\boldsymbol{K}_{\ldots}-2 \boldsymbol{K}_{., . . .}\right)^{\dagger} \circ\left(\boldsymbol{\epsilon}^{*}\right)^{\dagger} \circ\left(\boldsymbol{\epsilon}^{*}\right)^{\dagger} \circ\left(\boldsymbol{\epsilon}^{*}\right)^{\dagger}+3\left(\boldsymbol{K}_{\ldots}+2 \boldsymbol{K}_{., . .}\right)^{\dagger} \circ \boldsymbol{A}^{\dagger} \circ\left(\boldsymbol{\epsilon}^{*}\right)^{\dagger}\right. \\
& \left.+3\left(\boldsymbol{K}_{2 . .}+\boldsymbol{K}_{2, . .}\right)^{\dagger} \circ \boldsymbol{\epsilon} \circ\left(\boldsymbol{\epsilon}^{*}\right)^{\dagger} \circ\left(\boldsymbol{\epsilon}^{*}\right)^{\dagger}\right\} \text {, } \\
& a_{12}=-\frac{1}{6} \boldsymbol{K}_{., ., .}^{\dagger} \circ\left(\boldsymbol{\epsilon}^{*}\right)^{\dagger} \circ\left(\boldsymbol{\epsilon}^{*}\right)^{\dagger} \circ\left(\boldsymbol{\epsilon}^{*}\right)^{\dagger} \text {, } \\
& a_{13}=0 \text {, } \\
& a_{21}=\frac{1}{2}\left\{-\left(\boldsymbol{K}_{\ldots}+2 \boldsymbol{K}_{., . .}\right)^{\dagger} \circ\left(\boldsymbol{\epsilon}^{*}\right)^{\dagger} \circ\left(\boldsymbol{\epsilon}^{*}\right)^{\dagger} \circ\left(\boldsymbol{\epsilon}^{*}\right)^{\dagger}+2 \boldsymbol{K}_{., . .}^{\dagger} \circ\left(\boldsymbol{K}^{-1}-\boldsymbol{A}\right)^{\dagger} \circ\left(\boldsymbol{\epsilon}^{*}\right)^{\dagger}\right. \\
& \left.-\left(\boldsymbol{K}_{\ldots}+2 \boldsymbol{K}_{., .}\right)^{\dagger} \circ\left(\boldsymbol{K}^{-1}\right)^{\dagger} \circ\left(\boldsymbol{\epsilon}^{*}\right)^{\dagger}-\left(\boldsymbol{K}_{2 . .}+\boldsymbol{K}_{2, . .}\right)^{\dagger} \circ \boldsymbol{\epsilon} \circ\left(\boldsymbol{\epsilon}^{*}\right)^{\dagger} \circ\left(\boldsymbol{\epsilon}^{*}\right)^{\dagger}\right\} \text {, } \\
& a_{22}=\frac{1}{2}\left\{\boldsymbol{K}_{\ldots}^{\dagger} \circ\left(\boldsymbol{K}^{-1}-\boldsymbol{A}\right)^{\dagger} \circ\left(\boldsymbol{\epsilon}^{*}\right)^{\dagger}+\boldsymbol{K}_{. . . .}^{\dagger} \circ\left(\boldsymbol{\epsilon}^{*}\right)^{\dagger} \circ\left(\boldsymbol{\epsilon}^{*}\right)^{\dagger} \circ\left(\boldsymbol{\epsilon}^{*}\right)^{\dagger}\right\} \text {, } \\
& a_{23}=\frac{1}{6} \boldsymbol{K}_{\ldots}^{\dagger} \circ\left(\boldsymbol{\epsilon}^{*}\right)^{\dagger} \circ\left(\boldsymbol{\epsilon}^{*}\right)^{\dagger} \circ\left(\boldsymbol{\epsilon}^{*}\right)^{\dagger}, \\
& a_{31}=\frac{1}{6}\left\{-\left(\boldsymbol{K}_{\ldots}-2 \boldsymbol{K}_{., ., .}\right)^{\dagger} \circ\left(\boldsymbol{\epsilon}^{*}\right)^{\dagger} \circ\left(\boldsymbol{\epsilon}^{*}\right)^{\dagger} \circ\left(\boldsymbol{\epsilon}^{*}\right)^{\dagger}+3 \boldsymbol{K}_{., ., .}^{\dagger} \circ\left(\boldsymbol{K}^{-1}-\boldsymbol{A}\right)^{\dagger} \circ\left(\boldsymbol{\epsilon}^{*}\right)^{\dagger}\right. \\
& \left.-3\left(\boldsymbol{K}_{\ldots}+2 \boldsymbol{K}_{. . .}\right)^{\dagger} \circ \boldsymbol{A}^{\dagger} \circ\left(\boldsymbol{\epsilon}^{*}\right)^{\dagger}-3\left(\boldsymbol{K}_{2 . .}+\boldsymbol{K}_{2, . .}\right)^{\dagger} \circ \boldsymbol{\epsilon} \circ\left(\boldsymbol{\epsilon}^{*}\right)^{\dagger} \circ\left(\boldsymbol{\epsilon}^{*}\right)^{\dagger}\right\} \text {, } \\
& a_{32}=-\frac{1}{2} \boldsymbol{K}_{,,, .,}^{\dagger} \circ\left(\boldsymbol{K}^{-1}-\boldsymbol{A}\right)^{\dagger} \circ\left(\boldsymbol{\epsilon}^{*}\right)^{\dagger} \text {, } \\
& a_{33}=-\frac{1}{6} \boldsymbol{K}_{.,, .,}^{\dagger} \circ\left(\boldsymbol{\epsilon}^{*}\right)^{\dagger} \circ\left(\boldsymbol{\epsilon}^{*}\right)^{\dagger} \circ\left(\boldsymbol{\epsilon}^{*}\right)^{\dagger} \text {, }
\end{aligned}
$$

sendo os coeficientes $a_{i 0}$ 's obtidos como $a_{i 0}=-\left(a_{i 1}+a_{i 2}+a_{i 3}\right)$, para $i=1,2,3$. A expansão assintótica da distribuição da estatística $S_{4}$, isto é, $S_{T}$, foi obtida na seção anterior.

Podemos expressar as funções de poder (localmente) como

$$
\Pi_{i}=\Pi_{i}\left(x_{\alpha}\right)=1-\operatorname{Pr}\left(S_{i} \leq x_{\alpha}\right)=\operatorname{Pr}\left(S_{i}>x_{\alpha}\right), \quad i=1,2,3,4,
$$

em que $x_{\alpha}$ é obtido de $\operatorname{Pr}\left(\chi_{p-q}^{2} \leq x_{\alpha}\right)=1-\alpha$, sendo $\chi_{p-q}^{2}$ uma variável aleatória qui-quadrado central com $p-q$ graus de liberdade. O comportamento local das funções de poder em $\boldsymbol{\theta}_{2}=\boldsymbol{\theta}_{20}$ é refletido em suas derivadas (de primeira e segunda ordens) neste ponto. Todos os coeficientes apresentados anteriormente são complicados mas podemos tirar as seguintes conclusões gerais:

(a) Todos os quatro testes são localmente viesados em geral;

(b) Se $\boldsymbol{K}_{\ldots}=\mathbf{0}, S_{1}, S_{2}$ e $S_{4}$ têm propriedades de poder local idênticas; 
(c) Se $\boldsymbol{K}_{\ldots}=2 \boldsymbol{K}_{., ., .}, S_{3}$ e $S_{4}$ têm propriedades de poder local idênticas.

Mais classificações são possíveis se introduzirmos subespaços apropriados do espaço paramétrico. Desta análise, conclui-se que não é possível, em geral, afirmar que um dos testes é mais poderoso que os demais, ou seja, somente em situações particulares podem ser feitas comparações.

As conclusões gerais apresentadas anteriormente sobre o comportamente de poder local dos testes da razão de verossimilhanças, de Wald e escore de Rao, e o teste com base na estatística gradiente, leva-nos a concluir que o novo critério para testar hipóteses proposto por Terrell (2002), como C.R. Rao havia ressaltado, além de ser muito simples de ser calculado, apresenta, até ordem $n^{-1 / 2}$, poder local indêntico (em algumas situações particulares) ao poder local dos tradicionais testes de hipótese utilizados em aplicações práticas. Portanto, esperamos que a proposta de Terrell seja explorada em aplicações práticas e, com o passar do tempo, seja tão conhecida quanto os tradicionalíssimos testes da razão de verossimilhanças, de Wald e escore.

\subsection{Expansões Assintóticas na Família Exponencial Uniparamétrica}

Seja $\boldsymbol{x}=\left(x_{1}, x_{2}, \ldots, x_{n}\right)^{\top}$ uma amostra aleatória de tamanho $n$, em que cada $x_{i}$ possui distribuição pertencente a família uniparamétrica com função densidade de probabilidade (fdp) da forma

$$
\pi(x ; \theta)=\exp \{t(x ; \theta)\}
$$

sendo $\theta$ um parâmetro escalar.

Considere a notação

$$
\kappa_{\theta \theta}=\mathbb{E}\left\{t^{\prime \prime}(x ; \theta)\right\}, \quad \kappa_{\theta \theta \theta}=\mathbb{E}\left\{t^{\prime \prime \prime}(x ; \theta)\right\}, \quad \kappa_{\theta \theta, \theta}=\mathbb{E}\left\{t^{\prime \prime}(x ; \theta) t^{\prime}(x ; \theta)\right\}, \quad \kappa_{\theta \theta}^{(\theta)}=\frac{\mathrm{d} \kappa_{\theta \theta}}{\mathrm{d} \theta}, \quad \text { etc. },
$$

em que as linhas denotam derivadas com relação a $\theta$, por exemplo, $t^{\prime \prime}(x ; \theta)=\mathrm{d}^{2} t(x ; \theta) / \mathrm{d} \theta^{2}$. Os $\kappa$ 's satisfazem certas condições de regularidade, entre elas, temos

$$
\kappa_{\theta, \theta}=-\kappa_{\theta \theta}, \quad \kappa_{\theta \theta, \theta}=\kappa_{\theta \theta}^{(\theta)}-\kappa_{\theta \theta \theta}, \quad \kappa_{\theta, \theta, \theta}=2 \kappa_{\theta \theta \theta}-3 \kappa_{\theta \theta}^{(\theta)},
$$

entre outras. Vamos considerar o problema de testar a hipótese nula simples $\mathcal{H}_{0}: \theta=\theta^{(0)}$ contra a hipótese alternativa $\mathcal{H}_{1}: \theta \neq \theta^{(0)}$, sendo $\theta^{(0)}$ um escalar especificado. Para testar a hipótese nula anterior, as estatísticas da razão de verossimilhanças $(L R)$, de Wald $(W)$, escore de Rao $\left(S_{R}\right)$ e estatística gradiente $\left(S_{T}\right)$ assumem as formas

$$
\begin{gathered}
L R=2 \sum_{i=1}^{n}\left\{t\left(x_{i} ; \widehat{\theta}\right)-t\left(x_{i} ; \theta^{(0)}\right)\right\}, \quad W=n\left(\widehat{\theta}-\theta^{(0)}\right)^{2} I(\widehat{\theta}), \\
S_{R}=\left\{\frac{1}{\sqrt{n}} \sum_{i=1}^{n} t^{\prime}\left(x_{i} ; \theta^{(0)}\right)\right\}^{2} I\left(\theta^{(0)}\right)^{-1} \quad \text { e } \quad S_{T}=\left(\widehat{\theta}-\theta^{(0)}\right) \sum_{i=1}^{n} t^{\prime}\left(x_{i} ; \theta^{(0)}\right),
\end{gathered}
$$

em que $\widehat{\theta}$ é a estimativa de máxima verossimilhança de $\theta$ e $I(\theta)$ denota a informação de Fisher de $\theta$ para uma simples observação. Sob $\mathcal{H}_{0}: \theta=\theta^{(0)}$, as quatro estatísticas apresentadas acima possuem distribuição (assintótica) qui-quadrado com um grau de liberdade, isto é, $\chi_{1}^{2}$. 
A hipótese alternativa contígua considerada aqui é da forma

$$
\mathcal{H}_{1}: \theta=\theta^{(0)}+\frac{\epsilon}{\sqrt{n}},
$$

isto é, $\epsilon=n^{1 / 2}\left(\theta-\theta^{(0)}\right)$ assumido ser de ordem $O(1)$. Seja $S_{i}(i=1,2,3,4)$ representando, respectivamente, as estatísticas da razão de verossimilhanças, de Wald, escore de Rao e estatística gradiente. As expansões assintóticas das estatísticas $S_{1}, S_{2}, S_{3}$ e $S_{4}$, até ordem $n^{-1 / 2}$ e sob a hipótese alternativa contígua anterior, assumem a forma

$$
\operatorname{Pr}\left(S_{i} \leq x\right)=\operatorname{Pr}\left(\chi_{1, \lambda}^{2} \leq x\right)+\frac{1}{\sqrt{n}} \sum_{k=0}^{3} a_{i k} \operatorname{Pr}\left(\chi_{1+2 k, \lambda}^{2} \leq x\right)+O\left(n^{-1}\right),
$$

em que $\lambda=I \epsilon^{2} / 2, a_{13}=0$

$$
\begin{gathered}
a_{10}=a_{20}=a_{30}=-a_{23}=2 a_{43}=\frac{1}{6} \kappa_{\theta \theta \theta} \epsilon^{3}, \quad a_{11}=\frac{1}{2} \kappa_{\theta, \theta \theta} \epsilon^{3}, \quad a_{12}=a_{33}=-a_{40}=\frac{1}{6} \kappa_{\theta, \theta, \theta} \epsilon^{3}, \\
a_{21}=-a_{22}=\frac{1}{2} \kappa_{\theta, \theta \theta} \epsilon^{3}+\frac{1}{2} \kappa_{\theta \theta \theta} \kappa^{\theta, \theta} \epsilon, \quad a_{31}=\frac{1}{6}\left(\kappa_{\theta \theta \theta}-2 \kappa_{\theta, \theta, \theta}\right) \epsilon^{3}-\frac{1}{2}\left(\kappa_{\theta \theta \theta}+\kappa_{\theta, \theta \theta}\right) \epsilon^{3}-\frac{1}{2} \kappa_{\theta, \theta, \theta} \kappa^{\theta, \theta} \epsilon, \\
a_{32}=\frac{1}{2} \kappa_{\theta, \theta, \theta} \kappa^{\theta, \theta} \epsilon, \quad a_{41}=-\frac{1}{4} \kappa_{\theta \theta \theta} \kappa^{\theta, \theta} \epsilon+\frac{1}{2} \kappa_{\theta, \theta \theta} \epsilon^{3} \quad \mathrm{e} \quad a_{42}=\frac{1}{4} \kappa_{\theta \theta \theta} \kappa^{\theta, \theta} \epsilon-\frac{1}{4}\left(\kappa_{\theta \theta \theta}+2 \kappa_{\theta, \theta \theta}\right) \epsilon^{3},
\end{gathered}
$$

em que $\kappa^{\theta, \theta}=\kappa_{\theta, \theta}^{-1}=-\kappa_{\theta \theta}^{-1}$.

Considere agora a família exponencial uniparamética. Para tal, assuma que, em $(5.10), t(x ; \theta)=$ $-\log \zeta(\theta)-\alpha(\theta) d(x)+v(x)$, ou seja,

$$
\pi(x ; \theta)=\frac{1}{\zeta(\theta)} \exp \{-\alpha(\theta) d(x)+v(x)\}
$$

em que $\alpha(\cdot), \zeta(\cdot), d(\cdot)$ e $v(\cdot)$ são funções conhecidas. Estamos assumindo que o suporte de $\pi(x ; \theta)$ não depende do parâmetro $\theta$. Também, $\alpha(\cdot)$ e $\zeta(\cdot)$ são assumidas terem as três primeiras derivadas contínuas, $\zeta(\cdot)>0$ e $\alpha^{\prime}(\theta)$ e $\beta^{\prime}(\theta)$ são diferentes de zero para todo $\theta$ no espaço paramétrico, em que

$$
\beta(\theta)=\frac{\zeta^{\prime}(\theta)}{\zeta(\theta) \alpha^{\prime}(\theta)}
$$

Adicionalmente, pode ser mostrado que $\mathbb{E}\left\{d\left(x_{i}\right)\right\}=-\beta(\theta)$ e $\operatorname{Var}\left\{d\left(x_{i}\right)\right\}=\beta^{\prime}(\theta) / \alpha^{\prime}(\theta), i=1,2, \ldots, n$.

Uma vez que $I(\theta)=\alpha^{\prime}(\theta) \beta^{\prime}(\theta)$ e

$$
\sum_{i=1}^{n} t\left(x_{i} ; \theta\right)=-n\{\log \zeta(\theta)+\alpha(\theta) \bar{d}-\bar{v}\} \quad \text { e } \sum_{i=1}^{n} t^{\prime}\left(x_{i} ; \theta\right)=-n \alpha^{\prime}(\theta)\{\beta(\theta)+\bar{d}\},
$$

em que $\bar{d}=\sum_{i=1}^{n} d\left(x_{i}\right) / n$ e $\bar{v}=\sum_{i=1}^{n} v\left(x_{i}\right) / n$, as estatísticas $S_{1}, S_{2}, S_{3}$ e $S_{4}$ podem ser escritas da forma

$$
\begin{gathered}
S_{1}=2 n\left[\log \left\{\zeta\left(\theta^{(0)}\right) / \zeta(\widehat{\theta})\right\}+\left(\alpha\left(\theta^{(0)}\right)-\alpha(\widehat{\theta})\right) \bar{d}\right], \quad S_{2}=n\left(\widehat{\theta}-\theta^{(0)}\right)^{2} \alpha^{\prime}(\widehat{\theta}) \beta^{\prime}(\widehat{\theta}), \\
S_{3}=\frac{n \alpha^{\prime}\left(\theta^{(0)}\right)\left\{\beta\left(\theta^{(0)}\right)+\bar{d}\right\}^{2}}{\beta^{\prime}\left(\theta^{(0)}\right)} \text { e } \quad S_{4}=n\left(\theta^{(0)}-\widehat{\theta}\right) \alpha^{\prime}\left(\theta^{(0)}\right)\left\{\beta\left(\theta^{(0)}\right)+\bar{d}\right\} .
\end{gathered}
$$


Seja $\alpha^{\prime}=\alpha^{\prime}(\theta), \alpha^{\prime \prime}=\alpha^{\prime \prime}(\theta), \beta^{\prime}=\beta^{\prime}(\theta)$ e $\beta^{\prime \prime}=\beta^{\prime \prime}(\theta)$. É possível mostrar que

$$
\begin{gathered}
\kappa_{\theta \theta}=-\alpha^{\prime} \beta^{\prime}, \quad \kappa_{\theta \theta \theta}=-\left(2 \alpha^{\prime \prime} \beta^{\prime}+\alpha^{\prime} \beta^{\prime \prime}\right), \quad \kappa_{\theta \theta}^{(\theta)}=-\left(\alpha^{\prime \prime} \beta^{\prime}+\alpha^{\prime} \beta^{\prime \prime}\right), \\
\kappa_{\theta, \theta \theta}=\alpha^{\prime \prime} \beta^{\prime} \text { e } \kappa_{\theta, \theta, \theta}=\alpha^{\prime} \beta^{\prime \prime}-\alpha^{\prime \prime} \beta^{\prime} .
\end{gathered}
$$

Então, utilizando os cumulantes anteriores, temos que

$$
\begin{gathered}
a_{10}=a_{20}=a_{30}=-a_{23}=2 a_{43}=-\frac{\left(2 \alpha^{\prime \prime} \beta^{\prime}+\alpha^{\prime} \beta^{\prime \prime}\right) \epsilon^{3}}{6}, \quad a_{11}=\frac{\alpha^{\prime \prime} \beta^{\prime} \epsilon^{3}}{2}, \quad a_{13}=0, \\
a_{12}=a_{33}=-a_{40}=\frac{\left(\alpha^{\prime} \beta^{\prime \prime}-\alpha^{\prime \prime} \beta^{\prime}\right) \epsilon^{3}}{6}, \quad a_{21}=-a_{22}=\frac{\alpha^{\prime \prime} \beta^{\prime} \epsilon^{3}}{2}-\frac{\left(2 \alpha^{\prime \prime} \beta^{\prime}+\alpha^{\prime} \beta^{\prime \prime}\right) \epsilon}{2 \alpha^{\prime} \beta^{\prime}}, \\
a_{31}=\frac{\alpha^{\prime \prime} \beta^{\prime} \epsilon^{3}}{2}-\frac{\left(\alpha^{\prime} \beta^{\prime \prime}-\alpha^{\prime \prime} \beta^{\prime}\right) \epsilon}{2 \alpha^{\prime} \beta^{\prime}}, \quad a_{32}=\frac{\left(\alpha^{\prime} \beta^{\prime \prime}-\alpha^{\prime \prime} \beta^{\prime}\right) \epsilon}{2 \alpha^{\prime} \beta^{\prime}}, \\
a_{41}=\frac{\alpha^{\prime \prime} \beta^{\prime} \epsilon^{3}}{2}+\frac{\left(2 \alpha^{\prime \prime} \beta^{\prime}+\alpha^{\prime} \beta^{\prime \prime}\right) \epsilon}{4 \alpha^{\prime} \beta^{\prime}} \text { e } \quad a_{42}=\frac{\alpha^{\prime} \beta^{\prime \prime} \epsilon^{3}}{4}-\frac{\left(2 \alpha^{\prime \prime} \beta^{\prime}+\alpha^{\prime} \beta^{\prime \prime}\right) \epsilon}{4 \alpha^{\prime} \beta^{\prime}} .
\end{gathered}
$$

Quando $\alpha(\theta)=\theta$, isto é, $t(x ; \theta)=-\log \zeta(\theta)-\theta d(x)+v(x),(5.10)$ corresponde a família exponencial natural, isto é,

$$
\pi(x ; \theta)=\frac{1}{\zeta(\theta)} \exp \{-\theta d(x)+v(x)\} .
$$

Neste caso, os coeficientes $a_{i k}$ 's acima tomam a seguinte forma, uma vez que $\alpha^{\prime}=1$ e $\alpha^{\prime \prime}=0$,

$$
\begin{gathered}
a_{10}=a_{20}=a_{30}=-a_{23}=2 a_{43}=-\frac{\beta^{\prime \prime} \epsilon^{3}}{6}, \quad a_{11}=a_{13}=0, \\
a_{12}=a_{33}=-a_{40}=\frac{\beta^{\prime \prime} \epsilon^{3}}{6}, \quad a_{21}=-a_{22}=-\frac{\beta^{\prime \prime} \epsilon}{2 \beta^{\prime}}, \\
a_{31}=-\frac{\beta^{\prime \prime} \epsilon}{2 \beta^{\prime}}, \quad a_{32}=\frac{\beta^{\prime \prime} \epsilon}{2 \beta^{\prime}}, \quad a_{41}=\frac{\beta^{\prime \prime} \epsilon}{4 \beta^{\prime}} \quad \text { e } \quad a_{42}=\frac{\beta^{\prime \prime} \epsilon^{3}}{4}-\frac{\beta^{\prime \prime} \epsilon}{4 \beta^{\prime}} .
\end{gathered}
$$

A seguir, apresentamos comparações analíticas entre os poderes locais dos testes com base nas estatísticas da razão de verossimilhanças, Wald, escore de Rao e na estatística gradiente para algumas distribuições pertencentes à família exponencial uniparamétrica. Sejam $\Pi_{i}$ e $\Pi_{j}$ as funções de poder, até ordem $n^{-1 / 2}$, das estatísticas $S_{i}$ e $S_{j}$, respectivamente, com $i \neq j$ e $i, j=1,2,3,4$. Note que a partir de (5.11), temos que

$$
\Pi_{i}-\Pi_{j}=\frac{1}{\sqrt{n}} \sum_{k=1}^{3}\left(a_{j k}-a_{i k}\right) \operatorname{Pr}\left(\chi_{1+2 k, \lambda}^{2} \leq x\right) .
$$

É bem conhecido que

$$
\operatorname{Pr}\left(\chi_{m, \lambda}^{2} \leq x\right)-\operatorname{Pr}\left(\chi_{m+2, \lambda}^{2} \leq x\right)=2 f_{m+2}(x ; \lambda),
$$

sendo $f_{l}(x ; \lambda)$ a função densidade de uma variável aleatória qui-quadrado não-central com $l$ graus de liberdade e parâmetro de não-centralidade $\lambda$. Usando este fato e a expressão (5.12), é possível comparar, de forma analítica, os poderes locais dos testes com base em $S_{1}, S_{2}, S_{3}$ e $S_{4}$ para algumas distribuições pertencentes à família exponencial uniparamétrica, entre elas, temos: normal, normal 
inversa, gama, Pareto, power, Laplace e valor extremo truncado. Vale ressaltar que nas comparações das funções de poder $\Pi_{1}, \Pi_{2}, \Pi_{3}$ e $\Pi_{4}$ estamos considerando que $\theta>\theta^{(0)}$, no entanto, todas as desigualdades envolvendo os $\Pi_{i}$ 's se invertem se $\theta<\theta^{(0)}$. Por exemplo, se $\Pi_{1}>\Pi_{2}>\Pi_{3}>\Pi_{4}$ para $\theta>\theta^{(0)}$, então $\Pi_{1}<\Pi_{2}<\Pi_{3}<\Pi_{4}$ para $\theta<\theta^{(0)}$.

1. Normal $(\theta>0,-\infty \leq \mu \leq \infty$ e $x \in \mathbb{R})$ :

- $\mu$ conhecido: $\alpha(\theta)=(2 \theta)^{-1}, \zeta(\theta)=\theta^{1 / 2}, d(x)=(x-\mu)^{2}$ e $v(x)=-\{\log (2 \pi)\} / 2$. Neste caso nós temos que

$$
\begin{gathered}
a_{10}=a_{20}=a_{30}=-a_{23}=2 a_{43}=\frac{\epsilon^{3}}{3 \theta^{3}}, \quad a_{11}=-\frac{\epsilon^{3}}{2 \theta^{3}}, \quad a_{12}=a_{33}=-a_{40}=\frac{\epsilon^{3}}{6 \theta^{3}}, \\
a_{21}=-a_{22}=-\frac{\epsilon^{3}}{2 \theta^{3}}+\frac{2 \epsilon}{\theta}, \quad a_{31}=-\frac{\epsilon^{3}}{2 \theta^{3}}-\frac{\epsilon}{\theta}, \quad a_{32}=\frac{\epsilon}{\theta}, \quad a_{13}=0, \\
a_{41}=-\frac{\epsilon^{3}}{2 \theta^{3}}-\frac{\epsilon}{\theta} \quad \text { e } \quad a_{42}=\frac{\epsilon}{\theta} .
\end{gathered}
$$

Então, como $\epsilon=n^{1 / 2}\left(\theta-\theta^{(0)}\right)$, após álgebra simples, temos que $\Pi_{4}>\Pi_{3}>\Pi_{1}>\Pi_{2}$.

- $\theta$ conhecido: $\alpha(\mu)=-\mu / \theta, \zeta(\mu)=\exp \left\{\mu^{2} /(2 \theta)\right\}, d(x)=x$ e $v(x)=-\left\{x^{2}+\log (2 \pi \theta)\right\} / 2$. É fácil ver que $a_{10}=\cdots=a_{43}=0 \mathrm{e}$, como esperado, $\Pi_{1}=\Pi_{2}=\Pi_{3}=\Pi_{4}$ até ordem $n^{-1 / 2}$.

2. Normal inversa $(\theta>0, \mu>0$ e $x>0)$ :

- $\mu$ conhecido: $\alpha(\theta)=\theta, \zeta(\theta)=\theta^{-1 / 2}, d(x)=(x-\mu)^{2} /\left(2 \mu^{2} x\right)$ e $v(x)=-\left\{\log \left(2 \pi x^{3}\right)\right\} / 2$. Temos que

$$
\begin{gathered}
a_{10}=a_{20}=a_{30}=-a_{23}=2 a_{43}=\frac{\epsilon^{3}}{6 \theta^{3}}, \quad a_{11}=0, \quad a_{12}=a_{33}=-a_{40}=-\frac{\epsilon^{3}}{6 \theta^{3}}, \\
a_{21}=-a_{22}=\frac{\epsilon}{\theta}, \quad a_{31}=\frac{\epsilon}{\theta}, \quad a_{32}=-\frac{\epsilon}{\theta}, \quad a_{13}=0, \quad a_{41}=-\frac{\epsilon}{2 \theta} \quad \text { e } \quad a_{42}=-\frac{\epsilon^{3}}{4 \theta^{3}}+\frac{\epsilon}{2 \theta} .
\end{gathered}
$$

Então, tem-se que $\Pi_{1}>\Pi_{4}>\Pi_{2}=\Pi_{3}$.

- $\theta$ conhecido: $\left.\alpha(\mu)=\theta /\left(2 \mu^{2}\right), \zeta(\mu)=\exp \{-\theta / \mu)\right\}, d(x)=x$ e $v(x)=-\left\{\theta / x^{2}-\right.$ $\left.\log \left(\theta /\left(2 \pi x^{3}\right)\right)\right\} / 2$. Temos que

$$
\begin{gathered}
a_{10}=a_{20}=a_{30}=-a_{23}=2 a_{43}=\frac{\theta \epsilon^{3}}{\mu^{4}}, \quad a_{11}=-\frac{3 \theta \epsilon^{3}}{2 \mu^{4}}, \quad a_{12}=a_{33}=-a_{40}=\frac{\theta \epsilon^{3}}{2 \mu^{4}}, \\
a_{21}=-a_{22}=-\frac{3 \theta \epsilon^{3}}{2 \mu^{4}}+\frac{3 \epsilon}{\mu}, \quad a_{31}=-\frac{3 \theta \epsilon^{3}}{2 \mu^{4}}-\frac{3 \epsilon}{2 \mu}, \quad a_{32}=\frac{3 \epsilon}{2 \mu}, \quad a_{13}=0, \\
a_{41}=-\frac{3 \theta \epsilon^{3}}{2 \mu^{4}}-\frac{3 \epsilon}{2 \mu} \quad \text { e } \quad a_{42}=\frac{3 \epsilon}{2 \mu} .
\end{gathered}
$$

Assim, tem-se que $\Pi_{4}>\Pi_{3}>\Pi_{1}>\Pi_{2}$.

3. Gama $(k>0, k$ conhecido, $\theta>0$ e $x>0): \alpha(\theta)=\theta, \zeta(\theta)=\theta^{-k}, d(x)=x$ e $v(x)=$ 
$(k-1) \log (x)-\log \{\Gamma(k)\}$, sendo $\Gamma(\cdot)$ a função gama. Temos que

$$
\begin{gathered}
a_{10}=a_{20}=a_{30}=-a_{23}=2 a_{43}=\frac{k \epsilon^{3}}{3 \theta^{3}}, \quad a_{11}=a_{13}=0, \quad a_{12}=a_{33}=-a_{40}=-\frac{k \epsilon^{3}}{3 \theta^{3}}, \\
a_{21}=-a_{22}=\frac{\epsilon}{\theta}, \quad a_{31}=\frac{\epsilon}{\theta}, \quad a_{32}=-\frac{\epsilon}{\theta}, \quad a_{41}=-\frac{\epsilon}{2 \theta} \quad \text { e } \quad a_{42}=-\frac{k \epsilon^{3}}{2 \theta^{3}}+\frac{\epsilon}{2 \theta} .
\end{gathered}
$$

Então, tem-se que $\Pi_{4}>\Pi_{1}>\Pi_{2}=\Pi_{3}$.

4. Valor extremo truncado $(\theta>0$ e $x>0): \alpha(\theta)=\theta^{-1}, \zeta(\theta)=\theta, d(x)=\exp (x)-1$ e $v(x)=x$. Temos que

$$
\begin{gathered}
a_{10}=a_{20}=a_{30}=-a_{23}=2 a_{43}=\frac{2 \epsilon^{3}}{3 \theta^{3}}, \quad a_{11}=-\frac{\epsilon^{3}}{\theta^{3}}, \quad a_{12}=a_{33}=-a_{40}=\frac{\epsilon^{3}}{3 \theta^{3}}, \quad a_{13}=0, \\
a_{21}=-a_{22}=-\frac{\epsilon^{3}}{\theta^{3}}+\frac{2 \epsilon}{\theta}, \quad a_{31}=-\frac{\epsilon^{3}}{\theta^{3}}-\frac{\epsilon}{\theta}, \quad a_{32}=\frac{\epsilon}{\theta}, \quad a_{41}=-\frac{\epsilon^{3}}{\theta^{3}}-\frac{\epsilon}{\theta} \quad \text { e } \quad a_{42}=\frac{\epsilon}{\theta} .
\end{gathered}
$$

Assim, tem-se que $\Pi_{4}>\Pi_{3}>\Pi_{1}>\Pi_{2}$.

5. Pareto $(\theta>0, k>0, k$ conhecido e $x>k): \alpha(\theta)=1+\theta, \zeta(\theta)=\left(\theta k^{\theta}\right)^{-1}, d(x)=\log (x)$ e $v(x)=0$. Temos que

$$
\begin{gathered}
a_{10}=a_{20}=a_{30}=-a_{23}=2 a_{43}=\frac{\epsilon^{3}}{3 \theta^{3}}, \quad a_{11}=a_{13}=0, \quad a_{12}=a_{33}=-a_{40}=-\frac{\epsilon^{3}}{3 \theta^{3}}, \\
a_{21}=-a_{22}=\frac{\epsilon}{\theta}, \quad a_{31}=\frac{\epsilon}{\theta}, \quad a_{32}=-\frac{\epsilon}{\theta}, \quad a_{41}=-\frac{\epsilon}{2 \theta} \quad \text { e } \quad a_{42}=\frac{\epsilon}{2 \theta}-\frac{\epsilon^{3}}{2 \theta^{3}} .
\end{gathered}
$$

Então, tem-se que $\Pi_{4}>\Pi_{1}>\Pi_{2}=\Pi_{3}$.

6. Laplace $(\theta>0,-\infty<k<\infty, k$ conhecido e $x>0): \alpha(\theta)=\theta^{-1}, \zeta(\theta)=2 \theta, d(x)=|x-k|$ e $v(x)=0$. Temos que

$$
\begin{gathered}
a_{10}=a_{20}=a_{30}=-a_{23}=2 a_{43}=\frac{2 \epsilon^{3}}{3 \theta^{3}}, \quad a_{11}=-\frac{\epsilon^{3}}{\theta^{3}}, \quad a_{12}=a_{33}=-a_{40}=\frac{\epsilon^{3}}{3 \theta^{3}}, \quad a_{13}=0, \\
a_{21}=-a_{22}=-\frac{\epsilon^{3}}{\theta^{3}}+\frac{2 \epsilon}{\theta}, \quad a_{31}=-\frac{\epsilon^{3}}{\theta^{3}}-\frac{\epsilon}{\theta}, \quad a_{32}=\frac{\epsilon}{\theta}, \quad a_{41}=-\frac{\epsilon^{3}}{\theta^{3}}-\frac{\epsilon}{\theta} \quad \text { e } \quad a_{42}=\frac{\epsilon}{\theta} .
\end{gathered}
$$

Assim, tem-se que $\Pi_{4}>\Pi_{3}>\Pi_{1}>\Pi_{2}$.

7. Power $(\theta>0, \phi>0, \phi$ conhecido e $x>\phi): \alpha(\theta)=1-\theta, \zeta(\theta)=\theta^{-1} \phi^{\theta}, d(x)=\log (x)$ e $v(x)=0$. Temos que

$$
\begin{gathered}
a_{10}=a_{20}=a_{30}=-a_{23}=2 a_{43}=\frac{\epsilon^{3}}{3 \theta^{3}}, \quad a_{11}=a_{13}=0, \quad a_{12}=a_{33}=-a_{40}=-\frac{\epsilon^{3}}{3 \theta^{3}}, \\
a_{21}=-a_{22}=\frac{\epsilon}{\theta}, \quad a_{31}=\frac{\epsilon}{\theta}, \quad a_{32}=-\frac{\epsilon}{\theta}, \quad a_{41}=-\frac{\epsilon}{2 \theta} \quad \text { e } \quad a_{42}=\frac{\epsilon}{2 \theta}-\frac{\epsilon^{3}}{2 \theta^{3}} .
\end{gathered}
$$

Então, tem-se que $\Pi_{4}>\Pi_{1}>\Pi_{2}=\Pi_{3}$.

De acordo com os resultados obtidos acima, nota-se que nenhum teste é uniformemente melhor que os outros três. Isto está de acordo com os resultados obtidos, em generalidade, na Seção 5.2.4. 
No entanto, observe que na maioria das vezes, o teste com base na estatística gradiente foi o mais poderoso, até ordem $n^{-1 / 2}$ e sob a hipótese alternativa contígua $\mathcal{H}_{1 n}: \theta=\theta^{(0)}+n^{-1 / 2} \epsilon$.

\subsection{Expansões Assintóticas no Modelo de Regressão Birnbaum-Saunders}

O objetivo desta seção é apresentar expansões assintóticas, até ordem $n^{-1 / 2}$, para as funções de poder das estatísticas $L R, W, S_{R}$ e $S_{T}$, sob alternativas contíguas, para hipóteses sobre um subconjuto de parâmetros de $\boldsymbol{\beta}$ na classe de modelos de regressão Birnbaum-Saunders. Como descrito no Capítulo 1, considere o modelo de regressão

$$
y_{i}=\boldsymbol{x}_{i}^{\top} \boldsymbol{\beta}+\varepsilon_{i}, \quad i=1,2, \ldots, n,
$$

em que $\varepsilon_{i}$ são variáveis aleatórias independentes e identicamente distribuídas tendo distribuição senh-normal, isto é, $\varepsilon_{i} \sim \mathcal{S N}(\alpha, 0,2)$.

Assuma inicialmente que o parâmetro $\alpha$ é conhecido e considere a partição $\boldsymbol{\beta}=\left(\boldsymbol{\beta}_{1}^{\top}, \boldsymbol{\beta}_{2}^{\top}\right)^{\top}$ com $\boldsymbol{\beta}_{1}=\left(\beta_{1}, \ldots, \beta_{q}\right)^{\top}$ e $\boldsymbol{\beta}_{2}=\left(\beta_{q+1}, \ldots, \beta_{p}\right)^{\top}$. A função de log-verossimilhança pode ser expressa na forma

$$
\ell\left(\boldsymbol{\beta}_{1}, \boldsymbol{\beta}_{2}\right)=-\frac{n}{2} \log (8 \pi)+\sum_{i=1}^{n} \log \left(\xi_{i 1}\right)-\frac{1}{2} \sum_{i=1}^{n} \xi_{i 2}^{2} .
$$

Seja $\boldsymbol{X}=\left(\boldsymbol{x}_{1}, \boldsymbol{x}_{2}, \ldots, \boldsymbol{x}_{n}\right)^{\top}$ a matriz modelo de dimensão $n \times p$ e de posto completo. Estamos interessados em testar a hipótese nula $\mathcal{H}_{0}: \boldsymbol{\beta}_{2}=\boldsymbol{\beta}_{20}$ contra $\mathcal{H}_{1}: \boldsymbol{\beta}_{2} \neq \boldsymbol{\beta}_{20}$, sendo $\boldsymbol{\beta}_{20}$ um vetor $(p-q)$-dimensional de constantes especificadas. Particione a matriz modelo da forma $\boldsymbol{X}=\left(\boldsymbol{X}_{1} \boldsymbol{X}_{2}\right)$. A função escore e a matriz de informação de Fisher para o vetor de parâmetros $\boldsymbol{\beta}$ são dadas, respectivamente, por

$$
\boldsymbol{U}_{\boldsymbol{\beta}}=\frac{1}{2} \boldsymbol{X}^{\top} \boldsymbol{s} \quad \text { e } \quad \boldsymbol{K}_{\boldsymbol{\beta}}=\frac{h(\alpha)}{4}\left(\boldsymbol{X}^{\top} \boldsymbol{X}\right)
$$

em que $\boldsymbol{s}=\boldsymbol{s}(\boldsymbol{\beta})=\left(s_{1}, \ldots, s_{n}\right)^{\top}$ com $s_{i}=\xi_{i 1} \xi_{i 2}-\xi_{i 2} / \xi_{i 1}$ e $h(\alpha)=2+4 / \alpha^{2}-\sqrt{2 \pi} \alpha^{-1}\{1-$ $\operatorname{erf}(\sqrt{2} / \alpha)\} \exp \left(2 / \alpha^{2}\right)$; veja a Seção 1.3 para mais detalhes.

Seja $S_{i}(i=1,2,3,4)$ representando, respectivamente, as estatísticas da razão de verossimilhanças, de Wald, escore de Rao e estatística gradiente. Para testar $\mathcal{H}_{0}: \boldsymbol{\beta}_{2}=\boldsymbol{\beta}_{20}$, estas estatísticas são dadas, respectivamente, por

$$
\begin{gathered}
S_{1}=2\left\{\ell\left(\widehat{\boldsymbol{\beta}}_{1}, \widehat{\boldsymbol{\beta}}_{2}\right)-\ell\left(\widetilde{\boldsymbol{\beta}}_{1}, \boldsymbol{\beta}_{20}\right)\right\}, \quad S_{2}=\frac{h(\alpha)}{4}\left(\widehat{\boldsymbol{\beta}}_{2}-\boldsymbol{\beta}_{20}\right)^{\top}\left(\boldsymbol{R}^{\top} \boldsymbol{R}\right)\left(\widehat{\boldsymbol{\beta}}_{2}-\boldsymbol{\beta}_{20}\right), \\
S_{3}=h(\alpha)^{-1} \widetilde{\boldsymbol{s}}^{\top} \boldsymbol{X}_{2}\left(\boldsymbol{R}^{\top} \boldsymbol{R}\right)^{-1} \boldsymbol{X}_{2}^{\top} \widetilde{\boldsymbol{s}} \quad \text { e } \quad S_{4}=\frac{1}{2} \widetilde{\boldsymbol{s}}^{\top} \boldsymbol{X}_{2}\left(\widehat{\boldsymbol{\beta}}_{2}-\boldsymbol{\beta}_{20}\right),
\end{gathered}
$$

em que $\widehat{\boldsymbol{\beta}}=\left(\widehat{\boldsymbol{\beta}}_{1}^{\top}, \widehat{\boldsymbol{\beta}}_{2}^{\top}\right)^{\top}$ e $\widetilde{\boldsymbol{\beta}}=\left(\widehat{\boldsymbol{\beta}}_{1}^{\top}, \boldsymbol{\beta}_{20}^{\top}\right)^{\top}$ são os estimadores de máxima verossimilhança de $\boldsymbol{\beta}=$ $\left(\boldsymbol{\beta}_{1}^{\top}, \boldsymbol{\beta}_{2}^{\top}\right)^{\top}$ correspondentes às maximizações de $\ell(\boldsymbol{\beta})$ segundo $\mathcal{H}_{1}$ e $\mathcal{H}_{0}$, respectivamente, $\widetilde{\boldsymbol{s}}=\boldsymbol{s}(\widetilde{\boldsymbol{\beta}})$ e $\boldsymbol{R}=\boldsymbol{X}_{2}-\boldsymbol{X}_{1} \boldsymbol{C}$ com $\boldsymbol{C}=\left(\boldsymbol{X}_{1}^{\top} \boldsymbol{X}_{1}\right)^{-1} \boldsymbol{X}_{1}^{\top} \boldsymbol{X}_{2}$ representando uma matriz $q \times(p-q)$ cujas colunas são vetores dos coeficientes de uma regressão linear das colunas de $\boldsymbol{X}_{2}$ sobre a matriz modelo $\boldsymbol{X}_{1}$. Em particular, observe a simplicidade para o cálculo da estatística gradiente $\left(S_{4}\right)$ em relação ao cálculo das estatisticas dos testes de Wald $\left(S_{2}\right)$ e escore de Rao $\left(S_{3}\right)$. Adicionalmente, ao contrário das estatísticas de Wald e escore, a estatística gradiente não depende de $h(\alpha)$. 
Aqui, a sequência de hipóteses alternativas contíguas é da forma

$$
\mathcal{H}_{1 n}: \boldsymbol{\beta}_{2}=\boldsymbol{\beta}_{20}+\boldsymbol{\epsilon}
$$

em que $\boldsymbol{\epsilon}=\left(\epsilon_{q+1}, \ldots, \epsilon_{p}\right)^{\top}$, com $\epsilon_{r}=O\left(n^{-1 / 2}\right)$ para $r=q+1, \ldots, p$. Da Seção 5.2.4, as expansões assintóticas de $S_{1}, S_{2}, S_{3}$ e $S_{4}$ podem ser escritas da forma

$$
\operatorname{Pr}\left(S_{i} \leq x\right)=\operatorname{Pr}\left(\chi_{f, \lambda}^{2} \leq x\right)+\sum_{k=0}^{3} a_{i k} \operatorname{Pr}\left(\chi_{f+2 k, \lambda}^{2} \leq x\right)+O\left(n^{-1}\right),
$$

em que os $a_{i k}$ 's podem ser reescritos como

$$
\begin{aligned}
& a_{11}=-\frac{1}{6} \sum_{r, s, t=1}^{p}\left(\kappa_{r s t}-2 \kappa_{r, s, t}\right) \epsilon_{r}^{*} \epsilon_{s}^{*} \epsilon_{t}^{*}-\frac{1}{2} \sum_{r, s, t=1}^{p}\left(\kappa_{r s t}+2 \kappa_{r, s t}\right) a_{r s}^{*} \epsilon_{t}^{*}-\frac{1}{2} \sum_{r=q+1}^{p} \sum_{s, t=1}^{p}\left(\kappa_{r s t}+\kappa_{r, s t}\right) \epsilon_{r} \epsilon_{s}^{*} \epsilon_{t}^{*}, \\
& a_{12}=-\frac{1}{6} \sum_{r, s, t=1}^{p} \kappa_{r, s, t} \epsilon_{r}^{*} \epsilon_{s}^{*} \epsilon_{t}^{*}, \quad a_{13}=0, \\
& a_{21}=-\frac{1}{2} \sum_{r, s, t=1}^{p}\left(\kappa_{r s t}+2 \kappa_{r, s t}\right) \epsilon_{r}^{*} \epsilon_{s}^{*} \epsilon_{t}^{*}+\sum_{r, s, t=1}^{p} \kappa_{r, s t} m_{r s}^{*} \epsilon_{t}^{*}-\frac{1}{2} \sum_{r, s, t=1}^{p}\left(\kappa_{r s t}+2 \kappa_{r, s t}\right) \kappa^{r, s} \epsilon_{t}^{*} \\
& -\frac{1}{2} \sum_{r=q+1}^{p} \sum_{s, t=1}^{p}\left(\kappa_{r s t}+\kappa_{r, s t}\right) \epsilon_{r} \epsilon_{s}^{*} \epsilon_{t}^{*} \\
& a_{22}=\frac{1}{2} \sum_{r, s, t=1}^{p} \kappa_{r, s t} \epsilon_{r}^{*} \epsilon_{s}^{*} \epsilon_{t}^{*}+\frac{1}{2} \sum_{r, s, t=1}^{p} \kappa_{r, s t} m_{r s} \epsilon_{t}^{*}, \quad a_{23}=\frac{1}{6} \sum_{r, s, t=1}^{p} \kappa_{r s t} \epsilon_{r}^{*} \epsilon_{s}^{*} \epsilon_{t}^{*}, \\
& a_{31}=-\frac{1}{6} \sum_{r, s, t=1}^{p}\left(\kappa_{r s t}-2 \kappa_{r, s, t}\right) \epsilon_{r}^{*} \epsilon_{s}^{*} \epsilon_{t}^{*}+\frac{1}{2} \sum_{r, s, t=1}^{p} \kappa_{r, s, t} m_{r s}^{*} \epsilon_{t}^{*}-\frac{1}{2} \sum_{r, s, t=1}^{p}\left(\kappa_{r s t}+2 \kappa_{r, s t}\right) a_{r s} \epsilon_{t}^{*} \\
& -\frac{1}{2} \sum_{r=q+1}^{p} \sum_{s, t=1}^{p}\left(\kappa_{r s t}+\kappa_{r, s t}\right) \epsilon_{r} \epsilon_{s}^{*} \epsilon_{t}^{*} \\
& a_{32}=-\frac{1}{2} \sum_{r, s, t=1}^{p} \kappa_{r, s, t} m_{r s} \epsilon_{t}^{*}, \quad a_{33}=-\frac{1}{6} \sum_{r, s, t=1}^{p} \kappa_{r, s, t} \epsilon_{r}^{*} \epsilon_{s}^{*} \epsilon_{t}^{*}, \\
& a_{41}=\frac{1}{4} \sum_{r, s, t=1}^{p} \kappa_{r s t} \kappa^{r, s} \epsilon_{t}^{*}-\frac{1}{2} \sum_{r, s, t=1}^{p}\left(\kappa_{r s t}+2 \kappa_{r, s t}\right) \epsilon_{r}^{*} \epsilon_{s}^{*} \epsilon_{t}^{*}-\frac{1}{4} \sum_{r, s, t=1}^{p}\left(4 \kappa_{r, s t}+3 \kappa_{r, s t}\right) a_{r s} \epsilon_{t}^{*} \\
& -\frac{1}{2} \sum_{r=q+1}^{p} \sum_{s, t=1}^{p}\left(\kappa_{r s t}+\kappa_{r, s t}\right) \epsilon_{r} \epsilon_{s}^{*} \epsilon_{t}^{*} \\
& a_{42}=-\frac{1}{4} \sum_{r, s, t=1}^{p} \kappa_{r s t} m_{r s} \epsilon_{t}^{*}+\frac{1}{4} \sum_{r, s, t=1}^{p}\left(\kappa_{r s t}+2 \kappa_{r, s t}\right) \epsilon_{r}^{*} \epsilon_{s}^{*} \epsilon_{t}^{*}, \quad a_{43}=-\frac{1}{12} \sum_{r, s, t=1}^{p} \kappa_{r, s, t} \epsilon_{r}^{*} \epsilon_{s}^{*} \epsilon_{t}^{*} .
\end{aligned}
$$

Os coeficientes $a_{i 0}$ são obtidos da forma $a_{i 0}=-\left(a_{i 1}+a_{i 2}+a_{i 3}\right)$, para $i=1,2,3,4$. Nas expressões acima, $\epsilon_{r}^{*}$ é o $r$-ésimo elemento do vetor $\epsilon^{*}, a_{r s}$ e $m_{r s}$ representam os elementos $(r, s)$ das matrizes 
$\boldsymbol{A}$ e $\boldsymbol{M}$, respectivamente, em que

$$
\boldsymbol{K}_{\boldsymbol{\beta}}=\left[\begin{array}{ll}
\boldsymbol{K}_{11} & \boldsymbol{K}_{12} \\
\boldsymbol{K}_{21} & \boldsymbol{K}_{22}
\end{array}\right], \quad \boldsymbol{\epsilon}^{*}=\left[\begin{array}{c}
\boldsymbol{K}_{11}^{-1} \boldsymbol{K}_{12} \\
-\boldsymbol{I}_{p-q}
\end{array}\right] \boldsymbol{\epsilon}, \quad \boldsymbol{A}=\left[\begin{array}{cc}
\boldsymbol{K}_{11}^{-1} & \mathbf{0} \\
\mathbf{0} & \mathbf{0}
\end{array}\right] \quad \text { e } \quad \boldsymbol{M}=\boldsymbol{K}_{\boldsymbol{\beta}}^{-1}-\boldsymbol{A} .
$$

Nas expressões dos $a_{i k}$ 's todas as quantidades, exceto $\boldsymbol{\epsilon}$, devem ser avaliadas sob a hipótese nula $\mathcal{H}_{0}: \boldsymbol{\beta}_{2}=\boldsymbol{\beta}_{20}$. Aqui, estamos assumindo que todos os $\kappa$ 's presentes nos $a_{i k}$ 's são de ordem $O(n)$. Assim, note que, sob a hipótese contígua anterior, todos os $a_{i k}$ 's são de ordem $n^{-1 / 2}$.

A obtenção dos coeficientes que definem as expansões assinóticas, até ordem $n^{-1 / 2}$, das funções de poder das estatísticas $S_{1}, S_{2}, S_{3}$ e $S_{4}$ requer que encontremos os cumulantes $\kappa_{r s t}, \kappa_{r, s t}$ e $\kappa_{r, s, t}$ nesta classe de modelos. Do Apêndice A obtemos

$$
\kappa_{r s t}=\kappa_{r, s t}=\kappa_{r, s, t}=0, \quad r, s, t=1,2, \ldots, p .
$$

Assim, todos os $a_{i k}$ 's são iguais a zero. Então, os testes com base nas estatísticas $S_{1}, S_{2}, S_{3}$ e $S_{4}$ têm, até ordem $n^{-1 / 2}$, poder local idêntico. O parâmetro de não centralidade, $\lambda$, assume a forma $\lambda=h(\alpha) \boldsymbol{\epsilon}^{* \top}\left(\boldsymbol{X}^{\top} \boldsymbol{X}\right) \boldsymbol{\epsilon}^{*} / 8$.

Assuma agora que o parâmetro $\alpha$ é desconhecido e deve ser estimado. Neste caso, considere o vetor de parâmetros como $\boldsymbol{\theta}=\left(\boldsymbol{\beta}^{\top}, \alpha\right)^{\top}$. A matriz de informação de Fisher total para $\boldsymbol{\theta}$ assume a forma $\boldsymbol{K}_{\boldsymbol{\theta}}=\operatorname{diag}\left\{\boldsymbol{K}_{\boldsymbol{\beta}}, 2 n / \alpha^{2}\right\}$, em que $\boldsymbol{K}_{\boldsymbol{\beta}}$ é como antes. Defina as matrizes

$$
\boldsymbol{A}_{\boldsymbol{\beta}}=\left[\begin{array}{cc}
\boldsymbol{K}_{11}^{-1} & \mathbf{0} \\
\mathbf{0} & \mathbf{0}
\end{array}\right], \quad \boldsymbol{K}_{\boldsymbol{\beta}}^{-1}=\left[\begin{array}{ll}
\boldsymbol{K}^{11} & \boldsymbol{K}^{12} \\
\boldsymbol{K}^{21} & \boldsymbol{K}^{22}
\end{array}\right], \quad \boldsymbol{A}=\left[\begin{array}{cc}
\boldsymbol{A}_{\boldsymbol{\beta}} & \mathbf{0} \\
\mathbf{0} & \kappa_{\alpha, \alpha}^{-1}
\end{array}\right] \quad \text { e } \quad \boldsymbol{M}=\left[\begin{array}{cc}
\boldsymbol{M}_{\boldsymbol{\beta}} & \mathbf{0} \\
\mathbf{0} & 0
\end{array}\right],
$$

em que $\boldsymbol{M}_{\boldsymbol{\beta}}=\boldsymbol{K}_{\boldsymbol{\beta}}^{-1}-\boldsymbol{A}_{\boldsymbol{\beta}}$. Sejam $m_{r \alpha}$ e $a_{r \alpha}$ os $(r, p+1)$-ésimos elementos das matrizes $\boldsymbol{M}$ e $\boldsymbol{A}$, respectivamente, e sejam $m_{\alpha \alpha}$ e $a_{\alpha \alpha}$ os $(p+1, p+1)$-ésimos elementos das matrizes $\boldsymbol{M}$ e $\boldsymbol{A}$, respectivamente. Note que $m_{r \alpha}=m_{\alpha r}=m_{\alpha \alpha}=0$ e $a_{r \alpha}=a_{\alpha r}=0$, para $r=1,2, \ldots, p$, e $a_{\alpha \alpha}=\kappa_{\alpha, \alpha}^{-1}=\alpha^{2} / 2 n$. Adicionalmente, defina

$$
\boldsymbol{\epsilon}^{*}=\left[\begin{array}{c}
\boldsymbol{K}_{11}^{-1} \boldsymbol{K}_{12} \\
-\boldsymbol{I}_{p-q} \\
\mathbf{0}
\end{array}\right] \boldsymbol{\epsilon}
$$

em que 0 denota um vetor linha de zeros de dimensão $1 \times(p-q)$. Como o $(p+1)$-ésimo elemento do vetor $\epsilon^{*}$ é zero, $a_{\alpha \alpha}=\kappa_{\alpha, \alpha}^{-1}$ e $a_{r \alpha}=0(r=1,2, \ldots, p)$, após alguma álgebra, é possível mostrar que

$$
a_{11}=a_{11}^{0}+\gamma, \quad a_{21}=a_{21}^{0}+\gamma, \quad a_{31}=a_{31}^{0}+\gamma \quad \text { e } \quad a_{41}=a_{41}^{0}+\gamma,
$$

em que $a_{i 1}^{0}(i=1,2,3,4)$ denotam os coeficientes no caso em que o parâmetro $\alpha$ é conhecido e são como definidos anteriormente, e

$$
\gamma=-\frac{1}{2} \sum_{t=1}^{p}\left(\kappa_{\alpha \alpha t}+2 \kappa_{\alpha, \alpha t}\right) \kappa_{\alpha, \alpha}^{-1} \epsilon_{t}^{*} .
$$

Adicionalmente, $a_{1 k}=a_{1 k}^{0}, a_{2 k}=a_{2 k}^{0}, a_{3 k}=a_{3 k}^{0}$ e $a_{4 k}=a_{4 k}^{0}$, para $k=2,3$, e os coeficientes $a_{i 0}^{0}$ são obtidos da forma $a_{i 0}^{0}=-\left(a_{i 1}^{0}+a_{i 2}^{0}+a_{i 3}^{0}\right)$, para $i=1,2,3,4$. Também, $\lambda=\epsilon^{* \top} \boldsymbol{K} \boldsymbol{\epsilon}^{*} / 2$ e como $\boldsymbol{\epsilon}^{*}=\left(\boldsymbol{\epsilon}_{\boldsymbol{\beta}}^{* \top}, 0\right)^{\top}$, em que $\boldsymbol{\epsilon}_{\boldsymbol{\beta}}^{*}$ é o vetor $\boldsymbol{\epsilon}^{*}$ no caso em que $\alpha$ é conhecido, o parâmetro de não centralidade 
não muda quando o parâmetro $\alpha$ é desconhecido. Do Apêndice A obtemos

$$
\kappa_{\alpha, \alpha t}=\kappa_{\alpha \alpha, t}=0, \quad t=1,2, \ldots, p
$$

o que resulta em $\gamma=0$. Como mostrado anteriormente no caso em que $\alpha$ é conhecido, $a_{i k}^{0}=0$, para $i=1,2,3,4$ e $k=0,1,2,3$. Portanto, os testes com base nas estatísticas $S_{1}, S_{2}, S_{3}$ e $S_{4}$ têm, até ordem $n^{-1 / 2}$, poderes locais idênticos, independentemente se o parâmetro $\alpha$ é conhecido ou desconhecido, isto é, os coeficientes que definem as expansões assintóticas das distribuições das estatísticas $S_{1}, S_{2}, S_{3}$ e $S_{4}$ no caso em que $\alpha$ é conhecido são os mesmos quando este parâmetro é desconhecido. Em outras palavras, isto implica que $\Pi_{1}=\Pi_{2}=\Pi_{3}=\Pi_{4}$ até ordem $n^{-1 / 2}$.

\subsection{Expansões Assintóticas em Modelos Lineares Generalizados}

O objetivo desta seção é apresentar a expansão assintótica, até ordem $n^{-1 / 2}$ e em notação matricial, para a função de poder da estatística $S_{T}$, sob alternativas contíguas, em modelos lineares generalizados (MLG). Inicialmente, estaremos interessados em hipóteses sobre um subconjuto de parâmetros de $\boldsymbol{\beta}$ nesta classe de modelos assumindo primeiramente que o parâmetro $\phi$ é conhecido e, posteriormente, consideraremos este parâmetro desconhecido. Finalmente, derivaremos a expansão assintótica sob alternativas contíguas até ordem $n^{-1 / 2}$ da função de poder da estatística $S_{T}$ para hipóteses sobre o parâmetro $\phi$. As expansões assintóticas das funções de poder das estatísticas $L R$, $W$ e $S_{R}$ foram derivadas em Cordeiro, Botter \& Ferrari (1994) assumindo $\phi$ conhecido. No caso em que $\phi$ é desconhecido, as expansões assintóticas foram derivadas em Ferrari, Botter \& CribariNeto (1997). A seguir apresentaremos a classe dos MLG.

Considere um vetor de dimensão $n$ de variáveis aleatórias independentes $\boldsymbol{y}=\left(y_{1}, \ldots, y_{n}\right)^{\top}$ em que cada $y_{l}$ tem função densidade na família exponencial da forma

$$
\pi\left(y ; \theta_{l}, \phi\right)=\exp \left[\phi\left\{y \theta_{l}-b\left(\theta_{l}\right)+c(y)\right\}+a(y, \phi)\right],
$$

em que $a(\cdot, \cdot), b(\cdot)$ e $c(\cdot)$ são funções conhecidas. Adicionalmente, $\phi>0$ e chamamos $\phi^{-1}$ de parâmetro de dispersão. Temos as relações: $\mathbb{E}\left(y_{l}\right)=\mu_{l}=\mathrm{d} b\left(\theta_{l}\right) / \mathrm{d} \theta_{l}$, Var $\left(y_{l}\right)=\phi^{-1} V_{l}$, em que $V=V(\mu)=\mathrm{d} \mu / \mathrm{d} \theta$ é a função de variância e $\theta=\int V^{-1} \mathrm{~d} \mu=q(\mu)$, sendo $q(\cdot)$ uma função conhecida. As distribuições normal, Poisson, binomial, gama e normal inversa são casos especiais de (5.15). Para distribuições da família exponencial bi-paramétrica com parâmetros canônicos $\phi$ e $\phi \theta$, temos que $a(y, \phi)=a_{1}(\phi)+a_{2}(y)$. As distribuições contínuas normal, normal inversa e gama são exemplos em que $a(y, \phi)$ pode ser decomposto como acima. Por exemplo, para as distribuições normal e normal inversa, temos $a_{1}(\phi)=\frac{1}{2} \log (\phi)$, enquanto que para a distribuição gama, $a_{1}(\phi)=\phi \log (\phi)-\log \Gamma(\phi)$, sendo $\Gamma(\cdot)$ a função gama.

Suponha que variáveis auxiliares $\boldsymbol{x}_{1}, \boldsymbol{x}_{2}, \ldots, \boldsymbol{x}_{n}$, com $\boldsymbol{x}_{l}^{\top}=\left(x_{l 1}, x_{l 2}, \ldots, x_{l p}\right)$, produzam uma estrutura linear da forma $\boldsymbol{\eta}=\boldsymbol{X} \boldsymbol{\beta}$ sendo $\boldsymbol{X}=\left(\boldsymbol{x}_{1}, \boldsymbol{x}_{2}, \ldots, \boldsymbol{x}_{n}\right)^{\top}$ a matriz modelo conhecida e de posto completo, $\operatorname{rank}(\boldsymbol{X})=p(p<n)$, e $\boldsymbol{\beta}=\left(\beta_{1}, \beta_{2}, \ldots, \beta_{p}\right)^{\top}$ um vetor $p$-dimensional de parâmetros desconhecidos. Um MLG é definido por (5.15) e pela parte sistemática $d\left(\mu_{l}\right)=\eta_{l}, l=1,2, \ldots, n$, relacionando a média $\boldsymbol{\mu}=\left(\mu_{1}, \mu_{2}, \ldots, \mu_{n}\right)^{\top}$ com o preditor linear $\boldsymbol{\eta}=\left(\eta_{1}, \eta_{2}, \ldots, \eta_{n}\right)^{\top}$. Assumimos que $d(\cdot)$, denominada de função de ligação, é uma função biunívoca. 


\subsection{1 $\phi$ Conhecido}

Considere a partição $\boldsymbol{\beta}=\left(\boldsymbol{\beta}_{1}^{\top}, \boldsymbol{\beta}_{2}^{\top}\right)^{\top} \operatorname{com} \boldsymbol{\beta}_{1}=\left(\beta_{1}, \beta_{2}, \ldots, \beta_{q}\right)^{\top}$ e $\boldsymbol{\beta}_{2}=\left(\beta_{q+1}, \beta_{q+2}, \ldots, \beta_{p}\right)^{\top}$. A matriz modelo particionada de forma análoga é $\boldsymbol{X}=\left[\begin{array}{ll}\boldsymbol{X}_{1} & \boldsymbol{X}_{2}\end{array}\right]$. O interesse é testar a hipótese nula $\mathcal{H}_{0}: \boldsymbol{\beta}_{2}=\boldsymbol{\beta}_{20}$ contra $\mathcal{H}_{1}: \boldsymbol{\beta}_{2} \neq \boldsymbol{\beta}_{20}$, sendo $\boldsymbol{\beta}_{20}$ um vetor $(p-q)$-dimensional de constantes especificadas. A função de log-verossimilhança assume a forma

$$
\ell(\boldsymbol{\beta})=\phi \sum_{l=1}^{n}\left\{y_{l} \theta_{l}-b\left(\theta_{l}\right)+c\left(y_{l}\right)\right\}+\sum_{l=1}^{n} a\left(y_{l}, \phi\right) .
$$

A função escore e a matriz de informação de Fisher para o vetor de parâmetros $\boldsymbol{\beta}$ são dadas, respectivamente, por

$$
\boldsymbol{U}_{\boldsymbol{\beta}}=\phi \boldsymbol{X}^{\top} \boldsymbol{W}^{1 / 2} \boldsymbol{V}^{-1 / 2}(\boldsymbol{y}-\boldsymbol{\mu}) \quad \text { e } \quad \boldsymbol{K}_{\boldsymbol{\beta}}=\phi \boldsymbol{X}^{\top} \boldsymbol{W} \boldsymbol{X},
$$

em que $\boldsymbol{V}=\operatorname{diag}\left\{V_{1}, V_{2}, \ldots, V_{n}\right\}, \boldsymbol{W}=\operatorname{diag}\left\{w_{1}, w_{2}, \ldots, w_{n}\right\} \operatorname{com} w_{l}=\left(\mathrm{d} \mu_{l} / \mathrm{d} \eta_{l}\right)^{2} / V_{l}$. A matriz $\boldsymbol{K}_{\boldsymbol{\beta}}$ é particionada de acordo com a partição de $\boldsymbol{\beta}$, isto é, $\boldsymbol{K}_{11}=\phi \boldsymbol{X}_{1}^{\top} \boldsymbol{W} \boldsymbol{X}_{1}, \boldsymbol{K}_{12}=\boldsymbol{K}_{21}^{\top}=\phi \boldsymbol{X}_{1}^{\top} \boldsymbol{W} \boldsymbol{X}_{2}$ e $\boldsymbol{K}_{22}=\phi \boldsymbol{X}_{2}^{\top} \boldsymbol{W} \boldsymbol{X}_{2}$.

Como na seção anterior, seja $S_{i}(i=1,2,3,4)$ representando, respectivamente, as estatísticas da razão de verossimilhanças, de Wald, escore de Rao e estatística gradiente. Para testar $\mathcal{H}_{0}: \boldsymbol{\beta}_{2}=\boldsymbol{\beta}_{20}$, estas estatísticas são dadas, respectivamente, por

$$
\begin{aligned}
& S_{1}=2\left\{\ell\left(\widehat{\boldsymbol{\beta}}_{1}, \widehat{\boldsymbol{\beta}}_{2}\right)-\ell\left(\widetilde{\boldsymbol{\beta}}_{1}, \boldsymbol{\beta}_{20}\right)\right\}, \\
& S_{2}=\phi\left(\widehat{\boldsymbol{\beta}}_{2}-\boldsymbol{\beta}_{20}\right)^{\top}\left(\widehat{\boldsymbol{R}}^{\top} \widehat{\boldsymbol{W}} \widehat{\boldsymbol{R}}\right)\left(\widehat{\boldsymbol{\beta}}_{2}-\boldsymbol{\beta}_{20}\right), \\
& S_{3}=\widetilde{\boldsymbol{s}}^{\top} \widetilde{\boldsymbol{W}}^{1 / 2} \boldsymbol{X}_{2}\left(\widetilde{\boldsymbol{R}}^{\top} \widetilde{\boldsymbol{W}} \widetilde{\boldsymbol{R}}\right)^{-1} \boldsymbol{X}_{2}^{\top} \widetilde{\boldsymbol{W}}^{1 / 2} \widetilde{\boldsymbol{s}} \\
& S_{4}=\phi^{1 / 2} \widetilde{\boldsymbol{s}}^{\top} \widetilde{\boldsymbol{W}}^{1 / 2} \boldsymbol{X}_{2}\left(\widehat{\boldsymbol{\beta}}_{2}-\boldsymbol{\beta}_{20}\right),
\end{aligned}
$$

em que $\widehat{\boldsymbol{\beta}}=\left(\widehat{\boldsymbol{\beta}}_{1}^{\top}, \widehat{\boldsymbol{\beta}}_{2}^{\top}\right)^{\top}$ e $\widetilde{\boldsymbol{\beta}}=\left(\widehat{\boldsymbol{\beta}}_{1}^{\top}, \boldsymbol{\beta}_{20}^{\top}\right)^{\top}$ são os estimadores de máxima verossimilhança de $\boldsymbol{\beta}=$ $\left(\boldsymbol{\beta}_{1}^{\top}, \boldsymbol{\beta}_{2}^{\top}\right)^{\top}$ correspondentes às maximizações de $\ell(\boldsymbol{\beta})$ segundo $\mathcal{H}_{1}$ e $\mathcal{H}_{0}$, respectivamente. Também, $\boldsymbol{s}=\left(s_{1}, s_{2}, \ldots, s_{n}\right)^{\top}$ com $s_{l}=\phi^{1 / 2}\left(y_{l}-\mu_{l}\right) V_{l}^{-1 / 2}$, representa o resíduo de Pearson, e $\boldsymbol{R}=\boldsymbol{X}_{2}-\boldsymbol{X}_{1} \boldsymbol{C}$ com $\boldsymbol{C}=\left(\boldsymbol{X}_{1}^{\top} \boldsymbol{W} \boldsymbol{X}_{1}\right)^{-1} \boldsymbol{X}_{1}^{\top} \boldsymbol{W} \boldsymbol{X}_{2}$ representando uma matriz $q \times(p-q)$ cujas colunas são vetores dos coeficientes de uma regressão linear das colunas de $\boldsymbol{X}_{2}$ sobre a matriz modelo $\boldsymbol{X}_{1}$ com matriz de pesos $\boldsymbol{W}$. Adicionalmente, quantidades avaliadas em $\widetilde{\boldsymbol{\beta}}$ e $\widehat{\boldsymbol{\beta}}$ são distinguidas pela adição de " " e "^», respectivamente. Em particular, observe a simplicidade para o cáculo da estatística gradiente $\left(S_{4}\right)$ em relação ao cálculo das estatisticas dos testes de Wald $\left(S_{2}\right)$ e escore de Rao $\left(S_{3}\right)$.

Aqui, a sequência de hipóteses alternativas contíguas é da forma

$$
\mathcal{H}_{1 n}: \boldsymbol{\beta}_{2}=\boldsymbol{\beta}_{20}+\boldsymbol{\epsilon}
$$

em que $\boldsymbol{\epsilon}=\left(\epsilon_{q+1}, \ldots, \epsilon_{p}\right)^{\top}$, com $\epsilon_{r}=O\left(n^{-1 / 2}\right)$ para $r=q+1, \ldots, p$. As expansões assintóticas de $S_{1}$, $S_{2}, S_{3}$ e $S_{4}$ são como dado em (5.14). Adicionalmente, estamos assumindo que todos os $\kappa$ 's presentes nos $a_{i k}$ 's são de ordem $O(n)$. Assim, note que sob a hipótese contígua anterior, todos os $a_{i k}$ 's são de

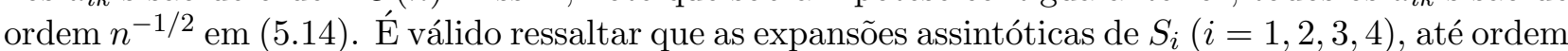
$n^{-1 / 2}$, foram derivadas para distribuições contínuas. Portanto, os resultados derivados nesta seção somente são válidos para modelos lineares generalizados contínuos como modelos normal, normal inversa e gama. 
A obtenção de fórmulas matriciais para os coeficientes que definem as expansões assinóticas, até ordem $n^{-1 / 2}$, das funções de poder das estatísticas $S_{1}, S_{2}, S_{3}$ e $S_{4}$ requer que encontremos os cumulantes $\kappa_{r s t}, \kappa_{r, s t}$ e $\kappa_{r, s, t}$ nesta classe de modelos. De Cordeiro, Ferrari \& Paula (1993) temos

$$
\kappa_{r s t}=-\phi \sum_{l=1}^{n}(f+2 g)_{l} x_{l r} x_{l s} x_{l t}, \quad \kappa_{r, s t}=\phi \sum_{l=1}^{n} g_{l} x_{l r} x_{l s} x_{l t} \quad \text { e } \quad \kappa_{r, s, t}=\phi \sum_{l=1}^{n}(f-g)_{l} x_{l r} x_{l s} x_{l t},
$$

em que $x_{l r}$ é o elemento $(l, r)$ da matriz $\boldsymbol{X}$ e os escalares $f_{l}$ e $g_{l}$ são dados por

$$
f=\frac{1}{V} \frac{\mathrm{d} \mu}{\mathrm{d} \eta} \frac{\mathrm{d}^{2} \mu}{\mathrm{d} \eta^{2}} \quad \text { e } \quad g=\frac{1}{V} \frac{\mathrm{d} \mu}{\mathrm{d} \eta} \frac{\mathrm{d}^{2} \mu}{\mathrm{d} \eta^{2}}-\frac{1}{V^{2}} \frac{\mathrm{d} V}{\mathrm{~d} \mu}\left(\frac{\mathrm{d} \mu}{\mathrm{d} \eta}\right)^{3} .
$$

Defina as matrizes $\boldsymbol{Z}=\boldsymbol{X}\left(\boldsymbol{X}^{\top} \boldsymbol{W} \boldsymbol{X}\right)^{-1} \boldsymbol{X}^{\top}=\left\{z_{l m}\right\}$ e $\boldsymbol{Z}_{1}=\boldsymbol{X}_{1}\left(\boldsymbol{X}_{1}^{\top} \boldsymbol{W} \boldsymbol{X}_{1}\right)^{-1} \boldsymbol{X}_{1}^{\top}=\left\{z_{1 l m}\right\}$. Note que, a menos do multiplicador $\phi^{-1}, \boldsymbol{Z}$ e $\boldsymbol{Z}_{1}$ podem ser interpretadas como estruturas de covariâncias assintóticas de $\widehat{\boldsymbol{\eta}}=\boldsymbol{X} \widehat{\boldsymbol{\beta}}$ e $\widetilde{\boldsymbol{\eta}}=\boldsymbol{X}_{1} \widetilde{\boldsymbol{\beta}}_{1}+\boldsymbol{X}_{2} \boldsymbol{\beta}_{20}$, respectivamente. Considere também as matrizes diagonais $\boldsymbol{Z}_{d}=\operatorname{diag}\left\{z_{11}, z_{22}, \ldots, z_{n n}\right\}, \boldsymbol{Z}_{1 d}=\operatorname{diag}\left\{z_{111}, z_{122}, \ldots, z_{1 n n}\right\}, \boldsymbol{F}=\operatorname{diag}\left\{f_{1}, f_{2}, \ldots, f_{n}\right\}$ e $\boldsymbol{G}=\operatorname{diag}\left\{g_{1}, g_{2}, \ldots, g_{n}\right\}$. Adicionalmente, usando a mesma notação adotada em Cordeiro, Botter \& Ferrari (1994), defina os vetores $\boldsymbol{t}=\left(t_{1}, t_{2}, \ldots, t_{n}\right)^{\top}=\boldsymbol{X} \boldsymbol{\epsilon}^{*}, \boldsymbol{e}=\left(e_{1}, e_{2}, \ldots, e_{n}\right)^{\top}=\boldsymbol{X}_{2} \boldsymbol{\epsilon}$ e as matrizes diagonais $\boldsymbol{T}=\operatorname{diag}\left\{t_{1}, t_{2}, \ldots, t_{n}\right\}, \boldsymbol{T}^{2}=\operatorname{diag}\left\{t_{1}^{2}, t_{2}^{2}, \ldots, t_{n}^{2}\right\}, \boldsymbol{T}^{3}=\operatorname{diag}\left\{t_{1}^{3}, t_{2}^{3}, \ldots, t_{n}^{3}\right\}$ e $\boldsymbol{E}=\operatorname{diag}\left\{e_{1}, e_{2}, \ldots, e_{n}\right\}$.

Para obter os coeficientes que definem as expansões assintóticas das funções de poder das estatísticas $L R, W, S_{R}$ e $S_{T}$, até ordem $n^{-1 / 2}$, procedemos da seguinte forma: substitua as expressões dos cumulantes $\kappa_{r s t}, \kappa_{r, s t}$ e $\kappa_{r, s, t}$ nas expressões dos $a_{i k}$ 's em (5.14) e calcule as somas sobre os parâmetros seguidas das somas sobre os dados utilizando as definições acima. (Este procedimento foi utilizado nos Capítulos 2 e 3, respectivamente, para derivar fatores de correção de Bartlett e tipoBartlett para as estatísticas da razão de verossimilhanças e escore de Rao em modelos de regressão Birnbaum-Saunders. Para mais detalhes veja os Apêndices B e C.)

Após alguma álgebra, encontramos as seguintes expressões para os $a_{4 k}$ 's da expansão assintótica da distribuição da estatística gradiente, até ordem $n^{-1 / 2}$ e sob alternativas contíguas, em MLG:

$$
\begin{gathered}
a_{41}=\frac{\phi}{2} \operatorname{tr}\left\{\boldsymbol{F} \boldsymbol{T}^{3}+(\boldsymbol{F}-\boldsymbol{G}) \boldsymbol{E} \boldsymbol{T}^{2}\right\}+\frac{1}{4} \operatorname{tr}\left\{(3 \boldsymbol{F}+2 \boldsymbol{G}) \boldsymbol{Z}_{1 d} \boldsymbol{T}-(\boldsymbol{F}+2 \boldsymbol{G}) \boldsymbol{Z}_{d} \boldsymbol{T}\right\}, \\
a_{42}=-\frac{\phi}{4} \operatorname{tr}\left\{\boldsymbol{F} \boldsymbol{T}^{3}\right\}+\frac{1}{4} \operatorname{tr}\left\{(\boldsymbol{F}+2 \boldsymbol{G})\left(\boldsymbol{Z}-\boldsymbol{Z}_{1}\right)_{d} \boldsymbol{T}\right\} \quad \text { e } \quad a_{43}=\frac{\phi}{12} \operatorname{tr}\left\{(\boldsymbol{F}+2 \boldsymbol{G}) \boldsymbol{T}^{3}\right\},
\end{gathered}
$$

em que $\operatorname{tr}(\cdot)$ denota o operador traço de uma matriz. Os coeficientes que definem as expansões assintóticas das funções de poder das estatísticas $L R, W$ e $S_{R}$ podem ser obtidos de Cordeiro, Botter \& Ferrari (1994) e são dados por:

$$
\begin{gathered}
a_{11}=\frac{\phi}{2} \operatorname{tr}\left\{(\boldsymbol{F}+\boldsymbol{G}) \boldsymbol{E} \boldsymbol{T}^{2}+\boldsymbol{F} \boldsymbol{T}^{3}\right\}+\frac{1}{2} \operatorname{tr}\left\{\boldsymbol{F} \boldsymbol{Z}_{1 d} \boldsymbol{T}\right\}, \quad a_{12}=-\frac{\phi}{6} \operatorname{tr}\left\{(\boldsymbol{F}-\boldsymbol{G}) \boldsymbol{T}^{3}\right\}, \quad a_{13}=0, \\
a_{21}=\frac{\phi}{2} \operatorname{tr}\left\{(\boldsymbol{F}+\boldsymbol{G}) \boldsymbol{E} \boldsymbol{T}^{2}+\boldsymbol{F} \boldsymbol{T}^{3}\right\}+\frac{1}{2} \operatorname{tr}\left\{\boldsymbol{F} \boldsymbol{Z}_{d} \boldsymbol{T}+2 \boldsymbol{G}\left(\boldsymbol{Z}-\boldsymbol{Z}_{1}\right)_{d} \boldsymbol{T}\right\}, \\
a_{22}=\frac{\phi}{2} \operatorname{tr}\left\{\boldsymbol{G} \boldsymbol{T}^{3}\right\}-\frac{1}{2} \operatorname{tr}\left\{(\boldsymbol{F}+2 \boldsymbol{G})\left(\boldsymbol{Z}-\boldsymbol{Z}_{1}\right)_{d} \boldsymbol{T}\right\}, \quad a_{23}=-\frac{\phi}{6} \operatorname{tr}\left\{(\boldsymbol{F}+2 \boldsymbol{G}) \boldsymbol{T}^{3}\right\},
\end{gathered}
$$




$$
\begin{gathered}
a_{31}=\frac{\phi}{2} \operatorname{tr}\left\{(\boldsymbol{F}+\boldsymbol{G}) \boldsymbol{E} \boldsymbol{T}^{2}+\boldsymbol{F} \boldsymbol{T}^{3}\right\}+\frac{1}{2} \operatorname{tr}\left\{(\boldsymbol{F}-\boldsymbol{G})\left(\boldsymbol{Z}-\boldsymbol{Z}_{1}\right)_{d} \boldsymbol{T}+\boldsymbol{F} \boldsymbol{Z}_{1 d} \boldsymbol{T}\right\} \\
a_{32}=-\frac{1}{2} \operatorname{tr}\left\{(\boldsymbol{F}-\boldsymbol{G})\left(\boldsymbol{Z}-\boldsymbol{Z}_{1}\right)_{d} \boldsymbol{T}\right\} \quad \text { e } \quad a_{33}=-\frac{\phi}{6} \operatorname{tr}\left\{(\boldsymbol{F}-\boldsymbol{G}) \boldsymbol{T}^{3}\right\} .
\end{gathered}
$$

Adicionalmente, os coeficientes $a_{i 0}$ 's são obtidos da forma $a_{i 0}=-\left(a_{i 1}+a_{i 2}+a_{i 3}\right)$, para $i=1,2,3,4$. O parâmetro de não centralidade, $\lambda$, assume a forma

$$
\lambda=\frac{\phi}{2} \boldsymbol{t}^{\top} \boldsymbol{W} \boldsymbol{t}=\frac{\phi}{2} \operatorname{tr}\{\boldsymbol{T} \boldsymbol{W} \boldsymbol{T}\}=\frac{\phi}{2} \operatorname{tr}\left\{\boldsymbol{W} \boldsymbol{T}^{2}\right\} .
$$

Como já descrito em Cordeiro, Botter \& Ferrari (1994, p. 714), note que as expressões para os $a_{i k}$ 's apresentadas acima são funções da matriz modelo $\boldsymbol{X}$, das médias desconhecidas e do parâmetro de dispersão. Estas expressões envolvem a função de ligação e suas primeira e segunda derivadas e a função de variância com sua primeira derivada. Em uma variedade de modelos lineares generalizados em que a matriz de informação tem forma fechada, é possível obter expressões em forma fechada para as matrizes de covariâncias assintóticas $\boldsymbol{Z}$ e $\boldsymbol{Z}_{1}$, e portanto para os $a_{i k}$ 's. Observe que as expressões acima são fáceis de serem operadas analiticamente pois só envolvem operações simples com matrizes. No entanto, estas expressões são difíceis de serem interpretadas, uma vez que cada termo das quantidades $a_{i k}$ depende do sistema de coordenadas escolhido, isto é, da parametrização adotada.

As distribuições de $S_{1}, S_{2}, S_{3}$ e $S_{4}$, até ordem $n^{-1 / 2}$ e sob alternativas contíguas da forma $\mathcal{H}_{1 n}: \boldsymbol{\beta}_{2}=\boldsymbol{\beta}_{20}+\boldsymbol{\epsilon}$, seguem portanto de (5.14) com os $a_{i k}$ 's dados anteriormente e parâmetro de não centralidade $\lambda$ dado acima. Estas expansões assintóticas são úteis quando estamos interessados em examinar as propriedades destes critérios em uma vizinhança do vetor hipotético $\boldsymbol{\beta}_{20}$.

Para um MLG com função de ligação identidade, isto é, $d\left(\mu_{l}\right)=\mu_{l}=\eta_{l}$, temos $\boldsymbol{F}=\mathbf{0}$ e $\boldsymbol{G}=-\operatorname{diag}\left\{1 / V_{1}^{2} \mathrm{~d} V_{1} / \mathrm{d} \mu_{1}, \ldots, 1 / V_{n}^{2} \mathrm{~d} V_{n} / \mathrm{d} \mu_{n}\right\}$. Então, podemos expressar os termos $a_{i k}$ 's como

$$
\begin{gathered}
a_{11}=\frac{\phi}{2} \operatorname{tr}\left\{\boldsymbol{G} \boldsymbol{E} \boldsymbol{T}^{2}\right\}, \quad a_{12}=\frac{\phi}{6} \operatorname{tr}\left\{\boldsymbol{G} \boldsymbol{T}^{3}\right\}, \quad a_{13}=0, \\
a_{21}=\frac{\phi}{2} \operatorname{tr}\left\{\boldsymbol{G} \boldsymbol{E} \boldsymbol{T}^{2}\right\}+\operatorname{tr}\left\{\boldsymbol{G}\left(\boldsymbol{Z}-\boldsymbol{Z}_{1}\right)_{d} \boldsymbol{T}\right\}, a_{22}=\frac{\phi}{2} \operatorname{tr}\left\{\boldsymbol{G} \boldsymbol{T}^{3}\right\}-\operatorname{tr}\left\{\boldsymbol{G}\left(\boldsymbol{Z}-\boldsymbol{Z}_{1}\right)_{d} \boldsymbol{T}\right\}, a_{23}=-\frac{\phi}{3} \operatorname{tr}\left\{\boldsymbol{G} \boldsymbol{T}^{3}\right\}, \\
a_{31}=\frac{\phi}{2} \operatorname{tr}\left\{\boldsymbol{G} \boldsymbol{E} \boldsymbol{T}^{2}\right\}-\frac{1}{2} \operatorname{tr}\left\{\boldsymbol{G}\left(\boldsymbol{Z}-\boldsymbol{Z}_{1}\right)_{d} \boldsymbol{T}\right\}, \quad a_{32}=\frac{1}{2} \operatorname{tr}\left\{\boldsymbol{G}\left(\boldsymbol{Z}-\boldsymbol{Z}_{1}\right)_{d} \boldsymbol{T}\right\}, \quad a_{33}=\frac{\phi}{6} \operatorname{tr}\left\{\boldsymbol{G} \boldsymbol{T}^{3}\right\}, \\
a_{41}=-\frac{\phi}{2} \operatorname{tr}\left\{\boldsymbol{G} \boldsymbol{E} \boldsymbol{T}^{2}\right\}-\frac{1}{2} \operatorname{tr}\left\{\boldsymbol{G}\left(\boldsymbol{Z}-\boldsymbol{Z}_{1}\right)_{d} \boldsymbol{T}\right\}, \quad a_{42}=\frac{1}{2} \operatorname{tr}\left\{\boldsymbol{G}\left(\boldsymbol{Z}-\boldsymbol{Z}_{1}\right)_{d} \boldsymbol{T}\right\} \quad \text { e } \quad a_{43}=\frac{\phi}{6} \operatorname{tr}\left\{\boldsymbol{G} \boldsymbol{T}^{3}\right\} .
\end{gathered}
$$

Note que para o modelo normal linear todos os $a_{i k}$ 's acima se anulam, uma vez que $\boldsymbol{G}=\mathbf{0}$ e, então, como esperado, as estatísticas $S_{1}, S_{2}, S_{3}$ e $S_{4}$, dadas em (5.16), respectivamente, têm exatamente distribuição $\chi_{p-q, \lambda}^{2}$ o que implica $\Pi_{1}=\Pi_{2}=\Pi_{3}=\Pi_{4}$ até ordem $n^{-1 / 2}$.

Considere, por exemplo, uma hipótese nula simples da forma $\mathcal{H}_{0}: \boldsymbol{\beta}=\boldsymbol{\beta}_{0}(q=0)$. Para testar esta hipótese nula, as estatísticas dadas em (5.16) assumem as formas

$$
\begin{gathered}
S_{1}=2\left\{\ell(\widehat{\boldsymbol{\beta}})-\ell\left(\boldsymbol{\beta}_{0}\right)\right\}, \quad S_{2}=\phi\left(\widehat{\boldsymbol{\eta}}-\boldsymbol{\eta}_{0}\right)^{\top} \widehat{\boldsymbol{W}}\left(\widehat{\boldsymbol{\eta}}-\boldsymbol{\eta}_{0}\right), \\
S_{3}=\phi\left(\boldsymbol{y}-\boldsymbol{\mu}_{0}\right)^{\top} \boldsymbol{P}_{0}\left(\boldsymbol{y}-\boldsymbol{\mu}_{0}\right) \quad \text { e } \quad S_{4}=\phi^{1 / 2} \boldsymbol{s}_{0}^{\top} \boldsymbol{W}_{0}^{1 / 2}\left(\widehat{\boldsymbol{\eta}}-\boldsymbol{\eta}_{0}\right),
\end{gathered}
$$


em que $\widehat{\boldsymbol{\eta}}=\boldsymbol{X} \widehat{\boldsymbol{\beta}}, \boldsymbol{\eta}_{0}=\boldsymbol{X} \boldsymbol{\beta}_{0}, \boldsymbol{\mu}_{0}=d^{-1}\left(\boldsymbol{\eta}_{0}\right), \boldsymbol{s}=\phi^{1 / 2} \boldsymbol{V}^{-1 / 2}(\boldsymbol{y}-\boldsymbol{\mu})$ e $\boldsymbol{P}=\boldsymbol{V}^{-1 / 2} \boldsymbol{W}^{1 / 2} \boldsymbol{Z} \boldsymbol{W}^{1 / 2} \boldsymbol{V}^{-1 / 2}$ sendo que $\boldsymbol{s}_{0}, \boldsymbol{P}_{0}$ e $\boldsymbol{W}_{0}$ denotam $\boldsymbol{s}, \boldsymbol{P}$ e $\boldsymbol{W}$, respectivamente, avaliados em $\mathcal{H}_{0}$. Observando que $\boldsymbol{Z}_{1}=\mathbf{0}, \boldsymbol{\epsilon}^{*}=-\boldsymbol{\epsilon}, \boldsymbol{t}=-\boldsymbol{X} \boldsymbol{\epsilon}$ e $\boldsymbol{e}=-\boldsymbol{t}$, temos que os $a_{i k}$ 's se reduzem a

$$
\begin{gathered}
a_{11}=-\frac{\phi}{2} \operatorname{tr}\left\{\boldsymbol{G} \boldsymbol{T}^{3}\right\}, \quad a_{12}=-\frac{\phi}{6} \operatorname{tr}\left\{(\boldsymbol{F}-\boldsymbol{G}) \boldsymbol{T}^{3}\right\}, \quad a_{13}=0, \\
a_{21}=-\frac{\phi}{2} \operatorname{tr}\left\{\boldsymbol{G} \boldsymbol{T}^{3}\right\}+\frac{1}{2} \operatorname{tr}\left\{(\boldsymbol{F}+2 \boldsymbol{G}) \boldsymbol{Z}_{d} \boldsymbol{T}\right\}, \quad a_{22}=\frac{\phi}{2} \operatorname{tr}\left\{\boldsymbol{G} \boldsymbol{T}^{3}\right\}-\frac{1}{2} \operatorname{tr}\left\{(\boldsymbol{F}+2 \boldsymbol{G}) \boldsymbol{Z}_{d} \boldsymbol{T}\right\}, \\
a_{23}=-\frac{\phi}{6} \operatorname{tr}\left\{(\boldsymbol{F}+2 \boldsymbol{G}) \boldsymbol{T}^{3}\right\}, \quad a_{31}=\frac{\phi}{2} \operatorname{tr}\left\{\boldsymbol{G} \boldsymbol{F} \boldsymbol{T}^{3}\right\}+\frac{1}{2} \operatorname{tr}\left\{(\boldsymbol{F}-\boldsymbol{G}) \boldsymbol{Z}_{d} \boldsymbol{T}\right\}, a_{32}=-\frac{1}{2} \operatorname{tr}\left\{(\boldsymbol{F}-\boldsymbol{G}) \boldsymbol{Z}_{d} \boldsymbol{T}\right\}, \\
a_{33}=-\frac{\phi}{6} \operatorname{tr}\left\{(\boldsymbol{F}-\boldsymbol{G}) \boldsymbol{T}^{3}\right\}, \quad a_{41}=\frac{\phi}{2} \operatorname{tr}\left\{\boldsymbol{G} \boldsymbol{T}^{3}\right\}-\frac{1}{4} \operatorname{tr}\left\{(\boldsymbol{F}+2 \boldsymbol{G}) \boldsymbol{Z}_{d} \boldsymbol{T}\right\}, \\
a_{42}=-\frac{\phi}{4} \operatorname{tr}\left\{\boldsymbol{F} \boldsymbol{T}^{3}\right\}+\frac{1}{4} \operatorname{tr}\left\{(\boldsymbol{F}+2 \boldsymbol{G}) \boldsymbol{Z}_{d} \boldsymbol{T}\right\} \quad \text { e } \quad a_{43}=\frac{\phi}{12} \operatorname{tr}\left\{(\boldsymbol{F}+2 \boldsymbol{G}) \boldsymbol{T}^{3}\right\} .
\end{gathered}
$$

Adicionalmente, os coeficientes $a_{i 0}$ são obtidos da forma $a_{i 0}=-\left(a_{i 1}+a_{i 2}+a_{i 3}\right)$, para $i=1,2,3,4$. O parâmetro de não centralidade é da forma $\lambda=\phi \operatorname{tr}\left\{\boldsymbol{W} \boldsymbol{T}^{2}\right\} / 2$.

\subsection{2 $\phi$ Desconhecido}

Considere o vetor de parâmetros desconhecido como $\boldsymbol{\theta}=\left(\boldsymbol{\beta}_{1}^{\top}, \boldsymbol{\beta}_{2}^{\top}, \phi\right)^{\top}$, em que $\phi$ é um parâmetro escalar, $\boldsymbol{\beta}=\left(\boldsymbol{\beta}_{1}^{\top}, \boldsymbol{\beta}_{2}^{\top}\right)^{\top}$ com $\boldsymbol{\beta}_{1}=\left(\beta_{1}, \ldots, \beta_{q}\right)^{\top}$ e $\boldsymbol{\beta}_{2}=\left(\beta_{q+1}, \ldots, \beta_{p}\right)^{\top}$. A hipótese nula é da forma $\mathcal{H}_{0}: \boldsymbol{\beta}_{2}=\boldsymbol{\beta}_{20}$. As estatísticas $S_{1}, S_{2}, S_{3}$ e $S_{4}$ para testar $\mathcal{H}_{0}$ são da forma

$$
\begin{aligned}
& S_{1}=2\left\{\ell\left(\widehat{\boldsymbol{\beta}}_{1}, \widehat{\boldsymbol{\beta}}_{2}, \widehat{\phi}\right)-\ell\left(\widetilde{\boldsymbol{\beta}}_{1}, \boldsymbol{\beta}_{20}, \widetilde{\phi}\right)\right\}, \quad S_{2}=\widehat{\phi}\left(\widehat{\boldsymbol{\beta}}_{2}-\boldsymbol{\beta}_{20}\right)^{\top}\left(\widehat{\boldsymbol{R}}^{\top} \widehat{\boldsymbol{W}} \widehat{\boldsymbol{R}}\right)\left(\widehat{\boldsymbol{\beta}}_{2}-\boldsymbol{\beta}_{20}\right), \\
& S_{3}=\widetilde{\boldsymbol{s}}^{\top} \widetilde{\boldsymbol{W}}^{1 / 2} \boldsymbol{X}_{2}\left(\widetilde{\boldsymbol{R}}^{\top} \widetilde{\boldsymbol{W}} \widetilde{\boldsymbol{R}}\right)^{-1} \boldsymbol{X}_{2}^{\top} \widetilde{\boldsymbol{W}}^{1 / 2} \widetilde{\boldsymbol{s}} \quad \text { e } \quad S_{4}=\widetilde{\phi}^{1 / 2} \widetilde{\boldsymbol{s}}^{\top} \widetilde{\boldsymbol{W}}^{1 / 2} \boldsymbol{X}_{2}\left(\widehat{\boldsymbol{\beta}}_{2}-\boldsymbol{\beta}_{20}\right),
\end{aligned}
$$

em que $\widehat{\boldsymbol{\theta}}=\left(\widehat{\boldsymbol{\beta}}_{1}^{\top}, \widehat{\boldsymbol{\beta}}_{2}^{\top}, \widehat{\phi}\right)^{\top}$ e $\widetilde{\boldsymbol{\theta}}=\left(\widehat{\boldsymbol{\beta}}_{1}^{\top}, \boldsymbol{\beta}_{20}^{\top}, \widetilde{\phi}\right)^{\top}$ são os estimadores de máxima verossimilhança de $\boldsymbol{\theta}=\left(\boldsymbol{\beta}_{1}^{\top}, \boldsymbol{\beta}_{2}^{\top}, \phi\right)^{\top}$ correspondentes às maximizações de $\ell(\boldsymbol{\theta})$ segundo $\mathcal{H}_{1}$ e $\mathcal{H}_{0}$, respectivamente. Adicionalmente, as quantidades $\boldsymbol{s}$ e $\boldsymbol{R}$ foram definadas na Seção 5.5.1, lembrando que quantidades avaliadas em $\widetilde{\boldsymbol{\theta}}$ e $\widehat{\boldsymbol{\theta}}$ são distinguidas pela adição de " " e "^", respectivamente.

A matriz de informação total de Fisher para $\boldsymbol{\theta}$ assume a forma $\boldsymbol{K}_{\boldsymbol{\theta}}=\operatorname{diag}\left\{\boldsymbol{K}_{\boldsymbol{\beta}}, \kappa_{\phi, \phi}\right\}$, em que $\boldsymbol{K}_{\boldsymbol{\beta}}$ é como na Seção 5.5.1. Adicionalmente, $\kappa_{\phi, \phi}=-n a_{1}^{\prime \prime}(\phi)$, com $a_{1}^{\prime \prime}(\phi)=\partial^{2} a_{1}(\phi) / \partial \phi^{2}$. A sequência de hipóteses alternativas contíguas é como na Seção 5.5.1. Da mesma forma, defina as matrizes

$$
\boldsymbol{A}_{\boldsymbol{\beta}}=\left[\begin{array}{cc}
\boldsymbol{K}_{11}^{-1} & \mathbf{0} \\
\mathbf{0} & \mathbf{0}
\end{array}\right], \quad \boldsymbol{K}_{\boldsymbol{\beta}}^{-1}=\left[\begin{array}{ll}
\boldsymbol{K}^{11} & \boldsymbol{K}^{12} \\
\boldsymbol{K}^{21} & \boldsymbol{K}^{22}
\end{array}\right], \quad \boldsymbol{A}=\left[\begin{array}{cc}
\boldsymbol{A}_{\boldsymbol{\beta}} & \mathbf{0} \\
\mathbf{0} & \kappa_{\phi, \phi}^{-1}
\end{array}\right] \quad \text { e } \quad \boldsymbol{M}=\left[\begin{array}{cc}
\boldsymbol{M}_{\boldsymbol{\beta}} & \mathbf{0} \\
\mathbf{0} & 0
\end{array}\right],
$$

em que $\boldsymbol{M}_{\boldsymbol{\beta}}=\boldsymbol{K}_{\boldsymbol{\beta}}^{-1}-\boldsymbol{A}_{\boldsymbol{\beta}}$. Sejam $m_{r \phi}$ e $a_{r \phi}$ os $(r, p+1)$-ésimos elementos das matrizes $\boldsymbol{M}$ e $\boldsymbol{A}$, respectivamente, e sejam $m_{\phi \phi}$ e $a_{\phi \phi}$ os $(p+1, p+1)$-ésimos elementos das matrizes $\boldsymbol{M}$ e $\boldsymbol{A}$, respectivamente. Note que $m_{r \phi}=m_{\phi r}=m_{\phi \phi}=0$ e $a_{r \phi}=a_{\phi r}=0$, para $r=1,2, \ldots, p$, e $a_{\phi \phi}=\kappa_{\phi, \phi}^{-1}=-\left\{n a_{1}^{\prime \prime}(\phi)\right\}^{-1}$. Adicionalmente, defina

$$
\boldsymbol{\epsilon}^{*}=\left[\begin{array}{c}
\boldsymbol{K}_{11}^{-1} \boldsymbol{K}_{12} \\
-\boldsymbol{I}_{p-q} \\
\mathbf{0}
\end{array}\right] \boldsymbol{\epsilon},
$$


em que 0 denota um vetor linha de zeros de dimensão $1 \times(p-q)$. Como o $(p+1)$-ésimo elemento do vetor $\epsilon^{*}$ é zero, $a_{\phi \phi}=\kappa_{\phi, \phi}^{-1}$ e $a_{r \phi}=0(r=1,2, \ldots, p)$, após alguma álgebra, é possível mostrar que

$$
a_{41}=a_{41}^{0}+\gamma, \quad a_{42}=a_{42}^{0}, \quad a_{43}=a_{43}^{0} \quad \text { e } \quad a_{40}^{0}=-\left(a_{41}^{0}+a_{42}^{0}+a_{43}^{0}\right),
$$

em que $a_{4 k}^{0}(k=1,2,3)$ denotam os coeficientes no caso em que o parâmetro $\phi$ é conhecido e são como definidos na Seção 5.5.1, e

$$
\gamma=-\frac{1}{2} \sum_{t=1}^{p}\left(\kappa_{\phi \phi t}+2 \kappa_{\phi, \phi t}\right) \kappa_{\phi, \phi}^{-1} \epsilon_{t}^{*}
$$

Os coeficientes que definem as expansões assintóticas das funções de poder, até ordem $n^{-1 / 2}$, para as estatísticas $S_{1}, S_{2}$ e $S_{3}$ podem ser encontrados em Ferrari, Botter \& Cribari-Neto (1997). Os autores deduzem, como mostrado nesta seção para a estatística gradiente, que

$$
a_{11}=a_{11}^{0}+\gamma, \quad a_{21}=a_{21}^{0}+\gamma \quad \text { e } \quad a_{31}=a_{31}^{0}+\gamma,
$$

em que $a_{i 1}^{0}(i=1,2,3)$ denotam os coeficientes no caso em que o parâmetro $\phi$ é conhecido e são como definidos na Seção 5.5.1. Adicionalmente, $a_{1 k}=a_{1 k}^{0}, a_{2 k}=a_{2 k}^{0}$ e $a_{3 k}=a_{3 k}^{0}$, para $k=2$, 3 , e os coeficientes $a_{i 0}^{0}$ são da forma $a_{i 0}^{0}=-\left(a_{i 1}^{0}+a_{i 2}^{0}+a_{i 3}^{0}\right)$, para $i=1,2,3$.

O parâmetro de não centralidade, $\lambda$, para as quatro estatísticas, é dado por $\lambda=\epsilon^{* \top} \boldsymbol{K} \epsilon^{*} / 2$ e como $\boldsymbol{\epsilon}^{*}=\left(\boldsymbol{\epsilon}_{\boldsymbol{\beta}}^{* \top}, 0\right)^{\top}$, em que $\boldsymbol{\epsilon}_{\boldsymbol{\beta}}^{*}$ é o vetor $\boldsymbol{\epsilon}^{*}$ no caso em que $\phi$ é conhecido, o parâmetro de não centralidade não muda quando o parâmetro $\phi$ é desconhecido. De Cordeiro (1987) temos que

$$
\kappa_{\phi, \phi t}=\kappa_{\phi \phi, t}=0, \quad t=1,2, \ldots, p,
$$

o que resulta em $\gamma=0$. Portanto, os coeficientes que definem as expansões assintóticas das distribuições das estatísticas $S_{1}, S_{2}, S_{3}$ e $S_{4}$ no caso em que $\phi$ é conhecido são os mesmos quando este parâmetro é desconhecido. Em outras palavras, até ordem $n^{-1 / 2}$ e sob a hipótese alternativa contígua $\mathcal{H}_{1 n}: \boldsymbol{\beta}_{2}=\boldsymbol{\beta}_{20}+\boldsymbol{\epsilon}$, as expansões assintóticas das funções de poder dos testes com base nas estatísticas $S_{1}, S_{2}, S_{3}$ e $S_{4}$ coincidem com as correspondentes expansões no caso em $\phi$ é suposto conhecido.

\subsubsection{Testes de Hipóteses sobre o Parâmetro de Dispersão}

O interesse é testar a hipótese nula $\mathcal{H}_{0}: \phi=\phi^{(0)}$ contra a hipótese alternativa $\mathcal{H}_{0}: \phi \neq \phi^{(0)}$, em que $\phi^{(0)}$ é uma constante positiva e especificada. Adicionalmente, $\boldsymbol{\beta}=\left(\beta_{1}, \ldots, \beta_{p}\right)^{\top}$ é o vetor de parâmetros de perturbação. A hipótese alternativa contígua é da forma $\mathcal{H}_{1 n}: \phi=\phi^{(0)}+\epsilon$, com $\epsilon=\phi-\phi^{(0)}$, em que estamos assumindo ser de ordem $n^{-1 / 2}$.

O parâmetro $\phi$ não está nas equações de estimação para $\widehat{\boldsymbol{\beta}}$, e $\widehat{\phi}$ é dado como uma função do desvio do modelo sob investigação, $D_{p}(\boldsymbol{y}, \widehat{\boldsymbol{\mu}})$. Cordeiro \& McCullagh (1991) escrevem a equação de máxima verossimilhança para $\widehat{\phi}$ como

$$
n a_{1}^{\prime}(\phi)=\frac{1}{2} D_{p}(\boldsymbol{y}, \widehat{\boldsymbol{\mu}})-\sum_{l=1}^{n}\left\{v\left(y_{l}\right)+c\left(y_{l}\right)\right\},
$$


em que

$$
v(y)=y q(y)-b(q(y)) \quad \text { e } \quad D_{p}(\boldsymbol{y}, \widehat{\boldsymbol{\mu}})=2 \sum_{l=1}^{n}\left\{v\left(y_{l}\right)-v\left(\widehat{\mu}_{l}\right)+\left(\widehat{\mu}_{l}-y_{l}\right) q\left(\widehat{\mu}_{l}\right)\right\} .
$$

O estimador de máxima verossimilhança de $\phi$ é obtido através da solução em $\phi$ da equação (5.18). Por exemplo, para o modelo gama, a estimativa de máxima verossimilhança de $\phi$ é obtida através da equação (Cordeiro \& McCullagh, 1991)

$$
\log (\widehat{\phi})-\psi(\widehat{\phi})=\frac{D_{p}(\boldsymbol{y}, \widehat{\boldsymbol{\mu}})}{2 n},
$$

em que $D_{p}(\boldsymbol{y}, \widehat{\boldsymbol{\mu}})=2 \sum_{l=1}^{n}\left\{\log \left(\widehat{\mu}_{l} / y_{l}\right)+\left(y_{l}-\widehat{\mu}_{l}\right) / \widehat{\mu}_{l}\right\}$ e $\psi(\cdot)$ é a função digama, isto é, $\psi(\phi)=$ $\Gamma^{\prime}(\phi) / \Gamma(\phi)$. Adicionalmente, para testar a hipótese nula $\mathcal{H}_{0}: \phi=\phi^{(0)}$, as estatísticas $S_{1}, S_{2}, S_{3}$ e $S_{4}$ podem ser escritas como

$$
\begin{gathered}
S_{1}=2 n\left\{a_{1}(\widehat{\phi})-a_{1}\left(\phi^{(0)}\right)-\left(\widehat{\phi}-\phi^{(0)}\right) a_{1}^{\prime}(\widehat{\phi})\right\}, \quad S_{2}=-n\left(\widehat{\phi}-\phi^{(0)}\right)^{2} a_{1}^{\prime \prime}(\widehat{\phi}), \\
S_{3}=-\frac{n\left\{a_{1}^{\prime}(\widehat{\phi})-a_{1}^{\prime}\left(\phi^{(0)}\right)\right\}^{2}}{a_{1}^{\prime \prime}\left(\phi^{(0)}\right)} \text { e } \quad S_{4}=n\left\{a_{1}^{\prime}\left(\phi^{(0)}\right)-a_{1}^{\prime}(\widehat{\phi})\right\}\left(\widehat{\phi}-\phi^{(0)}\right) .
\end{gathered}
$$

As expressões anteriores têm formas simples em alguns casos especiais. Por exemplo, $a_{1}=\frac{1}{2} \log \phi$ para as distribuições normal e normal inversa, então, temos que

$$
S_{1}=2 n\left\{\log \left(\frac{\widehat{\phi}}{\phi^{(0)}}\right)-\left(\frac{\widehat{\phi}-\phi^{(0)}}{\widehat{\phi}}\right)\right\}, S_{2}=S_{3}=\frac{n}{2}\left\{\frac{\widehat{\phi}-\phi^{(0)}}{\widehat{\phi}}\right\}^{2} \text { e } S_{4}=\frac{n}{2}\left\{\frac{\widehat{\phi}-\phi^{(0)}}{\phi^{(0)}}-\frac{\widehat{\phi}-\phi^{(0)}}{\widehat{\phi}}\right\} .
$$

Os coeficientes $a_{4 k}$ 's da expansão assintótica de $S_{T}$, até ordem $n^{-1 / 2}$ e sob alternativas contíguas, para o teste de $\mathcal{H}_{0}: \phi=\phi^{(0)}$ são simples de serem obtidos, uma vez que $\boldsymbol{\beta}$ e $\phi$ são ortogonais. Defina o vetor

$$
\boldsymbol{\epsilon}^{*}=\left[\begin{array}{c}
\mathbf{0} \\
-\epsilon
\end{array}\right]
$$

sendo $\mathbf{0}$ um vetor coluna $p$-dimensional de zeros, e as matrizes

$$
\boldsymbol{K}_{\boldsymbol{\theta}}=\left[\begin{array}{cc}
\boldsymbol{K}_{\boldsymbol{\beta}} & \mathbf{0} \\
\mathbf{0} & \kappa_{\phi, \phi}
\end{array}\right], \quad \boldsymbol{K}_{\boldsymbol{\theta}}^{-1}=\left[\begin{array}{cc}
\boldsymbol{K}_{\boldsymbol{\beta}}^{-1} & \mathbf{0} \\
\mathbf{0} & \kappa_{\phi, \phi}^{-1}
\end{array}\right], \quad \boldsymbol{A}=\left[\begin{array}{cc}
\boldsymbol{K}_{\boldsymbol{\theta}} & \mathbf{0} \\
\mathbf{0} & 0
\end{array}\right] \quad \text { e } \quad \boldsymbol{M}=\boldsymbol{K}_{\boldsymbol{\theta}}-\boldsymbol{A}=\left[\begin{array}{cc}
\mathbf{0} & \mathbf{0} \\
\mathbf{0} & \kappa_{\phi, \phi}^{-1}
\end{array}\right] .
$$

Sejam $m_{r \phi}$ e $a_{r \phi}$ os $(r, p+1)$-ésimos elementos das matrizes $\boldsymbol{M}$ e $\boldsymbol{A}$, respectivamente, e sejam $m_{\phi \phi}$ e $a_{\phi \phi}$ os $(p+1, p+1)$-ésimos elementos das matrizes $\boldsymbol{M}$ e $\boldsymbol{A}$, respectivamente. Note que $m_{r s}=m_{r \phi}=m_{\phi r}=0$ e $a_{r \phi}=a_{\phi r}=a_{\phi \phi}=0$, para $r=1,2, \ldots, p$, e $m_{\phi \phi}=\kappa_{\phi, \phi}^{-1}=-\left\{n a_{1}^{\prime \prime}(\phi)\right\}^{-1}$.

Após alguma álgebra, é possível mostrar que as expressões gerais para os coeficientes que definem a expansão assintótica, até ordem $n^{-1 / 2}$, da função de poder da estatística gradiente, $S_{4}$, assumem a forma

$$
\begin{gathered}
a_{41}=-\frac{1}{4} \sum_{r, s=1}^{p} \kappa_{r s \phi} \kappa^{r, s} \epsilon+\frac{1}{4} \sum_{r, s=1}^{p}\left(4 \kappa_{r, s \phi}+3 \kappa_{r s \phi}\right) \kappa^{r, s} \epsilon-\frac{1}{4} \kappa_{\phi \phi \phi} \kappa^{\phi, \phi} \epsilon+\frac{1}{2} \kappa_{\phi, \phi \phi} \epsilon^{3}, \\
a_{42}=\frac{1}{4} \kappa_{\phi \phi \phi} \kappa^{\phi, \phi} \epsilon-\frac{1}{4}\left(\kappa_{\phi \phi \phi}+2 \kappa_{\phi, \phi \phi}\right) \epsilon^{3} \quad \text { e } \quad a_{43}=\frac{1}{12} \kappa_{\phi \phi \phi} \epsilon^{3} .
\end{gathered}
$$


Adicionalmente, $a_{40}=-\left(a_{41}+a_{42}+a_{43}\right)$. Uma vez que $\kappa_{\phi, \phi \phi}=0, \kappa_{\phi, \phi, \phi}=-\kappa_{\phi \phi \phi}$ e $\kappa_{r s \phi}=-\kappa_{r, s \phi}$, as expressões anteriores se reduzem a

$$
a_{41}=\frac{1}{2} \sum_{r, s=1}^{p} \kappa_{r, s \phi} \kappa^{r, s} \epsilon-\frac{1}{4} \kappa_{\phi \phi \phi} \kappa^{\phi, \phi} \epsilon, \quad a_{42}=\frac{1}{4} \kappa_{\phi \phi \phi} \kappa^{\phi, \phi} \epsilon-\frac{1}{4} \kappa_{\phi \phi \phi} \epsilon^{3} \quad \text { e } \quad a_{43}=\frac{1}{12} \kappa_{\phi \phi \phi} \epsilon^{3} .
$$

De Cordeiro (1987), temos que

$$
\kappa_{r, \phi, \phi}=\kappa_{r s, \phi}=0, \quad \kappa_{r, s, \phi}=\kappa_{r s \phi}=-\kappa_{r, s \phi}=-\sum_{l=1}^{n} w_{l} x_{l r} x_{l s}, \quad r, s=1,2, \ldots, p .
$$

Adicionalmente, $\kappa_{\phi, \phi, \phi}=-\kappa_{\phi \phi \phi}=-n a_{1}^{\prime \prime \prime}(\phi)$. Após alguma álgebra, obtemos

$$
a_{41}=\frac{p \epsilon}{2 \phi}+\frac{a_{1}^{\prime \prime \prime}(\phi) \epsilon}{4 a_{1}^{\prime \prime}(\phi)}, \quad a_{42}=\frac{a_{1}^{\prime \prime \prime}(\phi) \epsilon}{4 a_{1}^{\prime \prime}(\phi)}-\frac{n a_{1}^{\prime \prime \prime}(\phi) \epsilon^{3}}{4} \quad \text { e } \quad a_{43}=\frac{n a_{1}^{\prime \prime \prime}(\phi) \epsilon^{3}}{12}
$$

sendo $a_{40}=-\left(a_{41}+a_{42}+a_{43}\right)$. Adicionalmente, o parâmetro de não centralidade é da forma $\lambda=$ $-n a_{1}^{\prime \prime}(\phi) \epsilon^{2}$. Todas as quantidades nos $a_{4 k}$ 's e $\lambda$ devem ser avaliadas sob a hipótese nula $\mathcal{H}_{0}: \phi=\phi^{(0)}$, exceto $\epsilon$ que deve ser avaliado sob a hipótese alternativa $\mathcal{H}_{0}: \phi \neq \phi^{(0)}$.

Os coeficientes $a_{i k}$ 's, $i=1,2,3$ e $k=0,1,2,3,4$, que definem as expansões assintóticas, até ordem $n^{-1 / 2}$ e sob a alternativa contígua $\mathcal{H}_{1 n}: \phi=\phi^{(0)}+\epsilon$, das funções de poder das estatísticas $S_{1}, S_{2}$ e $S_{3}$ foram derivados em Cordeiro, Botter \& Ferrari (1994), e são da forma

$$
\begin{gathered}
a_{11}=\frac{p \epsilon}{2 \phi}, \quad a_{12}=-\frac{n a_{1}^{\prime \prime \prime}(\phi) \epsilon^{3}}{6}, \quad a_{13}=0, \quad a_{21}=a_{31}=\frac{p \epsilon}{2 \phi}-\frac{a_{1}^{\prime \prime \prime}(\phi) \epsilon}{2 a_{1}^{\prime \prime}(\phi)}, \\
a_{22}=a_{32}=\frac{a_{1}^{\prime \prime \prime}(\phi) \epsilon}{2 a_{1}^{\prime \prime}(\phi)} \quad \text { e } \quad a_{23}=a_{33}=-\frac{n a_{1}^{\prime \prime \prime}(\phi) \epsilon^{3}}{6} .
\end{gathered}
$$

Observe que todos os $a_{i k}$ 's apresentados anteriormente dependem da matriz modelo somente através do seu posto e do parâmetro $\phi$. Adicionalmente, estes coeficientes não envolvem o vetor de parâmetros desconhecido $\boldsymbol{\beta}$. 


\section{Capítulo 6}

\section{Conclusões e Pesquisas Futuras}

Nesta tese obtivemos refinamentos de métodos assintóticos (correções de Bartlett e tipo-Bartlett, e estatísticas da razão de verossimilhanças sinalizada modificadas) no modelo de regressão BirnbaumSaunders. Adicionalmente, derivamos a expansão assintótica para a função de poder local, até ordem $n^{-1 / 2}$ e sob alternativas de Pitman, do teste gradiente.

Verificamos, através de estudos de simulações Monte Carlo, que testes de hipóteses com base nas estatísticas da razão de verossimilhanças $(L R)$ e escore $(S R)$ podem ser severamente liberais em amostras de tamanho pequeno e moderado na classe de modelos de regressão Birnbaum-Saunders, rejeitando a hipótese nula muito mais do que o esperado com base no nível nominal selecionado. Nos Capítulos 2 e 3, respectivamente, derivamos um fator de correção de Bartlett para a estatística $L R$ e um fator de correção tipo-Bartlett para a estatística $S R$ nesta classe de modelos. Estes ajustes melhoraram a aproximação da distribuição destas estatísticas pela distribuição qui-quadrado de referência. Em outras palavras, os fatores de correção (derivados na tese) fizeram com que as taxas de rejeição empíricas dos testes (corrigidos) ficassem mais próximas dos níveis nominais considerados. Observamos que o teste com base na estatística escore corrigida pelo fator de correção tipo-Bartlett apresentou resultados levemente melhores do que o teste com base na estatística da razão verossimilhanças ajustada pelo fator de correção de Bartlett.

No Capítulo 4, derivamos vários ajustes para a estatística da razão de verossimilhanças sinalizada, $R$, no modelo de regressão Birnbaum-Saunders. Tais ajustes melhoram a aproximação desta estatística pela distribuição normal padrão. Esta estatística é muito utilizada quando o interesse está em apenas um parâmetro (possivelmente na presença de parâmetros de perturbação) em testes unilaterais. Mostramos que as taxas de rejeição empíricas com base em $R$ são bastantes distorcidas em amostras de tamanho pequeno e moderado. Os ajustes (derivados na tese) fizeram com que as taxas de rejeição empíricas dos testes (ajustados) ficassem mais próximas dos níveis nominais considerados.

No Capítulo 5, derivamos a expansão assintótica da função de poder local, sob alternativas de Pitman e até ordem $n^{-1 / 2}$, para o teste com base na estatística gradiente, $S_{T}$, recentemente proposta em Terrell (2002). Esta estatística é muito simples de ser calculada e, em particular, não há a necessidade de se obter uma matriz de informação para o seu cálculo, como é o caso das estatísticas de Wald $(W)$ e escore. Fizemos um estudo de poder local do teste com base nesta estatística comparando-o com o poder local de três testes amplamente conhecidos: $L R, W$ e $S R$. Deste estudo, concluímos que não é possível, em geral, afirmar que um dos testes é mais poderoso que os demais até ordem $n^{-1 / 2}$. Adicionalmente, também particularizamos a expansão de $S_{T}$, até ordem $n^{-1 / 2}$, em algumas classes especiais de modelos: (i) modelos uniparamétricos da família exponencial; (ii) modelos de regressão 
Birnbaum-Saunders; (iii) modelos lineares generalizados.

Várias linhas de pesquisas ainda podem ser tratadas como possíveis trabalhos futuros:

- Generalisar os resultados derivados na tese considerando o modelo de regressão BirnbaumSaunders não linear recentemente proposto por Lemonte e Cordeiro (2009).

- Propor um modelo de regressão Birnbaum-Saunders multivariado.

- Desenvolver uma expansão assintótica, sob a hipótese nula e até ordem $n^{-1}$, para a distribuição da estatística $S_{T}$ e propor um estatística $S_{T}$ corrigida por um fator de correção tipo-Bartlett, $S_{T}^{*}$ digamos, que apresente distribuição sob a hipótese nula mais próxima da distribuição quiquadrado de referência do que a distribuição nula de $S_{T}$.

- Aplicar a estatística $S_{T}^{*}$ em muitos modelos particulares comparando-a com as correções existentes. 


\section{Apêndice A}

\section{Derivadas e Cumulantes}

Neste apêndice, iremos obter os cumulantes e derivadas destes cumulantes que são necessários para a obtenção das correções de Bartlett e tipo-Bartlett derivadas nos Capítulos 2 e 3, respectivamente, no modelo de regressão Birnbaum-Saunders.

\section{A.1 Função de Log-verossimilhança}

A função de log-verossimilhança para uma amostra aleatória $\boldsymbol{y}=\left(y_{1}, y_{2}, \ldots, y_{n}\right)^{\top}$ do modelo de regressão Birnbaum-Saunders pode ser expressa na forma

$$
\ell(\boldsymbol{\theta} ; \boldsymbol{y})=-\frac{n}{2} \log (8 \pi)+\sum_{i=1}^{n} \log \left(\xi_{i 1}\right)-\frac{1}{2} \sum_{i=1}^{n} \xi_{i 2}^{2},
$$

em que $\boldsymbol{\theta}=\left(\boldsymbol{\beta}^{\top}, \alpha\right)^{\top}$

$$
\xi_{i 1}=\xi_{i 1}(\boldsymbol{\theta})=\frac{2}{\alpha} \cosh \left(\frac{y_{i}-\mu_{i}}{2}\right), \quad \xi_{i 2}=\xi_{i 2}(\boldsymbol{\theta})=\frac{2}{\alpha} \operatorname{senh}\left(\frac{y_{i}-\mu_{i}}{2}\right),
$$

$\mu_{i}=\boldsymbol{x}_{i}^{\top} \boldsymbol{\beta}, \boldsymbol{\beta}=\left(\beta_{1}, \beta_{2}, \ldots, \beta_{p}\right)^{\top}$ e $\boldsymbol{x}_{i}^{\top}=\left(x_{i 1}, x_{i 2}, \ldots, x_{i p}\right)$, com $i=1,2, \ldots, n$.

As expressões para as derivadas da função de log-verossimilhança em relação aos componentes de $\boldsymbol{\theta}$ serão apresentadas a seguir, onde omitimos as passagens intermediárias, uma vez que algumas destas derivadas envolvem um trabalho algébrico bastante tedioso. Os índices $r, s, t$ e $u$ que aparecem variam de 1 até $p$.

\section{Derivadas de Primeira Ordem}

$$
U_{r}=\frac{\partial \ell(\boldsymbol{\theta})}{\partial \beta_{r}}=\frac{1}{2} \sum_{i=1}^{n} x_{i r}\left\{\xi_{i 1} \xi_{i 2}-\xi_{i 2} / \xi_{i 1}\right\} \quad \text { e } \quad U_{\alpha}=\frac{\partial \ell(\boldsymbol{\theta})}{\partial \alpha}=-\frac{n}{\alpha}+\frac{1}{\alpha} \sum_{i=1}^{n} \xi_{i 2}^{2}
$$

\section{Derivadas de Segunda Ordem}

$$
U_{r s}=\frac{\partial^{2} \ell(\boldsymbol{\theta})}{\partial \beta_{r} \partial \beta_{s}}=-\frac{1}{4} \sum_{i=1}^{n} x_{i r} x_{i s}\left\{2 \xi_{i 2}^{2}+\frac{4}{\alpha^{2}}-1+\frac{\xi_{i 2}^{2}}{\xi_{i 1}^{2}}\right\}
$$




$$
U_{r \alpha}=\frac{\partial^{2} \ell(\boldsymbol{\theta})}{\partial \beta_{r} \partial \alpha}=-\frac{1}{\alpha} \sum_{i=1}^{n} x_{i r} \xi_{i 1} \xi_{i 2} \quad \text { e } \quad U_{\alpha \alpha}=\frac{\partial^{2} \ell(\boldsymbol{\theta})}{\partial \alpha^{2}}=\frac{n}{\alpha^{2}}-\frac{3}{\alpha^{2}} \sum_{i=1}^{n} \xi_{i 2}^{2}
$$

\section{Derivadas de Terceira Ordem}

$$
\begin{gathered}
U_{r s t}=\frac{\partial^{3} \ell(\boldsymbol{\theta})}{\partial \beta_{r} \partial \beta_{s} \partial \beta_{t}}=\frac{1}{4} \sum_{i=1}^{n} x_{i r} x_{i s} x_{i t}\left\{2 \xi_{i 1} \xi_{i 2}+\frac{4 \xi_{i 2}}{\alpha^{2} \xi_{i 1}^{3}}\right\} \\
U_{r s \alpha}=\frac{\partial^{3} \ell(\boldsymbol{\theta})}{\partial \beta_{r} \partial \beta_{s} \partial \alpha}=\frac{1}{\alpha} \sum_{i=1}^{n} x_{i r} x_{i s}\left\{\frac{2}{\alpha^{2}}+\xi_{i 2}^{2}\right\}, \quad U_{r \alpha \alpha}=\frac{\partial^{3} \ell(\boldsymbol{\theta})}{\partial \beta_{r} \partial \alpha^{2}}=\frac{3}{\alpha^{2}} \sum_{i=1}^{n} x_{i r} \xi_{i 1} \xi_{i 2}
\end{gathered}
$$

e

$$
U_{\alpha \alpha \alpha}=\frac{\partial^{3} \ell(\boldsymbol{\theta})}{\partial \alpha^{3}}=-\frac{2 n}{\alpha^{3}}+\frac{12}{\alpha^{3}} \sum_{i=1}^{n} \xi_{i 2}^{2} .
$$

\section{Derivadas de Quarta Ordem}

$$
\begin{gathered}
U_{r s t u}=\frac{\partial^{4} \ell(\boldsymbol{\theta})}{\partial \beta_{r} \partial \beta_{s} \partial \beta_{t} \partial \beta_{u}}=-\frac{1}{4} \sum_{i=1}^{n} x_{i r} x_{i s} x_{i t} x_{i u}\left\{\frac{4}{\alpha^{2}}+2 \xi_{i 2}^{2}+\frac{2}{\alpha^{2} \xi_{i 1}^{2}}-\frac{6 \xi_{i 2}^{2}}{\alpha^{2} \xi_{i 1}^{4}}\right\}, \\
U_{r s t \alpha}=\frac{\partial^{4} \ell(\boldsymbol{\theta})}{\partial \beta_{r} \partial \beta_{s} \partial \beta_{t} \partial \alpha}=-\frac{1}{\alpha} \sum_{i=1}^{n} x_{i r} x_{i s} x_{i t} \xi_{i 1} \xi_{i 2}, \\
U_{r s \alpha \alpha}=\frac{\partial^{4} \ell(\boldsymbol{\theta})}{\partial \beta_{r} \partial \beta_{s} \partial \alpha^{2}}=-\frac{3}{\alpha^{2}} \sum_{i=1}^{n} x_{i r} x_{i s}\left\{\frac{2}{\alpha^{2}}+\xi_{i 2}^{2}\right\}, \quad U_{r \alpha \alpha \alpha}=\frac{\partial^{4} \ell(\boldsymbol{\theta})}{\partial \beta_{r} \partial \alpha^{3}}=-\frac{12}{\alpha^{3}} \sum_{i=1}^{n} x_{i r} \xi_{i 1} \xi_{i 2}
\end{gathered}
$$

$\mathrm{e}$

$$
U_{\alpha \alpha \alpha \alpha}=\frac{\partial^{4} \ell(\boldsymbol{\theta})}{\partial \alpha^{4}}=\frac{6 n}{\alpha^{4}}-\frac{60}{\alpha^{4}} \sum_{i=1}^{n} \xi_{i 2}^{2} .
$$

\section{A.2 Cálculo dos Cumulantes}

Considere $a_{0}(\alpha)=\{1-\operatorname{erf}(\sqrt{2} / \alpha)\} \exp \left(2 / \alpha^{2}\right)$, sendo erf $(\cdot)$ a error function. Tomando o valor esperado das derivadas acima, em que os índices $r, s, t$ e $u$ variam de 1 até $p$, temos

$$
\kappa_{r s}=-\frac{a_{1}(\alpha)}{4} \sum_{i=1}^{n} x_{i r} x_{i s}
$$

em que

$$
a_{1}(\alpha)=2+\frac{4}{\alpha^{2}}-\frac{\sqrt{2 \pi}}{\alpha} a_{0}(\alpha)
$$

Ainda,

$$
\kappa_{r \alpha}=0, \quad \kappa_{\alpha \alpha}=-\frac{2 n}{\alpha^{2}}, \quad \kappa_{r s t}=0, \quad \kappa_{r s \alpha}=\frac{2+\alpha^{2}}{\alpha^{3}} \sum_{i=1}^{n} x_{i r} x_{i s}, \quad \kappa_{r \alpha \alpha}=0 \quad \text { e } \quad \kappa_{\alpha \alpha \alpha}=\frac{10 n}{\alpha^{3}} .
$$


Também,

$$
\kappa_{r s t u}=a_{4}(\alpha) \sum_{i=1}^{n} x_{i r} x_{i s} x_{i t} x_{i u}
$$

em que

$$
a_{4}(\alpha)=-\frac{1}{4}\left\{2+\frac{7}{\alpha^{2}}-\sqrt{\frac{\pi}{2}}\left(\frac{1}{2 \alpha}+\frac{6}{\alpha^{3}}\right) a_{0}(\alpha)\right\} .
$$

Adicionalmente,

$$
\kappa_{r s t \alpha}=0, \quad \kappa_{r s \alpha \alpha}=-\frac{3\left(2+\alpha^{2}\right)}{\alpha^{4}} \sum_{i=1}^{n} x_{i r} x_{i s}, \quad \kappa_{r \alpha \alpha \alpha}=0 \quad \text { e } \quad \kappa_{\alpha \alpha \alpha \alpha}=-\frac{54 n}{\alpha^{4}} .
$$

\section{A.3 Derivadas dos Cumulantes}

Nesta seção, apresentamos as derivadas de alguns cumulantes utilizados na obtenção dos fatores de correções de Bartlett e tipo-Bartlett derivados nos Capítulos 2 e 3, respectivamente, onde omitimos as passagens intermediárias, uma vez que algumas destas derivadas envolvem um trabalho algébrico bastante tedioso, porém, elementar. Os índices $r, s, t$ e $u$ variam de 1 até $p$. Derivando, tem-se que

$$
\begin{gathered}
\kappa_{r s}^{(t)}=\frac{\partial \kappa_{r s}}{\partial \beta_{t}}=0, \quad \kappa_{r \alpha}^{(t)}=\frac{\partial \kappa_{r \alpha}}{\partial \beta_{t}}=0, \quad \kappa_{r \alpha}^{(\alpha)}=\frac{\partial \kappa_{r \alpha}}{\partial \alpha}=0, \quad \kappa_{\alpha \alpha}^{(t)}=\frac{\partial \kappa_{\alpha \alpha}}{\partial \beta_{t}}=0, \\
\kappa_{\alpha \alpha}^{(\alpha)}=\frac{\partial \kappa_{\alpha \alpha}}{\partial \alpha}=\frac{4 n}{\alpha^{3}}, \quad \kappa_{r s}^{(\alpha)}=\frac{\partial \kappa_{r s}}{\partial \alpha}=a_{2}(\alpha) \sum_{i=1}^{n} x_{i r} x_{i s}
\end{gathered}
$$

em que

$$
a_{2}(\alpha)=\frac{3}{\alpha^{3}}-\frac{\sqrt{2 \pi}}{4 \alpha^{2}}\left(1+\frac{4}{\alpha^{2}}\right) a_{0}(\alpha) .
$$

Ainda,

$$
\kappa_{r s}^{(t u)}=\frac{\partial^{2} \kappa_{r s}}{\partial \beta_{t} \partial \beta_{u}}=0, \quad \kappa_{r s}^{(t \alpha)}=\frac{\partial^{2} \kappa_{r s}}{\partial \beta_{t} \partial \alpha}=0, \quad \kappa_{r s}^{(\alpha \alpha)}=\frac{\partial^{2} \kappa_{r s}}{\partial \alpha^{2}}=a_{3}(\alpha) \sum_{i=1}^{n} x_{i r} x_{i s}
$$

em que

$$
a_{3}(\alpha)=-\frac{10}{\alpha^{4}}-\frac{4}{\alpha^{6}}+\frac{1}{\alpha^{7}} \sqrt{\frac{\pi}{2}}\left(\alpha^{4}+10 \alpha^{2}+8\right) a_{0}(\alpha) .
$$

Adicionalmente,

$$
\begin{gathered}
\kappa_{r \alpha}^{(t u)}=\frac{\partial^{2} \kappa_{r \alpha}}{\partial \beta_{t} \partial \beta_{u}}=0, \quad \kappa_{\alpha \alpha}^{(t u)}=\frac{\partial^{2} \kappa_{\alpha \alpha}}{\partial \beta_{t} \partial \beta_{u}}=0, \quad \kappa_{r \alpha}^{(t \alpha)}=\frac{\partial^{2} \kappa_{r \alpha}}{\partial \beta_{t} \partial \alpha}=0, \quad \kappa_{r \alpha}^{(\alpha \alpha)}=\frac{\partial^{2} \kappa_{r \alpha}}{\partial \alpha^{2}}=0, \\
\kappa_{\alpha \alpha}^{(t \alpha)}=\frac{\partial^{2} \kappa_{\alpha \alpha}}{\partial \beta_{t} \partial \alpha}=0, \quad \kappa_{\alpha \alpha}^{(\alpha \alpha)}=\frac{\partial^{2} \kappa_{\alpha \alpha}}{\partial \alpha^{2}}=-\frac{12 n}{\alpha^{4}}, \quad \kappa_{r s t}^{(u)}=\frac{\partial \kappa_{r s t}}{\partial \beta_{u}}=0, \quad \kappa_{r s \alpha}^{(u)}=\frac{\partial \kappa_{r s \alpha}}{\partial \beta_{u}}=0, \\
\kappa_{r \alpha \alpha}^{(u)}=\frac{\partial \kappa_{r \alpha \alpha}}{\partial \beta_{u}}=0, \quad \kappa_{\alpha \alpha \alpha}^{(u)}=\frac{\partial \kappa_{\alpha \alpha \alpha}}{\partial \beta_{u}}=0, \quad \kappa_{r s t}^{(\alpha)}=\frac{\partial \kappa_{r s t}}{\partial \alpha}=0, \\
\kappa_{r s \alpha}^{(\alpha)}=\frac{\partial \kappa_{r s \alpha}}{\partial \alpha}=-\left\{\frac{1}{\alpha^{2}}+\frac{6}{\alpha^{4}}\right\} \sum_{i=1}^{n} x_{i r} x_{i s}, \quad \kappa_{r \alpha \alpha}^{(\alpha)}=\frac{\partial \kappa_{r \alpha \alpha}}{\partial \alpha}=0 \quad \text { e } \quad \kappa_{\alpha \alpha \alpha}^{(\alpha)}=\frac{\partial \kappa_{\alpha \alpha \alpha}}{\partial \beta_{u}}=-\frac{30 n}{\alpha^{4}} .
\end{gathered}
$$




\section{A.4 Cumulantes Adicionais}

Os cumulantes apresentados nesta seção são exclusivamente usados para a obtenção do fator de correção tipo-Bartlett derivado no Capítulo 3. Os índices $r, s, t$ e $u$ variam de 1 até $p$. Dentre estes cumulantes, temos

$$
\begin{gathered}
\kappa_{r s, t}=\mathbb{E}\left(U_{r s} U_{t}\right), \quad \kappa_{r, s, t}=\mathbb{E}\left(U_{r} U_{s} U_{t}\right), \quad \kappa_{r, s, t u}=\mathbb{E}\left(U_{r} U_{s} U_{t u}\right)+\kappa_{r s} \kappa_{t u}, \\
\kappa_{r, s, t, u}=\mathbb{E}\left(U_{r} U_{s} U_{t} U_{u}\right)-\kappa_{r s} \kappa_{t u}-\kappa_{r t} \kappa_{s u}-\kappa_{r u} \kappa_{s t}, \quad \kappa_{r \alpha, \alpha}=\mathbb{E}\left(U_{r \alpha} U_{\alpha}\right), \\
\kappa_{r \alpha, s}=\mathbb{E}\left(U_{r \alpha} U_{s}\right), \quad \kappa_{r, s, \alpha}=\mathbb{E}\left(U_{r} U_{s} U_{\alpha}\right), \quad \kappa_{\alpha \alpha, \alpha}=\mathbb{E}\left(U_{\alpha \alpha} U_{\alpha}\right), \quad \kappa_{r s, \alpha}=\mathbb{E}\left(U_{r s} U_{\alpha}\right), \\
\kappa_{r, \alpha \alpha}=\mathbb{E}\left(U_{r} U_{\alpha \alpha}\right), \quad \kappa_{r, \alpha, \alpha}=\mathbb{E}\left(U_{r} U_{\alpha} U_{\alpha}\right), \quad \kappa_{r, s, \alpha \alpha}=\mathbb{E}\left(U_{r} U_{s} U_{\alpha \alpha}\right)+\kappa_{r s} \kappa_{\alpha \alpha}, \\
\kappa_{r, s, \alpha, \alpha}=\mathbb{E}\left(U_{r} U_{s} U_{\alpha} U_{\alpha}\right)-\kappa_{r s} \kappa_{\alpha \alpha}-\kappa_{r \alpha} \kappa_{s \alpha}-\kappa_{r \alpha} \kappa_{s \alpha}, \quad \kappa_{\alpha, \alpha, \alpha}=\mathbb{E}\left(U_{\alpha} U_{\alpha} U_{\alpha}\right), \\
\kappa_{\alpha, \alpha, \alpha, \alpha}=\mathbb{E}\left(U_{\alpha} U_{\alpha} U_{\alpha} U_{\alpha}\right)-3 \kappa_{\alpha \alpha}^{2} \quad \text { e } \quad \kappa_{\alpha, \alpha, t u}=\mathbb{E}\left(U_{\alpha} U_{\alpha} U_{t u}\right)+\kappa_{\alpha \alpha} \kappa_{t u} .
\end{gathered}
$$

Uma maneira menos trabalhosa para se obter estes cumulantes é utilizar agumas das identidades de Bartlett apresentadas na Seção 1.1.2. Utilizaremos as seguinte identidades:

$$
\begin{aligned}
\kappa_{r s, t} & =\kappa_{r s}^{(t)}-\kappa_{r s t}, \\
\kappa_{r, s, t} & =2 \kappa_{r s t}-\sum_{(3)} \kappa_{r s}^{(t)}, \\
\kappa_{r, s, t, u} & =-3 \kappa_{r s t u}+2 \sum_{(4)} \kappa_{r s t}^{(u)}-\sum_{(6)} \kappa_{r s}^{(t u)}+\sum_{(3)} \kappa_{r s, t u}, \\
\kappa_{r, s, t u} & =\kappa_{r s t u}-\kappa_{r t u}^{(s)}-\kappa_{s t u}^{(r)}+\kappa_{t u}^{(r s)}-\kappa_{r s, t u}, \\
\kappa_{r s, t u} & =\mathbb{E}\left(U_{r s} U_{t u}\right)-\kappa_{r s} \kappa_{t u},
\end{aligned}
$$

em que $\sum_{(j)}$ representa o somatório sobre todas as $j$-ésimas combinações de índices. Assim, considerando os cumulantes e derivadas destes cumulantes que foram calculados anteriormente, temos que

$$
\kappa_{r s, t}=0, \quad \kappa_{r, s, t}=0, \quad \kappa_{r, s, t u}=\left\{a_{4}(\alpha)-\frac{1}{16}\left[s_{1}(\alpha)-a_{1}(\alpha)^{2}\right]\right\} \sum_{i=1}^{n} x_{i r} x_{i s} x_{i t} x_{i u},
$$

em que

$$
s_{1}(\alpha)=12+\frac{2}{\alpha^{2}}+\frac{16}{\alpha^{4}}+\sqrt{\frac{\pi}{2}} \frac{1}{\alpha}\left(1+\frac{12}{\alpha^{2}}\right) a_{0}(\alpha) .
$$

Ainda,

$$
\kappa_{r, s, t, u}=\tilde{s}_{2}(\alpha) \sum_{i=1}^{n} x_{i r} x_{i s} x_{i t} x_{i u}
$$

em que

$$
\tilde{s}_{2}(\alpha)=-3\left\{a_{4}(\alpha)-\frac{1}{16}\left[s_{1}(\alpha)-a_{1}(\alpha)^{2}\right]\right\}
$$

Adicionalmente,

$$
\kappa_{r \alpha, \alpha}=0, \quad \kappa_{r \alpha, s}=-\frac{2+\alpha^{2}}{\alpha^{3}} \sum_{i=1}^{n} x_{i r} x_{i s} \quad \text { e } \quad \kappa_{r, s, \alpha}=\tilde{s}_{3}(\alpha) \sum_{i=1}^{n} x_{i r} x_{i s},
$$


em que

$$
\tilde{s}_{3}(\alpha)=\frac{2\left(2+\alpha^{2}\right)}{\alpha^{3}}-a_{2}(\alpha) .
$$

Também,

$$
\kappa_{\alpha \alpha, \alpha}=-\frac{6 n}{\alpha^{3}}, \quad \kappa_{r s, \alpha}=\left\{a_{2}(\alpha)-\frac{2+\alpha^{2}}{\alpha^{3}}\right\} \sum_{i=1}^{n} x_{i r} x_{i s}, \quad \kappa_{r, \alpha \alpha}=0 \quad \text { e } \quad \kappa_{r, \alpha, \alpha}=0 .
$$

Ainda,

$$
\kappa_{r s, \alpha \alpha}=\frac{3}{4 \alpha^{2}}\left\{s_{2}(\alpha)-a_{1}(\alpha)\right\} \sum_{i=1}^{n} x_{i r} x_{i s}
$$

com

$$
s_{2}(\alpha)=6+\frac{8}{\alpha^{3}} \sqrt{\frac{\pi}{2}} a_{0}(\alpha) .
$$

Também,

$$
\kappa_{r, s, \alpha \alpha}=-\frac{3}{\alpha^{2}}\left[\frac{2+\alpha^{2}}{\alpha^{2}}+\frac{1}{4}\left\{s_{2}(\alpha)-a_{1}(\alpha)\right\}\right] \sum_{i=1}^{n} x_{i r} x_{i s} .
$$

Adicionalmente,

$$
\kappa_{r \alpha, s \alpha}=\frac{4+3 \alpha^{2}}{\alpha^{4}} \sum_{i=1}^{n} x_{i r} x_{i s} \quad \text { e } \quad \kappa_{r, s, \alpha, \alpha}=\left\{\frac{2+11 \alpha^{2}}{\alpha^{4}}-a_{3}(\alpha)+\frac{3}{4 \alpha^{2}}\left\{s_{2}(\alpha)-a_{1}(\alpha)\right\}\right\} \sum_{i=1}^{n} x_{i r} x_{i s} .
$$

Ainda,

$$
\kappa_{\alpha, \alpha, \alpha}=\frac{8 n}{\alpha^{3}} \quad \text { e } \quad \kappa_{\alpha, \alpha, \alpha, \alpha}=\frac{48 n}{\alpha^{4}} .
$$

Finalmente,

$$
\kappa_{\alpha, \alpha, t u}=\left\{\frac{6-\alpha^{2}}{\alpha^{4}}+a_{3}(\alpha)-\frac{3}{4 \alpha^{2}}\left[s_{2}(\alpha)-a_{1}(\alpha)\right]\right\} \sum_{i=1}^{n} x_{i t} x_{i u} .
$$




\section{Apêndice B}

\section{Fator de Correção de Bartlett}

Neste apêndice apresentamos, com mais detalhes, o cálculo dos somatórios envolvidos na expressão de $\epsilon_{p}$ em (2.3), resultando na fórmula matricial de $\epsilon_{p+1} \mathrm{em}(2.6)$, para o modelo de regressão Birnbaum-Saunders. Para tal, utilizaremos os cumulantes e suas derivadas obtidos no Apêndice A.

\section{Obtenção de $\epsilon_{p+1}$}

A expressão de $\epsilon_{p+1}$, seguindo a expansão de Lawley (1956), é da forma

$$
\epsilon_{p+1}=\sum^{\prime}\left(\lambda_{r s t u}-\lambda_{r s t u v w}\right)
$$

em que $\sum^{\prime}$ é o somatório sobre todos os componentes do vetor $\boldsymbol{\theta}=\left(\boldsymbol{\beta}^{\top}, \alpha\right)^{\top}$, isto é, os índices $r, s, t, u, v$ e $w$ variam sobre todos os $p+1$ parâmetros, e os $\lambda$ 's são dados por

$$
\begin{aligned}
\lambda_{r s t u} & =\kappa^{r s} \kappa^{t u}\left\{\kappa_{r s t u} / 4-\kappa_{r s t}^{(u)}+\kappa_{r t}^{(s u)}\right\}, \\
\lambda_{r s t u v w} & =\kappa^{r s} \kappa^{t u} \kappa^{v w}\left\{\kappa_{r t v}\left(\kappa_{s u w} / 6-\kappa_{s w}^{(u)}\right)\right. \\
& \left.+\kappa_{r t u}\left(\kappa_{s v w} / 4-\kappa_{s w}^{(v)}\right)+\kappa_{r t}^{(v)} \kappa_{s w}^{(u)}+\kappa_{r t}^{(u)} \kappa_{s w}^{(v)}\right\} .
\end{aligned}
$$

Isto é, para obter a fórmula de $\epsilon_{p+1}$, necessitamos avaliar os seguintes somatórios:

$$
\sum_{\boldsymbol{\beta}, \alpha}^{\prime} \lambda_{r s t u}=\kappa^{r s} \kappa^{t u}\left\{\kappa_{r s t u} / 4-\kappa_{r s t}^{(u)}+\kappa_{r t}^{(s u)}\right\}
$$

$\mathrm{e}$

$$
\sum_{\boldsymbol{\beta}, \alpha}^{\prime} \lambda_{r s t u v w}=\kappa^{r s} \kappa^{t u} \kappa^{v w}\left\{\kappa_{r t v}\left(\kappa_{s u w} / 6-\kappa_{s w}^{(u)}\right)+\kappa_{r t u}\left(\kappa_{s v w} / 4-\kappa_{s w}^{(v)}\right)+\kappa_{r t}^{(v)} \kappa_{s w}^{(u)}+\kappa_{r t}^{(u)} \kappa_{s w}^{(v)}\right\}
$$

sendo que $\sum_{\boldsymbol{\beta}, \alpha}^{\prime}$ denota o somatório sobre todos os componentes do vetor $\boldsymbol{\beta}$ e de $\alpha$.

Como visto no Apêndice A, muitos cumulantes e derivadas destes cumulantes se anulam, devido à ortogonalidade global entre $\boldsymbol{\beta}$ e $\alpha$. Assim, para o cálculo de $\sum_{\boldsymbol{\beta}, \alpha}^{\prime} \lambda_{r s t u}$, apenas quatro termos necessitam ser avaliados, a saber:

1. $\sum_{\boldsymbol{\beta}}^{\prime} \ell_{r s t u}=\sum_{\boldsymbol{\beta}}^{\prime} \kappa^{r s} \kappa^{t u}\left\{\kappa_{r s t u} / 4-\kappa_{r s t}^{(u)}+\kappa_{r t}^{(s u)}\right\}$, 
2. $\sum_{\boldsymbol{\beta}, \alpha}^{\prime} \ell_{r s \alpha \alpha}=\sum_{\boldsymbol{\beta}, \alpha}^{\prime} \kappa^{r s} \kappa^{\alpha \alpha}\left\{\kappa_{r s \alpha \alpha} / 4-\kappa_{r s \alpha}^{(\alpha)}+\kappa_{r \alpha}^{(s \alpha)}\right\}$,

3. $\sum_{\boldsymbol{\beta}, \alpha}^{\prime} \ell_{\alpha \alpha t u}=\sum_{\boldsymbol{\beta}, \alpha}^{\prime} \kappa^{\alpha \alpha} \kappa^{t u}\left\{\kappa_{\alpha \alpha t u} / 4-\kappa_{\alpha \alpha t}^{(u)}+\kappa_{\alpha t}^{(\alpha u)}\right\}$,

4. $\sum_{\alpha}^{\prime} \ell_{\alpha \alpha \alpha \alpha}=\sum_{\alpha}^{\prime} \kappa^{\alpha \alpha} \kappa^{\alpha \alpha}\left\{\kappa_{\alpha \alpha \alpha \alpha} / 4-\kappa_{\alpha \alpha \alpha}^{(\alpha)}+\kappa_{\alpha \alpha}^{(\alpha \alpha)}\right\}=\left(\kappa^{\alpha \alpha}\right)^{2}\left\{\kappa_{\alpha \alpha \alpha \alpha} / 4-\kappa_{\alpha \alpha \alpha}^{(\alpha)}+\kappa_{\alpha \alpha}^{(\alpha \alpha)}\right\}$,

em que $\sum_{\boldsymbol{\beta}}^{\prime}$ e $\sum_{\alpha}^{\prime}$ representam, respectivamente, os somatórios sobre os componentes do vetor $\boldsymbol{\beta}$ e sobre o parâmetro escalar $\alpha$. A obtenção dos quatro termos acima é conseguida substituindo-se os cumulantes e derivadas destes cumulantes encontrados no Apêndice A nestas expressões. Como alguns cumulantes e derivadas destes cumlantes são nulos, tem-se que

1. $\sum_{\boldsymbol{\beta}}^{\prime} \ell_{r s t u}=\frac{1}{4} \sum_{\boldsymbol{\beta}}^{\prime} \kappa^{r s} \kappa^{t u} \kappa_{r s t u}$,

2. $\sum_{\boldsymbol{\beta}, \alpha}^{\prime} \ell_{r s \alpha \alpha}=\sum_{\boldsymbol{\beta}, \alpha}^{\prime} \kappa^{r s} \kappa^{\alpha \alpha}\left\{\kappa_{r s \alpha \alpha} / 4-\kappa_{r s \alpha}^{(\alpha)}\right\}$,

3. $\sum_{\boldsymbol{\beta}, \alpha}^{\prime} \ell_{\alpha \alpha t u}=\frac{1}{4} \sum_{\boldsymbol{\beta}, \alpha}^{\prime} \kappa^{\alpha \alpha} \kappa^{t u} \kappa_{\alpha \alpha t u}$,

4. $\sum_{\alpha}^{\prime} \ell_{\alpha \alpha \alpha \alpha}=\left(\kappa^{\alpha \alpha}\right)^{2}\left\{\kappa_{\alpha \alpha \alpha \alpha} / 4-\kappa_{\alpha \alpha \alpha}^{(\alpha)}+\kappa_{\alpha \alpha}^{(\alpha \alpha)}\right\}$.

Assim, temos que

$$
\begin{aligned}
\frac{1}{4} \sum_{\boldsymbol{\beta}}^{\prime} \kappa^{r s} \kappa^{t u} \kappa_{r s t u} & =\frac{1}{4} \sum_{\boldsymbol{\beta}}^{\prime} \kappa^{r s} \kappa^{t u}\left\{a_{4}(\alpha) \sum_{i=1}^{n} x_{i r} x_{i s} x_{i t} x_{i u}\right\} \\
& =\frac{a_{4}(\alpha)}{4} \sum_{\boldsymbol{\beta}}^{\prime} \kappa^{r s} \kappa^{t u} \sum_{i=1}^{n} x_{i r} x_{i s} x_{i t} x_{i u} .
\end{aligned}
$$

Invertendo a ordem dos somatórios, tem-se

$$
\begin{aligned}
\frac{1}{4} \sum_{\boldsymbol{\beta}}^{\prime} \kappa^{r s} \kappa^{t u} \kappa_{r s t u} & =\frac{a_{4}(\alpha)}{4} \sum_{i=1}^{n} \sum_{\boldsymbol{\beta}}^{\prime} \kappa^{r s} \kappa^{t u} x_{i r} x_{i s} x_{i t} x_{i u} \\
& =\frac{a_{4}(\alpha)}{4} \sum_{i=1}^{n}\left(\boldsymbol{x}_{i}^{\top} \boldsymbol{K}^{\boldsymbol{\beta} \boldsymbol{\beta}} \boldsymbol{x}_{i}\right)^{2},
\end{aligned}
$$

em que $\boldsymbol{x}_{i}^{\top}=\left(x_{i 1}, x_{i 2}, \ldots, x_{i p}\right)$ representa a $i$-ésima linha da matriz modelo $\boldsymbol{X}$ e $\boldsymbol{K}^{\boldsymbol{\beta} \boldsymbol{\beta}}=\boldsymbol{K}(\boldsymbol{\beta})^{-1}=$ $4\left(\boldsymbol{X}^{\top} \boldsymbol{X}\right)^{-1} / a_{1}(\alpha)$ representa a inversa da matriz de informação de Fisher de $\boldsymbol{\beta}$. Daí,

$$
\frac{1}{4} \sum_{\boldsymbol{\beta}}^{\prime} \kappa^{r s} \kappa^{t u} \kappa_{r s t u}=\frac{4 a_{4}(\alpha)}{a_{1}(\alpha)^{2}} \sum_{i=1}^{n}\left\{\boldsymbol{x}_{i}^{\top}\left(\boldsymbol{X}^{\top} \boldsymbol{X}\right)^{-1} \boldsymbol{x}_{i}\right\}^{2} .
$$

Agora, definindo as matrizes de dimensões $n \times n$

$$
\boldsymbol{Z}=\left\{z_{i j}\right\}=\boldsymbol{X}\left(\boldsymbol{X}^{\top} \boldsymbol{X}\right)^{-1} \boldsymbol{X}^{\top} \quad \text { e } \quad \boldsymbol{Z}_{d}=\operatorname{diag}\left\{z_{11}, z_{22}, \ldots, z_{n n}\right\},
$$


em que $z_{i j}=\boldsymbol{x}_{i}^{\top}\left(\boldsymbol{X}^{\top} \boldsymbol{X}\right)^{-1} \boldsymbol{x}_{j}$, temos que

$$
\sum_{\boldsymbol{\beta}}^{\prime} \ell_{r s t u}=\frac{4 a_{4}(\alpha)}{a_{1}(\alpha)^{2}} \sum_{i=1}^{n} z_{i i}^{2}=\frac{4 a_{4}(\alpha)}{a_{1}(\alpha)^{2}} \operatorname{tr}\left(\boldsymbol{Z}_{d}^{(2)}\right),
$$

em que $\operatorname{tr}(\cdot)$ representa o operador traço de uma matriz e $\boldsymbol{Z}_{d}^{(2)}$ é definida como segue: seja $\odot$ o produto de Hadamard de duas matrizes, então $\boldsymbol{Z}^{(2)}=\boldsymbol{Z} \odot \boldsymbol{Z}, \boldsymbol{Z}_{d}^{(2)}=\boldsymbol{Z}_{d} \odot \boldsymbol{Z}_{d}$, etc.

Da mesma forma,

$$
\begin{aligned}
\sum_{\boldsymbol{\beta}, \alpha}^{\prime} \kappa^{r s} \kappa^{\alpha \alpha}\left\{\kappa_{r s \alpha \alpha} / 4-\kappa_{r s \alpha}^{(\alpha)}\right\} & =\kappa^{\alpha \alpha} \sum_{\boldsymbol{\beta}}^{\prime} \kappa^{r s}\left\{\kappa_{r s \alpha \alpha} / 4-\kappa_{r s \alpha}^{(\alpha)}\right\} \\
& =\frac{18+\alpha^{2}}{2 n \alpha^{2} a_{1}(\alpha)} \sum_{i=1}^{n} z_{i i}=\frac{18+\alpha^{2}}{2 n \alpha^{2} a_{1}(\alpha)} \operatorname{tr}\left(\boldsymbol{Z}_{d}\right),
\end{aligned}
$$

mas como $\operatorname{tr}\left(\boldsymbol{Z}_{d}\right)=\operatorname{tr}(\boldsymbol{Z})=\operatorname{tr}\left(\boldsymbol{X}\left(\boldsymbol{X}^{\top} \boldsymbol{X}\right)^{-1} \boldsymbol{X}^{\top}\right)=\operatorname{tr}\left(\boldsymbol{X}^{\top} \boldsymbol{X}\left(\boldsymbol{X}^{\top} \boldsymbol{X}\right)^{-1}\right)=\operatorname{tr}\left(\boldsymbol{I}_{p}\right)=p$, temos que

$$
\sum_{\boldsymbol{\beta}, \alpha}^{\prime} \ell_{r s \alpha \alpha}=\frac{\left(18+\alpha^{2}\right) p}{2 n \alpha^{2} a_{1}(\alpha)} .
$$

Adicionalmente, temos que

$$
\begin{aligned}
\frac{1}{4} \sum_{\boldsymbol{\beta}, \alpha}^{\prime} \kappa^{\alpha \alpha} \kappa^{t u} \kappa_{\alpha \alpha t u} & =\frac{\kappa^{\alpha \alpha}}{4} \sum_{\boldsymbol{\beta}}^{\prime} \kappa^{t u} \kappa_{\alpha \alpha t u} \\
& =-\frac{3\left(2+\alpha^{2}\right)}{2 n \alpha^{2} a_{1}(\alpha)} \sum_{i=1}^{n} z_{i i}=-\frac{3\left(2+\alpha^{2}\right)}{2 n \alpha^{2} a_{1}(\alpha)} \operatorname{tr}\left(\boldsymbol{Z}_{d}\right),
\end{aligned}
$$

mas como $\operatorname{tr}\left(\boldsymbol{Z}_{d}\right)=p$, tem-se que

$$
\sum_{\boldsymbol{\beta}, \alpha}^{\prime} \ell_{\alpha \alpha t u}=-\frac{3\left(2+\alpha^{2}\right) p}{2 n \alpha^{2} a_{1}(\alpha)}
$$

Ainda,

$$
\sum_{\alpha}^{\prime} \ell_{\alpha \alpha \alpha \alpha}=\frac{\alpha^{4}}{4 n^{2}}\left\{-\frac{54 n}{4 \alpha^{4}}+\frac{30 n}{\alpha^{4}}-\frac{12 n}{\alpha^{4}}\right\}=\frac{9}{8 n} .
$$

Para o cálculo de $\sum_{\boldsymbol{\beta}, \alpha}^{\prime} \ell_{\text {rstuvw }}$ devemos desenvolver apenas os oito termos abaixo, devido à ortogonalidade global entre $\boldsymbol{\beta}$ e $\alpha$ :

1. $\sum_{\boldsymbol{\beta}}^{\prime} \lambda_{r s t u v w}=\sum_{\boldsymbol{\beta}}^{\prime} \kappa^{r s} \kappa^{t u} \kappa^{v w}\left\{\kappa_{r t v}\left(\kappa_{\text {suw }} / 6-\kappa_{s w}^{(u)}\right)+\kappa_{r t u}\left(\kappa_{s v w} / 4-\kappa_{s w}^{(v)}\right)+\kappa_{r t}^{(v)} \kappa_{s w}^{(u)}+\kappa_{r t}^{(u)} \kappa_{s w}^{(v)}\right\}$,

2. $\sum_{\boldsymbol{\beta}, \alpha}^{\prime} \lambda_{r s t u \alpha \alpha}=\sum_{\boldsymbol{\beta}, \alpha}^{\prime} \kappa^{r s} \kappa^{t u} \kappa^{\alpha \alpha}\left\{\kappa_{r t \alpha}\left(\kappa_{s u \alpha} / 6-\kappa_{s \alpha}^{(u)}\right)+\kappa_{r t u}\left(\kappa_{s \alpha \alpha} / 4-\kappa_{s \alpha}^{(\alpha)}\right)+\kappa_{r t}^{(\alpha)} \kappa_{s \alpha}^{(u)}+\kappa_{r t}^{(u)} \kappa_{s \alpha}^{(\alpha)}\right\}$,

3. $\sum_{\boldsymbol{\beta}, \alpha}^{\prime} \lambda_{r s \alpha \alpha v w}=\sum_{\boldsymbol{\beta}, \alpha}^{\prime} \kappa^{r s} \kappa^{\alpha \alpha} \kappa^{v w}\left\{\kappa_{r \alpha v}\left(\frac{\kappa_{s \alpha w}}{6}-\kappa_{s w}^{(\alpha)}\right)+\kappa_{r \alpha \alpha}\left(\frac{\kappa_{s v w}}{4}-\kappa_{s w}^{(v)}\right)+\kappa_{r \alpha}^{(v)} \kappa_{s w}^{(\alpha)}+\kappa_{r \alpha}^{(\alpha)} \kappa_{s w}^{(v)}\right\}$, 
4. $\sum_{\boldsymbol{\beta}, \alpha}^{\prime} \lambda_{\alpha \alpha t u v w}=\sum_{\boldsymbol{\beta}, \alpha}^{\prime} \kappa^{\alpha \alpha} \kappa^{t u} \kappa^{v w}\left\{\kappa_{\alpha t v}\left(\frac{\kappa_{\alpha u w}}{6}-\kappa_{\alpha w}^{(u)}\right)+\kappa_{\alpha t u}\left(\frac{\kappa_{\alpha v w}}{4}-\kappa_{\alpha w}^{(v)}\right)+\kappa_{\alpha t}^{(v)} \kappa_{\alpha w}^{(u)}+\kappa_{\alpha t}^{(u)} \kappa_{\alpha w}^{(v)}\right\}$,

5. $\sum_{\boldsymbol{\beta}, \alpha}^{\prime} \lambda_{r s \alpha \alpha \alpha \alpha}=\sum_{\boldsymbol{\beta}, \alpha}^{\prime} \kappa^{r s} \kappa^{\alpha \alpha} \kappa^{\alpha \alpha}\left\{\kappa_{r \alpha \alpha}\left(\frac{\kappa_{s \alpha \alpha}}{6}-\kappa_{s \alpha}^{(\alpha)}\right)+\kappa_{r \alpha \alpha}\left(\frac{\kappa_{s \alpha \alpha}}{4}-\kappa_{s \alpha}^{(\alpha)}\right)+\kappa_{r \alpha}^{(\alpha)} \kappa_{s \alpha}^{(\alpha)}+\kappa_{r \alpha}^{(\alpha)} \kappa_{s \alpha}^{(\alpha)}\right\}$,

6. $\sum_{\boldsymbol{\beta}, \alpha}^{\prime} \lambda_{\alpha \alpha t u \alpha \alpha}=\sum_{\boldsymbol{\beta}, \alpha}^{\prime} \kappa^{\alpha \alpha} \kappa^{t u} \kappa^{\alpha \alpha}\left\{\kappa_{\alpha t \alpha}\left(\frac{\kappa_{\alpha u \alpha}}{6}-\kappa_{\alpha \alpha}^{(u)}\right)+\kappa_{\alpha t u}\left(\frac{\kappa_{\alpha \alpha \alpha}}{4}-\kappa_{\alpha \alpha}^{(\alpha)}\right)+\kappa_{\alpha t}^{(\alpha)} \kappa_{\alpha \alpha}^{(u)}+\kappa_{\alpha t}^{(u)} \kappa_{\alpha \alpha}^{(\alpha \alpha)}\right\}$,

7. $\sum_{\boldsymbol{\beta}, \alpha}^{\prime} \lambda_{\alpha \alpha \alpha \alpha v w}=\sum_{\boldsymbol{\beta}, \alpha}^{\prime} \kappa^{\alpha \alpha} \kappa^{\alpha \alpha} \kappa^{v w}\left\{\kappa_{\alpha \alpha v}\left(\frac{\kappa_{\alpha \alpha w}}{6}-\kappa_{\alpha w}^{(\alpha)}\right)+\kappa_{\alpha \alpha \alpha}\left(\frac{\kappa_{\alpha v w}}{4}-\kappa_{\alpha w}^{(v)}\right)+\kappa_{\alpha \alpha}^{(v)} \kappa_{\alpha w}^{(\alpha)}+\kappa_{\alpha \alpha}^{(u)} \kappa_{\alpha w}^{(v)}\right\}$,

8. $\sum_{\alpha}^{\prime} \lambda_{\alpha \alpha \alpha \alpha \alpha \alpha}=\sum_{\alpha}^{\prime} \kappa^{\alpha \alpha} \kappa^{\alpha \alpha} \kappa^{\alpha \alpha}\left\{\kappa_{\alpha \alpha \alpha}\left(\kappa_{\alpha \alpha \alpha} / 6-\kappa_{\alpha \alpha}^{(\alpha)}\right)+\kappa_{\alpha \alpha \alpha}\left(\kappa_{\alpha \alpha \alpha} / 4-\kappa_{\alpha \alpha}^{(\alpha)}\right)+\kappa_{\alpha \alpha}^{(\alpha)} \kappa_{\alpha \alpha}^{(\alpha)}+\kappa_{\alpha \alpha}^{(\alpha)} \kappa_{\alpha \alpha}^{(\alpha)}\right\}$.

Dos oito termos acima, facilmente verificamos que os termos (1) e (5) se anulam, pois todas as quantidades entre chaves são nulas. Assim,

$$
\sum_{\boldsymbol{\beta}}^{\prime} \lambda_{r s t u v w}=0 \quad \text { e } \quad \sum_{\boldsymbol{\beta}, \alpha}^{\prime} \lambda_{r s \alpha \alpha \alpha \alpha}=0 .
$$

Como muitos dos cumulantes e suas derivadas são nulos, podemos escrever

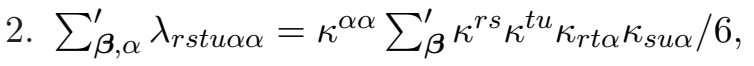

3. $\sum_{\boldsymbol{\beta}, \alpha}^{\prime} \lambda_{\text {rsa } \alpha v w}=\kappa^{\alpha \alpha} \sum_{\boldsymbol{\beta}}^{\prime} \kappa^{r s} \kappa^{v w} \kappa_{r \alpha v}\left(\kappa_{s \alpha w} / 6-\kappa_{s w}^{(\alpha)}\right)$,

4. $\sum_{\boldsymbol{\beta}, \alpha}^{\prime} \lambda_{\alpha \alpha t u v w}=\kappa^{\alpha \alpha} \sum_{\boldsymbol{\beta}}^{\prime} \kappa^{t u} \kappa^{v w}\left\{\kappa_{\alpha t v} \kappa_{\alpha u w} / 6+\kappa_{\alpha t u} \kappa_{\alpha v w} / 4\right\}$,

6. $\sum_{\boldsymbol{\beta}, \alpha}^{\prime} \lambda_{\alpha \alpha t u \alpha \alpha}=\left(\kappa^{\alpha \alpha}\right)^{2}\left(\kappa_{\alpha \alpha \alpha} / 4-\kappa_{\alpha \alpha}^{(\alpha)}\right) \sum_{\boldsymbol{\beta}}^{\prime} \kappa^{t u} \kappa_{\alpha t u}$,

7. $\sum_{\boldsymbol{\beta}, \alpha}^{\prime} \lambda_{\alpha \alpha \alpha \alpha v w}=\left(\kappa^{\alpha \alpha}\right)^{2} \kappa_{\alpha \alpha \alpha} \sum_{\boldsymbol{\beta}}^{\prime} \kappa^{v w} \kappa_{\alpha v w} / 4$,

8. $\sum_{\alpha}^{\prime} \lambda_{\alpha \alpha \alpha \alpha \alpha \alpha}=\left(\kappa^{\alpha \alpha}\right)^{3}\left\{\kappa_{\alpha \alpha \alpha}^{2} / 6+\kappa_{\alpha \alpha \alpha}^{2} / 4-2 \kappa_{\alpha \alpha \alpha} \kappa_{\alpha \alpha}^{(\alpha)}+2\left(\kappa_{\alpha \alpha}^{(\alpha)}\right)^{2}\right\}$.

O desenvolvimento dos termos (2), (3) e (4) são semelhantes e, assim, apresentamos, em detalhes, apenas a derivação do termo (4). Da mesma forma, o desenvolvimento dos termos (6) e (7) são parecidos e assim, desenvolveremos apenas o termo (7). A quantidade (8) só envolve componentes referentes ao parâmtro escalar $\alpha$, cujo desenvolvimento será deixado por último. Assim, temos que

$$
\begin{aligned}
\sum_{\boldsymbol{\beta}, \alpha}^{\prime} \lambda_{\alpha \alpha t u v w}= & -\frac{\alpha^{2}}{2 n} \sum_{\boldsymbol{\beta}}^{\prime} \kappa^{t u} \kappa^{v w}\left\{\frac{1}{6}\left[\frac{2+\alpha^{2}}{\alpha^{3}} \sum_{i=1}^{n} x_{i t} x_{i v}\right]\left[\frac{2+\alpha^{2}}{\alpha^{3}} \sum_{j=1}^{n} x_{j u} x_{j w}\right]\right. \\
& \left.+\frac{1}{4}\left[\frac{2+\alpha^{2}}{\alpha^{3}} \sum_{i=1}^{n} x_{i t} x_{i u}\right]\left[\frac{2+\alpha^{2}}{\alpha^{3}} \sum_{j=1}^{n} x_{j v} x_{j w}\right]\right\} \\
= & -\frac{\alpha^{2}\left(2+\alpha^{2}\right)^{2}}{2 n \alpha^{6}} \sum_{\boldsymbol{\beta}}^{\prime} \kappa^{t u} \kappa^{v w}\left\{\frac{1}{6} \sum_{i, j=1}^{n} x_{i t} x_{i v} x_{j u} x_{j w}+\frac{1}{4} \sum_{i, j=1}^{n} x_{i t} x_{i u} x_{j v} x_{j w}\right\} \\
= & -\frac{8\left(2+\alpha^{2}\right)^{2}}{n \alpha^{4} a_{1}(\alpha)^{2}}\left\{\frac{1}{6} \sum_{i, j=1}^{n} z_{i j}^{2}+\frac{1}{4} \sum_{i=1}^{n} z_{i i} \sum_{j=1}^{n} z_{j j}\right\} .
\end{aligned}
$$


Note que

$$
\sum_{i=1}^{n} z_{i i}=\operatorname{tr}\left(\boldsymbol{Z}_{d}\right)=p \quad \text { e } \quad \sum_{j=1}^{n} z_{j j}=\operatorname{tr}\left(\boldsymbol{Z}_{d}\right)=p .
$$

Ainda, como $\boldsymbol{Z}$ é simétrica e idempotente, isto é, $\boldsymbol{Z}^{\top}=\boldsymbol{Z}$ e $\boldsymbol{Z} \boldsymbol{Z}=\boldsymbol{Z}$, temos que, $\operatorname{posto}(\boldsymbol{Z})=$ $\operatorname{tr}(\boldsymbol{Z})=p$ e $\sum_{j=1}^{n} z_{i j}^{2}=z_{i i}$. Daí,

$$
\begin{aligned}
\sum_{i, j=1}^{n} z_{i j}^{2} & =\sum_{j=1}^{n} z_{1 j}^{2}+\sum_{j=1}^{n} z_{2 j}^{2}+\cdots+\sum_{j=1}^{n} z_{n j}^{2} \\
& =z_{11}+z_{22}+\cdots+z_{n n} \\
& =\sum_{i=1}^{n} z_{i i}=\operatorname{tr}\left(\boldsymbol{Z}_{d}\right)=p .
\end{aligned}
$$

Dessa forma, temos que

$$
\sum_{\boldsymbol{\beta}, \alpha}^{\prime} \lambda_{\alpha \alpha t u v w}=-\frac{8\left(2+\alpha^{2}\right)^{2}}{n \alpha^{4} a_{1}(\alpha)^{2}}\left\{\frac{1}{6} p+\frac{1}{4} p^{2}\right\} .
$$

Da mesma forma,

$$
\sum_{\boldsymbol{\beta}, \alpha}^{\prime} \lambda_{r s t u \alpha \alpha}=-\frac{4\left(2+\alpha^{2}\right)^{2} p}{3 n \alpha^{4} a_{1}(\alpha)^{2}}
$$

$$
\sum_{\boldsymbol{\beta}, \alpha}^{\prime} \lambda_{r s \alpha \alpha v w}=-\left\{\frac{4\left(2+\alpha^{2}\right)^{2}}{3 n \alpha^{4} a_{1}(\alpha)^{2}}-\frac{8\left(2+\alpha^{2}\right) a_{2}(\alpha)}{n \alpha a_{1}(\alpha)^{2}}\right\} p .
$$

Vamos agora calcular o termo (7). Tem-se que

$$
\begin{aligned}
\sum_{\boldsymbol{\beta}, \alpha}^{\prime} \lambda_{\alpha \alpha \alpha \alpha v w} & =\frac{\alpha^{4}}{4 n^{2}} \frac{5 n}{2 \alpha^{3}} \sum_{\boldsymbol{\beta}}^{\prime} \kappa^{v w}\left\{\frac{2+\alpha^{2}}{\alpha^{3}} \sum_{i=1}^{n} x_{i v} x_{i w}\right\} \\
& =-\frac{5\left(2+\alpha^{2}\right)}{2 n \alpha^{2} a_{1}(\alpha)} \sum_{i=1}^{n} z_{i i}=-\frac{5\left(2+\alpha^{2}\right)}{2 n \alpha^{2} a_{1}(\alpha)} \operatorname{tr}\left(\boldsymbol{Z}_{d}\right),
\end{aligned}
$$

mas como $\operatorname{tr}\left(\boldsymbol{Z}_{d}\right)=p$, temos que

$$
\sum_{\boldsymbol{\beta}, \alpha}^{\prime} \lambda_{\alpha \alpha \alpha \alpha v w}=-\frac{5\left(2+\alpha^{2}\right) p}{2 n \alpha^{2} a_{1}(\alpha)} .
$$

Da mesma forma,

$$
\sum_{\boldsymbol{\beta}, \alpha}^{\prime} \lambda_{\alpha \alpha t u \alpha \alpha}=-\frac{3\left(2+\alpha^{2}\right) p}{2 n \alpha^{2} a_{1}(\alpha)}
$$

Finalmente, temos que

$$
\sum_{\alpha}^{\prime} \lambda_{\alpha \alpha \alpha \alpha \alpha \alpha}=-\frac{\alpha^{6}}{8 n^{3}}\left\{\frac{100 n^{2}}{6 \alpha^{6}}+\frac{100 n^{2}}{4 \alpha^{6}}-\frac{80 n^{2}}{\alpha^{6}}+\frac{32 n^{2}}{\alpha^{6}}\right\}=\frac{19}{24 n} .
$$


Portanto, como

$$
\epsilon_{p+1}=\sum_{\boldsymbol{\beta}, \alpha}^{\prime} \lambda_{r s t u}-\sum_{\boldsymbol{\beta}, \alpha}^{\prime} \lambda_{r s t u v w},
$$

e todas as quantidades necessárias para o seu cálculo foram obtidas anteriormente, obtemos, após longas e tediosas manipulações algébricas, a expressão para $\epsilon_{p+1}$ dada em (2.6). 


\section{Apêndice C}

\section{Fator de Correção tipo-Bartlett}

Neste apêndice apresentamos, em detalhes, a obtenção das quantidades $A_{1}, A_{2}$ e $A_{3}$ referentes aos testes das hipóteses $\mathcal{H}_{0}: \boldsymbol{\beta}_{1}=\boldsymbol{\beta}_{1}^{(0)}$ versus $\mathcal{H}_{1}: \boldsymbol{\beta}_{1} \neq \boldsymbol{\beta}_{1}^{(0)}, \mathcal{H}_{0}: \alpha=\alpha^{(0)}$ versus $\mathcal{H}_{1}: \alpha \neq \alpha^{(0)}$ e $\mathcal{H}_{0}: \boldsymbol{\beta}=\boldsymbol{\beta}^{(0)}$ versus $\mathcal{H}_{1}: \boldsymbol{\beta} \neq \boldsymbol{\beta}^{(0)}$ no modelo de regressão Birnbaum-Saunders. Para tal, utilizaremos os cumulantes e suas derivadas encontrados no Apêndice A.

\section{C.1 Obtenção de $A_{1}, A_{2}$ e $A_{3}$ para testar $\mathcal{H}_{0}: \boldsymbol{\beta}_{1}=\boldsymbol{\beta}_{1}^{(0)}$}

Particione $\boldsymbol{\beta}$ da forma $\boldsymbol{\beta}=\left(\boldsymbol{\beta}_{1}^{\top}, \boldsymbol{\beta}_{2}^{\top}\right)^{\top}$, em que $\boldsymbol{\beta}_{1}=\left(\beta_{1}, \beta_{2}, \ldots, \beta_{q}\right)^{\top}$ e $\boldsymbol{\beta}_{2}=\left(\beta_{q+1}, \beta_{q+2}, \ldots, \beta_{p}\right)^{\top}$ são vetores $q$ e $p-q$ dimensionais, respectivamente. Seja $\boldsymbol{X}=\left[\boldsymbol{X}_{1} \boldsymbol{X}_{2}\right]$, cujas as dimensões de $\boldsymbol{X}_{1}$ e $\boldsymbol{X}_{2}$ são $n \times q$ e $n \times(p-q)$, respectivamente; essas matrizes são de postos completo. Devido à ortogonalidade global entre $\boldsymbol{\beta}$ e $\alpha$, a matriz de informação total de Fisher $\boldsymbol{K}(\boldsymbol{\theta})$ para $\boldsymbol{\theta}=\left(\boldsymbol{\beta}^{\top}, \alpha\right)^{\top}$ e sua inversa $\boldsymbol{K}(\boldsymbol{\theta})^{-1}$ são bloco-diagonais e podem ser escritas na forma

$$
\boldsymbol{K}(\boldsymbol{\theta})=\left[\begin{array}{ccc}
\boldsymbol{K}_{11} & \boldsymbol{K}_{12} & \mathbf{0} \\
\boldsymbol{K}_{21} & \boldsymbol{K}_{22} & \mathbf{0} \\
\mathbf{0} & \mathbf{0} & \kappa_{\alpha, \alpha}
\end{array}\right] \quad \text { e } \quad \boldsymbol{K}(\boldsymbol{\theta})^{-1}=\left[\begin{array}{ccc}
\boldsymbol{K}^{11} & \boldsymbol{K}^{12} & \mathbf{0} \\
\boldsymbol{K}^{21} & \boldsymbol{K}^{22} & \mathbf{0} \\
\mathbf{0} & \mathbf{0} & \kappa_{\alpha, \alpha}^{-1}
\end{array}\right] .
$$

Sejam as matrizes

$$
\boldsymbol{A}_{\boldsymbol{\beta}}=\left[\begin{array}{cc}
\mathbf{0} & \mathbf{0} \\
\mathbf{0} & \boldsymbol{K}_{22}^{-1}
\end{array}\right], \boldsymbol{K}_{\boldsymbol{\beta}}^{-1}=\left[\begin{array}{ll}
\boldsymbol{K}^{11} & \boldsymbol{K}^{12} \\
\boldsymbol{K}^{21} & \boldsymbol{K}^{22}
\end{array}\right], \boldsymbol{A}=\left[\begin{array}{cc}
\boldsymbol{A}_{\boldsymbol{\beta}} & \mathbf{0} \\
\mathbf{0} & \kappa_{\alpha, \alpha}^{-1}
\end{array}\right] \quad \text { e } \quad \boldsymbol{M}=\left[\begin{array}{cc}
\boldsymbol{M}_{\boldsymbol{\beta}} & \mathbf{0} \\
\mathbf{0} & 0
\end{array}\right],
$$

em que $\boldsymbol{M}_{\boldsymbol{\beta}}=\boldsymbol{K}_{\boldsymbol{\beta}}^{-1}-\boldsymbol{A}_{\boldsymbol{\beta}}$. Sejam $m_{r \alpha}$ e $a_{r \alpha}$ os $(r, p+1)$-ésimos elementos das matrizes $\boldsymbol{M}$ e $\boldsymbol{A}$, respectivamente, e sejam $m_{\alpha \alpha}$ e $a_{\alpha \alpha}$ os $(p+1, p+1)$-ésimos elementos das matrizes $\boldsymbol{M}$ e $\boldsymbol{A}$, respectivamente. Dessa forma, temos que $m_{r \alpha}=m_{\alpha r}=m_{\alpha \alpha}=0, a_{r \alpha}=a_{\alpha r}=0(r=1,2, \ldots, p)$ e $a_{\alpha \alpha}=\kappa_{\alpha, \alpha}^{-1}=\alpha^{2} / 2 n$.

As quantidades $A_{1}, A_{2}$ e $A_{3}$ são agora obtidas substituindo os cumulantes encontrados no Apêndice A nas expressões

$$
A_{1}=A_{1, \boldsymbol{\beta}}+A_{1, \boldsymbol{\beta} \alpha}, \quad A_{2}=A_{2, \boldsymbol{\beta}}+A_{2, \boldsymbol{\beta} \alpha} \quad \text { e } \quad A_{3}=A_{3, \boldsymbol{\beta}}+A_{3, \boldsymbol{\beta} \alpha},
$$


em que $A_{1, \boldsymbol{\beta}}, A_{2, \boldsymbol{\beta}}$ e $A_{3, \boldsymbol{\beta}}$ são como nas Equações (3.3), (3.4) e (3.5), respectivamente. Adicionalmente,

$$
\begin{aligned}
A_{1, \boldsymbol{\beta} \alpha} & =3 \sum^{\prime}\left(\kappa_{\alpha \alpha k}+2 \kappa_{\alpha, \alpha k}\right)\left(\kappa_{r s t}+2 \kappa_{r s, t}\right) a_{\alpha \alpha} a_{s t} m_{k r} \\
& +3 \sum^{\prime}\left(\kappa_{i j k}+2 \kappa_{i, j k}\right)\left(\kappa_{r \alpha \alpha}+2 \kappa_{r \alpha, \alpha}\right) a_{i j} a_{\alpha \alpha} m_{k r} \\
& +3 \sum^{\prime}\left(\kappa_{\alpha \alpha k}+2 \kappa_{\alpha, \alpha k}\right)\left(\kappa_{r \alpha \alpha}+2 \kappa_{r \alpha, \alpha}\right) a_{\alpha \alpha}^{2} m_{k r}-6 \sum^{\prime}\left(\kappa_{\alpha \alpha k}+2 \kappa_{\alpha, \alpha k}\right) \kappa_{r, s, t} a_{\alpha \alpha} a_{k r} m_{s t} \\
& -6 \sum^{\prime}\left(\kappa_{i j \alpha}+2 \kappa_{i, j \alpha}\right) \kappa_{\alpha, s, t} a_{i j} a_{\alpha \alpha} m_{s t}-6 \sum^{\prime}\left(\kappa_{\alpha \alpha \alpha}+2 \kappa_{\alpha, \alpha \alpha}\right) \kappa_{\alpha, s, t} a_{\alpha \alpha}^{2} m_{s t} \\
& +6 \sum^{\prime}\left(\kappa_{i, \alpha k}-\kappa_{i, \alpha, k}\right)\left(\kappa_{r \alpha t}+2 \kappa_{r \alpha, t}\right) a_{\alpha \alpha} a_{k t} m_{i r} \\
& +6 \sum^{\prime}\left(\kappa_{i, j \alpha}-\kappa_{i, j, \alpha}\right)\left(\kappa_{r s \alpha}+2 \kappa_{r s, \alpha}\right) a_{j s} a_{\alpha \alpha} m_{i r} \\
& +6 \sum^{\prime}\left(\kappa_{i, \alpha \alpha}-\kappa_{i, \alpha, \alpha}\right)\left(\kappa_{r \alpha \alpha}+2 \kappa_{r \alpha, \alpha}\right) a_{\alpha \alpha}^{2} m_{i r}-6 \sum^{\prime}\left(\kappa_{i, j, \alpha, \alpha}+\kappa_{i, j, \alpha \alpha}\right) a_{\alpha \alpha} m_{i j}, \\
& A_{2, \boldsymbol{\beta} \alpha}=-3 \sum^{\prime} \kappa_{i, j, \alpha} \kappa_{\alpha, s, t} a_{\alpha \alpha} m_{i j} m_{s t}+6 \sum^{\prime}\left(\kappa_{\alpha \alpha k}+2 \kappa_{\alpha, \alpha k}\right) \kappa_{r, s, t} a_{\alpha \alpha} m_{k r} m_{s t} \\
& \quad-6 \sum^{\prime} \kappa_{i, j, \alpha} \kappa_{r, s, \alpha} a_{\alpha \alpha} m_{i r} m_{j s}
\end{aligned}
$$

e

$$
A_{3, \boldsymbol{\beta} \alpha}=0 .
$$

Como muitos cumulantes são nulos, de (3.3) vem

$$
\begin{aligned}
A_{1, \boldsymbol{\beta}} & =-6 \sum^{\prime}\left(\kappa_{i, j, k, r}+\kappa_{i, j, k r}\right) a_{k r} m_{i j} \\
& =-6 \sum^{\prime}\left\{\tilde{s}_{1}(\alpha) \sum_{l=1}^{n} x_{l i} x_{l j} x_{l k} x_{l r}\right\} a_{k r} m_{i j} \\
& =-6 \tilde{s}_{1}(\alpha) \sum^{\prime} \sum_{l=1}^{n} x_{l i} x_{l j} x_{l k} x_{l r} a_{k r} m_{i j} .
\end{aligned}
$$

Invertendo a ordem dos somatórios, temos que

$$
\begin{aligned}
A_{1, \boldsymbol{\beta}} & =-6 \tilde{s}_{1}(\alpha) \sum_{l=1}^{n} \sum^{\prime} x_{l i} x_{l j} x_{l k} x_{l r} a_{k r} m_{i j} \\
& =-6 \tilde{s}_{1}(\alpha) \sum_{l=1}^{n}\left\{\sum_{k, r=1}^{p} x_{l k} x_{l r} a_{k r}\right\}\left\{\sum_{i, j=1}^{p} x_{l i} x_{l j} m_{i j}\right\} .
\end{aligned}
$$

Agora, os termos $\sum_{k, r=1}^{p} x_{l k} x_{m r} a_{k r}$ e $\sum_{k, r=1}^{p} x_{l i} x_{m j} m_{i j}$ representam os $(l, m)$-ésimos elementos das matrizes

$$
\frac{4}{a_{1}(\alpha)} \boldsymbol{Z}_{2} \quad \text { e } \quad \frac{4}{a_{1}(\alpha)}\left(\boldsymbol{Z}-\boldsymbol{Z}_{2}\right)
$$

respectivamente, sendo que as matrizes $\boldsymbol{Z}$ e $\boldsymbol{Z}_{2}$ estão definidas em (3.14). Daí,

$$
A_{1, \boldsymbol{\beta}}=-\frac{96 \tilde{s}_{1}(\alpha)}{a_{1}(\alpha)^{2}} \sum_{l=1}^{n} z_{2 l l}\left(z_{l l}-z_{2 l l}\right) .
$$


Portanto,

$$
A_{1, \boldsymbol{\beta}}=-\frac{96 \tilde{s}_{1}(\alpha)}{a_{1}(\alpha)^{2}} \operatorname{tr}\left\{\left(\boldsymbol{Z}-\boldsymbol{Z}_{2}\right)_{d} \boldsymbol{Z}_{2 d}\right\},
$$

em que a notação utilizada segue da Seção 3.2.

A obtenção de $A_{2, \boldsymbol{\beta}}$ e $A_{3, \boldsymbol{\beta}}$ é conduzida de maneira análoga. Como muitos cumulantes são nulos, de (3.4) vem

$$
\begin{aligned}
A_{2, \boldsymbol{\beta}} & =3 \sum^{\prime} \kappa_{i, j, k, r} m_{i j} m_{k r}=3 \sum^{\prime}\left\{\tilde{s}_{2}(\alpha) \sum_{l=1}^{n} x_{l i} x_{l j} x_{l k} x_{l r}\right\} m_{i j} m_{k r} \\
& =\frac{48 \tilde{s}_{2}(\alpha)}{a_{1}(\alpha)^{2}} \sum_{l=1}^{n}\left(z_{l l}-z_{2 l l}\right)\left(z_{l l}-z_{2 l l}\right)=\frac{48 \tilde{s}_{2}(\alpha)}{a_{1}(\alpha)^{2}} \sum_{l=1}^{n}\left(z_{l l}-z_{2 l l}\right)^{2} .
\end{aligned}
$$

Assim,

$$
A_{2, \boldsymbol{\beta}}=\frac{48 \tilde{s}_{2}(\alpha)}{a_{1}(\alpha)^{2}} \operatorname{tr}\left\{\left(\boldsymbol{Z}-\boldsymbol{Z}_{2}\right)_{d}^{(2)}\right\}
$$

Adicionalmente, de (3.5) vem

$$
A_{3, \boldsymbol{\beta}}=0,
$$

pois os cumulantes envolvidos nesta quantidade são nulos.

A obtenção de $A_{1, \beta}$ e $A_{2, \boldsymbol{\beta} \alpha}$ segue de forma análoga ao que foi feito anteriormente. Como alguns cumulantes são nulos, temos que

$$
\begin{aligned}
A_{1, \boldsymbol{\beta} \alpha} & =-6 \sum^{\prime}\left(\kappa_{i j \alpha}+2 \kappa_{i, j \alpha}\right) \kappa_{\alpha, s, t} a_{i j} a_{\alpha \alpha} m_{s t} \\
& -6 \sum^{\prime}\left(\kappa_{\alpha \alpha \alpha}+2 \kappa_{\alpha, \alpha \alpha}\right) \kappa_{\alpha, s, t} a_{\alpha \alpha}^{2} m_{s t} \\
& +6 \sum^{\prime}\left(\kappa_{i, \alpha k}-\kappa_{i, \alpha, k}\right)\left(\kappa_{r \alpha t}+2 \kappa_{r \alpha, t}\right) a_{\alpha \alpha} a_{k t} m_{i r} \\
& +6 \sum^{\prime}\left(\kappa_{i, j \alpha}-\kappa_{i, j, \alpha}\right)\left(\kappa_{r s \alpha}+2 \kappa_{r s, \alpha}\right) a_{j s} a_{\alpha \alpha} m_{i r} \\
& -6 \sum^{\prime}\left(\kappa_{i, j, \alpha, \alpha}+\kappa_{i, j, \alpha \alpha}\right) a_{\alpha \alpha} m_{i j} .
\end{aligned}
$$

Iremos apresentar a derivação em detalhes apenas do primeiro termo de $A_{1, \boldsymbol{\beta} \alpha}$, já que os outros termos são derivados de forma semelhante. Temos

$$
\begin{aligned}
-6 \sum^{\prime}\left(\kappa_{i j \alpha}+2 \kappa_{i, j \alpha}\right) \kappa_{\alpha, s, t} a_{i j} a_{\alpha \alpha} m_{s t} & =-6 \sum^{\prime}\left\{-\frac{2+\alpha^{2}}{\alpha^{3}} \sum_{l=1}^{n} x_{l i} x_{l j}\right\}\left\{\tilde{s}_{3}(\alpha) \sum_{m=1}^{n} x_{m s} x_{m t}\right\} a_{i j} a_{\alpha \alpha} m_{s t} \\
& =\frac{48\left(2+\alpha^{2}\right) \tilde{s}_{3}(\alpha)}{n \alpha a_{1}(\alpha)^{2}} \operatorname{tr}\left(\boldsymbol{Z}_{2}\right) \operatorname{tr}\left(\boldsymbol{Z}-\boldsymbol{Z}_{2}\right),
\end{aligned}
$$

mas $\operatorname{tr}\left(\boldsymbol{Z}_{2}\right)=\operatorname{tr}\left\{\boldsymbol{Z}_{2}\left(\boldsymbol{Z}_{2}^{\top} \boldsymbol{Z}_{2}\right)^{-1} \boldsymbol{Z}_{2}^{\top}\right\}=\operatorname{tr}\left\{\boldsymbol{Z}_{2}^{\top} \boldsymbol{Z}_{2}\left(\boldsymbol{Z}_{2}^{\top} \boldsymbol{Z}_{2}\right)^{-1}\right\}=\operatorname{tr}\left(\boldsymbol{I}_{p-q}\right)=p-q$. Da mesma forma, $\operatorname{tr}\left(\boldsymbol{Z}-\boldsymbol{Z}_{2}\right)=p-(p-q)=q$. Logo,

$$
-6 \sum^{\prime}\left(\kappa_{i j \alpha}+2 \kappa_{i, j \alpha}\right) \kappa_{\alpha, s, t} a_{i j} a_{\alpha \alpha} m_{s t}=\frac{48\left(2+\alpha^{2}\right) \tilde{s}_{3}(\alpha)}{n \alpha a_{1}(\alpha)^{2}} q(p-q) .
$$

Os termos restantes de $A_{1, \boldsymbol{\beta} \alpha}$ são obtidos da mesma maneira. Dessa forma, após alguma álgebra, 
temos que

$$
A_{1, \boldsymbol{\beta} \alpha}=\frac{12 q}{n a_{1}(\alpha)}\left\{\frac{4\left(2+\alpha^{2}\right) \tilde{s}_{3}(\alpha)}{a_{1}(\alpha) \alpha}(p-q)+\tilde{s}_{3}(\alpha) \alpha-\tilde{s}_{4}(\alpha) \alpha^{2}\right\}
$$

A obtenção de $A_{2, \boldsymbol{\beta} \alpha}$ segue de forma análoga. Como alguns cumulantes são nulos, temos que

$$
A_{2, \boldsymbol{\beta} \alpha}=-3 \sum^{\prime} \kappa_{i, j, \alpha} \kappa_{\alpha, s, t} a_{\alpha \alpha} m_{i j} m_{s t}-6 \sum^{\prime} \kappa_{i, j, \alpha} \kappa_{r, s, \alpha} a_{\alpha \alpha} m_{i r} m_{j s}
$$

Após algumas manipulações algébricas, tem-se que

$$
A_{2, \boldsymbol{\beta} \alpha}=-\frac{24 \tilde{s}_{3}(\alpha)^{2} \alpha^{2}}{n a_{1}(\alpha)^{2}} q(q+2) .
$$

Finalmente,

$$
A_{3, \boldsymbol{\beta} \alpha}=0 .
$$

Portanto,

$$
A_{1}=A_{1, \boldsymbol{\beta}}+A_{1, \boldsymbol{\beta} \alpha}, \quad A_{2}=A_{2, \boldsymbol{\beta}}+A_{2, \boldsymbol{\beta} \alpha} \quad \text { e } \quad A_{3}=0 .
$$

C.2 Obtenção de $A_{1}, A_{2}$ e $A_{3}$ para testar $\mathcal{H}_{0}: \alpha=\alpha^{(0)}$

Para obtermos $A_{1}, A_{2}$ e $A_{3}$, precisamos considerar as matrizes

$$
\begin{aligned}
& \boldsymbol{K}(\boldsymbol{\theta})=\left[\begin{array}{cc}
\boldsymbol{K}_{\boldsymbol{\beta}} & \mathbf{0} \\
\mathbf{0} & \kappa_{\alpha, \alpha}
\end{array}\right], \quad \boldsymbol{K}(\boldsymbol{\theta})^{-1}=\left[\begin{array}{cc}
\boldsymbol{K}_{\boldsymbol{\beta}}^{-1} & \mathbf{0} \\
\mathbf{0} & \kappa_{\alpha, \alpha}^{-1}
\end{array}\right], \\
& \boldsymbol{A}=\left[\begin{array}{cc}
\boldsymbol{K}_{\boldsymbol{\beta}}^{-1} & \mathbf{0} \\
\mathbf{0} & 0
\end{array}\right] \quad \text { e } \quad \boldsymbol{M}=\boldsymbol{K}_{\boldsymbol{\beta}}^{-1}-\boldsymbol{A}=\left[\begin{array}{cc}
\{\mathbf{0}\}_{p} & \mathbf{0} \\
\mathbf{0} & \kappa_{\alpha, \alpha}^{-1}
\end{array}\right]
\end{aligned}
$$

em que $\{\boldsymbol{0}\}_{p}$ representa uma matriz de zeros de dimensão $p \times p$. Vamos utilizar a seguinte notação, similarmente ao que já foi definido anteriormente: $m_{r \alpha}$ e $a_{r \alpha}$ representam os $(r, p+1)$-ésimos elementos de $\boldsymbol{M}$ e $\boldsymbol{A}$, respectivamente, e $m_{\alpha \alpha}$ e $a_{\alpha \alpha}$ os $(p+1, p+1)$-ésimos elementos das matrizes $\boldsymbol{M}$ e $\boldsymbol{A}$, respectivamente. Note que, $m_{r s}=m_{r \alpha}=m_{\alpha r}=0$ e $a_{r \alpha}=a_{\alpha r}=a_{\alpha \alpha}=0(r=1,2, \ldots, p)$. Ainda, $m_{\alpha \alpha}=\kappa_{\alpha, \alpha}^{-1}=\alpha^{2} / 2$.

Para testar a hipótese $\mathcal{H}_{0}: \alpha=\alpha^{(0)}$ contra $\mathcal{H}_{1}: \alpha \neq \alpha^{(0)}$, podemos escrever $A_{1}, A_{2}$ e $A_{3}$, dados, respectivamente, em (3.3), (3.4) e (3.5), da forma

$$
\begin{gathered}
A_{1}=3 \sum^{\prime}\left(\kappa_{i j \alpha}+2 \kappa_{i, j \alpha}\right)\left(\kappa_{\alpha s t}+2 \kappa_{\alpha s, t}\right) a_{i j} a_{s t} m_{\alpha \alpha} \\
-6 \sum^{\prime}\left(\kappa_{i j k}+2 \kappa_{i, j k}\right) \kappa_{r, \alpha, \alpha} a_{i j} a_{k r} m_{\alpha \alpha} \\
+6 \sum^{\prime}\left(\kappa_{\alpha, j k}-\kappa_{\alpha, j, k}\right)\left(\kappa_{\alpha s t}+2 \kappa_{\alpha s, t}\right) a_{j s} a_{k t} m_{\alpha \alpha} \\
-6 \sum^{\prime}\left(\kappa_{\alpha, \alpha, k, r}+\kappa_{\alpha, \alpha, k r}\right) a_{k r} m_{\alpha \alpha}, \\
A_{2}=-3 \sum^{\prime} \kappa_{\alpha, \alpha, k} \kappa_{r, \alpha, \alpha} a_{k r} m_{\alpha \alpha}^{2}+6 \sum^{\prime}\left(\kappa_{i j \alpha}+2 \kappa_{i, j \alpha}\right) \kappa_{\alpha, \alpha, \alpha} a_{i j} m_{\alpha \alpha}^{2} \\
-6 \sum^{\prime} \kappa_{\alpha, \alpha, k} \kappa_{\alpha, \alpha, t} a_{k t} m_{\alpha \alpha}^{2}+3 \sum^{\prime} \kappa_{\alpha, \alpha, \alpha, \alpha} m_{\alpha \alpha}^{2}
\end{gathered}
$$


$\mathrm{e}$

$$
A_{3}=5 \kappa_{\alpha, \alpha, \alpha}^{2} m_{\alpha \alpha}^{3} .
$$

Como alguns cumulantes são nulos, temos que

$$
\begin{aligned}
A_{1} & =3 \sum^{\prime}\left(\kappa_{i j \alpha}+2 \kappa_{i, j \alpha}\right)\left(\kappa_{\alpha s t}+2 \kappa_{\alpha s, t}\right) a_{i j} a_{s t} m_{\alpha \alpha} \\
& +6 \sum^{\prime}\left(\kappa_{\alpha, j k}-\kappa_{\alpha, j, k}\right)\left(\kappa_{\alpha s t}+2 \kappa_{\alpha s, t}\right) a_{j s} a_{k t} m_{\alpha \alpha} \\
& -6 \sum^{\prime}\left(\kappa_{\alpha, \alpha, k, r}+\kappa_{\alpha, \alpha, k r}\right) a_{k r} m_{\alpha \alpha} .
\end{aligned}
$$

Iremos apresentar a derivação em detalhes apenas para o segundo termos de $A_{1}$, já que outros termos são obtidos de forma semelhante. Assim,

$$
6 \sum^{\prime}\left(\kappa_{\alpha, j k}-\kappa_{\alpha, j, k}\right)\left(\kappa_{\alpha s t}+2 \kappa_{\alpha s, t}\right) a_{j s} a_{k t} m_{\alpha \alpha}
$$

é calculado da forma

$$
\begin{aligned}
6 \sum^{\prime} & {\left[\left\{2 a_{2}(\alpha)-\frac{3\left(2+\alpha^{2}\right)}{\alpha^{3}}\right\} \sum_{l=1}^{n} x_{l j} x_{l k}\right]\left[-\frac{2+\alpha^{2}}{\alpha^{3}} \sum_{m=1}^{n} x_{m s} x_{m t}\right] a_{j s} a_{k t} m_{\alpha \alpha} } \\
& =-\frac{3\left(2+\alpha^{2}\right)}{n \alpha}\left\{2 a_{2}(\alpha)-\frac{3\left(2+\alpha^{2}\right)}{\alpha^{3}}\right\} \sum^{\prime} \sum_{l, m=1}^{n} x_{l j} x_{l k} x_{m s} x_{m t} a_{j s} a_{k t} \\
& =-\frac{48\left(2+\alpha^{2}\right)}{n \alpha a_{1}(\alpha)^{2}}\left\{2 a_{2}(\alpha)-\frac{3\left(2+\alpha^{2}\right)}{\alpha^{3}}\right\} \operatorname{tr}(\boldsymbol{Z} \boldsymbol{Z}),
\end{aligned}
$$

mas como $\operatorname{tr}(\boldsymbol{Z} \boldsymbol{Z})=\operatorname{tr}(\boldsymbol{Z})=p$, temos que

$$
6 \sum^{\prime}\left(\kappa_{\alpha, j k}-\kappa_{\alpha, j, k}\right)\left(\kappa_{\alpha s t}+2 \kappa_{\alpha s, t}\right) a_{j s} a_{k t} m_{\alpha \alpha}=-\frac{48\left(2+\alpha^{2}\right)}{n \alpha a_{1}(\alpha)^{2}}\left\{2 a_{2}(\alpha)-\frac{3\left(2+\alpha^{2}\right)}{\alpha^{3}}\right\} p .
$$

Os termos restantes de $A_{1}$ são obtidos da mesma maneira. Dessa forma, após algumas manipulações algébricas, tem-se que

$$
A_{1}=\frac{24 p}{n a_{1}(\alpha)^{2} \alpha^{4}}\left\{\left(2+\alpha^{2}\right)^{2}(p+6)-\alpha^{2}\left[4\left(2+\alpha^{2}\right) a_{2}(\alpha) \alpha+\left(4+5 \alpha^{2}\right) a_{1}(\alpha)\right]\right\} .
$$

A obtenção de $A_{2}$ segue de forma análoga. Como alguns cumulantes são nulos, temos que

$$
A_{2}=6 \sum^{\prime}\left(\kappa_{i j \alpha}+2 \kappa_{i, j \alpha}\right) \kappa_{\alpha, \alpha, \alpha} a_{i j} m_{\alpha \alpha}^{2}+3 \kappa_{\alpha, \alpha, \alpha, \alpha} m_{\alpha \alpha}^{2}
$$

Após algumas manipulações algébricas, tem-se que

$$
A_{2}=\frac{12}{n}\left\{3-\frac{4\left(2+\alpha^{2}\right)}{a_{1}(\alpha) \alpha^{2}} p\right\} .
$$

Finalmente,

$$
A_{3}=5 \kappa_{\alpha, \alpha, \alpha}^{2} m_{\alpha \alpha}^{3}=5\left(\frac{8 n}{\alpha^{3}}\right)^{2}\left(\frac{\alpha^{2}}{2 n}\right)^{3}=\frac{40}{n}
$$


C.3 Obtenção de $A_{1}, A_{2}$ e $A_{3}$ para testar $\mathcal{H}_{0}: \boldsymbol{\beta}=\boldsymbol{\beta}^{(0)}$

Para obtermos $A_{1}, A_{2}$ e $A_{3}$, precisamos considerar as matrizes

$$
\begin{aligned}
& \boldsymbol{K}(\boldsymbol{\theta})=\left[\begin{array}{cc}
\boldsymbol{K}_{\boldsymbol{\beta}} & \mathbf{0} \\
\mathbf{0} & \kappa_{\alpha, \alpha}
\end{array}\right], \quad \boldsymbol{K}(\boldsymbol{\theta})^{-1}=\left[\begin{array}{cc}
\boldsymbol{K}_{\boldsymbol{\beta}}^{-1} & \mathbf{0} \\
\mathbf{0} & \kappa_{\alpha, \alpha}^{-1}
\end{array}\right], \\
& \boldsymbol{A}=\left[\begin{array}{cc}
\{\mathbf{0}\}_{p} & \mathbf{0} \\
\mathbf{0} & \kappa_{\alpha, \alpha}^{-1}
\end{array}\right] \quad \text { e } \quad \boldsymbol{M}=\boldsymbol{K}_{\boldsymbol{\beta}}^{-1}-\boldsymbol{A}=\left[\begin{array}{cc}
\boldsymbol{K}_{\boldsymbol{\beta}}^{-1} & \mathbf{0} \\
\mathbf{0} & 0
\end{array}\right]
\end{aligned}
$$

em que $\{\boldsymbol{0}\}_{p}$ representa uma matriz de zeros de dimensão $p \times p$. Vamos utilizar a seguinte notação, similarmente ao que já foi definido anteriormente: $m_{r \alpha}$ e $a_{r \alpha}$ representam os $(r, p+1)$-ésimos elementos de $\boldsymbol{M}$ e $\boldsymbol{A}$, respectivamente, e $m_{\alpha \alpha}$ e $a_{\alpha \alpha}$ os $(p+1, p+1)$-ésimos elementos das matrizes $\boldsymbol{M}$ e $\boldsymbol{A}$, respectivamente. Note que, $m_{r \alpha}=m_{\alpha s}=m_{\alpha \alpha}=0$ e $a_{r s}=a_{r \alpha}=a_{\alpha r}=0(r=1,2, \ldots, p)$. Ainda, $a_{\alpha \alpha}=\kappa_{\alpha, \alpha}^{-1}=\alpha^{2} / 2 n$.

Para testar a hipótese $\mathcal{H}_{0}: \boldsymbol{\beta}=\boldsymbol{\beta}^{(0)}$ contra $\mathcal{H}_{1}: \boldsymbol{\beta} \neq \boldsymbol{\beta}^{(0)}$, podemos escrever $A_{1}$ e $A_{2}$, dados, respectivamente, em (3.3) e (3.4), da forma

$$
\begin{aligned}
A_{1} & =3 \sum^{\prime}\left(\kappa_{\alpha \alpha k}+2 \kappa_{\alpha, \alpha k}\right)\left(\kappa_{r \alpha \alpha}+2 \kappa_{r \alpha, \alpha}\right) a_{\alpha \alpha}^{2} m_{k r} \\
& -6 \sum^{\prime}\left(\kappa_{\alpha \alpha \alpha}+2 \kappa_{\alpha, \alpha \alpha}\right) \kappa_{\alpha, s, t} a_{\alpha \alpha}^{2} m_{s t} \\
& +6 \sum^{\prime}\left(\kappa_{i, \alpha \alpha}-\kappa_{i, \alpha, \alpha}\right)\left(\kappa_{r \alpha \alpha}+2 \kappa_{r \alpha, \alpha}\right) a_{\alpha \alpha}^{2} m_{i r} \\
& -6 \sum^{\prime}\left(\kappa_{i, j, \alpha, \alpha}+\kappa_{i, j, \alpha, \alpha}\right) a_{\alpha \alpha} m_{i j}
\end{aligned}
$$

e

$$
\begin{aligned}
A_{2} & =-3 \sum^{\prime} \kappa_{i, j, \alpha} \kappa_{\alpha, s, t} a_{\alpha \alpha} m_{i j} m_{s t}+6 \sum^{\prime}\left(\kappa_{\alpha \alpha k}+2 \kappa_{\alpha, \alpha k}\right) \kappa_{r, s, t} a_{\alpha \alpha} m_{k r} m_{s t} \\
& -6 \sum^{\prime} \kappa_{i, j, \alpha} \kappa_{r, s, \alpha} a_{\alpha \alpha} m_{i r} m_{j s}+3 \sum^{\prime} \kappa_{i, j, k, r} m_{i j} m_{k r} .
\end{aligned}
$$

Ainda, $A_{3}$ fica da mesma forma que em (3.5). Vamos apresentar a derivação em detalhes apenas do segundo termo de $A_{1}$, pois os outros são obtidos de forma semelhante. Assim, temos que

$$
-6 \sum^{\prime}\left(\kappa_{\alpha \alpha \alpha}+2 \kappa_{\alpha, \alpha \alpha}\right) \kappa_{\alpha, s, t} a_{\alpha \alpha}^{2} m_{s t}
$$

fica dado por

$$
\begin{gathered}
-6 \sum^{\prime}\left[\frac{10 n}{\alpha^{3}}-\frac{12 n}{\alpha^{3}}\right]\left[\tilde{s}_{3}(\alpha) \sum_{l=1}^{n} x_{l s} x_{l t}\right] a_{\alpha \alpha}^{2} m_{s t} \\
=\frac{12 \tilde{s}_{3}(\alpha) \alpha}{n a_{1}(\alpha)} \operatorname{tr}(\boldsymbol{Z})=\frac{12 \tilde{s}_{3}(\alpha) \alpha}{n a_{1}(\alpha)} p .
\end{gathered}
$$

Logo, após algumas manipulações algébricas, tem-se que

$$
A_{1}=\frac{12 p}{n a_{1}(\alpha)}\left\{\tilde{s}_{3}(\alpha) \alpha-\tilde{s}_{4}(\alpha) \alpha^{2}\right\} .
$$


Da mesma forma, iremos desenvolver apenas o último termo de $A_{2}$, isto é,

$$
\begin{aligned}
3 \sum^{\prime} \kappa_{i, j, k, r} m_{i j} m_{k r} & =3 \sum^{\prime}\left[\tilde{s}_{2}(\alpha) \sum_{l=1}^{n} x_{l i} x_{l j} x_{l k} x_{l r}\right] m_{i j} m_{k r} \\
& =\frac{48 \tilde{s}_{2}(\alpha)}{a_{1}(\alpha)^{2}} \sum_{l=1}^{n} z_{l l}^{2}=\frac{48 \tilde{s}_{2}(\alpha)}{a_{1}(\alpha)^{2}} \operatorname{tr}\left(\boldsymbol{Z}_{d}^{(2)}\right),
\end{aligned}
$$

em que a notação utilizada segue da Seção 3.2. Logo, após algumas manipulações algébricas, temos que

$$
A_{2}=-\frac{24 \tilde{s}_{3}(\alpha)^{2} \alpha^{2}}{n a_{1}(\alpha)^{2}} p(p+2)+\frac{48 \tilde{s}_{2}(\alpha)}{a_{1}(\alpha)^{2}} \operatorname{tr}\left(\boldsymbol{Z}_{d}^{(2)}\right) .
$$

Finalmente, de (3.5) vem

$$
A_{3}=0,
$$

pois os cumulantes envolvidos nesta expressão são nulos. 


\section{Apêndice D}

\section{Modelo Birnbaum-Saunders Reparametrizado}

Neste apêndice, obteremos as derivadas de primeira e segunda ordens, a matriz de informação observada e a matriz de informação esperada para o modelo de regressão Birnbaum-Saunders reparametrizado definido na Seção 4.7.2. A função de log-verossimilhança é da forma

$$
l^{*}(\boldsymbol{\psi})=-\frac{n}{2} \log (8 \pi)+\sum_{i=1}^{n} \log \left(\xi_{i 1}^{*}\right)-\frac{1}{2} \sum_{i=1}^{n} \xi_{i 2}^{* 2},
$$

em que $\boldsymbol{\psi}=\left(\alpha, \gamma, \beta_{r}\right)$,

$$
\xi_{i 1}^{*}=\xi_{i 1}^{*}(\boldsymbol{\psi})=\frac{2}{\alpha} \cosh \left(\frac{y_{i}-\mu_{i}^{*}}{2}\right), \quad \xi_{i 2}^{*}=\xi_{i 2}^{*}(\boldsymbol{\psi})=\frac{2}{\alpha} \operatorname{senh}\left(\frac{y_{i}-\mu_{i}^{*}}{2}\right)
$$

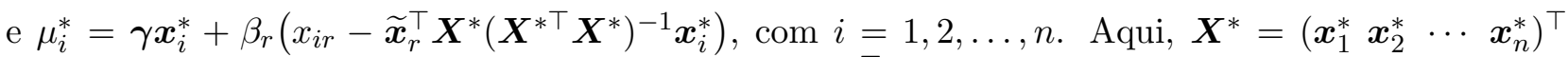
denota a matriz modelo $\boldsymbol{X}$ sem a $r$-ésima coluna, $\boldsymbol{x}_{i}^{* \top}=\left(x_{i 1}, \ldots, x_{i(p-1)}, x_{i(p+1)}, \ldots, x_{i p}\right)$ e $\widetilde{\boldsymbol{x}}_{r}=$ $\left(x_{1 r}, x_{2 r}, \ldots, x_{n r}\right)^{\top}$ representa a $r$-ésima coluna da matriz $\boldsymbol{X}$.

As expressões para as derivadas da função de log-verossimilhança em relação aos componentes de $\boldsymbol{\psi}$ serão aprentadas a seguir, onde omitimos as passagens intermediárias, uma vez que algumas destas derivadas envolvem um trabalho algébrico bastante tedioso. Os índices $R$ e $S$ que aparecem referem-se às derivadas de $l^{*}$ com relação aos componentes de $\gamma$. O índide $r$ refere-se à derivada com relação ao parâmetro de interesse $\beta_{r}$, enquanto que o índice $\alpha$ refere-se a diferenciação com respeito ao parâmetro $\alpha$.

\section{Derivadas de Primeira Ordem}

$$
l_{r}^{*}=\frac{\partial l^{*}}{\partial \beta_{r}}=\frac{1}{2} \sum_{i=1}^{n} \bar{x}_{i r}\left\{\xi_{i 1}^{*} \xi_{i 2}^{*}-\xi_{i 2}^{*} / \xi_{i 1}^{*}\right\},
$$

em que $\bar{x}_{i r}=x_{i r}-\widetilde{\boldsymbol{x}}_{r}^{\top} \boldsymbol{X}^{*}\left(\boldsymbol{X}^{* \top} \boldsymbol{X}^{*}\right)^{-1} \boldsymbol{x}_{i}^{*}$,

$$
l_{\alpha}^{*}=\frac{\partial l^{*}}{\partial \alpha}=-\frac{n}{\alpha}+\frac{1}{\alpha} \sum_{i=1}^{n} \xi_{i 2}^{* 2} \quad \text { e } \quad l_{R}^{*}=\frac{\partial l^{*}}{\partial \gamma_{R}}=\frac{1}{2} \sum_{i=1}^{n} x_{i R}\left\{\xi_{i 1}^{*} \xi_{i 2}^{*}-\xi_{i 2}^{*} / \xi_{i 1}^{*}\right\} .
$$

Aqui, $x_{i R}$ representa o $i$-ésimo elemento referente à $R$-ésima coluna da matriz modelo $\boldsymbol{X}$, sendo que $R \neq r$. 


\section{Derivadas de Segunda Ordem}

$$
\begin{gathered}
l_{r r}^{*}=\frac{\partial^{2} l^{*}}{\partial \beta_{r}^{2}}=-\frac{1}{4} \sum_{i=1}^{n} \bar{x}_{i r}^{2}\left\{2 \xi_{i 2}^{* 2}+\frac{4}{\alpha^{2}}-1+\frac{\xi_{i 2}^{* 2}}{\xi_{i 1}^{* 2}}\right\} \\
l_{r \alpha}^{*}=\frac{\partial^{2} l^{*}}{\partial \beta_{r} \partial \alpha}=-\frac{1}{\alpha} \sum_{i=1}^{n} \bar{x}_{i r} \xi_{i 1}^{*} \xi_{i 2}^{*}, \quad l_{\alpha \alpha}^{*}=\frac{\partial^{2} l^{*}}{\partial \alpha^{2}}=\frac{n}{\alpha^{2}}-\frac{3}{\alpha^{2}} \sum_{i=1}^{n} \xi_{i 2}^{* 2}, \\
l_{R S}^{*}=\frac{\partial^{2} l^{*}}{\partial \gamma_{R} \partial \gamma_{S}}=-\frac{1}{4} \sum_{i=1}^{n} x_{i R} x_{i S}\left\{2 \xi_{i 2}^{* 2}+\frac{4}{\alpha^{2}}-1+\frac{\xi_{i 2}^{* 2}}{\xi_{i 1}^{* 2}}\right\}, \\
l_{R r}^{*}=\frac{\partial^{2} l^{*}}{\partial \gamma_{R} \partial \beta_{r}}=-\frac{1}{4} \sum_{i=1}^{n} x_{i R} \bar{x}_{i r}\left\{2 \xi_{i 2}^{* 2}+\frac{4}{\alpha^{2}}-1+\frac{\xi_{i 2}^{* 2}}{\xi_{i 1}^{* 2}}\right\}
\end{gathered}
$$

e

$$
l_{R \alpha}^{*}=\frac{\partial^{2} l^{*}}{\partial \gamma_{R} \partial \alpha}=-\frac{1}{\alpha} \sum_{i=1}^{n} x_{i R} \xi_{i 1}^{*} \xi_{i 2}^{*}
$$

\section{Matriz de Informação Observada}

A matriz de informação observada, $\boldsymbol{j}^{*}(\boldsymbol{\psi})=-\partial^{2} l^{*} / \partial \boldsymbol{\psi} \partial \boldsymbol{\psi}^{\top}$, para o modelo de regressão BirnbaumSaunders (reparametrizado) é da forma

$$
\boldsymbol{j}^{*}(\boldsymbol{\psi})=\boldsymbol{j}^{*}\left(\alpha, \boldsymbol{\gamma}, \beta_{r}\right)=\left[\begin{array}{ccc}
\nu^{*} & \boldsymbol{k}^{* \top} \boldsymbol{X}^{*} & \boldsymbol{k}^{* \top} \overline{\boldsymbol{x}}_{r} \\
\boldsymbol{X}^{* \top} \boldsymbol{k}^{*} & \boldsymbol{X}^{* \top} \boldsymbol{W}^{*} \boldsymbol{X}^{*} & \boldsymbol{X}^{* \top} \boldsymbol{W}^{*} \overline{\boldsymbol{x}}_{r} \\
\overline{\boldsymbol{x}}_{r}^{\top} \boldsymbol{k}^{*} & \overline{\boldsymbol{x}}_{r}^{\top} \boldsymbol{W}^{*} \boldsymbol{X}^{*} & \overline{\boldsymbol{x}}_{r}^{\top} \boldsymbol{W}^{*} \overline{\boldsymbol{x}}_{r}
\end{array}\right]
$$

em que $\overline{\boldsymbol{x}}_{r}^{\top}=\widetilde{\boldsymbol{x}}_{r}^{\top}\left(\boldsymbol{I}_{n}-\boldsymbol{X}^{*}\left(\boldsymbol{X}^{* \top} \boldsymbol{X}^{*}\right)^{-1} \boldsymbol{X}^{* \top}\right)=\left(\bar{x}_{1 r}, \bar{x}_{2 r}, \ldots, \bar{x}_{n r}\right)$. Adicionalmente, $\boldsymbol{W}^{*}=\boldsymbol{W}^{*}(\boldsymbol{\psi})=$ $\operatorname{diag}\left\{w_{1}^{*}, w_{2}^{*}, \ldots, w_{n}^{*}\right\} \operatorname{com} w_{i}^{*}=w_{i}^{*}(\boldsymbol{\psi})=-0.25 \operatorname{sech}^{2}\left[\left(y_{i}-\mu_{i}^{*}\right) / 2\right]+\alpha^{-2} \cosh \left(y_{i}-\mu_{i}^{*}\right), \boldsymbol{k}^{*}=\boldsymbol{k}^{*}(\boldsymbol{\psi})=$ $\left(k_{1}^{*}, k_{2}^{*}, \ldots, k_{n}^{*}\right)^{\top} \operatorname{com} k_{i}^{*}=k_{i}^{*}(\boldsymbol{\psi})=2 \alpha^{-3} \operatorname{senh}\left(y_{i}-\mu_{i}^{*}\right)$ e $\nu^{*}=\nu^{*}(\boldsymbol{\psi})=n \alpha^{-2}\left\{3 \bar{\xi}_{2}^{*}-1\right\}$ com $\bar{\xi}_{2}^{*}=$ $\bar{\xi}_{2}^{*}(\boldsymbol{\psi})=\sum_{i=1}^{n} \xi_{i 2}^{* 2} / n$.

Seja $\boldsymbol{\lambda}=(\alpha, \gamma)$. Assim, por exemplo, de $\boldsymbol{j}^{*}\left(\alpha, \boldsymbol{\gamma}, \beta_{r}\right)$ temos que

$$
\boldsymbol{j}_{\boldsymbol{\lambda} \boldsymbol{\lambda}}^{*}(\psi)=\left[\begin{array}{cc}
\nu^{*} & \boldsymbol{k}^{* \top} \boldsymbol{X}^{*} \\
\boldsymbol{X}^{* \top} \boldsymbol{k}^{*} & \boldsymbol{X}^{* \top} \boldsymbol{W}^{*} \boldsymbol{X}^{*}
\end{array}\right] .
$$

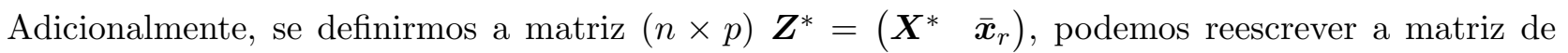
informação observada $\boldsymbol{j}^{*}(\boldsymbol{\psi})$ da forma

$$
\boldsymbol{j}^{*}(\boldsymbol{\psi})=\left[\begin{array}{cc}
\nu^{*} & \boldsymbol{k}^{* \top} \boldsymbol{Z}^{*} \\
\boldsymbol{Z}^{* \top} \boldsymbol{k}^{*} & \boldsymbol{Z}^{* \top} \boldsymbol{W}^{*} \boldsymbol{Z}^{*}
\end{array}\right] .
$$

Os determinantes de $\boldsymbol{j}_{\boldsymbol{\lambda} \boldsymbol{\lambda}}^{*}(\boldsymbol{\psi})$ e $\boldsymbol{j}^{*}(\boldsymbol{\psi})$ são obtidos como

$$
\left|\boldsymbol{j}_{\boldsymbol{\lambda} \boldsymbol{\lambda}}^{*}(\boldsymbol{\psi})\right|=\nu^{*}\left|\boldsymbol{X}^{* \top} \boldsymbol{H}^{*} \boldsymbol{X}^{*}\right| \quad \text { e } \quad\left|\boldsymbol{j}^{*}(\boldsymbol{\psi})\right|=\nu^{*}\left|\boldsymbol{Z}^{* \top} \boldsymbol{H}^{*} \boldsymbol{Z}^{*}\right|,
$$

em que $\boldsymbol{H}^{*}=\boldsymbol{H}^{*}(\boldsymbol{\psi})=\boldsymbol{W}^{*}-\nu^{*-1} \boldsymbol{k}^{*} \boldsymbol{k}^{* \top}$. 


\section{Matriz de Informação Esperada}

Tomando o valor esperado das derivadas de segunda ordens, temos que

$$
\begin{gathered}
\mathbb{E}\left(l_{r r}^{*}\right)=-\frac{h(\alpha)}{4} \sum_{i=1}^{n} \bar{x}_{i r}^{2}, \quad \mathbb{E}\left(l_{\alpha \alpha}^{*}\right)=-\frac{2 n}{\alpha^{2}}, \quad \mathbb{E}\left(U_{r \alpha}^{*}\right)=0, \quad \mathbb{E}\left(l_{R S}^{*}\right)=-\frac{h(\alpha)}{4} \sum_{i=1}^{n} x_{i R} x_{i S}, \\
\mathbb{E}\left(l_{R \alpha}^{*}\right)=0 \quad \text { e } \quad \mathbb{E}\left(l_{R r}^{*}\right)=-\frac{h(\alpha)}{4} \sum_{i=1}^{n} x_{i R} \bar{x}_{i r},
\end{gathered}
$$

em que

$$
h(\alpha)=2+\frac{4}{\alpha^{2}}-\frac{\sqrt{2 \pi}}{\alpha}\left\{1-\operatorname{erf}\left(\frac{\sqrt{2}}{\alpha}\right)\right\} \exp \left(\frac{2}{\alpha^{2}}\right) .
$$

Aqui, $\operatorname{erf}(\cdot)$ representa a error function, que é da forma

$$
\operatorname{erf}(x)=\frac{2}{\sqrt{\pi}} \int_{0}^{x} \mathrm{e}^{-t^{2}} \mathrm{~d} t
$$

Observe que

$$
\mathbb{E}\left(l_{R r}^{*}\right)=-\frac{h(\alpha)}{4} \sum_{i=1}^{n} x_{i R} \bar{x}_{i r}
$$

Em notação matricial, temos que

$$
\mathbb{E}\left(\boldsymbol{l}_{\boldsymbol{\gamma} \beta_{r}}^{*}\right)=-\frac{h(\alpha)}{4} \boldsymbol{X}^{* \top} \overline{\boldsymbol{x}}_{r}
$$

Entretanto, note que

$$
\boldsymbol{X}^{* \top} \overline{\boldsymbol{x}}_{r}=\boldsymbol{X}^{* \top}\left(\boldsymbol{I}_{n}-\boldsymbol{X}^{*}\left(\boldsymbol{X}^{* \top} \boldsymbol{X}^{*}\right)^{-1} \boldsymbol{X}^{* \top}\right) \widetilde{\boldsymbol{x}}_{r}=\left(\boldsymbol{X}^{* \top}-\boldsymbol{X}^{* \top}\right) \widetilde{\boldsymbol{x}}_{r}=\{\mathbf{0}\}_{(p-1) \times 1} .
$$

Portanto, $\beta_{r}$ é ortogonal a $\gamma$. Além, claro, de $\alpha$. Deste modo, a matriz de informação esperada $\boldsymbol{i}^{*}(\boldsymbol{\psi})=-\mathbb{E}\left(\partial^{2} l^{*} / \partial \boldsymbol{\psi} \partial \boldsymbol{\psi}^{\top}\right)$ é da forma

$$
\boldsymbol{i}^{*}\left(\alpha, \boldsymbol{\gamma}, \beta_{r}\right)=\left[\begin{array}{ccc}
i_{\alpha \alpha} & \boldsymbol{i}_{\alpha \boldsymbol{\gamma}} & i_{\alpha \beta_{r}} \\
\boldsymbol{i}_{\boldsymbol{\gamma} \alpha} & \boldsymbol{i}_{\boldsymbol{\gamma} \boldsymbol{\gamma}} & \boldsymbol{i}_{\boldsymbol{\gamma}_{\beta_{r}}} \\
i_{\beta_{r} \alpha} & \boldsymbol{i}_{\beta_{r} \boldsymbol{\gamma}} & i_{\beta_{r} \beta_{r}}
\end{array}\right]=\left[\begin{array}{ccc}
2 n / \alpha^{2} & \{\mathbf{0}\}_{1 \times(p-1)} & 0 \\
\{\mathbf{0}\}_{(p-1) \times 1} & h(\alpha) \boldsymbol{X}^{* \top} \boldsymbol{X}^{*} / 4 & \{\mathbf{0}\}_{(p-1) \times 1} \\
0 & \{\mathbf{0}\}_{1 \times(p-1)} & h(\alpha) \overline{\boldsymbol{x}}_{r}^{\top} \overline{\boldsymbol{x}}_{r} / 4
\end{array}\right] .
$$

Note que definindo $\widetilde{\boldsymbol{Z}}^{*}$ como uma matriz $(n+1) \times(p+1)$ da forma

$$
\widetilde{\boldsymbol{Z}}^{*}=\left[\begin{array}{cc}
1 & \{\mathbf{0}\}_{1 \times p} \\
\{\mathbf{0}\}_{n \times 1} & \boldsymbol{Z}^{*}
\end{array}\right]
$$

e considerando $\widetilde{\boldsymbol{D}}^{*}=\operatorname{diag}\left\{2 n / \alpha^{2}, h(\alpha) \boldsymbol{I}_{n} / 4\right\}$, pode-se escrever a matriz de informação total de Fisher para $\psi$ como

$$
\boldsymbol{i}^{*}(\boldsymbol{\psi})=\widetilde{\boldsymbol{Z}}^{* \top} \widetilde{\boldsymbol{D}}^{*} \widetilde{\boldsymbol{Z}}^{*}
$$




\section{Apêndice E}

\section{Estatística Gradiente $-S_{T}$}

Neste apêndice apresentamos, com mais detalhes, a obtenção da função geradora de momentos da estatística gradiente, $S_{T}$, até ordem $n^{-1 / 2}$ e sob uma hipótese alternativa local convergindo para a hipótese nula a uma taxa de convergência de $n^{-1 / 2}$, em que $n$ é o tamanho da amostra.

Utilizando a notação do Capítulo 5 , a estatística gradiente pode ser escrita, até ordem $O_{p}\left(n^{-1 / 2}\right)$, na forma

$$
\begin{aligned}
S_{T}= & -(\boldsymbol{Z} \boldsymbol{y}+\boldsymbol{\xi})^{\top} \boldsymbol{Y}(\boldsymbol{Z} \boldsymbol{y}+\boldsymbol{\xi})-\frac{1}{2 \sqrt{n}} \boldsymbol{K}_{\ldots} \circ(\boldsymbol{Z} \boldsymbol{y}+\boldsymbol{\xi}) \circ \boldsymbol{Y}^{-1} \boldsymbol{y} \circ \boldsymbol{Y}^{-1} \boldsymbol{y} \\
& -\frac{1}{2 \sqrt{n}} \boldsymbol{K}_{\ldots} \circ(\boldsymbol{Z} \boldsymbol{y}+\boldsymbol{\xi}) \circ\left(\boldsymbol{Z}_{0} \boldsymbol{y}-\boldsymbol{\xi}\right) \circ\left(\boldsymbol{Z}_{0} \boldsymbol{y}-\boldsymbol{\xi}\right)+O_{p}\left(n^{-1}\right) .
\end{aligned}
$$

A função geradora de momentos de $S_{T}, M(t)$, é dada por

$$
\begin{aligned}
M(t) & =\mathbb{E}\left(e^{t S_{T}}\right) \\
& =\int \cdots \int e^{t S_{T}} f_{1} \mathrm{~d} \boldsymbol{y} \mathrm{d} \boldsymbol{Y}+O\left(n^{-1}\right) \\
& =\int \cdots \int\left[\int \cdots \int e^{t S_{T}} f_{1} \mathrm{~d} \boldsymbol{Y}\right] \mathrm{d} \boldsymbol{y}+O\left(n^{-1}\right) \\
& =\int \cdots \int A(\boldsymbol{y}) \mathrm{d} \boldsymbol{y}+O\left(n^{-1}\right),
\end{aligned}
$$

em que

$$
A(\boldsymbol{y})=\int \cdots \int e^{t S_{T}} f_{1} \mathrm{~d} \boldsymbol{Y}
$$

Note que

$$
\begin{aligned}
A(\boldsymbol{y})= & \int \cdots \int e^{t S_{T}} f_{0}\left[1+\frac{1}{6 \sqrt{n}}\left(\boldsymbol{K}_{., ., .} \circ \boldsymbol{K}^{-1} \boldsymbol{y} \circ \boldsymbol{K}^{-1} \boldsymbol{y} \circ \boldsymbol{K}^{-1} \boldsymbol{y}-3 \boldsymbol{K}_{., ., .} \circ \boldsymbol{K}^{-1} \circ \boldsymbol{K}^{-1} \boldsymbol{y}\right)\right. \\
& \left.-\frac{1}{\sqrt{n}} \boldsymbol{K}_{., . .} \circ \boldsymbol{K}^{-1} \boldsymbol{y} \circ \boldsymbol{D}\right] \mathrm{d} \boldsymbol{Y} \\
= & (2 \pi)^{-p / 2}|\boldsymbol{K}|^{-1 / 2} \exp \left\{-\frac{1}{2} \boldsymbol{y}^{\top} \boldsymbol{K}^{-1} \boldsymbol{y}\right\} \mathcal{I},
\end{aligned}
$$


em que

$$
\begin{aligned}
\mathcal{I}= & \int \cdots \int e^{t S_{T}}\left[1+\frac{1}{6 \sqrt{n}}\left(\boldsymbol{K}_{., .,} \circ \boldsymbol{K}^{-1} \boldsymbol{y} \circ \boldsymbol{K}^{-1} \boldsymbol{y} \circ \boldsymbol{K}^{-1} \boldsymbol{y}-3 \boldsymbol{K}_{., ., .} \circ \boldsymbol{K}^{-1} \circ \boldsymbol{K}^{-1} \boldsymbol{y}\right)\right. \\
& \left.-\frac{1}{\sqrt{n}} \boldsymbol{K}_{., . .} \circ \boldsymbol{K}^{-1} \boldsymbol{y} \circ \boldsymbol{D}\right] \prod_{r, s=1}^{p} \delta\left(y_{r s}-\kappa_{r s}\right) \mathrm{d} \boldsymbol{Y} .
\end{aligned}
$$

Expandindo $e^{t S_{T}}$ em série de Taylor, com $S_{T}$ dado em (E.1), temos que

$$
\begin{aligned}
e^{t S_{T}}= & \exp \left\{-t(\boldsymbol{Z} \boldsymbol{y}+\boldsymbol{\xi})^{\top} \boldsymbol{Y}(\boldsymbol{Z} \boldsymbol{y}+\boldsymbol{\xi})\right\} \\
& {\left[1-\frac{t}{2 \sqrt{n}}\left[\boldsymbol{K} \ldots \circ(\boldsymbol{Z} \boldsymbol{y}+\boldsymbol{\xi})\left(\circ \boldsymbol{Y}^{-1} \boldsymbol{y}\right)^{2}+\boldsymbol{K}_{\ldots} \circ(\boldsymbol{Z} \boldsymbol{y}+\boldsymbol{\xi})\left(\circ\left(\boldsymbol{Z}_{0} \boldsymbol{y}-\boldsymbol{\xi}\right)\right)^{2}\right]\right]+O_{p}\left(n^{-1}\right) . }
\end{aligned}
$$

Inserindo esta última expressão em $\mathcal{I}$, vem

$$
\begin{aligned}
\mathcal{I}= & \int \cdots \int \exp \left\{-t(\boldsymbol{Z} \boldsymbol{y}+\boldsymbol{\xi})^{\top} \boldsymbol{Y}(\boldsymbol{Z} \boldsymbol{y}+\boldsymbol{\xi})\right\} \\
& {\left[1+\frac{1}{6 \sqrt{n}}\left(\boldsymbol{K}_{., ., .}\left(\circ \boldsymbol{K}^{-1} \boldsymbol{y}\right)^{3}-3 \boldsymbol{K}_{., ., .} \circ \boldsymbol{K}^{-1} \circ \boldsymbol{K}^{-1} \boldsymbol{y}\right)-\frac{1}{\sqrt{n}} \boldsymbol{K}_{., . .} \circ \boldsymbol{K}^{-1} \boldsymbol{y} \circ \boldsymbol{D}\right] \prod_{r, s=1}^{p} \delta\left(y_{r s}-\kappa_{r s}\right) \mathrm{d} \boldsymbol{Y} } \\
& -\frac{t}{2 \sqrt{n}} \int \cdots \int \exp \left\{-t(\boldsymbol{Z} \boldsymbol{y}+\boldsymbol{\xi})^{\top} \boldsymbol{Y}(\boldsymbol{Z} \boldsymbol{y}+\boldsymbol{\xi})\right\} \\
& {\left[\boldsymbol{K}_{\ldots .} \circ(\boldsymbol{Z} \boldsymbol{y}+\boldsymbol{\xi}) \circ \boldsymbol{Y}^{-1} \boldsymbol{y} \circ \boldsymbol{Y}^{-1} \boldsymbol{y}+\boldsymbol{K}_{\ldots} \circ(\boldsymbol{Z} \boldsymbol{y}+\boldsymbol{\xi}) \circ\left(\boldsymbol{Z}_{0} \boldsymbol{y}-\boldsymbol{\xi}\right) \circ\left(\boldsymbol{Z}_{0} \boldsymbol{y}-\boldsymbol{\xi}\right)\right] } \\
& \prod_{r, s=1}^{p} \delta\left(y_{r s}-\kappa_{r s}\right) \mathrm{d} \boldsymbol{Y}+O_{p}\left(n^{-1}\right) .
\end{aligned}
$$

Para resolver esta integral, vamos usar as seguintes propriedades da função delta de Dirac:

$$
\int h\left(\ldots, y_{r s}, \ldots\right) \delta\left(y_{r s}-\kappa_{r s}\right) \mathrm{d} y_{r s}=h\left(\ldots, \kappa_{r s}, \ldots\right)
$$

$\mathrm{e}$

$$
\int h\left(\ldots, y_{r s}, \ldots\right) \delta^{\prime}\left(y_{r s}-\kappa_{r s}\right) \mathrm{d} y_{r s}=-\left[\frac{\partial}{\partial y_{r s}} h\left(\ldots, y_{r s}, \ldots\right)\right]_{y_{r s}=\kappa_{r s}}
$$

em que $h(\cdot)$ é uma função diferenciável. Utilizando estas relações, após alguma álgebra, obtemos

$$
\begin{aligned}
\mathcal{I}= & \exp \left\{t\left(\boldsymbol{M} \boldsymbol{y}-\boldsymbol{\epsilon}^{*}\right)^{\top} \boldsymbol{K}\left(\boldsymbol{M} \boldsymbol{y}-\boldsymbol{\epsilon}^{*}\right)\right\} \\
& {\left[1+\frac{1}{6 \sqrt{n}}\left(\boldsymbol{K}_{., ., .} \circ \boldsymbol{K}^{-1} \boldsymbol{y} \circ \boldsymbol{K}^{-1} \boldsymbol{y} \circ \boldsymbol{K}^{-1} \boldsymbol{y}-3 \boldsymbol{K}_{., ., .} \circ \boldsymbol{K}^{-1} \circ \boldsymbol{K}^{-1} \boldsymbol{y}\right)\right.} \\
& +\frac{t}{\sqrt{n}} \boldsymbol{K}_{., . .} \circ \boldsymbol{K}^{-1} \boldsymbol{y} \circ\left[\boldsymbol{K}^{-1} \boldsymbol{y} \boldsymbol{y}^{\top} \boldsymbol{K}^{-1}-\left(\boldsymbol{A} \boldsymbol{y}+\boldsymbol{\epsilon}^{*}\right)\left(\boldsymbol{A} \boldsymbol{y}+\boldsymbol{\epsilon}^{*}\right)^{\top}\right] \\
& \left.+\frac{t}{2 \sqrt{n}}\left[\boldsymbol{K}_{\ldots} \circ\left(\boldsymbol{M} \boldsymbol{y}-\boldsymbol{\epsilon}^{*}\right) \circ \boldsymbol{K}^{-1} \boldsymbol{y} \circ \boldsymbol{K}^{-1} \boldsymbol{y}+\boldsymbol{K}_{\ldots} \circ\left(\boldsymbol{M} \boldsymbol{y}-\boldsymbol{\epsilon}^{*}\right) \circ\left(\boldsymbol{A} \boldsymbol{y}+\boldsymbol{\epsilon}^{*}\right) \circ\left(\boldsymbol{A} \boldsymbol{y}+\boldsymbol{\epsilon}^{*}\right)\right]\right] \\
& +O_{p}\left(n^{-1}\right),
\end{aligned}
$$


em que

$$
\boldsymbol{M}=\boldsymbol{K}^{-1}-\boldsymbol{A}, \quad \boldsymbol{A}=\left[\begin{array}{cc}
\boldsymbol{K}_{11}^{-1} & \mathbf{0} \\
\mathbf{0} & \mathbf{0}
\end{array}\right] \quad \text { e } \quad \boldsymbol{\epsilon}^{*}=\left[\begin{array}{c}
\boldsymbol{K}_{11}^{-1} \boldsymbol{K}_{12} \\
-\boldsymbol{I}_{p-q}
\end{array}\right] \boldsymbol{\epsilon}
$$

Então, temos que

$$
A(\boldsymbol{y})=(2 \pi)^{-p / 2}|\boldsymbol{K}|^{-1 / 2} \exp \left\{-\frac{1}{2} \boldsymbol{y}^{\top} \boldsymbol{K}^{-1} \boldsymbol{y}\right\} \mathcal{I}+O_{p}\left(n^{-1}\right)
$$

Como

$$
M(t)=\int \cdots \int A(\boldsymbol{y}) \mathrm{d} \boldsymbol{y}+O\left(n^{-1}\right)
$$

isto é,

$$
M(t)=(2 \pi)^{-p / 2}|\boldsymbol{K}|^{-1 / 2} \int \cdots \int \exp \left\{-\frac{1}{2} \boldsymbol{y}^{\top} \boldsymbol{K}^{-1} \boldsymbol{y}\right\} \mathcal{I} \mathrm{d} \boldsymbol{y}+O\left(n^{-1}\right),
$$

devemos resolver esta última integral para obter $M(t)$ explicitamente. Utilizando a transformação de variável dada em (5.6), com $\boldsymbol{y}=\boldsymbol{\Sigma}^{1 / 2} \boldsymbol{z}-2 t \boldsymbol{\Sigma} \boldsymbol{M} \boldsymbol{K} \boldsymbol{\epsilon}^{*}, \boldsymbol{\Sigma}=\boldsymbol{\Sigma}^{1 / 2} \boldsymbol{\Sigma}^{1 / 2}$,

$$
\boldsymbol{\Sigma}=\frac{1}{1-2 t} \boldsymbol{K}-\frac{2 t}{1-2 t} \boldsymbol{K} \boldsymbol{A} \boldsymbol{K}, \quad \boldsymbol{\eta}=2 t \boldsymbol{\Sigma} \boldsymbol{M} \boldsymbol{K} \boldsymbol{\epsilon}^{*}=\frac{2 t}{1-2 t} \boldsymbol{K} \boldsymbol{\epsilon}^{*} \quad \text { e } \quad \boldsymbol{K}_{22.1}=\boldsymbol{K}_{22}-\boldsymbol{K}_{21} \boldsymbol{K}_{11}^{-1} \boldsymbol{K}_{12}
$$

a fgm de $S_{T}, M(t)$, pode ser escrita na forma

$$
\begin{aligned}
M(t)= & (2 \pi)^{-p / 2}(1-2 t)^{-\frac{1}{2}(p-q)} \exp \left\{\frac{t}{1-2 t} \boldsymbol{\epsilon}^{\top} \boldsymbol{K}_{22.1} \boldsymbol{\epsilon}\right\} \\
& \int \cdots \int \exp \left\{-\frac{1}{2} \boldsymbol{z}^{\top} \boldsymbol{z}\right\}\left[1+\frac{1}{6 \sqrt{n}} \mathcal{A}_{1}+\frac{t}{\sqrt{n}} \mathcal{A}_{2}+\frac{t}{2 \sqrt{n}} \mathcal{A}_{3}\right] \mathrm{d} \boldsymbol{z}+O\left(n^{-1}\right),
\end{aligned}
$$

em que

$$
\begin{aligned}
& \mathcal{A}_{1}=\boldsymbol{K}_{., ., .}\left(\circ \boldsymbol{K}^{-1} \boldsymbol{\Sigma}^{1 / 2} \boldsymbol{z}\right)^{3}-3\left(\frac{2 t}{1-2 t}\right) \boldsymbol{K}_{., ., .} \circ \boldsymbol{K}^{-1} \boldsymbol{\Sigma}^{1 / 2} \boldsymbol{z} \circ \boldsymbol{K}^{-1} \boldsymbol{\Sigma}^{1 / 2} \boldsymbol{z} \circ \boldsymbol{\epsilon}^{*} \\
& +3\left(\frac{2 t}{1-2 t}\right)^{2} \boldsymbol{K}_{., ., .} \circ \boldsymbol{\epsilon}^{*} \circ \boldsymbol{\epsilon}^{*} \circ \boldsymbol{K}^{-1} \boldsymbol{\Sigma}^{1 / 2} \boldsymbol{z}-\left(\frac{2 t}{1-2 t}\right)^{3} \boldsymbol{K}_{., ., .}\left(\circ \boldsymbol{\epsilon}^{*}\right)^{3} \\
& -3 \boldsymbol{K}_{., ., .} \circ \boldsymbol{K}^{-1} \circ \boldsymbol{K}^{-1} \boldsymbol{\Sigma}^{1 / 2} \boldsymbol{z}+3\left(\frac{2 t}{1-2 t}\right) \boldsymbol{K}_{., ., .} \circ \boldsymbol{K}^{-1} \circ \boldsymbol{\epsilon}^{*}, \\
& \mathcal{A}_{2}=\boldsymbol{K}_{., .}\left(\circ \boldsymbol{K}^{-1} \boldsymbol{\Sigma}^{1 / 2} \boldsymbol{z}\right)^{3}-2\left(\frac{2 t}{1-2 t}\right) \boldsymbol{K}_{., .} \circ \boldsymbol{K}^{-1} \boldsymbol{\Sigma}^{1 / 2} \boldsymbol{z} \circ \boldsymbol{K}^{-1} \boldsymbol{\Sigma}^{1 / 2} \boldsymbol{z} \circ \boldsymbol{\epsilon}^{*} \\
& +\left(\frac{2 t}{1-2 t}\right)^{2} \boldsymbol{K}_{., . .} \circ \boldsymbol{K}^{-1} \boldsymbol{\Sigma}^{1 / 2} \boldsymbol{z} \circ \boldsymbol{\epsilon}^{*} \circ \boldsymbol{\epsilon}^{*}-\left(\frac{2 t}{1-2 t}\right) \boldsymbol{K}_{., . .} \circ \boldsymbol{\epsilon}^{*} \circ \boldsymbol{K}^{-1} \boldsymbol{\Sigma}^{1 / 2} \boldsymbol{z} \circ \boldsymbol{K}^{-1} \boldsymbol{\Sigma}^{1 / 2} \boldsymbol{z} \\
& +2\left(\frac{2 t}{1-2 t}\right)^{2} \boldsymbol{K}_{., . .} \circ \boldsymbol{\epsilon}^{*} \circ \boldsymbol{\epsilon}^{*} \circ \boldsymbol{K}^{-1} \boldsymbol{\Sigma}^{1 / 2} \boldsymbol{z}-\left(\frac{2 t}{1-2 t}\right)^{3} \boldsymbol{K}_{., . .}\left(\circ \boldsymbol{\epsilon}^{*}\right)^{3} \\
& -\boldsymbol{K}_{., . .} \circ \boldsymbol{K}^{-1} \boldsymbol{\Sigma}^{1 / 2} \boldsymbol{z} \circ \boldsymbol{A} \boldsymbol{\Sigma}^{1 / 2} \boldsymbol{z} \circ \boldsymbol{A} \boldsymbol{\Sigma}^{1 / 2} \boldsymbol{z}-2 \boldsymbol{K}_{., . .} \circ \boldsymbol{K}^{-1} \boldsymbol{\Sigma}^{1 / 2} \boldsymbol{z} \circ \boldsymbol{A} \boldsymbol{\Sigma}^{1 / 2} \boldsymbol{z} \circ \boldsymbol{\epsilon}^{*} \\
& -\boldsymbol{K}_{., . .} \circ \boldsymbol{K}^{-1} \boldsymbol{\Sigma}^{1 / 2} \boldsymbol{z} \circ \boldsymbol{\epsilon}^{*} \circ \boldsymbol{\epsilon}^{*}+2\left(\frac{2 t}{1-2 t}\right) \boldsymbol{K}_{., . .} \circ \boldsymbol{\epsilon}^{*} \circ \boldsymbol{A} \boldsymbol{\Sigma}^{1 / 2} \boldsymbol{z} \circ \boldsymbol{A} \boldsymbol{\Sigma}^{1 / 2} \boldsymbol{z} \\
& +2\left(\frac{2 t}{1-2 t}\right) \boldsymbol{K}_{., . .} \circ \boldsymbol{\epsilon}^{*} \circ \boldsymbol{\epsilon}^{*} \circ \boldsymbol{A} \boldsymbol{\Sigma}^{1 / 2} \boldsymbol{z}+\left(\frac{2 t}{1-2 t}\right) \boldsymbol{K}_{., . .}\left(\circ \boldsymbol{\epsilon}^{*}\right)^{3}
\end{aligned}
$$


e

$$
\begin{aligned}
\mathcal{A}_{3}= & \boldsymbol{K}_{\ldots} \circ \boldsymbol{M} \boldsymbol{\Sigma}^{1 / 2} \boldsymbol{z} \circ \boldsymbol{K}^{-1} \boldsymbol{\Sigma}^{1 / 2} \boldsymbol{z} \circ \boldsymbol{K}^{-1} \boldsymbol{\Sigma}^{1 / 2} \boldsymbol{z}-2\left(\frac{2 t}{1-2 t}\right) \boldsymbol{K}_{\ldots} \circ \boldsymbol{M} \boldsymbol{\Sigma}^{1 / 2} \boldsymbol{z} \circ \boldsymbol{K}^{-1} \boldsymbol{\Sigma}^{1 / 2} \circ \boldsymbol{\epsilon}^{*} \\
& +2\left(\frac{2 t}{1-2 t}\right)^{2} \boldsymbol{K} \ldots \circ \boldsymbol{M} \boldsymbol{\Sigma}^{1 / 2} \boldsymbol{z} \circ \boldsymbol{\epsilon}^{*} \circ \boldsymbol{\epsilon}^{*}-\frac{1}{1-2 t} \boldsymbol{K}_{\ldots} \circ \boldsymbol{\epsilon}^{*} \circ \boldsymbol{K}^{-1} \boldsymbol{\Sigma}^{1 / 2} \boldsymbol{z} \circ \boldsymbol{K}^{-1} \boldsymbol{\Sigma}^{1 / 2} \boldsymbol{z} \\
& +\frac{4 t}{(1-2 t)^{2}} \boldsymbol{K} \ldots \circ \boldsymbol{\epsilon}^{*} \circ \boldsymbol{\epsilon}^{*} \circ \boldsymbol{K}^{-1} \boldsymbol{\Sigma}^{1 / 2} \boldsymbol{z}-\frac{4 t^{2}}{(1-2 t)^{3}} \boldsymbol{K}_{\ldots}\left(\circ \boldsymbol{\epsilon}^{*}\right)^{3} \\
& +\boldsymbol{K}_{\ldots} \circ \boldsymbol{M} \boldsymbol{\Sigma}^{1 / 2} \boldsymbol{z} \circ \boldsymbol{A} \boldsymbol{\Sigma}^{1 / 2} \boldsymbol{z} \circ \boldsymbol{A} \boldsymbol{\Sigma}^{1 / 2} \boldsymbol{z}+2 \boldsymbol{K}_{\ldots} \circ \boldsymbol{M} \boldsymbol{\Sigma}^{1 / 2} \boldsymbol{z} \circ \boldsymbol{A} \boldsymbol{\Sigma}^{1 / 2} \boldsymbol{z} \circ \boldsymbol{\epsilon}^{*} \\
& +\boldsymbol{K}_{\ldots} \circ \boldsymbol{\epsilon}^{*} \circ \boldsymbol{\epsilon}^{*} \circ \boldsymbol{M} \boldsymbol{\Sigma}^{1 / 2} \boldsymbol{z}-\frac{1}{(1-2 t)} \boldsymbol{K}_{\ldots} \circ \boldsymbol{M} \boldsymbol{\Sigma}^{1 / 2} \boldsymbol{z} \circ \boldsymbol{A} \boldsymbol{\Sigma}^{1 / 2} \boldsymbol{z} \circ \boldsymbol{\epsilon}^{*} \\
& -\frac{2}{(1-2 t)} \boldsymbol{K}_{\ldots} \circ \boldsymbol{\epsilon}^{*} \circ \boldsymbol{\epsilon}^{*} \circ \boldsymbol{A} \boldsymbol{\Sigma}^{1 / 2} \boldsymbol{z}-\frac{1}{(1-2 t)} \boldsymbol{K}_{\ldots}\left(\circ \boldsymbol{\epsilon}^{*}\right)^{3} .
\end{aligned}
$$

A fim de obter $M(t)$ explicitamente, utilizaremos os resultados (Gradshteyn \& Ryzhik, 2007):

1.

$$
\int \cdots \int \exp \left\{-\frac{1}{2} \boldsymbol{z}^{\top} \boldsymbol{z}\right\} \prod_{r=1}^{p} \mathrm{~d} z_{r}=(2 \pi)^{p / 2}
$$

2.

$$
\int \cdots \int z_{i} \exp \left\{-\frac{1}{2} \boldsymbol{z}^{\top} \boldsymbol{z}\right\} \prod_{r=1}^{p} \mathrm{~d} z_{r}=0, \quad i=1,2, \ldots, p
$$

3.

$$
\int \cdots \int z_{i} z_{j} \exp \left\{-\frac{1}{2} \boldsymbol{z}^{\top} \boldsymbol{z}\right\} \prod_{r=1}^{p} \mathrm{~d} z_{r}=(2 \pi)^{p / 2}, \quad i=j=1,2, \ldots, p
$$

4.

$$
\int \cdots \int z_{i} z_{j} z_{k} \exp \left\{-\frac{1}{2} \boldsymbol{z}^{\top} \boldsymbol{z}\right\} \prod_{r=1}^{p} \mathrm{~d} z_{r}=0, \quad i, j, k=1,2, \ldots, p .
$$

Observe que muitas integrais devem ser resolvidas para obter $M(t)$ explicitamente. Resolveremos aqui algumas delas, sendo as outras integrais resolvidas de forma similar. Começaremos resolvendo a integral

$$
\int \cdots \int\left(\boldsymbol{K}_{., . . .}\left(\circ \boldsymbol{K}^{-1} \boldsymbol{\Sigma}^{1 / 2} \boldsymbol{z}\right)^{3}\right) \exp \left\{-\frac{1}{2} \boldsymbol{z}^{\top} \boldsymbol{z}\right\} \prod_{r=1}^{p} \mathrm{~d} z_{r} .
$$

Note que

$$
\boldsymbol{K}_{., ., .}\left(\circ \boldsymbol{K}^{-1} \boldsymbol{\Sigma}^{1 / 2} \boldsymbol{z}\right)^{3}=\boldsymbol{K}_{., ., .} \circ \boldsymbol{K}^{-1} \boldsymbol{\Sigma}^{1 / 2} \boldsymbol{z} \circ \boldsymbol{K}^{-1} \boldsymbol{\Sigma}^{1 / 2} \boldsymbol{z} \circ \boldsymbol{K}^{-1} \boldsymbol{\Sigma}^{1 / 2} \boldsymbol{z}=\sum_{r, s, t=1}^{p} \kappa_{r, s, t} a_{r} a_{s} a_{t},
$$

em que, por exemplo, $a_{r}$ corresponde ao $r$-ésimo elemento do vetor $\boldsymbol{K}^{-1} \boldsymbol{\Sigma}^{1 / 2} \boldsymbol{z}$. Observe que $a_{r}=$ $\sum_{i=1}^{p} \psi^{r, i} z_{i}$, com $r=1,2, \ldots, p$, em que $\psi^{r, i}$ é o $(r, i)$-ésimo elemento da matriz $\boldsymbol{K}^{-1} \boldsymbol{\Sigma}^{1 / 2}$ e $z_{i}$ o 
$i$-ésimo elemento do vetor $\boldsymbol{z}$. Assim,

$$
\begin{aligned}
\int \cdots \int\left(\boldsymbol{K}_{., ., .}\left(\circ \boldsymbol{K}^{-1} \boldsymbol{\Sigma}^{1 / 2} \boldsymbol{z}\right)^{3}\right) \exp \left\{-\frac{1}{2} \boldsymbol{z}^{\top} \boldsymbol{z}\right\} \prod_{r=1}^{p} \mathrm{~d} z_{r} \\
\quad=\sum_{r, s, t=1}^{p} \kappa_{r, s, t} \sum_{i, j, k=1}^{p} \psi^{r, i} \psi^{s, j} \psi^{t, k} \int \cdots \int z_{i} z_{j} z_{k} \exp \left\{-\frac{1}{2} \boldsymbol{z}^{\top} \boldsymbol{z}\right\} \prod_{r=1}^{p} \mathrm{~d} z_{i} \\
=0 .
\end{aligned}
$$

Também,

$$
\begin{gathered}
\int \cdots \int\left(\boldsymbol{K}_{., .,} \circ \boldsymbol{K}^{-1} \boldsymbol{\Sigma}^{1 / 2} \boldsymbol{z} \circ \boldsymbol{K}^{-1} \boldsymbol{\Sigma}^{1 / 2} \boldsymbol{z} \circ \boldsymbol{\epsilon}^{*}\right) \exp \left\{-\frac{1}{2} \boldsymbol{z}^{\top} \boldsymbol{z}\right\} \prod_{r=1}^{p} \mathrm{~d} z_{r} \\
=(2 \pi)^{p / 2} \boldsymbol{K}_{., ., .} \circ \boldsymbol{K}^{-1} \boldsymbol{\Sigma} \boldsymbol{K}^{-1} \circ \boldsymbol{\epsilon}^{*} .
\end{gathered}
$$

Note que

$$
\begin{aligned}
\boldsymbol{K}^{-1} \boldsymbol{\Sigma} \boldsymbol{K}^{-1} & =\boldsymbol{K}^{-1}\left[\frac{1}{1-2 t} \boldsymbol{K}-\frac{2 t}{1-2 t} \boldsymbol{K} \boldsymbol{A} \boldsymbol{K}\right] \boldsymbol{K}^{-1} \\
& =\frac{1}{1-2 t}\left(\boldsymbol{K}^{-1}-2 t \boldsymbol{A}\right)
\end{aligned}
$$

Assim,

$$
(2 \pi)^{p / 2} \boldsymbol{K}_{., ., .} \circ \boldsymbol{K}^{-1} \boldsymbol{\Sigma} \boldsymbol{K}^{-1} \circ \boldsymbol{\epsilon}^{*}=\frac{(2 \pi)^{p / 2}}{1-2 t} \boldsymbol{K}_{., ., .} \circ\left(\boldsymbol{K}^{-1}-\boldsymbol{A}\right) \circ \boldsymbol{\epsilon}^{*} .
$$

Adicionalmente,

$$
\begin{array}{r}
\int \ldots \int\left(\boldsymbol{K}_{., ., .}\left(\circ \boldsymbol{\epsilon}^{*}\right)^{3}\right) \exp \left\{-\frac{1}{2} \boldsymbol{z}^{\top} \boldsymbol{z}\right\} \prod_{i=1}^{p} d z_{i} \\
=(2 \pi)^{p / 2} \boldsymbol{K}_{., ., .} \circ \boldsymbol{\epsilon}^{*} \circ \boldsymbol{\epsilon}^{*} \circ \boldsymbol{\epsilon}^{*}
\end{array}
$$

Também,

$$
\begin{aligned}
\int \cdots \int\left(\boldsymbol{K}_{., . .} \circ \boldsymbol{K}^{-1} \boldsymbol{\Sigma}^{1 / 2} \boldsymbol{z} \circ \boldsymbol{A} \boldsymbol{\Sigma}^{1 / 2} \boldsymbol{z} \circ \boldsymbol{\epsilon}^{*}\right) \exp \left\{-\frac{1}{2} \boldsymbol{z}^{\top} \boldsymbol{z}\right\} \prod_{r=1}^{p} \mathrm{~d} z_{r} \\
=\int \cdots \int\left[\sum_{r, s, t=1}^{p} \kappa_{r, s t} a_{r} b_{s} \epsilon_{t}^{*}\right] \exp \left\{-\frac{1}{2} \boldsymbol{z}^{\top} \boldsymbol{z}\right\} \prod_{r=1}^{p} \mathrm{~d} z_{r},
\end{aligned}
$$

em que $b_{s}$ corresponde ao $s$-ésimo elemento do vetor $\boldsymbol{A} \boldsymbol{\Sigma}^{1 / 2} \boldsymbol{z}$. Observe que $b_{s}=\sum_{j=1}^{p} \varphi^{s, j} z_{j}$, com $s=1,2, \ldots, p$, em que $\varphi^{s, j}$ é o $(s, j)$-ésimo elemento da matriz $\boldsymbol{A} \boldsymbol{\Sigma}^{1 / 2}$ e $z_{j}$ o $j$-ésimo elemento do 
vetor $\boldsymbol{z}$. Assim,

$$
\begin{gathered}
\int \ldots \int\left(\boldsymbol{K}_{., . .} \circ \boldsymbol{K}^{-1} \boldsymbol{\Sigma}^{1 / 2} \boldsymbol{z} \circ \boldsymbol{A} \boldsymbol{\Sigma}^{1 / 2} \boldsymbol{z} \circ \boldsymbol{\epsilon}^{*}\right) \exp \left\{-\frac{1}{2} \boldsymbol{z}^{\top} \boldsymbol{z}\right\} \prod_{r=1}^{p} \mathrm{~d} z_{r} \\
=(2 \pi)^{p / 2} \boldsymbol{K}_{., . .} \circ \boldsymbol{K}^{-1} \boldsymbol{\Sigma} \boldsymbol{A} \circ \boldsymbol{\epsilon}^{*} .
\end{gathered}
$$

Note que

$$
\begin{aligned}
\boldsymbol{K}^{-1} \boldsymbol{\Sigma} \boldsymbol{A} & =\boldsymbol{K}^{-1}\left[\frac{1}{1-2 t} \boldsymbol{K}-\frac{2 t}{1-2 t} \boldsymbol{K} \boldsymbol{A} \boldsymbol{K}\right] \boldsymbol{A} \\
& =\boldsymbol{A}
\end{aligned}
$$

uma vez que $\boldsymbol{A} \boldsymbol{K} \boldsymbol{A}=\boldsymbol{A}$. Então,

$$
(2 \pi)^{p / 2} \boldsymbol{K}_{., . .} \circ \boldsymbol{K}^{-1} \boldsymbol{\Sigma} \boldsymbol{A} \circ \boldsymbol{\epsilon}^{*}=(2 \pi)^{p / 2} \boldsymbol{K}_{., . .} \circ \boldsymbol{A} \circ \boldsymbol{\epsilon}^{*} .
$$

Também,

$$
\begin{aligned}
\int \cdots \int\left(\boldsymbol{K}_{\ldots} \circ \boldsymbol{M} \boldsymbol{\Sigma}^{1 / 2} \boldsymbol{z} \circ \boldsymbol{K}^{-1} \boldsymbol{\Sigma}^{1 / 2} \boldsymbol{z} \circ \boldsymbol{\epsilon}^{*}\right) \exp \left\{-\frac{1}{2} \boldsymbol{z}^{\top} \boldsymbol{z}\right\} \prod_{r=1}^{p} \mathrm{~d} z_{r} \\
=\int \cdots \int\left[\sum_{r, s, t=1}^{p} \kappa_{r s t} c_{r} a_{s} \epsilon_{t}^{*}\right] \exp \left\{-\frac{1}{2} \boldsymbol{z}^{\top} \boldsymbol{z}\right\} \prod_{r=1}^{p} \mathrm{~d} z_{r}
\end{aligned}
$$

em que $c_{r}$ corresponde ao $r$-ésimo elemento do vetor $\boldsymbol{M} \boldsymbol{\Sigma}^{1 / 2} \boldsymbol{z}$, isto é, $c_{r}=\sum_{i=1}^{p} \zeta^{r, i} z_{i}$, com $r=$ $1,2, \ldots, p$, em que $\zeta^{r, i}$ é o $(r, i)$-ésimo elemento da matriz $\boldsymbol{M} \boldsymbol{\Sigma}^{1 / 2}$ e $z_{i}$ o $i$-ésimo elemento do vetor z. Assim,

$$
\begin{gathered}
\int \ldots \int\left(\boldsymbol{K}_{\ldots} \circ \boldsymbol{M} \boldsymbol{\Sigma}^{1 / 2} \boldsymbol{z} \circ \boldsymbol{K}^{-1} \boldsymbol{\Sigma}^{1 / 2} \boldsymbol{z} \circ \boldsymbol{\epsilon}^{*}\right) \exp \left\{-\frac{1}{2} \boldsymbol{z}^{\top} \boldsymbol{z}\right\} \prod_{r=1}^{p} \mathrm{~d} z_{r} \\
=(2 \pi)^{p / 2} \boldsymbol{K}_{\ldots} \circ \boldsymbol{M} \boldsymbol{\Sigma} \boldsymbol{K}^{-1} \circ \boldsymbol{\epsilon}^{*} .
\end{gathered}
$$

Note que

$$
\begin{aligned}
\boldsymbol{M} \boldsymbol{\Sigma} \boldsymbol{K}^{-1} & =\boldsymbol{M}\left[\frac{1}{1-2 t} \boldsymbol{K}-\frac{2 t}{1-2 t} \boldsymbol{K} \boldsymbol{A} \boldsymbol{K}\right] \boldsymbol{K}^{-1} \\
& =\frac{1}{1-2 t} \boldsymbol{M} .
\end{aligned}
$$

Então,

$$
(2 \pi)^{p / 2} \boldsymbol{K}_{\ldots} \circ \boldsymbol{M} \boldsymbol{\Sigma} \boldsymbol{K}^{-1} \circ \boldsymbol{\epsilon}^{*}=\frac{(2 \pi)^{p / 2}}{1-2 t} \boldsymbol{K}_{\ldots} \circ \boldsymbol{M} \circ \boldsymbol{\epsilon}^{*}
$$


Finalmente,

$$
\begin{gathered}
\int \cdots \int\left(\boldsymbol{K}_{\ldots} \circ \boldsymbol{M} \boldsymbol{\Sigma}^{1 / 2} \boldsymbol{z} \circ \boldsymbol{A} \boldsymbol{\Sigma}^{1 / 2} \boldsymbol{z} \circ \boldsymbol{\epsilon}^{*}\right) \exp \left\{-\frac{1}{2} \boldsymbol{z}^{\top} \boldsymbol{z}\right\} \prod_{r=1}^{p} \mathrm{~d} z_{r} \\
=(2 \pi)^{p / 2} \boldsymbol{K}_{\ldots} \circ \boldsymbol{M} \boldsymbol{\Sigma} \boldsymbol{A} \circ \boldsymbol{\epsilon}^{*}
\end{gathered}
$$

Note que

$$
M \Sigma A=0 .
$$

Então,

$$
\int \ldots \int\left(\boldsymbol{K}_{\ldots} \circ \boldsymbol{M} \boldsymbol{\Sigma}^{1 / 2} \boldsymbol{z} \circ \boldsymbol{A} \boldsymbol{\Sigma}^{1 / 2} \boldsymbol{z} \circ \boldsymbol{\epsilon}^{*}\right) \exp \left\{-\frac{1}{2} \boldsymbol{z}^{\top} \boldsymbol{z}\right\} \prod_{r=1}^{p} \mathrm{~d} z_{r}=0 .
$$

Após resolver todas as integrais necessárias e utilizando as relações

$$
\boldsymbol{K}_{\ldots} \circ \boldsymbol{\epsilon}^{*} \circ \boldsymbol{\epsilon}^{*} \circ \boldsymbol{\epsilon}^{*}+\boldsymbol{K}_{., ., .} \circ \boldsymbol{\epsilon}^{*} \circ \boldsymbol{\epsilon}^{*} \circ \boldsymbol{\epsilon}^{*}+3 \boldsymbol{K}_{., . .} \circ \boldsymbol{\epsilon}^{*} \circ \boldsymbol{\epsilon}^{*} \circ \boldsymbol{\epsilon}^{*}=0
$$

$\mathrm{e}$

$$
\boldsymbol{K}_{\ldots} \circ \boldsymbol{K}^{-1} \circ \boldsymbol{\epsilon}^{*}+\boldsymbol{K}_{., ., .} \circ \boldsymbol{K}^{-1} \circ \boldsymbol{\epsilon}^{*}+2 \boldsymbol{K}_{., . .} \circ \boldsymbol{K}^{-1} \circ \boldsymbol{\epsilon}^{*}+\boldsymbol{K}_{., .} \circ \boldsymbol{\epsilon}^{*} \circ \boldsymbol{K}^{-1}=0,
$$

temos, após longa e tediosa álgebra, que a função geradora de momentos de $S_{T}$, até ordem $n^{-1 / 2}$ e sob uma hipótese alternativa contígua, pode ser escrita na forma

$$
M(t)=(1-2 t)^{-\frac{1}{2}(p-q)} \exp \left(\frac{t}{1-2 t} \boldsymbol{\epsilon}^{\top} \boldsymbol{K}_{22.1} \boldsymbol{\epsilon}\right)\left[1+\frac{1}{\sqrt{n}}\left(A_{1} d+A_{2} d^{2}+A_{3} d^{3}\right)\right]+O\left(n^{-1}\right),
$$

em que $d=2 t /(1-2 t)$ e

$$
\begin{gathered}
A_{1}=-\frac{1}{4}\left(\boldsymbol{K}_{\ldots} \circ \boldsymbol{K}^{-1} \circ \boldsymbol{\epsilon}^{*}+4 \boldsymbol{K}_{., . .} \circ \boldsymbol{A} \circ \boldsymbol{\epsilon}^{*}+\boldsymbol{K}_{\ldots} \circ \boldsymbol{A} \circ \boldsymbol{\epsilon}^{*}+\boldsymbol{K}_{\ldots}\left(\circ \boldsymbol{\epsilon}^{*}\right)^{3}\right), \\
A_{2}=-\frac{1}{4}\left(\boldsymbol{K}_{\ldots} \circ \boldsymbol{K}^{-1} \circ \boldsymbol{\epsilon}^{*}-\boldsymbol{K}_{\ldots} \circ \boldsymbol{A} \circ \boldsymbol{\epsilon}^{*}-2 \boldsymbol{K}_{., . .}\left(\circ \boldsymbol{\epsilon}^{*}\right)^{3}\right) \quad \text { e } \quad A_{3}=-\frac{1}{12} \boldsymbol{K}_{\ldots}\left(\circ \boldsymbol{\epsilon}^{*}\right)^{3} .
\end{gathered}
$$




\section{Referências Bibliográficas}

Abell, M. L. \& Braselton, J. P. (1994). The Maple V Handbook. AP Professional, New York.

Abramowitz, M. \& Stegun, I. A. (1970). Handbook of Mathematical Functions. National Bureau of Standards, Washington.

Achcar, J. A. (1993). Inference for the Birnbaum-Saunders fatigue life model using bayesian methods. Computational Statistics and Data Analysis, 15, 367-380.

Aitchison, J. \& Silvey, S. D. (1958). Maximum-likelihood estimation of parameters subject to restraints. Annals of Mathematical Statistics, 29, 813-828.

Andrews, D. F. \& Stafford, J. E. (1993). Tools for the symbolic computation of asymptotic expansions. Journal of the Royal Statistical Society B, 55, 613-627.

Attfield, C. L. F. (1991). A Bartlett adjustment to the likelihood ratio test for homoskedasticity in the linear model. Economics Letters, 37, 119-123.

Barndorff-Nielsen, O. E. (1980). Conditionality resolutions. Biometrika, 67, 293-310.

Barndorff-Nielsen, O. E. (1983). On a formula for the distribution of the maximum likelihood estimator. Biometrika, 70, 343-365.

Barndorff-Nielsen, O. E. (1986). Inference on full and partial parameters based on the standadized signed log liklihood ratio. Biometrika, 73, 307-322.

Barndorff-Nielsen, O. E. (1990a). Approximate interval probabilities. Journal of the Royal Statistical Society $B$, 52, 485-496.

Barndorff-Nielsen, O. E. (1990b). Likelihood theory. In D. V. Hinkley, N. Reid, \& E. J. Snell, editors, Statistical Theory and Methods: In Honor to Sir David Cox. Chapman and Hall.

Barndorff-Nielsen, O. E. (1990c). A note on the standardized signed log likelihood ratio. Scandinavian Journal of Statistics, 17, 157-160.

Barndorff-Nielsen, O. E. (1991). Modified signed log likelihood ratio. Biometrika, 78, 557-563.

Barndorff-Nielsen, O. E. (1995). Stable and invariant adjusted profile likelihood and directed likelihood for curved exponential models. Biometrika, 82, 489-500.

Barndorff-Nielsen, O. E. \& Blaesild, P. (1986). A note on the calculation of Bartlett adjustments. Journal of the Royal Statistical Society B, 46, 483-495.

Barndorff-Nielsen, O. E. \& Cox, D. R. (1984). Bartlett adjustments to the likelihood ratio statistic and the distribution of the maximum likelihood estimator. Journal of the Royal Statistical Society B, 46, 484-495.

Barndorff-Nielsen, O. E. \& Cox, D. R. (1989). Asymptotics Techniques for Use in Statistics. Chapman and Hall, London. 
Barndorff-Nielsen, O. E. \& Cox, D. R. (1994). Inference and Asymptotics. Chapman and Hall, London.

Barndorff-Nielsen, O. E. \& Hall, P. (1988). On the level-error after Bartlett adjustment of the likelihood ratio statistic. Biometrika, 75, 374-378.

Barroso, L. P. \& Cordeiro, G. M. (2005). Bartlett corrections in heteroskedastic $t$ regression models. Statistics and Probability Letters, 75, 86-96.

Barroso, L. P., Cordeiro, G. M. \& Vasconcellos, K. L. P. (2001). Improved score tests for von mises regression models. Communications in Statistics - Theory and Methods, 30, 1295-1315.

Bartlett, M. S. (1937). Properties of sufficiency and statistical tests. Proceedings of the Royal Society A, 160, $268-282$.

Bartlett, M. S. (1947). Multivariate analysis. Journal of the Royal Statistical Society (Supplement), 9, $179-197$.

Bartlett, M. S. (1954). A note on the multiplying factors for various $\chi^{2}$ approximations. Journal of the Royal Statistical Society B, 16, 296-298.

Basu, D. (1964). Recovery of ancillary information. Sankhya A, 26, 223-226.

Bebu, I. \& Mathew, T. (2008). Comparing the means and variances of a bivariate log-normal distribution. Statistics in Medicine, 27, 2684-2696.

Bickel, P. J. \& Ghosh, J. K. (1990). A decomposition for the likelihood ratio and the Bartlett correction: a bayesian argument. Annals of Statistics, 18, 1-39.

Birnbaum, Z. W. \& Saunders, S. C. (1969a). A new family of life distributions. Journal of Applied Probability, 6, 319-327.

Birnbaum, Z. W. \& Saunders, S. C. (1969b). Estimation for a family of life distributions with applications to fatigue. Journal Applied Probability, 6, 328-347.

Botter, D. A. \& Cordeiro, G. M. (1997). Bartlett corrections for generalized linear models with dispersion covariates. Communications in Statistics - Theory and Methods, 26, 279-307.

Botter, D. A. \& Cordeiro, G. M. (1998). Improved estimators for generalized linear models with dispersion covariates. Journal of Statistical Computation and Simulation, 62, 91-104.

Box, G. E. P. (1949). A general distribution theory for a class of likelihood criteria. Biometrika, 36, 317-346.

Bracewell, R. (1999). The Fourier Transform and Its Applications. McGraw-Hill, New York, third edition.

Bradley, R. A. \& Gart, J. J. (1962). The asymptotic properties of ML estimators when sampling from associated populations. Biometrika, 46, 205-214.

Buse, A. (1982). The likelihood ratio, Wald, and lagrange multiplier tests: An expository note. The American Statistician, 36, 153-157.

Chanda, K. C. (1954). A note on the consistency and maxima of roots of likelihood equations. Biometrika, 41, 56-61.

Chandra, T. K. \& Ghosh, J. K. (1980). Valid asymptotic expasions for the likelihood ratio and other statistics under contiguos alternatives. Annals of the Institute of Statistical Mathematics A, 42, 170-184.

Chandra, T. K. \& Mukerjee, R. (1991). Bartlett-type modification for Rao's efficient score statistic. Journal of Multivariate Analysis, 36, 103-112. 
Chang, D. S. \& Tang, L. C. (1993). Reliability bounds and critical time for the Birnbaum-Saunders distribution. IEEE Transactions on Reliability, 42, 464-469.

Chang, D. S. \& Tang, L. C. (1994). Percentile bounds and tolerance limits for the Birnbaum-Saunders distribution. Communications in Statistics - Theory and Methods, 23, 2853-2863.

Chen, S. X. \& Hall, P. (1993). Smoothed empirical likelihood confidence intervals for quantiles. Annals of Statistics, 21, 1166-1181.

Chen, Y. \& Zhou, X. (2006). Interval estimates for the ratio and difference of two lognormal means. Statistics in Medicine, 25, 4099-4113.

Chesher, A. \& Smith, R. (1994). Bartlett corrections to likelihood ratio tests. Unpublished paper, Department of Economics, University of Bristol.

Cordeiro, G. M. (1982). Improved Likelihood Ratio Statistics for Generalized Linear Models. Ph.D. thesis, Imperial College of Science and Technology, University of London.

Cordeiro, G. M. (1983). Improved likelihood ratio statistics for generalized linear models. Journal of the Royal Statistical Society B, 45, 404-413.

Cordeiro, G. M. (1987). On the corrections to the likelihood ratio statistics. Biometrika, 74, 265-274.

Cordeiro, G. M. (1993a). General matrix formulae for computing Bartlett corrections. Statistics and Probability Letters, 16, 11-18.

Cordeiro, G. M. (1993b). Bartlett corrections and bias correction for two heteroscedastic regression models. Communications in Statistics - Theory and Methods, 22, 169-188.

Cordeiro, G. M. (2004). Corrected likelihood ratio tests in symmetric nonlinear regression models. Journal of Statistical Computation and Simulation, 74, 609-620.

Cordeiro, G. M. \& Ferrari, S. L. P. (1991). A modified score test statistic having chi-squared distribution to order $n^{-1}$. Biometrika, $\mathbf{7 8}, 573-582$.

Cordeiro, G. M. \& Ferrari, S. L. P. (1996). Bartlett-type corrections for some score tests in proper dispersion models. Communications in Statistics - Theory and Methods, 25, 29-48.

Cordeiro, G. M. \& McCulagh, P. (1991). Bias correction in generalized linear models. Journal of the Royal Statistical Society B, 53, 629-643.

Cordeiro, G. M. \& Paula, G. A. (1989). Improved likelihood ratio statistics for exponential family nonlinear models. Biometrika, $\mathbf{7 6}, 93-100$.

Cordeiro, G. M., Ferrari, S. L. P. \& Paula, G. A. (1993). Improved score tests for generalized linear models. Journal of the Royal Statistical Society B, 55, 661-674.

Cordeiro, G. M., Botter, D. A. \& Ferrari, S. L. P. (1994a). Nonnull asymptotic distributions of three classic criteria in generalised linear models. Biometrika, 81, 709-720.

Cordeiro, G. M., Paula, G. A. \& Botter, D. A. (1994b). Improved likelihood ratio tests for dispersion models. International Statistical Review, 62, 257-276.

Cordeiro, G. M., Cribari-Neto, F., Aubin, E. C. Q. \& Ferrari, S. L. P. (1995). Bartlett corrections for oneparameter exponential family models. Journal of Statistical Computation and Simulation, 53, 211-231.

Cordeiro, G. M., Botter, D. A., Barroso, L. P. \& Ferrari, S. L. P. (2003). Three corrected score tests for generalized linear models with dispersion covariates. Statistica Neerlandica, 57, 391-409. 
Cordeiro, G. M., Cysneiros, A. H. \& Cysneiros, F. J. (2006). Bartlett adjustments for overdispersed generalized linear models. Communications in Statistics - Theory and Methods, 35, 937-52.

Cox, D. R. (1971). The choice between alternative ancillary statistics. Journal of the Royal Statistical Society $B, 33,251-255$.

Cox, D. R. (1988). Some aspects of conditional and asymptotic inference: a review. Sankhyā A, 50, 314-337.

Cox, D. R. \& Hinkley, D. V. (1974). Theoretical Statistics. Chapman and Hall, London.

Cox, D. R. \& Reid, N. (1987). Parameter orthogonality and approximate conditional inference. Journal of the Royal Statistical Society B, 40, 1-39.

Cox, D. R. \& Reid, N. (1993). A note on the calculation of adjusted profile likelihood. Journal of the Royal Statistical Society B, 55, 467-471.

Cribari-Neto, F. \& Cordeiro, G. M. (1996). On Bartlett and Bartlett-type corrections. Econometric Reviews, 15, 339-367.

Cribari-Neto, F. \& Ferrari, S. L. P. (1995a). Bartlett-corrected tests for heteroskedastic linear models. Economics Letters, 48, 113-118.

Cribari-Neto, F. \& Ferrari, S. L. P. (1995b). Second order asymptotics for score tests in generalised linear models. Biometrika, 82, 426-432.

Cribari-Neto, F. \& Ferrari, S. L. P. (1995c). An improved lagrange multiplier test of heteroskedasticity. Communications in Statistics - Simulation and Computation, 24, 31-44.

Cribari-Neto, F. \& Zarkos, S. (1995). Improved test statistics for multivariate regression. Economics Letters, 49, 113-120.

Cribari-Neto, F. \& Zarkos, S. (2003). Econometric and statistical computing using Ox. Computational Economics, 21, 277-295.

Desmond, A. F. (1985). Stochastic models of failure in random environments. Canadian Journal of Statistics, 13, 171-183.

Desmond, A. F. (1986). On the relationship between two fatigue-life models. IEEE Transactions on Reliability, 35, 167-169.

Díaz-García, J. A. \& Leiva, V. (2005). A new family of life distributions based on the elliptically contoured distributions. Journal of Statistical Planning and Inference, 128, 445-457.

DiCiccio, T. J. (1984). On parameter transformations and interval estimation. Biometrika, 71, 477-485.

DiCiccio, T. J. \& Martin, M. A. (1993). Simple modifications for signed roots of likelihood ratio statistics. Journal of the Royal Statistical Society B, 55, 305-316.

DiCiccio, T. J., Hall, P. \& Romano, J. P. (1991). Empirical likelihood is Bartlett-correctable. Annals of Statistics, 19, 1053-1061.

Doornik, J. A. (2006). Ox: An Object-Oriented Matrix Language. Timberlake Consultants Press, London. 5th ed.

Engelhardt, M., Bain, L. J. \& Wright, F. T. (1981). Inferences on the parameters of the Birnbaum-Saunders fatigue life distribution based on maximum likelihood estimation. Technometrics, 23, 251-255. 
Ferrari, S. L. P. \& Arellano-Valle, R. B. (1993). Bartlett-corrected tests for regression models with student- $t$ independent errors. Technical Report Working Paper 9310, Department of Statistics, University of Sao Paulo.

Ferrari, S. L. P. \& Arellano-Valle, R. B. (1996). Modified likelihood ratio and score tests in regression models using the $t$ distribution. Brazilian Journal of Probability and Statistics, 10, 15-33.

Ferrari, S. L. P. \& Cordeiro, G. M. (1994). Matrix formulae for computing improved score tests. Journal of Statistical Computation and Simulation, 49, 196-206.

Ferrari, S. L. P. \& Cordeiro, G. M. (1996). Corrected score tests for exponential family nonlinear models. Statistics and Probability Letters, 26, 7-12.

Ferrari, S. L. P. \& Uribe-Opazo, M. A. (2001). Corrected likelihood ratio tests in a class of symmetric linear regression models. Brazilian Journal of Probability and Statistics, 15, 49-67.

Ferrari, S. L. P., Botter, D. A. \& Cribari-Neto, F. (1997). Local power of three classic criteria in generalised linear models with unknown dispersion. Biometrika, 84, 482-485.

Ferrari, S. L. P., Cordeiro, G. M. \& Cribari-Neto, F. (2001). Higher order asymptotic refinements for score tests in proper dispersion models. Journal of Statistical Planning and Inference, 97, 177-190.

Fisher, R. A. (1925). Theory of statistical estimation. Proceedings of the Cambridge Philosophical Society, 22, 700-725.

Fisher, R. A. (1934). Two new properties ok mathematical likelihood. Proceedings of the Royal Philosophical Society A, 144, 285-307.

Fisher, R. A. (1935). The logic of indutive inference. Journal of the Royal Statistical Society B, 98, 39-54.

Fraser, D. A. S. (1990). Tail probabilities from observed likelihoods. Biometrika, 77, 65-76.

Fraser, D. A. S. \& Reid, N. (1995). Ancillaries and third-order significance. Utilitas Mathematica, 47, 33-53.

Fraser, D. A. S. \& Reid, N. (1999). Ancillary information for statistical inference. Proceedings of a CRM Symposium on Empirical Bayes and Likelihood Inference. eds. E. Ahmad and N. Reid. New York: SpringerVerlag.

Fraser, D. A. S., Reid, N. \& Wu, J. (1999a). A simple general formula for tail probabilities for frequentist and bayesian inference. Biometrika, 86, 249-264.

Fraser, D. A. S., Wong, A. \& Wu, J. (1999b). Regression analysis, nonlinear or nonnormal: Simple and accurate p values from likelihood analysis. Journal of the American Statistical Association, 94, 1286-1295.

Frydenberg, M. \& Jensen, J. L. (1989). Is the 'improved likelihood statistic' really improved in the discrete case? Biometrika, 76, 655-661.

Galea, M., Leiva, V. \& Paula, G. A. (2004). Influence diagnostics in log-Birnbaum-Saunders regression models. Journal of Applied Statistics, 31, 1049-1064.

Gamerman, D. \& Lopes, E. F. (2006). Markov Chain Monte Carlo: Stochastic Simulation for Bayesian Inference. Chapman and Hall, London, second edition.

Ghosh, M., Reid, N. \& Fraser, D. A. S. (2009). Ancillary statistics: a review. Statistica Sinica. A aparecer.

Gradshteyn, I. S. \& Ryzhik, I. M. (2007). Table of Integrals, Series, and Products. Academic Press, New York.

Guiraud, P., Leiva, V. \& Fierro, R. (2009). A non-central version of the Birnbaum-Saunders distribution for reliability analysis. IEEE Transactions on Reliability, 58, 152-160. 
Harris, P. (1985). An asymptotic expansion for the null distribution of the efficient score statistic. Biometrika, 72, 653-659.

Harris, P. (1986). A note on Bartlett adjustments to likelihood ratio tests. Biometrika, 73, 735-737.

Harris, P. \& Peers, H. W. (1980). The power of the efficient score test statistic. Biometrika, 67, 525-529.

Hayakawa, T. (1975). The likelihood ratio criterion for a composite hypothesis under a local alternative. Biometrika, 37, 95-108.

Hayakawa, T. (1977). The likelihood ratio criterion and the asymptotic expansion of its distribution. Annals of the Institute of Statistical Mathematics A, 29, 359-378.

Hayakawa, T. (1987). Correction to 'The likelihood ratio criterion and the asymptotic expansion of its distribution'. Annals of the Institute of Statistical Mathematics A, 39, 681.

Hayakawa, T. \& Puri, M. L. (1985). Asymptotic expansions of the distributions of some test statistics. Annals of the Institute of Statistical Mathematics A, 62, 451-460.

He, H. \& Severini, T. A. (2007). Higher-order asymptotic normality of approximations to the modified signed likelihood ratio statistic for regular models. Annals of Statistics, 35, 2054-2074.

Hill, G. W. \& Davis, A. W. (1968). Generalized asymptotic expansions of Cornish-Fisher type. The Annals of Mathematical Statistics, 39, 1246-1273.

Jensen, J. L. (1986). Similar tests and the standardized log-likelihood ratio statistic. Biometrika, 73, 567-572.

Jensen, J. L. (1987). Standardized log-likelihood ratio statistics for mixtures of discrete and continuous observations. Annals of Statistics, 15, 314-324.

Jensen, J. L. (1992). The modified signed likelihood ratio and saddlepoint approximations. Biometrika, 79, 693-703.

Jensen, J. L. (1993). A historical sketch and some new results on the improved log likelihood ratio statistics. Scandinavian Journal of Statistics, 93, 1-15.

Jensen, J. L. (1995). Saddlepoint approximations. Oxford University Press, Oxford.

Jensen, J. L. (1997). A simple derivation of $r^{*}$ for curved exponential families. Scandinavian Journal of Statistics, 24, 33-46.

Jim, X. \& Kawczak, J. (2003). Birnbaum-Saunders and lognormal kernel estimators for modelling durations in high frequency financial data. Annals of Economics and Finance, 4, 103-124.

Johnson, N., Kotz, S. \& Balakrishnan, N. (1995). Continuous Univariate Distributions - Volume 2. John Wiley and Sons, New York. 2th ed.

Jorgensen, B. (1987). Small dispersion asymptotics. Brazilian Journal of Probability and Statistics, 1, 59-90.

Jorgensen, B. (1997). The theory of dispersion models. Chapman and Hall, London.

Kendall, M. G. \& Stuart, A. (1977). The Advanced Theory of Statistics. Griffin, London, fourth edition.

Kundu, D., Kannan, N. \& Balakrishnan, N. (2008). On the function of Birnbaum-Saunders distribution and associated inference. Computational Statistics and Data Analysis, 52, 2692-2702.

Lagos, B. M. \& Morettin, P. A. (2004). Improved of the likelihood ratio test statistic in ARMA models. Journal of Time Series Analysis, 25, 83-101. 
Lagos, B. M., Morettin, P. A. \& Barroso, L. P. (2009). On Bartlett-type corrections of the score test statistics for autoregressive moving average models. Brazilian Journal of Probability and Statistics. A aparecer.

Larsen, P. V., Blaesild, P. \& Sorensen, M. K. (2002). Improved likelihood ratio tests on the von Mises-Fisher distribution. Biometrika, 89, 947-951.

Lawley, D. (1956). A general method for approximating to the distribution of likelihood ratio criteria. Biometrika, 43, 295-303.

Lehmann, E. L. (1999). Elements of Large-Sample Theory. Springer-Verlag, New York.

Lehmann, E. L. \& Casella, E. (1998). Theory of Point Estimation. Springer-Verlag, New York.

Leiva, V., Barros, M. K., Paula, G. A. \& Galea, M. (2007). Influence diagnostics in log-Birnbaum-Saunders regression models with censored data. Computational Statistics and Data Analysis, 51, 5694-5707.

Leiva, V., Riquelme, M., Balakrishnan, N. \& Sanhueza, A. (2008). Lifetime analysis based on the generalized Birnbaum-Saunders distribution. Computational Statistics and Data Analysis, 69, 533-539.

Lemonte, A. J. \& Cordeiro, G. M. (2009). Birnbaum-Saunders nonlinear regression models. Computational Statistics and Data Analysis, 53, 4441-4452.

Lemonte, A. J., Cribari-Neto, F. \& Vasconcellos, K. L. P. (2007). Improved statistical inference for the twoparameter Birnbaum-Saunders distribution. Computational Statistics and Data Analysis, 51, 4656-4681.

Lemonte, A. J., Simas, A. B. \& Cribari-Neto, F. (2008). Bootstrap-based improved estimators for the twoparameter Birnbaum-Saunders distribution. Journal of Statistical Computation and Simulation, 78, 37-49.

Lepadatu, D., Kobi, A., Hambli, R. \& Barreau, A. (2005). Lifetime multiple response optimization of metal extrusion die. Proceedings of the Annual Reliability and Maintainability Symposium. 37-42.

Lugannani, R. \& Rice, S. (1980). Saddlepoint approximation for the distribution of the sum of independent random variables. Advances in Applied Probability, 12, 475-490.

McCarter, K. S. (1999). Estimation and prediction for the Birnbaum-Saunders distribution using Type-II censored samples, with a comparison to the Inverse Gaussian Distribution. Ph.D. thesis, Kansas State University.

McCullagh, P. (1984). Local sufficiency. Biometrika, 71, 233-244.

McCullagh, P. (1987). Tensor Methods in Statistics. Chapman and Hall, London.

McCullagh, P. \& Cox, D. R. (1986). Invariants and the likelihood ratio statistic. Annals of Statistics, 14, 1419-1430.

McCullagh, P. \& Nelder, J. A. (1989). Generalized linear models. Chapman and Hall, London. 2 th ed.

Mukerjee, R. (1992). Parametric orthogonality and a Bartlett-type modification for Rao's statistic in the presence of a nuisance parameter. Statistics and Probability Letters, 13, 397-400.

Nelson, W. (1990). Accelerated Testing, Statistical Models, Test Plans and Data Analisys. John Wiley and Sons, New York.

Ng, H. K. T., Kundu, D. \& Balakrishnan, N. (2003). Modifiel moment estimation for the two-parameter Birnbaum-Saunders distribution. Computational Statistics and Data Analysis, 43, 283-298.

Owen, A. B. (1988). Empirical likelihood confidence intervals for a single functional. Biometrika, 75, $237-249$.

Owen, A. B. (1990). Empirical likelihood ratio confidence regions. Annals of Statistics, 18, 90-120. 
Owen, W. J. \& Padgett, W. J. (1999). Accelerated test models for system strength based on BirnbaumSaunders distributions. Lifetime Data Analysis, 5, 133-147.

Owen, W. J. \& Padgett, W. J. (2000). A Birnbaum-Saunders accelerated life model. IEEE Transactions on Reliability, 49, 224-229.

Pace, L. \& Salvan, A. (1997). Principles of statistical inference. World Scientific, Singapore.

Peers, H. W. (1971). Likelihood ratio and associated test criteria. Biometrika, 58, 577-587.

Pena, E. A., Rohatgi, V. K. \& Szekely, G. J. (1992). On the non-existence of ancillary statistics. Statistics and Probability Letter, 15, 357-360.

Press, W. H., Teulosky, S. A., Vetterling, W. T. \& Flannery, B. P. (1992). Numerical Recipes in C: The Art of Scientific Computing. Prentice Hall, London. 2th ed.

R Development Core Team (2008). R: A Language and Environment for Statistical Computing. R Foundation for Statistical Computing, Vienna, Austria. ISBN 3-900051-07-0.

Rao, C. R. (1948). Large sample tests of statistical hypotheses concerning several parameters with applications to problens of estimation. Proceedings of the Cambridge Philosophical Society, 44, 50-57.

Rao, C. R. (1973). Linear Statistical Inference and Its Applications. John Wiley and Sons, New York.

Rao, C. R. (2005). Score test: historical review and recent developments. In N. Balakrishnan, N. Kannan, \& H. N. Nagaraja, editors, Advances in Ranking and Selection, Multiple Comparisons, and Reliability. Birkhuser, Boston.

Rao, C. R. \& Mitra, S. K. (1971). Generalized Inverse of Matrices and Its Applications. Wiley, New York.

Rao, C. R. \& Mukerjee, R. (1995). Comparison of Bartlett-type adjustments for the efficient score statistic. Journal of Statistical Planning and Inference, 46, 137-146.

Reid, N. (1995). The roles of conditioning in inference. Statistical Science, 10, 138-157.

Reid, N. (1996). Likelihood and higher-order approximations to tail areas: a review and annotated bibliography. Canadian Journal of Statistics, 24, 141-166.

Reid, N. (2003). Asymptotics and the theory of inference. Annals of Statistics, 31, 1695-1731.

Rieck, J. R. (1989). Statistical Analysis for the Birnbaum-Saunders Fatigue Life Distribution. Ph.D. thesis, Clemson University, Dept. of Mathematical Sciences.

Rieck, J. R. (1995). Parametric estimation for the Birnbaum-Saunders distribution based on symmetrically censored samples. Communications in Statistics - Theory and Methods, 24, 1721-1736.

Rieck, J. R. (1999). A moment-generating function with application to the Birnbaum-Saunders distribution. Communications in Statistics - Theory and Methods, 28, 2213-2222.

Rieck, J. R. \& Nedelman, J. R. (1991). A log-linear model for the Birnbaum-Saunders distribution. Technometrics, 33, 51-60.

Saunders, S. C. (1974). A family of random variables closed under reciprocation. Journal of the American Statistical Association, 69, 533-539.

Severini, T. A. (1998). An approximation to the modified profile likelihood function. Biometrika, 85, $403-411$.

Severini, T. A. (1999). An empirical adjustment to the likelihood ratio statistic. Biometrika, 86, $235-247$. 
Severini, T. A. (2000). Likelihood methods in statistics. Oxford University Press, New York.

Silvey, S. D. (1959). The lagrangian multipler test. Annals of Mathematical Statistics, 30, 389-407.

Skovgaard, I. M. (1990). On the density of minimum contrast estimators. Annals of Statistics, 18, 779-789.

Skovgaard, I. M. (1996). An explicit large-deviance approximation to one-parameter tests. Bernoulli, 2, $145-165$.

Skovgaard, I. M. (2001). Likelihood asymptotics. Scandinavian Journal of Statistics, 28, 3-32.

Stafford, J. E. \& Andrews, D. F. (1993). A symbolic algorithm for studying adjustments to the profile likelihood. Biometrika, 80, 715-730.

Taniguchi, M. (1991). Third-order asymptotic properties of a class of test statistics under a local alternative. Journal of Multivariate Analysis, 37, 223-238.

Terrell, G. R. (2002). The gradient statistic. Computing Science and Statistics, 34, 206-215.

Tisionas, E. G. (2001). Bayesian inference in Birnbaum-Saunders regression. Communications in Statistics Theory and Methods, 30, 179-193.

Tu, D., Chen, J. \& Shi, P. Wu, Y. (2005). A Bartlett type correction for Rao's score test in Cox regression model. Sankhyā, 67, 722-735.

Uribe-Opazo, M. A., Ferrari, S. L. P. \& Cordeiro, G. M. (2008). Improved score tests in symmetric linear regression models. Communications in Statistics - Theory and Methods, 37, 261-276.

Vilca-Labra, F. E. \& Leiva, V. (2006). A new fatigue life model based on the family of skew-elliptical distributions. Communications in Statistics - Theory and Methods, 35, 229-244.

Wald, A. (1943). Tests of statistical hypotesis concerning several parameters when the number of observations is large. Trans. Ann. Math. Soc., 54, 426-482.

Wang, Z., Desmond, A. F. \& Lu, X. (2006). Modified censored moment estimation for the two-parameter Birnbaum-Saunders distribution. Computational Statistics and Data Analysis, 50, 1033-1051.

Wei, B. C. (1998). Exponential Family Nonlinear Models. Springer, Singapore.

Wilks, S. S. (1938). The large-sample distribution of the likelihood ratio for testing composite hypotesis. Annals of Mathematical Statistics, 9, 60-62.

Wong, A. C. M. \& Wu, J. (2002). Small sample asymptotic inference for the coefficient of variation: normal and nonnormal models. Journal of Statistical Planning and Inference, 104, 73-82.

Wong, M. Y. (1991). Bartlett adjustment to the likelihood ratio statistic for testing several slopes. Biometrika, 78, 221-224.

Wu, J. \& Jiang, G. (2007). Confidence intervals of effect size for paired studies. Biometrical Journal, 49, $765-773$.

Wu, J. \& Wong, A. C. M. (2004). Improved interval estimation for the two-parameter Birnbaum-Saunders distribution. Computational Statistics and Data Analysis, 47, 809-821.

Wu, J., Jiang, G., Wong, A. C. M. \& Sun, X. (2002). Likelihood analysis for the ratio of means of two independent log-normal distributions. Biometrics, 58, 463-469.

Wu, J., Wong, A. C. M. \& Jiang, G. (2003). Likelihood-based confidence intervals for a log-normal mean. Statistics in Medicine, 22, 1849-1860. 
Wu, J., Jiang, G. \& Wei, W. (2006a). Confidence intervals of effect size in randomized comparative parallelgroup studies. Statistics in Medicine, 25, 639-651.

Wu, J., Wong, A. C. M. \& Wei, W. (2006b). Interval estimation of the mean response in a log-regression model. Statistics in Medicine, 25, 2125-2135.

Xi, F. C. \& Wei, B. C. (2007). Diagnostics analysis for log-Birnbaum-Saunders regression models. Computational Statistics and Data Analysis, 51, 4692-4706.

Xu, A. \& Tang, Y. (2010). Reference analysis for Birnbaum-Saunders distribution. Computational Statistics and Data Analysis, 54, 185-192. 